\title{
Enabling Technology for Monitoring \& Predicting Gas Turbine Health $\&$ Performance in IGCC Powerplants
}

\section{Final Technical Report}

Period of Performance

Beginning March 1, 2003

Ending September 30, 2005

Submitted To: US Department of Energy

National Energy Technology Laboratory

Morgantown, WV 26507-0880

Submitted By: GE Energy

Schenectady, NY 12345

December 1, 2005

DOE Cooperative Agreement No. DE-FC26-03NT41448 


\section{DISCLAIMER}

This report was prepared as an account of work sponsored by an agency of the United States Government. Neither the United States Government nor any agency thereof, nor any of their employees, makes any warranty, express or implied, or assumes any legal liability or responsibility for the accuracy, completeness, or usefulness of any information, apparatus, product, or process disclosed, or represents that its use would not infringe privately owned rights. Reference herein to any specific commercial product, process, or service by trade name, trademark, manufacturer, or otherwise does not necessarily constitute or imply its endorsement, recommendation, or favoring by the United States Government or any agency thereof. The views and opinions of authors expressed herein do not necessarily state or reflect those of the United States Government or any agency thereof. 


\section{ABSTRACT}

The "Enabling \& Information Technology To Increase RAM for Advanced Powerplants" program, by DOE request, was re-directed, de-scoped to two tasks, shortened to a 2-year period of performance, and refocused to develop, validate and accelerate the commercial use of enabling materials technologies and sensors for coal/IGCC powerplants. The new program was re-titled "Enabling Technology for Monitoring \& Predicting Gas Turbine Health \& Performance in IGCC Powerplants". This final report summarizes the work accomplished from March 1, 2003 to March 31, 2004 on the four original tasks, and the work accomplished from April 1, 2004 to July 30, 2005 on the two re-directed tasks. The program Tasks are summarized below:

Task 1 - IGCC Environmental Impact on high Temperature Materials:

The first task was refocused to address IGCC environmental impacts on high temperature materials used in gas turbines. This task screened material performance and quantified the effects of high temperature erosion and corrosion of hot gas path materials in coal/IGCC applications. The materials of interest included those in current service as well as advanced, high-performance alloys and coatings.

Task 2 - Material In-Service Health Monitoring:

The second task was reduced in scope to demonstrate new technologies to determine the inservice health of advanced technology coal/IGCC powerplants. The task focused on two critical sensing needs for advanced coal/IGCC gas turbines: 1) Fuel Quality Sensor to rapidly determine the fuel heating value for more precise control of the gas turbine, and detection of fuel impurities that could lead to rapid component degradation. 2) Infra-Red Pyrometer to continuously measure the temperature of gas turbine buckets, nozzles, and combustor hardware.

Task 3 - Advanced Methods for Combustion Monitoring and Control:

The third task was originally to develop and validate advanced monitoring and control methods for coal/IGCC gas turbine combustion systems. This task was refocused to address pre-mixed combustion phenomenon for IGCC applications. The work effort on this task was shifted to another joint GE Energy/DOE-NETL program investigation, High Hydrogen Pre-mixer Designs, as of April 1, 2004.

Task 4 - Information Technology (IT) Integration:

The fourth task was originally to demonstrate Information Technology (IT) tools for advanced technology coal/IGCC powerplant condition assessment and condition based maintenance. The task focused on development of GateCycle ${ }^{\mathrm{TM}}$ software to model complete-plant IGCC systems, and the Universal On-Site Monitor (UOSM) to collect and integrate data from multiple condition monitoring applications at a power plant. The work on this task was stopped as of April 1, 2004. 


\section{TABLE OF CONTENTS}

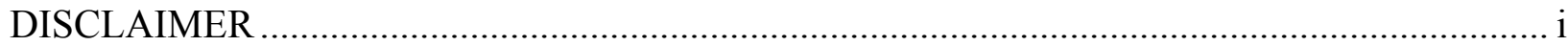

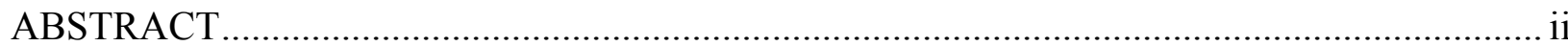

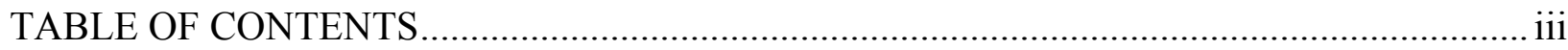

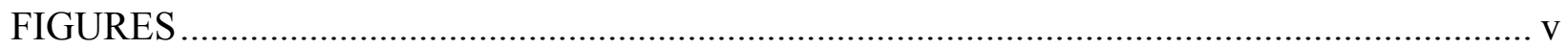

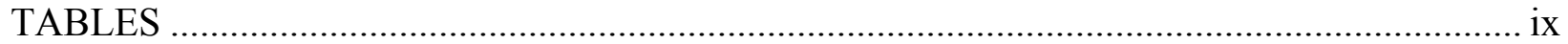

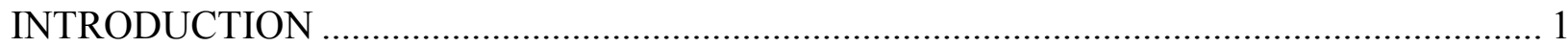

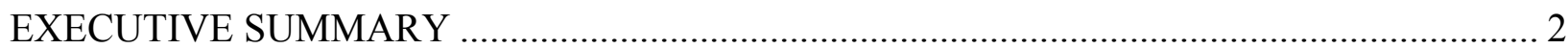

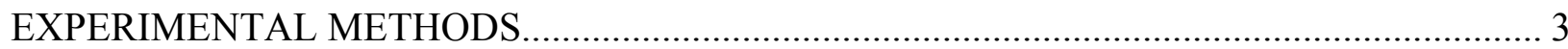

Task 1 - IGCC Environmental Impact on High Temperature Materials:................................ 3

Sub-Task 1.1 - High Temperature Erosion/Corrosion Data Collection ............................... 3

Sub-Task 1.2 - High Temperature Erosion/Corrosion Mechanism Quantification ............ 12

Task 2 - Material In-Service Health Monitoring:........................................................... 13

Subtask 2.1 - Define Coal IGCC Power Plant Requirements ......................................... 13

Subtask 2.2 - Sensor Capability Studies..................................................................... 13

Subtask 2.3 - IR Pyrometer for Condition Based Maintenance ......................................... 18

Subtask 2.4 - Field Deployment and System Validation .............................................. 25

Task 3 - Advanced Methods for Combustion Monitoring and Control: ................................ 28

Sub-Task 3.1 - Physics-based Combustion Modeling ................................................... 28

Sub-Task 3.2 - Development of Control Methods ....................................................... 30

Task 4 - Information Technology (IT) Integration: ........................................................ 33

Sub-Task 4.1 - Performance Modeling for Coal/IGCC Powerplants................................ 33

Sub-Task 4.2 - Coal/IGCC Powerplant Data Integration.............................................. 35

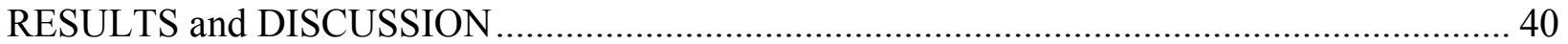

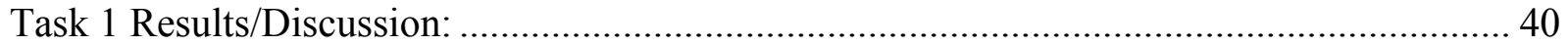

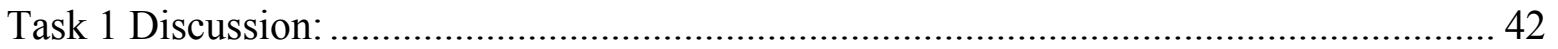

Sub-Task 1.1 - High Temperature Erosion/Corrosion Data Collection ............................. 42

Sub-Task 1.2 - High Temperature Erosion/Corrosion Mechanism Quantification ............. 54

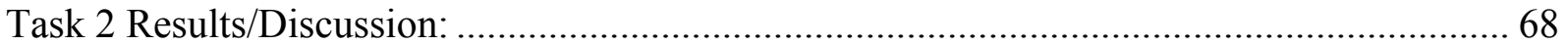

Subtask 2.1 - Define Coal IGCC Power Plant Requirements ......................................... 69

Subtask 2.2 - Sensor Capability Studies...................................................................... 70

Subtask 2.3 - IR Pyrometer for Condition Based Maintenance ....................................... 80

Subtask 2.4 - Field Deployment and System Validation ......................................... 85

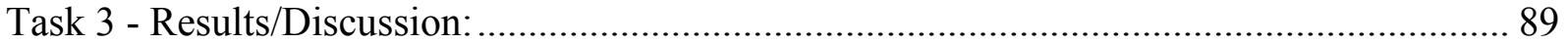

Sub-Task 3.1 - Physics-based Combustion Modeling .................................................. 89

Sub-Task 3.2 - Development of Control Methods ....................................................... 103

Task 4 - Results/Discussion: .......................................................................................... 107

Sub-Task 4.1 - Performance Modeling for Coal/IGCC Powerplants............................... 107

Sub-Task 4.2 - Coal/IGCC Powerplant Data Integration................................................ 109

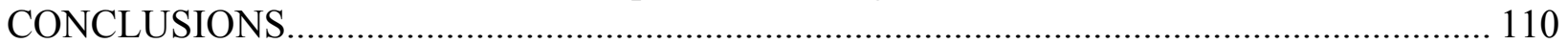

Task 1 - IGCC Environmental Impact on High Temperature Materials .............................. 110

Task 2 - Material In-Service Health Monitoring.......................................................... 112

Task 3 - Advanced Methods for Combustion Modeling ................................................ 112

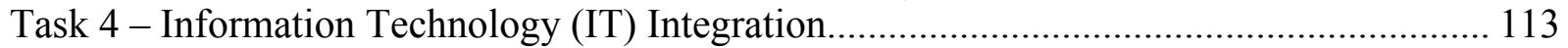

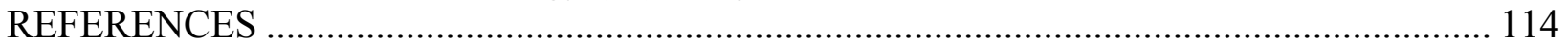

Cooperative Agreement No: $\quad$ iii

DE-FC26-03NT41448 
Appendix A: Photographs of a typical specimen of each of the nine materials, as-received Appendix B: Photographs of specimens following exposure to different test environments Appendix C: Photomicrographs of transverse cross-sections of specimens following exposure to different environments

Appendix D: Georgia Institute of Technology final technical report 


\title{
FIGURES
}

Figure 1: Test Set-Up Showing Furnace with Reactor Tube, Temperature Controller, and Gas

Bubbler at Exit

Figure 2: Set-Up Schematic for High-Temperature Tests in Air........................................... 6

Figure 3: Set-Up Schematic For High-Temperature Tests In Dry $\mathrm{H}_{2} \mathrm{~S}$ Environment .................. 7

Figure 4: Set-Up Schematic for High-Temperature Tests in Wet $\mathrm{H}_{2} \mathrm{~S}$ Environment ................... 7

Figure 5: Drawing of Holder for Specimens, showing (a) Identification Code, and (b) Specimen

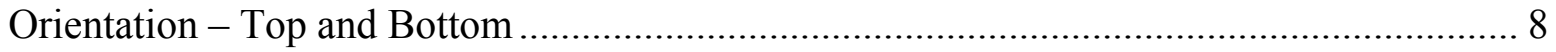

Figure 6: Photograph of Alumina Holder with Specimens Before Exposure............................ 9

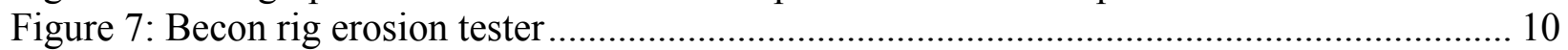

Figure 8: TECO Polk IGCC sample locations ................................................................... 10

Figure 9: Fuel Lower Heating Value (LHV) sensor fixture ............................................... 14

Figure 10: Dimensioned drawings of the sensor fixture ................................................ 15

Figure 11: Laboratory based testing station showing four mass flow controllers (MFCs) at the top

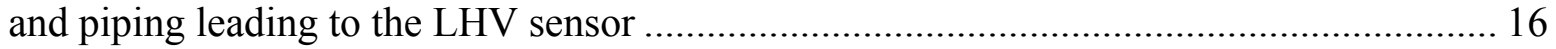

Figure 12: Fishbone diagram of factors that effect reliability and life of the LHV sensor hotplates

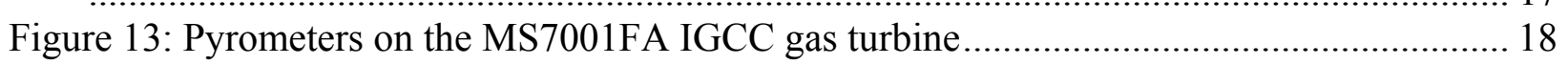

Figure 14: Alignment flange positioned on black body furnace during calibration .................... 19

Figure 15: Optical fiber and alignment flange installed on turbine ....................................... 19

Figure 16: Modified Pyrometer Line-Of-Sight................................................................ 20

Figure 17: Pyrometer lens fogging error measurement setup .............................................. 21

Figure 18: Pyrometer lens fogging error transfer function ............................................... 22

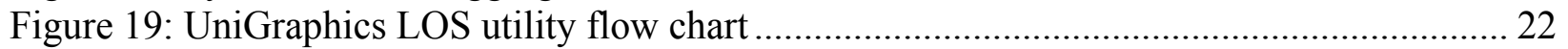

Figure 20: Plenum flow chamber with bucket affixed to the top ........................................... 23

Figure 21: Flow chart of the bucket reliability management strategy using pyrometer

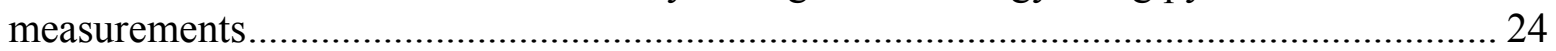

Figure 22: Sensor fixture with orifices and related plumbing ............................................ 25

Figure 23: Fuel LHV sensor installation at the TECO Polk 1 station. Shown are sensor enclosure

(left), data acquisition enclosure (middle) and data acquisition computer (right).............. 26

Figure 24: Schematic diagram of single-nozzle combustion rig ............................................ 28

Figure 25: Fuel premixer used in single-nozzle experiments .............................................. 29

Figure 26: Fast response fuel valve used for control experiments........................................... 30

Figure 27: Fast response fuel actuator coupled to DLN 2 premixer..................................... 31

Figure 28: Hot film probe positioned near premixer fuel orifice........................................... 32

Figure 29: TECO Polk 1 IGCC Heat Balance Diagram and Process Variables .......................... 34

Figure 30: TECO Polk 1 IGCC Power Plant GateCycle Model .............................................. 34

Figure 31: Universal On-Site Monitor hardware (typical) .................................................... 37

Figure 32: Universal On-Site Monitor hardware at TECO Polk 1 Powerplant .......................... 38

Figure 33: Universal On-Site Monitor Data Network at Polk 1 Powerplant (new hardware and

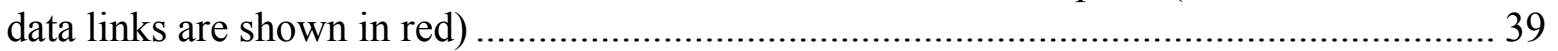

Figure 34: Photograph of Alumina Holder with Specimens After 300-Hour Exposure to Wet $\mathrm{H}_{2} \mathrm{~S}$

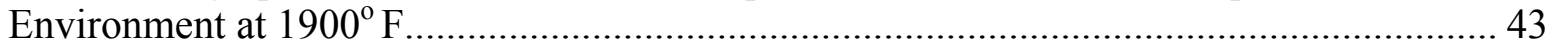

Figure 35: Nimonic-263 + CoNiCrAlY bondcoat + air plasma sprayed TBC specimens after

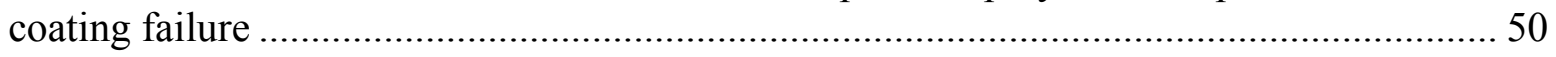

Cooperative Agreement No: V

DE-FC26-03NT41448 
Figure 36: GTD111 + CoNiCrAlY bondcoat + air plasma sprayed TBC specimens after coating failure 50

Figure 37: Rene N5 + NiCrAlY bondcoat + air plasma sprayed TBC specimens after coating failure

Figure 38: Cross -sectional micrograph of GT-29+ coating on TECO Polk 1 first stage bucket after approximately 24000 hours of service. Typical surface reactions were observed in this region of the bucket.

Figure 39: Cross -sectional micrograph of GT-29+ coating on TECO Polk 1 first stage bucket after approximately 24000 hours of service. Atypical surface reactions were observed in this region of the bucket...

Figure 40: Cross -sectional micrograph of GT-29+ coating on TECO Polk 1 first stage bucket after approximately 24000 hours of service. Higher magnification of the same region as shown in Figure 39 (see text for explanation).

Figure 41: Optical macrograph of the suction side of the airfoil (bucket tip towards top and leading edge on right). The circles indicate the locations at which analyses were performed.

Figure 42: Optical macrograph of the pressure side of the airfoil (bucket tip towards bottom and leading edge on right). The circles indicate the locations at which analyses were performed.

Figure 43: EDS (25kV) spectra for the following locations indicated in Figure 41 and Figure 42:

(i) 1, (ii) 2 (similar spectra for locations 3 and 4), (iii) 5, (iv) 6, (v) 7 (similar spectrum for location 9), (vi) 8 and (vii) 10 .

Figure 44: High magnification micrographs of the airfoil surface at Figure 42, location 7

(pressure side) by (a) backscatter SEM and (b) EDS map for iron

Figure 45: Optical micrographs of a cross-section of the CoCrAlY coating. Sample taken from the suction side of the leading edge, about 3.25" from tip, etched for about 15 seconds in Kallings etch $(5 \mathrm{gm} \mathrm{CuCl} 2,100 \mathrm{ml} \mathrm{HCL}, 100 \mathrm{ml}$ Ethanol). Dark spots are pits that form immediately after contact with etch.

Figure 46: EBSD micrographs of the CoCrAlY coating, (a) Euler orientation map (colors indicate crystal orientation) and (b) grain boundary misorientation map: $\operatorname{Red}=1^{\circ}<\theta<3^{\circ}$, Yellow $=3^{\circ}<\theta<10^{\circ}$, Black $=10^{\circ}<\theta<60^{\circ}$, Green $=$ Twin $\left(60^{\circ}\right)$

Figure 47: EDS maps for aluminum at (a) suction side and (b) pressure side by the trailing edge and (c) suction side and (d) pressure side by the leading edge (Substrate to the right of image, except in (a), where it is to the left)

Figure 48: Micrographs of craze cracks found along the suction side of the leading edge ( $\sim 3.25$ " from tip) obtained by (a) backscatter SEM and (b) EDS elemental maps..... 62

Figure 49: EBSD analysis of the oxide structure of a craze crack on the pressure side 0.5 " from the leading edge, about 3.25 " from tip.

Figure 50: Evidence of sulfur-rich precipitates at the leading edge, about 3.25" from tip, obtained by means of (a) backscatter SEM and (b) EDS map 63

Figure 51: EBSD analyses of sulfur-rich precipitates. (a) and (b) indicate the area sampled in (c), located on the leading edge, about 3.25 " from the tip. 64

Figure 52: Summary of a series of EDS analyses of a cross-section of the leading edge 3.5" from tip . 65

Figure 53: Backscatter SEM images of the airfoil surface near area 10 of Figure 42 with summary of EDS analyses ( 0.6 " from tip and 0.5 " from trailing edge). 66 
Figure 54: SEM backscatter micrograph of a trailing edge cross-section of the area analyzed in

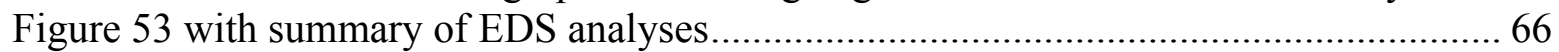

Figure 55: (a) Mass gain and (b) mass gain squared histories of parabolic weight gain of DS

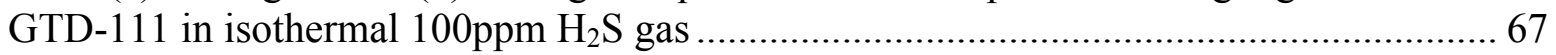

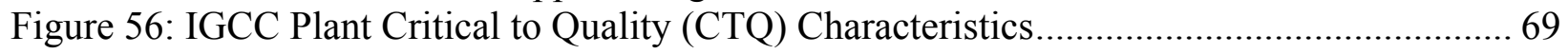

Figure 57: IGCC Gas Turbine Critical to Quality (CTQ) Characteristics ...................................... 69

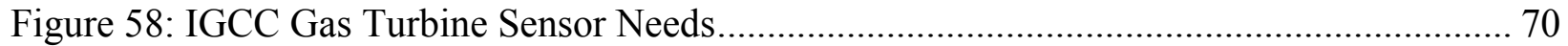

Figure 59: Screening DoE (design of experiment) to determine flows for which combustion

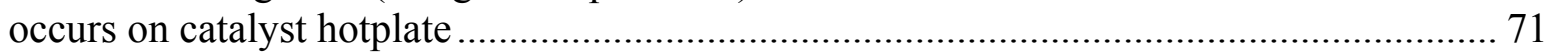

Figure 60: LHV sensor power as a function of lambda (fuel to air mixture) ................................ 71

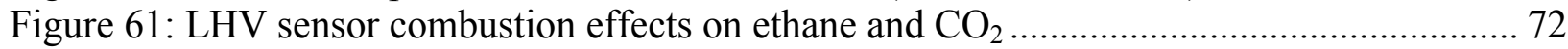

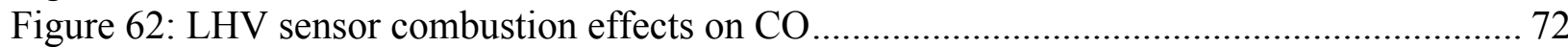

Figure 63: LHV sensor signal responding to varying concentrations of ethane............................ 73

Figure 64: Percent change of natural gas constituents by concentration...................................... 74

Figure 65: Finite element model of an optimized heater in the sensor hotplate. This design

shows a significant increase in the area of uniform heating................................................ 75

Figure 66: Sensor output as a function of hotplate temperature for two different size catalyst

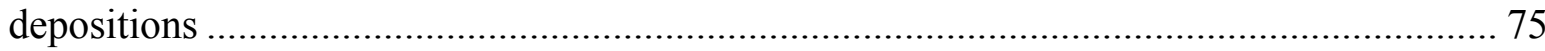

Figure 67: Sensor response for gas mixture with varying fuel:air mixtures. The left plot is for a

$10 \% \mathrm{Pt}$ catalyst and the right plot is for a 1\% Pt catalyst. ................................................. 76

Figure 68: Sensor output versus time for Syngas fuel. Each plateau corresponds to a change in

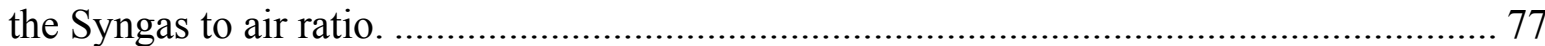

Figure 69: Micrographs of back and front of hotplate SiN membrane with Alumina reference coating. Note the wrinkled appeared of the backside due to conflicting stress between hotplate layers. .......................................................................................................... 77

Figure 70: Infra-Red temperature plot of reference (left) and catalyst (right) hotplate during operation. Temperature scale is in degrees $\mathrm{C}$. Flow conditions: $\Phi=0.95 ; \mathrm{T}_{\text {cat } \_ \text {average }}=400 \mathrm{C}$,

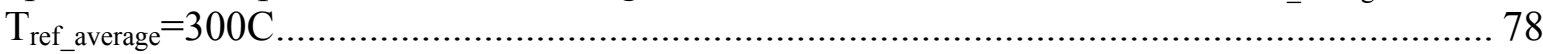

Figure 71: Stress measurements on hotplate heater layer as a function of temperature. Blue is repeat number 1, pink line is repeat number two............................................................ 79

Figure 72: Stress measurements on hotplate capping layer as a function of temperature. Run

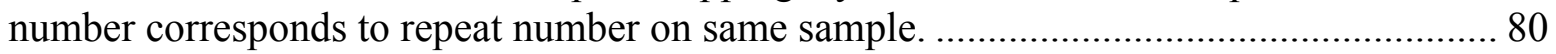

Figure 73: Spectra corrected for spectrometer efficiency ....................................................... 82

Figure 74: Average wheel emissivity of the buckets back-calculated from the temperature difference between the Land pyrometer and the spectrometer measurements ...................... 83

Figure 75: Infra-red images of the leading edge of pressure side of bucket................................. 83

Figure 76: Bucket corners identified with red '+' symbols for alignment purposes ..................... 84

Figure 77: Alignment of the IR transient temperature data to the ANSYS model....................... 84

Figure 78: LHV sensor output versus time at PSDF. At the left of the plot the Syngas was generated via an $\mathrm{O}_{2}$-blown process, while on the right it was generated via an air-blown process.............................................................................................................. 85

Figure 79: LHV value on a reciprocating engine as fuel type was changed. See the text for

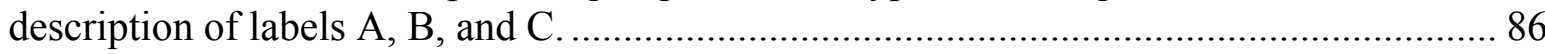

Figure 80: Catalyst hotplate power while combusting syngas at TECO Polk 1. Only air flowed through the sensor until the fuel valve was opened at the blue line. 
Figure 81: Syngas fuel heating values determined using the LHV sensor and mass spectrometer

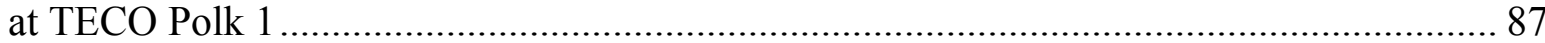

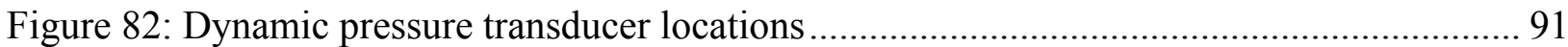

Figure 83: Measured dynamics data in SNR at the baseline operation condition ........................ 92

Figure 84: Dynamic modes predicted by the model ............................................................... 92

Figure 85: Calculated dynamics modal shape compared with experimental data ....................... 93

Figure 86: Block diagram of Delay Differential Equation thermo-acoustic model. The block K

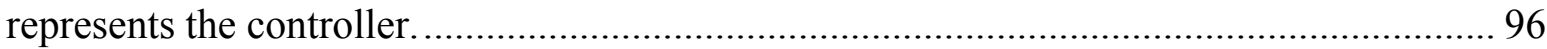

Figure 87: Lean blowout limits measured with and without fuel modulation............................... 97

Figure 88: Thermo-acoustic oscillation with (red) and without (blue) fuel modulation .............. 97

Figure 89: Power spectral density plots of pressure inside the combustion chamber with no fuel

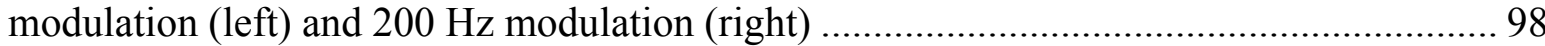

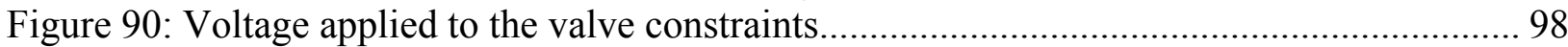

Figure 91: Block diagram of the Delay Differential Equation (DDE) thermo-acoustic model.... 99

Figure 92: Combustor response to fuel forcing - peak amplitude of pressure spectra at forcing

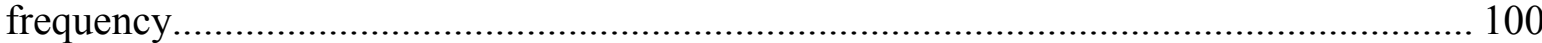

Figure 93: Effect of fuel forcing on amplitude of primary combustion dynamics tone ............. 101

Figure 94: Thermo-acoustic oscillations with (red) and without (blue) fuel modulation............ 102

Figure 95: Comparison between experimental (above) and predicted (below) pressure fluctuation

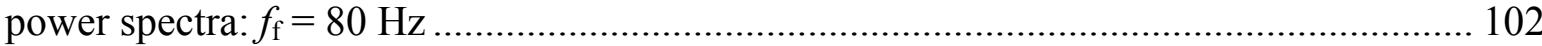

Figure 96: Lean blowout limits measured with and without fuel modulation............................. 103

Figure 97: Frequency response of valve-pre-mixer assembly .................................................. 104

Figure 98: Mass flow perturbation in fuel system .............................................................. 105

Figure 99: Power spectral density and time-series plots of pressure inside the combustion chamber with no fuel modulation (upper/blue) and $130 \mathrm{~Hz}$ modulation (middle/red)....... 106

Cooperative Agreement No: viii 


\section{TABLES}

Table 1: Alphanumeric Code for Specimen Identification ................................................ 5

Table 2: Snapshot of the most hazardous failure modes of the LHV sensor from FMEA .......... 27

Table 3: TECO Polk 1 IGCC Heat Balance Diagram and Process Variables ............................ 35

Table 4: Overall Assessment of Scale After Exposure to Three Different Environments at $1900^{\circ} \mathrm{F}$

Table 5: Weight Change in Specimens Following Exposure to Different Environments ........... 45

Table 6: Summary of Average Weight Gain, Sorted by Alloy and Exposure Period ................. 48

Table 7: Scale Thickness Measured from Photomicrographs of Transverse Cross-Sections of

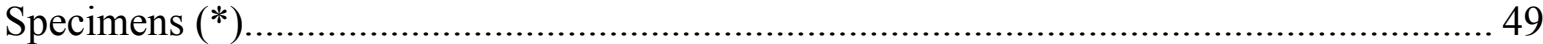

Table 8: MS7001FA IGCC gas turbine pyrometer calibration details .................................. 80

Table 9: Pyrometer calibrations on MS7001FA+E gas turbines ......................................... 81

Cooperative Agreement No: ix 


\section{INTRODUCTION}

\section{Enabling Technology for Monitoring \& Predicting Gas Turbine Health \& Performance in IGCC Powerplants}

\section{Objective:}

Materials and sensors for advanced technology coal/IGCC (Integrated Gasification Combined Cycle) powerplants will be evaluated and demonstrated at a U.S powerplant. These technologies are critical for meeting the DOE targets for coal/IGCC powerplant performance, emissions, reliability, and cost. The project will also benefit advanced technology gas-fired and dual fuel powerplants via spin-off of new technologies.

\section{Background:}

The project will develop, validate and accelerate the commercial use of enabling technologies for advanced technology coal/IGCC powerplant condition assessment and condition based maintenance. The purpose of condition assessment is the real-time, automatic extraction of useful information from operating data to guide decisions, which differentiates it from traditional powerplant monitoring. By contrast, condition based maintenance relies upon the use of this derived information, along with analytical models of part degradation, to accurately predict capital parts consumption and plan maintenance outage schedules

\section{Relevancy:}

Significant benefits to the U.S. public will be additional electricity delivered to the power grid, with fewer interruptions, particularly during periods of peak demand; and reduced generation cost resulting from lower equipment maintenance costs, lower insurance premiums, more stable and reliable power transmission and distribution, and shorter learning curves when new power generation technologies are introduced to the market. Additional benefits include significant reduction in pollutants and improved capability for burning a wide variety of gaseous and liquid fuels in advanced power plants. These advances will benefit future coal/IGCC powerplants and existing natural gas powerplants.

Period of Performance: March 01, 2003 to July 30, 2005 


\section{EXECUTIVE SUMMARY}

The "Enabling \& Information Technology To Increase RAM for Advanced Powerplants" program, by DOE request, was re-directed, de-scoped to two tasks, shortened to a 2-year period of performance, and refocused to develop, validate and accelerate the commercial use of enabling materials technologies and sensors for coal/IGCC powerplants. The new program was re-titled "Enabling Technology for Monitoring \& Predicting Gas Turbine Health \& Performance in IGCC Powerplants".

This final report summarizes the work accomplished from September 1, 2003 to March 31, 2004 on the four original tasks, and the work accomplished from April 1, 2004 to July 30, 2005 on the two re-directed tasks. The program Tasks are summarized below:

Task 1 - IGCC Environmental Impact on high Temperature Materials:

The first task was refocused to address IGCC environmental impacts on high temperature materials used in gas turbines. This task screened material performance and quantified the effects of high temperature erosion and corrosion of hot gas path materials in coal/IGCC applications. The materials of interest included those in current service as well as advanced, high-performance alloys and coatings. The contributors were GE Energy, GE Global Research Center, Georgia Institute of Technology, and Tampa Electric Company.

Task 2 - Material In-Service Health Monitoring:

The second task was reduced in scope to demonstrate new technologies to determine the inservice health of advanced technology coal/IGCC powerplants. The task focused on two critical sensing needs for advanced coal/IGCC gas turbines: 1) Fuel Quality Sensor to rapidly determine the fuel heating value for more precise control of the gas turbine, and detection of fuel impurities that could lead to rapid component degradation. 2) Infra-Red Pyrometer to continuously measure the temperature of gas turbine buckets, nozzles, and combustor hardware. The contributors were GE Energy, GE Global Research Center, Sandia National Laboratory, Tampa Electric Company, Duke Energy, and Cinergy.

Task 3 - Advanced Methods for Combustion Monitoring and Control:

The third task was originally to develop and validate advanced monitoring and control methods for coal/IGCC gas turbine combustion systems. This task was refocused to address pre-mixed combustion phenomenon for IGCC applications. The work effort on this task was shifted to another joint GE Energy/DOE-NETL program investigation, High Hydrogen Pre-mixer Designs, as of April 1, 2004. The contributors were GE Energy, GE Global Research Center, and Georgia Institute of Technology.

Task 4 - Information Technology (IT) Integration:

The fourth task was originally to demonstrate Information Technology (IT) tools for advanced technology coal/IGCC powerplant condition assessment and condition based maintenance. The task focused on development of GateCycle ${ }^{\mathrm{TM}}$ software to model complete-plant IGCC systems, and the Universal On-Site Monitor (UOSM) to collect and integrate data from multiple condition monitoring applications at a power plant. The contributors were GE Energy and Tampa Electric Company. The work on this task was stopped as of April 1, 2004. 


\section{EXPERIMENTAL METHODS}

This section presents a summary of the experimental methods used for each sub-task.

$\underline{\text { Task } 1 \text { - IGCC Environmental Impact on High Temperature Materials: }}$

Sub-Task 1.1 - High Temperature Erosion/Corrosion Data Collection

Environment Effects

CC Technologies of Dublin, $\mathrm{OH}$ was supplied nine sets of test specimens, representing nine different alloys and/or coatings, for exposure to air and $\mathrm{H}_{2} \mathrm{~S}$ containing gaseous environments at a temperature of $1900^{\circ} \mathrm{F}$. These are candidate alloys for gas turbines of an integrated gasification combined cycle (IGCC) power plant. The objectives of this work were to expose the alloys/coatings to simulated gas turbine environments for different periods of time and determine their scaling characteristics from visual inspection, gravimetric measurements and metallographic examination of the specimens.

The candidate alloys and/or coatings were identified as follows: (A) Alloy FSX-414, (B) Alloy GTD222, (C) Alloy Hastelloy-X, (D) Alloy Rene N5, (E) Alloy Nimonic-263, (F) Alloy GTD111, (G) Alloy Rene N5 + Coating of NiCrAlY, (H) Alloy Nimonic-263 + Coating of CoNiCrAlY, and (K) Alloy GTD111 + Coating of CoNiCrAlY.

The test specimens were in the form of cylindrical pins having nominal 3/16-in. diameter. The specimens of base alloys (uncoated) were 1.0-in. long whereas the coated alloys were only 0.9 in. long. The external surface of the specimens without coating had a smooth finish with metallic luster, whereas the coated specimens had matted surface and gray appearance. In addition, the mid-section of each coated specimen had a different texture than either end.

There were three test environments as follows:

1. Oxidation Test: Pre-purified air.

2. Dry Sulfidation Test: $100 \mathrm{ppm} \mathrm{H}_{2} \mathrm{~S}$ in $\mathrm{N}_{2}$.

3. Wet Sulfidation Test: 100 ppm $\mathrm{H}_{2} \mathrm{~S}$ in $\mathrm{N}_{2}$ and saturated with $\mathrm{H}_{2} \mathrm{O}$ vapor at room temperature.

The specimens were exposed in 10-, 30-, 100-, and 300-hour runs to each of the three environments. A new set of triplicate specimens was used for each exposure period. The three environments and four exposure periods resulted in twelve different test runs for each set of nine materials. Thus, 324 specimens were tested in 12 runs with 27 specimens per run. The 27 specimens in each run represented the 9 different materials in triplicate. The specimens were assigned alphanumeric codes for identification, according to the scheme shown in Table 1. An example of the identification code is given below the table.

Two, once-through flow systems with tubular reactors were used for testing of the materials. A photograph of one of the systems is shown in Figure 1. Each system consisted of a horizontal 2- 
inch diameter alumina reactor housed in a tubular furnace. It comprised three independently controlled heating zones equipped with independent temperature controllers and a hightemperature alarm. Gas connections to and from the alumina reactor were made with stainless steel tubing and end caps. End caps were sealed to the reactor with ceramic cement. Schematics of the set-ups for the three different high temperature gaseous environments are shown in Figure 2, Figure 3, and Figure 4.

The system was operated in a once-through flow mode with the appropriate gas mixture, i.e. prepurified air or $\mathrm{N} 2+100 \mathrm{ppm} \mathrm{H} 2 \mathrm{~S}$. The latter gas mixture was procured pre mixed. The flow rate of the gas into the reactor was set at $40 \mathrm{ml} / \mathrm{min}$. At this setting, the specimen holding zone of the reactor was refreshed almost every three minutes at $1900^{\circ} \mathrm{F}$. This flow rate was considered sufficient to keep the reactants replenished in the reactor. In the case of the wet gas mixture (environment \#3), the dry gas mixture was first bubbled through a deionized (DI) water saturator that was maintained at room temperature. This saturated the gas mixture with water vapor prior to entry into the reactor.

The working zone in each reactor was at least 12 -in. long to amply accommodate the specimen holder at the desired temperature. The working zone operated within $\pm 5^{\circ} \mathrm{F}$ of the test temperature of $1900^{\circ} \mathrm{F}$, as estimated from a pre-run calibration of the reactor in which the set point of each of the three heating zones was finely tuned. Custom-made 12-in. long holders of high-purity, high-density alumina with drilled holes were used to hold specimens in the working zone. Figure 5 shows drawings of the boat-type holder and specimen loading details. Two arrays of 7/32-in. diameter holes in the boat deck accommodated 27 specimens vertically. The specimens leaned against the holder at approximately one-third of their vertical length. Thus, the bottom face of the specimens and the side contact points were probably partially shielded from the test environment.

Prior to testing, each base alloy specimen was cleaned with methanol and weighed on an analytical balance. The coated specimens were weighed in the as-received condition, without cleaning. Each measurement was repeated three times, and the average was recorded for the specimen. The specimens were then placed into respective allotted holes of the specimen holder, as shown in Figure 6, so that each specimen, because of its unique position, could be traced after the testing period. After loading the holder with specimens into a reactor, the system was sealed and flushed with argon to drive off air. At the same time, the furnace was switched on and the temperature on all the three heating zones was raised to $250^{\circ} \mathrm{F}$. Argon was continually flushed as the temperature of the reactor was raised in steps of about $250^{\circ} \mathrm{F}$ to the set point $\left(1900^{\circ} \mathrm{F}\right)$, over a period of 4 to 6 hours.

Once the furnace reached the desired temperature, argon flushing was stopped and depending upon the environment either purified air or $\mathrm{H} 2 \mathrm{~S}$ mixture was introduced into the system. The exposure periods for different runs were 10, 30, 100, and 300 hours. At the end of the predetermined exposure period, the system was flushed again with argon. The reactor was then cooled in steps of about $250^{\circ} \mathrm{F}$ to the room temperature, which took about 2 days. The alumina holder loaded with the specimens was removed and stored in a dessicator until needed for specimen evaluation. 
After the exposure test, each specimen was weighed again and photographed to document its appearance. The specimen was photographed in the weighing cup itself to minimize handling and undue spalling of the scale. The weight gain in each specimen was determined from the difference between its final and initial weights. (Some specimens showed negative weight gain, i.e. weight loss, which was probably caused by spalling of the scales).

The work scope of the project required a subset of nine specimens from each run to be examined metallographically. Thus, one specimen of each material was mounted in epoxy, sectioned at its mid-length, and one of the sectioned faces metallographically polished to a final finish using $0.05-\mu \mathrm{m}$ colloidal alumina. The prepared cross-section was examined on a metallograph having magnifications up to $1000 \mathrm{X}$. The scale thickness as well as any intergranular penetration (IGP) into the base metal was measured and documented with a photomicrograph.

Table 1: Alphanumeric Code for Specimen Identification

\begin{tabular}{|c|c|c|c|}
\hline Order & & Property & Code \\
\hline \multirow{3}{*}{1} & \multirow{3}{*}{ Environment } & Air & A \\
\hline & & Dry $\mathrm{H}_{2} \mathrm{~S}$ & $\mathrm{H}$ \\
\hline & & Wet $\mathrm{H}_{2} \mathrm{~S}$ & $\mathrm{~W}$ \\
\hline \multirow{4}{*}{2} & \multirow{4}{*}{$\begin{array}{l}\text { Exposure } \\
\text { Period }\end{array}$} & 10 hours & 10 \\
\hline & & 30 hours & 30 \\
\hline & & 100 hours & 100 \\
\hline & & 300 hours & 300 \\
\hline \multirow{9}{*}{3} & \multirow{9}{*}{ Material } & Alloy FSX-414 & A \\
\hline & & Alloy GTD222 & $\mathrm{B}$ \\
\hline & & Alloy Hastelloy X & $\mathrm{C}$ \\
\hline & & Alloy N5 & $\mathrm{D}$ \\
\hline & & Alloy Nimonic 263 & $\mathrm{E}$ \\
\hline & & Alloy GTD111 & $\mathrm{F}$ \\
\hline & & Alloy N5 + NiCrAlY Coating & $\mathrm{G}$ \\
\hline & & Alloy Nimonic $263+$ CoNiCrAlY Coating & $\mathrm{H}$ \\
\hline & & Alloy GTD111 + CoNiCrAlY Coating & $\mathrm{K}$ \\
\hline \multirow{3}{*}{4} & \multirow{3}{*}{$\begin{array}{l}\text { Individual } \\
\text { Specimen }\end{array}$} & $\# 1$ & 1 \\
\hline & & $\# 2$ & 2 \\
\hline & & $\# 3$ & 3 \\
\hline
\end{tabular}

Example: Air / 10 hours / FSX-414 / Specimen \#1 = A10A1. 


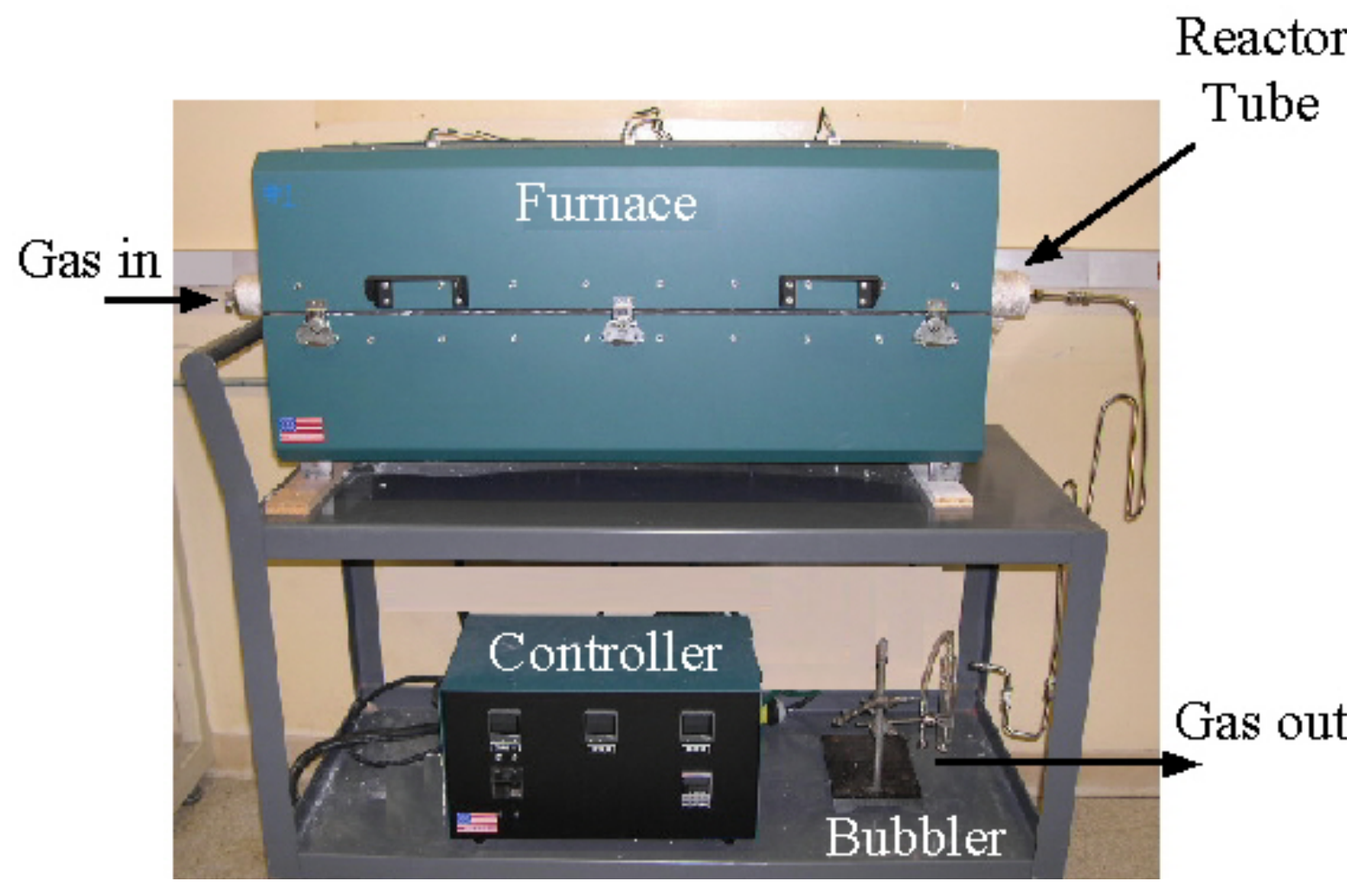

Figure 1: Test Set-Up Showing Furnace with Reactor Tube, Temperature Controller, and Gas Bubbler at Exit

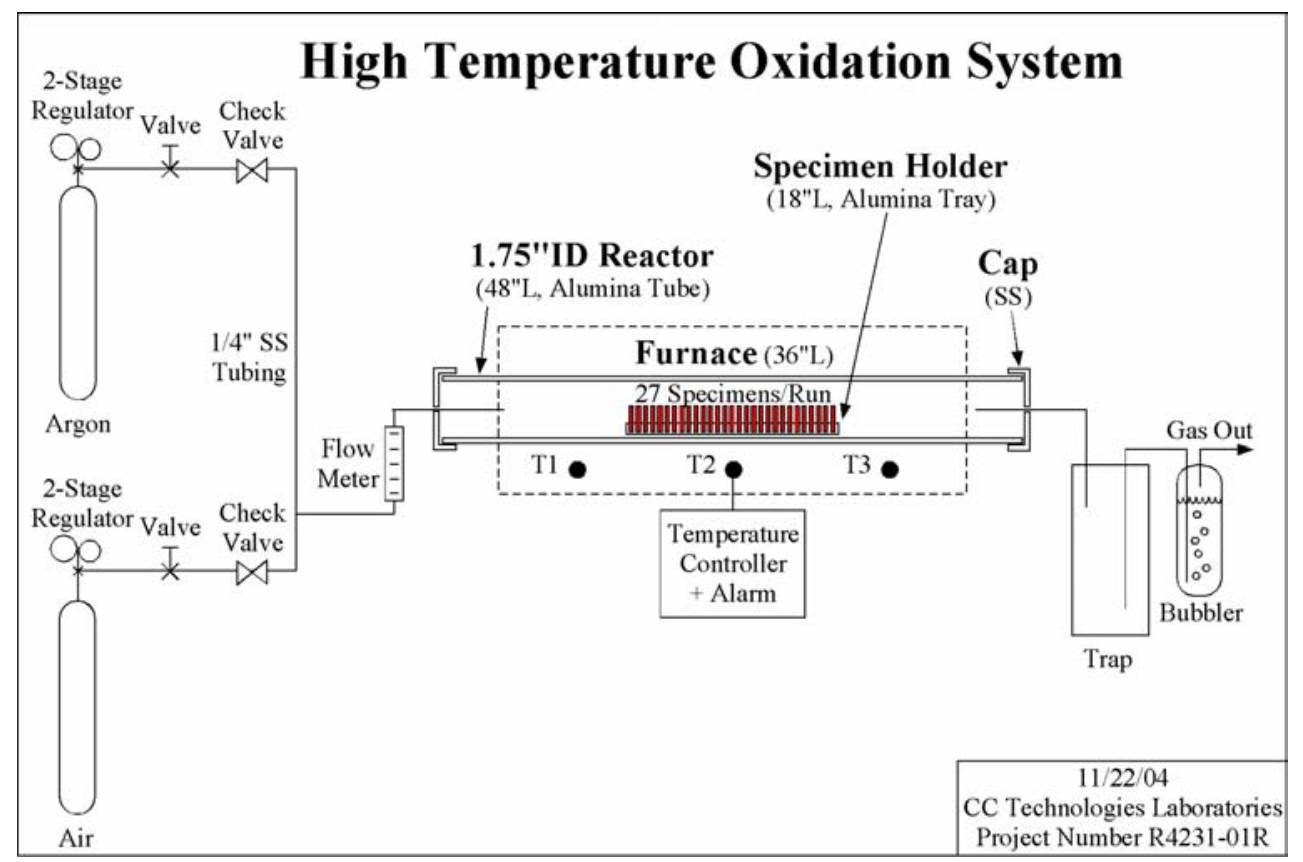

Figure 2: Set-Up Schematic for High-Temperature Tests in Air 


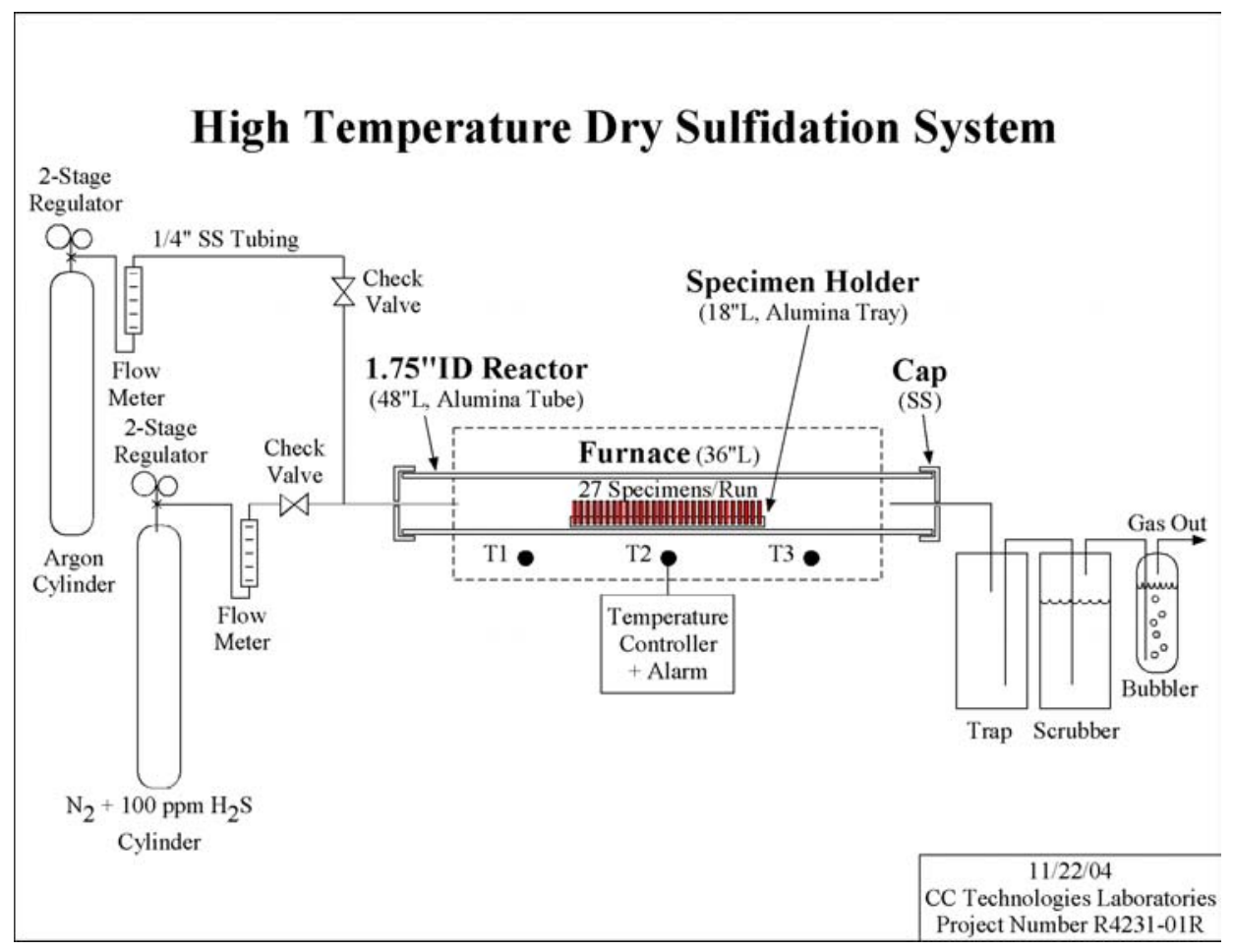

Figure 3: Set-Up Schematic For High-Temperature Tests In Dry $\mathrm{H}_{2} \mathrm{~S}$ Environment

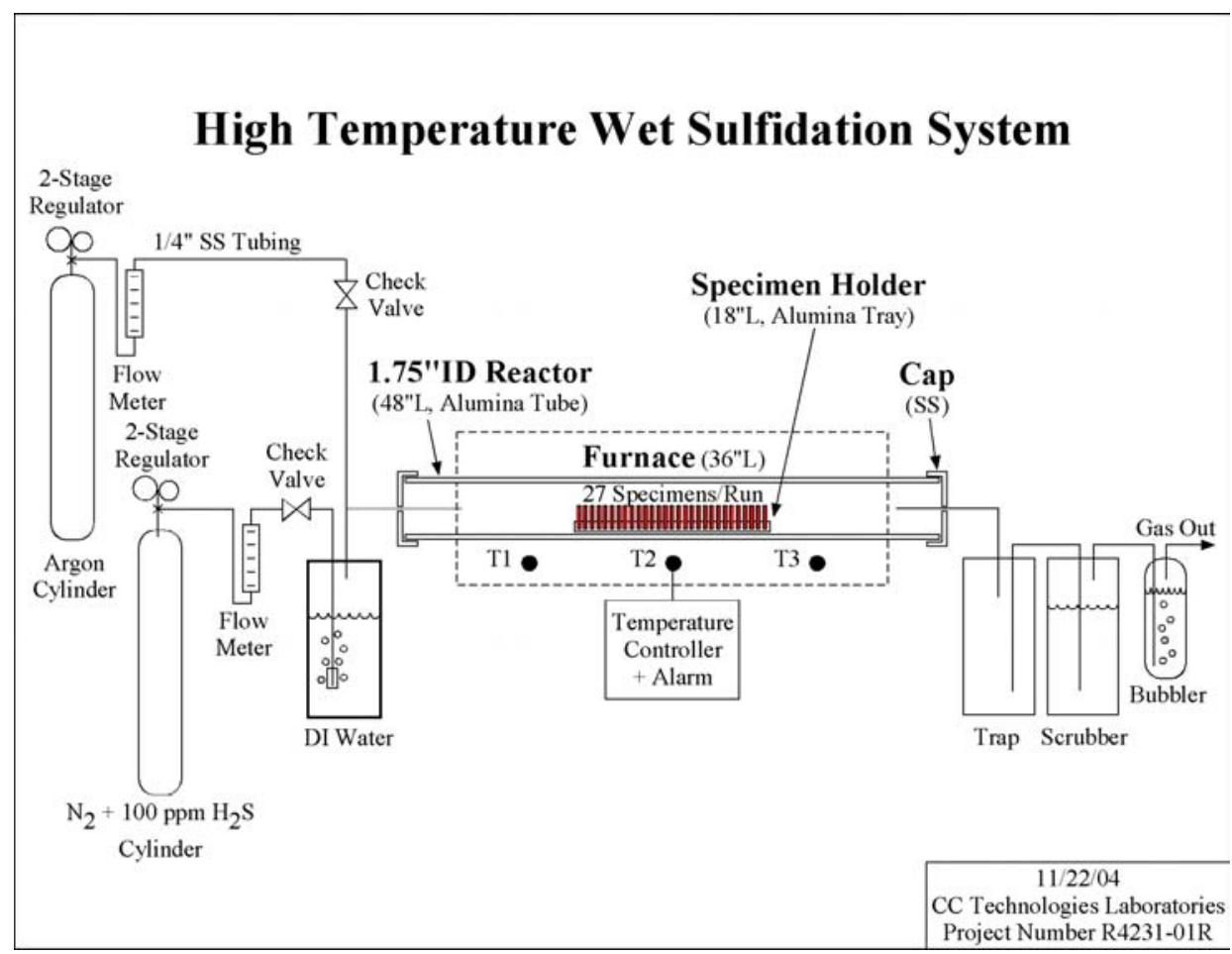

Figure 4: Set-Up Schematic for High-Temperature Tests in Wet $\mathrm{H}_{2} \mathrm{~S}$ Environment 


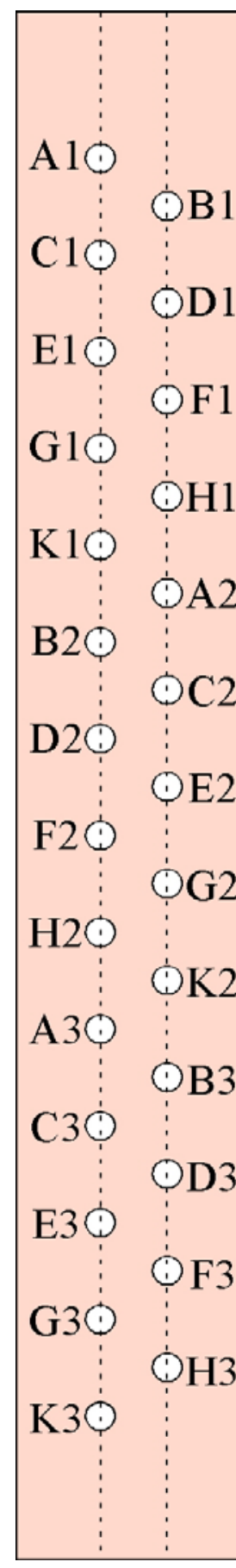

(a)

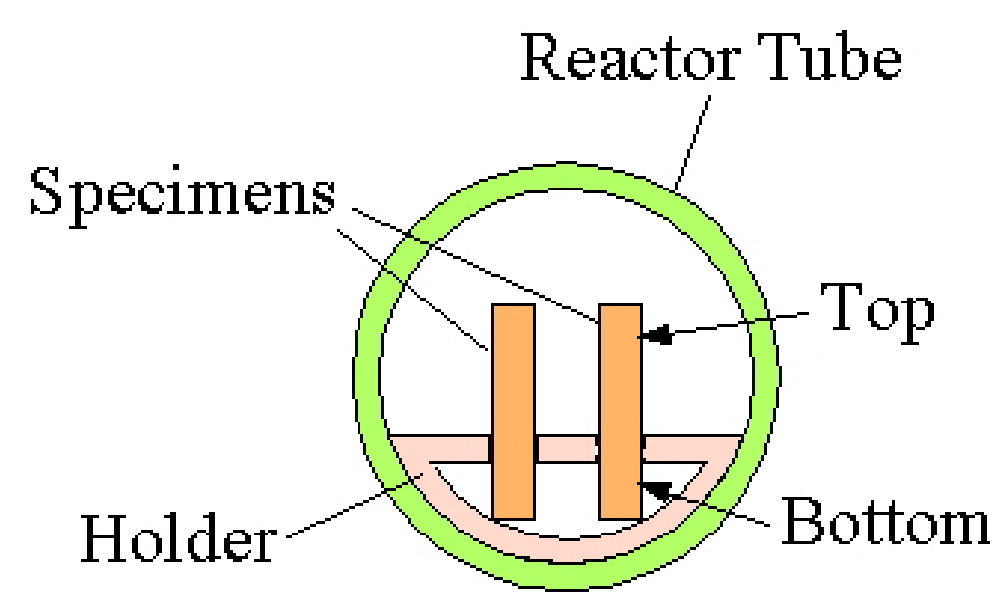

(b)

Figure 5: Drawing of Holder for Specimens, showing (a) Identification Code, and (b) Specimen Orientation - Top and Bottom 


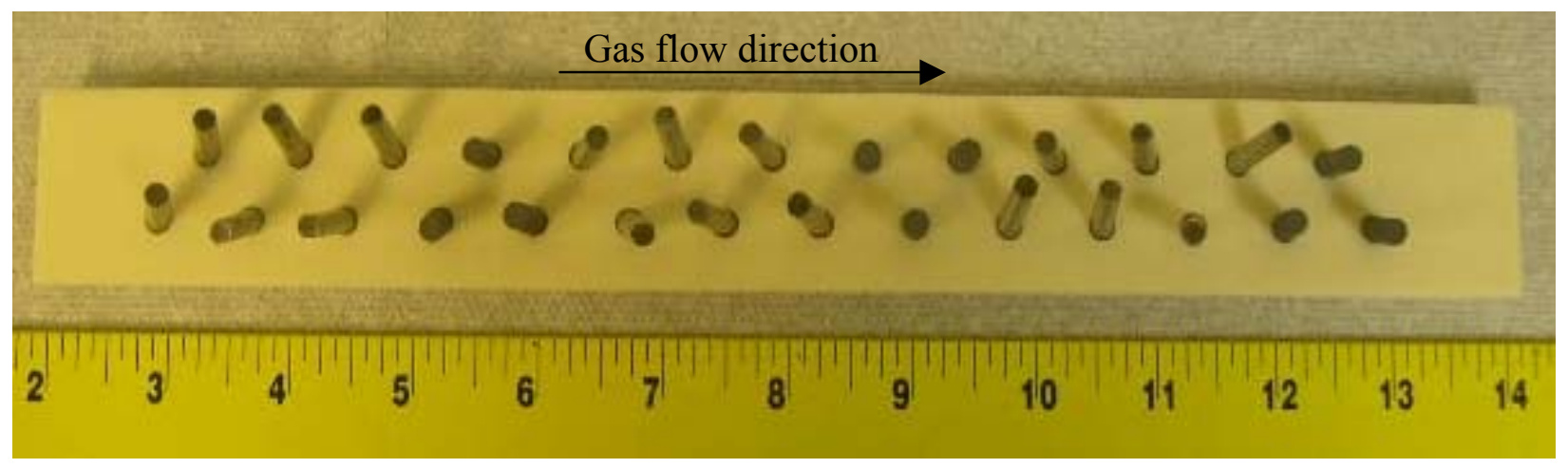

Figure 6: Photograph of Alumina Holder with Specimens Before Exposure

Erosion Experiments

GE Aircraft Engines in Evendale, $\mathrm{OH}$ conducted high temperature erosion experiments on the following material/coating systems:

- Rene N5 + NiCrAlY bondcoat + air plasma sprayed TBC

- GTD111 + CoNiCrAlY bondcoat + air plasma sprayed TBC

- Nimonic-263 + CoNiCrAlY bondcoat + air plasma sprayed TBC

The tests determined the amount of erosion resistance the above coatings systems possess, under conditions that simulate the gas turbine. These conditions were simulated through the use of a Becon rig. The Becon rig operations were as follows; coated pin specimens (diameter of $0.230 \mathrm{in}$ $+0.000 \mathrm{in} /-0.003 \mathrm{in}$ and length of 3.500in $\pm 0.010 \mathrm{in}$ ) were placed in a rotating carousel in front of a combustion can, shown in Figure 7. During each experimental cycle the specimens were brought to temperature and $50 \mu \mathrm{m}$ alumina particles were accelerated towards the specimens. One cycle was determined by the amount of time needed to accelerate 100 grams of particulates towards the specimens ( $\sim 5 \mathrm{~min})$. After each cycle each specimen was photographed, weighed, the diameter was measured and the cycle number was noted.

\section{Syngas Sampling}

Syngas fuel sampling tests were performed on June 5-7, 2004 and April 29 to May 1, 2005 at the Tampa Electric Company (TECO) Polk 1 powerplant, located near Mulberry, FL. The purpose of the fuel sampling was to evaluate the effect of Syngas fuel constituents and contaminants on the service lives of gas turbine combustion and downstream turbine components during actual operating conditions. Typical Coal IGCC Syngas contains a gaseous mixture of mostly combustible constituents such as $\mathrm{CO}, \mathrm{H}_{2}$, and $\mathrm{CH}_{4}$, plus non-combustible gases such as $\mathrm{CO}_{2}, \mathrm{~N}_{2}$, Ar and $\mathrm{H}_{2} \mathrm{O}$, and small quantities of compounds of sulfur (20 to $200 \mathrm{ppm}$ ). During gasification plant start up and operational transients, the Syngas may contain additional contaminants, such as compounds of iron, nickel, calcium, sodium and potassium, ammonia, volatile compounds of mercury, $\mathrm{HCl}$, arsenic, etc. The gas turbine fuel and exhaust gas streams were sampled at regular intervals. Results from this task allowed the formulation of predictive models to correlate the observed fuel variation with the observed part condition. A schematic diagram of the fuel sampling locations is shown in Figure 8. 


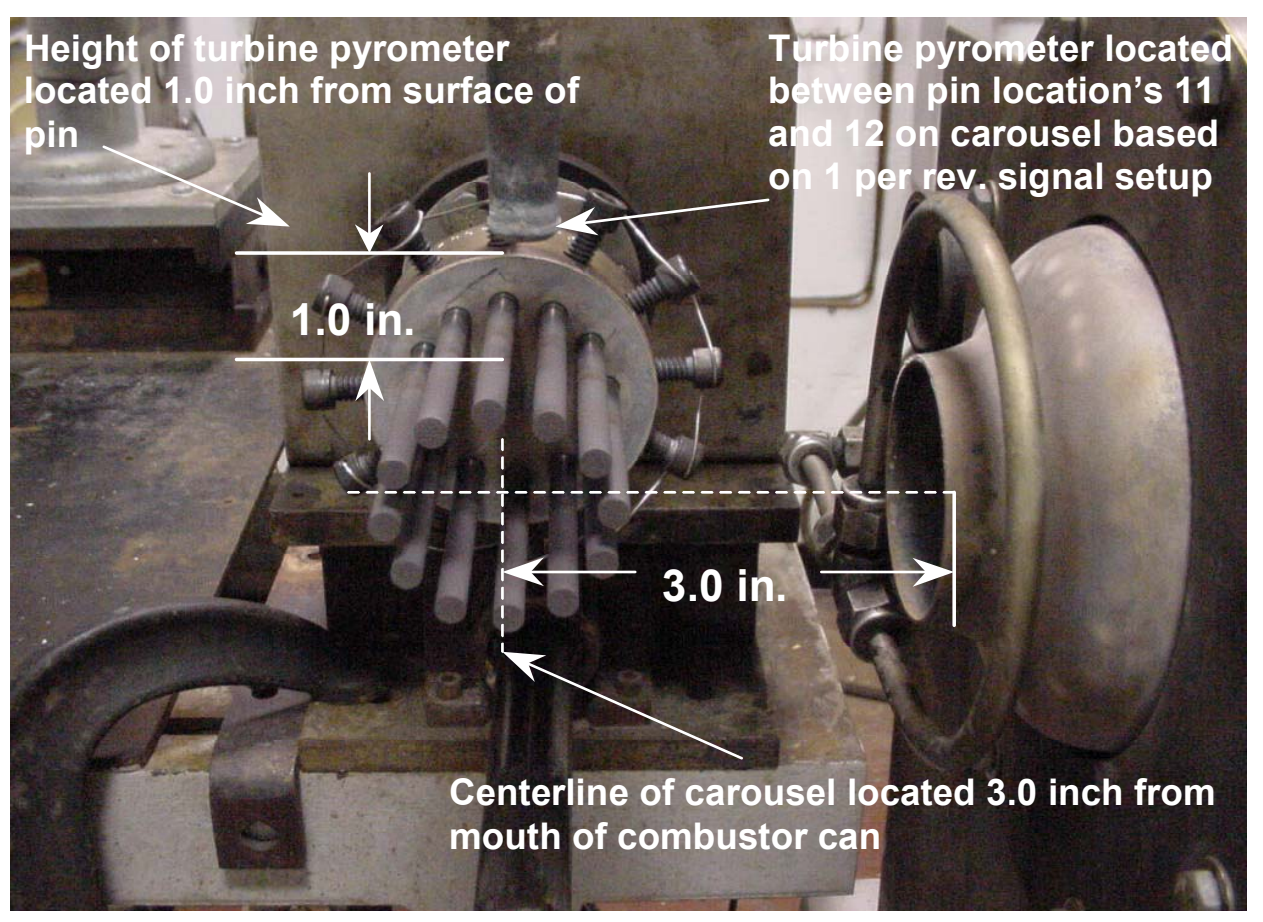

Figure 7: Becon rig erosion tester

Cubix Corporation sampled for combustible gases, particulate matter (PM), metals, $\mathrm{HCl}$, Ammonia $\left(\mathrm{NH}_{3}\right)$, and sulfur species at locations 1-3 in Figure 8. Three test runs were performed simultaneously at each location. The first two runs were performed during startup and partial loading of the gas turbine. The third run was performed when the turbine was at full load. A total of six Syngas samples were taken.

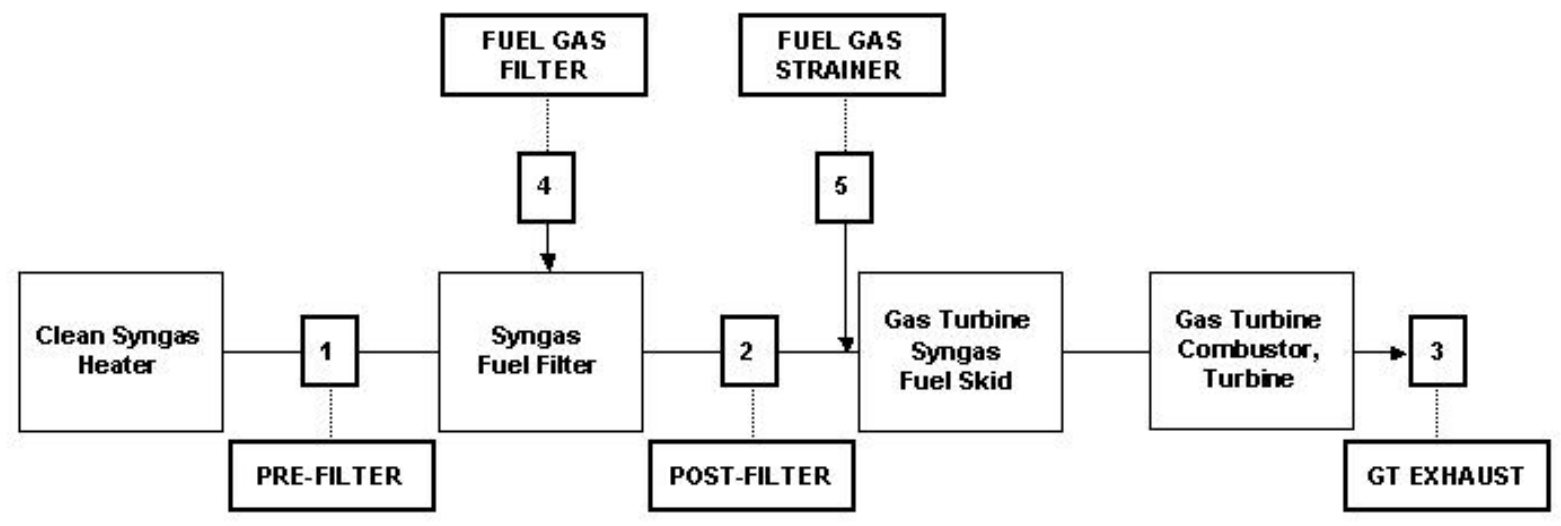

Figure 8: TECO Polk IGCC sample locations 
Syngas fuel residue samples were taken from the fuel filter and fuel strainer, i.e. at locations 4 and 5 in Figure 8, during gasifier/gas turbine shutdown. The fuel strainer was removed and rinsed with deionized (DI) water and $0.1 \mathrm{~N}$ Nitric acid. Each sample was collected in stainless steel sample cylinders. The DI water was analyzed for $\mathrm{Cl}, \mathrm{NO}_{3}$ and $\mathrm{SO}_{4}$. The DI waste and the nitric acid were mixed, and this mixture was analyzed for metals by Chester LabNet. The list of metals included $\mathrm{As}, \mathrm{Ca}, \mathrm{Fe}, \mathrm{Hg}, \mathrm{Ni}, \mathrm{K}$ and $\mathrm{Na}$. These samples were analyzed for sulfur species (ASTM D-5504) and fuel composition (UOP-539) by Core Laboratories of Houston, TX.

Particulate matter was collected over two sampling periods. The first sampling period included runs 1 and 2. The second sampling period included run 3. The particulate matter samples were analyzed for gravimetric weight gain and PM metals ( $\mathrm{As}, \mathrm{Ca}, \mathrm{Fe}, \mathrm{Hg}, \mathrm{Ni}, \mathrm{K}$ and $\mathrm{Na}$ ). Particulate matter testing followed EPA isokinetic techniques. However, due to the unusual source and sampling conditions, a custom sampling apparatus was used to accommodate the high flow necessary to sample isokinetically. The sample was extracted from the Syngas fuel line through a 1/4" ID sampling nozzle installed in the pipe. The sample flowed from the nozzle through stainless steel tubing and several shut off valves to the PM sampling apparatus. PM was collected on a $47 \mathrm{~mm}, 0.2 \mu \mathrm{m}$ porosity, quartz fiber filter contained in a heated enclosure. Sample flow was measured with a $3 / 8$ " orifice plate. The flow was controlled using stainless steel needle valves. Isokinetic sample rates were determined from the sample flow and the Syngas fuel flow in the fuel line. The particulate matter filters were analyzed by Chester LabNet of Tigard, OR for gravimetric weight gain and metals.

Ammonia $\left(\mathrm{NH}_{3}\right)$ and $\mathrm{HCl}$ was collected from the Syngas into an impinger train containing $0.1 \mathrm{~N}$ sulfuric acid. Impinger samples were analyzed for $\mathrm{NH}_{3}$ analysis was performed at Cubix's Austin Laboratory. An aliquot of the impinger solution was sent to Chester $\mathrm{LabNet}$ for $\mathrm{HCl}$ analysis. The $\mathrm{HCl}$ analysis was performed using Ion Chromatography (IC) following the procedures in EPA Method 26A. The analysis for gaseous metals followed the procedures of EPA Method 29. As, $\mathrm{Ca}, \mathrm{Fe}, \mathrm{Hg}, \mathrm{Ni}, \mathrm{K}$ and $\mathrm{Na}$ were collected from the Syngas into an impinger train. The impinger samples were sent to Chester LabNet for analysis. Hg was analyzed using cold vapor atomic absorption. The other metals were analyzed by a Inductively-Coupled Plasmaspectrometer (ICP).

Test Deliverables: Actual and corrected experimental results of test data (flow, pressure, temperature and composition) for the following streams:

1. Fuel Gas stream immediately upstream of Syngas Fuel Filter

2. Fuel Gas stream immediately upstream of Syngas Fuel Skid

3. Gas stream immediately downstream of Gas Turbine Exhaust Duct

4. Residue composition and constituents of Syngas Fuel Filter

5. Residue composition and constituents of Gas Turbine Fuel Gas Strainer

\section{Service Exposed Parts Evaluation}

To understand the environmental effects on high temperature materials in an IGCC power plant, two sets of data were analyzed. The first was to determine the corrosion products present on the surface of the gas turbine parts after a known service exposure time. Tampa Electric Company (TECO) provided two stage one buckets and two stage two buckets from their IGCC gas turbine for destructive analysis. This analysis consisted of microstructural observation of the surface and 
cross-section of the part and spectroscopy to determine the composition of the surface and near surface layers. The second data set needed were the environmental conditions responsible for producing the surface reactions observed on the service parts. Both of these sets of data were collected and analyzed to facilitate the correlation of service data to laboratory data.

The analysis of the coatings and the corrosion and oxidation products after service exposure of a TECO Polk 1 IGCC first stage bucket was performed using destructive and non-destructive analytical methods. Four distinct objectives were identified for this task, each involving different sample preparation and analysis techniques as described below.

1) Surface analysis of the as-received (service exposed) airfoil: The objective was to identify foreign matter on the coating surface that would be indicative of a detrimental effect of the IGCC process on the airfoil coating. No specific surface preparation was performed for this analysis. The analytical methods consisted of Scanning Electron Microscopy (SEM) imaging, Energy Dispersive Spectroscopy (EDS), and X-Ray Diffraction (XRD) analyses of various locations on the pressure and suction sides of the airfoil.

2) XRD analyses of oxide particles removed from internal cooling passages were performed after removal of the particles by extractive replication (using acetate tape and high purity acetone).

3) Cross-section analyses of the coating and the underlying secondary reaction zone were performed to identify oxidation and corrosion products, determine the state of distress of the coating after service exposure and analyze the structure of craze cracks in the coating. SEM, EDS and Electron Back-Scattered Diffraction (EBSD) techniques were applied in this task. Coupons were removed from the trailing edge and leading edge, about 3.25" from the tip, and were mounted and polished for the cross-section analyses.

4) The analysis of corrosion products related to the presence of sulfur was performed on samples from the leading and trailing edges, 3.5" and 0.6" from the tip, respectively, where sulfur was detected on the airfoil surface. Analytical techniques included SEM, EDS, EBSD, and Wavelength Dispersive Spectroscopy (WDS) to allow an accurate identification of phases found in the coating and underlying diffusion zone.

\section{$\underline{\text { Sub-Task 1.2 - High Temperature Erosion/Corrosion Mechanism Quantification }}$}

The experimental methods for Sub-Task 1.2 were based upon the data collected in Sub-Task 1.1, as reported above, and by the Georgia Institute of Technology as reported in Appendix D. 


\section{Task 2 - Material In-Service Health Monitoring:}

\section{Subtask 2.1 - Define Coal IGCC Power Plant Requirements}

Needs were identified and prioritized using Quality Function Deployment (QFD) and other "Six Sigma" quality tools that are now used widely throughout GE. The Critical-To-Quality (CTQ) characteristics of the required sensors, systems, and controls were identified during this process.

\section{Subtask 2.2 - Sensor Capability Studies}

Fuel Lower Heating Value (LHV) Sensor

Sandia National Laboratory developed a prototype sensor to measure the lower heating value (LHV) of gaseous fuels as part of the DOE Smart Turbine Program. This device uses a pair of Micro-Electro-Mechanical Systems (MEMS) micro hot plates that produce a uniform temperature across their surface via a power control circuit and resistive heating. A catalytic material applied to one of the hotplates promotes combustion of a fuel/air mixture passed over its surface. The hotplates are maintained at a constant temperature, so reduced power is required when energy is released during fuel combustion. The other hotplate is used to measure the convective heat transfer due to the fluid flow. The overall change in the power supplied to these two hotplates is directly related to the fuel LHV. The time response of the micro hot plates is on the order of milliseconds, so LHV is measured nearly in real time.

The development effort by Sandia National Laboratory primarily consisted of the production of the prototype MEMS device and initial performance measurements. These were conducted in a controlled environment with pure methane as fuel. The technology was transitioned to the GE Global Research Center (GRC) to fully develop the sensor for industrial applications.

\section{Fuel LHV Sensor Development by Sandia National Laboratory}

While the fundamental device designs had already been determined, further design work was performed by Sandia to develop a more efficient heater wiring configuration. The wiring from the micro hot plates connects to an external circuit board. This circuit control logic acts to maintain the hotplate resistance at a constant value, which sets the device temperature. By setting an input voltage to the circuit, the hotplate temperature can be raised from room levels to the operating conditions.

A large area of uniformly heated catalyst was necessary for maximum signal response and repeatability. Thermal modeling using ANSYS software showed that the design possessed a fairly uniformly heated central area. Additional modeling work was done to further optimize the catalyst heater design. Repeatable catalyst deposition was required to reduce signal variation between devices. Additionally, membrane deflection produced considerable stress in the deposited catalysts, which required considerable effort to control.

A gas chromatography (GC) system (Agilent 3000 MicroGC) was used to study the fuels and exhaust gas constituents from the LHV sensor rig. The purpose of these experiments was to better correlate the LHV sensor signal with the actual BTU content of the fuel, understand combustion in the sensor rig, and determine the combustion efficiencies of fuel constituents. The 
testing encompassed four different natural gas standards, several singular natural gas components, and syngas mixtures. Prior to the combustion studies, modifications were made to the LHV sensor rig that would allow for both pre-combustion and post-combustion sampling of the air/fuel stream.

The GC system was calibrated for all components expected in testing. Calibration resulted in linear signal response for $\mathrm{C} 1-\mathrm{C} 5$, nitrogen, oxygen, carbon dioxide, carbon monoxide, and hydrogen over a wide range of concentrations. Water was difficult to measure given the lack of heat tracing on the sample lines; however, a calibration for water was developed. The experimental procedure entailed pre-combustion sampling to establish a baseline, followed by post-combustion sampling to record the affects of the catalytic combustion. In addition to having good signal linearity, the GC system showed a reasonable noise floor and signal repeatability.

\section{Fuel LHV Sensor Development by GE Global Research Center}

Studies conducted at GRC concentrated on two aspects. The first was to find the optimal flow rates and fuel:air ratios to calibrate the sensor to an absolute LHV value. The second was to develop the sensor system as a whole; which included fixture design, flow control, fuel temperature control, and safety considerations.

The two hotplates were mounted in a fixture crafted from 304 stainless steel. The fixture is shown in Figure 9 and the fixture dimensions are shown in Figure 10. The air-fuel mixture first passes over the reference hotplate, removing energy by convective heat transfer. The catalyst hotplate is immediately downstream of the reference hotplate.

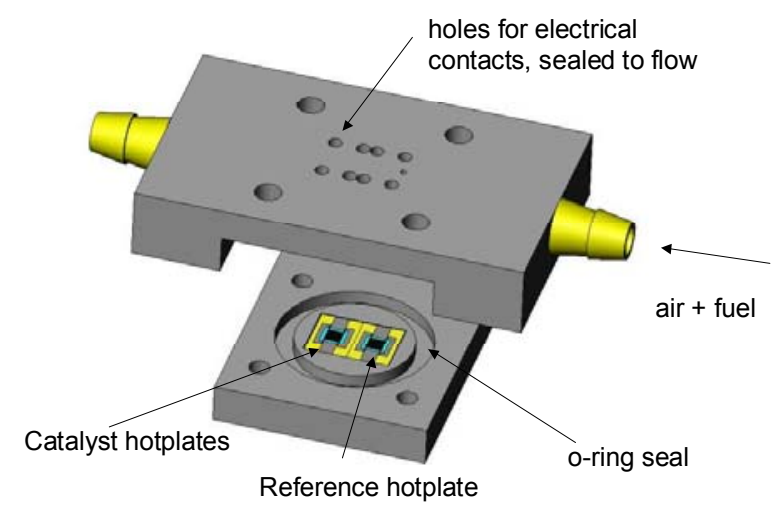

Figure 9: Fuel Lower Heating Value (LHV) sensor fixture 


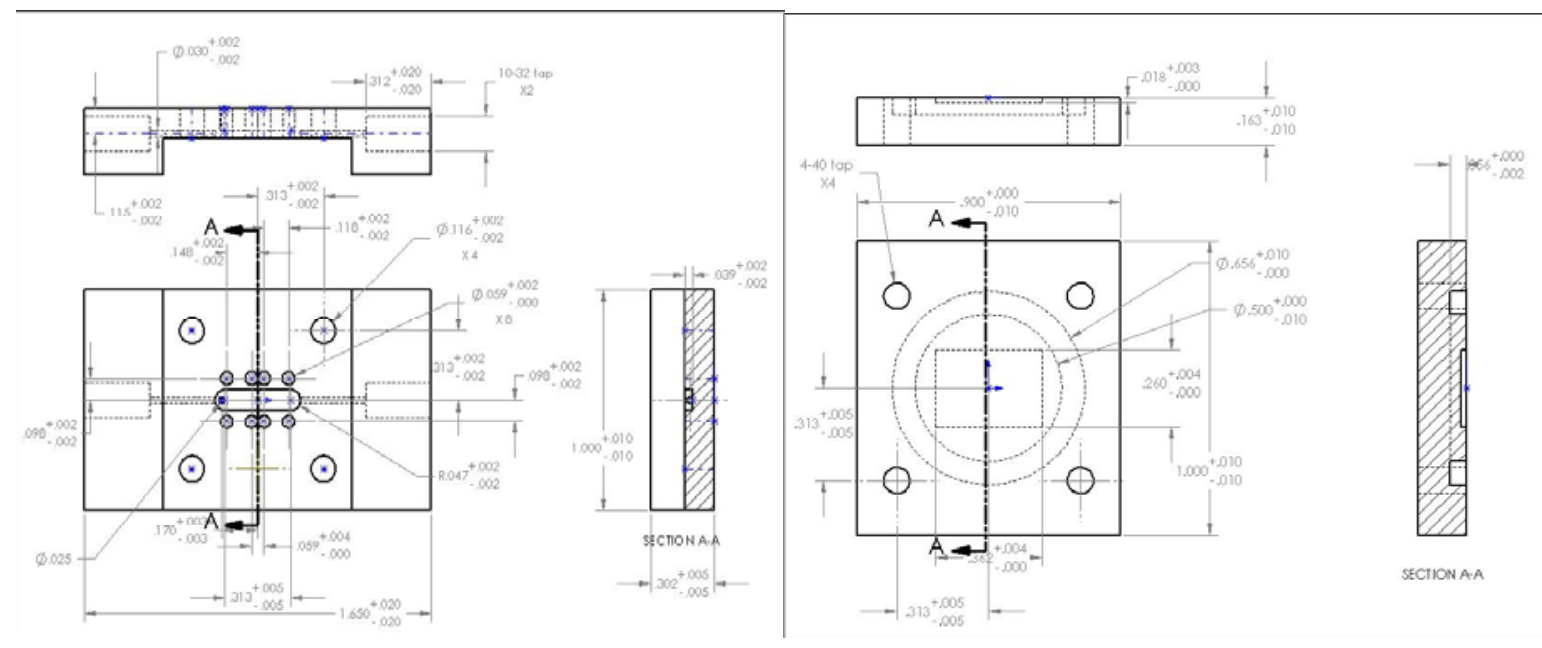

Figure 10: Dimensioned drawings of the sensor fixture

The inlet fuel/air mixture to the sensor was regulated using MKS 1179A mass flow controllers (MFCs). The MFCs fix the volume flow rates that pass through the devices and enter the downstream piping. There were three MFCs for fuel gases and one for air. With this setup, shown in Figure 11, it was possible to generate mixtures of gases including simulated syngas. The lab at GRC was upgraded to two full testing stations, each with four MFCs. The first station was for experimental work and the second station was for unattended, long-term reliability testing of the sensor.

Experiments were also conducted to investigate using choked flow through an orifice to control the gas flow, as MFCs are not feasible outside the laboratory environment. MFCs are ideal for use in the laboratory because they precisely control the flow of a known gas. However, in the field, the sensor must respond to the fuel gas that is present. Here, the goal is to control the mass flow of that gas and to balance it versus the flow of air to achieve a particular fuel to air ratio. Additionally, the flow control device must be adequate for high temperature fuels (up to $200^{\circ} \mathrm{C}$ ) and fuels with contaminants (particulates, tars, etc).

Choked flow through an orifice was identified as the lowest cost and most robust solution. When the pressure upstream of an orifice is sufficiently higher than the pressure downstream, the velocity of the gas flowing through the orifice will be constant, or "choked." However, it is possible to have changes in mass flow if the density of the gas changes, so an independent measurement was made to determine mass flow. During field tests (sub-task 2.4) an orifice was chosen such that the fuel flows were allowed over pre-determined limits and would be sufficient for sensor operation. 


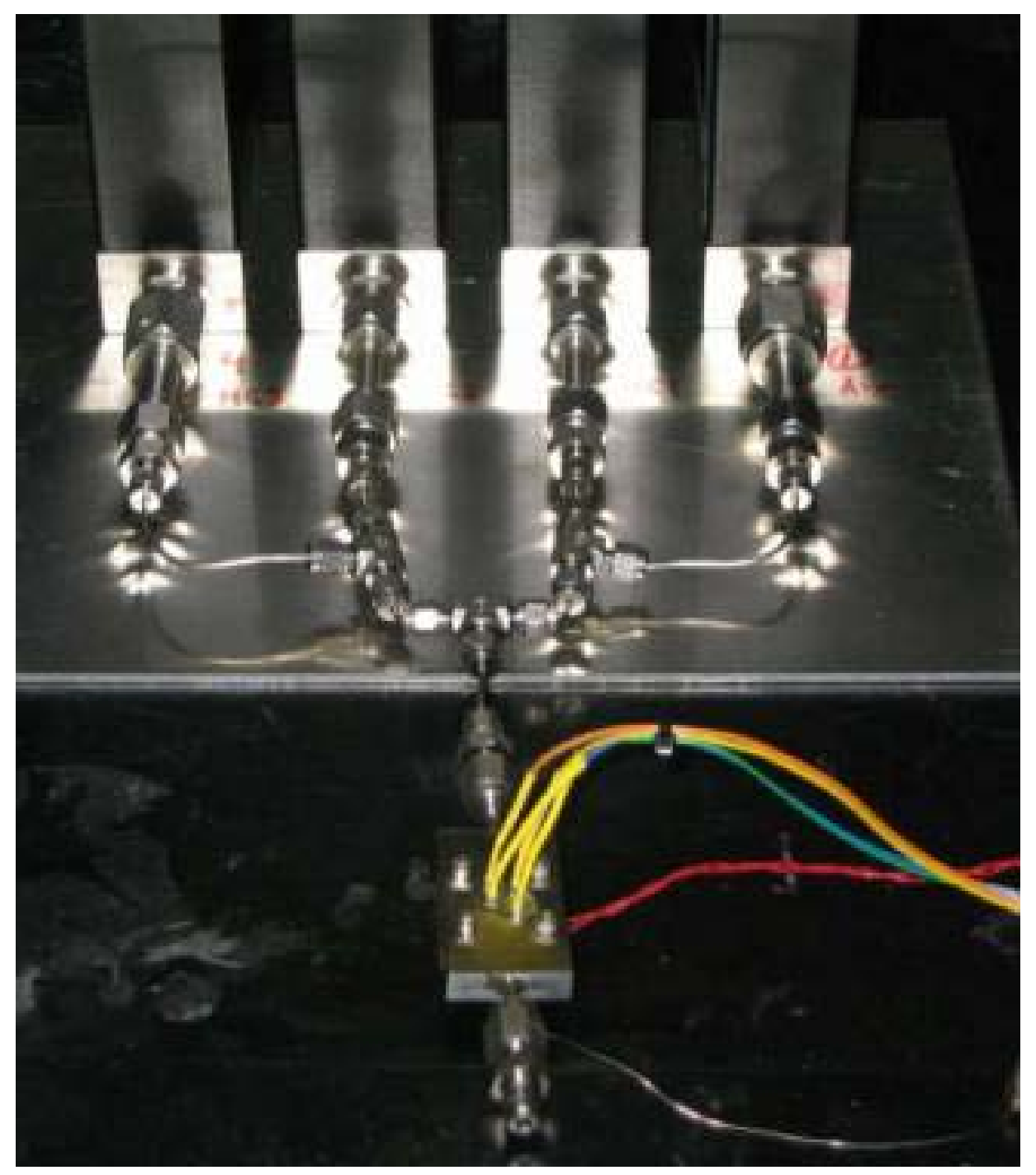

Figure 11: Laboratory based testing station showing four mass flow controllers (MFCs) at the top and piping leading to the LHV sensor

\section{Fuel LHV Sensor Reliability}

During the course of testing the fuel LHV sensor, in both the laboratory and in the field, the hotplate lifetime was not long enough to meet the requirements of a commercial sensor. Thus, an effort was undertaken to analyze the cause of the failure of these hotplates. In order to organize all of the possible causes of premature hotplate failure, the failure mechanisms were listed using a cause-effect diagram (also referred to as a fishbone), shown in Figure 12. The majority of the possible causes were related to hotplate manufacturing. Additionally, some of the possible causes were due to the environment the hotplates were operated in. 


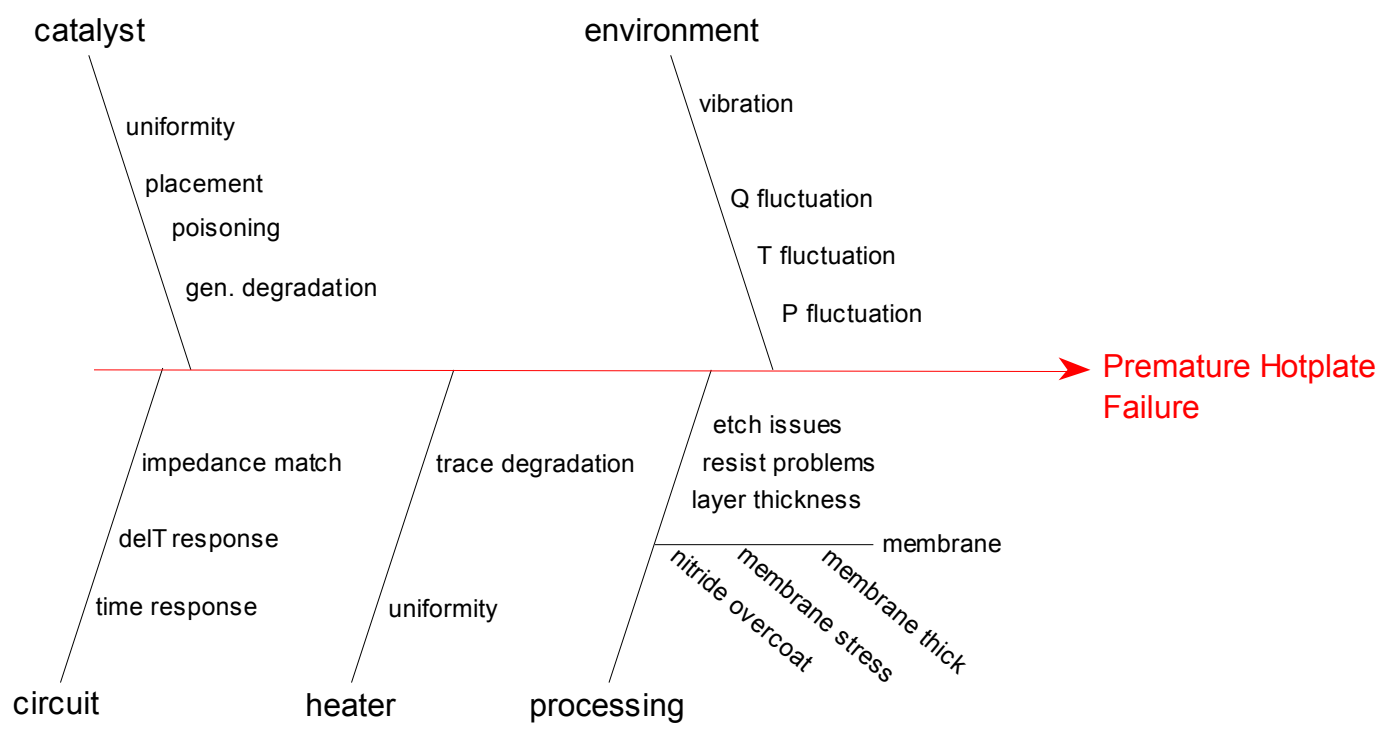

\section{Figure 12: Fishbone diagram of factors that effect reliability and life of the LHV sensor hotplates}

Micrographs were taken of each hotplate before and after each experimental run to establish if there was a correlation between hotplate life and variations introduced by the manufacturing process. The thermal performance of the hotplate was also investigated using a wafer bow apparatus with a heated stage and infra-red micrographs, in order to determine the stresses in the thin film layers as a function of temperature.

Environmental effects were investigated in the laboratory to simulate the pressure and flow variations observed in the field. This rig used a diaphragm pump whose flow is choked down to induce flow variations and pressure variations into the fuel flow stream. Additionally, the sensor fixture was vibrated by coupling it mechanically to a loudspeaker. The feedback circuit's response time was also investigated to ascertain if hotplate temperature could track with observed flow oscillations. 


\section{Subtask 2.3 - IR Pyrometer for Condition Based Maintenance}

Improved procedures and methodologies for using Infra-Red (IR) pyrometers in the gas turbine were developed. Pyrometers were installed and/or maintained in four GE F-class gas turbines.

MS7001FA IGCC Gas Turbine

Five pyrometers were previously installed to monitor three stages of an MS7001FA IGCC gas turbine. Figure 13 shows the locations of the pyrometers. The pyrometers were calibrated and data acquisition was connected to the GE network.

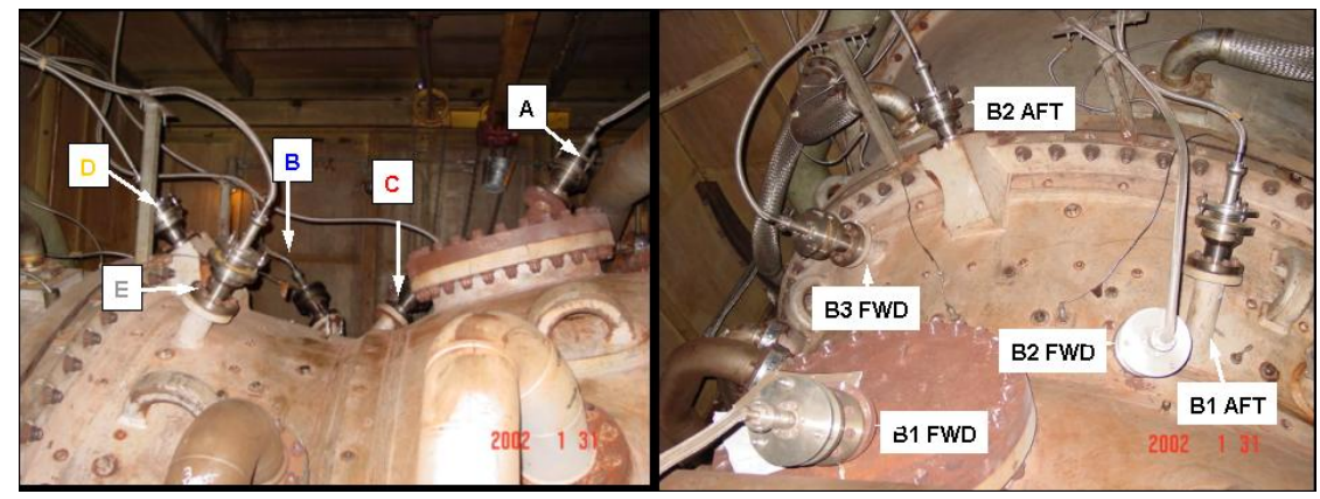

Pyrometer A: B1FWD - monitors Stage 1 buckets from forward Pyrometer B: B1AFT - monitors Stage 1 buckets from aft Pyrometer C: B2FWD - monitors Stage 2 buckets from forward Pyrometer D: B2AFT - monitors Stage 2 buckets from aft Pyrometer E: B3FWD - monitors Stage 2 buckets from forward

Figure 13: Pyrometers on the MS7001FA IGCC gas turbine

The five pyrometer fiber optic light guides and alignment flanges at the site were removed during a planned gas turbine outage. The following tasks were performed:

- Pyrometer calibration checked and adjusted

- Alignment flanges re-installed and aligned

- Computer connectivity to the GE network re-established

- Pyrometer connectivity to data acquisition systems confirmed

- Bucket indexing performed

MS7001FA+E Gas Turbines

Pyrometers were installed to monitor the stage one buckets on three MS7001FA+E gas turbines. The pyrometers and fiber-optic cables were maintained and calibrated (Figure 14 and Figure 15). The calibrations of the detector electronics were adjusted after cable replacement. Modifications to the Line-Of-Sight (LOS) were also done, shown in Figure 16. 


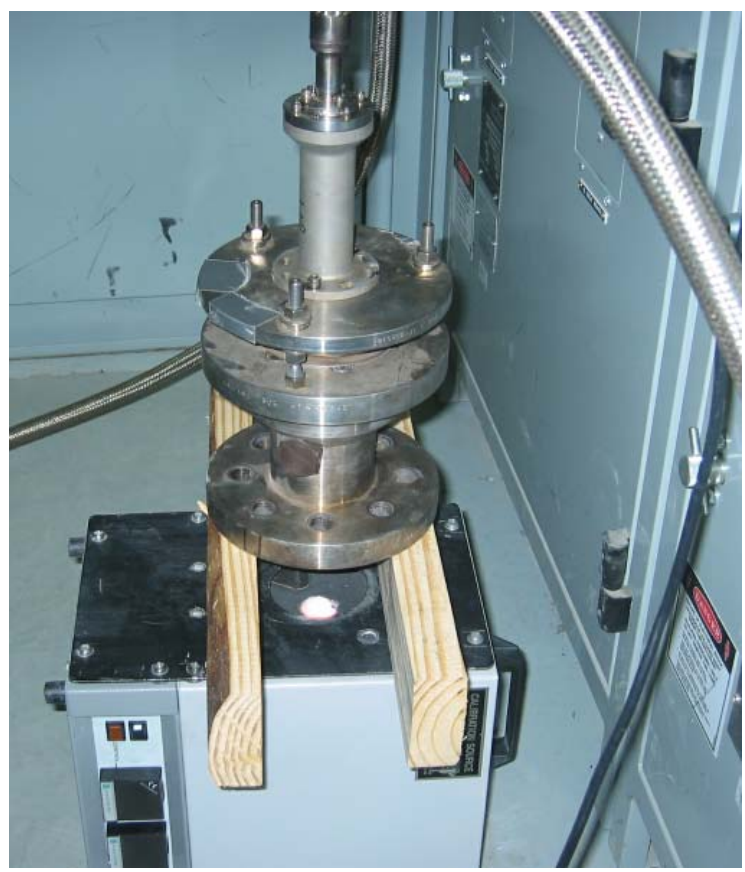

Figure 14: Alignment flange positioned on black body furnace during calibration

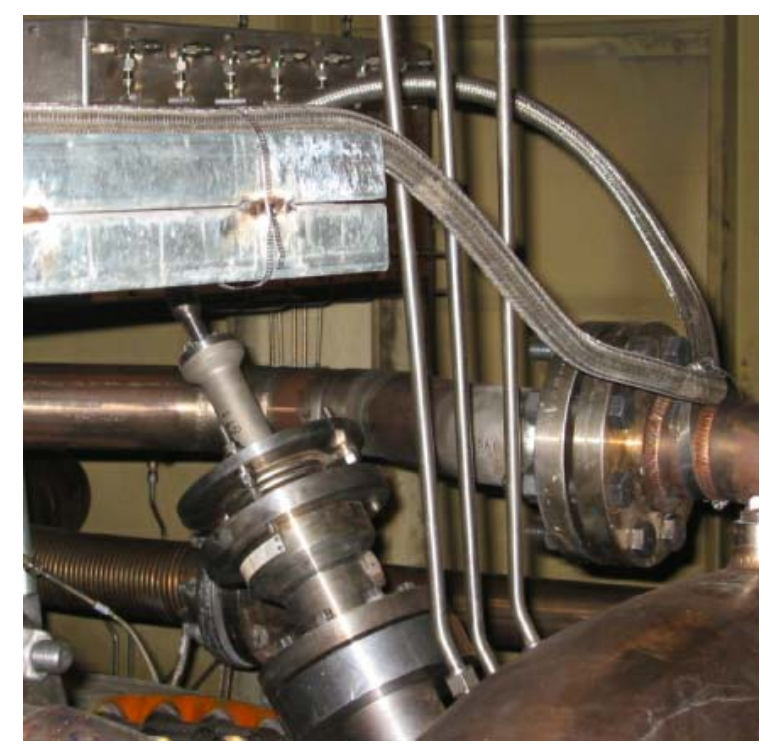

Figure 15: Optical fiber and alignment flange installed on turbine 


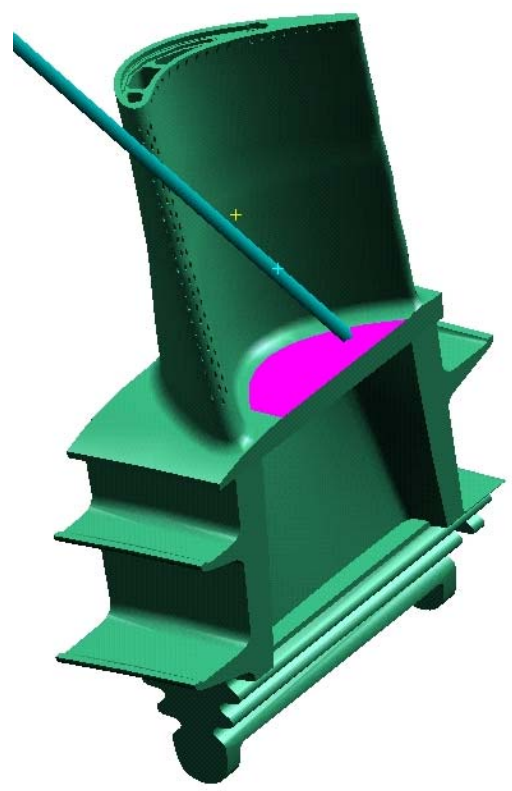

Figure 16: Modified Pyrometer Line-Of-Sight

Boroscope inspections were performed as part of the long-term IR pyrometer data collection activity. Two main tasks were accomplished during these inspections: 1) routine pyrometer maintenance and 2) visual inspection of the Stage 1 buckets via boroscope for comparison with pyrometer and thermal imaging data.

Determining Absolute Temperature and Emissivity using Spectroscopy

Land single wavelength pyrometers were installed on three MS7001FA+E gas turbines. These pyrometers return a calibrated high speed radiance signal to a data acquisition system. Thus we are able to obtain a radiance profile of each bucket along a single line of site. However, the conversion from radiance to temperature assumes that the emissivity of the buckets is 1.0. This is not the case in reality, which leads to an offset in absolute value. Outlined here is a method to determine absolute temperature without a priori knowing the emissivity of the buckets.

The IR radiance $(\mathrm{R})$ emitted by the first stage buckets (and indeed any hot object) is given by the Plank relation:

$$
R=\frac{\varepsilon \mathrm{C}_{1}}{\lambda^{5}} \frac{1}{\exp \left[\mathrm{C}_{2} / \lambda \mathrm{T}\right]-1} \approx \frac{\varepsilon \mathrm{C}_{1}}{\lambda^{5}} \frac{1}{\exp \left[\mathrm{C}_{2} / \lambda \mathrm{T}\right]}
$$

where $\varepsilon$ is emissivity, $\lambda$ is wavelength, $\mathrm{T}$ is temperature, and $\mathrm{C} 1$ and $\mathrm{C} 2$ are the first and second optical constants equal to $3.74 \mathrm{e} 8$ and $1.44 \mathrm{e} 4$ respectively. The Land pyrometer measures radiance at a fixed wavelength ( $\sim 1 \mathrm{um})$. To determine an accurate temperature, the emissivity of the bucket must be known. By measuring a spectrum from the buckets we obtain radiance as a 
function of wavelength. If this spectra is fit to the Plank relation, the emissivity is an irrelevant scaling factor. However, it should be noted that this is true if it is assumed that the emissivity is a constant spatially on the bucket and a constant with temperature. In the case of the MS7001FA+E gas turbine buckets, both of these are reasonable assumptions, thus it is possible to obtain a temperature from the fitted spectra.

There are several factors involved that complicate the conversion from theory to experimental reality. First, the spectrometer is not able to collect data at the high speed rate of the Land pyrometer. To obtain good statistics, a scan length of several seconds is required. Given the gas turbine is spinning at $3600 \mathrm{RPM}$, the radiance we measure is an average of that emitted by all the buckets. Thus it is only possible to obtain an average temperature for the wheel. The second factor has to do with the fact that there is a temperature gradient across the buckets. Each point that is at a different temperature is emitting a different Planck curve. A sum of Planck curves is not equal to a Planck curve. However, in the analysis this is ignored. Investigation of the errors associated with ignoring this indicates an error of $0.2 \%$.

Reducing the Effect of Lens Fogging on Pyrometer Measurements

Fogging of the pyrometer optical system during on-line operation causes an error. The fogging error was historically measured during the pyrometer maintenance. The measurement setup is shown in Figure 17.
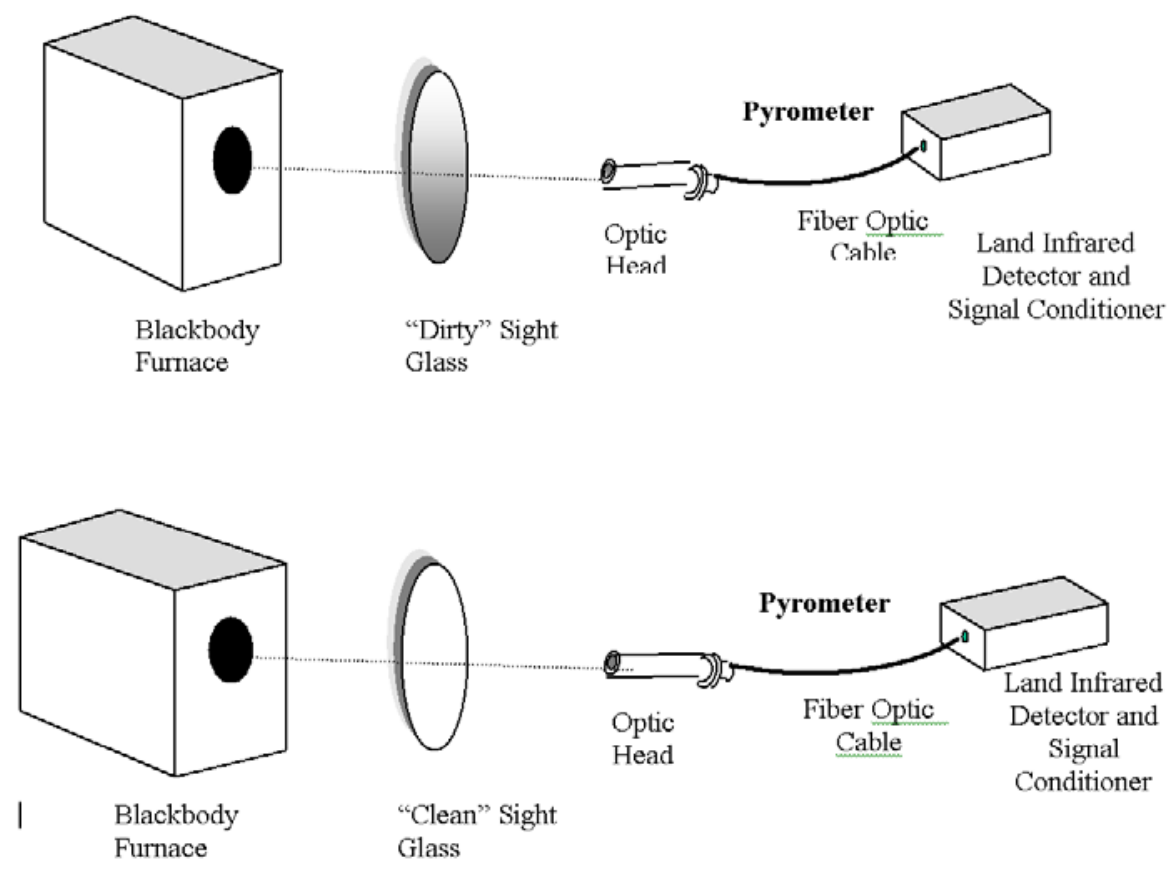

Figure 17: Pyrometer lens fogging error measurement setup

To quantify the error and compensate for it, all data was compiled in a table with fired hours associated with a measured error. A transfer function between the fogging error and fired hours was then developed (Figure 18). Together with regular optical system cleaning at each shutdown, the transfer function will be used to correct the error between maintenances. 


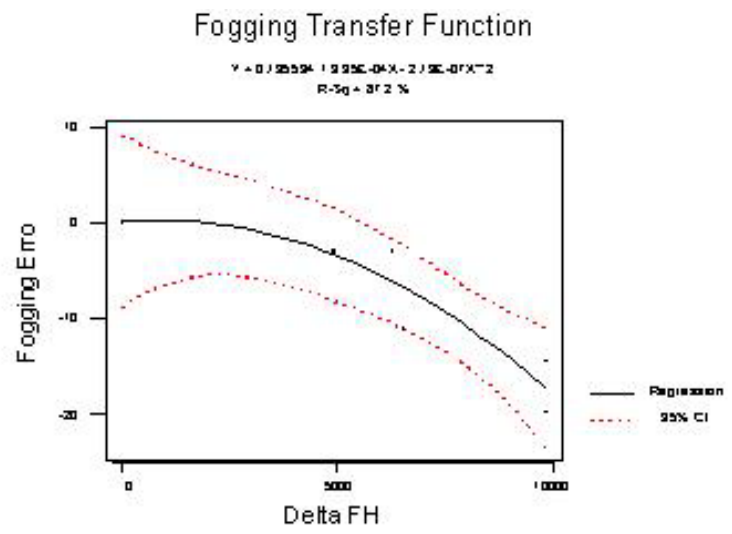

Figure 18: Pyrometer lens fogging error transfer function

Methodology to Predict Temperature of the Buckets

The pyrometer field of view is small compared with the total surface area of the gas turbine buckets. In order to extend the real time temperature measurements to the entire bucket, a UniGraphics LOS utility has been developed to rapidly transfer the pyrometer measurements to a finite element model of the bucket. The flow chart of the utility illustrated in Figure 19.

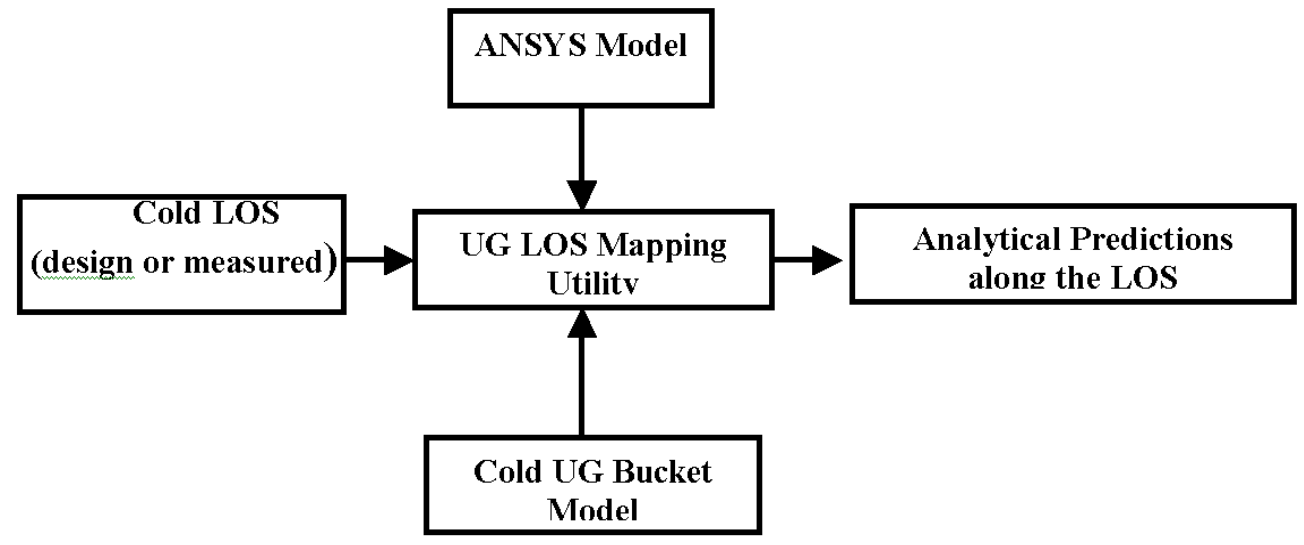

Figure 19: UniGraphics LOS utility flow chart

The quality of the temperature validation is highly dependent on the reliability and fidelity of the analytical models developed to support the surface temperature predictions. Therefore, the initial focus of this effort will be to update the aerodynamic, heat transfer, thermo-mechanical models used to validate the original design configuration. 
The UG LOS utility has been developed with the following capabilities:

$>$ LOS path definition along the blade surface based on predefined LOS vector and UG solid.

$>$ LOS vector definition based on either GUI user input or mouse click position.

$>$ Optical pyrometer signal processing to convert the signal measurements to a surface temperatures.

$>$ Length scale normalization defining the exact position of each of the temperature measurements

$>$ Metal temperature extraction from ANSYS thermo-mechanical solution along a defined LOS path

$>$ Summarization of measured/ analytical temperatures in EXCEL spreadsheet format

$>$ Plot creation of measured vs analytical temperature predictions in normalized length scale

$>$ GUI user interface soliciting the following information: hot-to-cold displacements, steady state rotational velocity, LOS vector coordinates, number of parts, and Filename and path of required UG and ANSYS models/ data

The UG LOS utility was tested and validated. As a result of the validation work, several modifications/ upgrades to the code were necessary, including the following:

$>$ Additional user input required to utilize mouse click LOS definition option

$>$ Modify code logic to improve the extraction of temperature predictions from ANSYS solution

$>$ Increase the number of characters accepted in GUI interface to accommodate longer path names

$>$ Upgrade the code logic utility to accommodate either (1) or (2) shadow geometric configurations

Pre-determining the Thermal Variation of Gas Turbine Buckets

The methodology involves placing individual buckets on a flow check stand, heating them to an elevated temperature, then inducing a transient cooling period by supplying internal cooling air to the bucket, over which time the part is thermally imaged with an Infra-Red (IR) camera to capture the external surface temperature. The measurement apparatus is shown in Figure 20. Analysis of the data is done per the method described in Ref. 2.1.

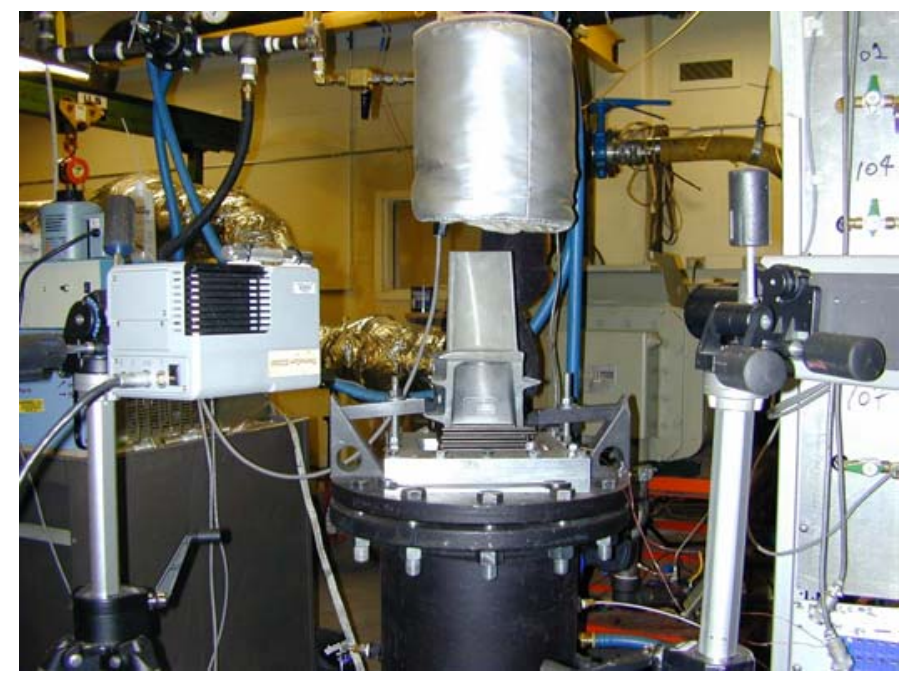

Figure 20: Plenum flow chamber with bucket affixed to the top 
$\underline{\text { Strategy for Bucket Reliability Management }}$

A strategy for identifying bucket anomalies was developed, as illustrated in Figure 21.

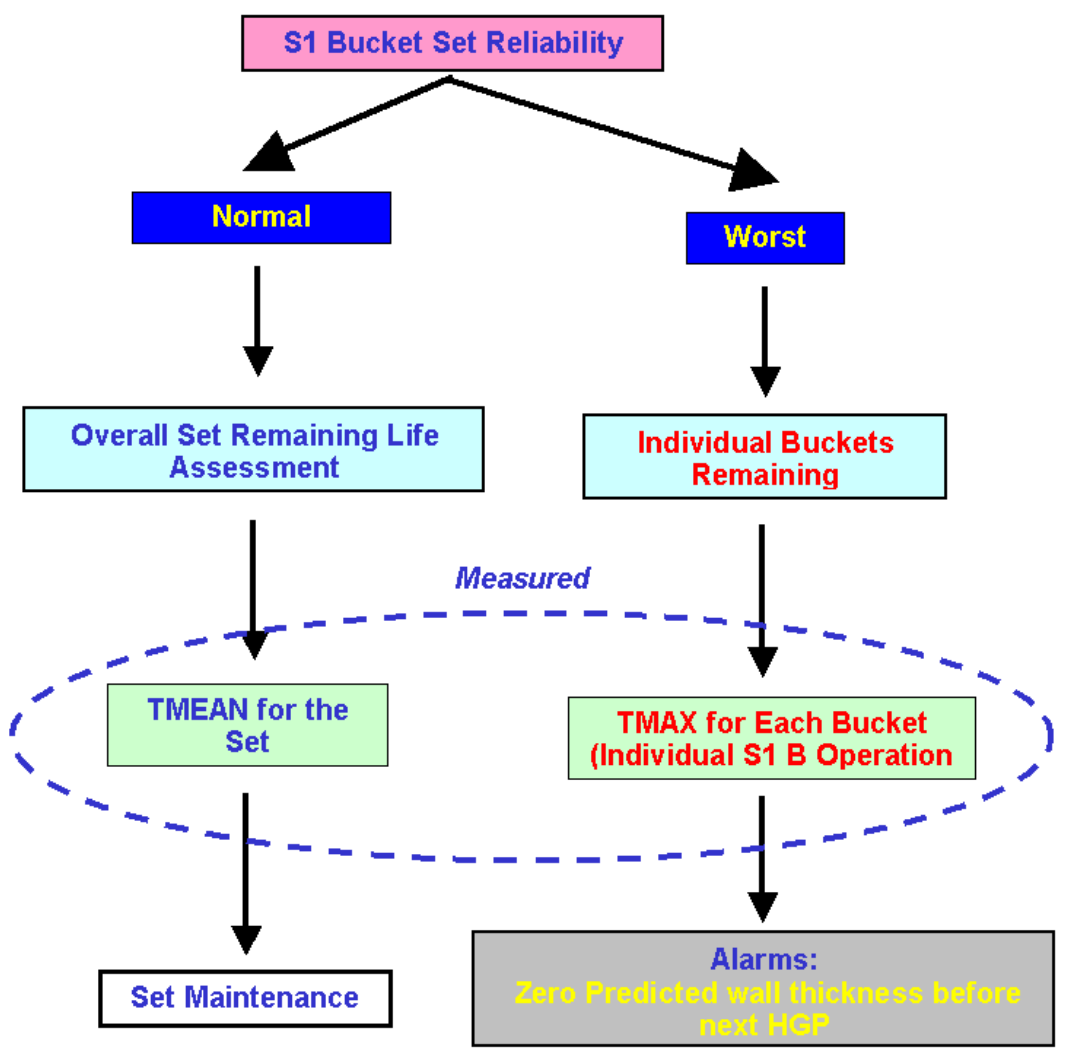

Figure 21: Flow chart of the bucket reliability management strategy using pyrometer measurements

Software was installed on the pyrometer data acquisition computer to communicate anomalies to the gas turbine on-site monitoring (OSM) system. These anomalies are then routed to the GE Energy remote monitoring and diagnostic center in Atlanta, GA. There are two classes of anomalies reported: anomalies with the pyrometer and anomalies with the gas turbine parts. 


\section{Subtask 2.4 - Field Deployment and System Validation}

Fuel LHV Sensor Field Test at Power Systems Development Facility

The fuel LHV sensor was extensively operated on syngas fuels during a field test at the Power Systems Development Facility (PSDF), located in Wilsonville, AL. This is a Department of Energy funded site administrated by Southern Company, and consists of a small-scale gasifier that is used to test different processes of syngas production. Given its research and development role, the entire process is more heavily instrumented than a commercial Coal IGCC plant. This includes a number of gas chromatographs (GCs) measuring the fuel stream constituents, from which an independent value of the LHV was obtained. The PSDF provided a unique environment in which to test the LHV sensor, as the supplementary data was needed to fully evaluate and interpret the sensor's performance with a syngas fuel stream.

The testing had two main goals: 1) evaluation of the reliability of the sensor and 2) evaluation of the accuracy of the sensor output versus that of the GCs. The test was conducted over five days using two different gas streams, during both $\mathrm{O}_{2}$-blown and Air-blown gasifier operation. The first was a low temperature $\left(65.5^{\circ} \mathrm{C}\right)$ gas stream, which had passed through a series of condensing tanks to remove a significant portion of the water vapor and contaminants present in the raw, unconditioned gas stream. The second was a high temperature $\left(260^{\circ} \mathrm{C}\right)$ gas stream from sampling the raw syngas. As the gas was passed through the sensor it was further reduced in temperature to $176.7^{\circ} \mathrm{C}$. Orifices and choked flow were used to regulate the mass flow through the sensor during all tests. The fixture and flow control hardware is shown in Figure 22.

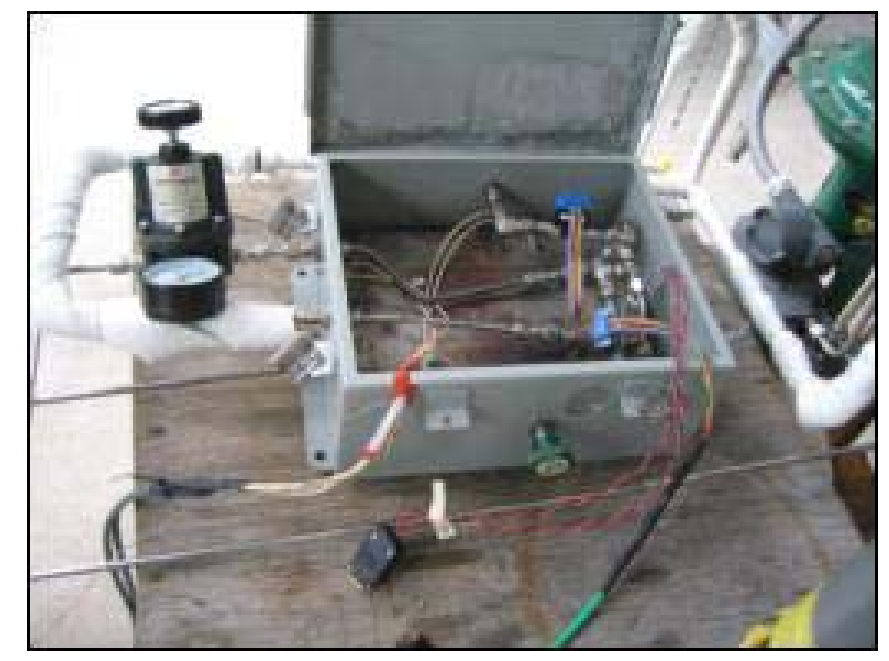

Figure 22: Sensor fixture with orifices and related plumbing

\section{Fuel LHV Sensor Test at Reciprocating Engine Facility}

The fuel LHV sensor was operated on mixed natural gas / propane fuels in a reciprocating engine at GE Jenbacher in Jenbach, Austria. The engine was located in a test cell. Fuel variations for this engine consisted of a mixture ranging from pure city natural gas through to bottled propane. Additionally, it was possible to dilute the fuel stream with carbon dioxide. The sensor was 
installed downstream of the fuel mixing apparatus and upstream of the turbo-charger. Due to the suction of the turbo-charger, there was a small negative pressure in the fuel line to the sensor. To overcome this, a small diaphragm pump was used to provide the backing pressure for the sensor's fuel orifice. Air for the sensor was provided via instrument air. As an independent confirmation of the sensor's performance, the LHV values were compared to those calculated via a GC installed on site.

\section{Fuel LHV Sensor Field Test at Tampa Electric Company Polk 1}

The fuel LHV sensor was operated on syngas fuel during a field test at the Tampa Electric Company (TECO) Polk 1 station located in Mulberry, FL. The goals of this test were:

1. Determine sensor accuracy, repeatability and response time

2. Determine sensor reliability and failure mechanisms

3. Develop field calibration procedures

4. Develop field installation and support procedures

In preparation for this test, a new field test rig for the sensor was developed. The sensor installation is shown in Figure 23. Considerable work was spent on the design of the test rig, to ensure both accurate sensor performance and safe operation for extended periods. The test rig was housed in the analyzer hut where the gas chromatograph and other gas analysis equipment were located. This location was chosen to take advantage of the fuel handling and air handling already installed.

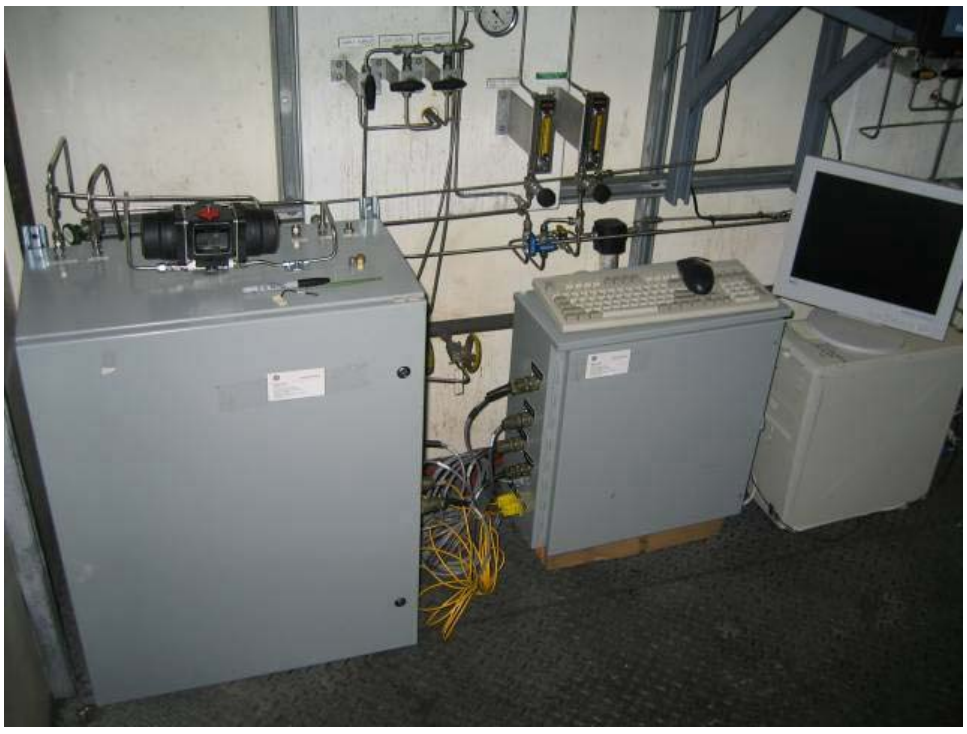

Figure 23: Fuel LHV sensor installation at the TECO Polk 1 station. Shown are sensor enclosure (left), data acquisition enclosure (middle) and data acquisition computer (right). 
To examine the safety aspects of the sensor in detail, a Failure Modes and Effect Analysis (FMEA) review of the system was conducted. Approximately thirty possible failure modes were examined and the severity of each failure examined. A snapshot of the more hazardous failure modes is shown in Table 2. Based on this FMEA, a number of safety issues associated with the operation of the sensor in a commercial environment were addressed.

Table 2: Snapshot of the most hazardous failure modes of the LHV sensor from FMEA

\begin{tabular}{|c|c|c|c|c|c|c|c|c|}
\hline $\begin{array}{l}\text { Process } \\
\text { Step/Part } \\
\text { Number }\end{array}$ & Potential Failure Mode & Potential Failure Effects & $\begin{array}{l}S \\
E \\
V\end{array}$ & Potential Causes & $\begin{array}{l}\mathrm{o} \\
\mathrm{c} \\
\mathrm{c}\end{array}$ & Current Controls & $\begin{array}{l}D \\
E \\
T\end{array}$ & $\begin{array}{l}\mathrm{R} \\
\mathbf{P} \\
\mathrm{N}\end{array}$ \\
\hline Gas Supply & Gas Leakage & $\begin{array}{l}\text { Leak combustable gas into } \\
\text { enclosure. Explosion risk. }\end{array}$ & 10 & Piping rupture / loose fittings & 3 & None & 9 & 270 \\
\hline Gas Supply & $\begin{array}{l}\begin{array}{l}\text { Flame propogates upstream } \\
\text { (flashback) }\end{array} \\
\end{array}$ & $\begin{array}{l}\text { Burning of upstream } \\
\text { components. Gas leaks. } \\
\text { Explosion risk. }\end{array}$ & 10 & $\begin{array}{l}\text { Loss of pressure or pressure } \\
\text { oscillations in gas supply }\end{array}$ & 3 & Pressure regulator & 9 & 270 \\
\hline Gas Supply & $\begin{array}{l}\text { Water or Liquid Fuel } \\
\text { (condensate) in Gas Line }\end{array}$ & $\begin{array}{l}\text { Inaccurate LHV / Wobbe Index } \\
\text { values. Degrade sensor or } \\
\text { electronics. Explosion risk. }\end{array}$ & 7 & $\begin{array}{l}\text { Installation and startup / } \\
\text { shutdown of system }\end{array}$ & 5 & None & 7 & 245 \\
\hline Gas Supply & Contaminants in Gas Line & $\begin{array}{l}\text { Damaged gas valves or } \\
\text { sensor / potential leaks }\end{array}$ & 7 & $\begin{array}{l}\text { Uncleaned fuel gas piping, } \\
\text { damaged fuel filter }\end{array}$ & 3 & Gas Filter & 7 & 147 \\
\hline
\end{tabular}




\section{$\underline{\text { Task } 3 \text { - Advanced Methods for Combustion Monitoring and Control: }}$}

\section{Sub-Task 3.1 - Physics-based Combustion Modeling}

The model development process consisted of both analytical and experimental components. An analytical framework was devised using fundamental conservation laws, and from this a model was synthesized. In the case of combustion dynamics in a gas turbine, several simplifying assumptions were made to render a tractable set of equations. The simplified model was then subjected to experimental validation. The following section describes the experimental techniques used to acquire data for model validation. Comparison of validation data with model predictions is shown in the results and discussion section.

Experiments were performed using an existing laboratory-scale combustor. The combustor scale makes it suitable for testing individual nozzles from a multi-nozzle can combustion system. The experimental device is traditionally used for dry low-NOx (DLN) nozzle development. The single-nozzle rig (SNR) was chosen for performing combustion dynamics validation and control experiments because it contains many design features relevant to full-scale can combustors and can be operated at a small fraction of the cost. Specific features relevant to full-scale combustors include reverse flow liner, similar flame stabilization characteristics, and ability to run under simulated full-load conditions of an F-class turbine.

Figure 24 shows a schematic diagram of the SNR combustor facility. Preheated air enters the combustor test section after passing through passages formed by a reverse flow baffle.

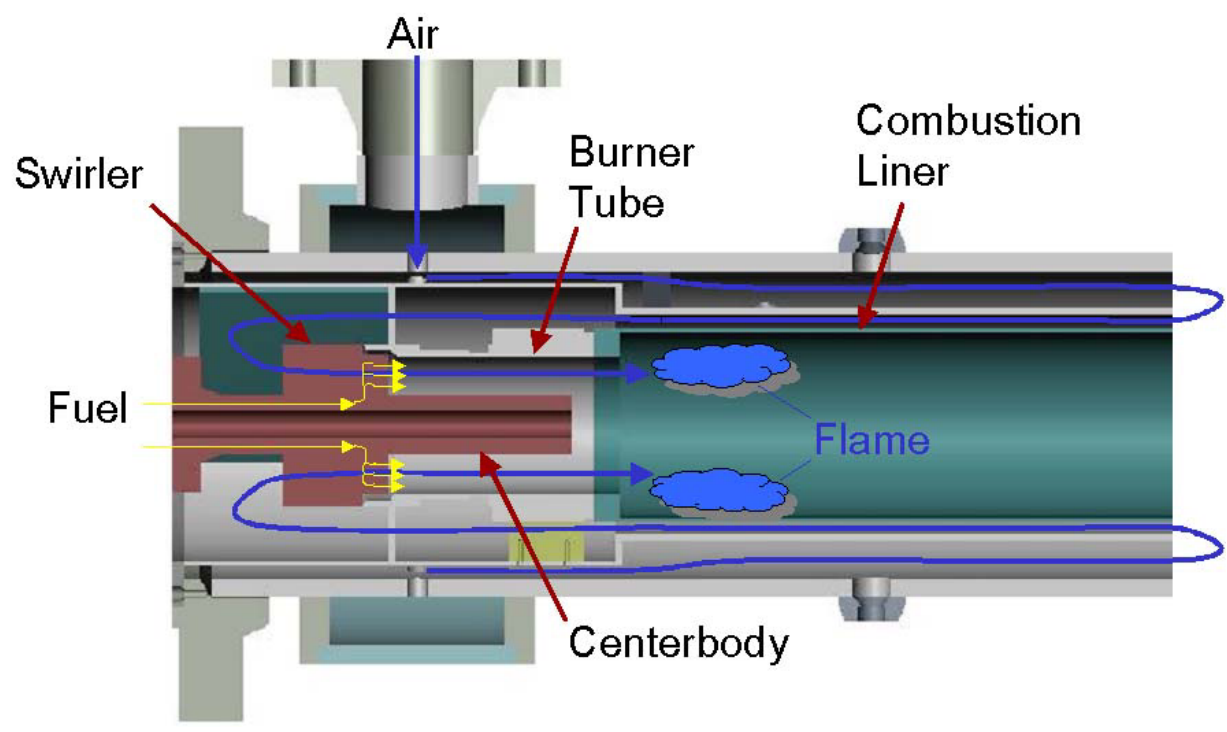

Figure 24: Schematic diagram of single-nozzle combustion rig 
This airflow arrangement allows cooling of the combustion liner by the incoming primary combustion air without need for secondary cooling flow. After passing through the reverse flow passages, the air enters a plenum section before passing through a fuel premixer. The premixer consists of an annular flow passage with a set of swirl vanes to create vortical motion in the flow before it enters the combustor. Fuel is injected into the swirling flow through a set of faired fuel spokes protruding radially from the premixer centerbody (see Figure 25).

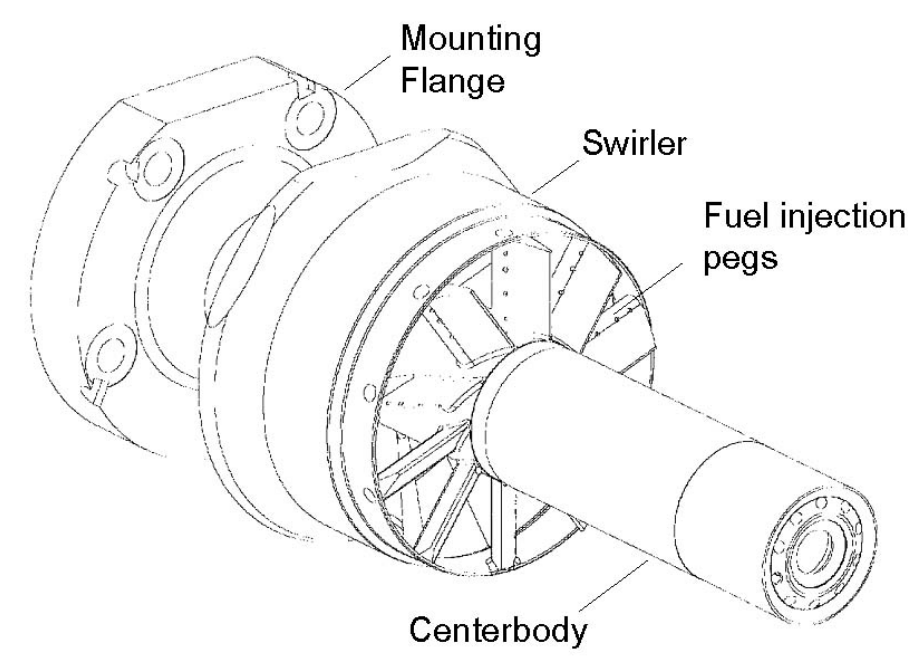

Figure 25: Fuel premixer used in single-nozzle experiments

The well-mixed fuel-air stream enters a combustion chamber with a sudden flow expansion near the upstream end to facilitate flame stabilization. Typical air and fuel flow rates for the facility are $\mathrm{m}_{\text {air }}=3$ to $5 \mathrm{lb}_{\mathrm{m}} / \mathrm{s}$ and $\mathrm{m}_{\text {fuel }}=0.1$ to $0.17 \mathrm{lb} / \mathrm{s}$, respectively. The air and fuel flows are measured with venturi flow meters. The air and fuel flow measurements uncertainties are typically $3 \%$ and $1.3 \%$, respectively. The uncertainty in fuel-to-air ratio is typically $3 \%$.

The SNR is fitted with several probes to detect pressure oscillations associated with combustion dynamics. The probes consist of fast response pressure transducers (PCB Model 102M43) coupled to sense tubes that protrude into various places inside the facility flow path. The sense tubes are $1 / 4$ in diameter stainless steel and are terminated with a long coil of copper tubing to suppress natural resonance in the frequency range where combustion dynamics typically occur $(f>1 \mathrm{~Hz})$. The pressure probes were distributed along the flow path and combustion chamber to allow identification of resonant acoustic standing waves that are excited during combustion dynamics events.

Data from the experiments were acquired using two separate recording systems. Pressure, temperature and flow rate data were acquired using a PC-based data acquisition system. The data logging rate on this system is approximately $3 \mathrm{~Hz}$ has the capacity to read approximately 100 data channels at this rate. The fast response pressure transducer signals are recorded using a digital tape recorder with a bandwidth of $10 \mathrm{kHz}$. 
The SNR facility and pressure measurement systems described in the preceding paragraphs were used to perform combustion experiments to determine the characteristics of thermo-acoustic instabilities (combustion dynamics) under various operating conditions. The data derived from these experiments were used to validate a combustion dynamics modeling tool. The dynamics modeling tool, the validation results and the control scheme to be developed later in the program are described in the following section.

\section{Sub-Task 3.2 - Development of Control Methods}

Development and testing of a fuel valve for combustion control applications was performed. The valve was implemented in the single nozzle rig, described under sub-task 3.1, to examine its effectiveness as an actuator element for active control of combustion dynamics. In addition, valve flow capacity and transient response characteristics were examined in the context of its use as an IGCC fuel control valve. The experimental arrangement is described in the following section and results from cold flow bench tests and combustion control tests are given in the results and discussion section.

To enable fuel flow rate modulation for control experiments, the fuel supply system was fitted with a fast response fuel valve (see Figure 26). The valve was designed to allow modulation of the total fuel mass flow to a single premixer nozzle. The valve seat incorporated a spring-loaded assembly that could be displaced from its mean position by activating a voice coil linear actuator (BEI Model LA-30). This valve action was effective in creating periodic variations in the total fuel flow passing through the premixer while maintaining a constant time averaged flow. The time-averaged flow could be adjusted by supplying a DC offset to the voice coil or by adjusting the spring preload on the valve seat.

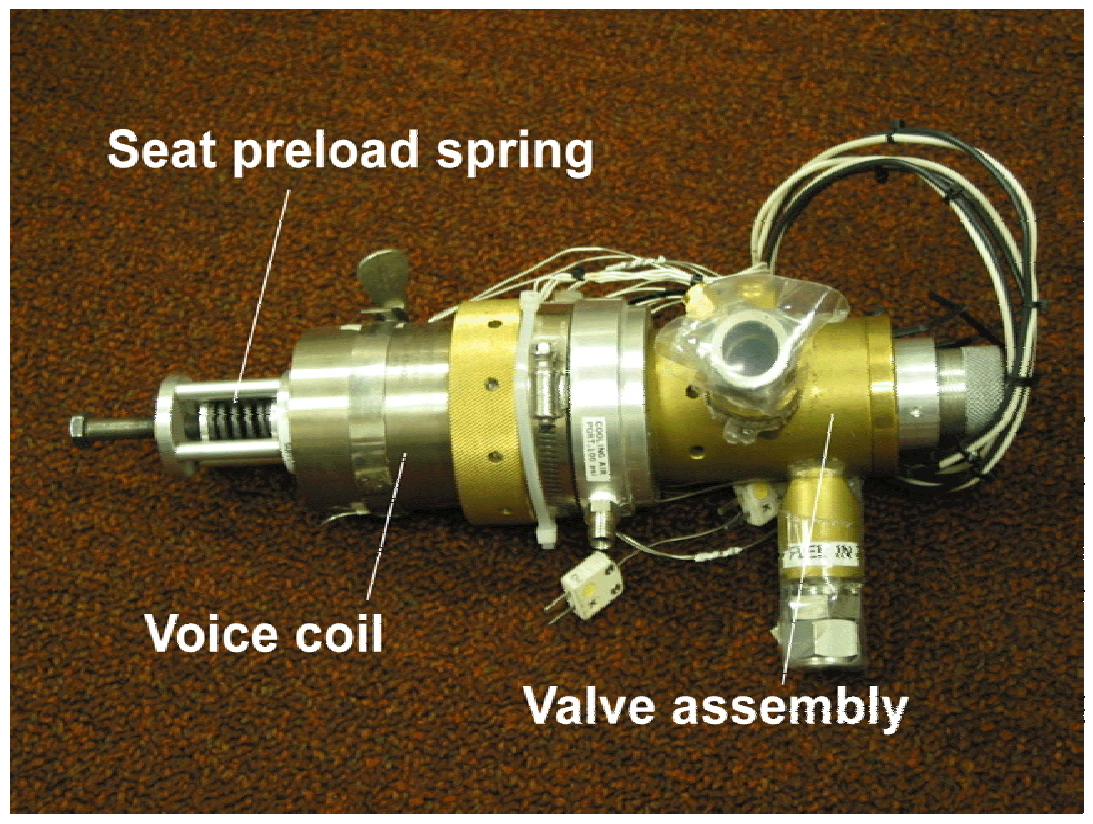

Figure 26: Fast response fuel valve used for control experiments 
The valve was positioned approximately $10 \mathrm{~cm}$ upstream from the premixer mounting flange, which incorporates the premixer fuel inlet passages (Figure 27). The time-averaged flow through the valve was measured far upstream from the valve/premixer assembly using an orifice flow meter. The frequency response of the valved premixer was measured on a bench using a calibrated hot-film anemometer (TSI, Model IFA100) that detected the fuel pulse amplitudes at the fuel injection orifices. The hot film instrument had a frequency response of approximately DC to $20 \mathrm{kHz}$ and this response range was more than sufficient for the present experiments where fuel modulation frequencies were limited to $350 \mathrm{~Hz}$. The hot film element was placed in close proximity to a fuel injection orifice (see Figure 28). The hot film output was analyzed to determine the frequency response of the valve/premixer assembly. Additionally, the unsteady pressure in the flow line between the valve and the premixer was measured using a fast response pressure transducer (PCB model 112A). This allowed measurement of the response transfer function, $R=u(t) / p(t)$, across the premixer. The signals from the pressure transducer and the hot film anemometer were acquired using an HP signal analyzer (HP, model 35639A), which was used to produce Bode plots of frequency response and transfer functions between the two signals.

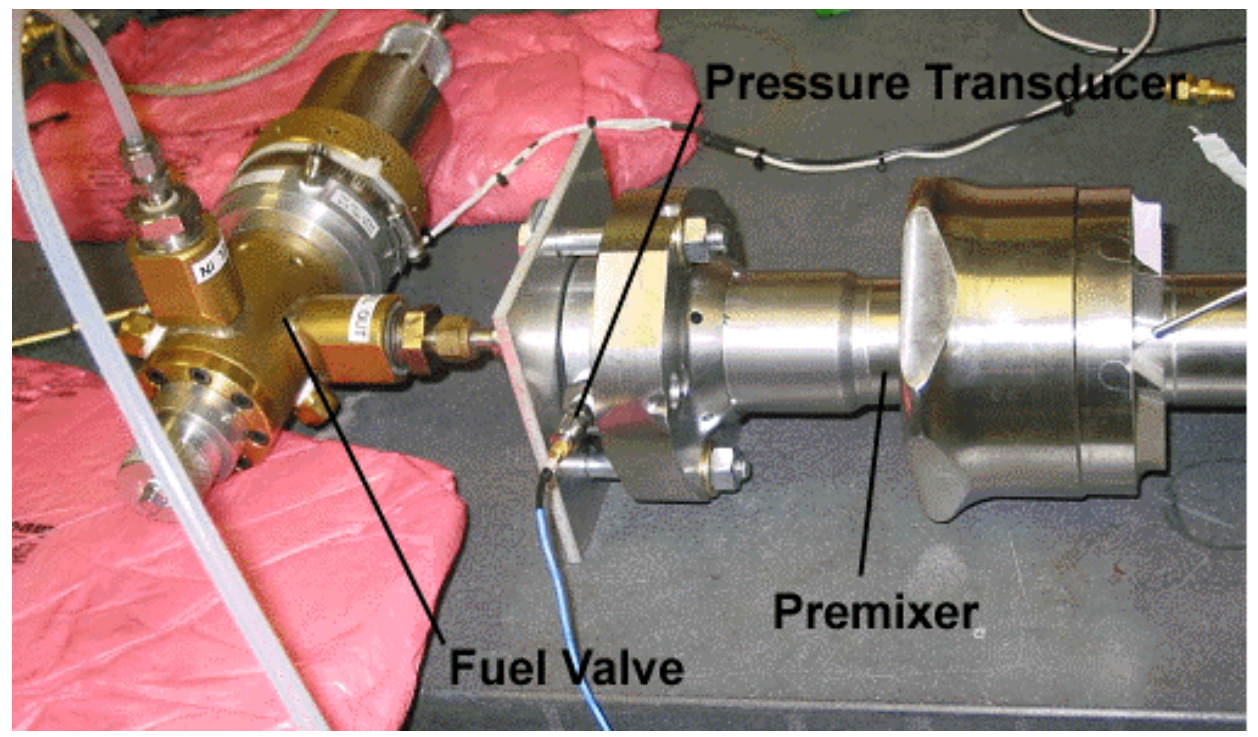

Figure 27: Fast response fuel actuator coupled to DLN 2 premixer 


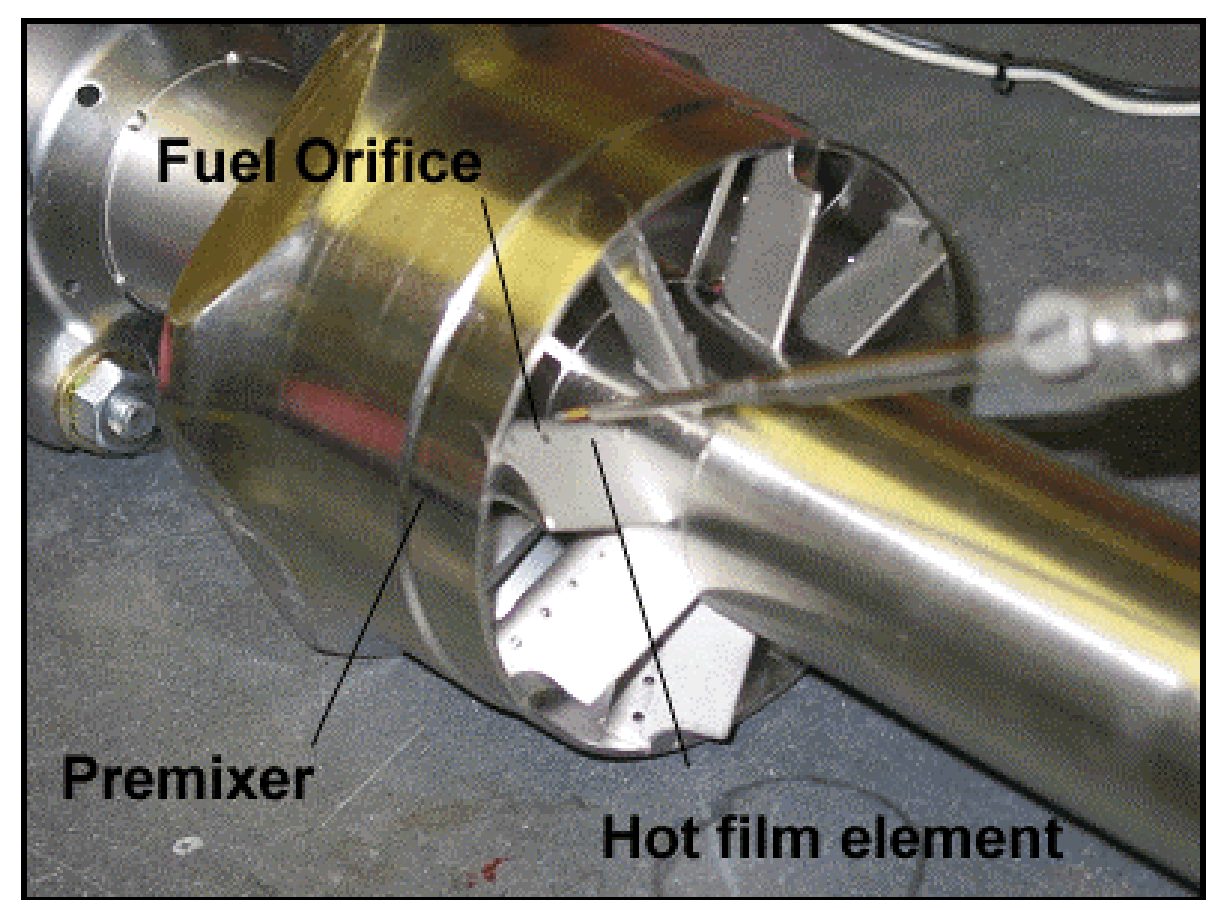

Figure 28: Hot film probe positioned near premixer fuel orifice

The combustor used for the control experiments was identical to that described under sub-task 3.1. Preheated air enters the combustor test section after passing through passages formed by a reverse flow baffle. This airflow arrangement allows cooling of the combustion liner by the incoming primary combustion air without need for secondary cooling flow. After passing through the reverse flow passages, the air enters a plenum section before passing through a fuel premixer. The premixer consists of an annular flow passage with a set of swirl vanes to create vortical motion in the flow before it enters the combustor. Natural gas fuel is injected into the swirling flow through a set of faired fuel spokes protruding radially from the premixer center body. The well-mixed fuel-air stream enters a combustion chamber with a sudden flow expansion near the upstream end to facilitate flame stabilization. Typical air and fuel flow rates for the facility are $\mathrm{m}_{\text {air }}=3$ to $5 \mathrm{lb}_{\mathrm{m}} / \mathrm{s}$ and $\mathrm{m}_{\text {fuel }}=0.1$ to $0.17 \mathrm{lb} / \mathrm{s}$, respectively. The air and fuel flows are measured with venturi flow meters. The air and fuel flow measurements uncertainties are typically $3 \%$ and $1.3 \%$, respectively. The uncertainty in fuel-to-air ratio is typically $3 \%$. The combustion gases pass through a 30 in long cylindrical chamber before passing through a watercooled exhaust nozzle. The nozzle accelerates the gasses to approximately $M=0.3$ before they are expelled into an exhaust plenum. The plenum then leads to a choked backpressure valve.

The SNR facility and pressure measurement systems described in the preceding paragraphs were used to perform combustion experiments to determine the characteristics of thermo-acoustic instabilities under various operating conditions. The data derived from these experiments were used to validate a combustion dynamics modeling tool. In addition, the response of the combustion system to external fuel supply perturbations was examined. 


\section{Task 4 - Information Technology (IT) Integration:}

\section{Sub-Task 4.1 - Performance Modeling for Coal/IGCC Powerplants}

To extend the GateCycle ${ }^{\mathrm{TM}}$ software to model complete IGCC power plants, it was necessary first to rework the database foundation in the code and enable the code to be restructured to eliminate the data structure limitations that were preventing major extensions from being made to the analysis and property calculations. The database system embedded in the current commercial release of GateCycle ${ }^{\mathrm{TM}}$ is based on EASE+, a commercial, proprietary flat-file binary database system that is no longer viable or supported. A SQL-based database system was selected as a more robust and extensible platform that would enable the code structures to be extended to support modeling of IGCC technologies. The SQL framework selected was Microsoft MSDE, since GateCycle ${ }^{\mathrm{TM}}$ is a Microsoft Windows ${ }^{\mathrm{TM}}{ }^{-b a s e d}$ application, MSDE has no run-time license fees, and MSDE is upgradeable to an enterprise-level, multi-user database (Microsoft SQL Server).

The basic functionality of the SQL database for GateCycle ${ }^{T M}$ has been completed and verified. The current functionality of the EASE + version of GateCycle ${ }^{\mathrm{TM}}$ has been reproduced, while at the same time making sure that the architecture and design framework will support the needed IGCC extensions. All of the basic database functionality is now in place in the MSDE version of GateCycle ${ }^{\mathrm{TM}}$; that is, models can be created, run and saved. In addition, basic import and export functionality via XML has been implemented and tested. The import of existing GateCycle ${ }^{\mathrm{TM}}$ models (importing directly from the current EASE+ database system) to the new SQL database version has been verified and tested.

The methodology of the extension of the database structures in GateCycle ${ }^{\mathrm{TM}}$ to support the modeling extensions of IGCC technologies has been completed. Specifically, a new GateCycle $^{\mathrm{TM}}$ modeling data structures has been selected utilizing a new GUI interface based on Microsoft .Net architecture and a new universal Stream Function Icon added, which would enable support of increased number of gaseous constituents (the limit in the current database implementation is 11 constituents in each gas stream), and support property calculations of solid streams, and mixed streams (gas/solid, gas/liquid and liquid/solid). The task of evaluating external property databases and code sets for the property extensions that need to be integrated into the GateCycle ${ }^{\mathrm{TM}}$ database and analysis framework to support the calculations for solid properties and sulfur-removal chemical process modeling has been completed. Several alternatives were considered to incorporate an existing thermodynamic property package for modeling IGCC components. It was decided that the quickest approach would be to convert and use PC DOS version of PRO ASPEN/PC software property package available though P\&P Innovative Software Inc.

A list of IGCC process variables and heat balance diagram showing their locations were prepared, as shown in Figure 29 and Table 3. This was necessary for the collection of data from the Tampa Electric Company (TECO) Polk 1 powerplant to support the IGCC components process modeling into the GateCycle ${ }^{\mathrm{TM}}$. A model of TECO Polk 1 on the current GateCycle ${ }^{\mathrm{TM}}$ was constructed, as shown in Figure 30. This was needed for integration and validation of the complete IGCC model, and was checked and verified against existing plant operating data. 


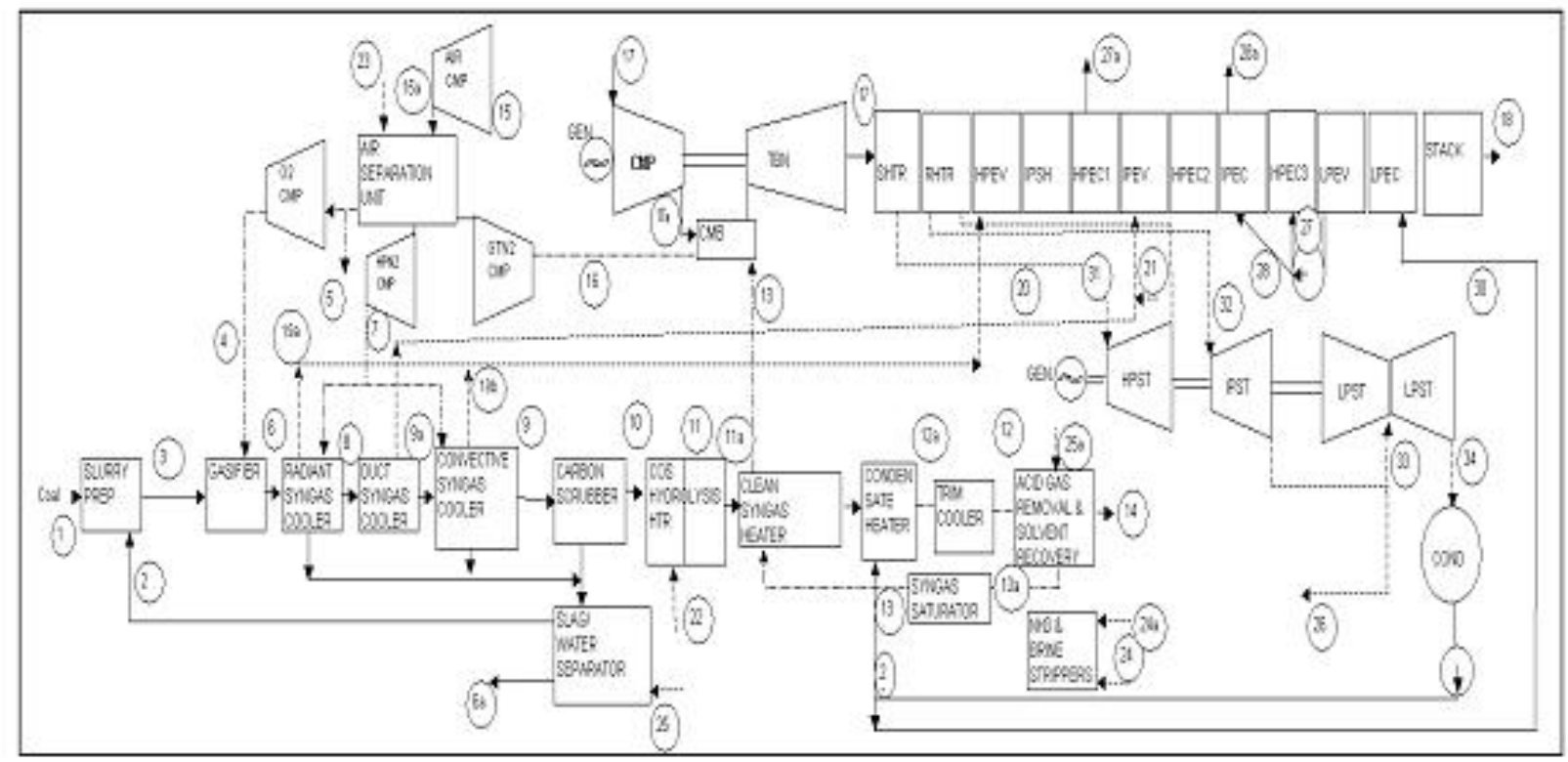

Figure 29: TECO Polk 1 IGCC Heat Balance Diagram and Process Variables

$\ddot{z}=$

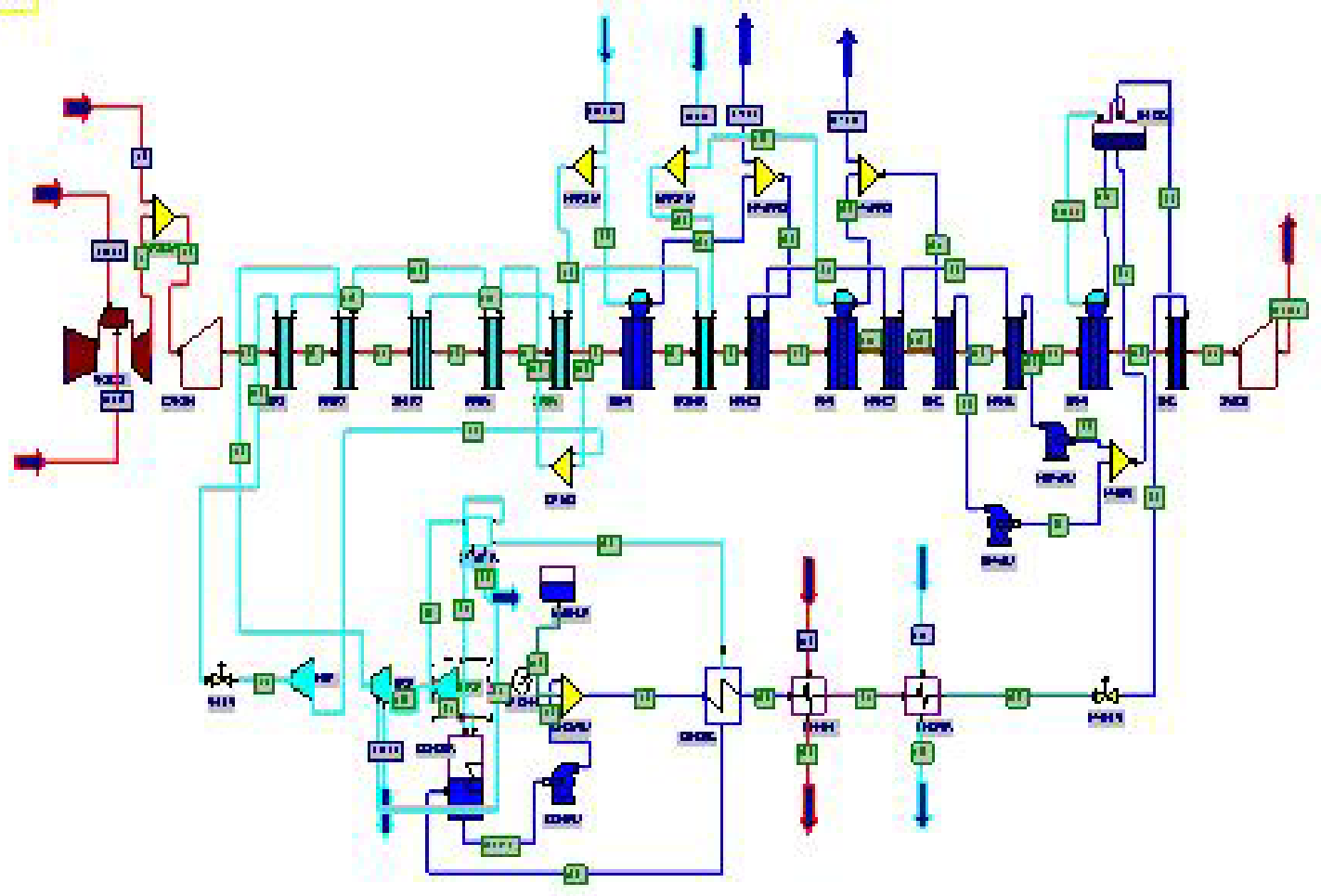

Figure 30: TECO Polk 1 IGCC Power Plant GateCycle Model 
Table 3: TECO Polk 1 IGCC Heat Balance Diagram and Process Variables

\begin{tabular}{|c|c|c|c|c|c|c|}
\hline atrame & Desription & Fbwkilbhr & Presyre paid & Te npotheF & 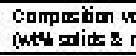 & $\begin{array}{l}\text { of Pow Cong mpion IW } \\
\text { ures) }\end{array}$ \\
\hline 1 & Cogl To Jumy Prap & $x$ & $x$ & $x$ & 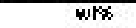 & Gumy Pump \\
\hline$z$ & Penotefine & $x$ & $x$ & $x$ & w/s & Proj土 Pump \\
\hline 3 & aumy fead azita & $x$ & $x$ & $x$ & whs & \\
\hline 4 & ongon To Gziter & $x$ & $x$ & $x$ & vols & $0 z$ compressor \\
\hline 5 & Ooyjor To 3ultur Remby & $x$ & $x$ & $x$ & vols & \\
\hline 6 & Syroz cosila Exit & $x$ & $x$ & $x$ & vols & \\
\hline 6 & and Fine Disptel & $x$ & $x$ & $x$ & vols & \\
\hline$\overline{7}$ & HP Puाए Hitrog & $x$ & $x$ & $x$ & uds & HPGAM Compressor \\
\hline $\mathbf{z}$ & Pulint Contergzaxit & $x$ & $x$ & $x$ & vols & \\
\hline 50 & Duntcond Gra Ext & $x$ & $x$ & $x$ & usis & \\
\hline 9 & Convertie contr Gzexì & $x$ & $x$ & $x$ & uds & \\
\hline 10 & 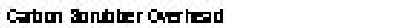 & $x$ & $x$ & $x$ & vols & Equber crc Pump \\
\hline 11 & Cod Hydralysic Ges htet & $x$ & $x$ & $x$ & vels & \\
\hline $11 \mathbf{a}$ & Cod Hydrdysis Cos Ext & $x$ & $x$ & $x$ & vols & \\
\hline 12 & Synz AQRIIIt & $x$ & $x$ & $x$ & vols & Lean EoluenI Rump \\
\hline 128 & 3yr Condenctr Hetrexit & $x$ & $x$ & $x$ & vols & \\
\hline 13 & 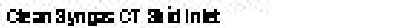 & $x$ & $x$ & $x$ & vols & \\
\hline 13 & Cten Iyntos MQR EXt & $x$ & $x$ & $x$ & uds & \\
\hline 13 & 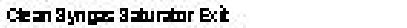 & $x$ & $x$ & $x$ & vols & Ealusabr Pump \\
\hline 14 & AER Adid GX To TRU & $x$ & $x$ & $x$ & udis & \\
\hline 15 & hetsir To cau co mpresar & $x$ & $x$ & $x$ & vols & \\
\hline 15 & cou Comprear Exit & $x$ & $x$ & $x$ & vols & AEU Alr compressor \\
\hline 16 & Dìuet Hitrugen Ta cT & $x$ & $x$ & $x$ & vols & DGAM COmpRESOR \\
\hline 17 & Combution Turbire Intet is & $x$ & $x$ & $x$ & vols & \\
\hline $17 \mathrm{a}$ & CT Co mp esor Ext & $x$ & $x$ & $x$ & vols & \\
\hline $17 \mathrm{~b}$ & CT Exhast & $x$ & $x$ & $x$ & vols & CT Gererabr \\
\hline 18 & Bad Extaut & $x$ & $x$ & $x$ & vols & \\
\hline 150 & Padint Comterater mexit & $\mathrm{x}$ & $x$ & $x$ & & \\
\hline 156 & Convertie Contrsate mexit & $x$ & $x$ & $x$ & & \\
\hline D] & Dutasc atemexit & $x$ & $x$ & $x$ & & \\
\hline 21 & If atemautur Plart & $x$ & $x$ & $x$ & & \\
\hline$z z$ & of atromTo 000 Urit & $x$ & $x$ & $x$ & & \\
\hline$z$ & UP anam, & $x$ & $x$ & $x$ & & \\
\hline 24 & Uि ateamTo E rine Urit & $x$ & $x$ & $x$ & & Brire connentalor \\
\hline 240 & LP 3itamTa H H\& Gipg & $x$ & $x$ & $x$ & & \\
\hline 25 & 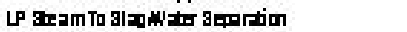 & $x$ & $x$ & $x$ & & \\
\hline 25 & 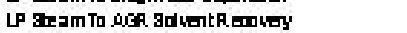 & $x$ & $x$ & $x$ & & AOGR PJdI BotuenI REdre. Pump \\
\hline 25 & up anamTurtine Extration & $x$ & $x$ & $x$ & & \\
\hline $\mathrm{zr}$ & HP FW TO HP Emm mis & $x$ & $\mathrm{x}$ & $x$ & & Bcle feed Pump \\
\hline$z i a$ & HP EFW TOCOS PInt & $x$ & $x$ & $x$ & & \\
\hline $\boldsymbol{z}$ & IP EFW TO IP Enmarim & $x$ & $x$ & $x$ & & \\
\hline$\infty$ & IP EFW TO GX Pbrt & $x$ & $x$ & $x$ & & \\
\hline 요 & Ferd Wtertafromíz Heat & $x$ & $x$ & $x$ & & Feed untekr circ. Aump \\
\hline $\bar{x}$ & 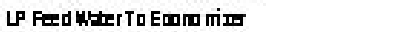 & $x$ & $x$ & $x$ & & condereal Pump \\
\hline 31 & HP atromTo atemTutine & $x$ & $x$ & $x$ & & \\
\hline 표 & P ateamTurtine het & $x$ & $x$ & $x$ & & \\
\hline \multirow{7}{*}{34} & Uf anam Turtine het & $x$ & $x$ & $x$ & & stam Tuthe Gereabr \\
\hline & If atramTurtine Exlaut & $x$ & $x$ & $x$ & & \\
\hline & AGR-ADE Ga RemoGs Uril & & & & & \\
\hline & AEU- Ar Eeparalon Uril & & & & & \\
\hline & 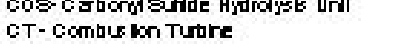 & & & & & \\
\hline & 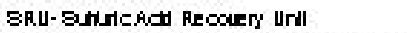 & & & & & \\
\hline & H PGAM - HIDh RESUE Gessous WItogen & & & & & \\
\hline
\end{tabular}

\section{Sub-Task 4.2 - Coal/IGCC Powerplant Data Integration}

The Universal On-Site Monitor (UOSM) has been developed to collect and integrate data from multiple condition monitoring applications at a power plant: turbine-generator controls, plant distributed controls, emissions monitoring, etc. Typical hardware is shown in Figure 31.

A team from GE Energy Services Technology and GE Bently Nevada visited the Tampa Electric Company (TECO) Polk 1 powerplant during a planned shutdown from February 23 to March 3 to upgrade the existing Generation II OSMs to a UOSM configuration. This site is located near Mulberry, Florida. There are three gas turbine units installed: 
- Unit 1 includes a PG72221FA gas turbine and a D11-26 steam turbine. The gas turbine is fueled by syngas from the coal/IGCC gasifier.

- Units 2 and 3 are PG7241FA+e simple cycle natural gas fired gas turbines.

All three units were originally equipped with Gen II OSMs. The plant uses a Distributed Control System (DCS) to acquire Balance of Plant (BOP) data for Unit 1. The three Gen II OSMs were replaced by one UOSM, consisting of two 7765 VME computers, a VMIC hardware rack, and a SCSI drive array; which are located in Unit 1 control cabinet (also known as a PEECC). The hardware cabinet is shown in Figure 32.

The scope of work included networking the three gas turbines, one steam turbine, and the plant DCS to the UOSM. The upgraded data network is shown in Figure 33. The data from the Unit 2 and 3 gas turbines and Unit 1 steam turbine Mark V control systems are acquired via Modhub extensions and optical fiber. The data from the Unit 1 gas turbine Mark V control system are acquired via an existing coax cable. The data from all of the Bently 3500 vibration monitors are acquired by serial connections to the VME computers; Units 2 and 3 are connected via optical fiber. The DCS was previously integrated into the UOSM system, and was not reconfigured.

The following is a summary of the site integration work performed:

$>$ Install and configure serial to fiber converters for fiber runs between PEECC \#1 and PEECC \#2, and between PEECC \#1 and PEECC \#3

$>$ Using fiber media (Modhubs), extend the Stagelink network for Unit \#2 in PEECC \#2, Unit \#3 in PEECC \#3, and the Steam Turbine in the Steam Turbine PEECC to the PEECC \#1

$>$ Using fiber media, extend the serial connectivity from the Bently monitors in the Steam Turbine PEECC, PEECC \#2, and PEECC \#3 to the UOSM location in PEECC \#1

$>$ Test and verify all communications between and among Stagelink and serial devices

$>$ Install the hard drives, cables, and terminating resistors for the SCSI Hard Drive (HD) array; this system will use two 7765 computers and use both channels of the SCSI Hard Drive Array

$>$ Configure the ARCNet interface card for compatibility with the customer Stagelink/ARCNet system

$>$ Start up and configure the UOSM computer presently located in the VME cabinet

$>$ Transfer the appropriate Unit and Prom data from the existing OSM computers to the UOSM computer; combine unit data file information from the various config.dat files

$>$ Reconfigure the existing PSDM data to be provided to the UOSM

$>$ Configure the UOSM to provide data to the GE Atlanta Data Highway (ADH)

$>$ Verify that there is at least a one fiber pair (i.e., two fibers) run between the ST PEECC and PEECC \#1, a distance of approximately 350 feet

$>$ Verify that there is at least a two fiber pair (i.e., four fibers) run between PEECC \#3 and PEECC \#1, a distance of approximately 1,000 feet

$>$ Verify that there is at least a two fiber pair (i.e., four fibers) run between PEECC \#2 and PEECC \#1, a distance of approximately 700 feet 
$>$ Subcontractor will install fiber junction boxes in all four PEECCs to terminate the installed fiber, as needed; fiber runs and terminations will be managed by site personnel

$>$ Provide or install 110 vac power for media converters in each PEECC

$>$ Re-verify data availability to Atlanta via the ADH with OSW and M\&D staff personnel

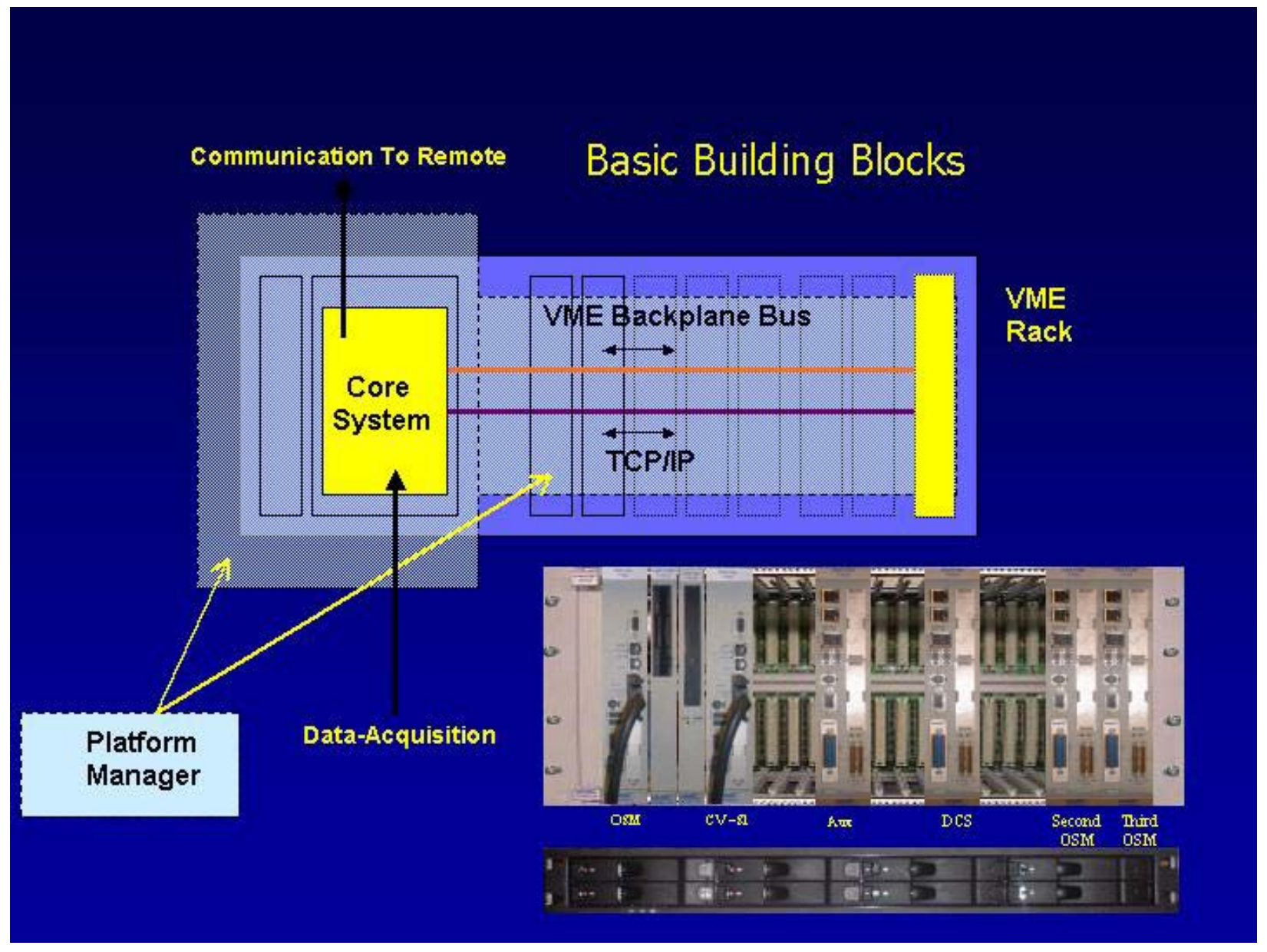

Figure 31: Universal On-Site Monitor hardware (typical) 


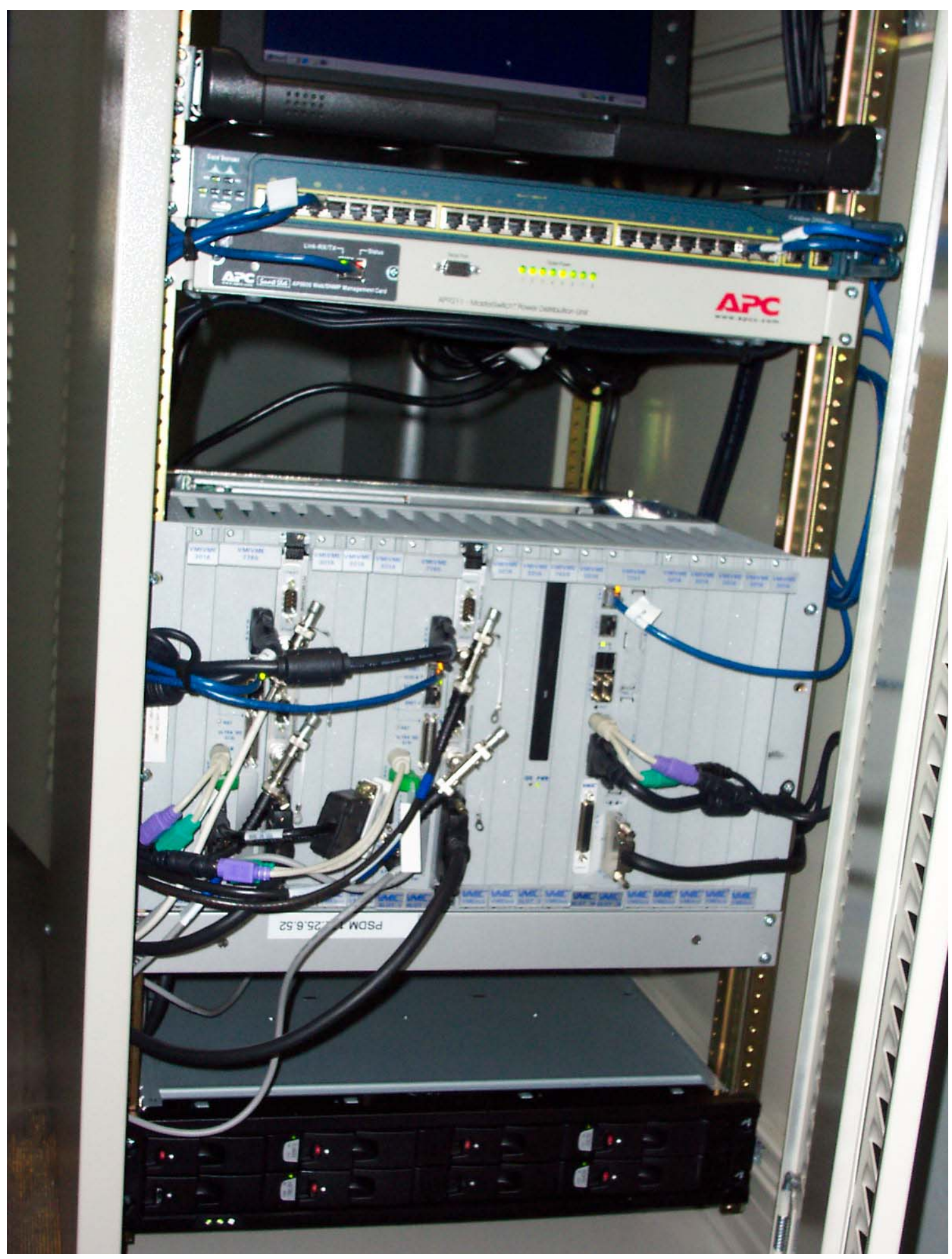

Figure 32: Universal On-Site Monitor hardware at TECO Polk 1 Powerplant 


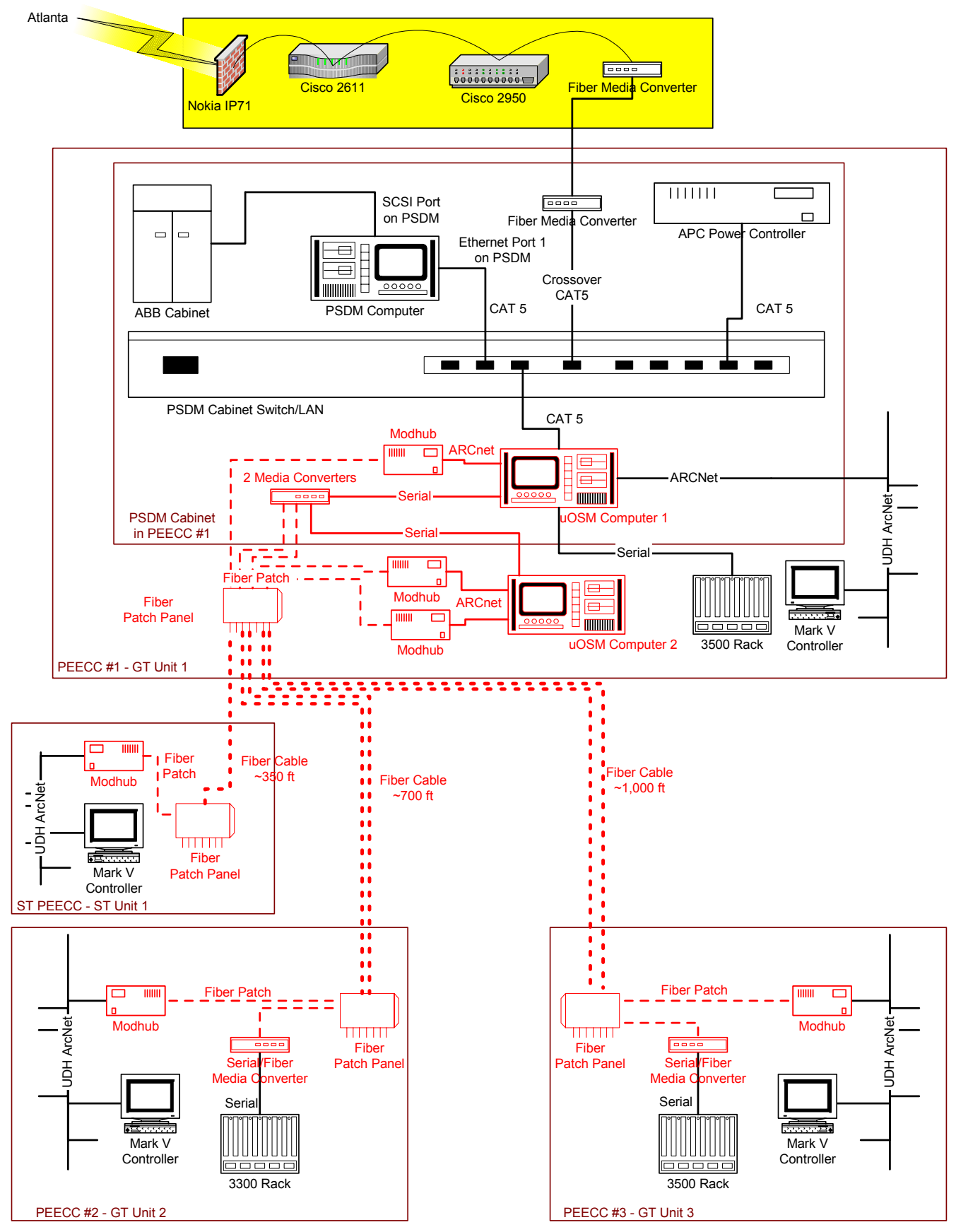

Figure 33: Universal On-Site Monitor Data Network at Polk 1 Powerplant (new hardware and data links are shown in red) 


\section{RESULTS and DISCUSSION}

This section presents a summary and discussion of the results for each sub-task. An overview section is included to clarify the goals of each sub-task.

\section{Task 1 Results/Discussion:}

\section{IGCC Environmental Impact on High temperature Materials}

\section{Task 1 Overview:}

The first task addressed environmental impacts on high temperature materials used in IGCC gas turbines. Material performance and the effects of high temperature erosion and corrosion of hot gas path materials were evaluated. The materials of interest included those in current service as well as advanced, high-performance alloys and coatings.

The fuel used to fire gas turbines for integrated gasification combined cycle (IGCC) applications contains particulates and contaminates that are not present under normal natural gas firing. The important material degradation issues present in IGCC applications change because of the presence of the particulates and contaminates in the fuel as well as high levels of moisture in the combustion gas. The particulates in the fuel cause erosion, and the contaminates and moisture cause corrosion reactions not common to natural gas fired turbines. Erosion and corrosion may also reduce mechanical properties such as fatigue and creep rupture.

The contributors to this task were GE Energy, GE Global Research Center (GRC), Georgia Institute of Technology, and Tampa Electric Company (TECO). The work was directed by GE Energy. Detailed investigations of serviced parts and materials behavior were performed by GRC and Georgia Institute of Technology, respectively. Serviced gas turbine parts and fuel samples were provided by TECO.

Original Scope: The original Task 1 scope consisted of 4 Sub-tasks:

Sub-Task 1.1: Physics based Metallurgical Tool Development: The mechanical behavior of combustor and HGP materials under complex load / transient histories will be characterized for advanced coal/IGCC gas turbine parts. An investigation of the service history of selected gas turbine combustor and HGP parts will be performed to determine those parts that are not meeting design life and/or causing unscheduled maintenance of the gas turbine. Laboratory mechanical property testing will be conducted to characterize the material behavior under the mechanical/thermal/environmental conditions during gas turbine operation and to develop the analytical models in Task 1.2. Parameters will be defined to mathematically correlate the microstructural state to residual mechanical properties and part life via transfer functions, which will be experimentally verified in Task 1.3.

Sub-Task 1.2: Analytical Life Assessment Model Development: Improved constitutive equations of materials behavior will be developed based on the mechanical property data generated in Task 1.1. Lifing models that take into account complex material behavior 
interactions such as creep/fatigue, $\mathrm{HCF} / \mathrm{LCF}$, and hold time effects will be developed to replace the simple linear superposition models currently used today. In addition, improved fracture mechanics models will be studied to address damage tolerance in the lifing of fracture-critical parts.

Sub-Task 1.3: Experimental Verification of Life Assessment Methodology: Transfer functions that correlate the metallurgical state of the materials (i.e. service/exposure history) to residual part life will be experimentally verified. These transfer functions will accurately represent changes in microstructure resulting from service exposure under conditions representative of the gas turbine operating environment, including transient and off-design conditions.

Sub-Task 1.4: Field Validation of Life Assessment Methodology: Combustor and HGP parts will be removed from powerplants and extensively evaluated to validate the predicted part life. The parts will be evaluated using destructive and NDE techniques to determine microstructural changes, damage accumulation and/or failure modes in service. Residual mechanical properties will be measured. These data will ensure that the models developed in Task 1.2 represent the true physics of the materials behavior.

Re-Directed Scope: The re-directed Task 1 scope consisted of 2 Sub-tasks:

Subtask 1.1 - High Temperature Erosion/Corrosion Data Collection: The high temperature erosion and corrosion behavior of a wide variety of materials commonly used in gas turbine buckets, nozzles, and combustor hardware was characterized. Laboratory erosion and corrosion experiments at elevated temperatures were conducted in order to document the environmental effects associated with an IGCC application. Corrosion kinetics were recorded as well as the corrosion product morphology evolution as a function of time, temperature and gas environment. Erosion experiments were conducted at elevated temperatures and the effect of foreign object impact on coatings and native corrosion scales was documented. Mechanical properties experiments were conducted on alloys exposed to erosive and corrosive environments. Fuel and exhaust gas sampling was performed at a coal/IGCC powerplant to develop correlations between the syngas composition and gasifier / gas turbine load level. Additionally, hot gas path (HGP) parts were removed from an IGCC gas turbine for evaluation. The condition of the parts removed from service was correlated to the fuel sampling results.

Subtask 1.2 - High Temperature Erosion/Corrosion Mechanism Quantification: Using the data collected in subtask 1.1 along with micro-structural analysis of virgin materials and service-exposed materials, scale growth mechanisms and erosion effects were determined by comparing to a control. Corrosion scale product was determined along with growth kinetics. Corrosion scale growth and failure were quantified. The effect of erosion on coating performance was determined. Foreign object impact failure mechanism was determined. The effect of environmental exposure (erosion and/or corrosion) on mechanical properties was quantified. 


\section{$\underline{\text { Task } 1 \text { Discussion: }}$}

\section{$\underline{\text { Sub-Task 1.1 - High Temperature Erosion/Corrosion Data Collection }}$}

\section{Environment Effects}

Figure 34 shows a typical batch of specimens as-removed from the reactor following exposure to the test environment. In general, the high temperature scale on most of the specimens was friable and some scale spalled on to the alumina holder. Spalling of scale increased with the longer exposure periods in all three test environments, i.e. air, dry $\mathrm{H}_{2} \mathrm{~S}$, and wet $\mathrm{H}_{2} \mathrm{~S}$.

Individual photographs of the specimens are shown in Appendices A and B. The material is shown in the as received condition in Appendix A, and as tested in Appendix B. The "top" of the specimens is identified in the photographs to indicate which side was "up" in the holder. A distinct band formed on each specimen on its bottom section that was inside the holder. It is possible that the gas flow was somewhat restricted inside the holder and produced differential oxidation/sulfidation on the specimen below the deck area.

In general, the coated specimens appeared to have thinner scales and showed less spalling than the uncoated specimens in all three environments. The uncoated Alloy N5 showed least scaling among the different base alloys. The other five base alloys with thicker scales showed the most spalling. As mentioned earlier, the runs in dry $\mathrm{H}_{2} \mathrm{~S}$ had higher flow rates than the other runs. As a result, those specimens closest to the gas inlet in the dry $\mathrm{H}_{2} \mathrm{~S}$ runs yielded thicker scale and some specimens grew nodules on their leading faces, see Appendix B. It is possible that the gas stream impinged directly on some of the specimens that were in the front section of the holder.

The weight gains in specimens from various runs are given in Table 4, Table 5, and Table 6 for the air, dry $\mathrm{H}_{2} \mathrm{~S}$ and wet $\mathrm{H}_{2} \mathrm{~S}$ environments, respectively. The maximum weight gain (or most loss) for each group of triplicate specimens of the same material exposed in the same run was extracted from the above tables and summarized in Table 7. The maximum weight gain may not reflect the original scale thickness because many specimens experienced spalling and some yielded negative weight gains. Therefore, the weight gain data cannot be used in any quantitative manner, e.g., to develop rate models.

In general, the weight change of specimens exposed to air indicated thinner scales than those exposed to either of the $\mathrm{H}_{2} \mathrm{~S}$ containing environments, see Table 7. The dry $\mathrm{H}_{2} \mathrm{~S}$ environment produced the most weight gain/loss in the specimens, most likely from excessive sulfidation. The wet $\mathrm{H}_{2} \mathrm{~S}$ environment possibly produced oxidation as well as some sulfidation in specimens. Thus, the weight change values of the specimens from the wet $\mathrm{H}_{2} \mathrm{~S}$ environment were relatively closer to that for the corresponding specimens exposed to air.

The coated specimens showed relatively smaller weight gains in all three environments, indicating better resistance to scaling than the corresponding base alloys. The weight gain was comparable in all three coated materials, regardless of the environment. Amongst the base alloys, Alloy N5 performed the best in air, but its performance was not so distinctive in the dry or wet $\mathrm{H}_{2} \mathrm{~S}$ environment. A ranking of the other base alloys cannot be made solely based on the weight change data. 
Photomicrographs of the individual specimens (108) are shown in Appendix C, arranged according to the material, exposure period, and test environment. The metallographic examination often showed non-uniform scale thickness and IGP around the circumference of the specimen because of spalling. The results in the table represent the maximum scale thickness and the maximum IGP observed around the circumference of the individual specimens in the plane examined. In general, the scale thickness measured on specimens exposed to air was less than on the corresponding specimens exposed to the $\mathrm{H}_{2} \mathrm{~S}$ containing environments. This trend is in accord with that reported earlier based on the weight change results.

Alloy N5 exhibited practically the least scale thickness amongst the base alloys, in all three environments. The coated Alloy N5 produced results not much different from the other coated alloys. In general, the coated specimens of Alloys Nimonic-263 and GTD111 exhibited less scaling than the corresponding base alloys, irrespective of the environment. This latter trend was not applicable to Alloy N5. None of the coated specimens exhibited IGP in any of the three environments. The IGP depth affects material strength and, thus, may be more important than the scaling characteristics of the alloys.

No IGP was observed in uncoated specimens of Hastelloy-X either in air or wet $\mathrm{H}_{2} \mathrm{~S}$, but the depth was very significant (IGP $>>10 \mu \mathrm{m}$ ) in the dry $\mathrm{H}_{2} \mathrm{~S}$ environment. The uncoated Alloy N5 specimens behaved similarly, except for the one specimen that was exposed for 300-hours in wet $\mathrm{H}_{2} \mathrm{~S}$. The latter specimen for unexplainable reason contained very deep IGP. The other four base Alloys FSX-414, GTD222, Nimonic-263 and GTD111, all of them experienced considerable IGP in air, wet and dry $\mathrm{H}_{2} \mathrm{~S}$ environments. The dry $\mathrm{H}_{2} \mathrm{~S}$ environment produced the deepest attack in all six alloys.

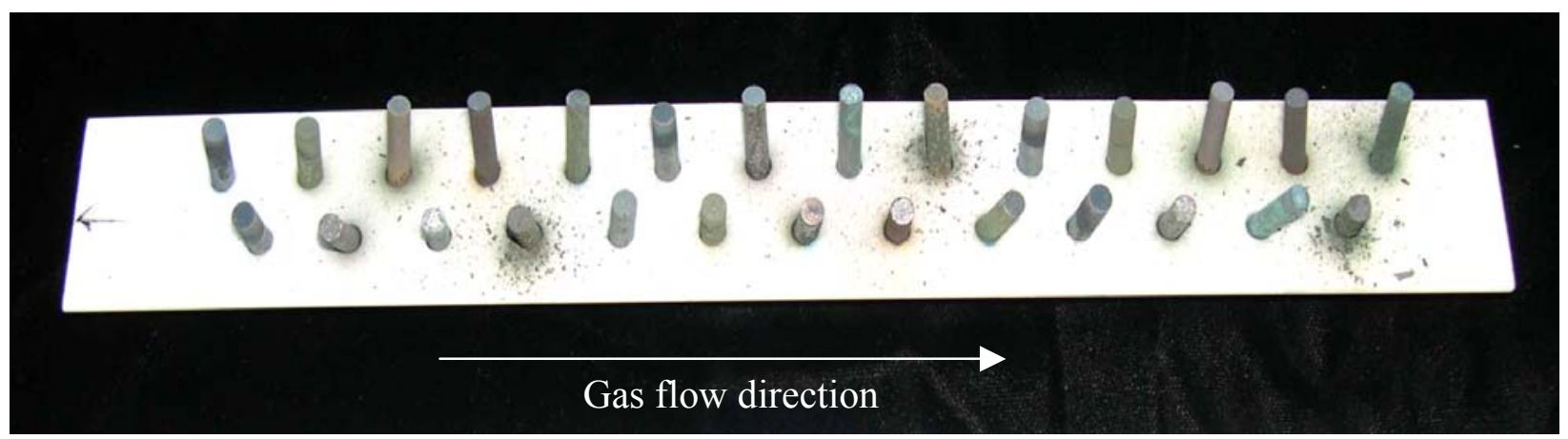

Figure 34: Photograph of Alumina Holder with Specimens After 300-Hour Exposure to Wet $\mathrm{H}_{2} \mathrm{~S}$ Environment at $1900^{\circ} \mathrm{F}$ 
Table 4: Overall Assessment of Scale After Exposure to Three Different Environments at $\mathbf{1 9 0 0}^{\circ} \mathrm{F}$

\begin{tabular}{|c|c|c|c|c|c|}
\hline \multirow{2}{*}{\multicolumn{2}{|c|}{ Material }} & \multirow{3}{*}{$\begin{array}{c}\begin{array}{c}\text { Scale } \\
\text { Assessment }\end{array} \\
\text { Thickness } \\
\end{array}$} & \multicolumn{3}{|c|}{ Environment } \\
\hline & & & Air & Dry $\mathrm{H}_{2} \mathrm{~S}$ & Wet $\mathrm{H}_{2} \mathrm{~S}$ \\
\hline \multirow{3}{*}{ A } & \multirow{3}{*}{ Alloy FSX-414 } & & Thin & Thick & Medium \\
\hline & & Spalling & 100,300 & 300 & 300 \\
\hline & & Penetration & Intergranular & Intergranular & Intergranular \\
\hline \multirow{3}{*}{ B } & \multirow{3}{*}{ Alloy GTD222 } & Thickness & Medium & Thick & Medium \\
\hline & & Spalling & 100,300 & - & - \\
\hline & & Penetration & Intergranular & Intergranular & Intergranular \\
\hline \multirow{3}{*}{$\mathrm{C}$} & \multirow{3}{*}{ Alloy Hastelloy X } & Thickness & Thin & Thick & Medium \\
\hline & & Spalling & 300 & 300 & - \\
\hline & & Penetration & Surface Layer & Intergranular & Surface Layer \\
\hline \multirow{3}{*}{ D } & \multirow{3}{*}{ Alloy N5 } & Thickness & Thin & Thick & Medium \\
\hline & & Spalling & 300 & 300 & 10 \\
\hline & & Penetration & Surface Layer & Surface Layer & Surface Layer \\
\hline \multirow{3}{*}{$\mathbf{E}$} & \multirow{3}{*}{ Alloy Nimonic 263} & Thickness & Medium & Thick & Thick \\
\hline & & Spalling & 300 & 300 & - \\
\hline & & Penetration & Intergranular & Intergranular & Intergranular \\
\hline \multirow{3}{*}{$\mathbf{F}$} & \multirow{3}{*}{ Alloy GTD111 } & Thickness & Medium & Thick & Medium \\
\hline & & Spalling & 300 & 300 & 300 \\
\hline & & Penetration & Intergranular & Intergranular & Intergranular \\
\hline \multirow{3}{*}{$\mathbf{G}$} & \multirow{3}{*}{$\begin{array}{c}\text { Alloy N5 + } \\
\text { NiCrAlY Coating }\end{array}$} & Thickness & Medium & Medium & Medium \\
\hline & & Spalling & - & 10,300 & - \\
\hline & & Penetration & Surface Layer & Surface Layer & Surface Layer \\
\hline \multirow{3}{*}{$\mathbf{H}$} & \multirow{3}{*}{$\begin{array}{l}\text { Alloy Nimonic } 263+ \\
\text { CoNiCrAlY Coating }\end{array}$} & Thickness & Medium & Thick & Medium \\
\hline & & Spalling & - & - & - \\
\hline & & Penetration & Surface Layer & Surface Layer & Surface Layer \\
\hline \multirow{3}{*}{ K } & \multirow{3}{*}{$\begin{array}{c}\text { Alloy GTD111 + } \\
\text { CoNiCrAlY Coating }\end{array}$} & Thickness & Thin & Thick & Medium \\
\hline & & Spalling & - & 300 & - \\
\hline & & Penetration & Surface Layer & Surface Layer & Surface Layer \\
\hline
\end{tabular}

(*) Scale thickness based on largest value measured from Photomicrographs - refer to Table 5. Thin = scale 1-10 micron, Medium = scale 10-100 micron, Thick = scale $100-1000$ micron

(**) Scale spalling is assessed from Negative Weight Gain - refer to Table 4.

$10=$ spalling in $10 \mathrm{hrs}, 30=$ spalling in $30 \mathrm{hrs}, 100=$ spalling in $100 \mathrm{hrs}, 300=$ spalling in $300 \mathrm{hrs}$

$(* * *)$ Scale penetration is assessed from photomicrographs - refer to Appendix B. 
Table 5: Weight Change in Specimens Following Exposure to Different Environments

\begin{tabular}{|c|c|c|c|c|c|c|c|c|}
\hline \multicolumn{3}{|c|}{ Air } & \multicolumn{3}{|c|}{ Dry $\mathrm{H}_{2} \mathrm{~S}$} & \multicolumn{3}{|c|}{ Wet $\mathrm{H}_{2} \mathrm{~S}$} \\
\hline \multirow[t]{2}{*}{ ID } & Gain(*) & $\begin{array}{l}\text { Avg. } \\
\text { Gain }\end{array}$ & ID & Gain(*) & $\begin{array}{l}\text { Avg. } \\
\text { Gain }\end{array}$ & ID & Gain(*) & $\begin{array}{l}\text { Avg. } \\
\text { Gain }\end{array}$ \\
\hline & mg & mg & & mg & mg & & mg & mg \\
\hline A10A1 & 1.9 & \multirow{3}{*}{1.9} & H10A1 & 40.9 & \multirow{3}{*}{34.8} & W10A1 & 1.7 & \multirow{3}{*}{5.6} \\
\hline A10A2 & 1.6 & & H10A2 & 49.4 & & W10A2 & 6.0 & \\
\hline A10A3 & 2.1 & & H10A3 & 14.2 & & W10A3 & 9.3 & \\
\hline A10B1 & 7.1 & \multirow{3}{*}{5.8} & H10B1 & 92.9 & \multirow{3}{*}{17.6} & W10B1 & 14.1 & \multirow{3}{*}{12.5} \\
\hline A10B2 & 5.4 & & H10B2 & 23.2 & & W10B2 & 14.6 & \\
\hline A10B3 & 4.8 & & \begin{tabular}{|l|} 
H10B3 \\
\end{tabular} & $(63.4)$ & & W10B3 & 8.8 & \\
\hline $\mathrm{A} 10 \mathrm{C} 1$ & 3.0 & \multirow{3}{*}{2.5} & H10C1 & 150.0 & \multirow{3}{*}{111.1} & W10C1 & 10.7 & \multirow{3}{*}{4.9} \\
\hline $\mathrm{A} 10 \mathrm{C} 2$ & 3.0 & & H10C2 & 86.1 & & W10C2 & $(2.4)$ & \\
\hline A10C3 & 1.6 & & H10C3 & 97.1 & & W10C3 & 6.4 & \\
\hline A10D1 & 2.0 & \multirow{3}{*}{1.6} & \begin{tabular}{|l|} 
H10D1 \\
\end{tabular} & 141.5 & \multirow{3}{*}{225.1} & W10D1 & 7.6 & \multirow{3}{*}{$(4.7)$} \\
\hline A10D2 & 1.9 & & \begin{tabular}{|l|} 
H10D2 \\
\end{tabular} & 99.9 & & W10D2 & $(20.7)$ & \\
\hline A10D3 & 1.0 & & \begin{tabular}{|l} 
H10D3 \\
\end{tabular} & 434.0 & & W10D3 & $(0.8)$ & \\
\hline A10E1 & 4.6 & \multirow{3}{*}{3.7} & \begin{tabular}{|l|} 
H10E1 \\
\end{tabular} & 77.5 & \multirow{3}{*}{54.6} & W10E1 & 13.9 & \multirow{3}{*}{6.5} \\
\hline A10E2 & 3.3 & & H10E2 & 40.8 & & W10E2 & 6.4 & \\
\hline A10E3 & 3.3 & & H10E3 & 45.4 & & W10E3 & $(0.8)$ & \\
\hline A10F1 & 9.8 & \multirow{3}{*}{9.0} & H10F1 & 56.3 & \multirow{3}{*}{23.8} & W10F1 & 12.2 & \multirow{3}{*}{11.0} \\
\hline A10F2 & 9.6 & & H10F2 & 27.9 & & W10F2 & 9.2 & \\
\hline A10F3 & 7.7 & & H10F3 & $(12.9)$ & & W10F3 & 11.6 & \\
\hline$\overline{\text { A10G1 }}$ & 2.1 & \multirow{3}{*}{4.1} & H10G1 & 11.3 & \multirow{3}{*}{$(9.6)$} & W10G1 & 11.7 & \multirow{3}{*}{4.4} \\
\hline A10G2 & 5.3 & & H10G2 & 7.3 & & W10G2 & 7.5 & \\
\hline A10G3 & 4.8 & & H10G3 & $(47.4)$ & & W10G3 & $(6.1)$ & \\
\hline $\mathrm{A} 10 \mathrm{H} 1$ & 2.2 & & \begin{tabular}{|l}
$\mathrm{H} 10 \mathrm{H} 1$ \\
\end{tabular} & 60.5 & & $\mathrm{~W} 10 \mathrm{H} 1$ & 6.7 & \\
\hline $\mathrm{A} 10 \mathrm{H} 2$ & 1.6 & 2.0 & $\mathrm{H} 10 \mathrm{H} 2$ & 50.6 & 48.8 & $\mathrm{~W} 10 \mathrm{H} 2$ & 8.6 & 3.2 \\
\hline $\mathrm{A} 10 \mathrm{H} 3$ & 2.2 & & $\mathrm{H} 10 \mathrm{H} 3$ & 35.4 & & $\mathrm{~W} 10 \mathrm{H} 3$ & $(5.8)$ & \\
\hline A10K1 & 2.7 & & \begin{tabular}{|l}
$\mathrm{H} 10 \mathrm{~K} 1$ \\
\end{tabular} & 58.6 & & $\mathrm{~W} 10 \mathrm{~K} 1$ & 11.6 & \\
\hline A10K2 & 1.3 & 1.9 & \begin{tabular}{|l|}
$\mathrm{H} 10 \mathrm{~K} 2$ \\
\end{tabular} & 8.9 & 16.0 & $\mathrm{~W} 10 \mathrm{~K} 2$ & 4.8 & 11.1 \\
\hline A10K3 & 1.7 & & H10K3 & $(19.3)$ & & W10K3 & 16.9 & \\
\hline A30A1 & 2.6 & & \begin{tabular}{|l|} 
H30A 1 \\
\end{tabular} & $(* *)$ & & W30A1 & 2.0 & \\
\hline A30A2 & 1.3 & 2.0 & \begin{tabular}{|l|} 
H30A2 \\
\end{tabular} & 11.6 & 11.9 & W30A2 & 3.1 & 6.3 \\
\hline A30A3 & 2.1 & & \begin{tabular}{|l|} 
H30A3 \\
\end{tabular} & 12.3 & & W30A3 & 13.8 & \\
\hline A30B1 & 4.0 & & H30B1 & 424.9 & & W30B1 & 6.7 & \\
\hline A30B2 & 4.7 & 5.2 & H30B2 & 37.1 & 171.8 & W30B2 & 22.1 & 15.1 \\
\hline A30B3 & 7.0 & & H30B3 & 53.4 & & W30B3 & 16.3 & \\
\hline $\mathrm{A} 30 \mathrm{C} 1$ & 2.8 & & H30C1 & 256.6 & & W30C1 & $(3.1)$ & \\
\hline $\mathrm{A} 30 \mathrm{C} 2$ & 1.9 & 2.5 & H30C2 & 6.7 & 89.8 & W30C2 & 25.3 & 11.9 \\
\hline A30C3 & 2.7 & & \begin{tabular}{|l}
$\mathrm{H} 30 \mathrm{C} 3$ \\
\end{tabular} & 6.0 & & W30C3 & 13.4 & \\
\hline
\end{tabular}


Table 5 Weight Change in Specimens Following Exposure to Different Environments (continued)

\begin{tabular}{|c|c|c|c|c|c|c|c|c|}
\hline \multicolumn{3}{|c|}{ Air } & \multicolumn{3}{|c|}{ Dry $\mathrm{H}_{2} \mathrm{~S}$} & \multicolumn{3}{|c|}{ Wet $\mathrm{H}_{2} \mathrm{~S}$} \\
\hline ID & Gain(*) & $\begin{array}{l}\text { Avg. } \\
\text { Gain }\end{array}$ & ID & Gain(*) & $\begin{array}{l}\text { Avg. } \\
\text { Gain }\end{array}$ & ID & Gain(*) & $\begin{array}{l}\text { Avg. } \\
\text { Gain }\end{array}$ \\
\hline & mg & mg & & mg & mg & & mg & mg \\
\hline A30D1 & 0.2 & \multirow{3}{*}{0.5} & H30D1 & 151.5 & \multirow{3}{*}{83.7} & W30D1 & 7.4 & \multirow{3}{*}{12.1} \\
\hline A30D2 & 0.8 & & H30D2 & 59.3 & & W30D2 & 20.5 & \\
\hline A30D3 & 0.5 & & H30D3 & 40.4 & & W30D3 & 8.5 & \\
\hline A30E1 & 6.2 & \multirow{3}{*}{6.3} & H30E1 & 32.9 & \multirow{3}{*}{15.8} & W30E1 & 14.0 & \multirow{3}{*}{18.2} \\
\hline A30E2 & 5.8 & & H30E2 & 4.3 & & W30E2 & 25.3 & \\
\hline A30E3 & 6.8 & & H30E3 & 10.1 & & W30E3 & 15.2 & \\
\hline A30F1 & 11.2 & \multirow{3}{*}{11.2} & H30F1 & 6.6 & \multirow{3}{*}{24.9} & W30F1 & 15.5 & \multirow{3}{*}{20.6} \\
\hline A30F2 & 11.1 & & H30F2 & 55.3 & & W30F2 & 29.1 & \\
\hline A30F3 & 11.3 & & H30F3 & 12.8 & & W30F3 & 17.3 & \\
\hline A30G1 & 3.2 & \multirow{3}{*}{3.7} & H30G1 & 11.3 & \multirow{3}{*}{6.7} & W30G1 & 2.3 & \multirow{3}{*}{10.2} \\
\hline A30G2 & 4.1 & & H30G2 & 1.8 & & W30G2 & 18.3 & \\
\hline A30G3 & 3.8 & & H30G3 & 7.1 & & W30G3 & 9.9 & \\
\hline $\mathrm{A} 30 \mathrm{H} 1$ & 2.6 & \multirow{3}{*}{2.8} & $\mathrm{H} 30 \mathrm{H} 1$ & 1.4 & \multirow{3}{*}{4.3} & W30H1 & 1.9 & \multirow{3}{*}{8.9} \\
\hline $\mathrm{A} 30 \mathrm{H} 2$ & 3.3 & & $\mathrm{H} 30 \mathrm{H} 2$ & 8.3 & & $\mathrm{~W} 30 \mathrm{H} 2$ & 14.7 & \\
\hline $\mathrm{A} 30 \mathrm{H} 3$ & 2.5 & & $\mathrm{H} 30 \mathrm{H} 3$ & 3.2 & & W30H3 & 10.1 & \\
\hline A30K1 & 2.7 & \multirow{3}{*}{2.3} & H30K1 & 5.5 & \multirow{3}{*}{18.6} & W30K1 & 2.1 & \multirow{3}{*}{8.7} \\
\hline $\mathrm{A} 30 \mathrm{~K} 2$ & 1.7 & & H30K2 & 48.6 & & $\mathrm{~W} 30 \mathrm{~K} 2$ & 15.3 & \\
\hline A30K3 & 2.6 & & H30K3 & 1.7 & & W30K3 & 8.6 & \\
\hline A100A1 & 0.0 & \multirow{3}{*}{$(0.1)$} & H100A1 & 114.7 & \multirow{3}{*}{51.9} & W100A1 & 2.3 & \multirow{3}{*}{4.8} \\
\hline A100A2 & $(0.5)$ & & H100A2 & 31.0 & & W100A2 & 6.6 & \\
\hline A100A3 & 0.2 & & H100A3 & 9.9 & & W100A3 & 5.4 & \\
\hline A100B1 & $(4.1)$ & \multirow{3}{*}{$(2.5)$} & H100B1 & $(18.7)$ & & W100B1 & 9.6 & \\
\hline A100B2 & $(1.5)$ & & H100B2 & 26.0 & 3.1 & W100B2 & 8.4 & 9.1 \\
\hline A100B3 & $(1.8)$ & & H100B3 & 2.1 & & W100B3 & 9.2 & \\
\hline $\mathrm{A} 100 \mathrm{C} 1$ & 4.2 & & $\mathrm{H} 100 \mathrm{C} 1$ & 129.5 & & $\mathrm{~W} 100 \mathrm{C} 1$ & 4.8 & \\
\hline $\mathrm{A} 100 \mathrm{C} 2$ & 4.5 & 4.3 & $\mathrm{H} 100 \mathrm{C} 2$ & 18.0 & 59.2 & $\mathrm{~W} 100 \mathrm{C} 2$ & 5.4 & 5.2 \\
\hline A100C3 & 4.3 & & H100C3 & 30.2 & & W100C3 & 5.3 & \\
\hline A100D1 & 0.6 & & H100D1 & 18.9 & & W100D1 & 0.8 & \\
\hline A100D2 & 0.2 & 0.3 & H100D2 & 15.2 & 12.2 & W100D2 & 4.1 & 2.0 \\
\hline A100D3 & 0.0 & & H100D3 & 2.5 & & W100D3 & 1.2 & \\
\hline A100E1 & 8.5 & & H100E1 & 27.7 & & W100E1 & 10.8 & \\
\hline A100E2 & 8.3 & 8.3 & H100E2 & 21.8 & 22.9 & W100E2 & 11.2 & 10.6 \\
\hline A100E3 & 8.3 & & H100E3 & 19.2 & & W100E3 & 9.9 & \\
\hline A100F1 & 6.2 & & H100F1 & $(16.8)$ & & W100F1 & 10.2 & \\
\hline A100F2 & 5.2 & 5.8 & H100F2 & 2.3 & 9.8 & W100F2 & 9.6 & 8.9 \\
\hline A100F3 & 5.9 & & H100F3 & 44.1 & & W100F3 & 7.0 & \\
\hline
\end{tabular}


Table 5 Weight Change in Specimens Following Exposure to Different Environments (continued)

\begin{tabular}{|c|c|c|c|c|c|c|c|c|}
\hline \multicolumn{3}{|c|}{ Air } & \multicolumn{3}{|c|}{ Dry $\mathrm{H}_{2} \mathrm{~S}$} & \multicolumn{3}{|c|}{ Wet $\mathrm{H}_{2} \mathrm{~S}$} \\
\hline \multirow[t]{2}{*}{ ID } & Gain(*) & $\begin{array}{l}\text { Avg. } \\
\text { Gain }\end{array}$ & ID & Gain(*) & $\begin{array}{l}\text { Avg. } \\
\text { Gain }\end{array}$ & ID & Gain(*) & $\begin{array}{l}\text { Avg. } \\
\text { Gain }\end{array}$ \\
\hline & mg & mg & & mg & mg & & mg & mg \\
\hline A100G1 & 4.7 & \multirow{3}{*}{4.8} & H100G1 & 9.7 & \multirow{3}{*}{21.7} & W100G1 & 4.5 & \multirow{3}{*}{5.0} \\
\hline A100G2 & 5.5 & & H100G2 & 40.3 & & W100G2 & 5.1 & \\
\hline A100G3 & 4.4 & & H100G3 & 15.0 & & W100G3 & 5.3 & \\
\hline $\mathrm{A} 100 \mathrm{H} 1$ & 4.1 & \multirow{3}{*}{3.6} & $\mathrm{H} 100 \mathrm{H} 1$ & 12.4 & \multirow{3}{*}{12.5} & W100H1 & 3.0 & \multirow{3}{*}{3.8} \\
\hline $\mathrm{A} 100 \mathrm{H} 2$ & 3.4 & & $\mathrm{H} 100 \mathrm{H} 2$ & 15.3 & & $\mathrm{~W} 100 \mathrm{H} 2$ & 4.6 & \\
\hline $\mathrm{A} 100 \mathrm{H} 3$ & 3.2 & & $\mathrm{H} 100 \mathrm{H} 3$ & 9.6 & & $\mathrm{~W} 100 \mathrm{H} 3$ & 3.9 & \\
\hline $\mathrm{A} 100 \mathrm{~K} 1$ & 3.5 & \multirow{3}{*}{3.4} & $\mathrm{H} 100 \mathrm{~K} 1$ & 2.8 & \multirow{3}{*}{13.2} & W100K1 & 3.8 & \multirow{3}{*}{3.8} \\
\hline $\mathrm{A} 100 \mathrm{~K} 2$ & 4.5 & & $\mathrm{H} 100 \mathrm{~K} 2$ & 34.7 & & $\mathrm{~W} 100 \mathrm{~K} 2$ & 3.2 & \\
\hline $\mathrm{A} 100 \mathrm{~K} 3$ & 2.3 & & $\mathrm{H} 100 \mathrm{~K} 3$ & 2.1 & & $\mathrm{~W} 100 \mathrm{~K} 3$ & 4.3 & \\
\hline A300A1 & $(1.8)$ & \multirow{3}{*}{$(1.8)$} & H300A1 & $(1.0)$ & \multirow{3}{*}{$(0.3)$} & W300A1 & 0.4 & \multirow{3}{*}{$(1.4)$} \\
\hline A300A2 & $(3.0)$ & & H300A2 & 2.2 & & W300A2 & $(3.3)$ & \\
\hline A300A3 & $(0.7)$ & & H300A3 & $(2.0)$ & & W300A3 & $(1.5)$ & \\
\hline A300B1 & $(13.4)$ & \multirow{3}{*}{$(15.4)$} & H300B1 & 14.1 & \multirow{3}{*}{3.8} & W300B1 & 7.0 & \multirow{3}{*}{6.1} \\
\hline $\mathrm{A} 300 \mathrm{~B} 2$ & $(16.3)$ & & H300B2 & $(4.1)$ & & W300B2 & 7.0 & \\
\hline A300B3 & $(16.4)$ & & H300B3 & 1.4 & & W300B3 & 4.3 & \\
\hline $\mathrm{A} 300 \mathrm{C} 1$ & $(2.8)$ & \multirow{3}{*}{$(1.3)$} & $\mathrm{H} 300 \mathrm{C} 1$ & $(27.6)$ & \multirow{3}{*}{ (16.3) } & W300C1 & 5.5 & \multirow{3}{*}{5.7} \\
\hline $\mathrm{A} 300 \mathrm{C} 2$ & $(2.4)$ & & $\mathrm{H} 300 \mathrm{C} 2$ & $(21.8)$ & & W300C2 & 5.0 & \\
\hline $\mathrm{A} 300 \mathrm{C} 3$ & 1.2 & & $\mathrm{H} 300 \mathrm{C} 3$ & 0.5 & & W300C3 & 6.6 & \\
\hline A300D1 & $(0.8)$ & \multirow{3}{*}{$(0.3)$} & H300D1 & 3.5 & \multirow{3}{*}{$(8.7)$} & W300D1 & 5.8 & \multirow{3}{*}{3.7} \\
\hline A300D2 & $(0.0)$ & & H300D2 & $(22.2)$ & & W300D2 & 3.9 & \\
\hline A300D3 & $(0.1)$ & & H300D3 & $(7.3)$ & & W300D3 & 1.5 & \\
\hline A300E1 & 1.5 & & H300E1 & $(24.1)$ & & W300E1 & 11.7 & \\
\hline A300E2 & $(13.0)$ & $(9.3)$ & H300E2 & $(8.1)$ & $(6.5)$ & W300E2 & 7.2 & 11.1 \\
\hline A300E3 & $(16.6)$ & & H300E3 & 12.6 & & W300E3 & 14.3 & \\
\hline A300F1 & 3.3 & & H300F1 & 0.3 & & W300F1 & $(10.3)$ & \\
\hline A300F2 & $(21.3)$ & $(16.7)$ & H300F2 & $(18.4)$ & $(9.1)$ & W300F2 & $(8.8)$ & $(6.5)$ \\
\hline A300F3 & $(32.0)$ & & H300F3 & $(9.1)$ & & W300F3 & $(0.3)$ & \\
\hline A300G1 & 5.4 & & H300G1 & $(12.5)$ & & W300G1 & 6.5 & \\
\hline A300G2 & 5.8 & 6.1 & H300G2 & $(9.1)$ & $(4.7)$ & W300G2 & 6.7 & 6.8 \\
\hline A300G3 & 7.0 & & H300G3 & 7.5 & & W300G3 & 7.3 & \\
\hline $\mathrm{A} 300 \mathrm{H} 1$ & 3.3 & & $\mathrm{H} 300 \mathrm{H} 1$ & 20.3 & & W300H1 & 4.6 & \\
\hline $\mathrm{A} 300 \mathrm{H} 2$ & 3.7 & 3.5 & $\mathrm{H} 300 \mathrm{H} 2$ & $(4.8)$ & 7.4 & W300H2 & 5.3 & 4.8 \\
\hline $\mathrm{A} 300 \mathrm{H} 3$ & 3.4 & & $\mathrm{H} 300 \mathrm{H} 3$ & 6.5 & & W300H3 & 4.6 & \\
\hline $\mathrm{A} 300 \mathrm{~K} 1$ & 2.9 & & $\mathrm{H} 300 \mathrm{~K} 1$ & $(15.0)$ & & W300K1 & 5.2 & \\
\hline $\mathrm{A} 300 \mathrm{~K} 2$ & 4.4 & 4.0 & $\mathrm{H} 300 \mathrm{~K} 2$ & $(7.4)$ & $(5.5)$ & W300K2 & 3.3 & 4.3 \\
\hline $\mathrm{A} 300 \mathrm{~K} 3$ & 4.7 & & H300K3 & 5.7 & & W300K3 & 4.6 & \\
\hline
\end{tabular}

(*) Numbers in brackets indicate weight loss, i.e., negative gain.

(*) Specimen H30A1 was fused to holder due to reactor with gas in high gas flow rate. 
Table 6: Summary of Average Weight Gain, Sorted by Alloy and Exposure Period

\begin{tabular}{|c|c|c|c|c|c|}
\hline & \multirow{2}{*}{ Material } & \multirow{2}{*}{$\begin{array}{c}\text { Exposure } \\
\text { Period, hrs. }\end{array}$} & \multicolumn{3}{|c|}{ Average Weight Gain(*), mg } \\
\hline & & & Air & Dry & Wet \\
\hline \multirow{4}{*}{$\mathbf{A}$} & \multirow{4}{*}{ Alloy FSX-414 } & 10 & 1.9 & 34.8 & 5.6 \\
\hline & & 30 & 2.0 & 11.9 & 6.3 \\
\hline & & 100 & $(0.1)$ & 51.9 & 4.8 \\
\hline & & 300 & $(1.8)$ & $(0.3)$ & $(1.4)$ \\
\hline \multirow{4}{*}{ B } & \multirow{4}{*}{ Alloy GTD222 } & 10 & 5.8 & 17.6 & 12.5 \\
\hline & & 30 & 5.2 & 171.8 & 15.1 \\
\hline & & 100 & $(2.5)$ & 3.1 & 9.1 \\
\hline & & 300 & (15.4) & 3.8 & 6.1 \\
\hline \multirow{4}{*}{$\mathbf{C}$} & \multirow{4}{*}{ Alloy Hastelloy X } & 10 & 2.5 & 111.1 & 4.9 \\
\hline & & 30 & 2.5 & 89.8 & 11.9 \\
\hline & & 100 & 4.3 & 59.2 & 5.2 \\
\hline & & 300 & $(1.3)$ & $(16.3)$ & 5.7 \\
\hline \multirow{4}{*}{ D } & \multirow{4}{*}{ Alloy N5 } & 10 & 1.6 & 225.1 & $(4.7)$ \\
\hline & & 30 & 0.5 & 83.7 & 12.1 \\
\hline & & 100 & 0.3 & 12.2 & 2.0 \\
\hline & & 300 & $(0.3)$ & $(8.7)$ & 3.7 \\
\hline \multirow{4}{*}{$\mathbf{E}$} & \multirow{4}{*}{ Alloy Nimonic 263} & 10 & 3.7 & 54.6 & 6.5 \\
\hline & & 30 & 6.3 & 15.8 & 18.2 \\
\hline & & 100 & 8.3 & 22.9 & 10.6 \\
\hline & & 300 & $(9.3)$ & $(6.5)$ & 11.1 \\
\hline \multirow{4}{*}{$\mathbf{F}$} & \multirow{4}{*}{ Alloy GTD111 } & 10 & 9.0 & 23.8 & 11.0 \\
\hline & & 30 & 11.2 & 24.9 & 20.6 \\
\hline & & 100 & 5.8 & 9.8 & 8.9 \\
\hline & & 300 & $(16.7)$ & $(9.1)$ & $(6.5)$ \\
\hline \multirow{4}{*}{ G } & \multirow{4}{*}{$\begin{array}{l}\text { Alloy N5 + } \\
\text { NiCrAlY Coating }\end{array}$} & 10 & 4.1 & $(9.6)$ & 4.4 \\
\hline & & 30 & 3.7 & 6.7 & 10.2 \\
\hline & & 100 & 4.8 & 21.7 & 5.0 \\
\hline & & 300 & 6.1 & (4.7) & 6.8 \\
\hline \multirow{4}{*}{$\mathbf{H}$} & \multirow{4}{*}{$\begin{array}{l}\text { Alloy Nimonic } 263+ \\
\text { CoNiCrAlY Coating }\end{array}$} & 10 & 2.0 & 48.8 & 3.2 \\
\hline & & 30 & 2.8 & 4.3 & 8.9 \\
\hline & & 100 & 3.6 & 12.5 & 3.8 \\
\hline & & 300 & 3.5 & 7.4 & 4.8 \\
\hline \multirow{4}{*}{$\mathbf{K}$} & \multirow{4}{*}{$\begin{array}{l}\text { Alloy GTD111 + } \\
\text { CoNiCrAlY Coating }\end{array}$} & 10 & 1.9 & 16.0 & 11.1 \\
\hline & & 30 & 2.3 & 18.6 & 8.7 \\
\hline & & 100 & 3.4 & 13.2 & 3.8 \\
\hline & & 300 & 4.0 & (5.5) & 4.3 \\
\hline
\end{tabular}

(*) Average of triplicate specimens for each test condition - refer to Table 3. 
Table 7: Scale Thickness Measured from Photomicrographs of Transverse Cross-Sections of Specimens (*)

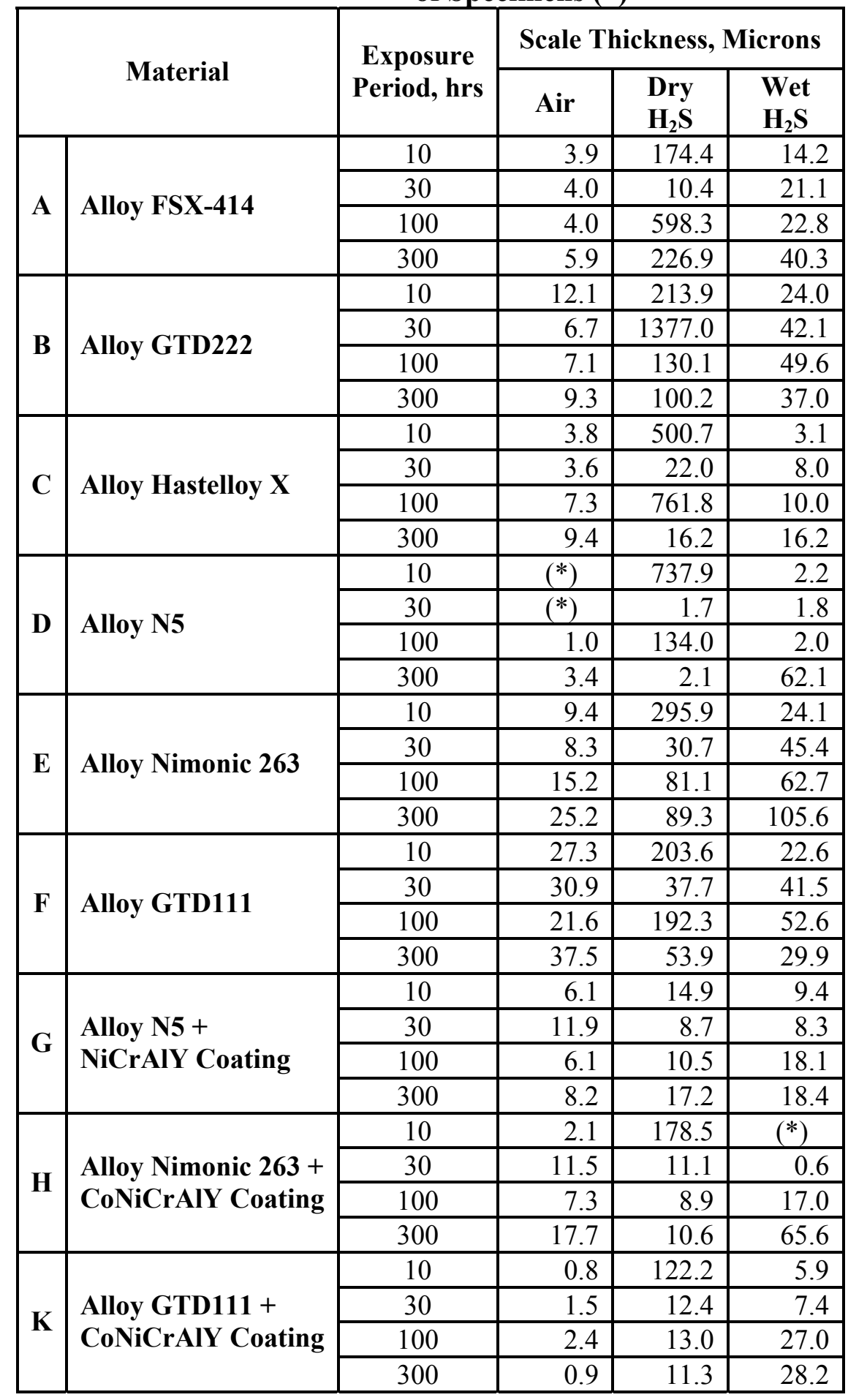

(*) Only one specimen from each set of triplicates was examined metallographically.

(**) Scale was undetectable using light microscope. 


\section{Erosion Experiments}

12 specimens were exposed in the Becon rig, four with Nimonic-263 substrate, four with GTD111 substrate and four with N5 substrate. Figure 35 shows the four Nimonic-263 specimens after failure. Three of the four specimens failed on the first cycle and the fourth on the second cycle. The TBC seemed to fail by a spallation mode in all four cases. Figure 36 shows the four GTD111 specimens after failure. As in the case of the Nimonic-263 specimens, three of the four specimens failed on the first cycle and the fourth on the second cycle. The TBC seemed to fail by a spallation mode in all four cases as well. Figure 37 shows the four N5 specimens after failure. In this case only one of the specimens failed on the first cycle. The other three specimens failed after two, six and ten cycles (considerably more cycles than both the Nimonic-263 specimens and the GTD111 specimens). Also, the TBC seemed to fail by an erosion mode rather than spallation.

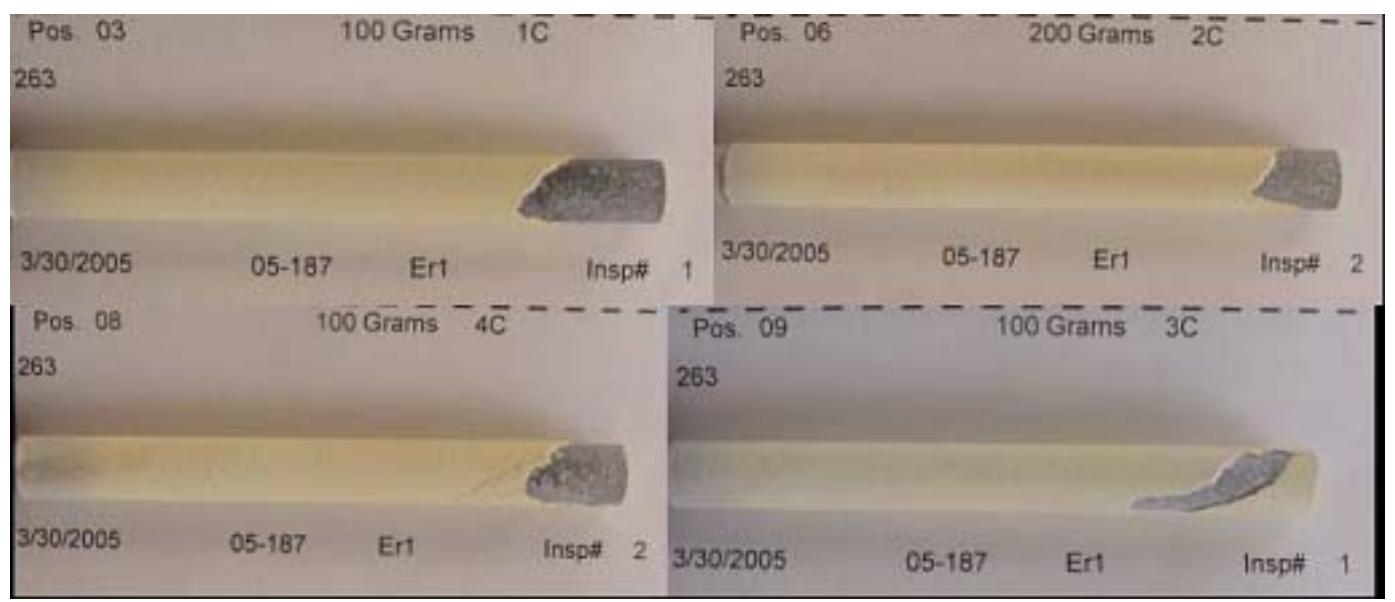

Figure 35: Nimonic-263 + CoNiCrAIY bondcoat + air plasma sprayed TBC specimens after coating failure

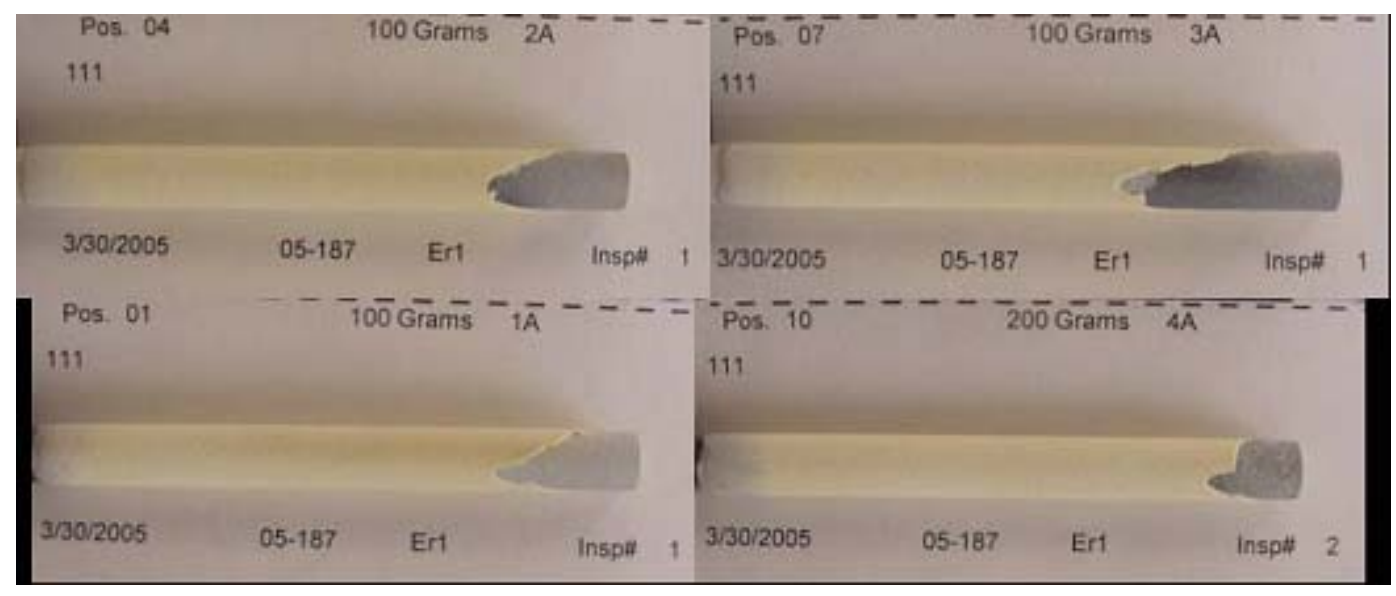

Figure 36: GTD111 + CoNiCrAlY bondcoat + air plasma sprayed TBC specimens after coating failure 


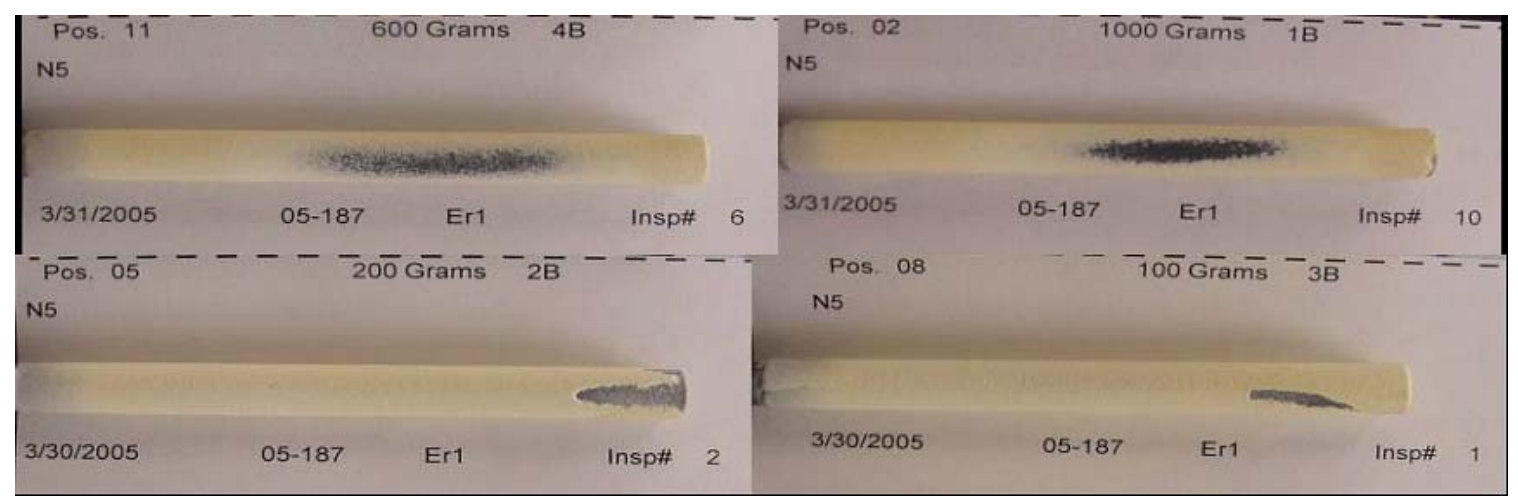

Figure 37: Rene N5 + NiCrAIY bondcoat + air plasma sprayed TBC specimens after coating failure

Syngas Sampling

Syngas fuel sampling tests were performed on June 5-7, 2004 and April 29 to May 1, 2005 at the Tampa Electric Company (TECO) Polk 1 powerplant, located near Mulberry, FL. The purpose of the fuel sampling was to evaluate the effect of Syngas fuel constituents and contaminants on the service lives of gas turbine combustion and downstream turbine components during actual operating conditions. 


\section{$\underline{\text { Service Exposed Parts }}$}

Tampa Electric Company (TECO) provided two first stage buckets and two second stage buckets removed from the MS7001FA IGCC gas turbine following service. All of the buckets were in service under the following conditions:

\begin{tabular}{|l|l|}
\hline Customer & TECO-Polk County \\
\hline Machine Type (Model Series) & MS7221FA \\
\hline Turbine S/N & 296436 \\
\hline Steam/Water Injection & $0 \%$ \\
\hline Fuel Type & Syngas and \#2 distillate \\
\hline Plant Type & IGCC \\
\hline Fired Starts & 238 \\
\hline Emergency Trips & 117 \\
\hline Fired Hours & 22975 \\
\hline
\end{tabular}

Preliminary microscopic analysis was performed on the leading edge of a first stage bucket from TECO (directionally solidified GTD-111 + CoCrAlY coating with over-aluminide). At the leading edge of the bucket, two distinct surface reactions were observed. Figure 38 shows the cross-section of the surface of the first stage bucket. The surface reaction is what is commonly observed in a high temperature oxidizing environment (typical natural gas fired turbine surface condition). Figure 39 shows the cross-section of the surface of the first stage bucket at a slightly different area of the leading edge. The surface reaction in this case is radically different from what is typically observed on the surface of hot gas path parts in gas turbines fired by natural gas. Figure 40 is a higher magnification of the same area depicted in Figure 39. Noted on the photomicrograph are the presence of sulfides and the absence of a continuous alumina layer.

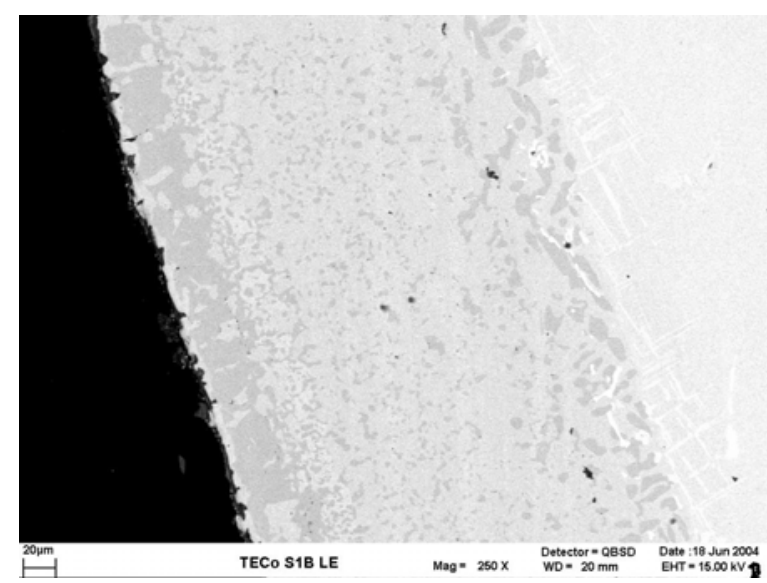

Figure 38: Cross -sectional micrograph of GT-29+ coating on TECO Polk 1 first stage bucket after approximately 24000 hours of service. Typical surface reactions were observed in this region of the bucket. 


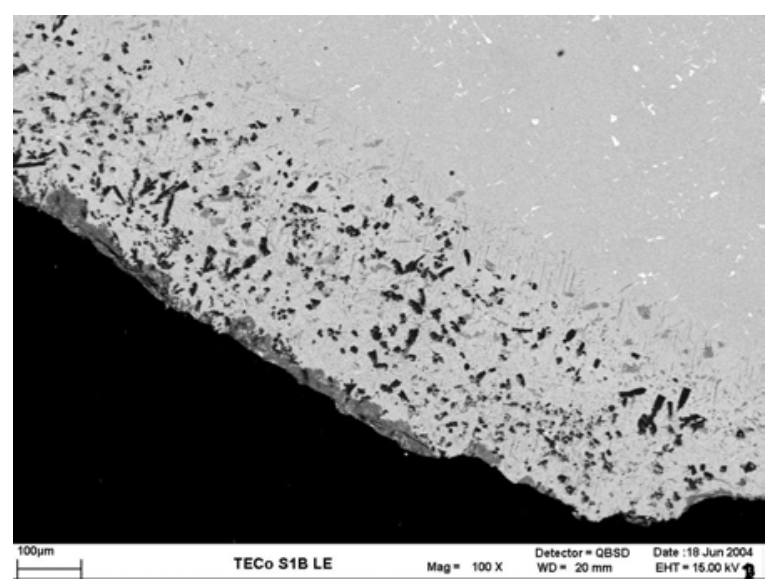

Figure 39: Cross -sectional micrograph of GT-29+ coating on TECO Polk 1 first stage bucket after approximately 24000 hours of service. Atypical surface reactions were observed in this region of the bucket.

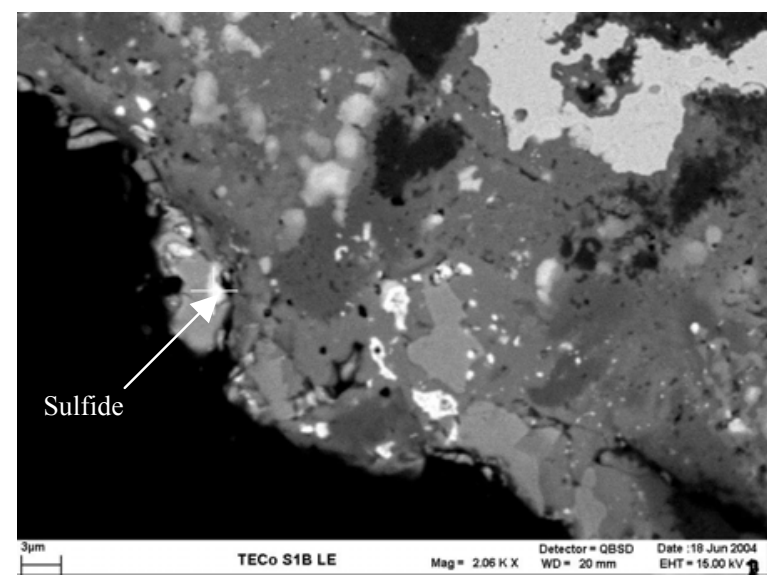

Figure 40: Cross -sectional micrograph of GT-29+ coating on TECO Polk 1 first stage bucket after approximately 24000 hours of service. Higher magnification of the same region as shown in Figure 39 (see text for explanation).

The preliminary analysis of a stage one bucket indicated that the environment the part encountered altered the corrosion product on the surface. In some areas the coating microstructure and composition indicated typical oxidation behavior seen in natural gas fired turbines. In other areas the coating microstructure and composition indicated a combination of oxidation and sulfidation. Metals sulfidize rather than oxidize only when it is more thermodynamically and kinetically favorable to do so. There are three major factors that influence the oxidation/sulfidation behavior of an alloy: the partial pressure of oxygen, the partial pressure of sulfur and the temperature. The partial pressure of oxygen and the temperature of the part are both relatively constant during firing. This leaves the sulfur as the main variable in the equation. It is believed that the sulfur that reached the hot gas path parts in the TECO Polk 1 gas turbine originated from the syngas fuel under certain operating conditions. When the amount of sulfur in the fuel reached a high enough value, sulfides formed on the 
surface rather than oxides. The location of sulfidation was also dependent upon the local temperature and pressure. As a result, the sulfidation that was observed on these parts was not uniformly distributed.

\section{$\underline{\text { Sub-Task 1.2 - High Temperature Erosion/Corrosion Mechanism Quantification }}$}

\section{Service Exposed Parts}

The analysis of parts removed from the TECO Polk 1 MS7001FA IGCC gas turbine revealed the presence of both oxides and sulfides on the surface. The formation of sulfides on the surface of hot gas path parts is not desired. Three major factors were identified that influence the oxidation/sulfidation behavior of an alloy in a syngas environment: the partial pressure of oxygen, the partial pressure of sulfur and the temperature.

Detailed structural and compositional analysis of the surface scale of the serviced parts from TECO Polk 1 was performed at the GE Global Research Center (GRC). Additional evaluations were performed on coupons by the Georgia Institute of Technology, as described below and in Appendix D. A preliminary model for syngas firing effects on hot gas path parts life was defined based on results of these evaluations. The results are divided in three distinct parts, (i) airfoil surface, (ii) coating structure and (iii) sulfur precipitates analyses.

\section{(i) Bucket Airfoil Surface Analysis}

The evaluation of the airfoil surface composition was performed at various locations that had distinct visual characteristics, as shown in Figure 45 and Figure 46 for the suction and pressure sides, respectively. Figure 47 summarizes the EDS spectra and maps collected at specific locations. A normal variation in the relative peak heights of the elements found in the original coating composition was observed, representative of the evolution of the coating during service. This evolution consists mainly of outward diffusion of substrate elements, such as titanium, or depletion of aluminum, which serves to form the protective alumina scale. The aluminum depletion is clearly illustrated in locations 5, 7 and 9, where the coating was significantly altered. On the other hand, even though the coating was severely damaged in location 10, the EDS analysis did not detect aluminum depletion because the severe material loss due to erosion went beyond the thickness of the coating, revealing the underlying base metal with the nominal GTD111 aluminum content. Additional analyses of the surface and a cross-section presented in part (iii) further substantiate this statement.

Besides coating deterioration, deposits of sulfur and iron were detected on the airfoil surface. Sulfur was found only on the surface of the pressure side, with the highest concentration found at location 10. This corresponds to the corner towards the tip on the trailing edge, where the coating is severely damaged. EDS spectra at locations 5, 7, 8 and 9 (coating deterioration visible) also detected sulfur, while a very faint sulfur peak was detected at location 6 . EDS analyses of the leading edge surface were not reliable due to the sharp curvature at that location. However, sulfur was detected along the leading edge in cross-section analyses, with results presented in part (iii) below. No specific sulfur-rich particles were identified on the surface of the airfoil but, instead, the sulfur seemed to be included in small patches over the surface. Details of this analysis are shown below in part (iii). This is consistent with the hot corrosion 
mechanisms that lead to the failure of MCrAlY coatings [e.g. see Refs. 1.1, 1.2], which are characterized by a segregation of impurities, here sulfur based, at the alumina-MCrAlY interface. The fact that sulfur is only found at specific locations of the bucket may indicate that certain conditions, e.g. a combination of temperature and gas velocity at the location of impact with the airfoil surface, must be met for the absorption of sulfur into the surface.

Iron was detected at every location sampled on the surface of the bucket. The surface coverage by iron deposits was not uniform, but speckled with clusters of iron oxides, as shown in Figure 44. It was not possible to identify the source of the iron. XRD analyses confirmed the presence of hematite $\mathrm{Fe}_{2} \mathrm{O}_{3}$, along with other oxide species that varied with location on the bucket (per Figure 42):

- Location 11: corundum $\left(\mathrm{Al}_{2} \mathrm{O}_{3}\right)$ and CoCrAlY were the main phases, with hematite $\left(\mathrm{Fe}_{2} \mathrm{O}_{3}\right)$, rutile $\left(\mathrm{TiO}_{2}\right)$ and $\mathrm{NiCr}_{2} \mathrm{O}_{4}$ spinel also present.

- Location 6: Mainly $\mathrm{NiCr}_{2} \mathrm{O}_{4}$ spinel. No corundum $\left(\mathrm{Al}_{2} \mathrm{O}_{3}\right)$ was detected in this area. A small amount of another spinel, possibly $\mathrm{Co}_{3} \mathrm{O}_{4}$ or $\mathrm{CoAl}_{2} \mathrm{O}_{4}$ was also present, as well as hematite $\left(\mathrm{Fe}_{2} \mathrm{O}_{3}\right)$.

- Apex of leading edge next to location 6: Mainly $\mathrm{NiO}$, with possibly a $\mathrm{NiCr}_{2} \mathrm{O}_{4}$ spinel. Additional phases were rutile $\left(\mathrm{TiO}_{2}\right), \mathrm{Cr}_{2} \mathrm{O}_{3}$ and hematite $\left(\mathrm{Fe}_{2} \mathrm{O}_{3}\right)$

\section{(2) Bucket Coating Structure Analysis}

The objective of this section was to analyze the state of distress of the coating after service exposure, characterize the grain structure in the coating and determine if a relationship exists between craze cracks and the microstructure. Coupons were removed from the trailing edge and leading edge, about 3.25" from the tip, and were mounted and polished for cross-section analyses.

A typical illustration of the coating microstructure and the occurrence of craze cracks is shown in optical and EBSD images, Figure 45 and Figure 46. The grain structure of the coating was fairly uniform over three quarters of its thickness, with a $10 \mu \mathrm{m}$ grain size determined by visual standard overlays, and became bimodal with coarser grains by the outer surface. The EBSD analysis did not show any texturing of the grains in the coating, Figure 46 (a). However, a band of grains with significant residual strain was observed parallel to the coating-substrate interface about $75 \mu \mathrm{m}$ into the coating, Figure 46 (b). This band of strain was probably the result from the thermal expansion mismatch between the substrate and the coating. Residual strains were also concentrated in the vicinity of the craze cracks, where a mismatch in thermal expansion also existed. The depletion of the aluminum-rich $\beta$ phase, illustrated in the aluminum EDS maps of Figure 47, did not occur uniformly across the entire airfoil. At about 3.25" from the tip, a more severe depletion of aluminum was measured on the suction side, both at the trailing and leading edges, indicating that the coating was close to the end of its life in those regions. On the other hand, the pressure side showed a healthier coating with significant amount of $\beta$ phase still present. 
The formation of cracks from thermal cycling during service was more prominent along the leading edge, while the coating along the trailing edge was left fairly intact. Craze cracks typically form in the brittle outer $\mathrm{Al}_{2} \mathrm{O}_{3}$ layer and propagate into the bond coat where they eventually arrest. It was not possible to establish a correlation between the crack paths or density and the coating microstructure, mainly because of the high level of oxidation in those areas.

The high density of craze cracks on the pressure side of the leading edge is illustrated in Figure 48 (a) and a higher magnification image of suction side craze cracks is shown in Figure 48 (b). In most cases, the craze cracks were fully oxidized, with an oxide scale that completely filled the crack and extended up to about $50 \mu \mathrm{m}$ into the coating. The elemental EDS maps in Figure 48 (c) indicated that the center of the oxidized crack was rich in chromium and cobalt with some nickel and aluminum, while the area immediately surrounding it consisted mainly of aluminum oxide. This was supported by the EBSD analysis of a craze crack on the pressure side of the leading edge, Figure 49, which indicated that the center of the crack matched a $\mathrm{CoAl}_{2} \mathrm{O}_{4}$ spinel structure adjacent to an $\mathrm{Al}_{2} \mathrm{O}_{3}$ layer. The detection of chromium in the spinel is explained by its high solubility in the spinel phase. Beyond the $\mathrm{Al}_{2} \mathrm{O}_{3}$ layer, oxidation progressed into the bulk of the coating and consisted of a mixture of oxides. A portion was identified as the same $\mathrm{CoAl}_{2} \mathrm{O}_{4}$ spinel as in the center of the crack, while the remaining oxides were difficult to identify by EBSD due the poor diffraction conditions. These partial EBSD results in conjunction with EDS data seemed to match a $\mathrm{Ni}, \mathrm{Co}, \mathrm{O}$ spinel.

(3) Sulfur Precipitate Analysis

Sulfur was detected in various regions of surface of the pressure side of the airfoil, mainly by the trailing edge and where severe distress of the coating was observed. Since the leading edge of the airfoil experienced significant coating damage, a mount was removed 3.25 " from the tip to sample a cross-section of the material. Sulfur-rich precipitates were detected in the diffusion zone of the substrate directly beneath the coating, Figure 50. WDS microprobe analyses of these precipitates were performed to confirm the presence of sulfur, which may be confused by EDS due to a peak overlap with molybdenum (GTD111 substrate contains $1.5 \mathrm{wt} \% \mathrm{Mo}$ ). EBSD analyses of these particles clearly showed chromium sulfides $\mathrm{Cr}_{8} \mathrm{~S}_{7}$ in a $\gamma$ matrix, Figure 51.

Extensive EDS analyses performed along the apex of the leading edge revealed that sulfur-rich precipitates formed in the diffusion zone of the substrate over the entire area that suffered severe coating distress, Figure 52. A high density of sulfur-rich particles was observed in the region where the substrate is exposed at the very apex. Beyond that region, over $\sim 0.080$ " $(\sim 2 \mathrm{~mm})$ on both pressure and suction sides, the coating remnant consisted essentially of a thick layer of oxides, underneath which chromium sulfides were detected within 0.010 " $(250 \mathrm{~mm})$ of the surface of the substrate. On the suction side, some sulfur was detected in the upper layer of the oxidized coating and also below the sound coating further along the airfoil, about $\sim 0.180$ " (4.5 $\mathrm{mm}$ ) from the leading edge apex. Beyond that area, no sulfides were detected below the healthy layer of the coating, indicating that either the breach in coating was a leading cause in the formation of sulfides, or that enhanced sulfur absorption in those regions caused the premature failure of the coating. 
A detailed EDS analysis of the airfoil surface was performed by the trailing edge close to the tip where significant levels of sulfur were detected in part (i). Foreign matter deposits on the exposed base metal included sulfur, iron and chlorine, Figure 53. Calcium and potassium were also detected, but this type of contamination is often the result of sample handling and, therefore, is not relevant in this study. Sulfur was detected in darker patches than the background surface in SEM backscatter mode. Other areas reflected the chemistry of the substrate with the addition of iron, an impurity that is readily found on the airfoil surface, as established in part (i).

A cross-section of the same area was analyzed by EDS and revealed a stratified structure of oxide layers and precipitates, Figure 54. On the outer surface, a 20 to $50 \mu \mathrm{m}$ thick oxide scale rich in chromium, nickel and cobalt was found, directly followed by a discontinuous layer of chromium and titanium rich oxides. Below the oxide scale, oxide precipitates of chromium and titanium and alumina were detected within $150 \mu \mathrm{m}$ of the surface. Beyond this level, between about 150 and $250 \mu \mathrm{m}$ from the surface, titanium-rich needles and chromium sulfides were observed, which is indicative of the high diffusivity of sulfur in the $\gamma$ matrix. The number density of sulfides by the trailing edge was similar to that observed on the apex of the leading edge where no coating remained on the surface.

\section{Environmental Effects on Mechanical Properties}

The chief goal of this sub-task was to characterize the conditions and microstructures associated with crack initiation in directionally-solidified Ni-base superalloys. The goals of the research were as follows:

- Distinguish the dominant microstructural mechanisms that develop and enhance damage.

- Determine the influence of material orientation on crack initiation.

- Define the role of thermal/mechanical pre-exposure on crack initiation life.

- Characterize damage mechanisms and relate them to crack initiation behavior.

Results showed that kinetics of oxidation in DS GTD-111 are parabolic and have an estimated activation energy of $4.02 \mathrm{~J}$ in dry $100 \mathrm{ppm} \mathrm{H}_{2} \mathrm{~S}$ in $\mathrm{N}_{2}$ gas. Thin sulfide scales suggest that corrosion was minimal. This is in accordance with sulfidation behavior of samples pre-exposed in wet $\mathrm{H}_{2} \mathrm{~S}$ for $100 \mathrm{hr}$ at $982^{\circ} \mathrm{C}$, shown in Figure 55. Based on these observations, it is concluded that 100 hours was not a sufficient time to investigate the kinetics base on the low $\mathrm{H}_{2} \mathrm{~S}$ concentration. Additionally, a concentration of $0.01 \%$ was too low for adequate characterization of sulfidation testing. Typically, concentrations between 1-40\% are used. Further discussion of the environmental effects on mechanical properties is found in Appendix D. 


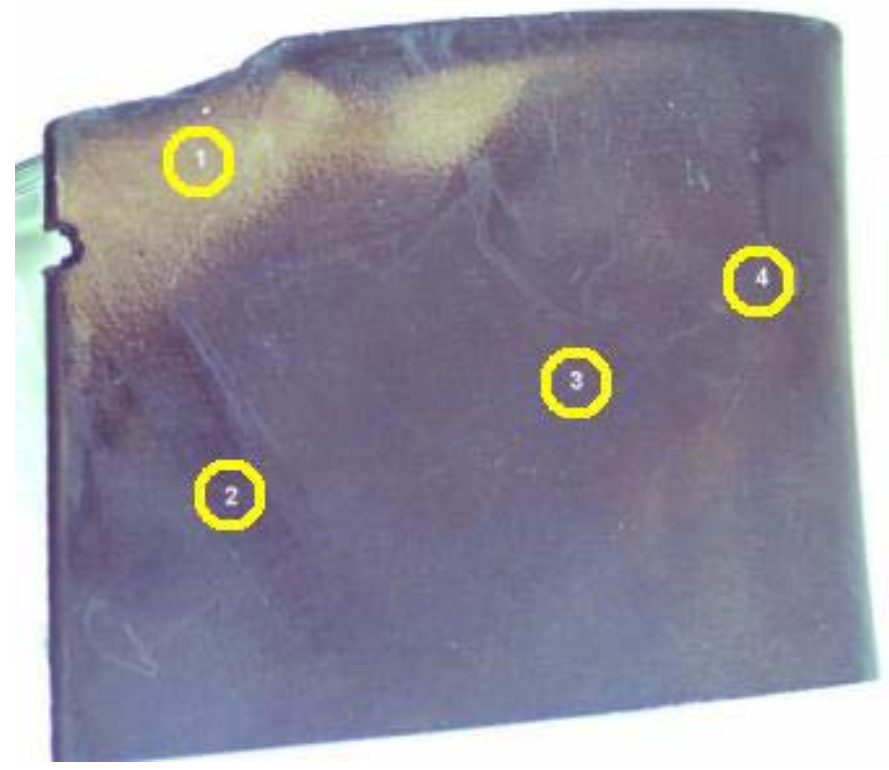

Figure 41: Optical macrograph of the suction side of the airfoil (bucket tip towards top and leading edge on right). The circles indicate the locations at which analyses were performed.

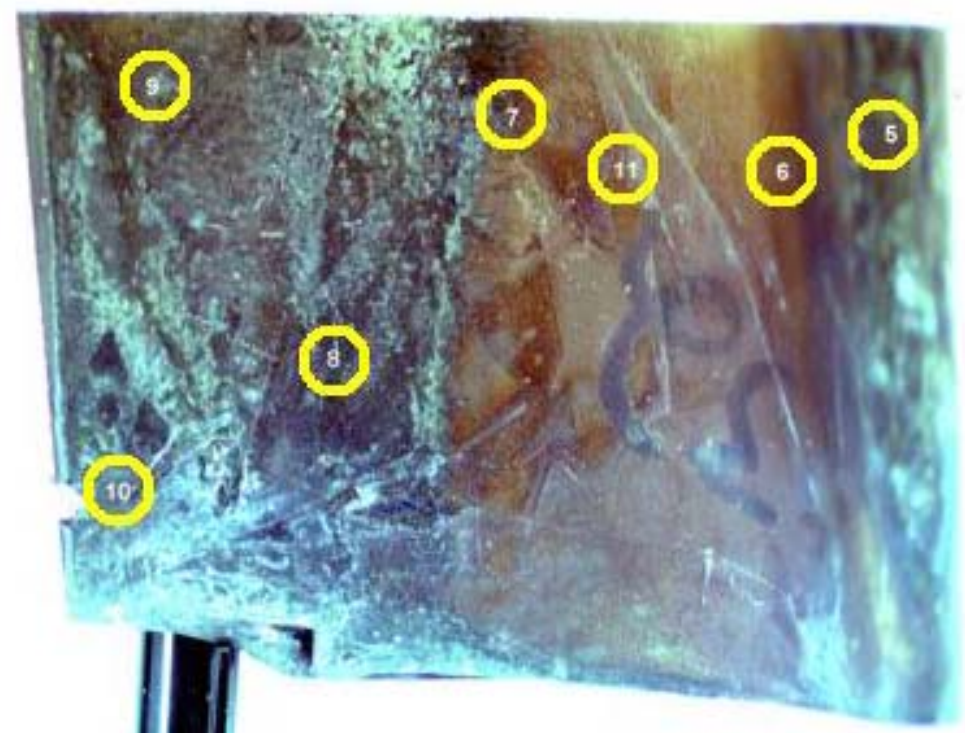

Figure 42: Optical macrograph of the pressure side of the airfoil (bucket tip towards bottom and leading edge on right). The circles indicate the locations at which analyses were performed. 


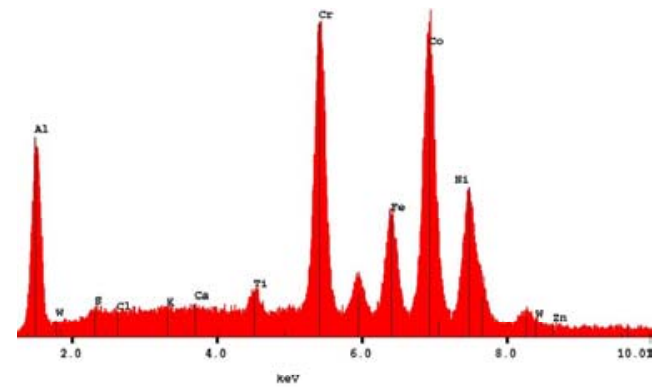

(i)

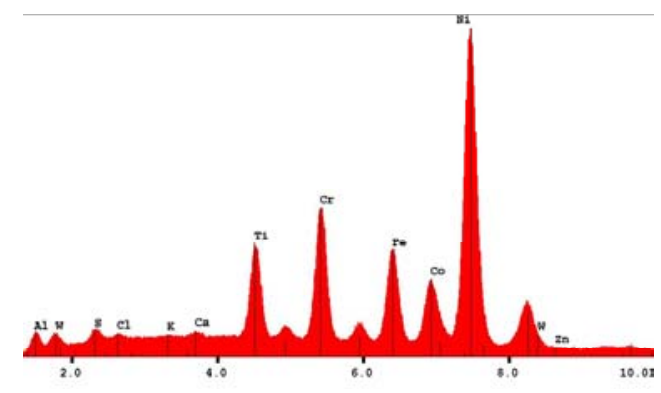

(iii)

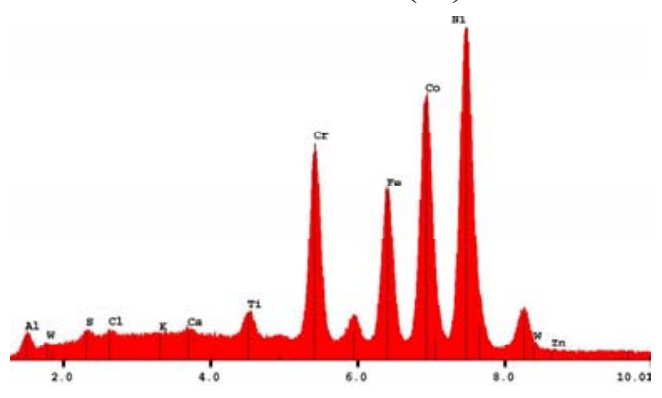

(v)

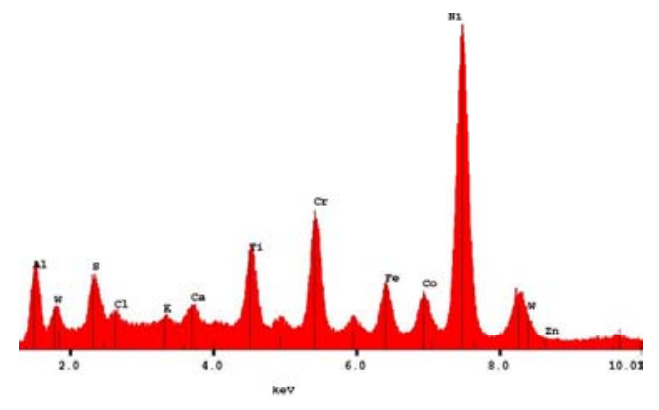

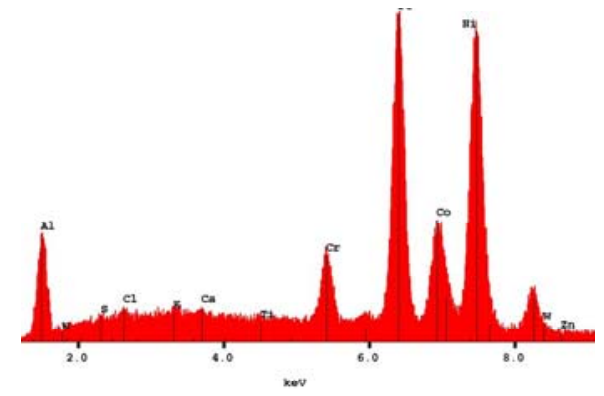

(ii)

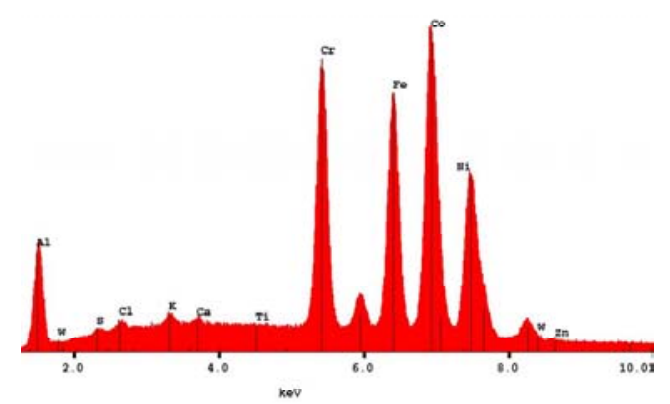

(iv)

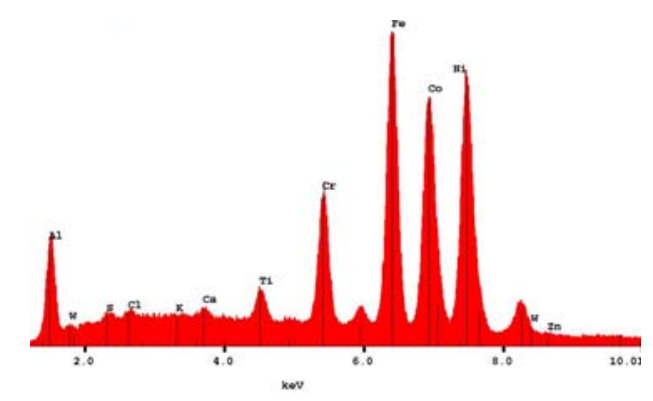

(vi)

(vii)

Figure 43: EDS $(25 \mathrm{kV})$ spectra for the following locations indicated in Figure 41 and Figure 42: (i) 1, (ii) 2 (similar spectra for locations 3 and 4), (iii) 5, (iv) 6, (v) 7 (similar spectrum for location 9), (vi) 8 and (vii) 10 


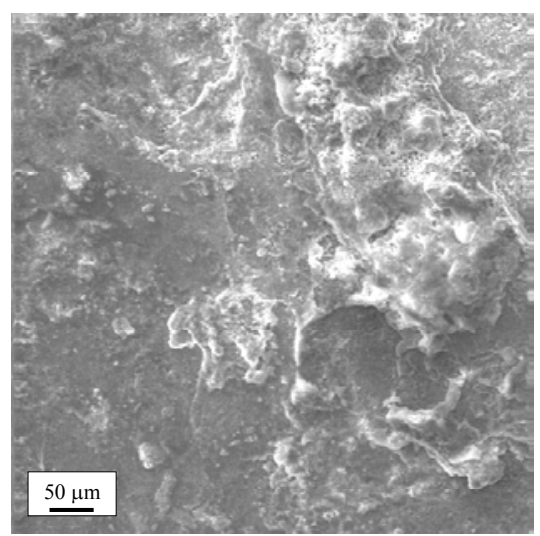

(a)

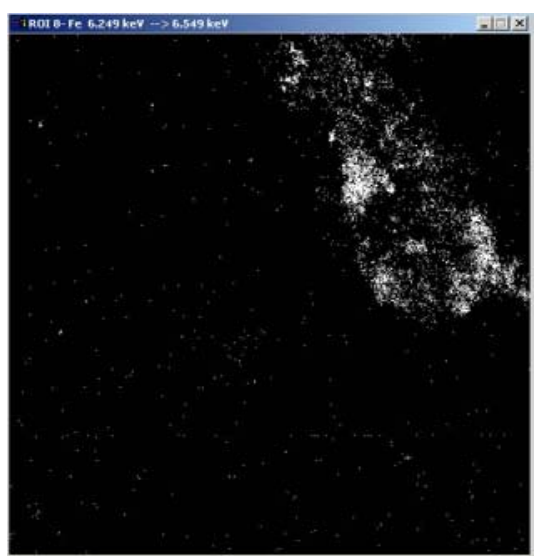

(b)

Figure 44: High magnification micrographs of the airfoil surface at Figure 42, location 7 (pressure side) by (a) backscatter SEM and (b) EDS map for iron 

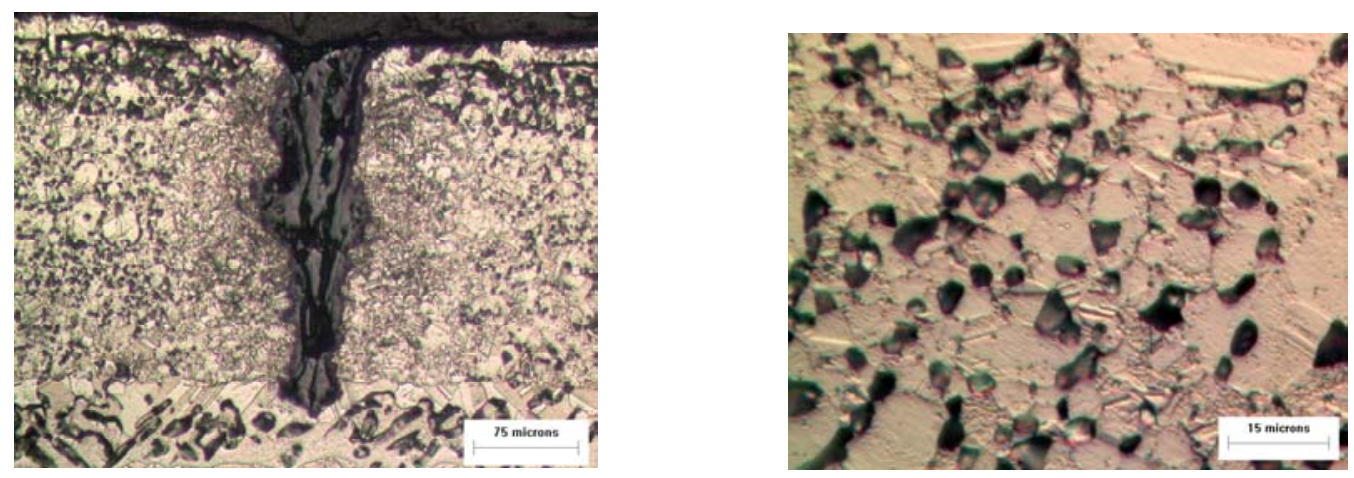

Figure 45: Optical micrographs of a cross-section of the CoCrAIY coating. Sample taken from the suction side of the leading edge, about 3.25" from tip, etched for about 15 seconds in Kallings etch (5gm CuCl2, 100ml HCL, 100ml Ethanol). Dark spots are pits that form immediately after contact with etch.

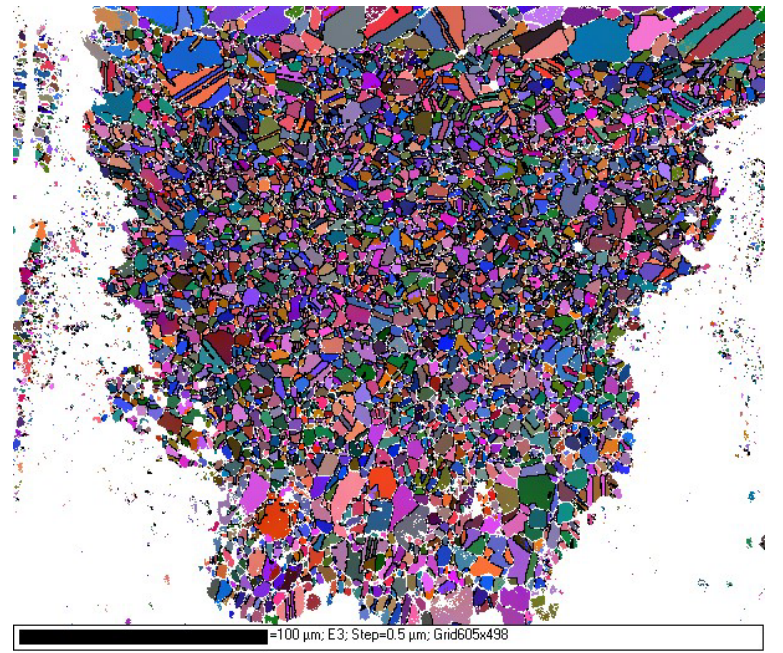

(a)

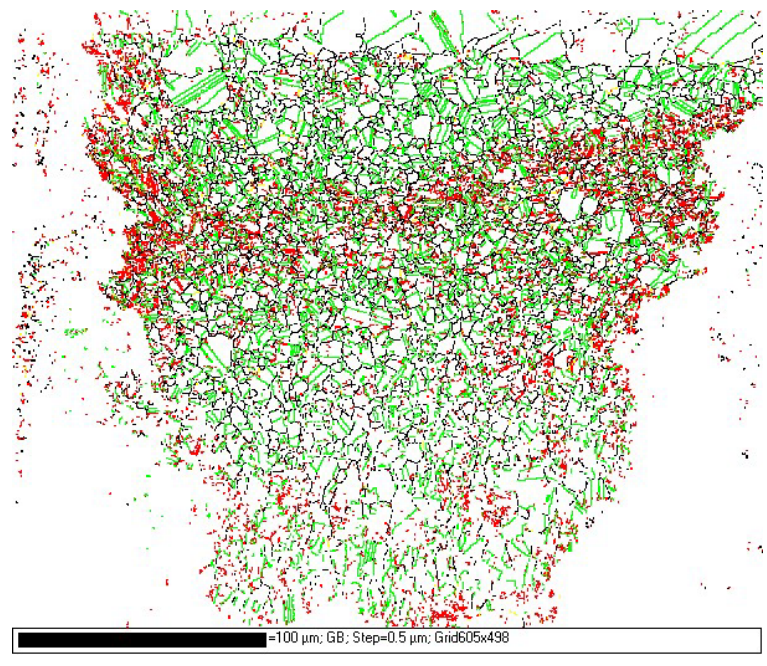

(b)

Figure 46: EBSD micrographs of the CoCrAIY coating, (a) Euler orientation map (colors indicate crystal orientation) and (b) grain boundary misorientation map: Red $=1^{\circ}<\theta<3^{\circ}$, Yellow $=3^{\circ}<\theta<10^{\circ}$, Black $=10^{\circ}<\theta<60^{\circ}$, Green $=$ Twin $\left(60^{\circ}\right)$ 


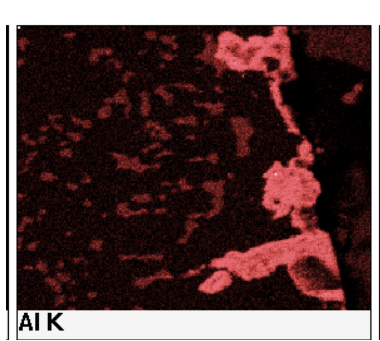

(a)

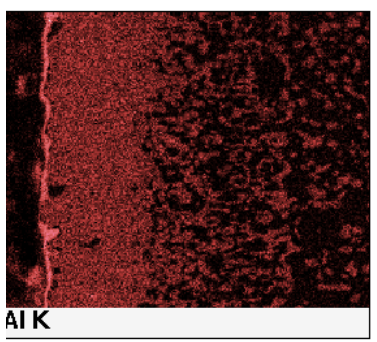

(b)

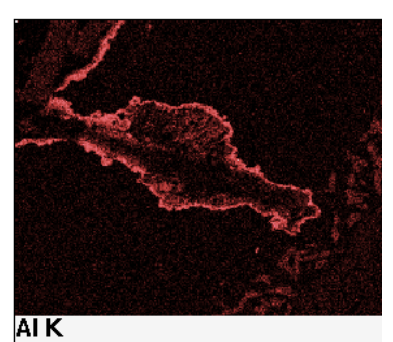

(c)

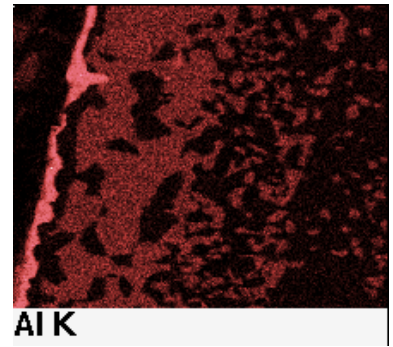

(d)

Figure 47: EDS maps for aluminum at (a) suction side and (b) pressure side by the trailing edge and (c) suction side and (d) pressure side by the leading edge (Substrate to the right of image, except in (a), where it is to the left)

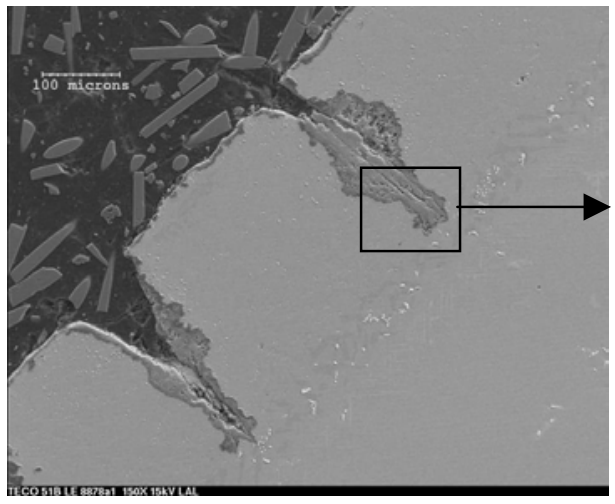

(a)
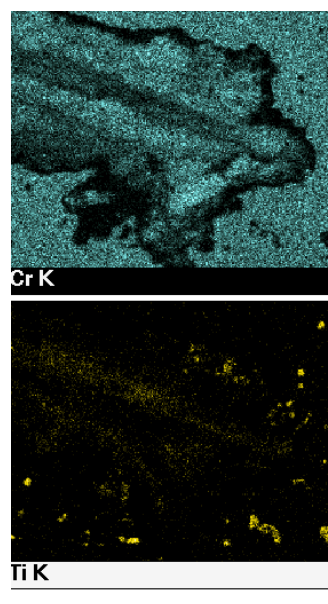

(b)

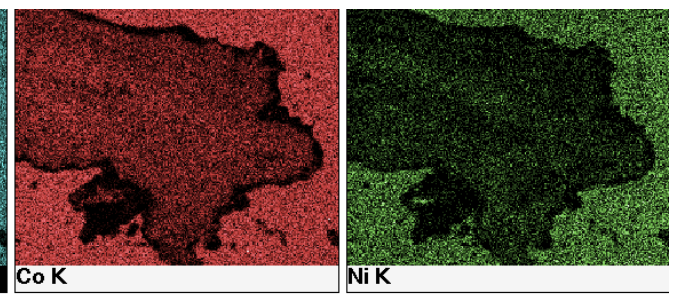

CoK

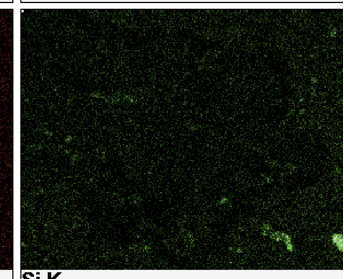

AIK

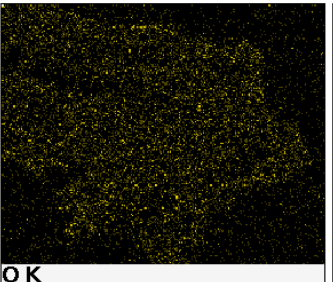

Si K

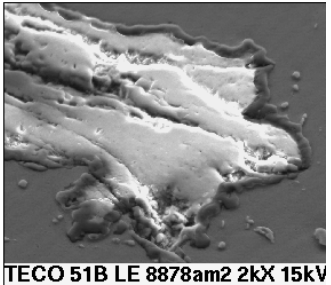

Figure 48: Micrographs of craze cracks found along the suction side of the leading edge ( 3.25" from tip) obtained by (a) backscatter SEM and (b) EDS elemental maps 


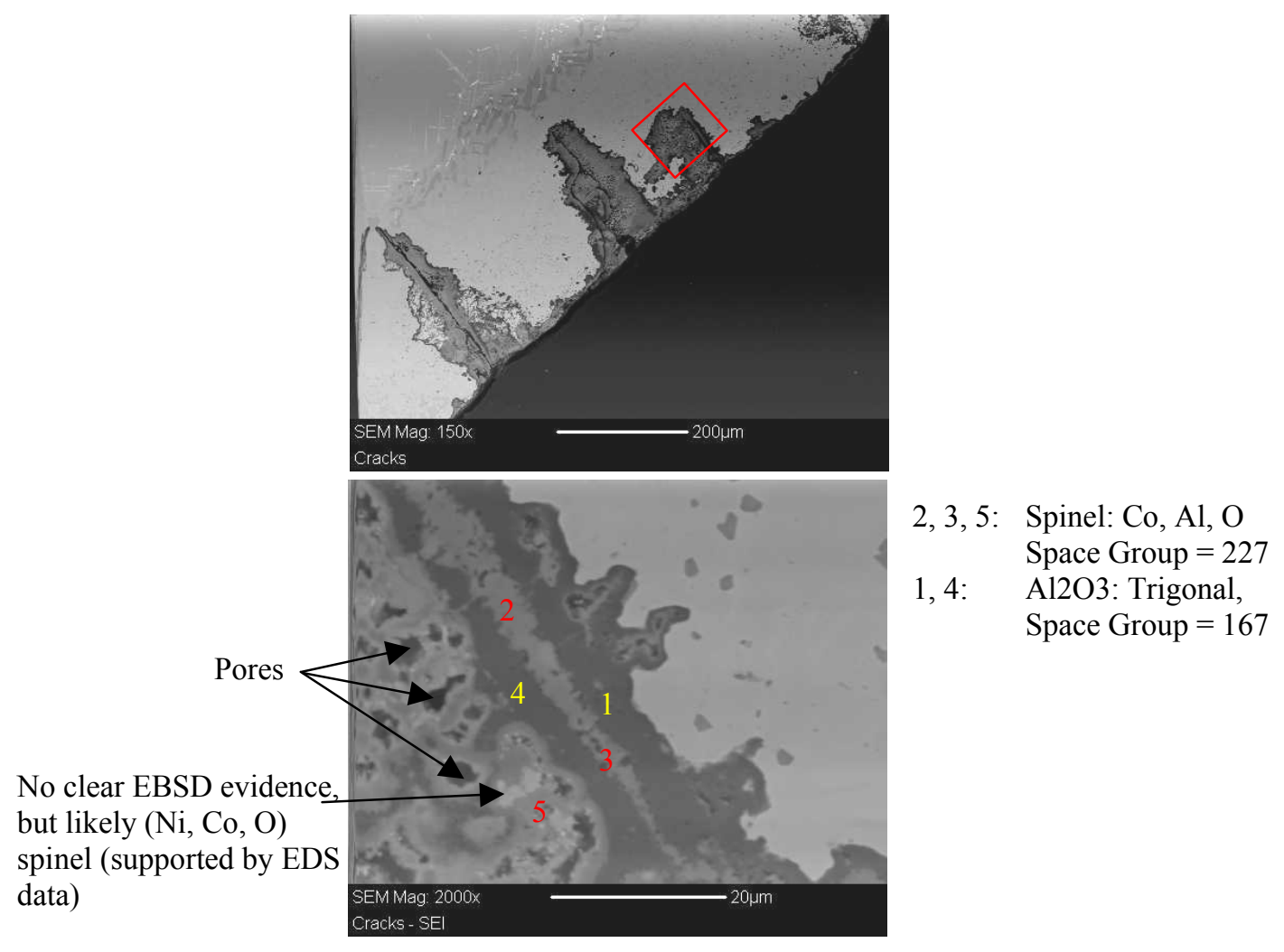

Figure 49: EBSD analysis of the oxide structure of a craze crack on the pressure side 0.5" from the leading edge, about 3.25 " from tip

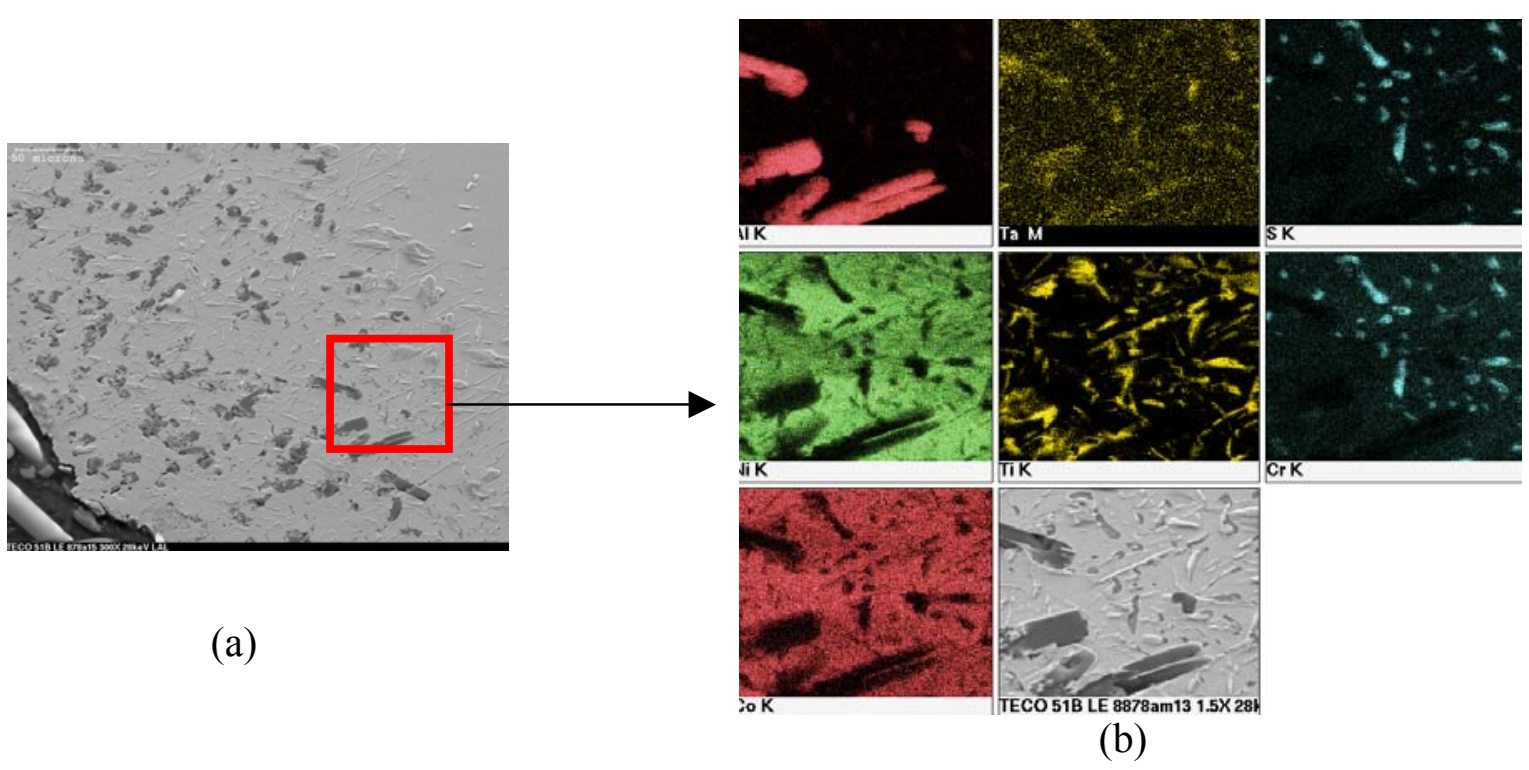

Figure 50: Evidence of sulfur-rich precipitates at the leading edge, about 3.25" from tip, obtained by means of (a) backscatter SEM and (b) EDS map 


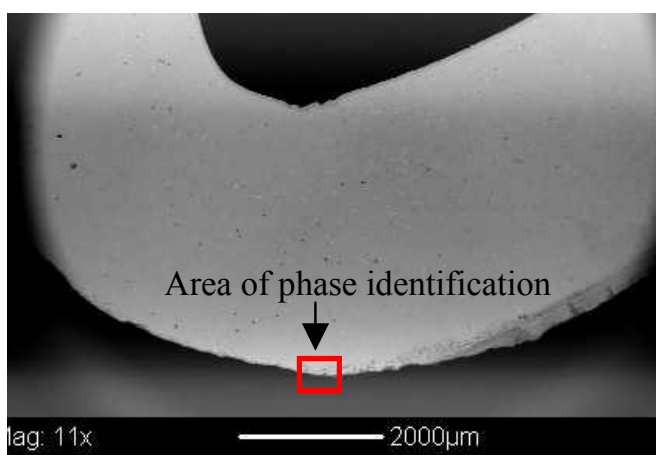

(a)

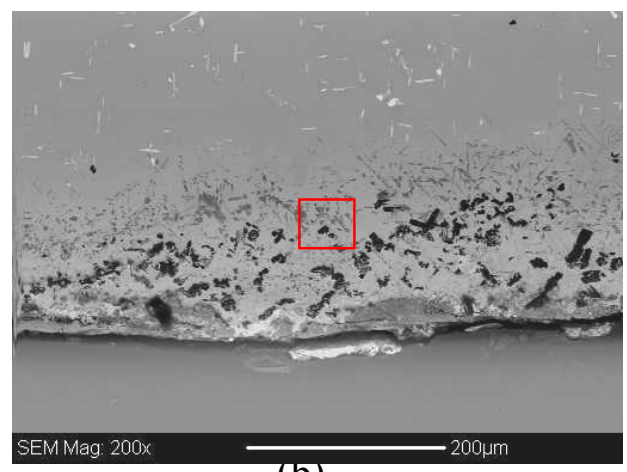

(b)

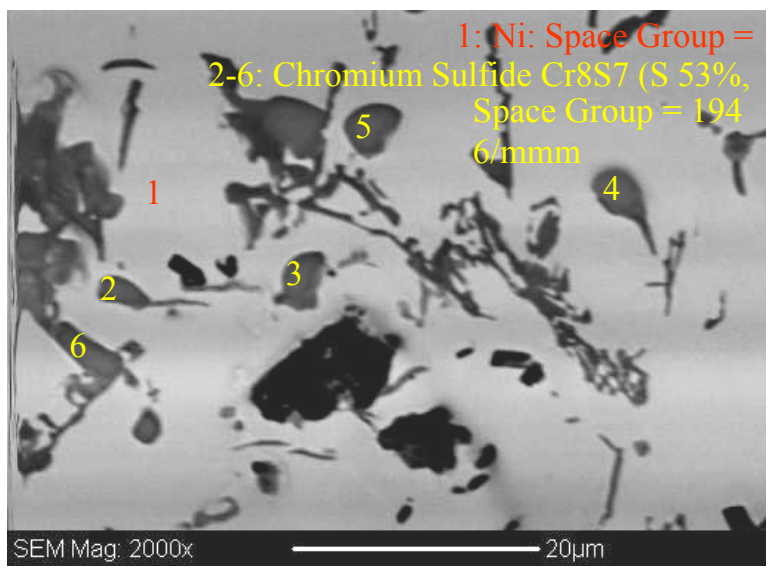

(c)

Figure 51: EBSD analyses of sulfur-rich precipitates. (a) and (b) indicate the area sampled in (c), located on the leading edge, about 3.25" from the tip. 


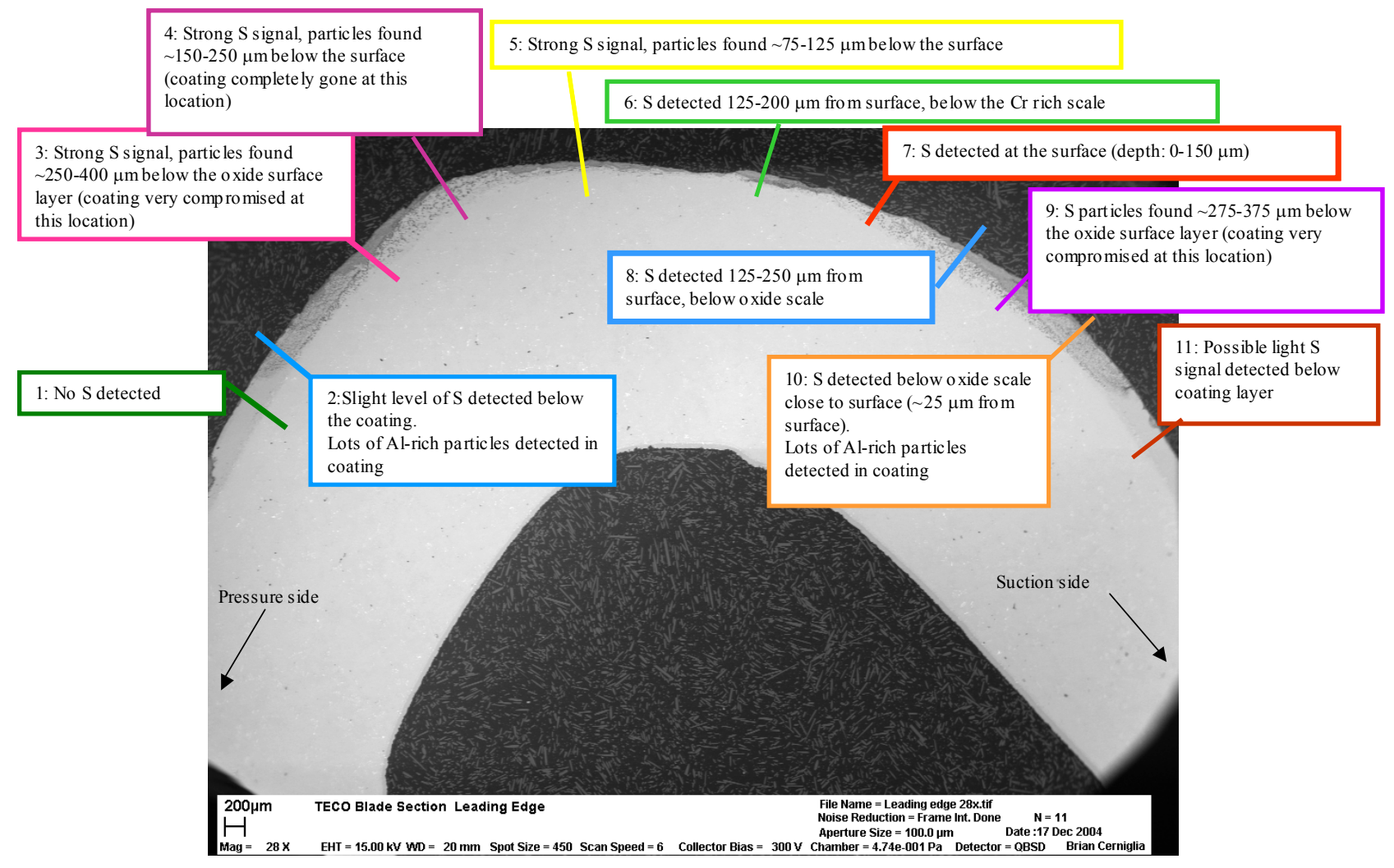

Figure 52: Summary of a series of EDS analyses of a cross-section of the leading edge 3.5" from tip 


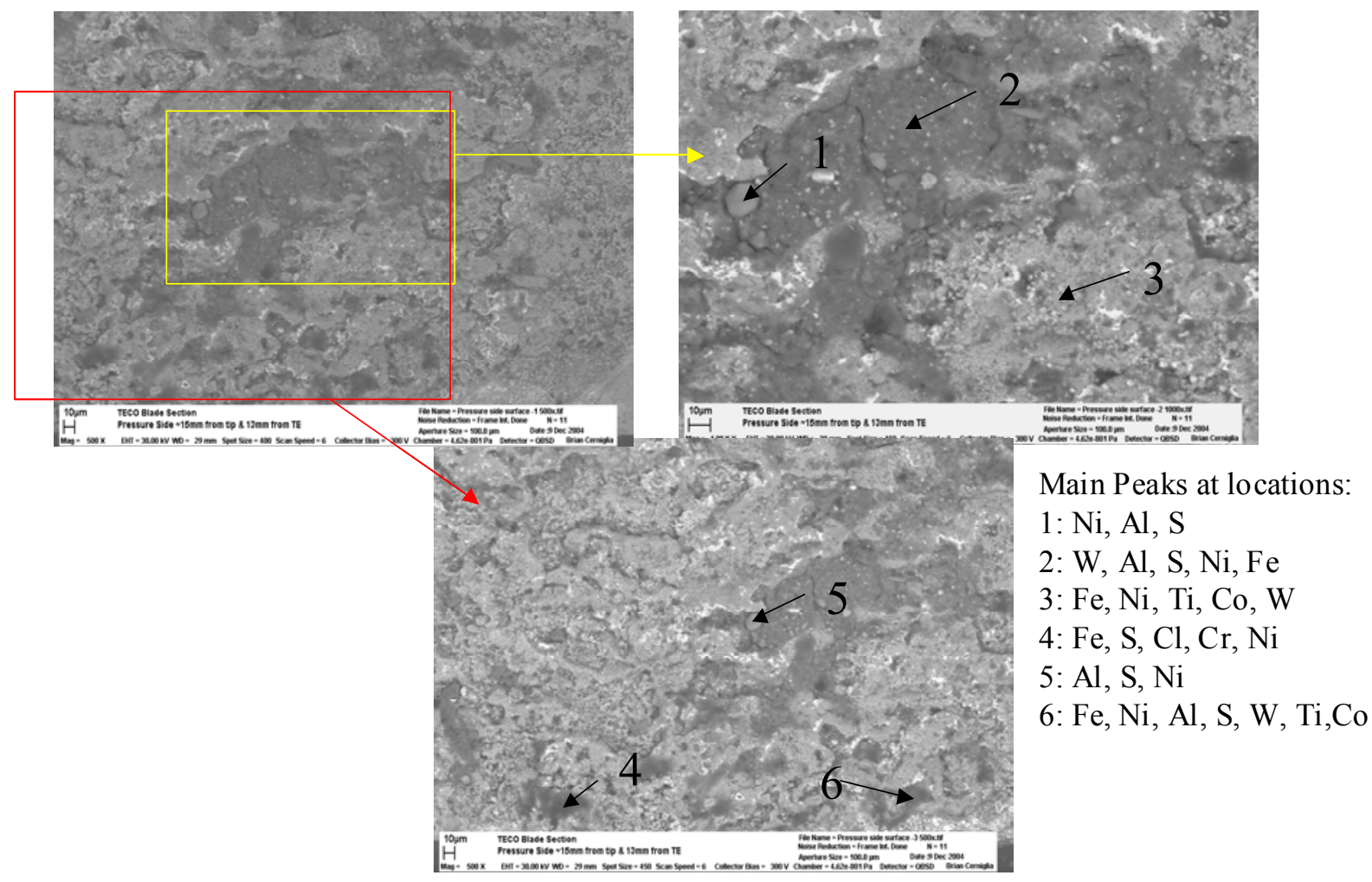

Figure 53: Backscatter SEM images of the airfoil surface near area 10 of Figure 42 with summary of EDS analyses (0.6" from tip and 0.5" from trailing edge).

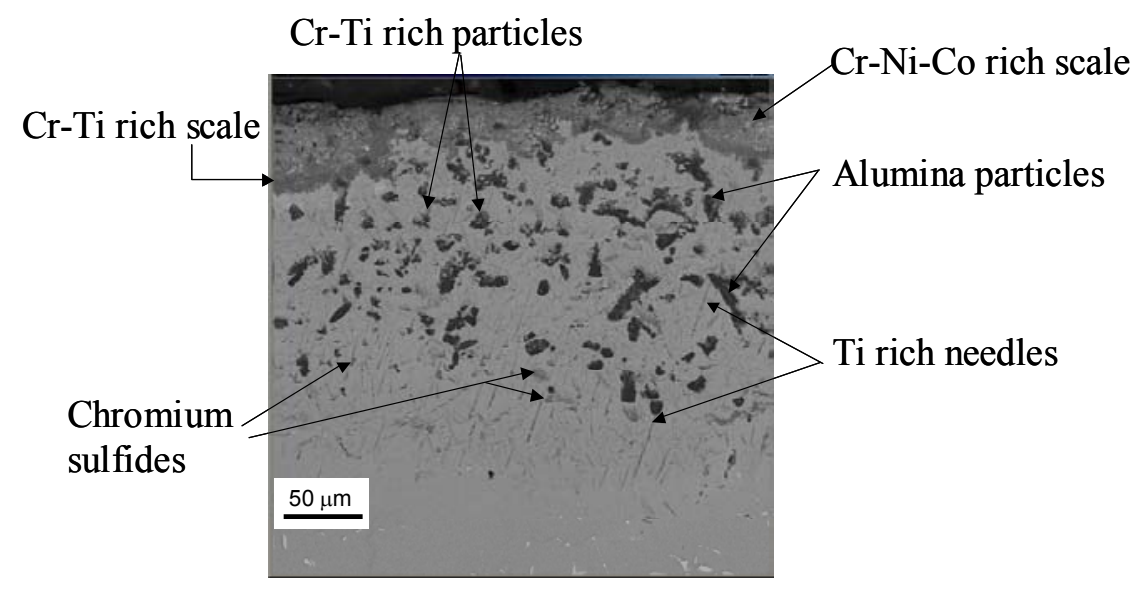

Figure 54: SEM backscatter micrograph of a trailing edge cross-section of the area analyzed in Figure 53 with summary of EDS analyses 

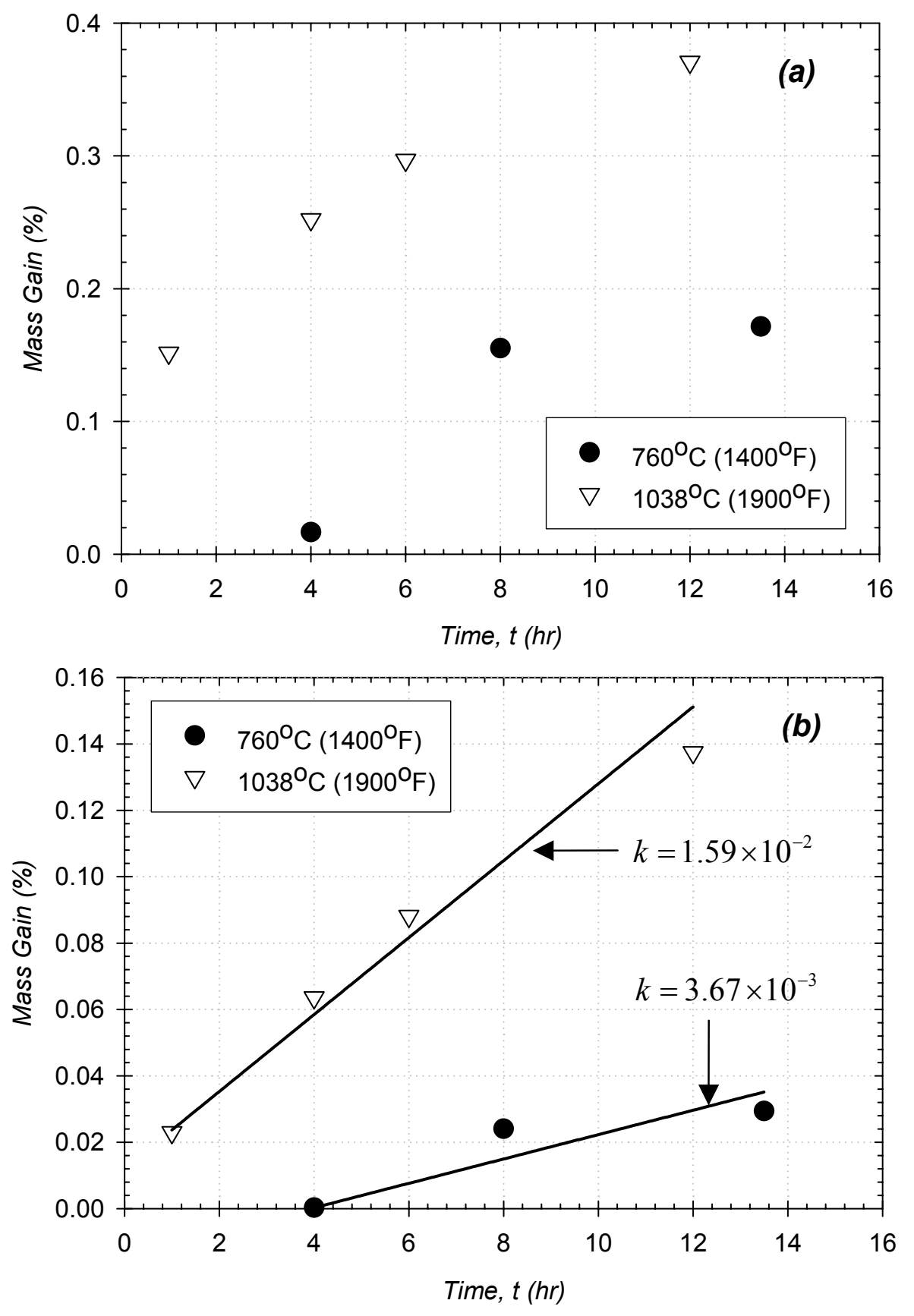

Figure 55: (a) Mass gain and (b) mass gain squared histories of parabolic weight gain of DS GTD-111 in isothermal 100ppm $\mathrm{H}_{2} \mathrm{~S}$ gas 


\section{Task 2 Results/Discussion:}

\section{Material In-Service Health Monitoring}

\section{Task 2 Overview:}

The second task demonstrated new technologies to determine the in-service health of advanced technology coal/IGCC powerplants. The task focused on two critical sensing needs for advanced coal/IGCC gas turbines: 1) Fuel Quality Sensor to rapidly determine the fuel heating value for more precise control of the gas turbine, and detection of fuel impurities that could lead to rapid component degradation. 2) Infra-Red Pyrometer to continuously measure the temperature of gas turbine buckets, nozzles, and combustor hardware.

The contributors to this task were GE Energy, GE Global Research Center (GRC), Sandia National Laboratory(SNL), Tampa Electric Company (TECO), Duke Energy, and Cinergy. Sensor development was performed by GRC and SNL. Sensor validation was performed by GE Energy, GRC and SNL using gas turbines owned by TECO, Duke Energy, and Cinergy.

The Task 2 scope consisted of 4 Sub-tasks:

Subtask 2.1 - Define Coal IGCC Powerplant Requirements: Monitoring requirements were established for coal IGCC and natural gas powerplants. Needs were identified and prioritized using Quality Function Deployment (QFD) and other "Six Sigma" quality tools that are now used widely throughout GE. The Critical-To-Quality (CTQ) characteristics of the required sensors, systems, and controls were identified during this process.

Subtask 2.2 - Sensor Capability Studies: Laboratory trials were performed to baseline the accuracy and repeatability of the fuel quality sensor. The capability of the fuel quality sensor was tailored specifically for coal IGCC fuel constituents.

Subtask 2.3 - IR Pyrometer for Condition Based Maintenance: Infra-Red (IR) pyrometer temperature measurements were validated using experimental data from the laboratory and field data from four gas turbine installations. The pyrometer data were compared to predictions of metal temperature from aerodynamic and heat transfer models. Factors that affect the pyrometer temperature measurement and causes of errors were evaluated.

Subtask 2.4 - Field Deployment and System Validation: Field trials of the fuel quality sensor were performed at coal IGCC facilities. Experiments to verify that the advanced sensor technologies meet the requirements of the power plant environment were performed. 


\section{Task 2 Discussion:}

\section{Subtask 2.1 - Define Coal IGCC Power Plant Requirements}

The GE team conducted a Six Sigma based Quality Function Deployment (QFD) to identify and rank the sensor requirements of a coal/IGCC power plant. IGCC Plant Monitoring Critical to Quality (CTQ) goals (Figure 56) were developed from customer requirements to IGCC gas turbine sub-system CTQs (Figure 57) to gas turbine sensor requirements (Figure 58). Sensors needed for development were prioritized.

Coal/IGCC gas turbine monitoring sensors are needed for, in order of priority, measurement of fuel quality, fuel heating value, hot gas path metal temperature, firing temperature, thermal barrier coating (TBC) loss, combustion flame temperature, exhaust emissions, and combustion dynamics. Some of these requirements can be met simultaneously with one sensor; for example, hot gas path metal temperature, firing temperature, and TBC coating loss can all be determined using the pyrometer.

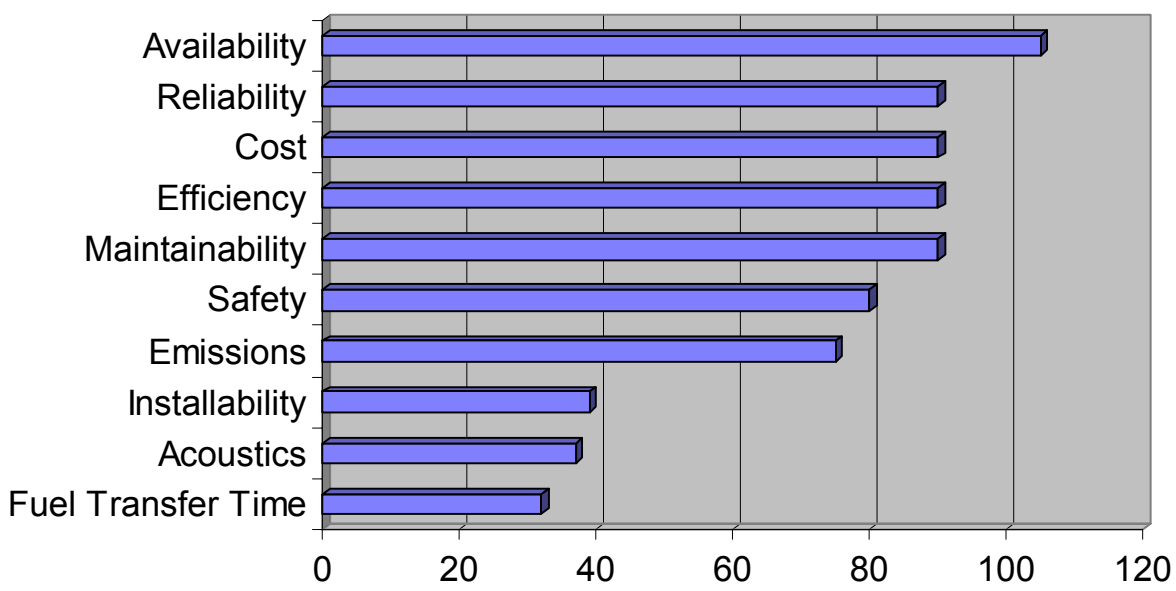

Figure 56: IGCC Plant Critical to Quality (CTQ) Characteristics

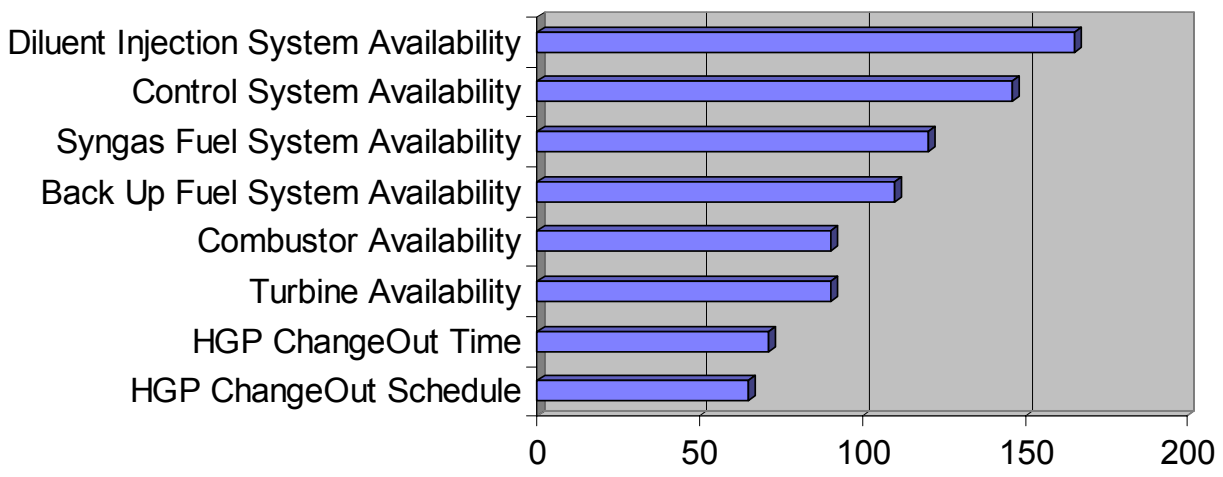

Figure 57: IGCC Gas Turbine Critical to Quality (CTQ) Characteristics 


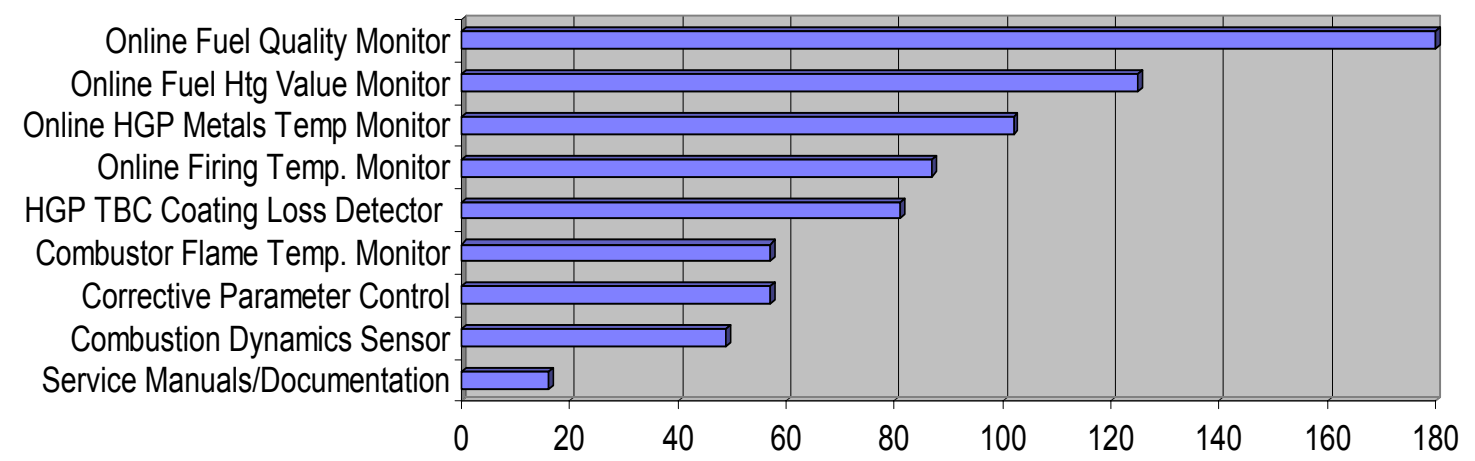

Figure 58: IGCC Gas Turbine Sensor Needs

\section{Subtask 2.2 - Sensor Capability Studies}

Gaseous fuels used by gas turbines and reciprocating engines can have significant variations in energy content, which can significantly affect performance and reliability. In particular, variation in syngas fuel quality from coal IGCC processes is of great concern. Additionally reciprocating engines commonly use special fuels that contain bio-gas and waste gases, which can change significantly over short time periods. Hence, subtask 2.2 concentrated on a sensor design to rapidly and accurately determine the lower heating value (LHV) of gaseous fuels.

Fuel LHV Sensor Flow Optimization and Calibration

Figure 59 shows data from an initial screening experiment of flow conditions to identify those regions of fuel:air ratio for which combustion occurs. The top plot shows the power required to keep the catalyst hotplate at constant temperature. The bottom plot is the difference between the catalyst hotplate power and the reference hotplate power. Regions of combustion can be identified using the bottom plot; e.g. these are the 4 regions that show a large positive signal (30sccm: $40 \%$ and 50\% fuel, 50sccm: $40 \%$ and 50\% fuel). These data were used to plan further tests to optimize the flow in those regions where combustion takes place.

Figure 60 shows the influence of mixture $\lambda$ on the final power difference (difference between the catalyst and reference hotplate powers). The trend decreases linearly as the mixture becomes leaner, with only one data point deviating slightly from the curve. The power decreased with increased $\lambda$, as was expected for this leaner mixture.

Tests of individual constituents; including methane $\left(\mathrm{CH}_{4}\right)$, ethane $\left(\mathrm{C}_{2} \mathrm{H}_{8}\right)$, and $\mathrm{CO}$; were performed. Figure 61 shows the signals for pre-combustion ethane, post-combustion ethane, and post-combustion $\mathrm{CO}_{2}$. The $\mathrm{CO}_{2}$ results indicate an increase in combustion until $\sim 6 \%$ ethane concentration, after which the combustion decreased slowly. CO was not present in the exhaust at appreciable concentrations, indicating that combustion was proceeding primarily to $\mathrm{CO}_{2}$. Figure 62 shows results for $\mathrm{CO}$; in this case, combustion appeared to maintain its efficiency independent of concentration, as indicated by increasing $\mathrm{CO}_{2}$ in the exhaust. 


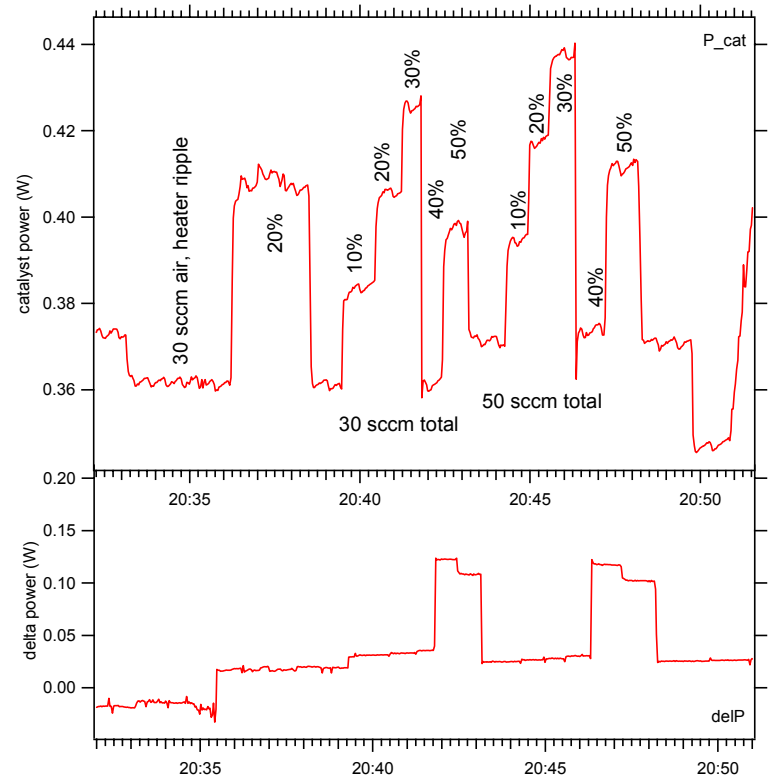

Figure 59: Screening DoE (design of experiment) to determine flows for which combustion occurs on catalyst hotplate

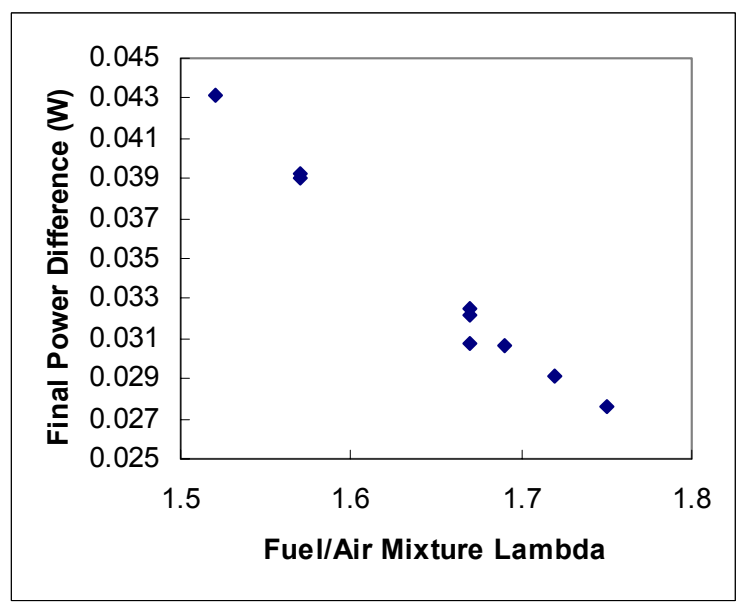

Figure 60: LHV sensor power as a function of lambda (fuel to air mixture) 


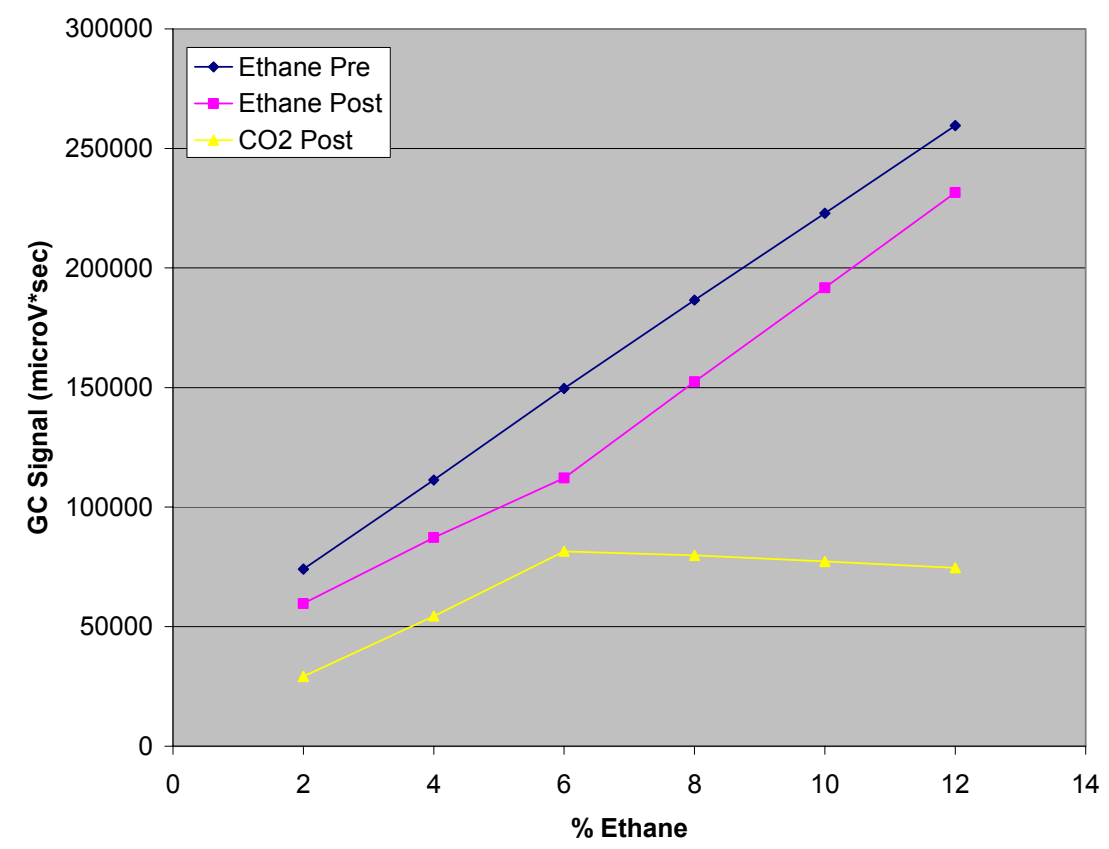

Figure 61: LHV sensor combustion effects on ethane and $\mathrm{CO}_{2}$

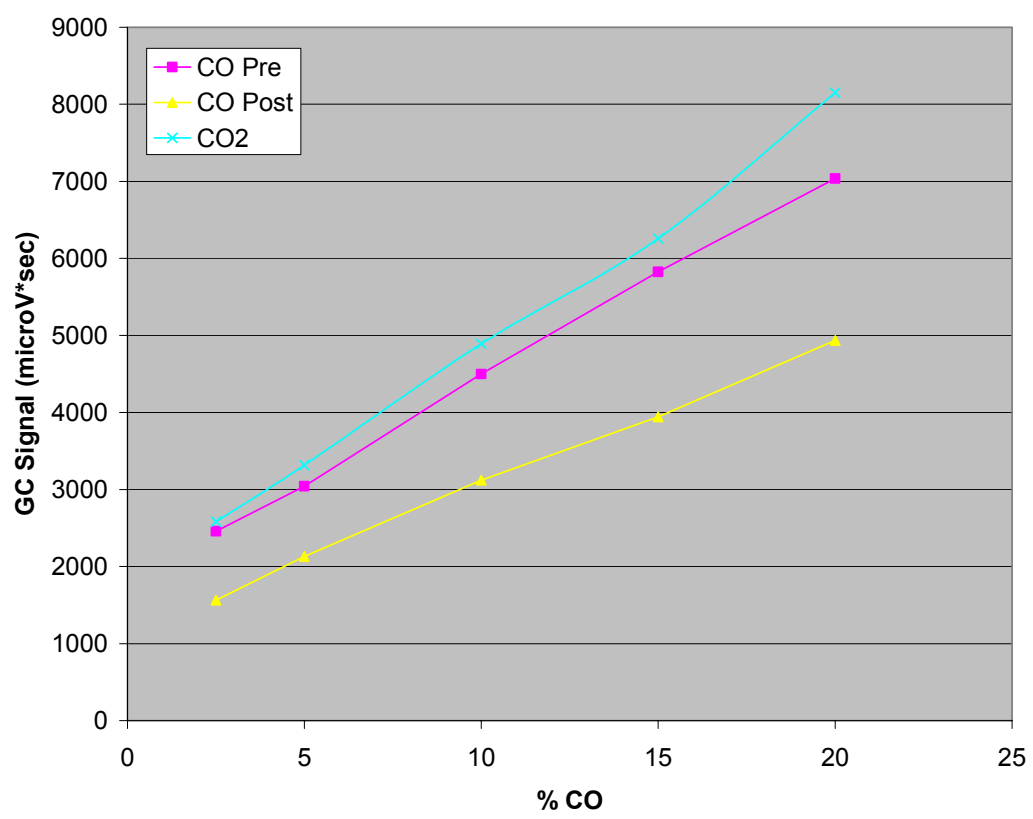

Figure 62: LHV sensor combustion effects on CO 
Figure 63 shows the LHV sensor signal over the same range of concentrations, and validated the interpretation of decreasing combustion with higher ethane concentrations.

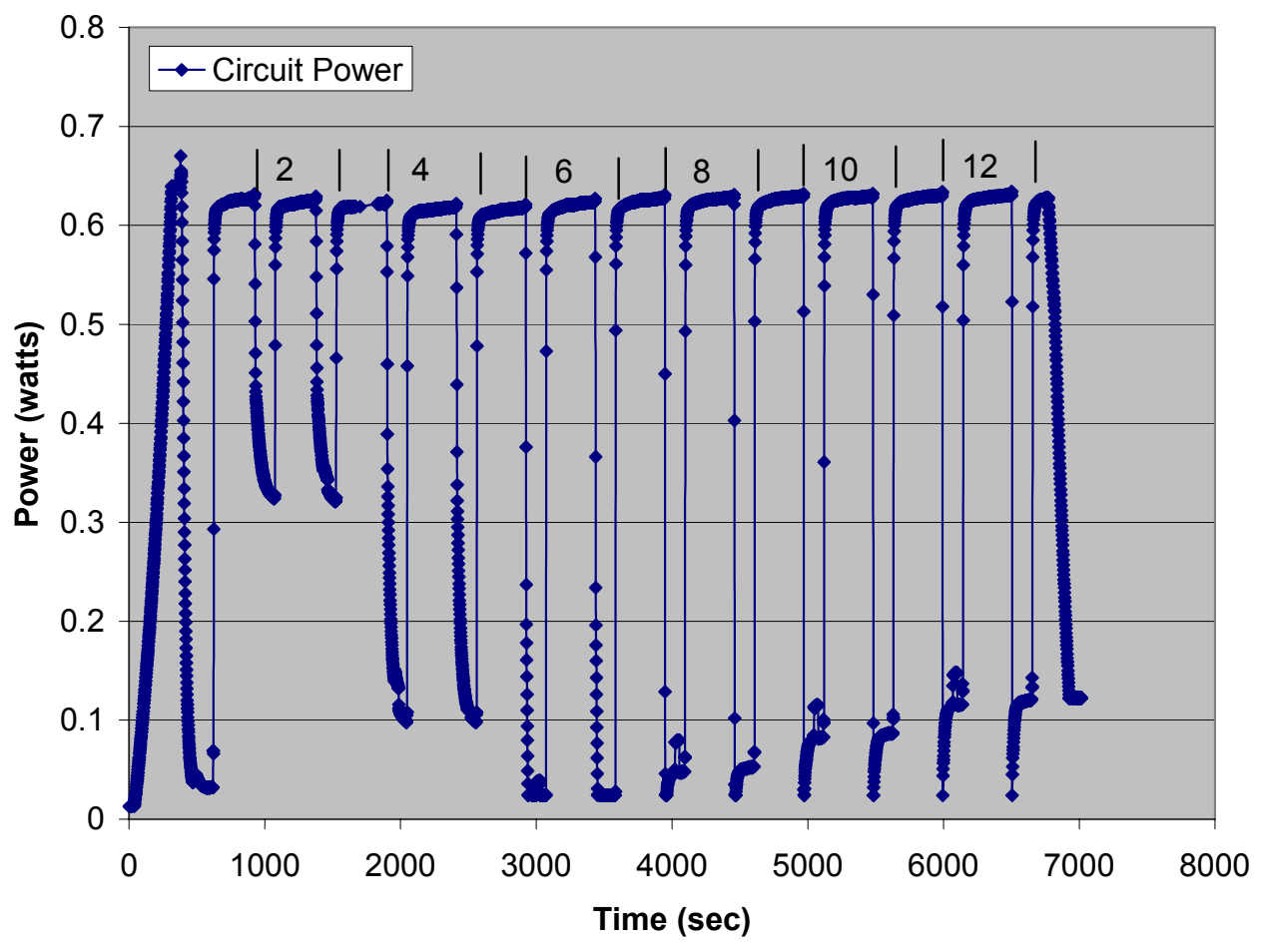

Figure 63: LHV sensor signal responding to varying concentrations of ethane

Analysis of test results using the natural gas standards, which are more complex hydrocarbon mixtures of $\mathrm{C} 1-\mathrm{C} 5$, showed similar results. Figure 64 shows the \% change of each hydrocarbon constituent. This indicates that combustion efficiency is a function of both the hydrocarbon order and the natural gas concentration. Of note is the poor combustion of methane, independent of concentration. When methane is tested alone, it exhibits both stronger conversion and a point of maximum combustion at $\sim 15 \%$. The difference in combustion efficiencies between methane in a fuel mixture and singularly indicates the presence of competing reaction mechanisms. The point of maximum combustion for the natural gas standard 303102, indicated by a peak in the carbon dioxide concentration, appeared to be around 8\%. Carbon monoxide emissions in the exhaust were extremely small, indicating nearly complete combustion for those constituents that undergo reaction. 


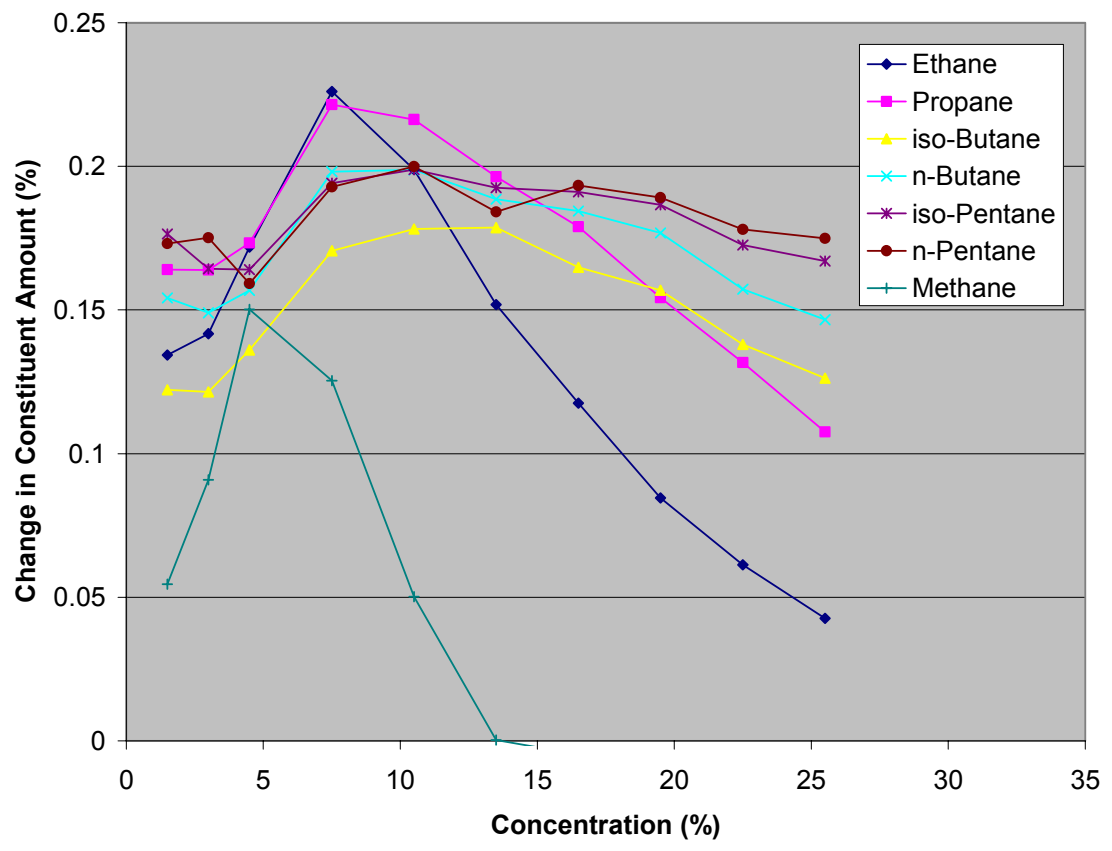

Figure 64: Percent change of natural gas constituents by concentration

Fuel LHV Sensor Hotplate Heater Optimization

Thermal modeling using ANSYS showed that the previous design possessed a fairly uniformly heated central area; however, it was believed designs with a more uniformly heated device area could be created. ANSYS modeling showed that a single heater with a variable width wiring configuration could improve the heating uniformity and reduce the temperature gradients across the device. The addition of metallization between the wires to distribute heat away from the center of the membrane further improved the design. The final design, showed as a half-model in Figure 65, provides an almost two-fold increase in the uniformly heated device area.

\section{Fuel LHV Sensor Catalyst Optimization}

There was a relatively large signal variation caused by minor dimensional changes in the deposited catalyst, as seen in Figure 66. To facilitate the desired repeatability, a spray-coating system was used that consists of a computer controlled stage and a nebulizer. The nebulizer was used to spray catalyst slurry through a shadow mask onto the device. Initially, the high liquid content of the slurry caused catalyst spot distortion. Catalyst spot cracking could also occur due to the stresses induced during drying of the slurry.

To address these problems, a heated stage was developed that would boil the liquid from the catalyst slurry as it was deposited. A kapton heater and temperature controller was used to increase the stage temperature to $177^{\circ} \mathrm{C}\left(350^{\circ} \mathrm{F}\right)$. Results indicated that the deposited catalyst is significantly more robust and has better adhesion to the device surface. The dimensional control of the spot sizes also improved. 


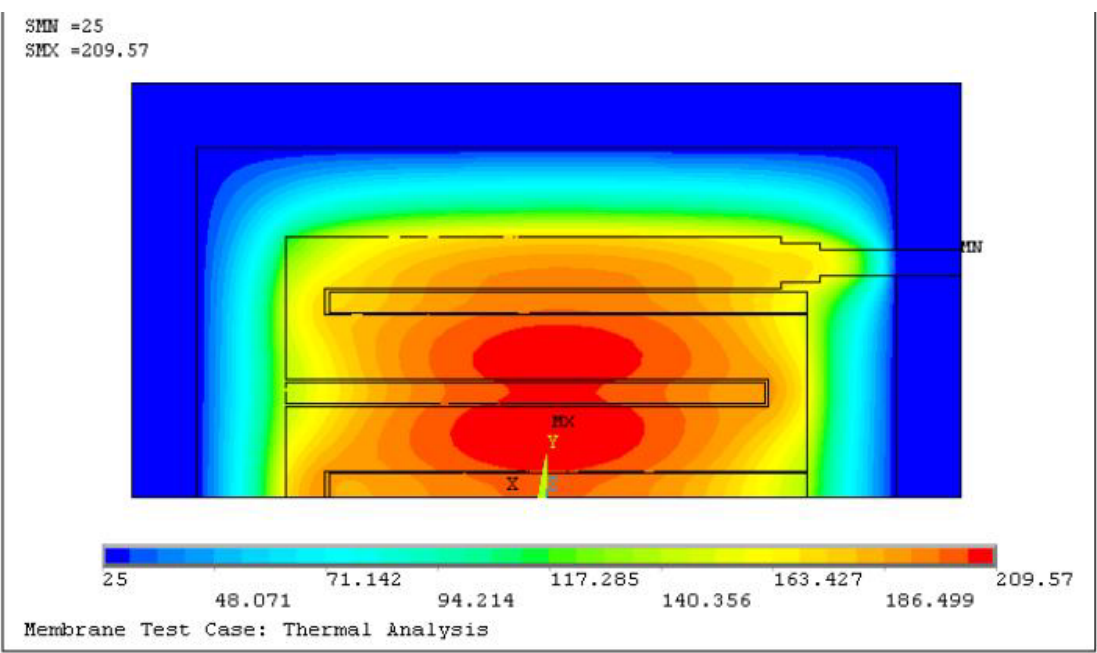

Figure 65: Finite element model of an optimized heater in the sensor hotplate. This design shows a significant increase in the area of uniform heating.

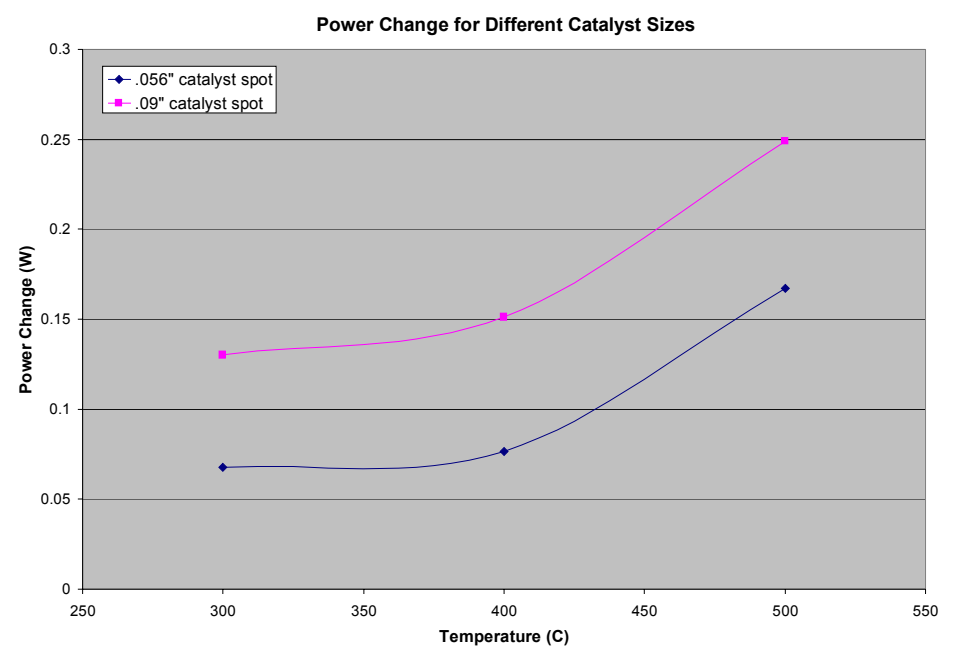

Figure 66: Sensor output as a function of hotplate temperature for two different size catalyst depositions

The value of a catalyst with higher Platinum content was demonstrated. Current catalyst sources consist of Alumina combined with $1 \%$ Pt by weight. A test of a catalyst with $10 \% \mathrm{Pt}$. by weight has been completed, and the results are shown in Figure 67. Keeping all other testing conditions constant, such as sample flow and natural gas concentrations, the signal change caused by the $10 \%$ weight catalyst was greater by a factor of 2 to 3 . This increased signal results from the greater availability of catalyst reaction sites in the newer catalyst formulation. The increased sensitivity of the device could be used to test lower concentrations of hydrocarbons, and could result in more accurate BTU measurement. 

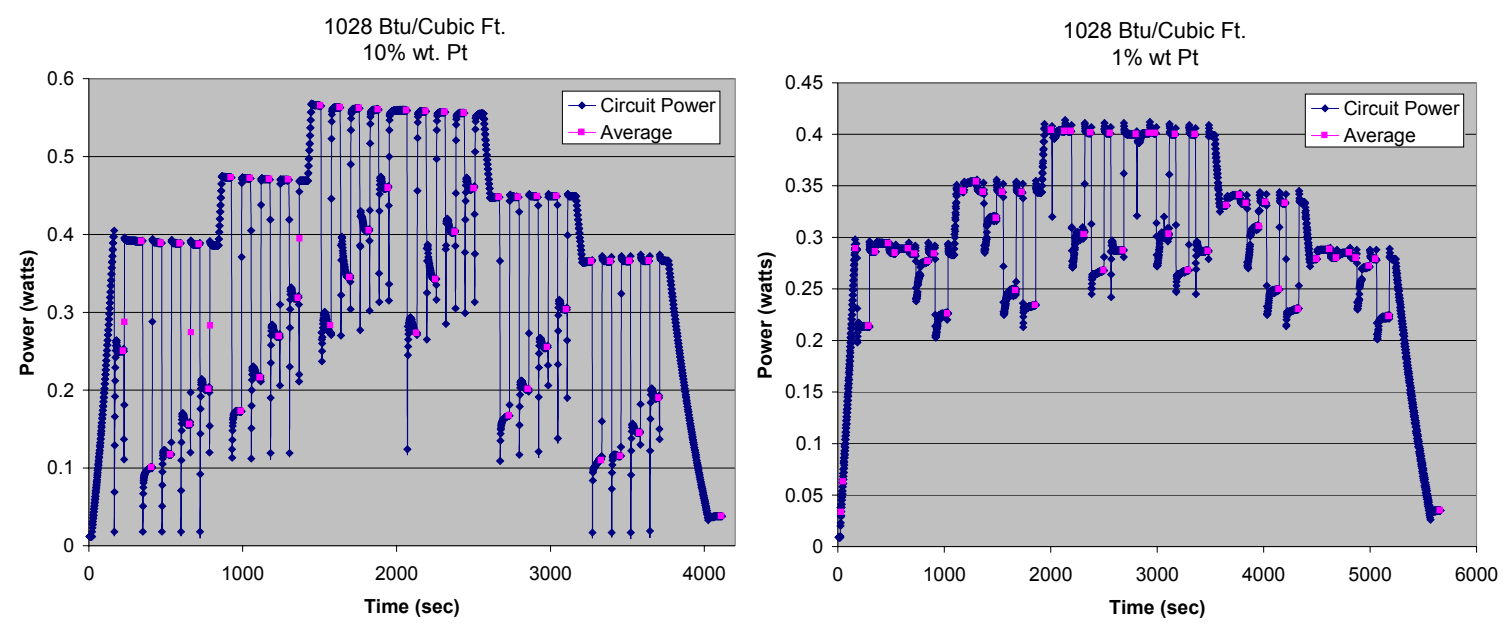

Figure 67: Sensor response for gas mixture with varying fuel:air mixtures. The left plot is for a $10 \%$ Pt catalyst and the right plot is for a $1 \%$ Pt catalyst.

\section{Optimization of Sensor Parameters for Syngas Fuels}

Representative data from the optimization experiments are shown in Figure 68. A number of designed experiments were run to find the ideal temperature and equivalence ratio (phi) at which to operate the sensor with Syngas fuels. In the plot shown, the percentage of Syngas in the total flow is increased with time moving from left to right (i.e. phi is increasing). Each peak is a different percentage, with the fuel flow stopped in between each. When there was no fuel flowing, the base amount of air $(30 \mathrm{sccm})$ was flowing. For each peak, an average value and standard deviation was obtained. The overall goal was to maximize the average value and minimize the standard deviation. From these experiments, it was determined that the sensor can be operated over a rather broad range of phi values, ranging from about 0.4 (lean mixture) to 1.1 (rich mixture). Obviously, to obtain accurate measurements of LHV, total combustion of the fuel needs to take place in the sensor; thus, flows were kept in the lean regime. Finally, from the optimum flow ratios, ideal orifice sizes were calculated for the field tests.

\section{Fuel LHV Sensor Reliability}

A number of issues were investigated to identify the root cause(s) of the short hotplate lifetime observed in some tests. The power circuit was found to track oscillations greatly in excess of those seen in the field, and the other environmental causes were found to have no effect on hotplate lifetime. Our preliminary conclusion is that residual stresses in the hotplate layers are leading to premature failure. Work has been done to vary the materials used, thickness, and deposition parameters to reduce these stresses.

Two micrographs of a reference hotplate are shown in Figure 69. Images such as these were taken for each of the hotplates before it was used in experiments. In the left hand image, the back of the SiN thin membrane is pictured after the hotplate has been run for a period of time. Notice the appearance of a rippled texture. This will be addressed further below. In the right hand micrograph, note that the alumina spot is offset to the left of center of the SiN membrane (the lighter background area). In general, it has been seen that membranes typically have split or burst, with the splits originating in the uncoated area and the corners of the membrane. Thus, the offset alumina spot causes a thermal stress in the membrane due to unequal heating. 


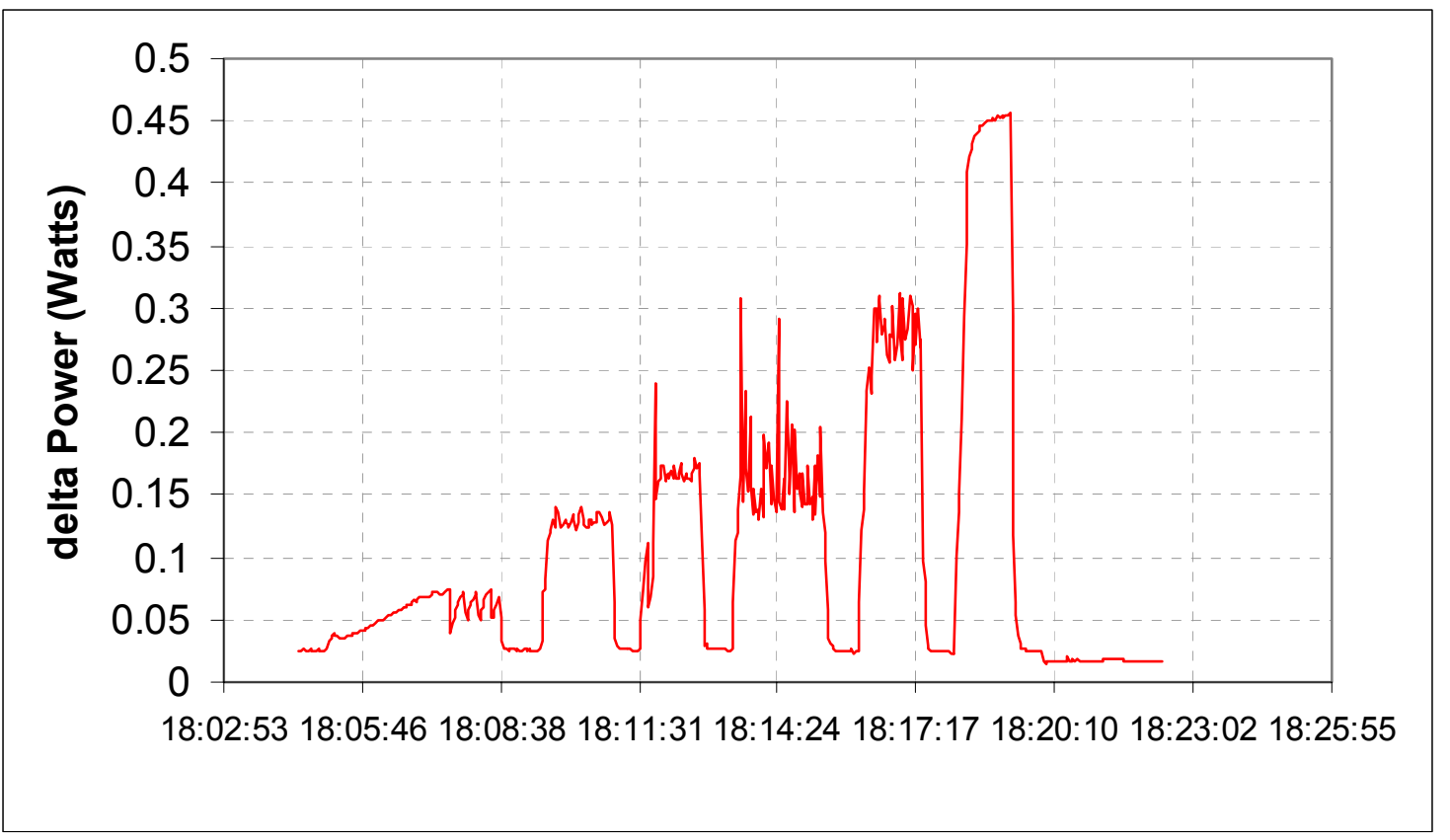

Figure 68: Sensor output versus time for Syngas fuel. Each plateau corresponds to a change in the Syngas to air ratio.

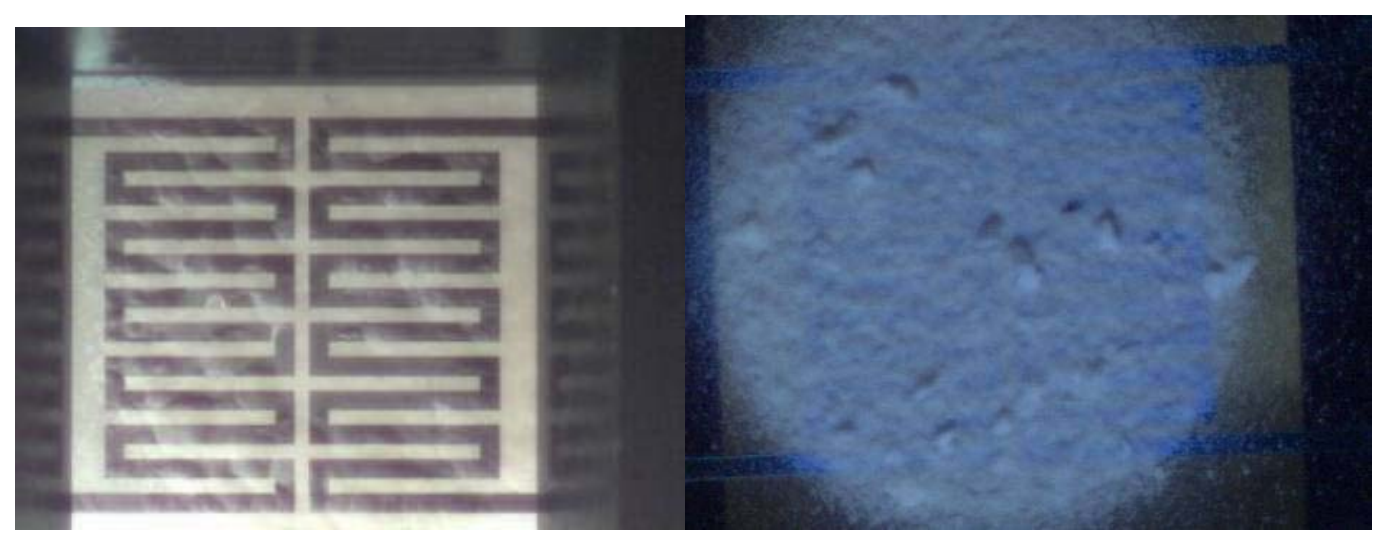

Figure 69: Micrographs of back and front of hotplate SiN membrane with Alumina reference coating. Note the wrinkled appeared of the backside due to conflicting stress between hotplate layers. 
Infra-Red (IR) micrographs of the hotplates during operation are shown in Figure 70. These images were taken to validate the ANSYS finite element modeling of the hotplate temperature distribution.

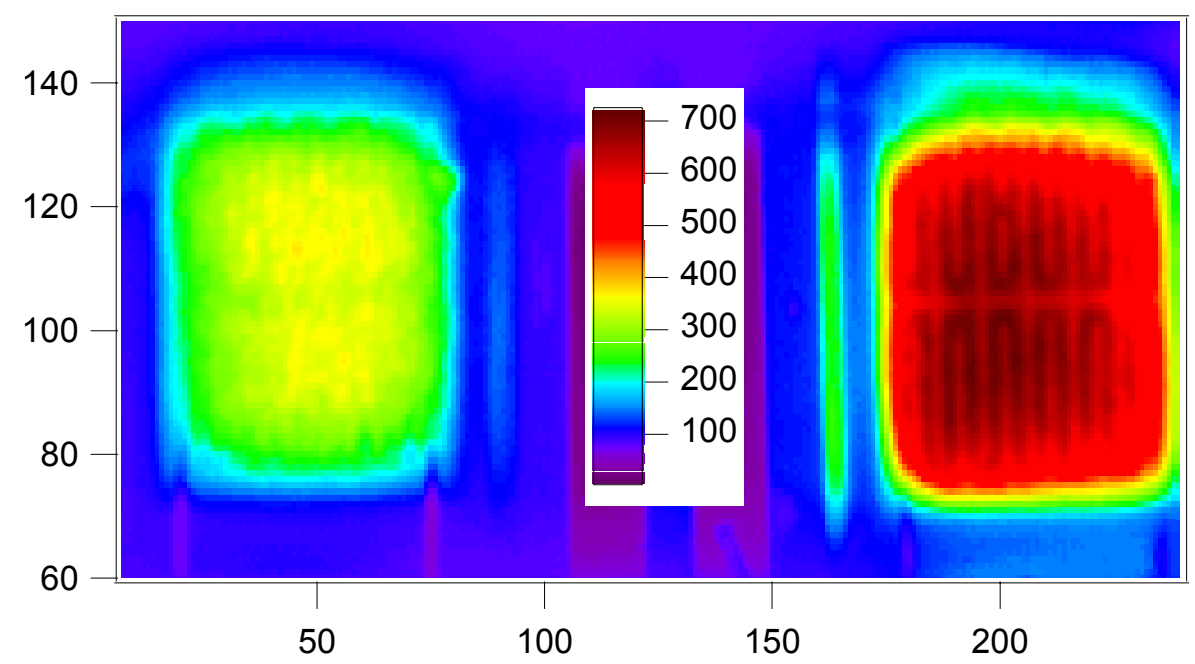

\section{Figure 70: Infra-Red temperature plot of reference (left) and catalyst (right) hotplate during operation. Temperature scale is in degrees $\mathrm{C}$. Flow conditions: $\Phi=0.95 ; . T_{\text {cat_average }}=400 \mathrm{C}, \mathrm{T}_{\text {ref_average }}=300 \mathrm{C}$}

Wafer bow stress measurements for the heater thin film layer and the capping dielectric stack are shown in Figure 71 and Figure 72. Both of these plots show the layer stress as a function of temperature, note that positive values in both diagrams indicate tensile stress. Additionally, multiple measurements are show on each plot. These correspond to subsequent temperature ramps of the sample. For example Run 1 is the first temperature ramp, Run 2 is the second temperature ramp of the same sample.

The stress of the heater layer is shown in Figure 71. Notice that at room temperature that the stress in the layer is tensile, however as the temperature is increased the stress becomes slightly compressive. Comparing these results with the temperatures seen in the IR image is of interest. While the hotplate has been designed to have a uniform temperature profile, there is of a course a profile from hot to cold near the edges of the SiN membrane. Comparing this temperature range with the stress diagram, it can be observed that the stress in the heater layer varies over the heater from compressive to null. It is possible that this variation in stress could have an effect on the overall fatigue failure of the hotplate. However, this is considered to be unlikely, given that the compressive stress values are relatively small.

The stress in the dielectric stack that is used to prevent oxidation of the heater wires is shown in Figure 72. The first fact to note from this plot is that there is a large change in the layer's characteristics with each subsequent temperature ramp. However, after the first several repetitions, it can be seen that the stress settles onto a constant line with very little hysteresis. Note that the absolute value of the stress after annealing is all positive, which corresponds to 
tensile stress. Comparing this data set with that shown in the previous figure, a likely cause for membrane buckling and rupture can be seen. At the operating temperature of the hotplates, the stress in the heater layer is almost purely compressive, while that of the capping layers is tensile. These competing stresses can lead to fatigue in the SiN membrane and the other layers.

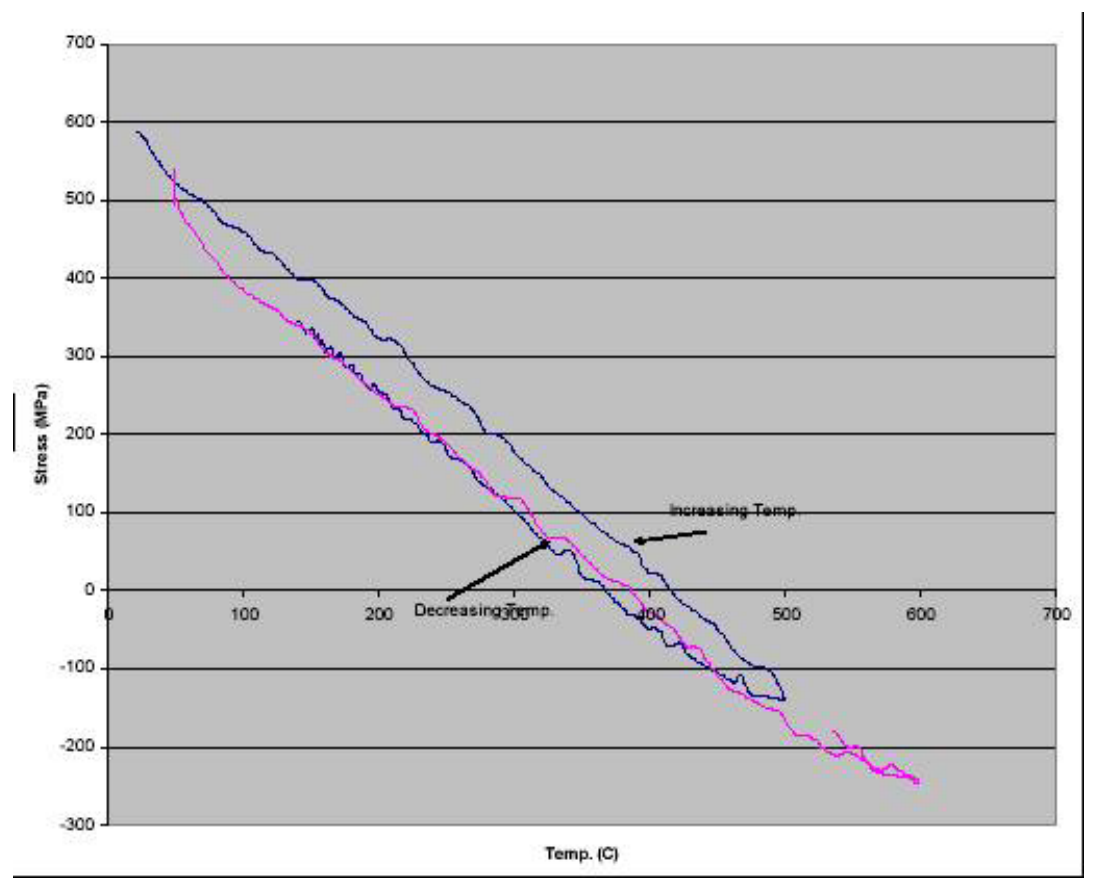

Figure 71: Stress measurements on hotplate heater layer as a function of temperature. Blue is repeat number 1 , pink line is repeat number two. 


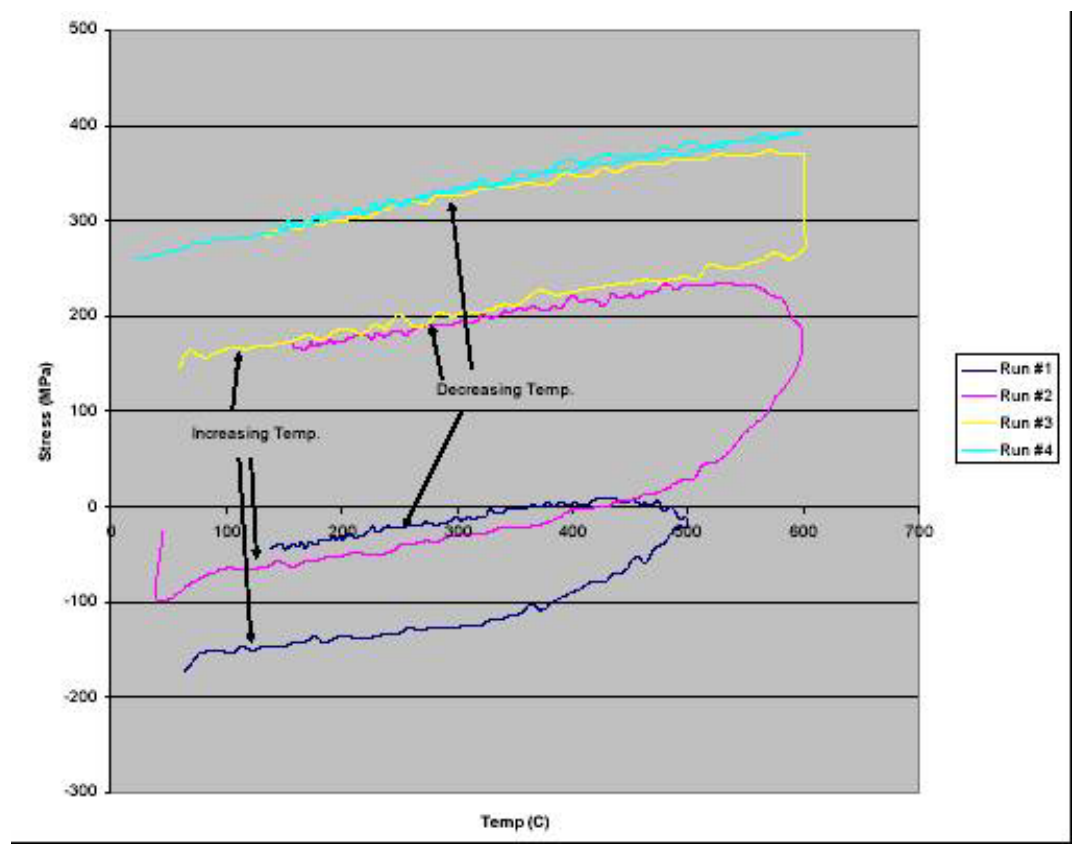

Figure 72: Stress measurements on hotplate capping layer as a function of temperature. Run number corresponds to repeat number on same sample.

\section{Subtask 2.3 - IR Pyrometer for Condition Based Maintenance}

MS7001FA IGCC Gas Turbine

Five pyrometers were previously installed to monitor three stages of an MS7001FA IGCC gas turbine. The pyrometers were calibrated and data acquisition was connected to the GE network. Details of the calibration are shown in Table 8.

Table 8: MS7001FA IGCC gas turbine pyrometer calibration details

\begin{tabular}{|l|c|c|c|}
\hline Position & $\begin{array}{c}\text { Before Cleaning } \\
{ }^{\circ} \mathrm{C}\end{array}$ & $\begin{array}{c}\text { Clean Top } \\
{ }^{\circ} \mathrm{C}\end{array}$ & $\begin{array}{c}\text { Clean Bottom } \\
{ }^{\circ} \mathrm{C}\end{array}$ \\
\hline B1F & 862 & 909 & 953 \\
\hline B1A & 928 & 941 & $953 / 947^{*}$ \\
\hline B2F & 923 & 944 & 953 \\
\hline B2A & 926 & 939 & 952 \\
\hline B3F & 913 & 940 & 951 \\
\hline
\end{tabular}

* B1A sightglass was found to have a baked on stain that could not easily be removed. The two readings indicate the best and worst readings measured by the Cyclops 152 pyrometer. 
MS7001FA+E Gas Turbines

Pyrometers were installed to monitor the stage one buckets on three MS7001FA+E gas turbines. Analysis of the long-term pyrometer data was performed. A relationship was developed between the bucket bulk cooling effectiveness and other gas turbine operating parameters. This relationship will be used for detecting anomalies in bucket condition, as well as improving the accuracy of gas turbine performance models.

Inspections were performed during planned gas turbine outages. Three main tasks were accomplished during the inspections: 1) routine pyrometer maintenance, 2) visual inspection of the Stage 1 buckets via boroscope for comparison with pyrometer and thermal imaging data, and 3) collection of spectroscopy data (see the next section). The pyrometer hardware was found to be in good condition on all three units. The windows appeared to minor fogging, and were cleaned. Calibrations were performed using a Land black body before and after the windows were cleaned. The calibration results are shown in Table 9. After the calibrations were completed, the alignment flanges were re-installed on the gas turbines and the pyrometers were re-aligned using a Land telescope.

There was noise in the once-per-rev keyphasor signal during the startup of one of the gas turbines. The location of the noise moved as a function of load. At least at lower power levels, it was seen that the noise propagated into the pyrometer signal. The result was that the buckets under the noise spikes were seen to have an artificially higher temperature.

Table 9: Pyrometer calibrations on MS7001FA+E gas turbines

\begin{tabular}{|cccc|cccc|}
\hline \multicolumn{4}{|c|}{ Unit 1 } & \multicolumn{4}{c|}{ Unit 2 } \\
Oven & Cyclops & Pyro & Case & Oven & Cyclops & Pyro & Case \\
\hline 1785 & 1802 & & oven & 1787 & 1803 & & oven \\
1785 & 1776 & 1778 & dirty glass & 1785 & 1729 & 1746 & dirty glass \\
1787 & 1786 & 1788 & clean glass & 1787 & 1789 & 1806 & clean glass \\
\hline
\end{tabular}

The Stage 1 buckets on the three gas turbines were imaged via a boroscope through the plug on the side of the pyrometer alignment flange. A laser pointer, shown through the pyrometer flange, was used to indicate the cold pyrometer line of sight. To obtain the single bucket motion of the turbine, the turbine was hand cranked with a ratchet. The once-per-revolution key phasor signal was monitored while the turbine was rotated until a negative voltage was observed from the connected oscilloscope. The triggering of the key phasor indicates bucket number 1 in relation to the pyrometer data acquisition system. From this point, all of the buckets were imaged to observe known areas of wear and other damage. Overall, the buckets appeared to be in very good condition.

Determining Absolute Temperature and Emissivity using Spectroscopy

Spectroscopy measurements were performed on two MS7001FA+E gas turbines at various power levels. The data for twelve different power levels are shown in Figure 73. 
The emissivity values calculated for each power level are shown in Figure 74. These values represent an average emissivity over the entire wheel. If the lowest power level is ignored (where there are large errors) there is a close agreement between the values at each power level, indicating an average emissivity of 0.54 .

Methodology to Predict Temperature of the Buckets

Comparisons of optical pyrometer temperature measurements to analytical predictions were completed using the upgraded UG LOS utility.

Pre-determining the Thermal Variation of Gas Turbine Buckets

Infra-Red (IR) images of a stage one bucket are shown in Figure 75.

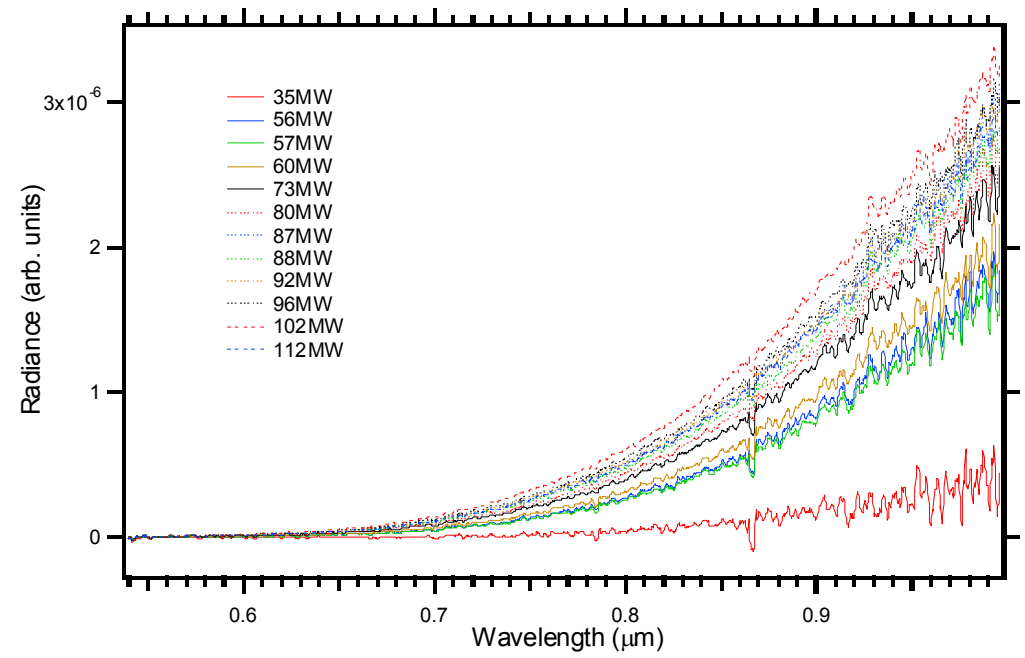

Figure 73: Spectra corrected for spectrometer efficiency 


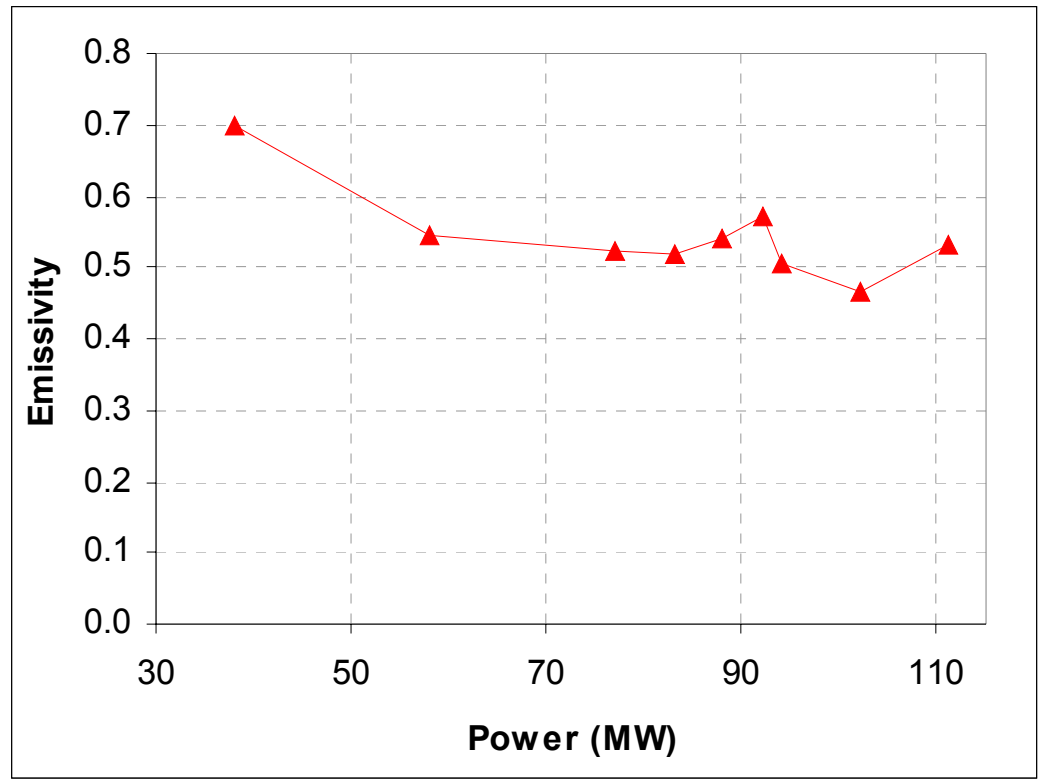

Figure 74: Average wheel emissivity of the buckets back-calculated from the temperature difference between the Land pyrometer and the spectrometer measurements
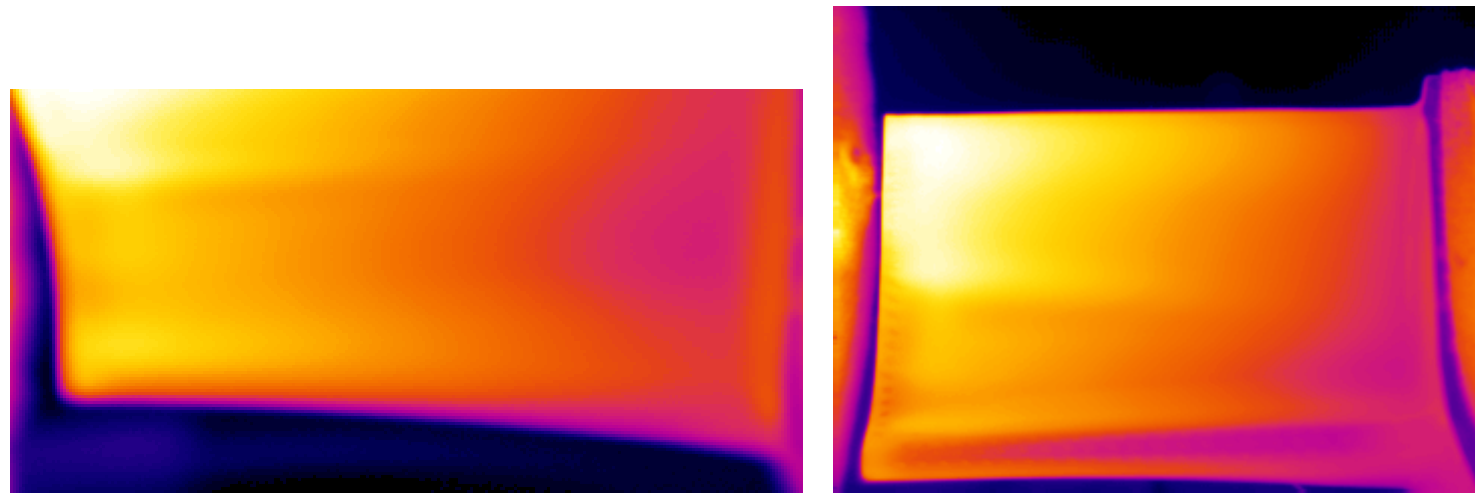

Figure 75: Infra-red images of the leading edge of pressure side of bucket

Analysis of the data was done per the method described in Ref. 1, although improvements were made. Rather than simple flat plate geometry, a full 3D model of the bucket was used. Additionally, variations in wall thickness from bucket to bucket were accounted for. A flow network was also incorporated into the model to account for the heat pickup in the fluid throughout the circuit in order to obtain a more accurate wall-to-fluid heat balance. Finally, the procedure for updating the internal heat transfer coefficients depends not only on the temperature ratio at one location, as stated in Ref. 1, but it depends on the ratio at multiple locations. 
To analyze the two full sets of MS7001FA+E gas turbine buckets efficiently, a routine was implemented to allow for sequential running of the analyses. The routine involved three main sections: querying the data acquisition information, alignment of the IR data, and running the ANSYS finite element analysis. The data acquisition query step involved retrieval of information from a compiled spreadsheet for modification of ANSYS macros for items such as flow and ambient conditions, as well as data file names and locations. Within the IR data alignment section, the buckets were located within the IR image (as shown in Figure 76), the transient temperature data was read into ANSYS and aligned with the model (as shown in Figure 77). The final step involved running the model and writing out the solution before moving onto the next bucket in the sequence.

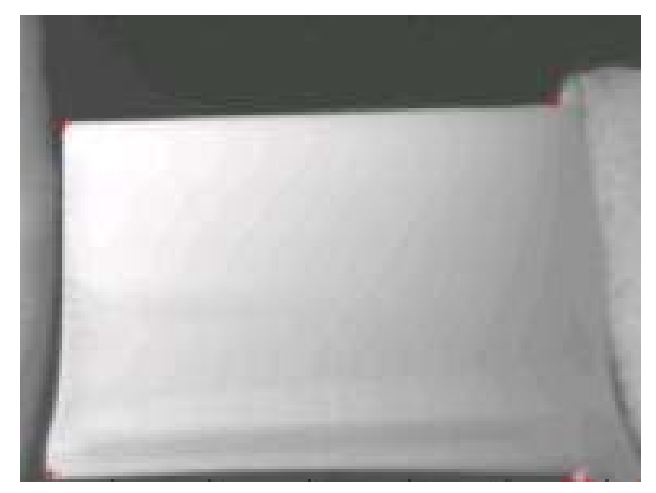

Figure 76: Bucket corners identified with red '+' symbols for alignment purposes

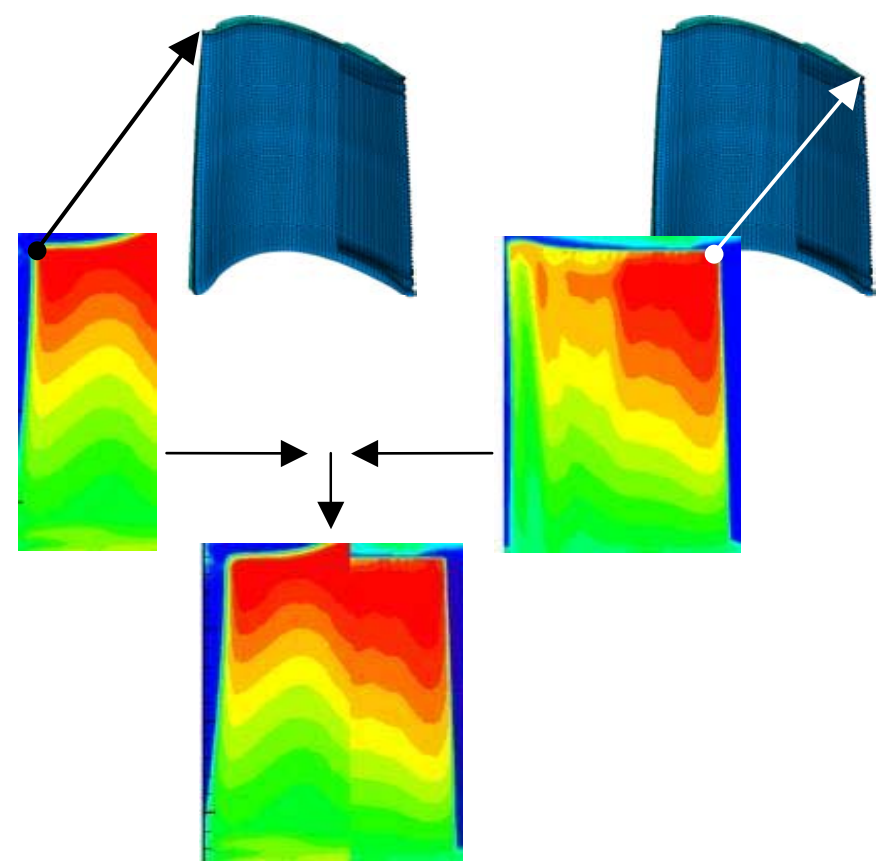

Figure 77: Alignment of the IR transient temperature data to the ANSYS model 
A characterization study was performed to determine the test variation in the method. A Design of Experiment (DoE) was performed on the identified sources of part and test variation, then a Monte Carlo simulation was performed to evaluate the effect of removing different sources of test variation. A test error of $6 \%$ at a $95 \%$ confidence level was identified.

\section{$\underline{\text { Subtask 2.4 - Field Deployment and System Validation }}$}

Fuel LHV Sensor Field Test at Power Systems Development Facility

The field test at the Power Systems Development Facility (PSDF) was our first experience using the fuel LHV sensor in a harsh environment. The sensor was very robust and reliable over the duration of the testing, which included operation on the raw syngas stream at a temperature of $176.7^{\circ} \mathrm{C}$, and which contained approximately $20 \%$ water vapor and approximately $200 \mathrm{ppm}$ of $\mathrm{H}_{2} \mathrm{~S}$. The same set of sensing elements was used for the entire test.

A representative series of data that shows the sensor's response to changes in gas conditions is shown in Figure 78. At the left of the plot, an $\mathrm{O}_{2}$-blown gasification process was used, while at the right of the plot, an Air-blown process was used. During this transition, the heating value of the fuel stream dropped by approximately one half. The transition between the two processes took place over a 15-minute period. The LHV sensor took a longer time to respond than the GC, although the GC required additional time to stabilize to the correct value. After the GC stabilized, the relative change in sensor output versus the relative change in heating value measured by the GC showed a fairly good correlation.

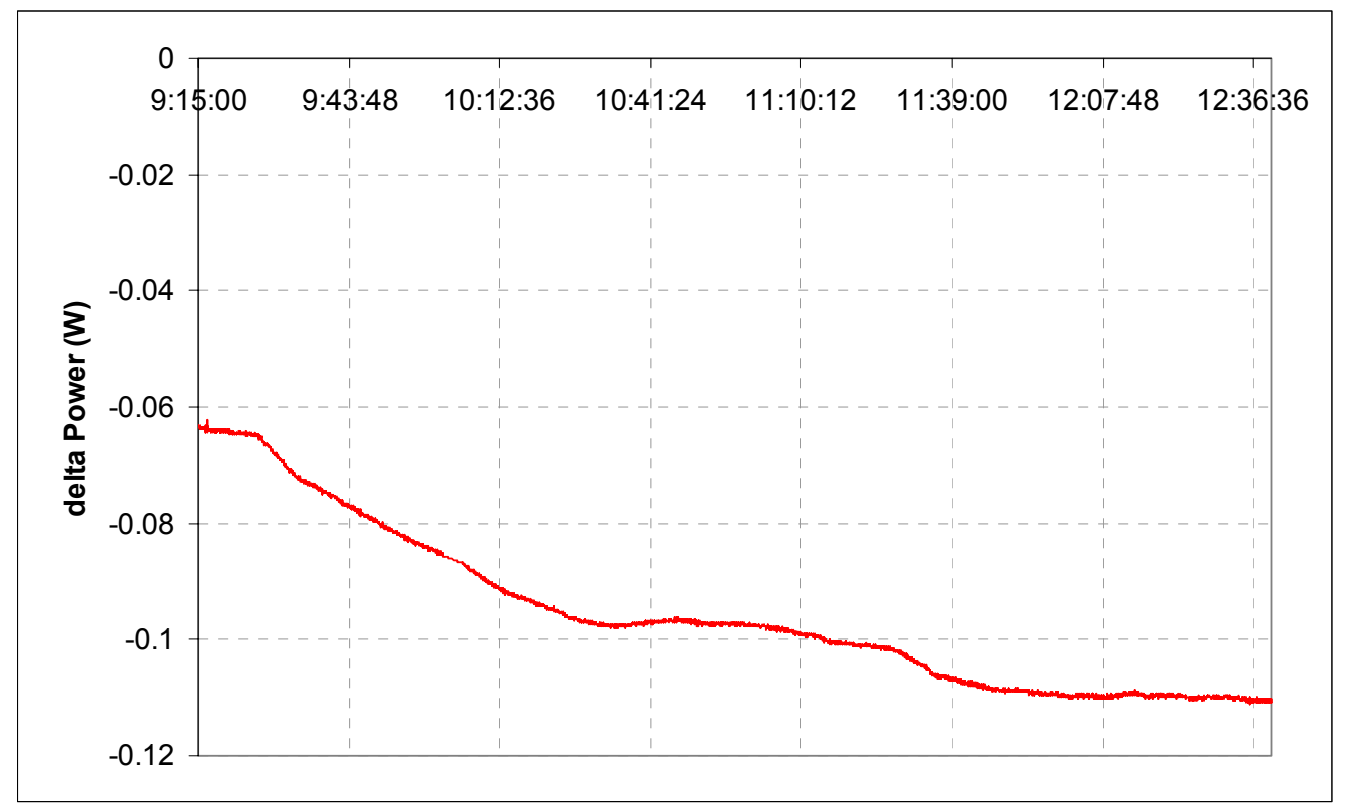

Figure 78: LHV sensor output versus time at PSDF. At the left of the plot the Syngas was generated via an $\mathrm{O}_{2}$-blown process, while on the right it was generated via an air-blown process. 


\section{Fuel LHV Sensor Test at Reciprocating Engine Facility}

Data from the reciprocating engine test at GE Jenbacher is shown in Figure 79. The data presented here is a calibrated LHV value from the sensor as a function of time. During this time period the fuel type was changed from $0.502 \mathrm{CH}_{4} / 0.498 \mathrm{C}_{3} \mathrm{H}_{8}$ to $0.37 \mathrm{CH}_{4} / 0.63 \mathrm{C}_{3} \mathrm{H}_{8}$. The corresponding LHV values for these two mixtures, as determined using a $\mathrm{GC}$, are indicated in the figure.

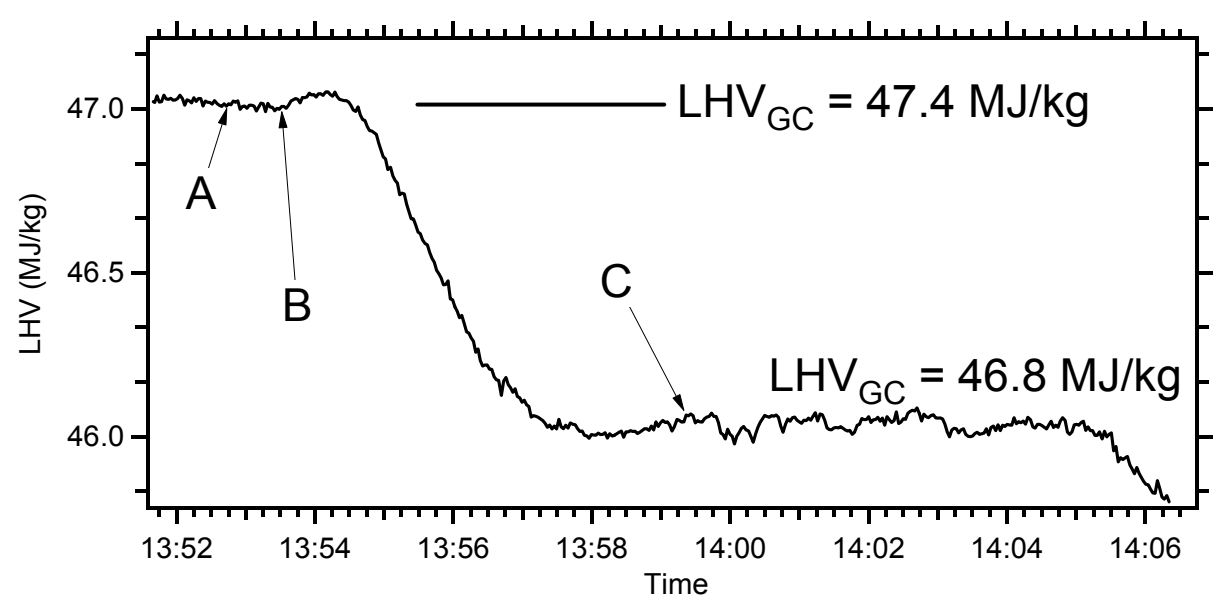

Figure 79: LHV value on a reciprocating engine as fuel type was changed. See the text for description of labels $\mathrm{A}, \mathrm{B}$, and $\mathrm{C}$.

Several things can be inferred from Figure 79. First is the time response of the sensor. The hotplates themselves respond on the millisecond time scale to changes in fuel concentration. However, the fuel had to flow through supporting plumbing, which greatly slowed the sensor response. At point $\mathrm{A}$, the command was sent to the engine control system to begin changing the fuel concentration. Due to the nature of the controls, the fuel change continued until point C. It can be seen that approximately one minute after the fuel change was initiated, a response was seen by the LHV sensor (at point B). This trend was repeated during a series of subsequent fuel concentration changes (not shown). Thus, it was concluded that the sensor system, in its current configuration, had a time response of approximately 1 minute.

It is also instructive to consider the LHV values indicated by the sensor versus those determined from the GC measurements. As is indicated on the figure, the GC determined LHV values dropped $1.3 \%$ from a value of $47.4 \mathrm{MJ} / \mathrm{kg}$ to a value of $46.8 \mathrm{MJ} / \mathrm{kg}$. Over the same time period, the LHV sensor showed a drop of $2.1 \%$. Additionally, the absolute value of the LHV from the sensor was not in exact agreement with that of the GC. However, it should be noted that the sensor's reading is within approximately $1 \%$ of the GC value. Thus, the sensor was demonstrated to provide a continuous reading that was in close agreement with the GC.

Fuel LHV Sensor Field Test at Tampa Electric Company Polk 1

Data from the field test at the Tampa Electric Company (TECO) Polk 1 station are shown in Figure 80 and Figure 81. 


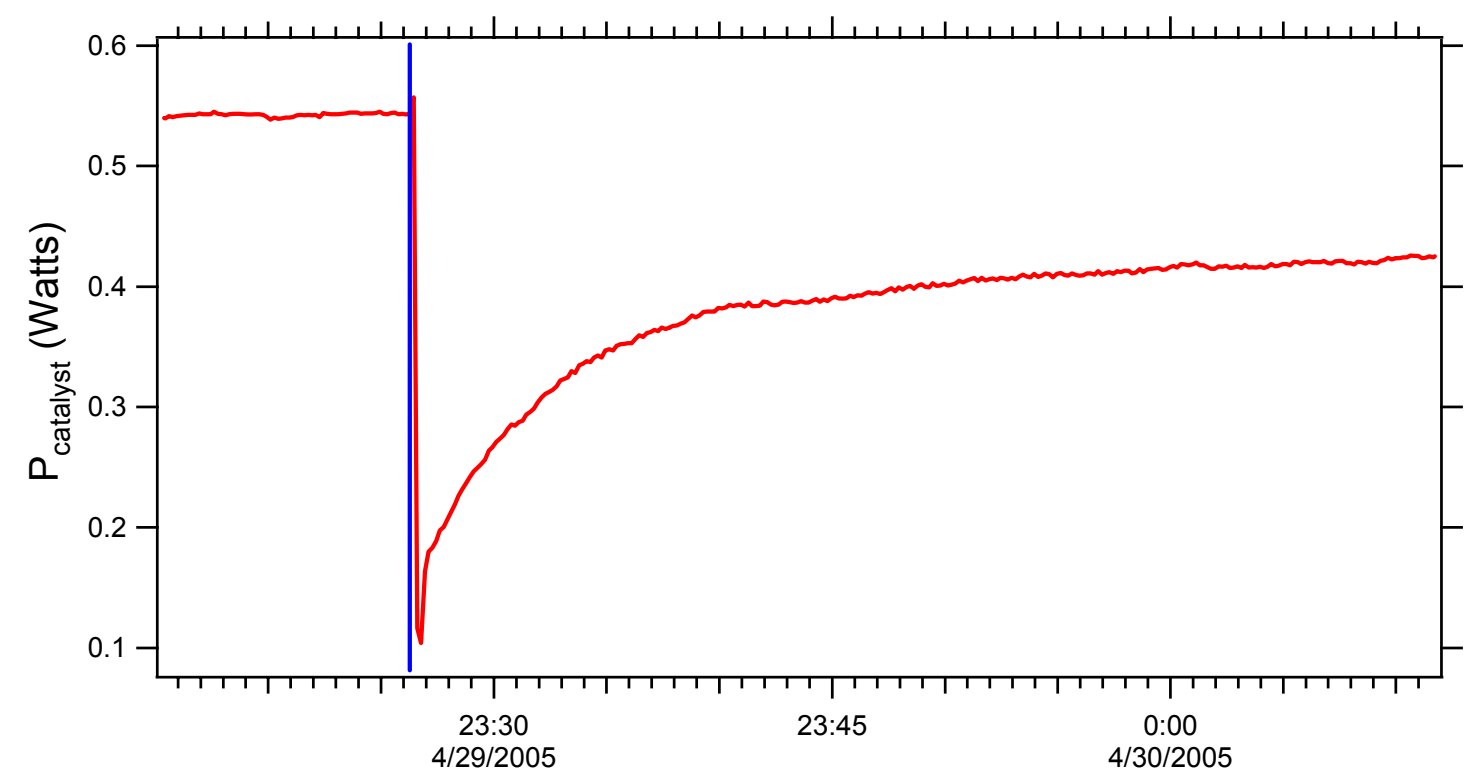

Figure 80: Catalyst hotplate power while combusting syngas at TECO Polk 1. Only air flowed through the sensor until the fuel valve was opened at the blue line.

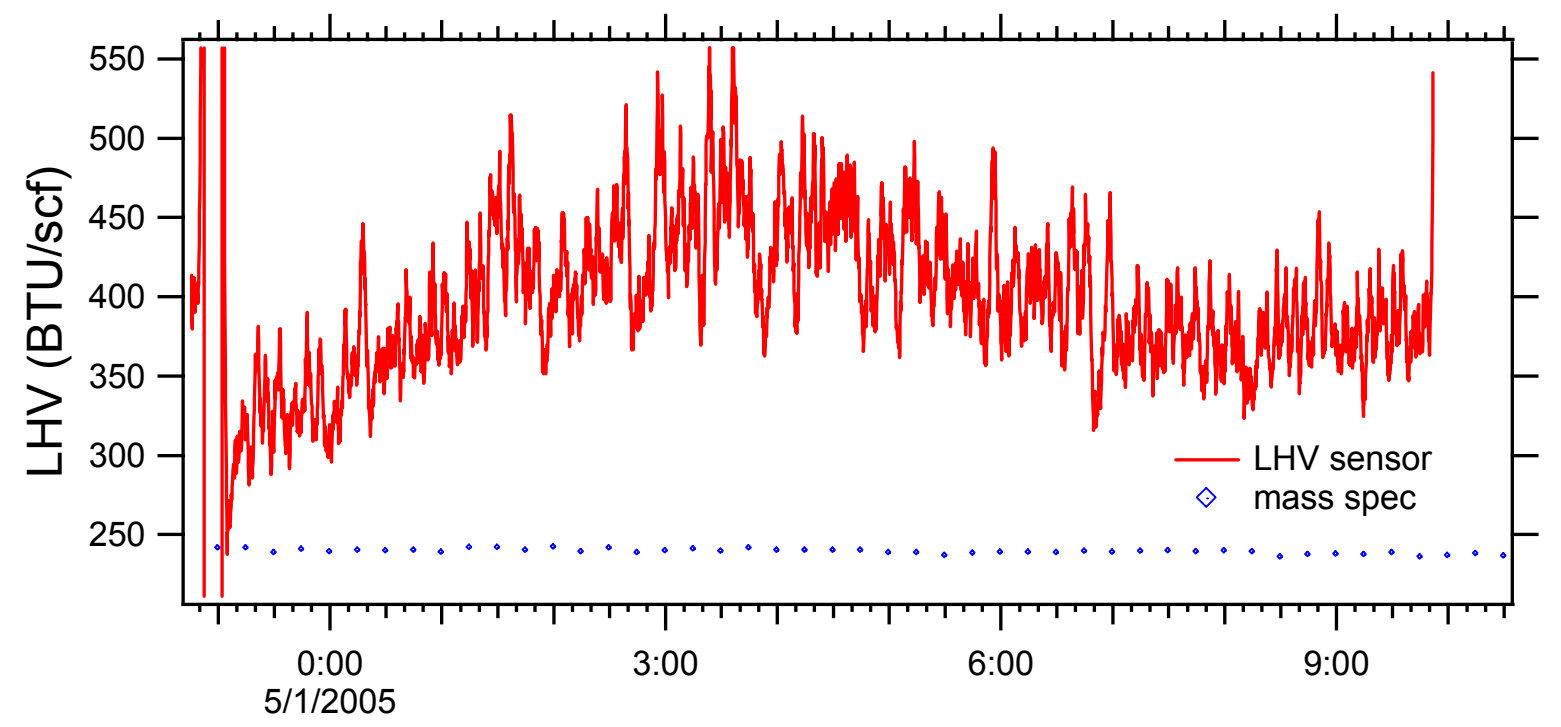

Figure 81: Syngas fuel heating values determined using the LHV sensor and mass spectrometer at TECO Polk 1 
The power supplied to the catalyst hotplate as a function of time is shown in Figure 80. At the time denoted by the blue line in the plot, the fuel valve to the sensor was opened for the first time during the test. As can be seen, there was a sharp drop in the power, signifying that combustion had initiated. The signal took about 20 minutes to reach a steady state condition due to the large volume of piping through which the fuel flowed before reaching the sensor, and the very low flow rates within the sensor.

The syngas fuel heating values as a function of time, for both the LHV sensor and for a mass spectrometer, are shown in Figure 81. The mass spectrometer data was obtained using a commercial instrument located on site. Over the time period shown, the average LHV was 239 $\mathrm{BTU} / \mathrm{scf}$ and the mass spectrometer indicated a change in LHV of around $1 \%$.

The calibration of the LHV sensor was performed after the test was completed, using scaling values obtained using a calibration gas while at TECO Polk 1. A simple multiplicative scaling was used during calibration of the LHV sensor, which results in the noise being grossly exaggerated. In addition, it was not possible to simulate the $3 \% \mathrm{H}_{2} \mathrm{O}$ content in the TECO fuel, which seemed to play a role in the combustion characteristics of the sensor. As a result, the accuracy of the LHV sensor during the test performed at TECO Polk 1 was poorer than the accuracy observed in previous tests. These issues notwithstanding, the results were encouraging, and demonstrated the capability of the LHV sensor to operate on a wide range of fuels, including syngas from an operating coal IGCC powerplant. 


\section{$\underline{\text { Task } 3 \text { - Results/Discussion: }}$}

\section{$\underline{\text { Advanced Methods for Combustion Monitoring and Control }}$}

\section{Task 3 Overview:}

The third task addressed pre-mixed combustion phenomenon for IGCC applications. The work effort on this task was shifted to another joint GE Energy/DOE-NETL program investigation, High Hydrogen Pre-mixer Designs (CA-41893), as of April 1, 2004. The contributors were GE Energy, GE Global Research Center, and Georgia Institute of Technology.

Sub-Task 3.1 - Physics-based Combustion Modeling: Physics-based models of combustor and fuel system dynamics critical for improving the understanding of performance characteristics for coal IGCC combustors were developed. Theoretical modeling was combined with system identification experiments to develop physics-based models for predicting combustion performance and operability.

Sub-Task 3.2 - Development of Control Methods: Control strategies for improving combustor operability and performance while reducing or maintaining emissions levels were developed and tested. System models developed in sub-task 3.1 were used to develop and test open- and closed-loop active controllers in computer simulations. Robust control components, including feedback sensors and control actuators, were identified and tested.

\section{Task 3 Discussion:}

\section{Sub-Task 3.1 - Physics-based Combustion Modeling}

Combustion Dynamics Modeling

Combustion dynamics (or instability) arises from acoustic-combustion interaction. When acoustic perturbations gain energy from flame heat release, perturbations tend to grow in amplitudes, resulting in large amplitude acoustic oscillations. The oscillations are usually at discrete frequencies that are very close to combustor acoustic system natural frequencies. Combustion driven oscillations often pose significant problems in the operation of gas turbines. To develop effective methods to eliminate these combustion instabilities in a system, one requires a good model of system dynamics that includes the interactions between different combustor components. The purpose of the model is two fold. First, it should validate our understanding of combustion dynamics including the dynamic effects of fuel modulation. Second, it should provide a simple but solid foundation for nonlinear dynamical analysis and control design tools to be applied.

A physics-based combustor dynamics-modeling tool was developed in GE Global Research Center (GRC). In this model, the physical processes including fundamental acoustic characteristics of the combustor systems, fuel supply system, acoustic-flame interaction, and flame heat release are captured. The acoustics of the combustor system is modeled using the 
following standard acoustic equations that account for the effect of heat release, mean flow, and temperature variations upon wave propagation:

Mass: $\quad \frac{\partial \rho^{\prime}}{\partial t}+\nabla \cdot\left(\bar{\rho} \vec{u}^{\prime}+\rho^{\prime} \overline{\vec{u}}\right)=0$

Momentum:

$$
\bar{\rho}\left(\frac{\partial \vec{u}^{\prime}}{\partial t}+\vec{u}^{\prime} \cdot \nabla \overline{\vec{u}}+\overline{\vec{u}} \cdot \nabla \vec{u}^{\prime}\right)+\rho^{\prime} \overline{\vec{u}} \cdot \nabla \overline{\vec{u}}=-\nabla p^{\prime}
$$

Energy:

$$
\frac{\partial p^{\prime}}{\partial t}+\nabla \cdot\left(p^{\prime} \overline{\vec{u}}+\bar{p} \vec{u}^{\prime}\right)=\frac{\gamma-1}{\gamma} q^{\prime}
$$

where a prime denotes a fluctuating quantity and an overbar a mean quantity. These equations can be simplified in various regions of the combustion and fuel supply system. In ducts and piping with diameters much smaller than the wavelength of oscillations and with constant or slowly varying temperatures, the acoustic field is a one-dimensional longitudinal acoustic wave field. In each of these approximately one-dimensional regions, the exact solution for the acoustic field can be written as:

$$
\begin{aligned}
& p_{j}{ }^{\prime}=\left(A_{j} e^{-i k_{j} x /(1+M)}+B_{j} e^{i k_{j} x /(1-M)}\right) e^{i \omega t} \\
& u_{j}{ }^{\prime}=\frac{1}{\overline{\rho_{j} c}}{ }_{j}\left(A_{j} e^{-i k_{j} x /(1+M)}-B_{j} e^{i k_{j} x /(1-M)}\right) e^{i \omega t}
\end{aligned}
$$

where $\mathrm{A}$ and $\mathrm{B}$ are the magnitudes of acoustic waves propagating in the positive and negative $\mathrm{x}$ directions at speeds of $\mathrm{c}+\mathrm{U}$ and $\mathrm{c}-\mathrm{U}$, respectively, $\omega$ is the angular frequency, $k=\omega / c$ is the acoustic wavenumber, and the subscript, $\mathrm{j}$, denotes a region of the system.

Using boundary conditions at the combustion flow inlet and outlet, and matching conditions for equation (4) and (5) at the interfaces of different combustion sub-systems, one obtains a model of coupled algebraic and differential equations. The model can be used to calculate system natural resonant frequencies, growth rates, and modal shapes. Some of these acoustic modes will gain energy from flame heat release and grow in amplitudes. These modes are called unstable modes (growth rate $>0$ ). The other modes interact with flame in such a way that their amplitudes will decay from initial perturbations. These are called stable modes (growth rate $<0$ ). The unstable modes are associated with combustion dynamics. The structure of the model has been supported by initial experimental results described below and also has been compared with other published works in this field.

\section{Model Validation}

The model described above was applied to the single nozzle rig (SNR) described in the previous section. Dynamics data were obtained from the rig using 6 dynamic pressure transducers. The locations of the transducers were shown in Figure 82. 


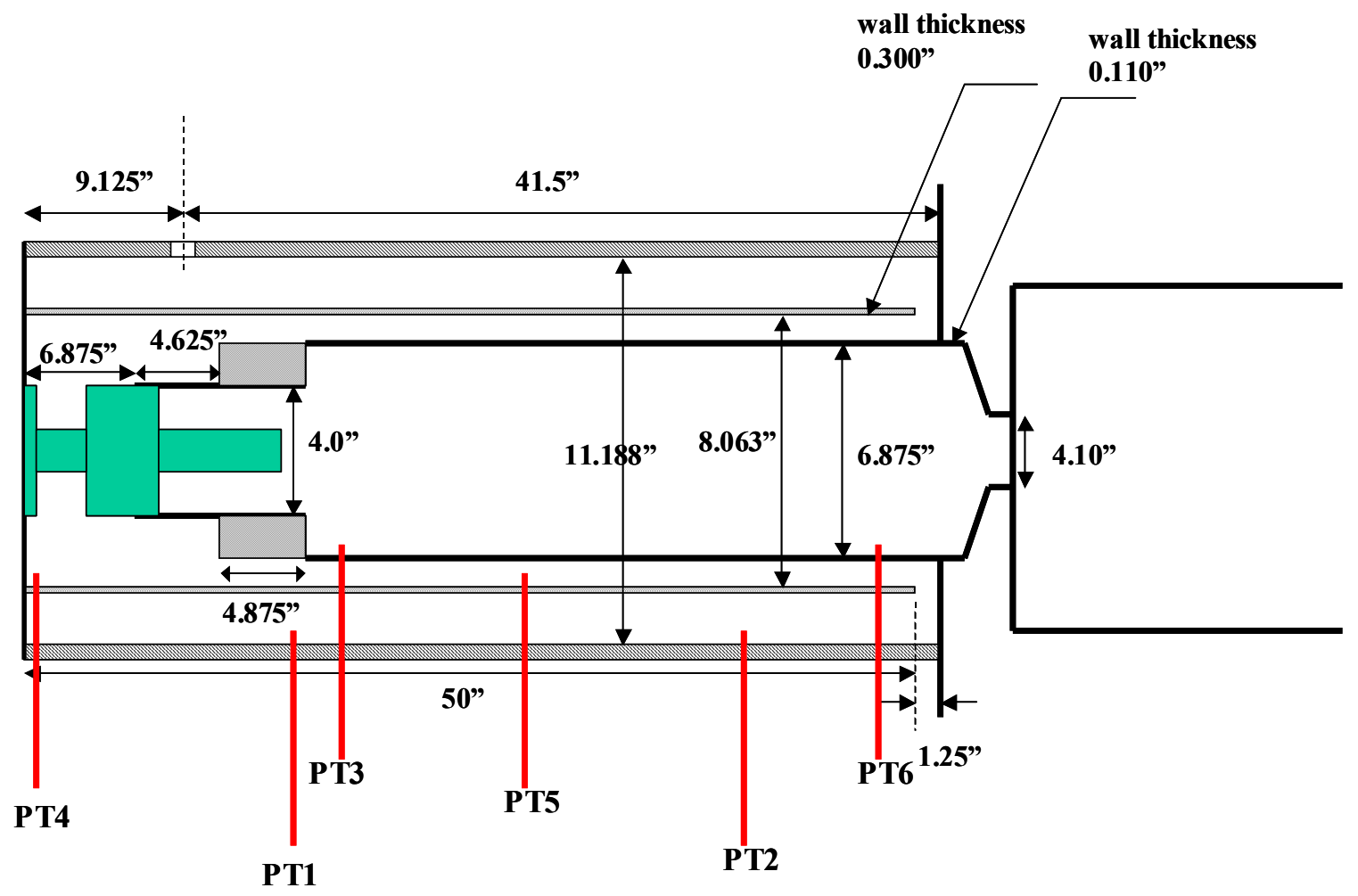

Figure 82: Dynamic pressure transducer locations

At the baseline operation condition, the combustor rig has a distinctive tone of around $180 \mathrm{~Hz}$, with amplitudes at certain locations above 0.8 psi, as shown in Figure 83 . 


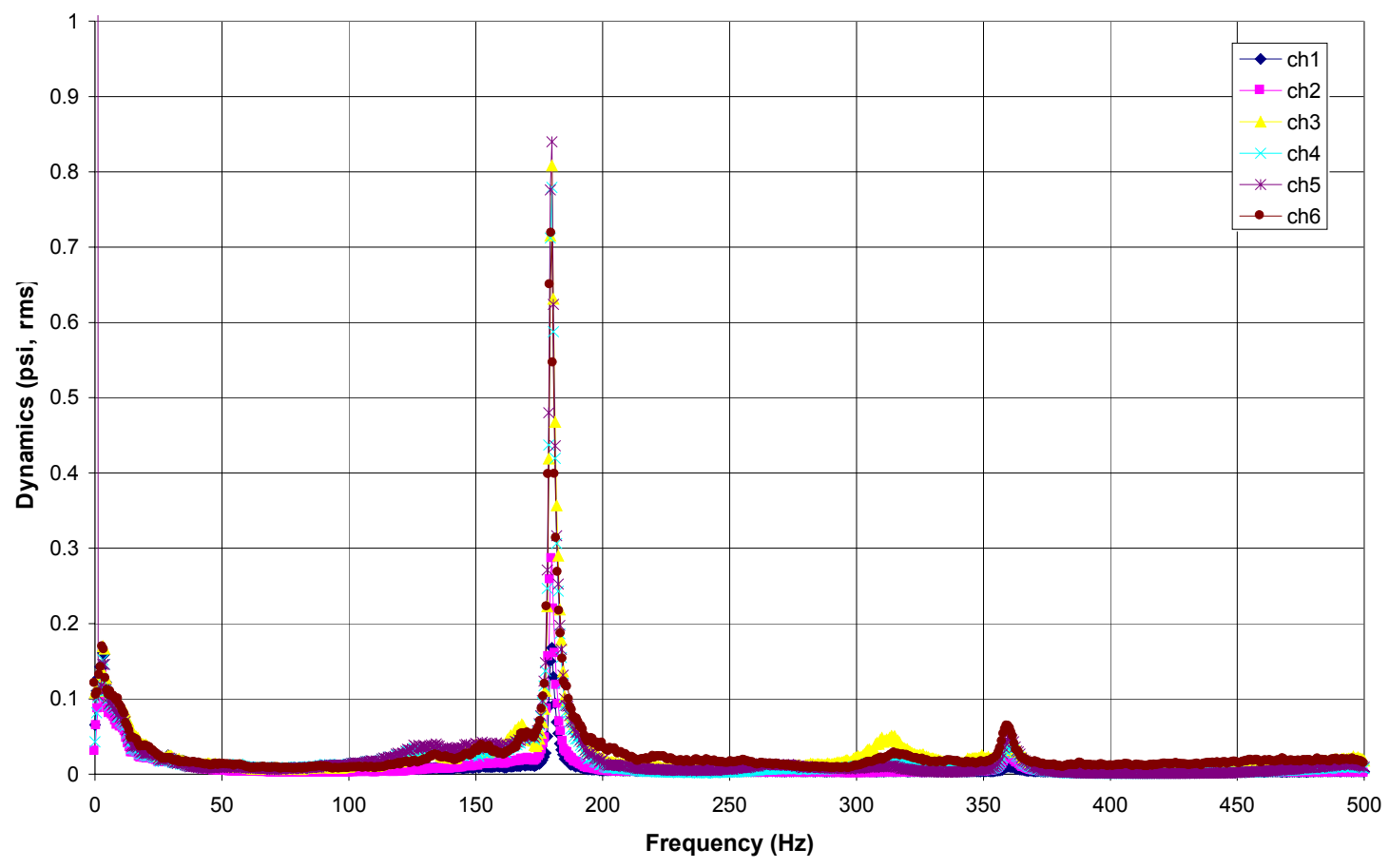

Figure 83: Measured dynamics data in SNR at the baseline operation condition

The frequencies and growth rates of all the dynamic modes below $300 \mathrm{~Hz}$ predicted by the model at the same rig operation condition are shown in Figure 84.

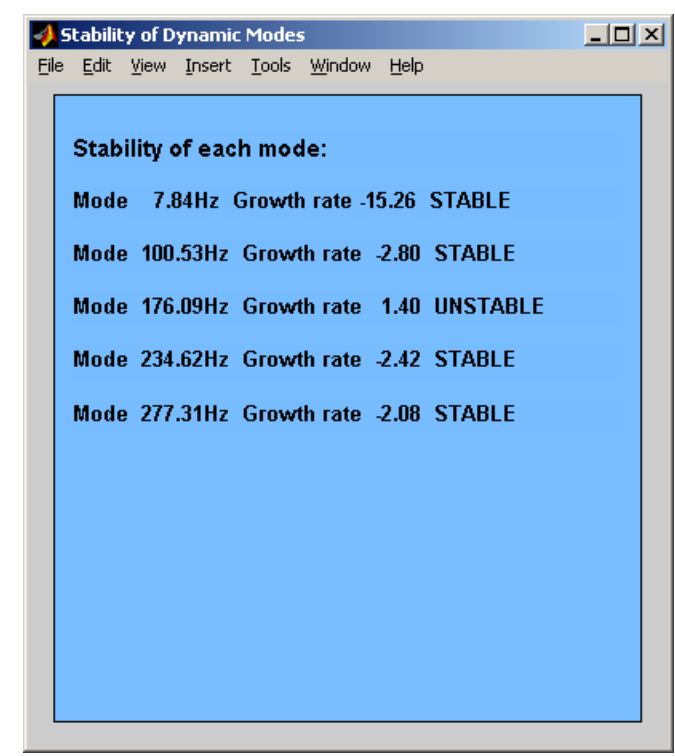

Figure 84: Dynamic modes predicted by the model 
There are totally 5 modes for the combustor system below $300 \mathrm{~Hz}$. The mode at $176 \mathrm{~Hz}$ is an unstable mode - all others are stable modes. The model successfully predicted the dynamics observed in tests.

The modal shape at $176 \mathrm{~Hz}$ predicted by the model is also compared with the measured dynamics at the six pressure transducer locations. The results are shown in Figure 85 . It can be seen that both the magnitude and phase of the $176 \mathrm{~Hz}$ mode calculated by the model agree very well the test data.

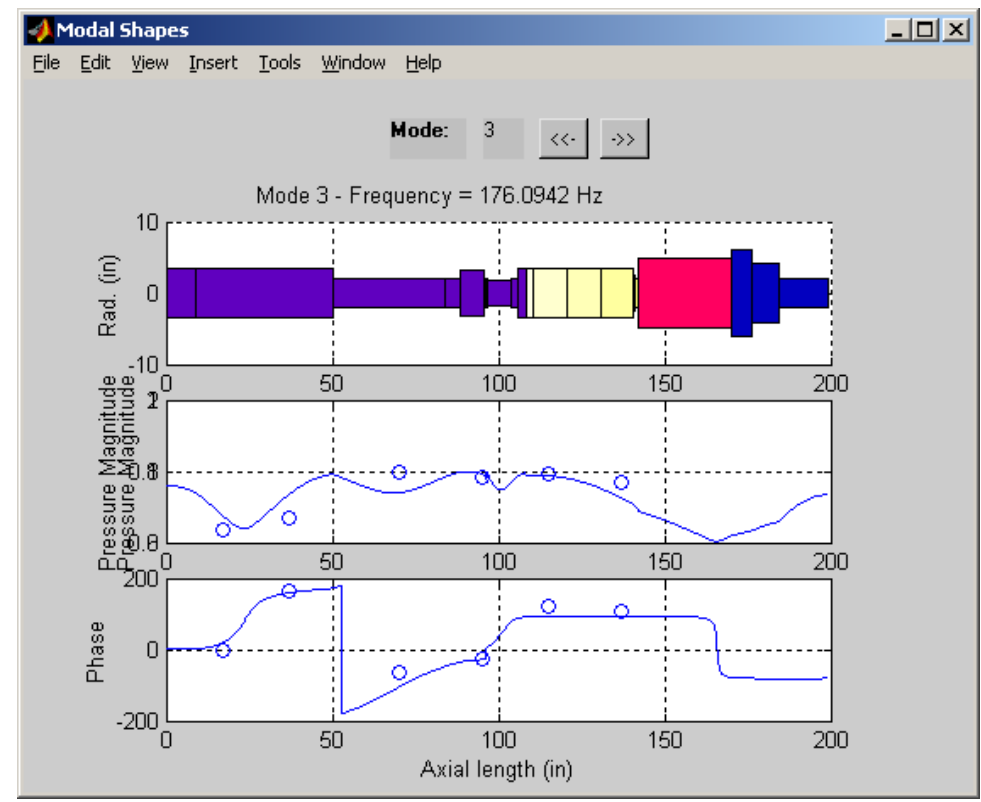

Figure 85: Calculated dynamics modal shape compared with experimental data

Validations using the SNR data show that the model is accurate in calculating the dynamics frequencies, stability, and modal shapes. The model will be used to design dynamics active control schemes.

\section{System Dynamics Time Domain Equations}

The model must first be converted into differential equations that can capture dynamic processes in a combustion system in time domain in order to be used for active control design. The details of this transformation are given below.

Using the mass, momentum, and the energy equations $(1,2,3)$ and partially differentiating equation (2) with $x$ and differentiate equation (3) with $t$, and combining the resulting two equations yield

$$
\frac{1}{c^{2}} \frac{\partial^{2} p^{\prime}}{\partial t^{2}}-\frac{\partial^{2} p^{\prime}}{\partial x^{2}}=\frac{\gamma-1}{c^{2}} \frac{\partial q^{\prime}}{\partial t}-\frac{\gamma R}{c^{2}} \bar{\rho} \frac{\partial}{\partial t}\left(u^{\prime} \frac{\partial \bar{T}}{\partial x}\right)
$$


Using the modal analysis method the acoustic pressure p' can be written in Fourier series as

$$
p^{\prime}(x, t)=\bar{p} \sum_{n} \eta_{n}(t) \psi_{n}(x)
$$

where $\eta_{n}(t)$ is the magnitude of the $n^{\text {th }}$ dynamics mode of the combustor, and $\psi_{n}(x)$ is the weighting function or modal shape. Substituting equation (7) into equation (6) gives

$$
\ddot{\eta}_{n}(t)+2 \zeta_{n} \omega_{n} \dot{\eta}_{n}(t)+\omega_{n}^{2} \eta_{n}(t)=\frac{\gamma-1}{\bar{p}} \frac{1}{\int_{0}^{L} \psi_{n}^{2} d x} \int_{0}^{L} \psi_{n}(x) \frac{\partial q^{\prime}}{\partial t} d x
$$

where $\zeta_{n}$ is the system damping coefficient of the $n^{\text {th }}$ mode and $L$ is the whole combustor length. The term on the right hand side of equation (8) is the source term of the flame heat release.

Using the new heat release model developed jointly by GRC and Georgia Institute of Technology, we have

$$
\frac{Q^{\prime}}{\bar{Q}}(t)=f_{n} \frac{u_{f}^{\prime}}{\bar{u}_{f}}\left(t-\tau_{f}\right)+g_{n} \frac{\phi_{f}^{\prime}}{\bar{\phi}_{f}}\left(t-\tau_{g}\right)
$$

The first term on the right hand side of the equation represents heat release fluctuation caused by flow velocity fluctuation at the flame base, $u_{f}^{\prime}$, the second term is heat release fluctuation caused by fuel-air ratio fluctuation at the flame base, $\phi_{f}^{\prime}$. Due to distributed flame shape, heat release will react to velocity fluctuation and fuel-air ratio fluctuation at flame base with time delays of $\tau_{\mathrm{f}}$ and $\tau_{\mathrm{g}}$, respectively. Fuel-air ratio at the flame base is related to fuel air ratio at the fuel injection location by the flow convection speed:

$$
\frac{\phi_{f}^{\prime}}{\bar{\phi}_{f}}(t)=\frac{\phi_{i n j}^{\prime}}{\bar{\phi}_{i n j}}\left(t-\tau_{c}\right)
$$

Fuel air ratio fluctuation can be written as fuel fluctuation and air fluctuation at the fuel injection location using

$$
\frac{\phi_{i n j}^{\prime}}{\bar{\phi}_{i n j}}=\frac{\frac{m_{f u e l}^{\prime}}{\bar{m}_{f u e l}}-\frac{m_{a i r}^{\prime}}{\bar{m}_{a i r}}}{1+\frac{m_{a i r}^{\prime}}{\bar{m}_{\text {air }}}}
$$

where $m_{\text {fuel }}^{\prime}$ is mass flow fluctuations of fuel from nozzle fuel injection holes and $m^{\prime}$ air is air flow fluctuations on the air side of the nozzle at the fuel injection location.

Fuel fluctuation comes from modulation by air pressure fluctuation and by dynamics source located upstream the fuel line (in this case, the active control valve)

$$
\frac{m_{\text {fuel }}^{\prime}}{\bar{m}_{\text {fuel }}}(t)=a_{n} \frac{m_{\text {valve }}^{\prime}}{\bar{m}_{\text {fuel }}}\left(t-\tau_{a}\right)+b_{n} \frac{p_{\text {air }}^{\prime}}{2 \overline{\Delta P}}\left(t-\tau_{b}\right)
$$


where $m^{\prime}$ valve is mass flow fluctuations generated by the valve and $\Delta \mathrm{P}$ is the fuel side pressure drop across fuel injection holes. $\mathrm{p}_{\text {air }}$ is air pressure fluctuations on the air side of the nozzle at the fuel injection location. $\tau_{\mathrm{a}}$ is the time delay from valve to fuel injection fluctuation and $\tau_{\mathrm{b}}$ is the time delay from pressure fluctuation to fuel injection fluctuation. The values of $a_{n}$ and $b_{n}$ change with acoustic modes.

Combining equations (9-12) yields

$$
\frac{Q^{\prime}}{\bar{Q}}(t)=f_{n} \frac{u_{f}^{\prime}}{\bar{u}_{f}}\left(t-\tau_{f}\right)+g_{n} \frac{a_{n} \frac{m_{\text {valve }}^{\prime}}{\bar{m}_{\text {fuel }}}\left(t-\tau_{a}-\tau_{c}-\tau_{g}\right)+b_{n} \frac{p_{i n j}^{\prime}}{2 \overline{\Delta P}}\left(t-\tau_{b}-\tau_{c}-\tau_{g}\right)-\frac{u_{i n j}^{\prime}}{\bar{u}_{i n j}}\left(t-\tau_{c}-\tau_{g}\right)}{1+\frac{u_{i n j}^{\prime}}{\bar{u}_{i n j}}\left(t-\tau_{c}-\tau_{g}\right)}
$$

Equation (8) describes the dynamics amplitude growing process in time domain as a result of flame heat release. The flame heat release process in time domain is described in detail in equation (13). They can be used combined to develop an active control strategy to reduce combustion dynamics.

\section{Control Design Considerations}

A combustion dynamics control strategy based on unsteady fuel addition is being developed. The technique has been demonstrated extensively on small-scale combustion systems and results of these studies may be found in the literature (see Ref. 3.1). In the present work, this strategy is being adapted for use in full-scale power generation gas turbines. To this end, detailed controller design is being performed through the use of the system model described above. In addition, experiments are being performed to understand the limits of control authority, or actuator effectiveness when using high-frequency fuel modulation to effect control in current DLN combustion hardware. Preliminary results from these activities are described below.

The combustion dynamics model described above has been used extensively to predict unstable modes in various combustor designs. In addition, it is being used to develop model-based control schemes for suppressing combustion dynamics. To be effective for the latter, it is recognized that the model must adequately represent certain key behavior in the combustion system including:

- linear behavior during weak thermo-acoustic instability

- nonlinear behavior during strong thermo-acoustic instability

- the effects of fuel forcing on overall system dynamics

In order to apply nonlinear dynamical analysis tools (such as bifurcation theory and manifold descriptions), the model was transformed to an analytical form in the time-domain (see Eqs. 8-13 above). The resulting equation is a closed-loop delay-differential-equation (DDE) with a forced input, the block diagram for which is shown in Figure 86. 


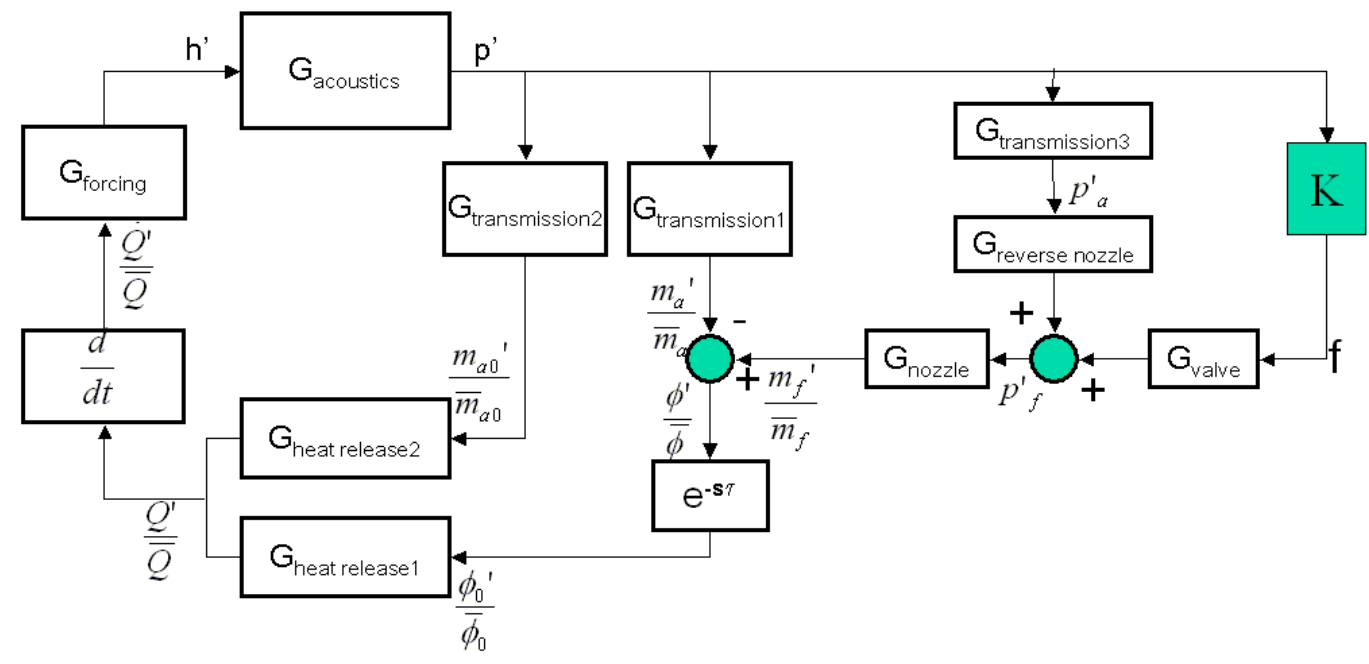

\section{Figure 86: Block diagram of Delay Differential Equation thermo-acoustic model. The block $\mathrm{K}$ represents the controller.}

Preliminary Investigation of Fuel Forcing

Several experiments were performed to gain understanding of the effects of unsteady fuel modulation on the behavior of the combustor. A high-bandwidth fuel control valve was coupled to the fuel premixer on the single-nozzle rig. The effects of fixed-frequency fuel modulation on the combustor operability and dynamics was examined. Control strategies using fuel modulation generally require the ability to modulate fuel flow at frequencies in the range of those where combustion dynamics occur. Because the identified thermo-acoustic oscillation frequencies were significantly higher than those which off-the-shelf fuel valves could achieve, high frequency modulation valve technologies were explored and an experimental valve from Georgia Institute of Technology was selected for trials. The GaTech fuel valve was coupled to the DLN premixer and open loop fuel forcing experiments were performed over a range of frequencies from 50 to $200 \mathrm{~Hz}$.

Initial experiments focused on general combustor operability characteristics. An important measure of operability is the combustor lean blowout (LBO) limit. Results from a series of LBO experiments both with and without fuel modulation are shown in Figure 87. 


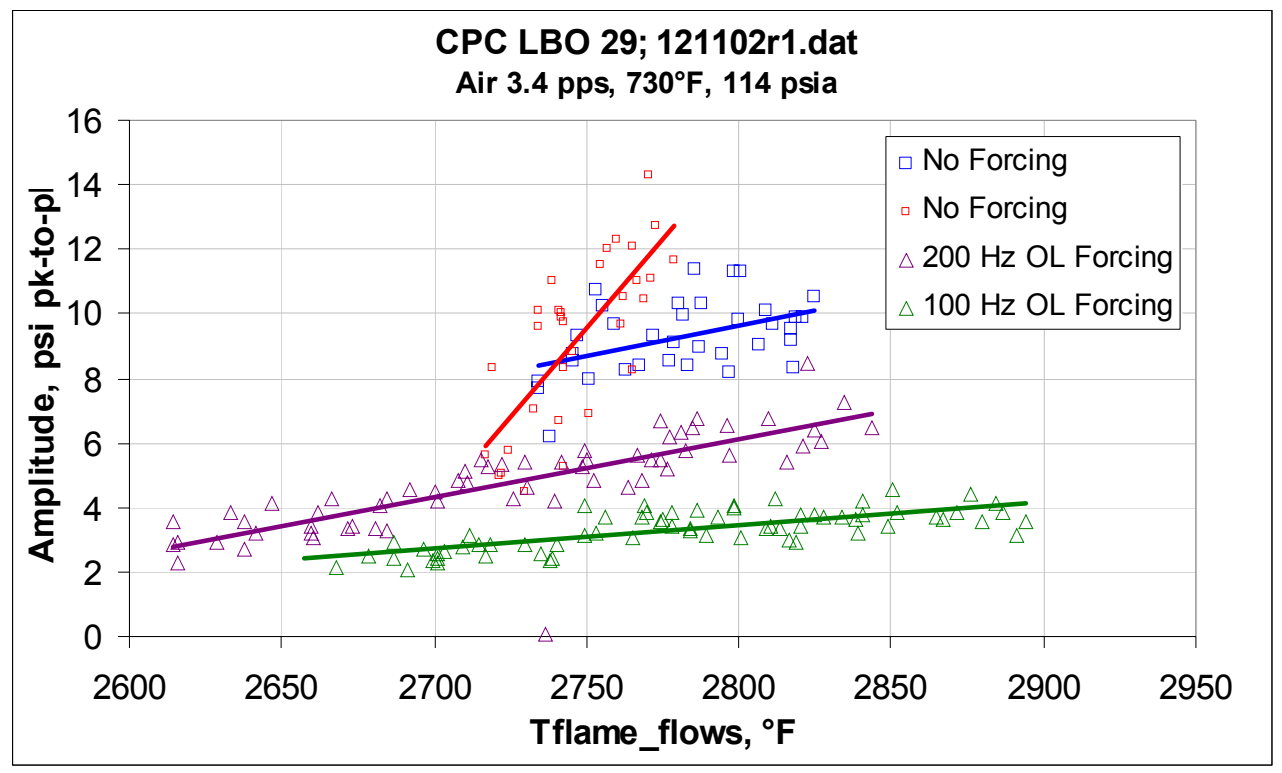

Figure 87: Lean blowout limits measured with and without fuel modulation

Extension (improvement) in LBO is seen when fuel modulation is incorporated. This suggests that there is an intrinsic change in combustion stability when the fuel flow is modulated at highfrequency. The reason for this is unclear at present and further experiments are planned to elucidate the physical mechanism responsible for this performance improvement.

The second set of experiments aimed to characterize the fuel valve dynamic properties (frequency response if a linear system). Initial results indicate the valve to have an effective bandwidth of $300 \mathrm{~Hz}$ - well above the oscillating frequency of the thermo-acoustic instability. As part of the second set of experiments, the effect of open-loop forcing using fuel modulation was investigated. Qualitatively there is an attenuation effect observed at various forcing frequencies and amplitudes, as in shown in Figure 88.

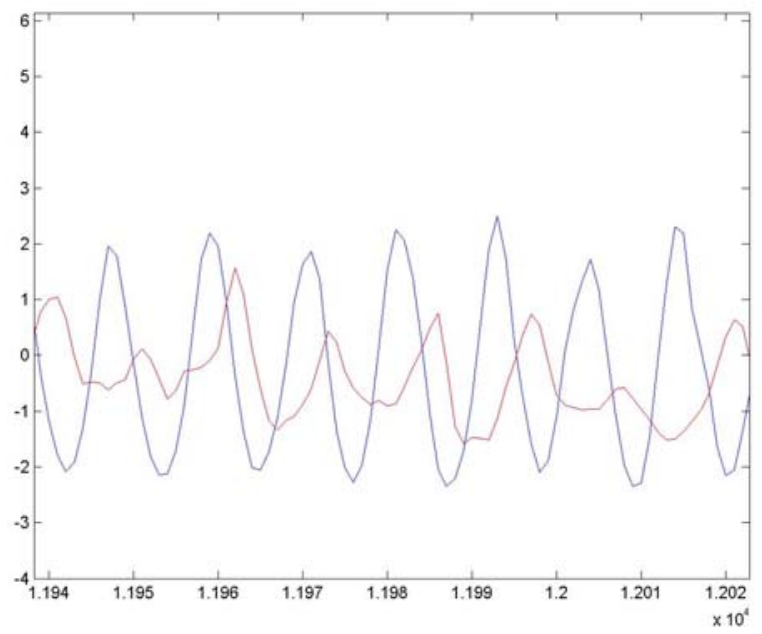

Figure 88: Thermo-acoustic oscillation with (red) and without (blue) fuel modulation 
However, new dynamics appear and grow as the forcing is increased. This is illustrated in the Power Spectral Density comparison in Figure 89.
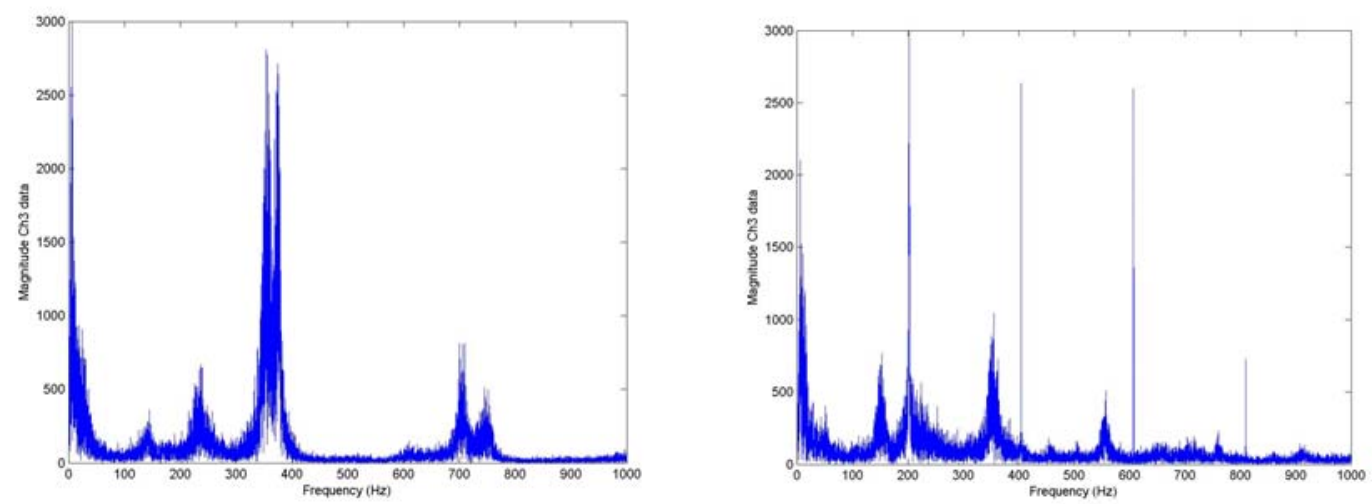

Figure 89: Power spectral density plots of pressure inside the combustion chamber with no fuel modulation (left) and $200 \mathrm{~Hz}$ modulation (right)

The results from these experiments were used to modify the model structure to incorporate the fuel forcing mechanism.

The third set of experiments were cold flow experiments to further characterize the valve and amplifier which drives the modulating signal. The intent is to establish a valve operating profile that satisfies thermal constraints, current/voltage constraints, as in Figure 90, inlet static flow variability and downstream back propagation effects from the nozzle. The amplifier is being tested for nonlinear effects and harmonic distortion.

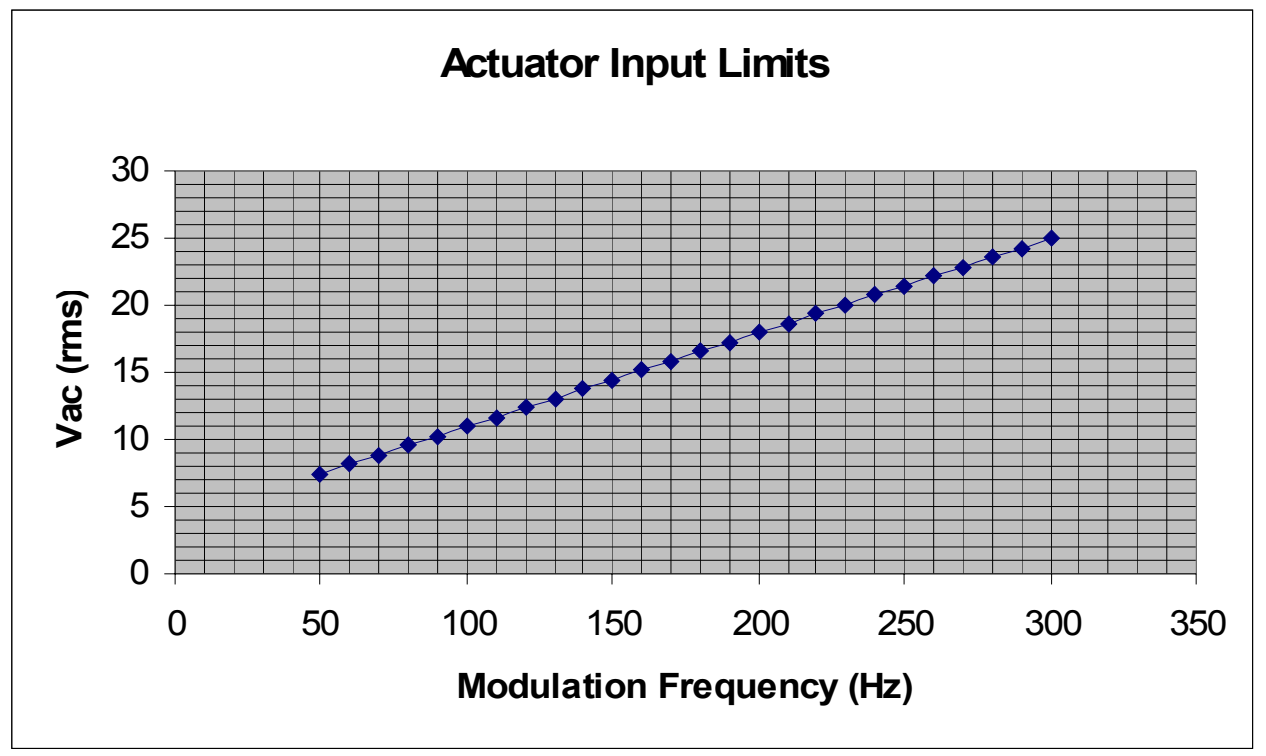

Figure 90: Voltage applied to the valve constraints 
The fourth set of experiments used the bifurcation diagram of the model and hot flow conditions to explore the dynamic system behavior including the actuator effects. The aim was to have a predictive capability, using the model, of how the system will react to a given fuel forcing condition. This thorough experiment tested various fuel forcing amplitudes and frequencies over a range of equivalence ratios, encompassing both lean and rich induced thermo-acoustic instabilities. The data from Experiment set four was used to calibrate the model, and validate our assumptions regarding the fuel forcing mechanism.

Non-model based control designs were also evaluated, though they are expected to give larger level of conservatism, and hence lower performance. They are also unable to explain secondary peaking phenomena that tend to appear in the controlled system. Nevertheless they have been demonstrated to work in the literature.

\section{Combustion Dynamics Modeling Validation}

The combustion dynamics model has been successfully used to predict unstable modes in various combustor designs. In addition, it has been used to examine control schemes for suppressing combustion dynamics. To be effective for the latter, it is recognized that the model must adequately represent certain key behavior in the combustion system including:

- linear behavior during weak thermo-acoustic instability

- nonlinear behavior during strong thermo-acoustic instability (limit cycle)

- effects of fuel forcing on overall system dynamics

The model performance in each of these areas must be adequate to reproduce the combustor response, both in forced and unforced modes. In order to facilitate comparison of the model predictions with experimental results, the model was transformed to an analytical form in the time-domain. The resulting equation is a closed-loop delay-differential-equation (DDE) with a forced input. The model structure used is shown in Figure 91.

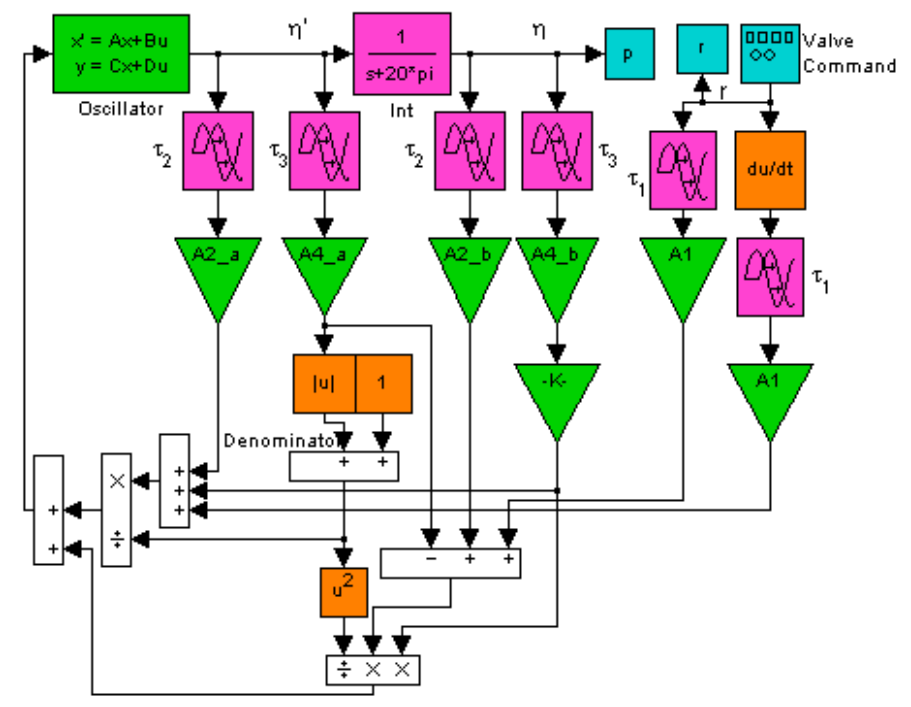

Figure 91: Block diagram of the Delay Differential Equation (DDE) thermo-acoustic model 
Several experiments were performed to gain understanding of the effects of unsteady fuel modulation on the behavior of the combustor. Control strategies using fuel modulation generally require the ability to modulate fuel flow at frequencies in the range of those where combustion dynamics occur. Because the identified thermo-acoustic oscillation frequencies were significantly higher than those which off-the-shelf fuel valves could achieve, high frequency modulation valve technologies were explored and an experimental valve utilizing a linear voice coil actuator was selected for trials. The fuel valve was coupled to the DLN pre-mixer, and open loop fuel forcing experiments were performed over a range of frequencies from 40 to $250 \mathrm{~Hz}$.

The effects of forcing frequency and amplitude on combustion dynamics were investigated through a series of experiments. The fuel flow to the combustor was modulated using a command signal with fixed frequency and amplitude $\left(\mathrm{A}_{0}\right)$ for a period of approximately $10 \mathrm{~s}$ before changing to the next setting. During the experiments, data were collected from the pressure transducers in the combustion chamber to examine the response to the excitation signal. Figure 92 shows the response of the combustor to fuel forcing.

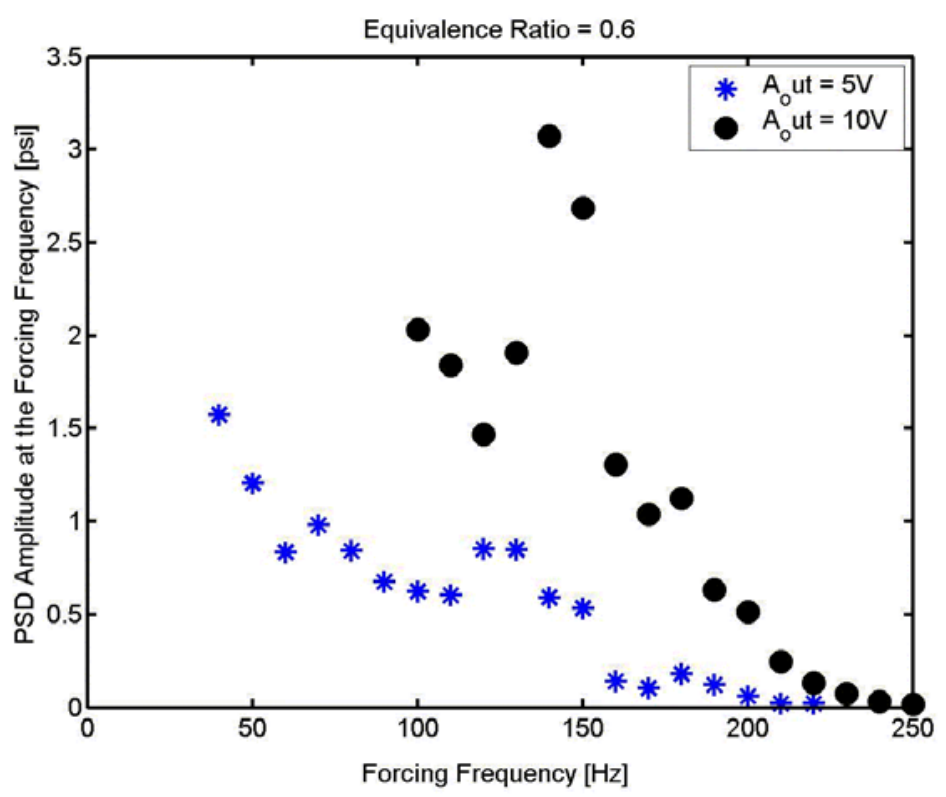

Figure 92: Combustor response to fuel forcing - peak amplitude of pressure spectra at forcing frequency

The data shown represent the peak amplitudes observed in the pressure fluctuation power spectra at the forcing frequency. Data for two different command signal amplitudes are shown. The fuel mass flow perturbation for fixed command signal amplitude varies with frequency. For the case with $\mathrm{A}_{0}=5 \mathrm{~V}$, the ratio of the fuel mass flow perturbation to the mean fuel flow $\left(\mathrm{m}_{\mathrm{f}}^{\prime} / \mathrm{m}_{\mathrm{f}}\right)$ varied from approximately $35 \%$ to $6 \%$ when $f_{\mathrm{f}}$ was increased from 40 to $250 \mathrm{~Hz}$. Similarly, for $\mathrm{A}_{0}=$ $10 \mathrm{~V}, \mathrm{~m}_{\mathrm{f}}^{\prime} / \mathrm{m}_{\mathrm{f}}$ changed from $42 \%$ to $11 \%$ with $f_{\mathrm{f}}$ increased from 100 to $250 \mathrm{~Hz}$. In general, the combustor response to fuel excitation decreased with increased forcing frequency. This can likely be attributed to the measured decrease in actuator response with increasing frequency. 
The data show regions of increased pressure response in the vicinity of $140-150 \mathrm{~Hz}$ forcing frequency. This frequency corresponds to an actuator resonance as identified in frequency response measurements. The slight increase in response shown near $180 \mathrm{~Hz}$ suggests that the fuel-forcing signal is amplified slightly by the natural combustor resonance.

Figure 93 shows the effects of fuel forcing on the amplitude of the natural combustor resonance near $170 \mathrm{~Hz}$, where strong combustion dynamics normally occur. The strength of the dominant dynamics mode near $170 \mathrm{~Hz}$ is approximately 2.3 psi without fuel modulation for the operating conditions in this example. In general, the amplitude of the resonant dynamics peak increases slightly as forcing frequency is increased from 40 to $240 \mathrm{~Hz}$ (varies from approximately 1.5 to $2.5 \mathrm{psi}$ ). However, for the $10 \mathrm{~V}$ gain setting, and with forcing frequencies in the range of approximately $100 \mathrm{~Hz}<f_{\mathrm{f}}<140 \mathrm{~Hz}$, there is a substantial decrease in the amplitude of pressure fluctuations at the natural resonance.

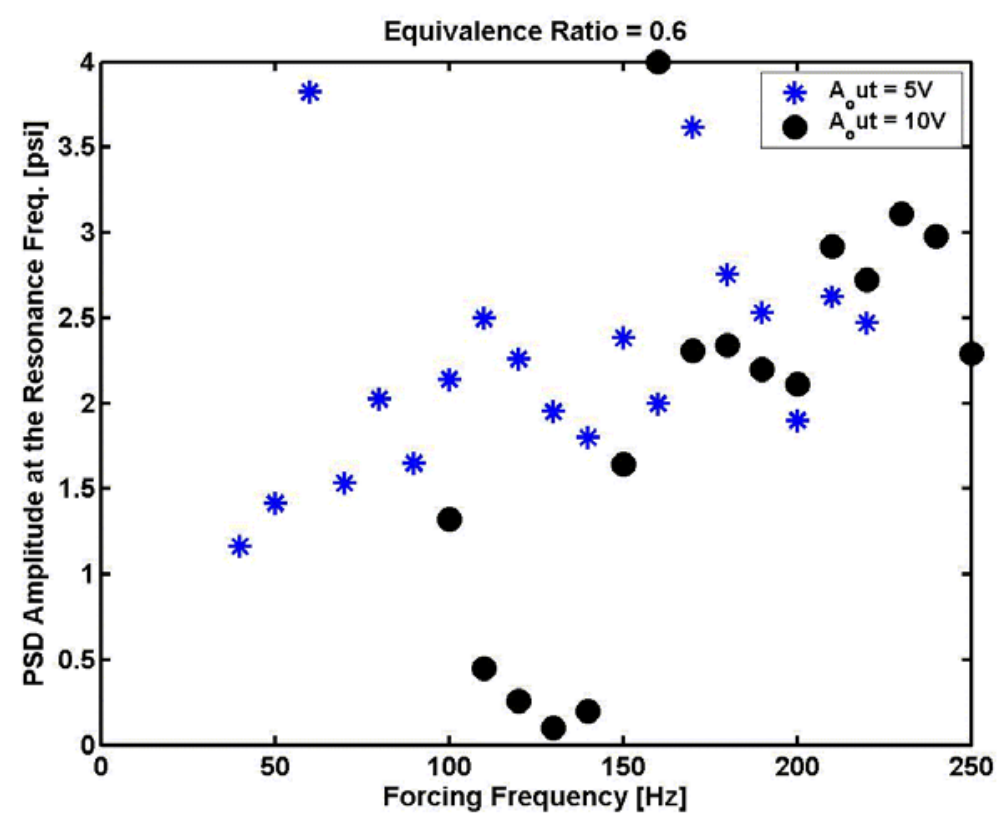

Figure 93: Effect of fuel forcing on amplitude of primary combustion dynamics tone

Figure 94 compares pressure oscillation time traces for cases with and without fuel modulation. The data indicate that there is suppression in the overall amplitude of the pressure fluctuation levels as well as a slight distortion of the waveform. The trends shown in this figure are representative of all cases where attenuation of dynamics levels was observed. 


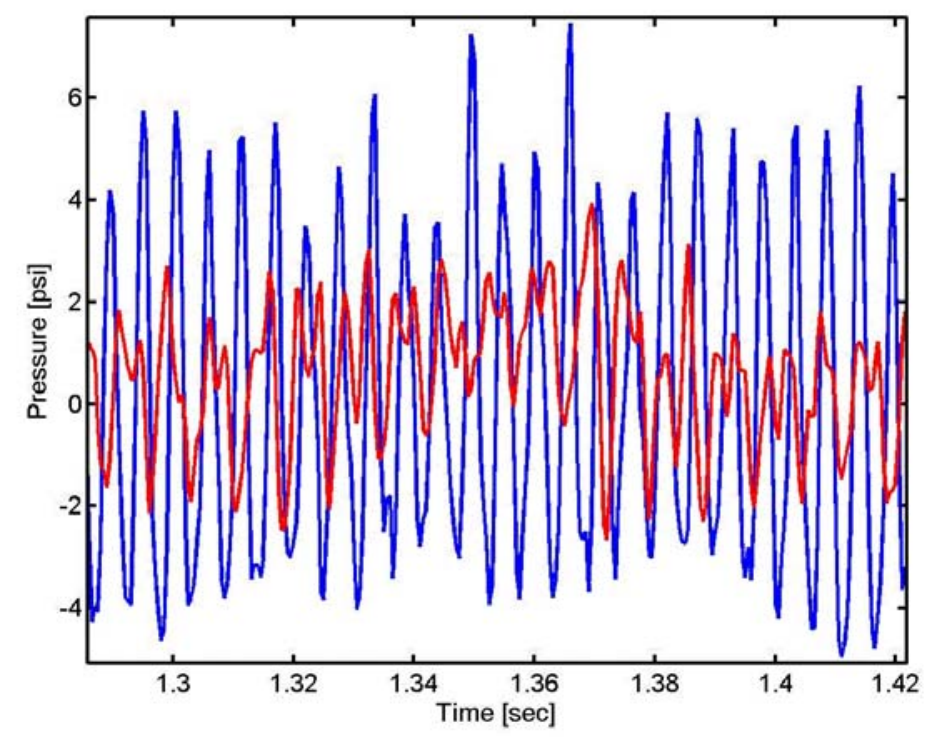

Figure 94: Thermo-acoustic oscillations with (red) and without (blue) fuel modulation

The dynamics model was used to predict the response of the combustor to fuel flow forcing. The model parameters describing fuel modulation were adjusted to match a specific set of experimental conditions and the predicted pressure fluctuation levels were compared with those observed in experiments. Figure 95 shows a comparison between the experimentally observed pressure fluctuation power spectrum and that predicted by the model. The forcing frequency is at $f_{\mathrm{f}}=80 \mathrm{~Hz}$ and pressure fluctuations at this frequency are seen both in the experimental data and the prediction.
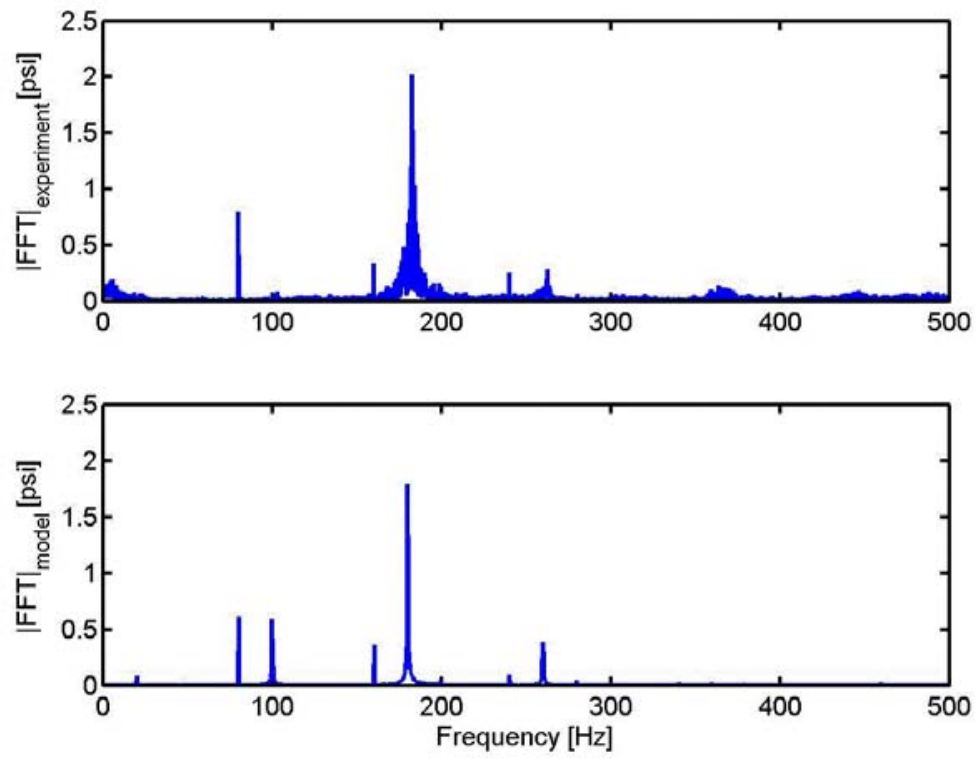

Figure 95: Comparison between experimental (above) and predicted (below) pressure fluctuation power spectra: $f_{\mathrm{f}}=80 \mathrm{~Hz}$ 
The model accurately captures the features of the spectral peak associated with the dominant dynamics tone. In addition, both the model and the experimental data exhibit side-band peaks at both $f_{\mathrm{r}}+f_{\mathrm{f}}$ and $f_{\mathrm{r}}-f_{\mathrm{f}}$ where $f_{\mathrm{r}}$ is the resonant frequency at approximately $190 \mathrm{~Hz}$.

\section{Effects of Fuel Forcing on Combustor Operability}

Several experiments were performed that focused on general combustor operability. An important measure of operability is the combustor lean blowout (LBO) limit. Results from a series of LBO experiments both with and without fuel modulation are shown in Figure 96. There are two sets of data shown where no fuel forcing was applied and these represent two separate experimental runs. These two data sets give an indication of the typical run-to-run variation in the pressure fluctuation amplitudes as well as the LBO limit. We see an LBO enhancement (improvement) when fuel modulation is incorporated. This suggests that there is an intrinsic change in combustion stability when the fuel flow is modulated at high frequency. The reason for this is unclear at present and further experiments are planned to elucidate the physical mechanism responsible for this performance improvement.

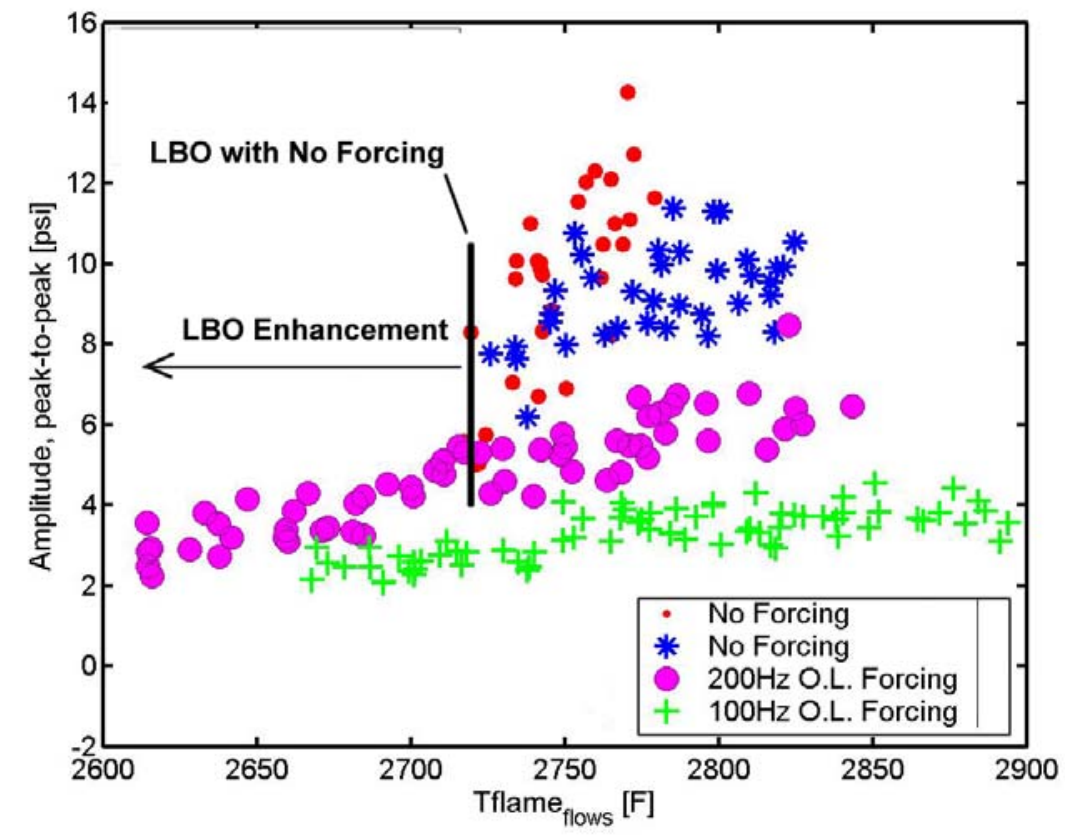

Figure 96: Lean blowout limits measured with and without fuel modulation

\section{$\underline{\text { Sub-Task 3.2 - Development of Control Methods }}$}

This sub-task concerns the development and implementation of active control techniques to improve combustor operability and robustness. Initial experiments were conducted to evaluate the effectiveness of open and closed-loop fuel flow modulation on combustion pressure oscillations. 


\section{Fuel Valve Response}

The fuel valve and pre-mixer assembly frequency response was measured using a hot film anemometer and pressure transducer. The fuel valve actuator was supplied with a sinusoidal signal whose frequency was varied over a range from approximately 50 to $350 \mathrm{~Hz}$. The fuel jet velocity and unsteady pressure signal taken upstream from the pre-mixer were correlated in frequency and the transfer function was computed. Figure 97 shows the pressure-velocity transfer function. This transfer function indicates that the pressure perturbation produced by the valve upstream of the fuel pre-mixer is more efficiently transmitted through the pre-mixer at low frequencies and the transmission efficiency decreases with increasing frequency. Also shown in the figure is a calculated frequency response based on a one-dimensional acoustic model. The model shows a system resonance at approximately $400 \mathrm{~Hz}$. which is not apparent in the measurement. This discrepancy is likely due to a degradation of the velocity and pressure signals that was apparent in this frequency range.

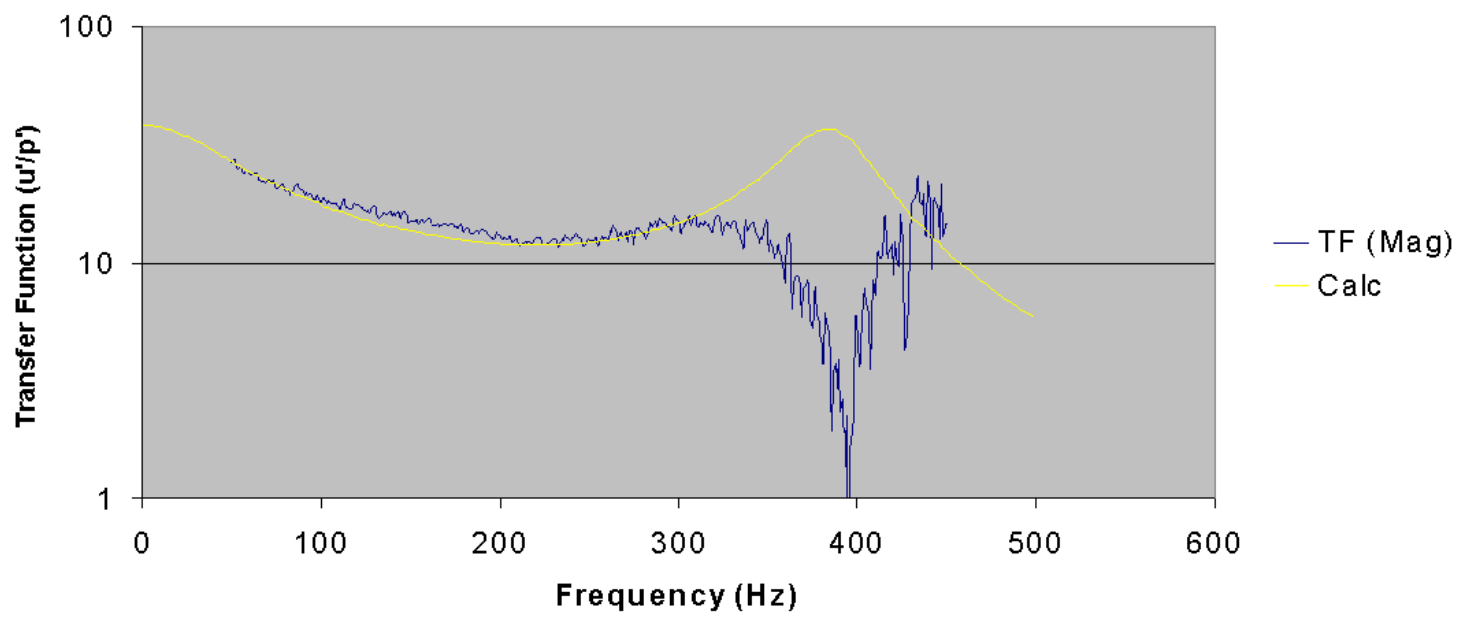

Figure 97: Frequency response of valve-pre-mixer assembly

Additional experiments were conducted using the fuel valve coupled to the DLN2 pre-mixer nozzle to determine the level of mass flow fluctuation in the fuel line during a single cycle. For this experiment, two pressure transducers were positioned in series in the tube coupling the valve with the pre-mixer. The transducers were used to measure the convection velocity of the mass flow perturbation created by the valve action. The variation in convection speed could then be used to compute the mass flow perturbation. Figure 98 shows the results from these experiments for two different values of actuator input signal amplitude. The data show that the perturbation amplitude decreases with increasing forcing frequency and this is consistent with the fuel jet velocity measurement described above. Maximum perturbation amplitudes $\left(\mathrm{m}^{\prime} / \mathrm{m}(\mathrm{ave})\right)$ were approximately $40 \%$ This level of mass flow control is consistent with the expected level of variations in fuel quality entering an IGCC combustion system. The valve would therefore provide a viable means to stabilize and control the combustor in the face of fuel quality variations. Experiments to demonstrate this capability are planned for the HEET Phase II program and would constitute the continuation of the present effort. 


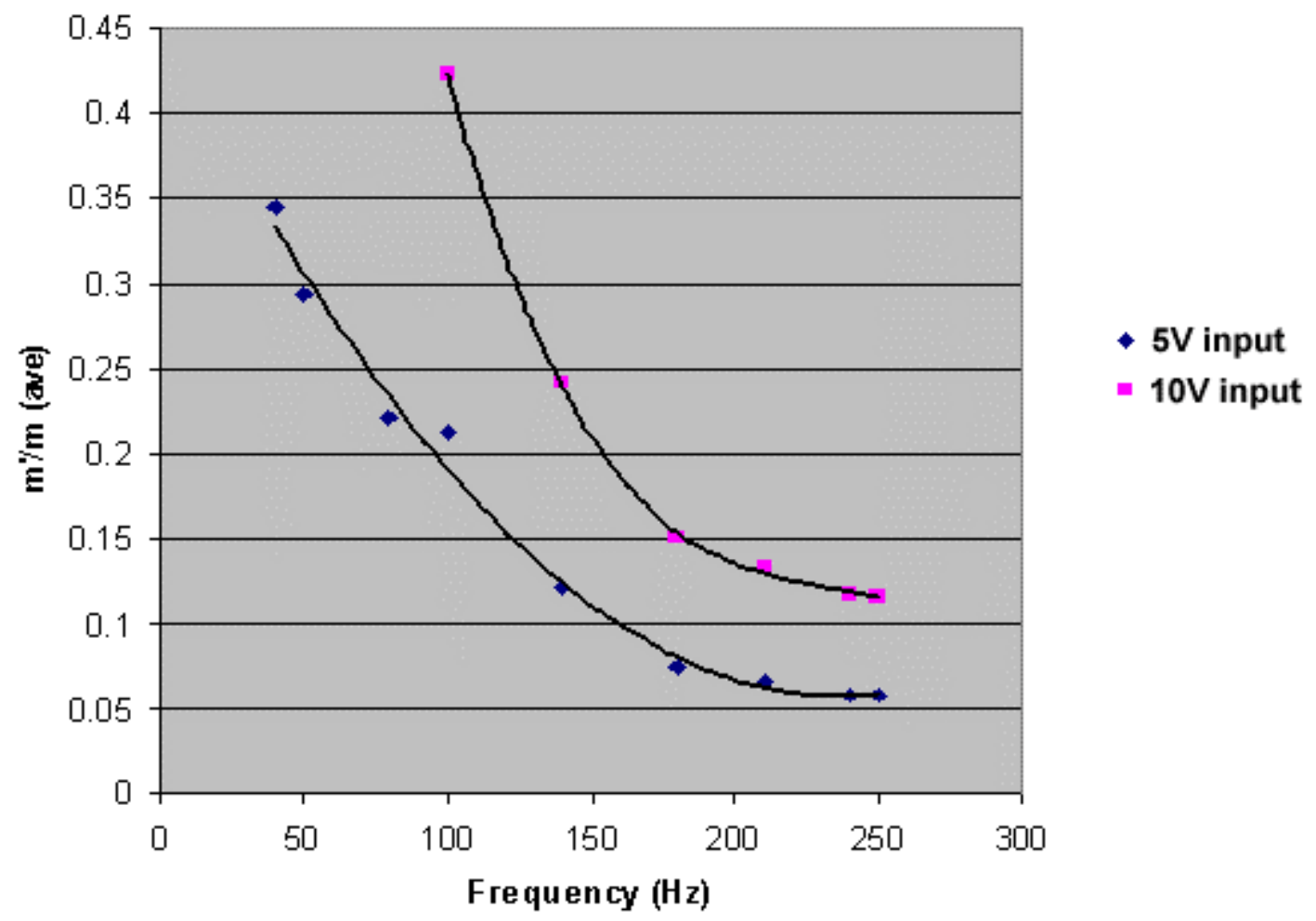

Figure 98: Mass flow perturbation in fuel system

Combustor Response

Experiments were performed using the SNR described above to determine the combustor response to open-loop fuel flow perturbations. The technique of reducing pressure fluctuations through open-loop forcing has been demonstrated extensively on small-scale combustion systems. A model-based control strategy is being pursued using the system model described above. The experiments provided data for model validation and development; in addition, they elucidated limits of control authority, or actuator effectiveness, in a typical DLN combustion system.

The results indicate that the dynamics of a laboratory combustor can be manipulated through open-loop flow modulation applied to the main fuel supply. The system under study exhibited interesting dynamic response to harmonic fuel flow modulation at certain frequencies and showed very low response in other frequency ranges.

In cases where the pressure fluctuation levels at the dominant unstable frequency were suppressed with fuel forcing applied, there was a simultaneous growth of oscillations at the forcing frequency and decrease of oscillation at the unforced resonant peak. This result suggests that fuel forcing created a transfer of thermo-acoustic energy out of the resonant mode and into a non-resonant mode. In general, when this occurred in a robust manner, there was a result net decrease in the overall acoustic energy, or rms pressure fluctuation level. This phenomenon is illustrated in Figure 99. Accompanied by the reduction in acoustic energy in the resonant mode 
was an improvement in combustor LBO. This result suggests that there was an intrinsic improvement in flame stabilization enhancing overall operability of the system.
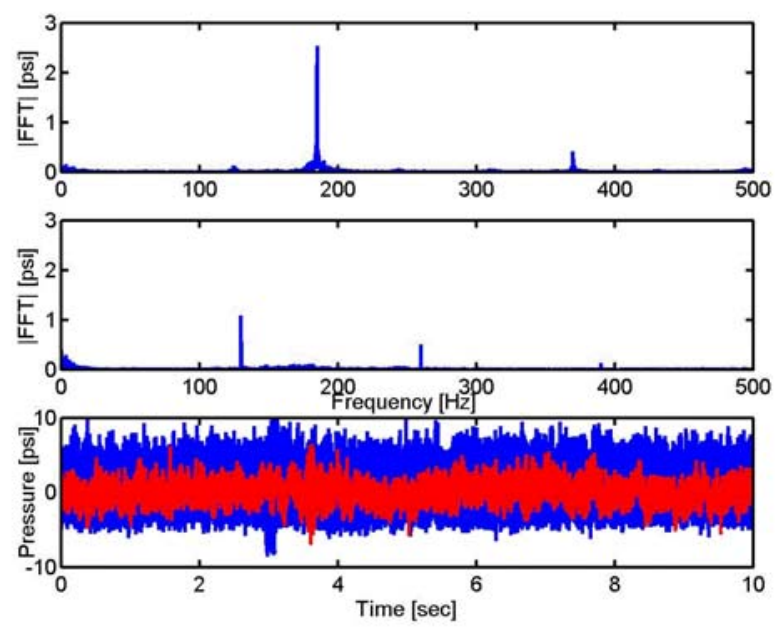

Figure 99: Power spectral density and time-series plots of pressure inside the combustion chamber with no fuel modulation (upper/blue) and $130 \mathrm{~Hz}$ modulation (middle/red) 


\section{Task 4 - Results/Discussion:}

\section{$\underline{\text { Information Technology (IT) Integration }}$}

\section{Task 4 Overview:}

The fourth task demonstrated Information Technology (IT) tools for advanced technology coal/IGCC powerplant condition assessment and condition based maintenance. The task focused on development of GateCycle ${ }^{\mathrm{TM}}$ software to model complete-plant IGCC systems, and the Universal On-Site Monitor (UOSM) to collect and integrate data from multiple condition monitoring applications at a power plant. The contributors were GE Energy and Tampa Electric Company. The work on this task was stopped as of April 1, 2004.

Sub-Task 4.1 - Performance Modeling of Coal IGCC Powerplants: The functionality of GE's GateCycle ${ }^{\mathrm{TM}}$ software was extended in order to model the complete Integrated Gasification Combined-Cycle (IGCC) power plant, to provide coal/IGCC powerplant performance data in real time.

Sub-Task 4.2 - Powerplant Data Integration: A Universal On-Site Monitor (UOSM) was installed at the Tampa Electric Company's Polk 1 coal/IGCC powerplant for data collection and integration.

\section{Task 4 Discussion:}

\section{$\underline{\text { Sub-Task 4.1 - Performance Modeling for Coal/IGCC Powerplants }}$}

The primary focus of this sub-task is to extend the functionality of GE's GateCycle ${ }^{\mathrm{TM}}$ commercial heat-balance simulation software in order to model complete Integrated Gasification Combined-Cycle (IGCC) power plants, and to use this extended GateCycle ${ }^{\mathrm{TM}}$ software to build and configure the online EfficiencyMap ${ }^{\mathrm{TM}}$ performance monitoring software system at a selected IGCC site.

Currently, there is no available commercial software, which can simulate the performance of an IGCC power plant. This is urgently needed for designing, optimizing, testing and technology improvements of IGCC power plants, which have complex integration of energy and flows between gasification and power plant. Improved integration in new IGCC power plants are expected to increase efficiency and decrease equipment and operating costs significantly thus making them available commercially and operate economically. In support of this work, it is necessary to enhance the database foundation, property calculations, convergence routines and unit operation equipment models of the GateCycle ${ }^{\mathrm{TM}}$ software.

During the development of the SQL database version of GateCycle, a key issue that had to be resolved was the speed of database access. With the new SQL database design, considerably more of the configuration and program data is now stored in the database, rather than compiled directly in code structures. In addition, additional data structures have now been added that have 
considerably increased the complexity of the data structures needed, including more complex streams, table structures, the concept of libraries, as well as configuration constants and default data. The basic structures were modified to move constants that had been compiled in the code itself instead into the SQL database (such as default data values, user-selected engineering methods for equipment models, etc.).

The basic functionality of the SQL database for GateCycle ${ }^{T M}$ has been completed and verified. The current functionality of the EASE+ version of GateCycle ${ }^{\mathrm{TM}}$ has been reproduced, while at the same time making sure that the architecture and design framework will support the needed IGCC extensions. All of the basic database functionality is now in place in the MSDE version of GateCycle ${ }^{\mathrm{TM}}$; that is, models can be created, run and saved. In addition, basic import and export functionality via XML has been implemented and tested. The import of existing GateCycle ${ }^{\mathrm{TM}}$ models (importing directly from the current EASE+ database system) to the new SQL database version has been verified and tested. Now that the data structure is in place to support extended stream modeling, the property calculations and unit operation models can be designed and implemented.

Requirements gathering and initial architectural design work was completed for the database integration and analysis extensions needed to support IGCC modeling. The key enhancements to be included are:

- GateCycle ${ }^{T M}$ stream data structures must be extended to increase the number of constituents supported in a gas stream (the current limit is 11 constituents)

- Database and property calculation routines must be added for solid streams

- Database and property calculation routines must be added for mixed-phase streams (gas/solid, gas/liquid and liquid/solid)

- Current GateCycle ${ }^{\mathrm{TM}}$ unit operation models for gasifiers and gas saturators will be enhanced

- Unit operation models will be added for syngas coolers, solid and slurry handling equipment, distillation/absorption columns and gas cleanup equipment

- Core convergence and analysis routines will need to be extended and tested for the more complex chemical system and plant integration inherent in IGCC systems.

The extension of the database structures in GateCycle ${ }^{T M}$ to support the modeling extensions of IGCC technologies required a new methodology. Specifically, a new GateCycle ${ }^{\mathrm{TM}}$ stream data structures has been decided utilizing a new GUI interface based on Microsoft .Net architecture and new universal Stream Function Icon which would be able to support increased number of gaseous constituents (the limit in the current database implementation is 11 constituents in each gas stream), and support property calculations of solid streams, and mixed streams (gas/solid, gas/liquid and liquid/solid).

The task of evaluating external property databases and code sets for the property extensions that need to be integrated into the GateCycle ${ }^{\mathrm{TM}}$ database and analysis framework to support the calculations for solid properties and sulfur-removal chemical process modeling has been completed. The task focused on evaluating external real gas property packages that would need to be integrated into GateCycle ${ }^{\mathrm{TM}}$ to provide an IGCC model. A number of property packages were identified as possible options to provide the required additional functionality to 
GateCycle ${ }^{\mathrm{TM}}$. Two particular property packages were examined in detail. The investigation looked at the suitability for integration into the GateCycle ${ }^{\mathrm{TM}}$ code.

The two property packages identified were:

(a) ASPEN (Advance System for Process Engineering) Physical Property package.

(b) VMG (Virtual Materials Group) VMG Thermo Physical Property System.

The ASPEN physical property package is open source code written in FORTRAN for VAX computers. A preliminary investigation into converting the FORTRAN code into a more useable software language such as $\mathrm{C}++$ indicated that considerable time would be required before this code could be integrated into GateCycle ${ }^{\mathrm{TM}}$. (A commercial PC version of the ASPEN code called PRO Aspen/PCTM, owned and licensed by P\&P Innovative Software, Inc was considered as a possible alternative, however purchasing and licensing issues were not resolved during this reporting period.)

The VMG property package called VMG Thermo includes support for Sulfuric++ and Amines++ but does not have support for solids. It was decided to pursue a detailed investigation of the VMG software to determine if any risks existed to integrate this software package into the existing GateCycle ${ }^{\mathrm{TM}}$ product.

The work on this sub-task was stopped as of April 1, 2004.

\section{$\underline{\text { Sub-Task 4.2 - Coal/IGCC Powerplant Data Integration }}$}

The Universal On-Site Monitor (UOSM) was developed to collect and integrate data from multiple condition monitoring applications at a power plant: turbine-generator controls, plant distributed controls, vibration monitoring, emissions monitoring, etc. UOSMs have been installed at approximately 20 natural gas and dual fuel powerplants to date. The Tampa Electric Company Polk 1 powerplant was the first coal/IGCC powerplant to be equipped with the UOSM and GE's upgraded condition assessment software.

The work on this sub-task was stopped as of April 1, 2004. 


\section{CONCLUSIONS}

\section{$\underline{\text { Task } 1 \text { - IGCC Environmental Impact on High Temperature Materials }}$}

The environmental impacts on high temperature materials used in advanced IGCC gas turbines were extensively evaluated. A wide variety of hot gas path and combustion alloys and alloy/coating systems were tested in simulated IGCC environments. Parts removed from a MS7001FA IGCC gas turbine were evaluated and the environmental impact was described mechanistically. Syngas and filter residues were sampled from multiple locations in a MS7001FA IGCC gas turbine powerplant, and the results were correlated with the material condition of the parts. The part condition was also used to validate the environmental simulations performed in the laboratory. The ability to correlate laboratory data to gas turbine parts in service was of paramount importance.

\section{Environment Effects}

The laboratory environmental simulation tests demonstrated that the latest generation bucket alloy and coatings performed better in oxidation, corrosion and erosion testing than the older generation materials. The investigation compared scaling characteristics of nine candidate materials in three different gaseous environments - air, dry $\mathrm{H}_{2} \mathrm{~S}$ and wet $\mathrm{H}_{2} \mathrm{~S}$ at $1900^{\circ} \mathrm{F}-$ with exposures up to 300 hours. The test materials were: (A) Alloy FSX-414, (B) Alloy GTD222, (C) Alloy Hastelloy-X, (D) Alloy N5, (E) Alloy Nimonic-263, (F) Alloy GTD111, (G) Alloy N5 + Coating of NiCrAlY, (H) Alloy Nimonic-263 + Coating of CoNiCrAlY, and (K) Alloy GTD111

+ Coating of CoNiCrAlY. The findings based on gravimetric measurements and metallographic examinations of the specimens are as follows.

1. Scales formed on the various materials were friable and spalled; the loss increased with thicker scales and longer exposure periods.

2. Air environment:

a. Alloy N5 exhibited the least amount of scaling of all the systems evaluated.

b. The coated alloys experienced less scaling than the corresponding base alloys.

c. No intergranular penetration (IGP) was seen on the coated alloys nor the base alloys N5 and Hastelloy-X. All of the remaining alloys experienced significant depths of IGP, typically $>10 \mu \mathrm{m}$.

3. Dry $\mathrm{H}_{2} \mathrm{~S}$ environment:

a. All of the base alloys experienced significant scaling, typically $>10 \mu \mathrm{m}$.

b. Overall, the coated alloys exhibited less scaling than the corresponding base alloys.

c. Severe IGP $(>>10 \mu \mathrm{m})$ was noticed on the base alloys but not on the coated alloys.

d. Direct impingement of the gas (extreme turbulence) appeared to accelerate sulfidation, forming nodules on the leading faces of the specimens.

4. Wet $\mathrm{H}_{2} \mathrm{~S}$ environment:

a. Alloy N5 and Hastelloy-X produced thinner scales than the other base alloys.

b. Overall, the coated alloys produced thinner scales than the corresponding base alloys, except for base Alloy N5.

c. No IGP was noticed on the coated alloys or on base Alloy Hastelloy-X, but moderate attack was present on the other base alloys, typically $>10 \mu \mathrm{m}$. 
d. Alloy N5 exhibited deep IGP in only one of the four specimens examined. The reason for this anomaly is unknown.

5. The severity of the test environments based on scaling tendency and IGP attack in various materials can be ranked as follows: dry $\mathrm{H}_{2} \mathrm{~S}>$ wet $\mathrm{H}_{2} \mathrm{~S}>$ air. Water vapor in wet $\mathrm{H}_{2} \mathrm{~S}$ likely acted as an oxidizer and, thus, oxidation of the alloys hindered sulfidation.

\section{Erosion Experiments}

Significant erosion was also observed in some of the parts removed from the MS7001FA IGCC gas turbine. The relative erosion resistance of several thermal barrier coating (TBC) systems was compared using a high temperature test apparatus, because new IGCC gas turbine designs will extensively utilize TBC for thermal protection of hot gas path parts. The results were not conclusive, however, because the mode of failure for the $\mathrm{TBC}$ systems differed. In two of the three coatings (GTD111 + CoNiCrAlY bondcoat + TBC, Nimonic-263 + CoNiCrAlY bondcoat $+\mathrm{TBC}$ ), the TBC appeared to have spalled from the surface, indicating that another failure mechanism was competing with the erosion mechanism. In the third coating (Rene N5 + NiCrAlY bondcoat $+\mathrm{TBC}$ ), the TBC wore off the surface in the typical erosion manner. It was concluded that the Rene N5 specimens had superior high temperature erosion resistance on the basis of these results, but additional tests may be needed to verify this.

\section{Syngas Sampling}

Syngas fuel sampling tests performed at the Tampa Electric Company (TECO) Polk 1 powerplant showed that some fuel contaminants were significantly higher during powerplant start up and partial load operation than during steady state operation. Fuel contaminants may reduce the life of gas turbine hot parts or be a possible health hazard for maintenance personnel. Therefore, future IGCC powerplant designs should seek to reduce the levels of fuel contaminants during non-steady operating conditions.

\section{Service Exposed Parts}

Stage one buckets removed from the MS7001FA IGCC gas turbine at TECO Polk 1 were evaluated to analyze the condition of the material (directionally solidified GTD-111 + CoCrAlY coating with over-aluminide) and identify foreign matter that may have deposited or diffused into the coating and substrate. Significant coating distress was observed around the leading edge and pressure side of the airfoil, with significant erosion at the leading and trailing edges. In surface analyses, traces of sulfur were detected in these areas predominantly. On the other hand, iron deposits were found in most locations of the airfoil. The origin of the iron was not identified, but was likely to be from the erosion of upstream steel components.

A detailed investigation of the presence of sulfur in the areas where the coating was severely distressed, i.e. on the leading edge and towards the tip of the trailing edge on the pressure side, revealed the formation of $\mathrm{Cr}_{8} \mathrm{~S}_{7}$ sulfides in the diffusion zone of the substrate just below the coating. On the leading edge, a high number density of sulfides was found along the apex where the coating was removed, became less numerous as some coating, albeit highly oxidized, remained on the surface and completely disappeared away from the apex where a sound coating covered the substrate. The density of sulfides by the trailing edge was similar to that observed on the leading edge where no coating remained on the surface. Aluminum depletion in the coating was more prominent on the suction side, while the formation of craze cracks that 
propagated into the coating seemed to be confined to the leading edge area, both on the suction and pressure sides. No correlation between the crack paths and the coating microstructure was observed due to the severe oxidation surrounding the cracks.

The results of this investigation point to sulfidation as a possible failure mechanism of the coating. The areas where the coating was completely removed during service showed a high concentration of sulfides in the diffusion zone of the substrate, while areas sampled under a healthy coating did not reveal these precipitates. However, these results are not sufficient to determine whether sulfur was the main cause of the coating failure, or if the coating failed from a different mechanism, which allowed the subsequent diffusion of sulfur in the substrate.

\section{Task 2 - Material In-Service Health Monitoring}

Sensing needs for advanced coal/IGCC gas turbines were evaluated and prioritized. The two most critical needs were: 1) Fuel Quality Sensor to rapidly determine the fuel heating value for more precise control of the gas turbine, and detection of fuel impurities that could lead to rapid component degradation. 2) Infra-Red Pyrometer to continuously measure the temperature of gas turbine buckets, nozzles, and combustor hardware.

The most common method to determine the fuel heating value involves the use of a gas chromatograph (GC) with a glass capillary system to separate the fuel constituents and a thermal conductivity detector and/or a flame ionization detector to quantitatively identify the constituents. However, a conventional GC has several drawbacks: it is relatively expensive (\$30k or more), difficult to operate, continuous measurement is usually not practical, and analysis times of several minutes are normally required. The Fuel Quality Sensor is needed to address these drawbacks. The sensor was demonstrated on natural gas, as well as actual and simulated coal IGCC syngas fuels, and showed great promise. However, sensor reliability issues were identified and must be overcome before the sensor can be offered commercially.

Infra-Red optical pyrometers were demonstrated in four gas turbines, and proved to be capable of measuring temperatures within the hot gas path for extended periods of time. Analysis tools and techniques were developed to allow the temperatures measured using the pyrometer to be used to validate design models and for real time monitoring of turbine performance and part life. A second optical technique, using an Infra-Red camera for measuring transient temperature response, was used to characterize the thermal variation of two sets of stage 1 turbine buckets.

\section{Task 3 - Advanced Methods for Combustion Modeling}

A fast response fuel valve for the implementation of active combustion control of Coal/IGCC turbines has been evaluated. The valve was bench tested in non-reacting experiments to determine its frequency and amplitude response and then was implemented in reacting flow experiments using a single nozzle combustion rig. Fuel modulation experiments have demonstrated operability improvements in a Dry Low NOx (DLN) combustor, including improved lean blowout. Further work in this Task is being directed towards the development of a fuel pre-mixer for Coal/IGCC application. The active control development started under this 
program is being continued in GE's Pre-mixer Design for High Hydrogen Fuels program under DOE sponsorship.

\section{Task 4 - Information Technology (IT) Integration}

The functionality of GE's GateCycle ${ }^{\mathrm{TM}}$ commercial heat balance simulation software was extended to model simulation of solid fuel gasification components and their complex integration for Coal/IGCC power plants. Key progress was made in: the enhancement of the database foundation, methodology to add thermodynamic properties and stream object integration for IGCC components, and new graphical user interface (GUI). Yet to be accomplished were: the addition of solid and multiphase real liquid and gaseous mixture properties, improved convergence algorithms and physics based models of gasification components, and final model validation at TECO Polk IGCC power plant.

A team from GE Energy Services Technology and GE Bently Nevada visited the Tampa Electric Company Polk 1 powerplant during a planned shutdown to upgrade the existing Generation II OSMs to a UOSM configuration. The UOSM was developed to collect and integrate data from multiple condition monitoring applications at a power plant: turbine-generator controls, plant distributed controls, vibration monitoring, emissions monitoring, etc. The Tampa Electric Company Polk 1 powerplant is the first Coal/IGCC powerplant to be equipped with the UOSM and GE's upgraded condition assessment software. These advanced monitoring and diagnostics capabilities were extensively leveraged during the syngas sampling tests performed in Task 1 . 


\section{REFERENCES}

1.1. J.M. Gallardo, J.A. Rodríguez, E.J. Herrera, Wear 252, pp. 264-268, 2002.

1.2. G.W. Goward, Surface and Coatings Technology 108-109, pp. 73-79, 1998.

2.1. "The Measurement of Full-Surface Internal Heat Transfer Coefficients for Turbine Airfoils Using a Nondestructive Thermal Inertia Technique," Nirmalan, Bunker, and Hedlund, Journal of Turbomachinery, Volume 125, pp. 83-89, 2003.

3.1. McManus, K.R., Poinsot, T. and Candel, S.M.,"A Review of Active Control of Combustion Instabilities," Prog. Energy Combust. Sci. Vol. 19, pp. 1-29, 1993. 


\section{ACRONYMS and ABBREVIATIONS}

$\mathrm{ADH}$ - Atlanta Data Highway

BOP - Balance of Plant

CTQ - Six Sigma: Critical To Quality

EDS - Energy Dispersive Spectroscopy

EBSD - Electron Back-Scattered Diffraction

FMEA - Failure Mode and Effects Analysis

DCS - Distributed Control System

DDE - Delay-Differential-Equation

DLN - Dry Low NOx

DoE - Design of Experiment

GC - Gas Chromatograph

GEAE - GE Aircraft Engines

GEPS - GE Power Systems

GRC - GE Global Research Center

GT - Gas Turbine

GUI - Graphical User Interface

IGCC - Integrated Gasification Combined Cycle

HGP - Hot Gas Path

IGP - InterGranular Penetration

IR - Infra-Red

IT - Information Technology

LBO - Lean Blow Out

LHV - Lower Heating Value

LIBS - Laser Induced Breakdown Spectrometry

LOS - Line-Of-Sight

MEMS - Micro-Electro-Mechanical System

MFC - Mass Flow Controller

OSM - On-Site Monitor

PSDF - Power Systems Development Facility

QFD - Six Sigma: Quality Function Deployment

RAM - Reliability, Availability, and Maintainability

SEM - Scanning Electron Microscopy

SNL - Sandia National Laboratory

SNR - Single Nozzle Rig

TBC - Thermal Barrier Coating 
TECO - Tampa Electric Company

UOSM - Universal On-Site Monitor

WDS - Wavelength Dispersive Spectroscopy

XRD - X-Ray Diffraction 
APPENDIX A.

PHOTOGRAPHS OF A TYPICAL SPECIMEN OF EACH OF THE NINE MATERIALS, AS-RECEIVED 


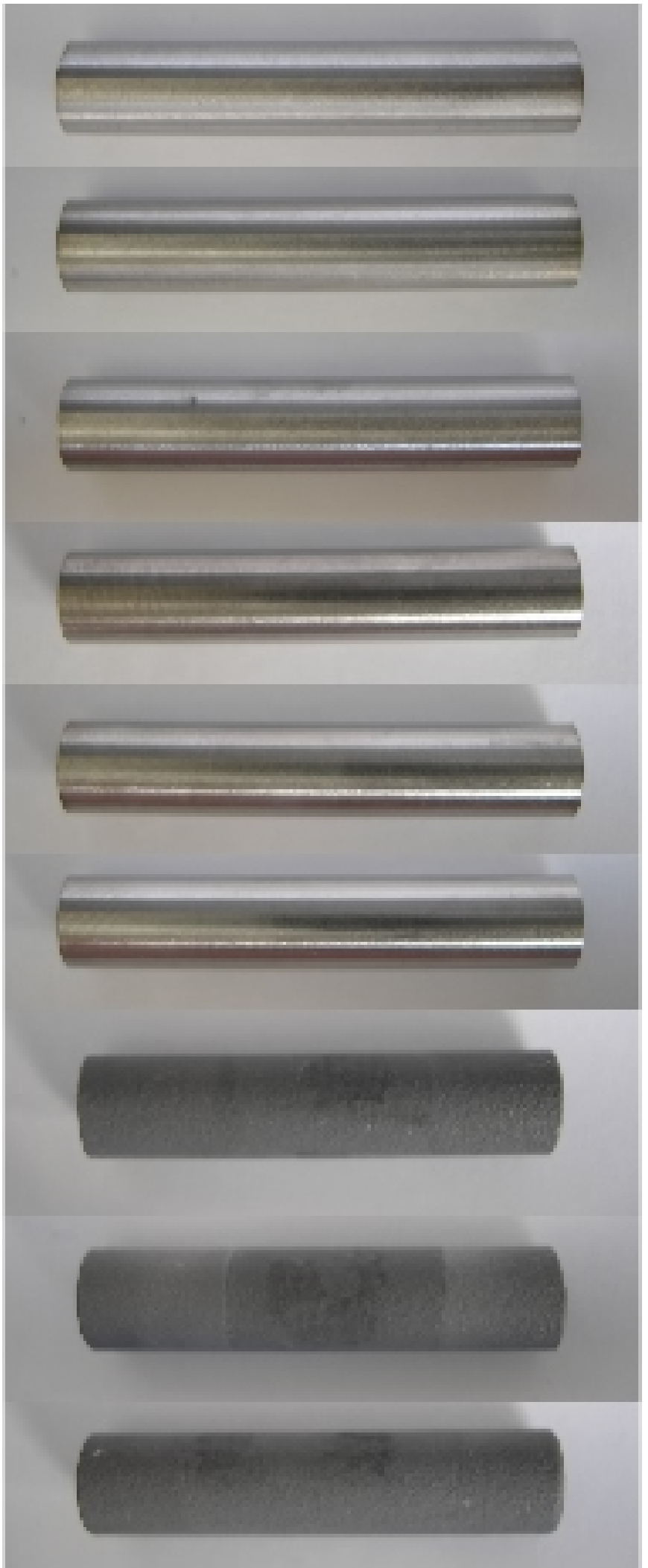

Alloy $\mathrm{FSC4} 14$

Alloy GTD222

Alloy Hastelloy X

Alloy $\mathbf{N} 5$

Alloy Nimonic 263

Alloy GTD111

Alloy N5 + NiCrAlY Coating

Alloy Nimonic $263+$ CoNiCrAlY Coating

Alloy GTD111 + CoNiCrAlY Coating

Photographs Of A Typical Specimen Of Each Of The Nine Materials, As-Received. 


\section{APPENDIX B}

Photographs of Specimens Following Exposure to Different Test Environments

(Photographs are presented in the ascending order of materials code (A-K) for each test environment - air, dry $\mathrm{H}_{2} \mathrm{~S}$, wet $\mathrm{H}_{2} \mathrm{~S}$ ). 
10 hours

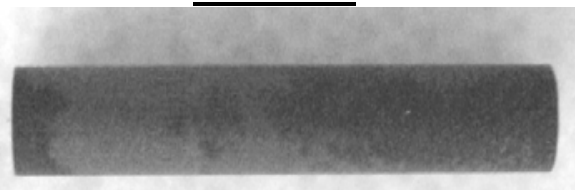

(a) A10A1

(b) $\mathrm{A} 10 \mathrm{~A} 2$

(c) $\mathrm{A} 10 \mathrm{~A} 3$

30 hours

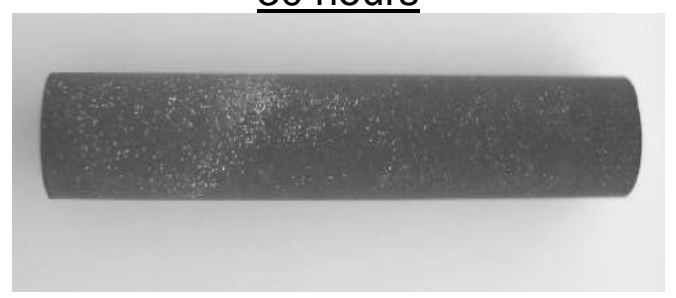

(d) A30A1

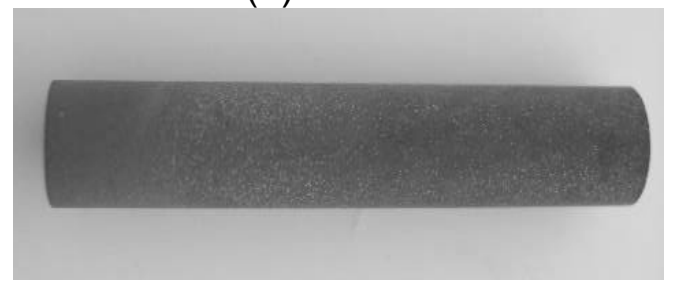

(e) $\mathrm{A} 30 \mathrm{~A} 2$

(f) $\mathrm{A} 30 \mathrm{~A} 3$
100 hours

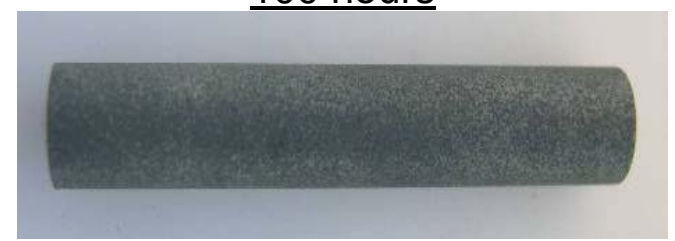

(g) A100A1

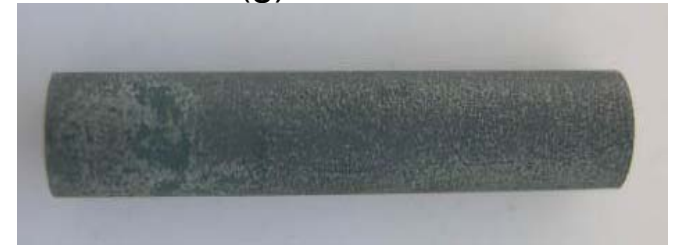

(h) A100A2

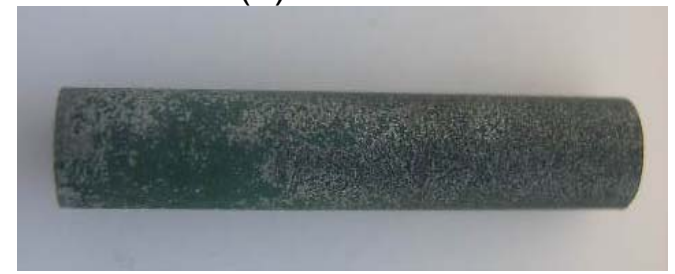

(i) $\mathrm{A} 100 \mathrm{~A} 3$

300 hours

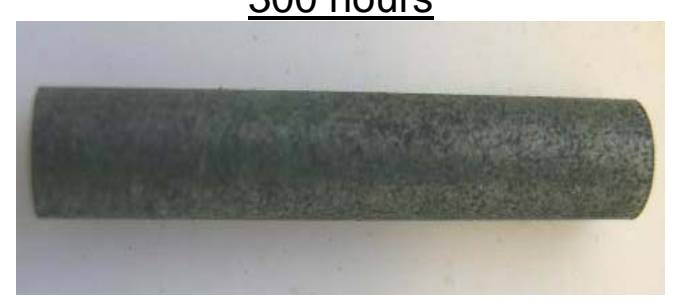

(j) $\mathrm{A} 300 \mathrm{~A} 1$

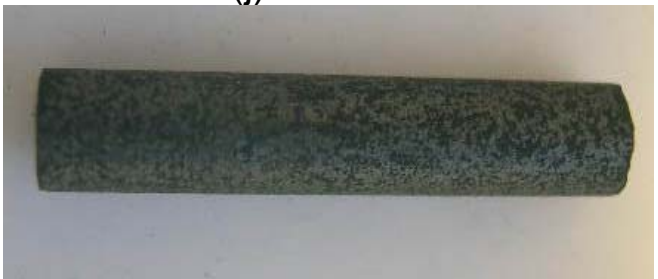

(k) A300A2

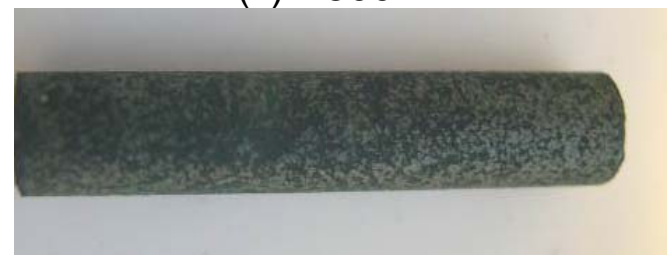

(I) $\mathrm{A} 300 \mathrm{~A} 3$

Photographs of Triplicate Specimens of FSC-414 Exposed to Air at $1900{ }^{\circ} \mathrm{F}$ for 10, 30, 100 and 300 Hours. Note: Top of Specimens is on the Right in Each Photograph. 
10 hours

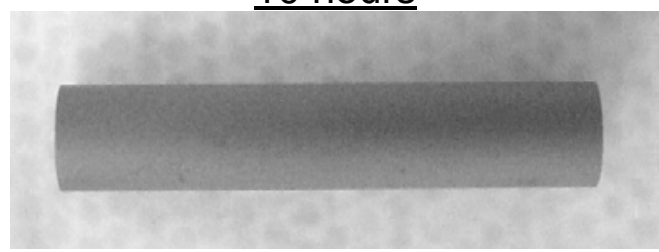

(a) $\mathrm{A} 10 \mathrm{~B} 1$

(b) $\mathrm{A} 10 \mathrm{~B} 2$

(c) A10B3

$\underline{30 \text { hours }}$

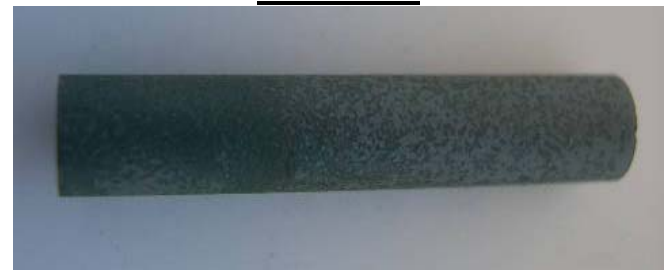

(d) A30B1

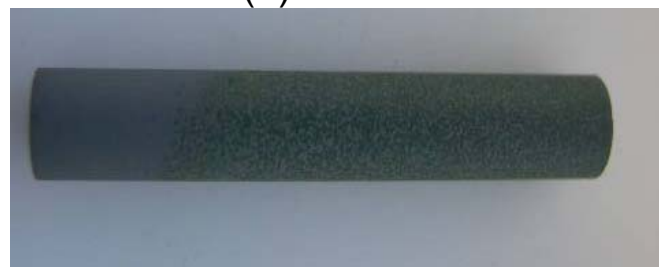

(e) A30B2

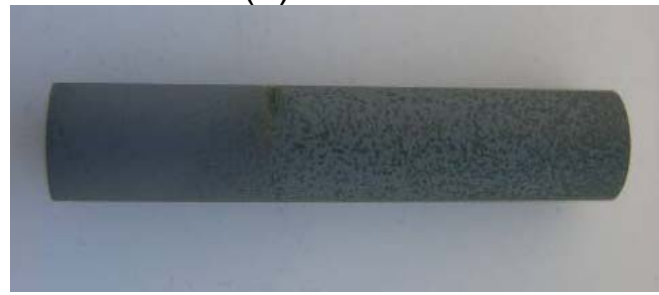

(f) A30B3
100 hours

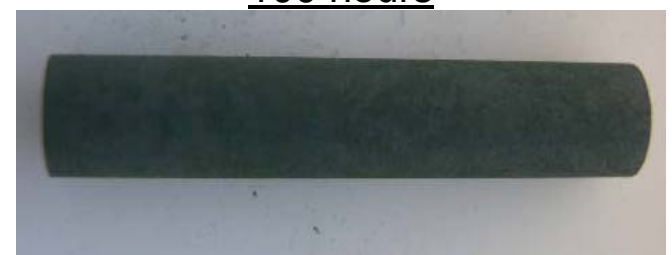

(g) A100B1

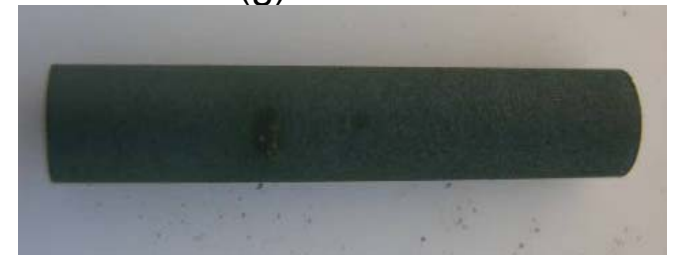

(h) A100B2

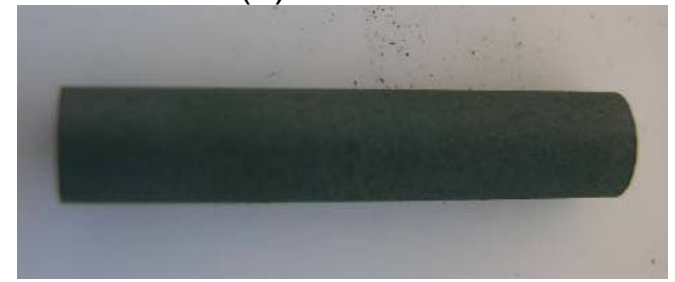

(i) A100B3

300 hours

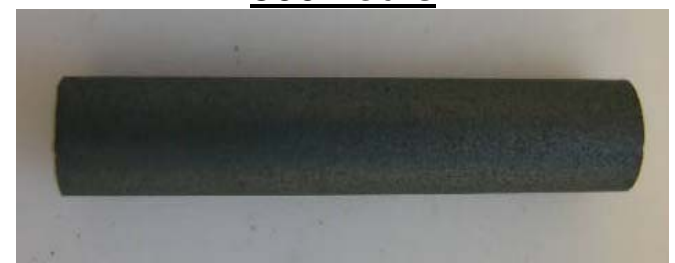

(j) $\mathrm{A} 300 \mathrm{~B} 1$

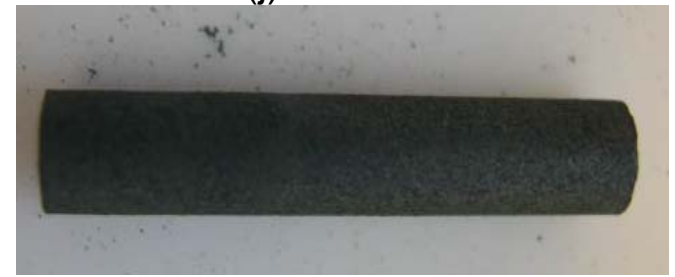

(k) A300B2

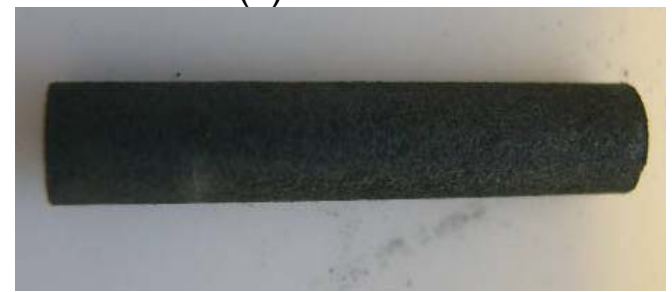

(I) A300B3

Photographs of Triplicate Specimens of GTD222 Exposed to Air at $1900{ }^{\circ} \mathrm{F}$ for 10, 30, 100 and 300 Hours. Note: Top of Specimens is on the Right in Each Photograph. 
10 hours

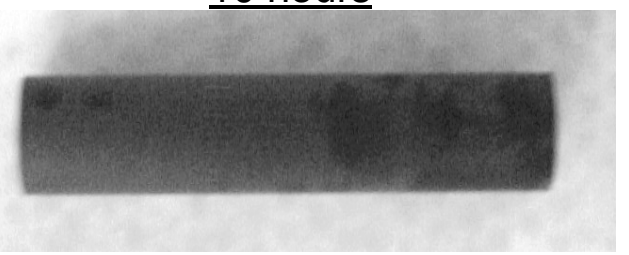

(a) $\mathrm{A} 10 \mathrm{C} 1$

(b) A10C2

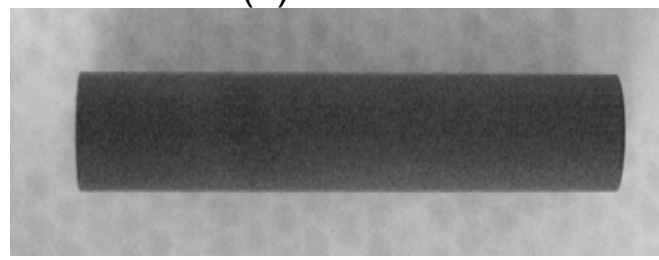

(c) $\mathrm{A} 10 \mathrm{C} 3$

30 hours

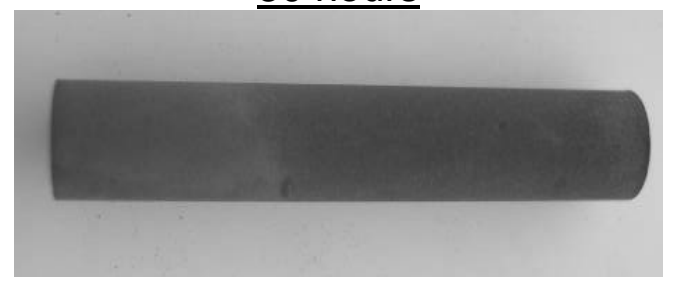

(d) A30C1

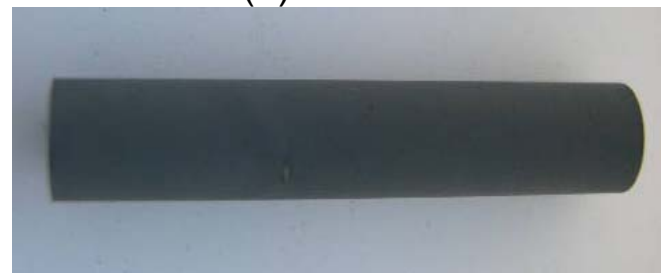

(e) A30C2

(f) $\mathrm{A} 30 \mathrm{C} 3$
100 hours

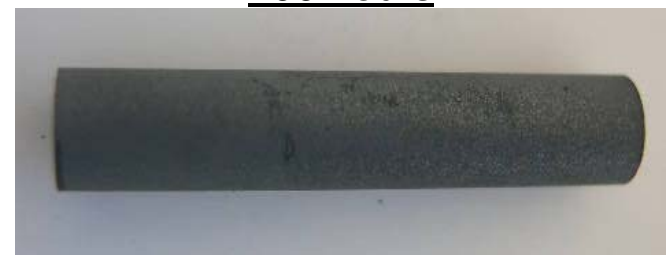

(g) $\mathrm{A} 100 \mathrm{C} 1$

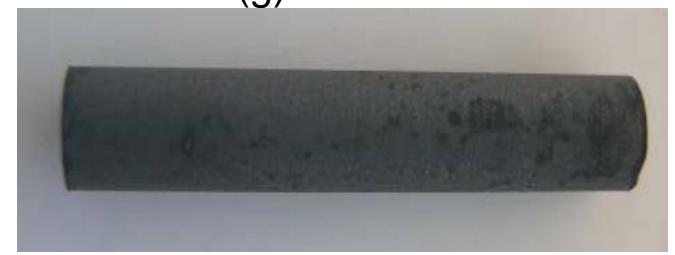

(h) A100C2

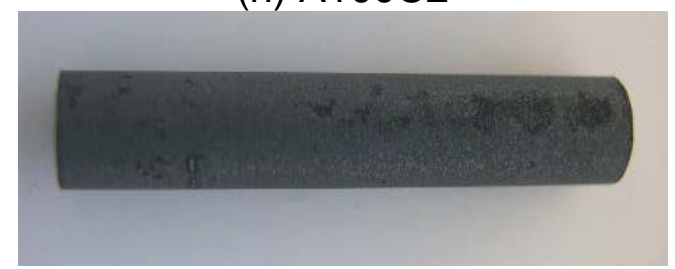

(i) $\mathrm{A} 100 \mathrm{C} 3$

300 hours

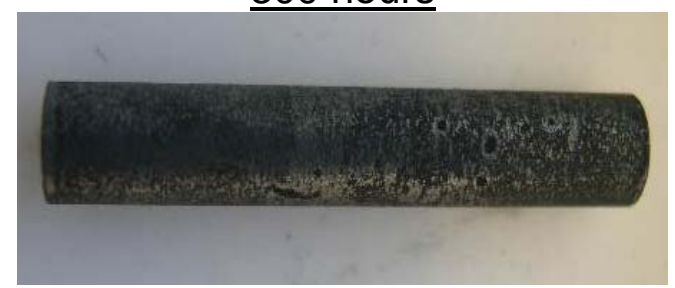

(j) $\mathrm{A} 300 \mathrm{C} 1$

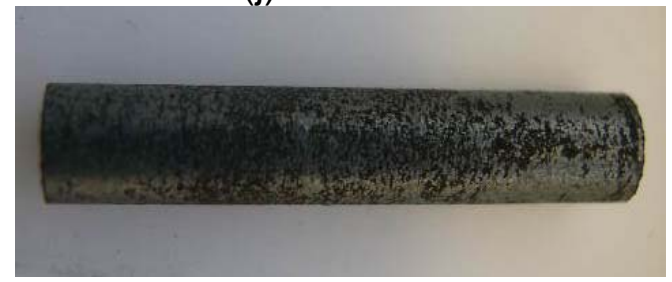

(k) A300C2

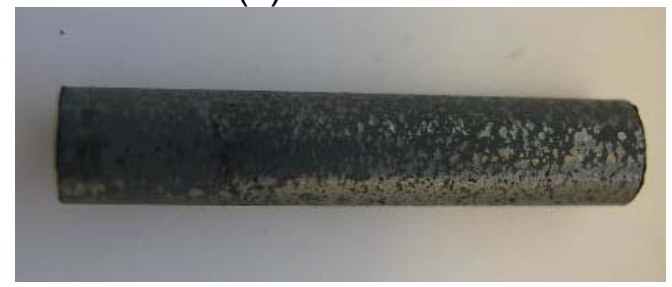

(I) $\mathrm{A} 300 \mathrm{C} 3$

Photographs of Triplicate Specimens of Hastelloy-X Exposed to Air at $1900{ }^{\circ} \mathrm{F}$ for 10, 30, 100 and 300 Hours. Note: Top of Specimens is on the Right in Each Photograph. 
10 hours

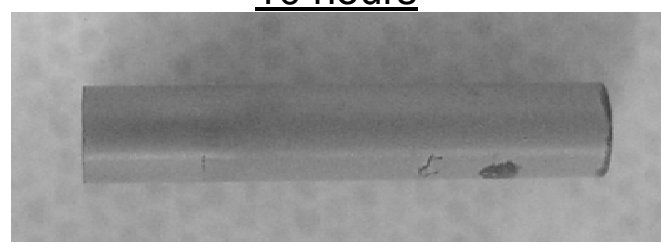

(a) A10D1

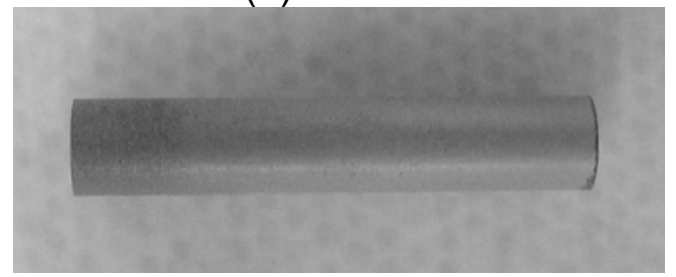

(b) A10D2

(c) A10D3

30 hours

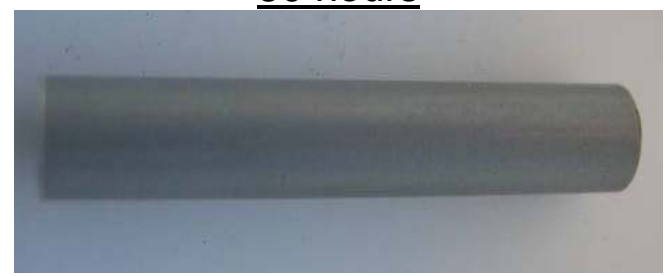

(d) A30D1

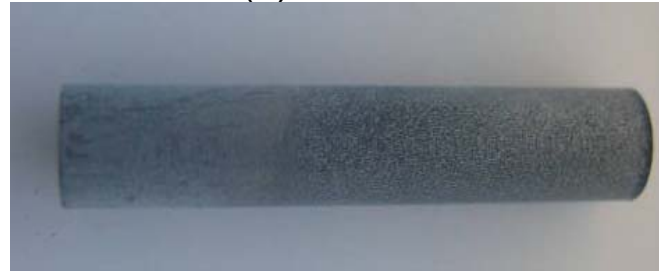

(e) A30D2

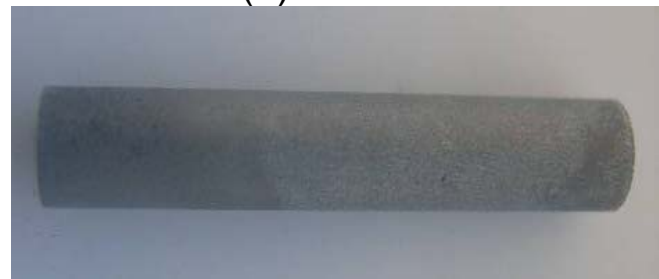

(f) A30D3
100 hours

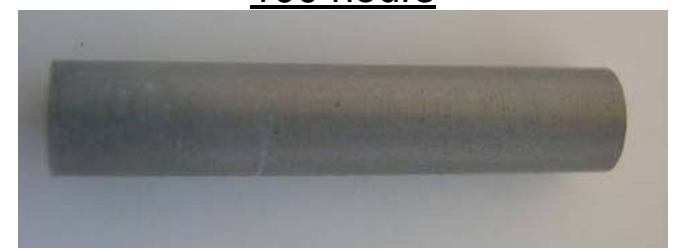

(g) A100D1

(h) A100D2

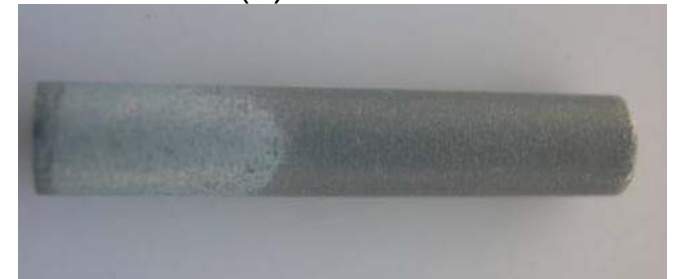

(i) A100D3

300 hours

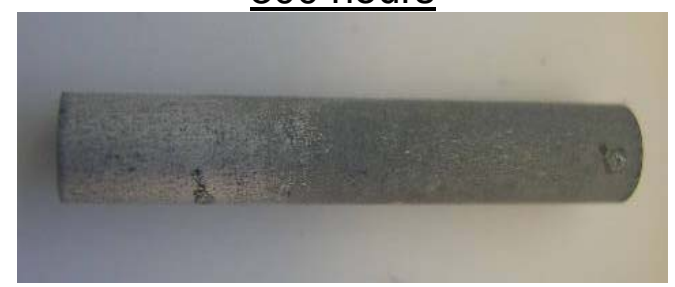

(j) A300D1

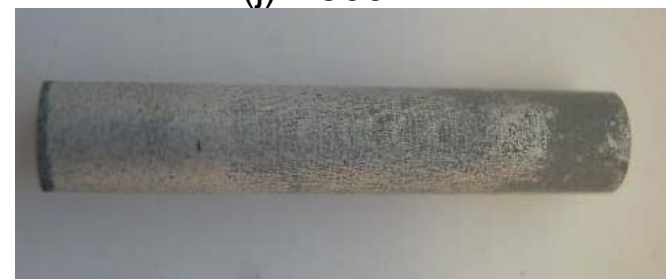

(k) A300D2

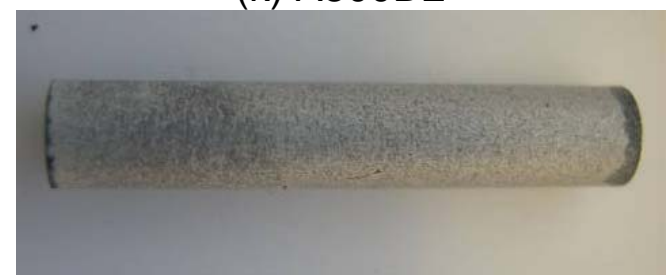

(I) A300D3

Photographs of Triplicate Specimens of Alloy N5 Exposed to Air at $1900{ }^{\circ} \mathrm{F}$ for 10, 30, 100 and 300 Hours. Note: Top of Specimens is on the Right in Each Photograph. 
10 hours

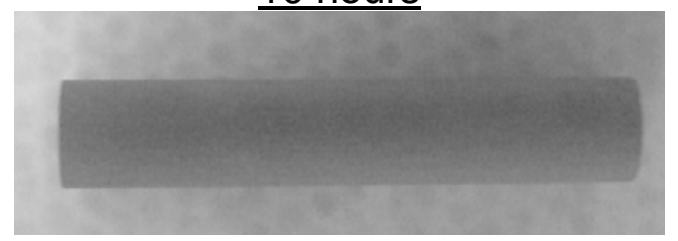

(a) A10E1

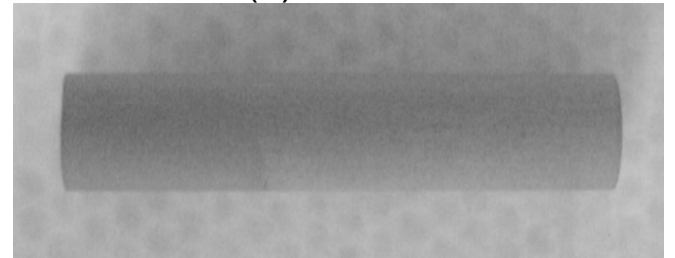

(b) A10E2

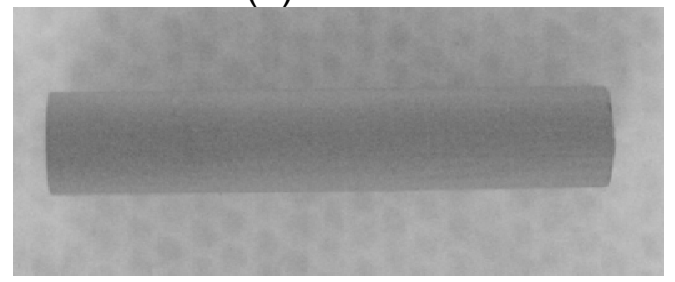

(c) A10E3

30 hours

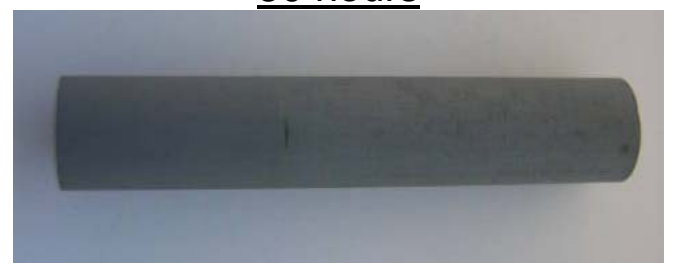

(d) A30E1

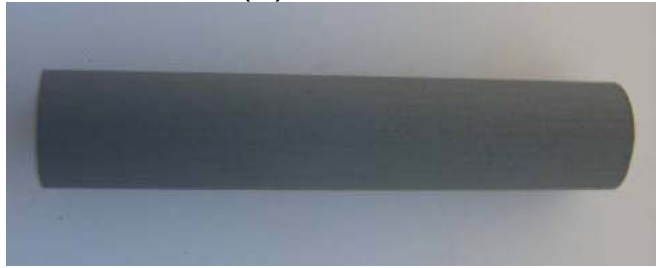

(e) A30E2

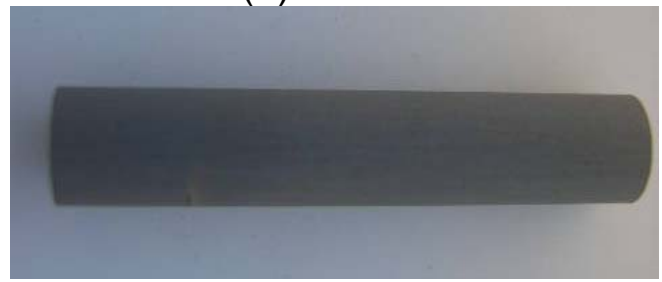

(f) A30E3
100 hours

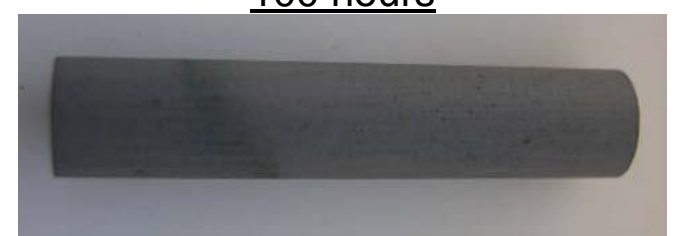

(g) A100E1

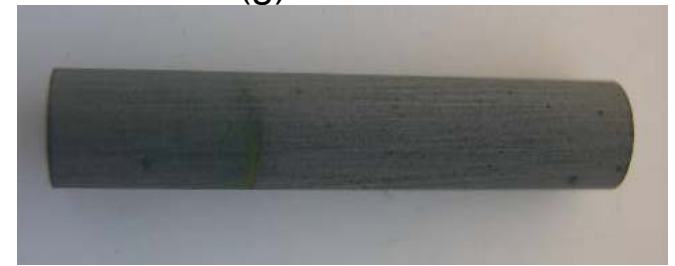

(h) A100E2

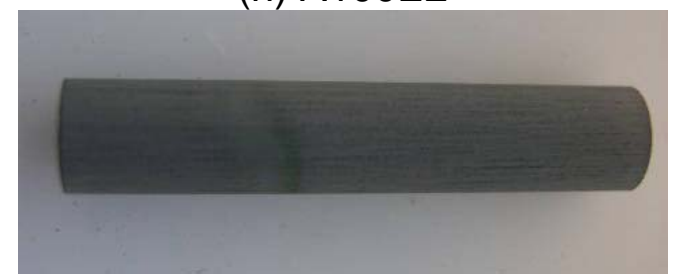

(i) A100E3

300 hours

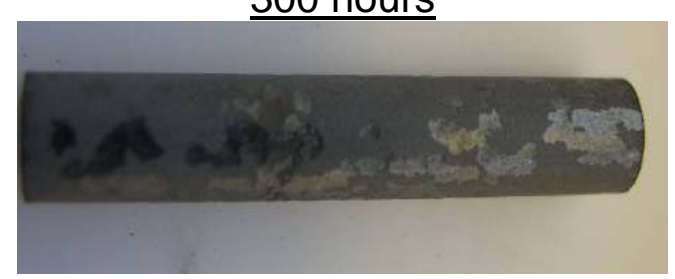

(j) A300E1

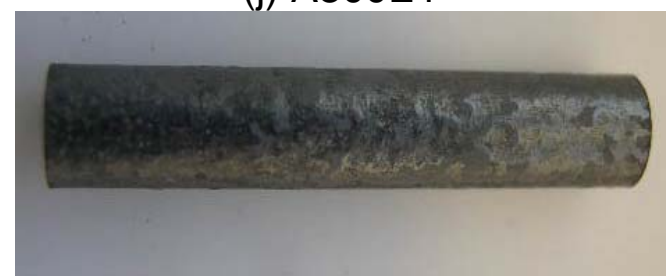

(k) A300E2

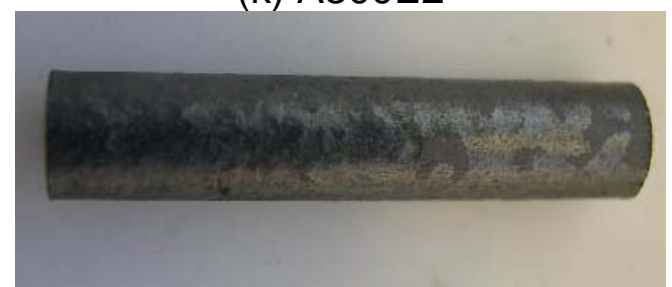

(I) A300E3

Photographs of Triplicate Specimens of Nimonic-263 Exposed to Air at $1900{ }^{\circ} \mathrm{F}$ for 10, 30, 100 and 300 Hours. Note: Top of Specimens is on the Right in Each Photograph. 
10 hours

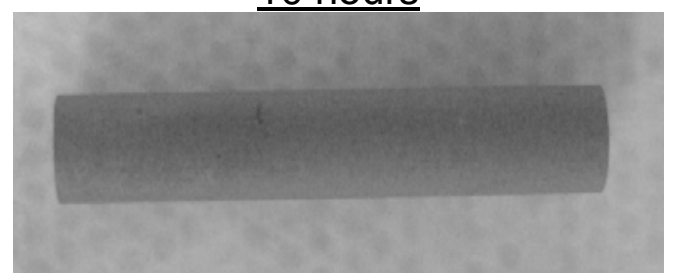

(a) A10F1

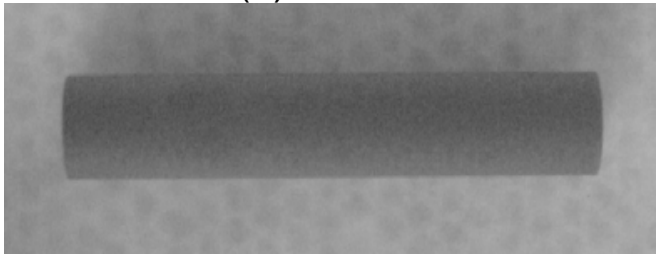

(b) A10F2

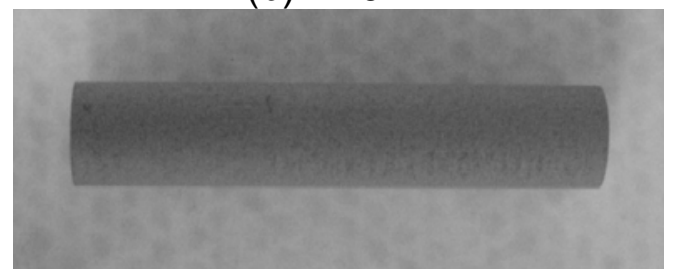

(c) $\mathrm{A} 10 \mathrm{~F} 3$

30 hours

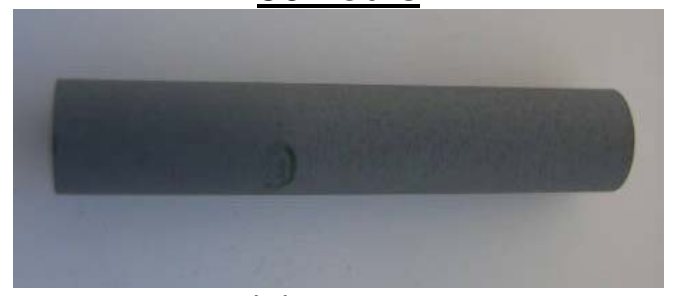

(d) A30F1

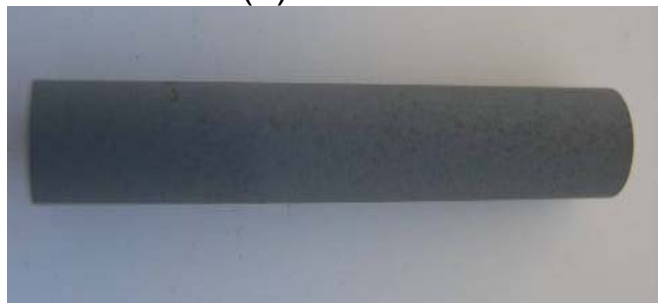

(e) A30F2

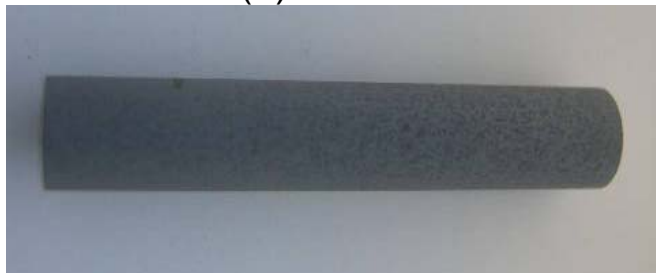

(f) A30F3
100 hours

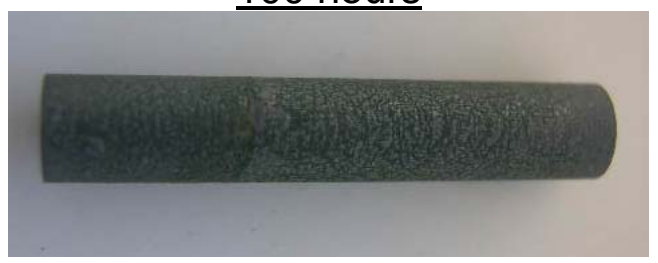

(g) A100F1

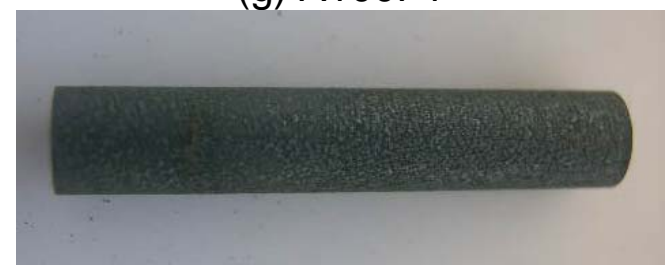

(h) A100F2

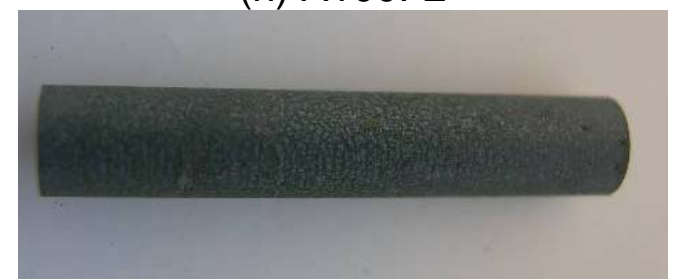

(i) $\mathrm{A} 100 \mathrm{~F} 3$

$\underline{300 \text { hours }}$

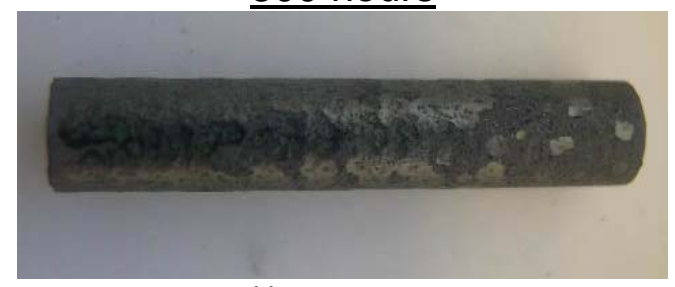

(j) $\mathrm{A} 300 \mathrm{~F} 1$

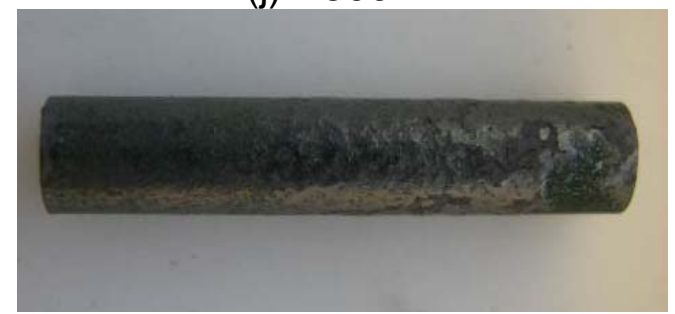

(k) A300F2

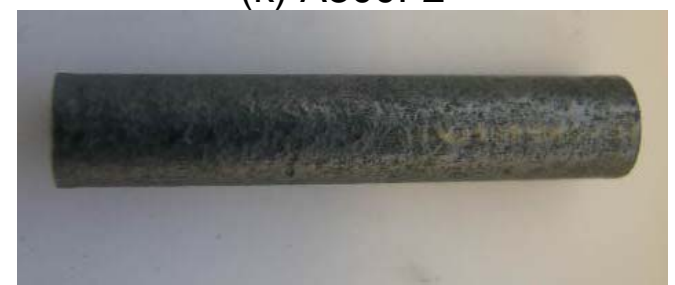

(I) A300F3

Photographs of Triplicate Specimens of GTD111 Exposed to Air at $1900{ }^{\circ} \mathrm{F}$ for 10, 30, 100 and 300 Hours. Note: Top of Specimens is on the Right in Each Photograph. 
10 hours

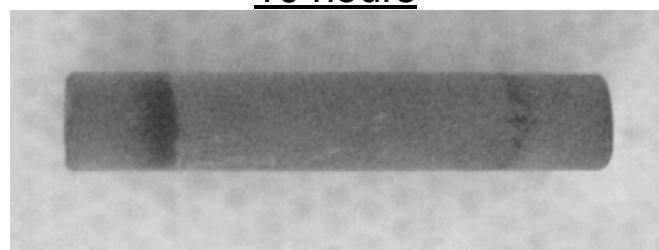

(a) A10G1

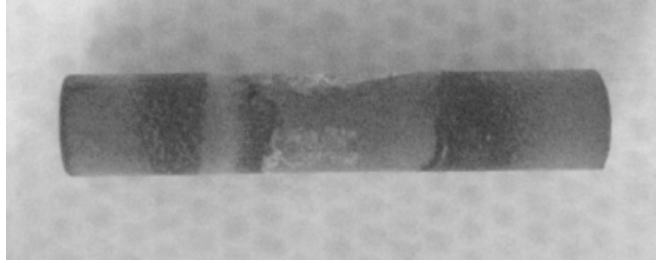

(b) A10G2

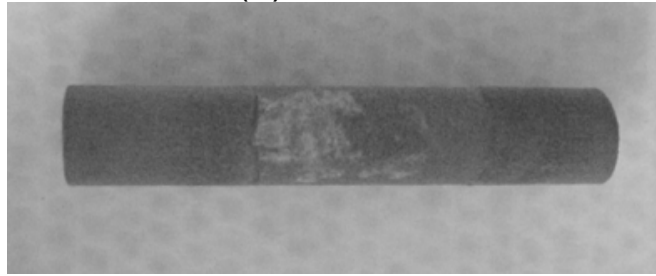

(c) A10G3

30 hours

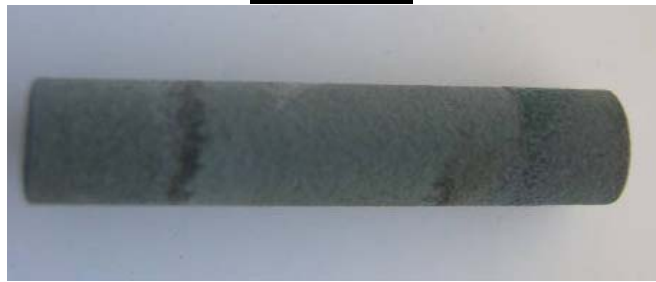

(d) A30G1

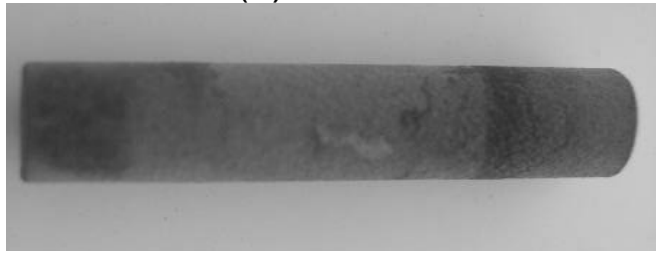

(e) A30G2

(f) A30G3
100 hours

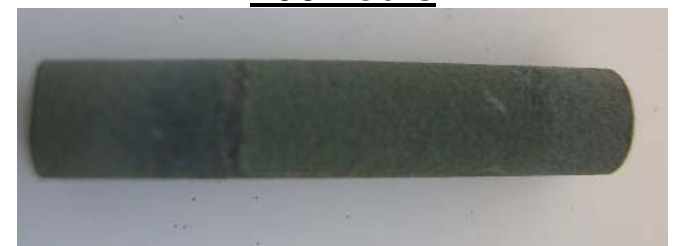

(g) A100G1

(h) A100G2

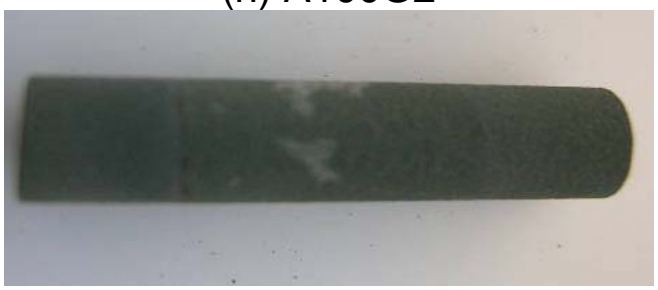

(i) A100G3

300 hours

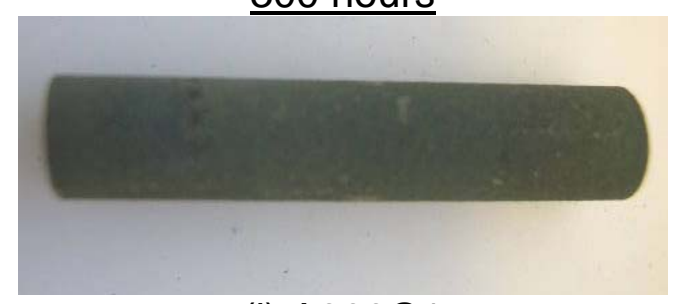

(j) A300G1

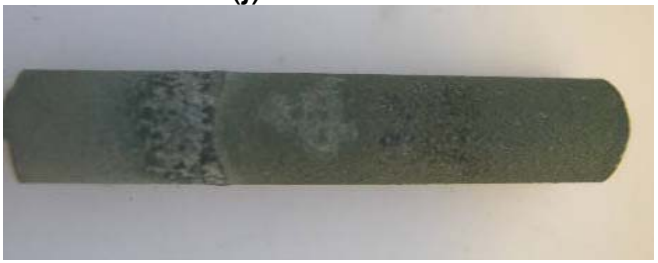

(k) A300G2

(I) A300G3

Photographs of Triplicate Specimens of Alloy N5 + NiCrAlY Coating Exposed to Air at $1900{ }^{\circ} \mathrm{F}$ for $10,30,100$ and 300 Hours. Note: Top of Specimens is on the Right in Each Photograph. 
10 hours

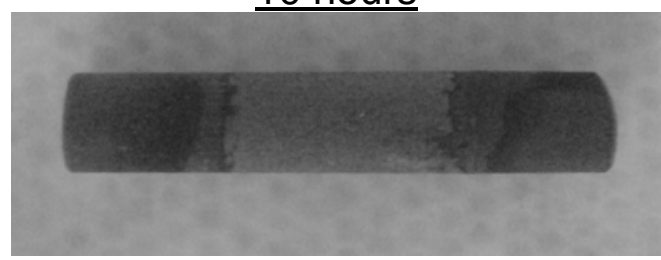

(a) $\mathrm{A} 10 \mathrm{H} 1$

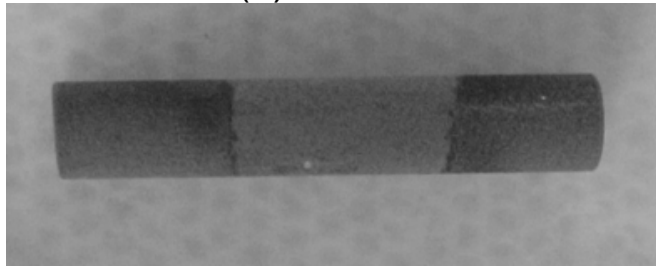

(b) $\mathrm{A} 10 \mathrm{H} 2$

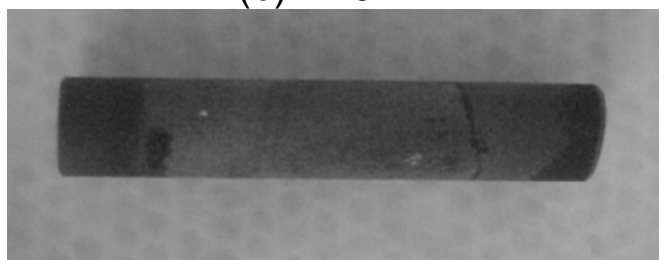

(c) $\mathrm{A} 10 \mathrm{H} 3$

30 hours

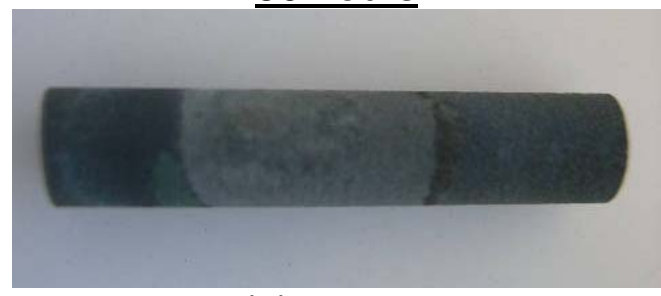

(d) $\mathrm{A} 30 \mathrm{H} 1$

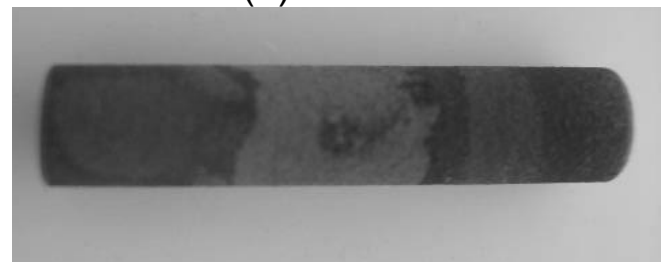

(e) $\mathrm{A} 30 \mathrm{H} 2$

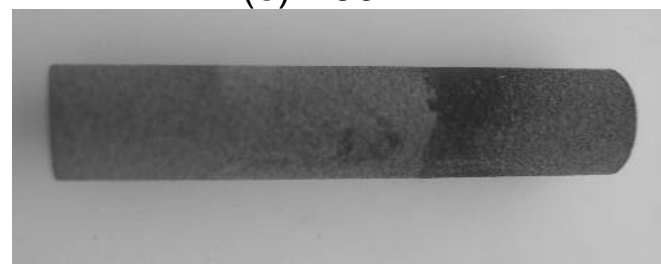

(f) $\mathrm{A} 30 \mathrm{H} 3$
100 hours

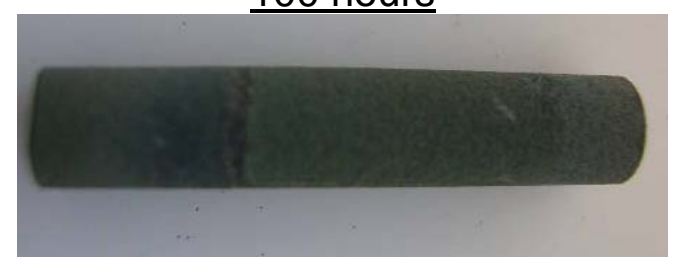

(g) A100G1

(h) A100G2

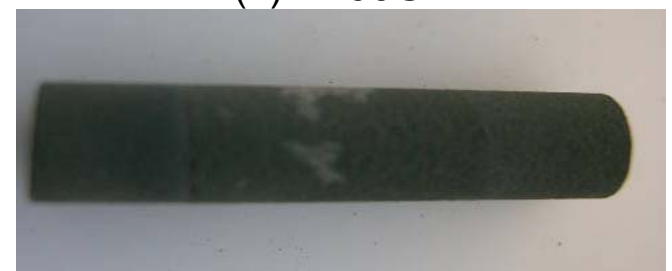

(i) A100G3

300 hours

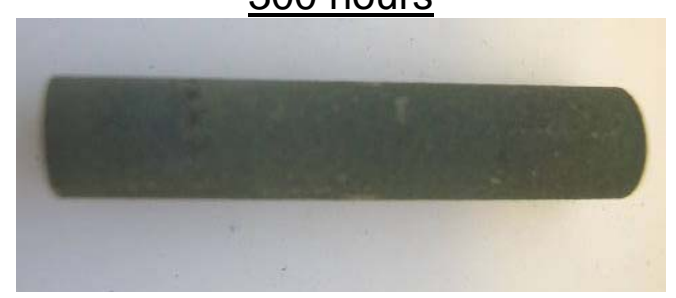

(j) A300G1

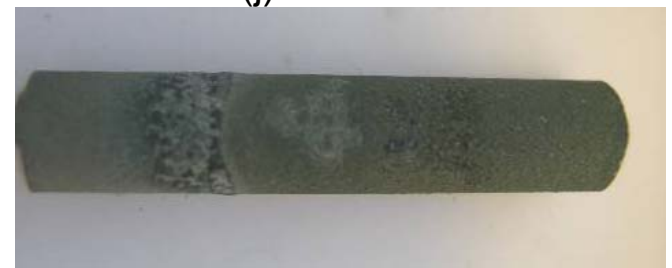

(k) A300G2

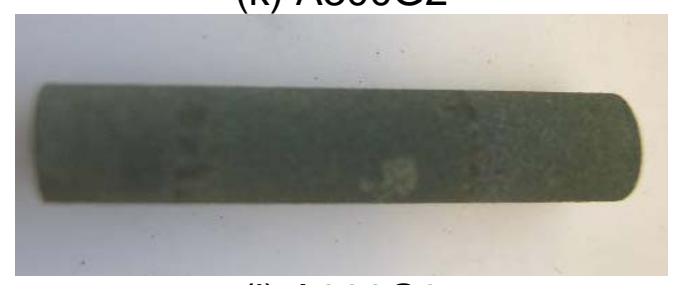

(I) A300G3

Photographs of Triplicate Specimens of Nimonic 263 + CoNiCrAlY Coating Exposed to Air at $1900{ }^{\circ} \mathrm{F}$ for 10, 30, 100 and 300 Hours. Note: Top of Specimens is on the Right in Each Photograph. 
10 hours

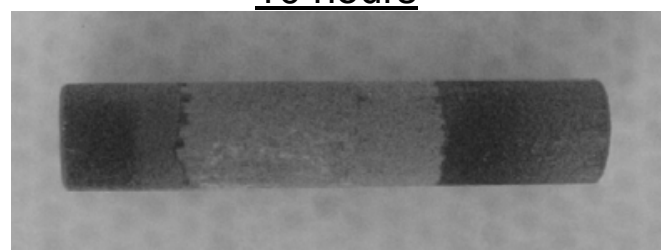

(a) A10K1

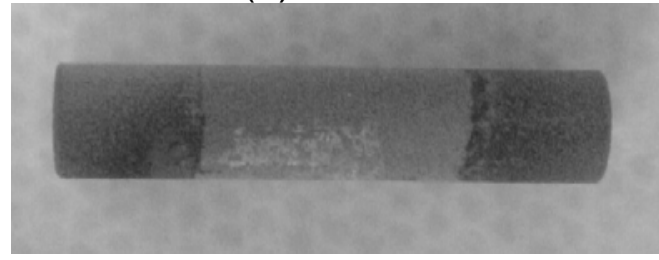

(b) A10K2

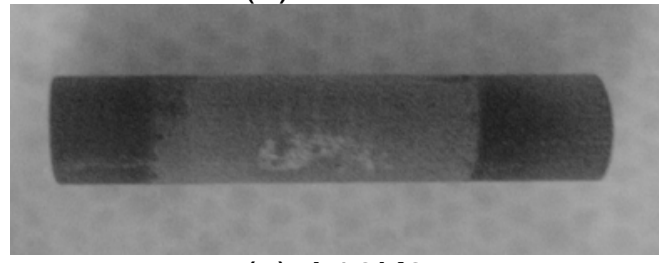

(c) $\mathrm{A} 10 \mathrm{~K} 3$

30 hours

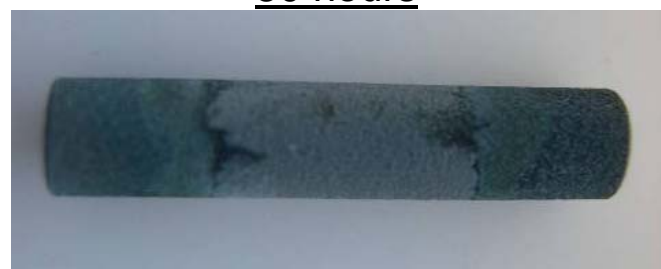

(d) A30K1

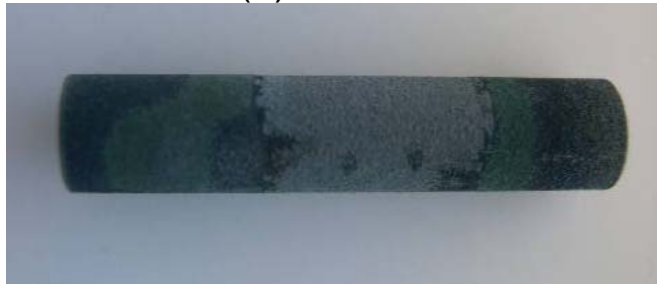

(e) A30K2

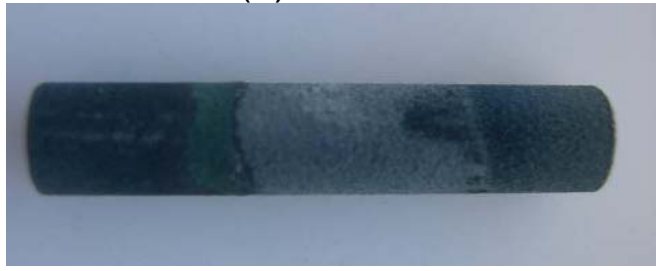

(f) $\mathrm{A} 30 \mathrm{~K} 3$
100 hours

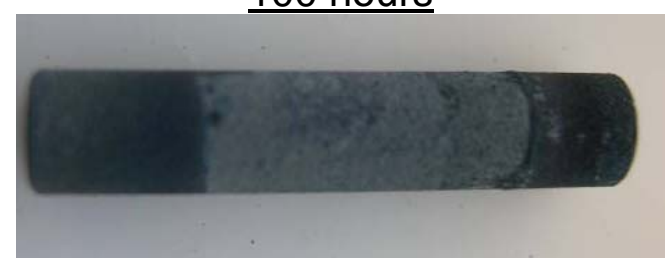

(g) A100K1

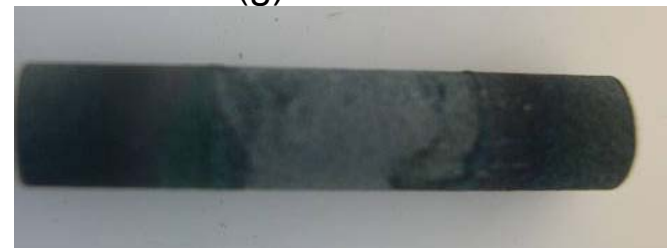

(h) A100K2

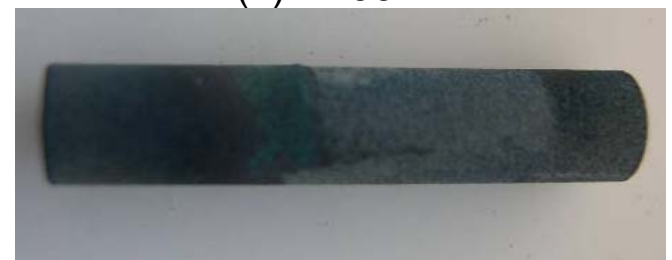

(i) $\mathrm{A} 100 \mathrm{~K} 3$

300 hours

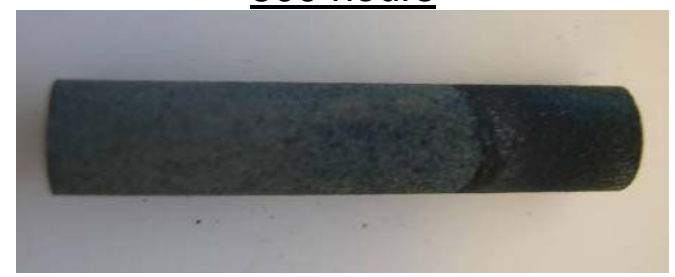

(j) $\mathrm{A} 300 \mathrm{~K} 1$

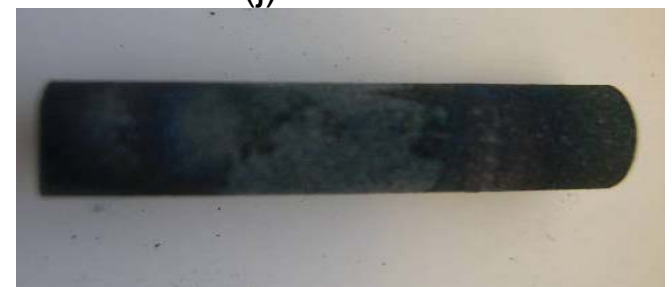

(k) A300K2

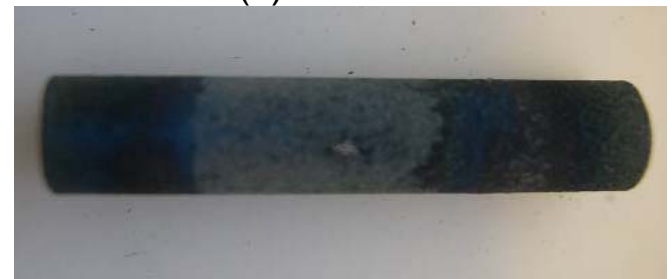

(I) $\mathrm{A} 300 \mathrm{~K} 3$

Photographs of Triplicate Specimens of GTD111 + CoNiCrAlY Coating Exposed to Air at $1900{ }^{\circ} \mathrm{F}$ for $10,30,100$ and 300 Hours. Note: Top of Specimens is on the Right in Each Photograph. 


\section{Dry $\mathrm{H}_{2} \underline{S}$}

10 hours

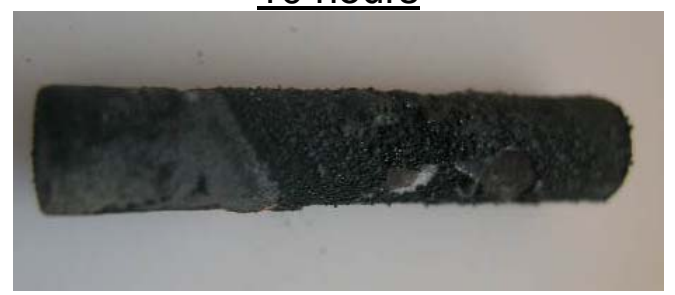

(a) H10A1

(b) $\mathrm{H} 10 \mathrm{~A} 2$

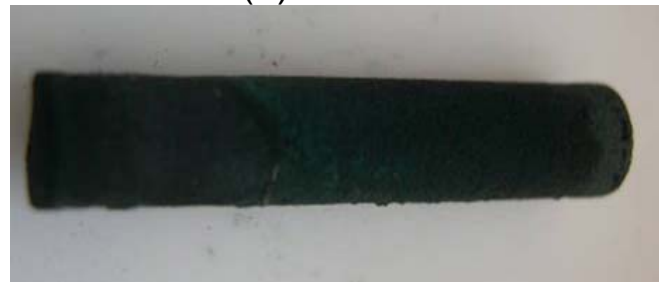

(c) $\mathrm{H} 10 \mathrm{~A} 3$

30 hours

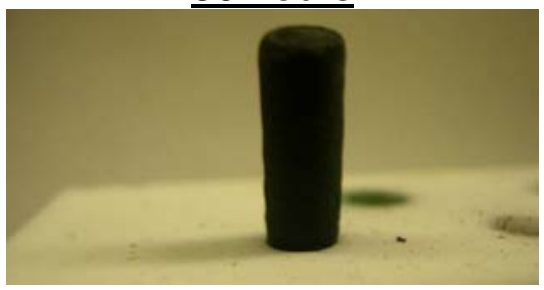

(d) H30A1

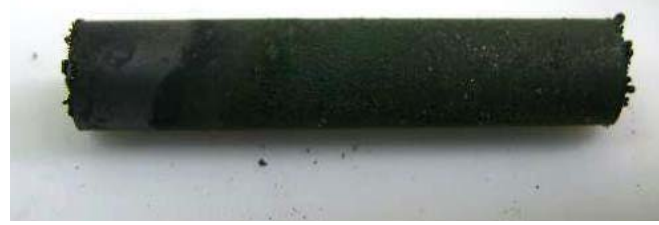

(e) $\mathrm{H} 30 \mathrm{~A} 2$

(f) $\mathrm{H} 30 \mathrm{~A} 3$

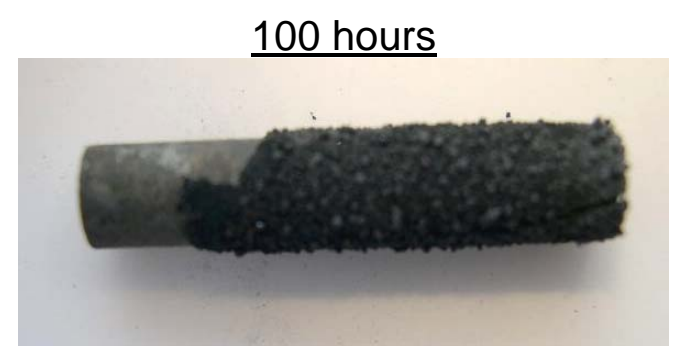

(g) H100A1

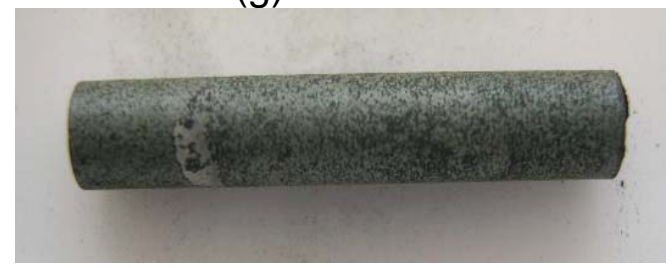

(h) $\mathrm{H} 100 \mathrm{~A} 2$

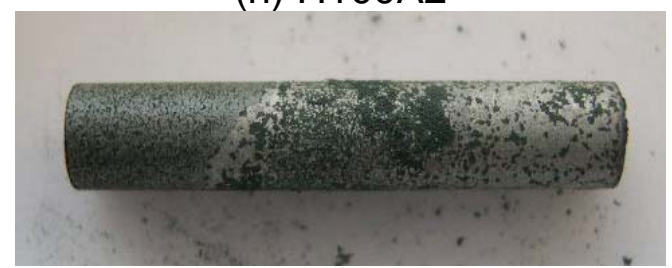

(i) $\mathrm{H} 100 \mathrm{~A} 3$

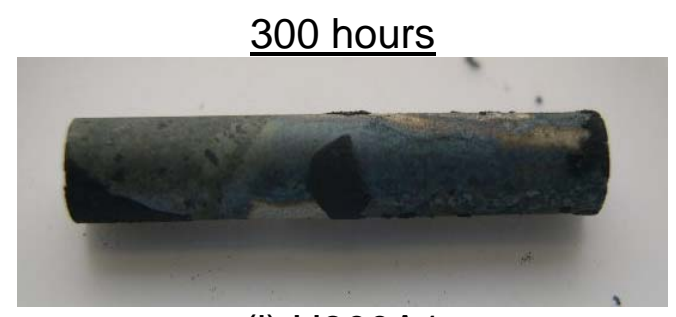

(j) $\mathrm{H} 300 \mathrm{~A} 1$

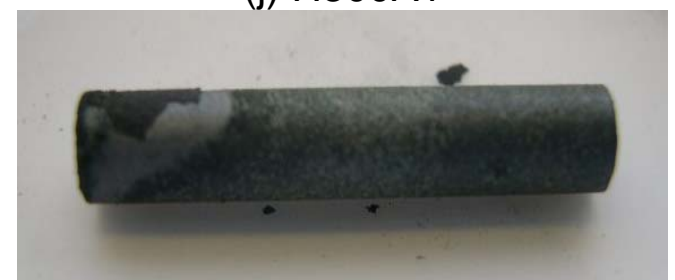

(k) H300A2

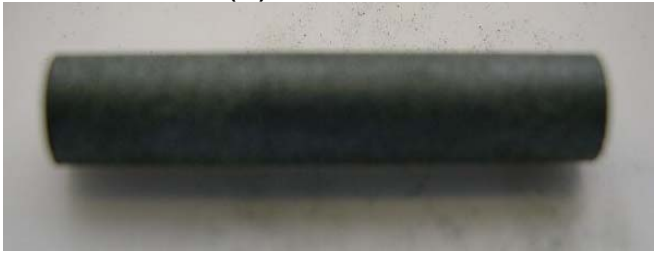

(I) $\mathrm{H} 300 \mathrm{~A} 3$

Photographs of Triplicate Specimens of FSC-414 Exposed to $100 \mathrm{ppm} \mathrm{H}_{2} \mathrm{~S}$ / Balance $\mathrm{N}_{2}$ at $1900^{\circ} \mathrm{F}$ for $10,30,100$ and 300 Hours. Note: Top of Specimens is on the Right in Each Photograph. 


\section{Dry $\mathrm{H}_{2} \underline{S}$}

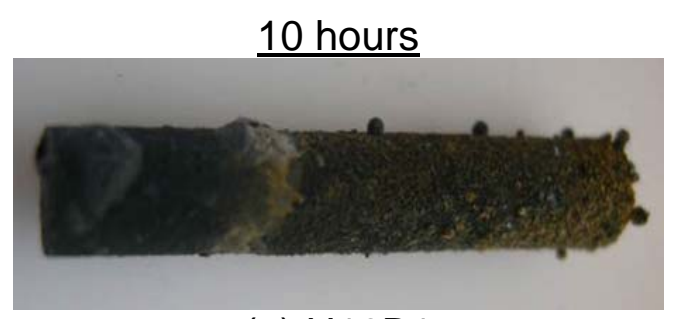

(a) H10B1

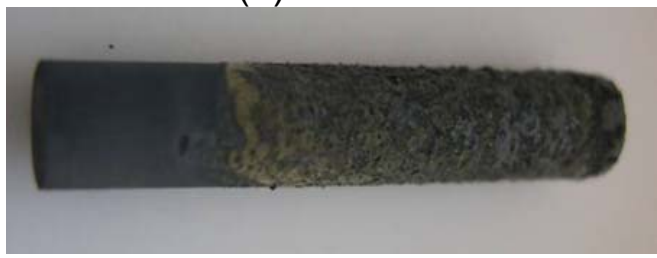

(b) H10B2

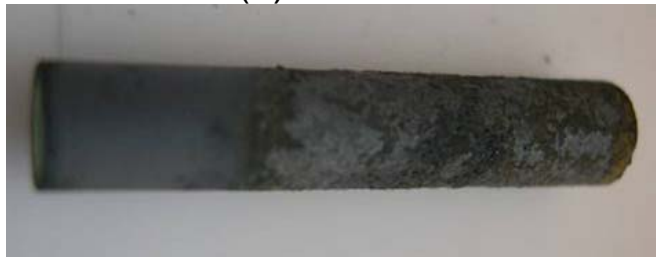

(c) $\mathrm{H} 10 \mathrm{~B} 3$

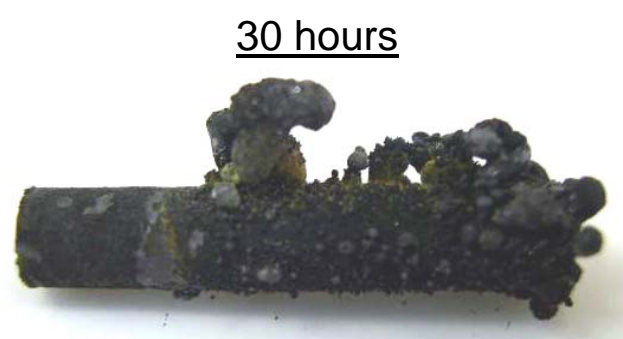

(d) H30B1

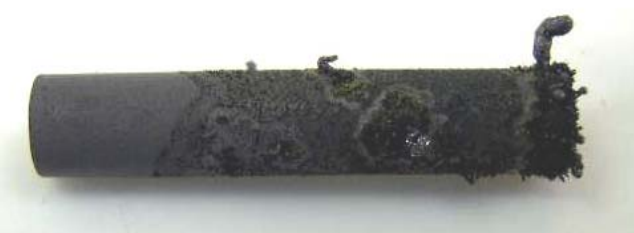

(e) H30B2

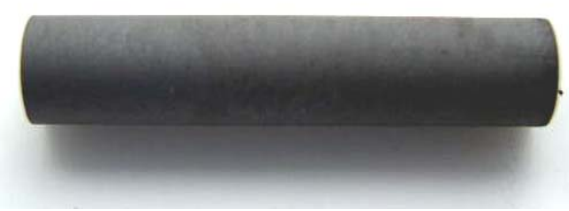

(f) H30B3
100 hours

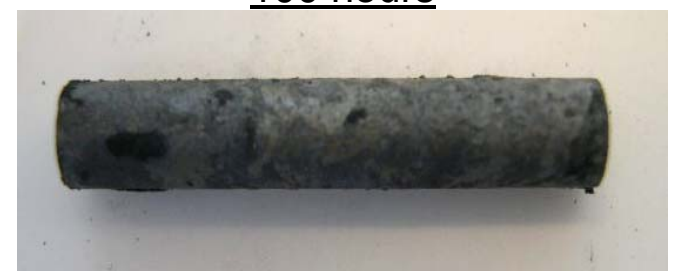

(g) H100B1

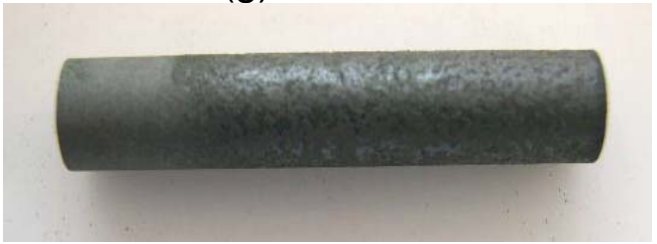

(h) H100B2

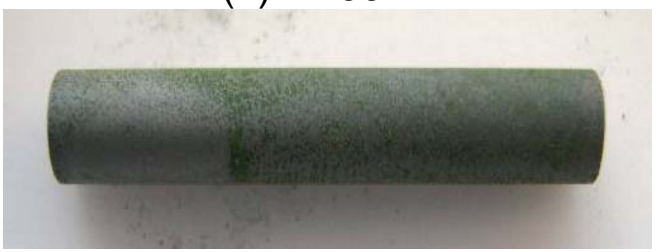

(i) $\mathrm{H} 100 \mathrm{~B} 3$

300 hours

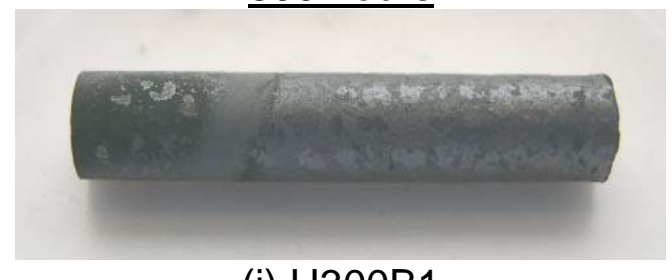

(j) $\mathrm{H} 300 \mathrm{~B} 1$

(k) H300B2

(I) H300B3

Photographs of Triplicate Specimens of GTD222 Exposed to $100 \mathrm{ppm} \mathrm{H}_{2} \mathrm{~S}$ / Balance $\mathrm{N}_{2}$ at $1900^{\circ} \mathrm{F}$ for $10,30,100$ and 300 Hours. Note: Top of Specimens is on the Right in Each Photograph. 


\section{Dry $\mathrm{H}_{2} \underline{S}$}

10 hours

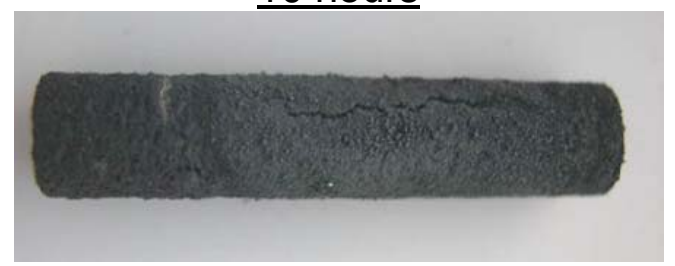

(a) $\mathrm{H} 10 \mathrm{C} 1$

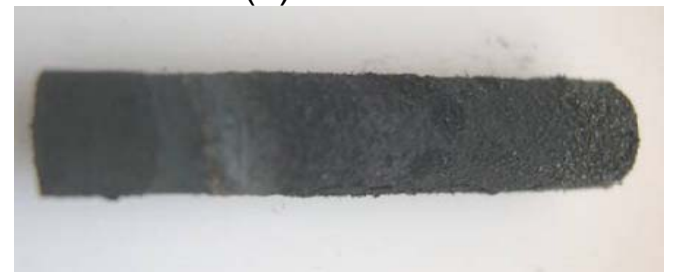

(b) $\mathrm{H} 10 \mathrm{C} 2$

(c) $\mathrm{H} 10 \mathrm{C} 3$

30 hours

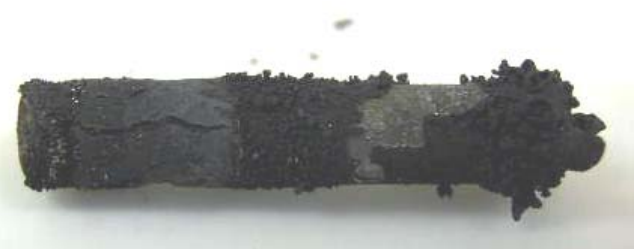

(d) $\mathrm{H} 30 \mathrm{C} 1$

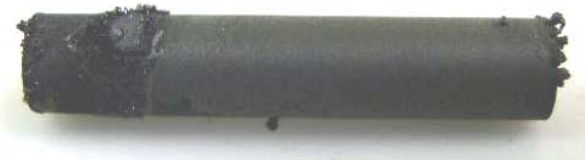

(e) $\mathrm{H} 30 \mathrm{C} 2$

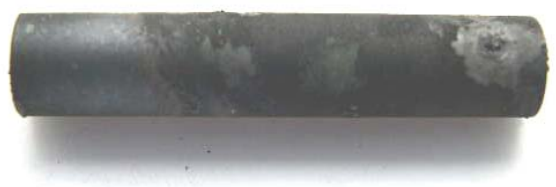

(f) $\mathrm{H} 30 \mathrm{C} 3$
100 hours

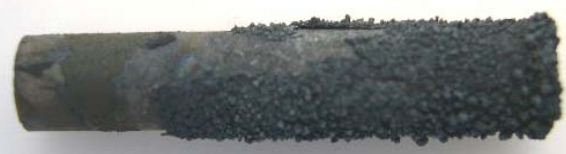

(g) $\mathrm{H} 100 \mathrm{Cl}$

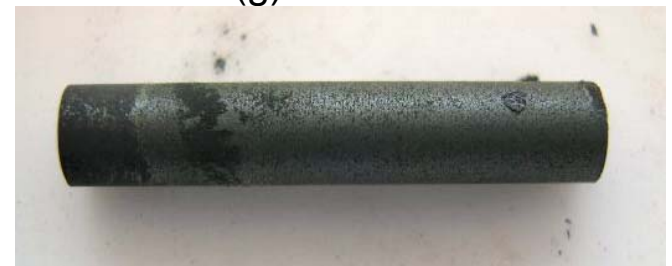

(h) $\mathrm{H} 100 \mathrm{C} 2$

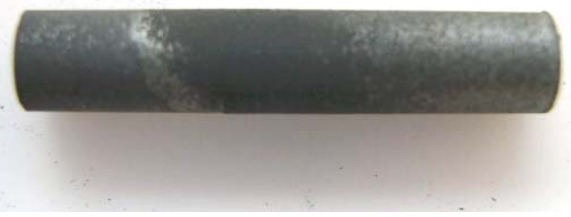

(i) $\mathrm{H} 100 \mathrm{C} 3$

$\underline{300 \text { hours }}$

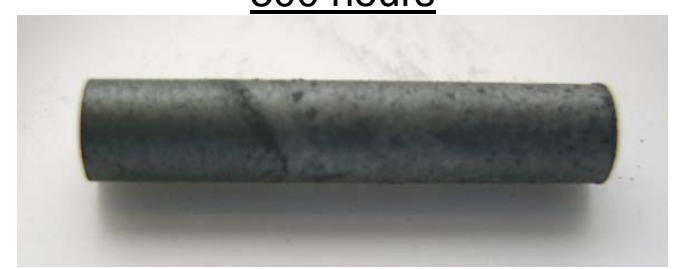

(j) $\mathrm{H} 300 \mathrm{C} 1$

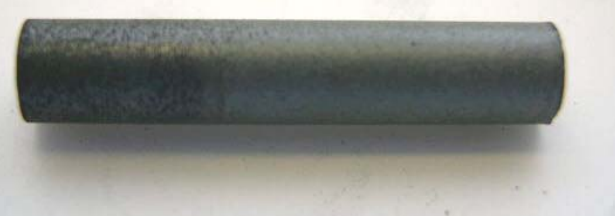

(k) $\mathrm{H} 300 \mathrm{C} 2$

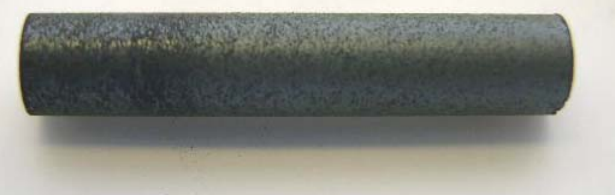

(I) $\mathrm{H} 300 \mathrm{C} 3$

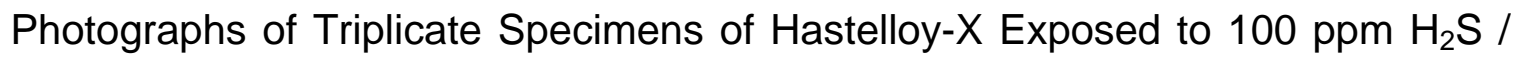
Balance $\mathrm{N}_{2}$ at $1900^{\circ} \mathrm{F}$ for $10,30,100$ and 300 Hours. Note: Top of Specimens is on the Right in Each Photograph. 


\section{Dry $\mathrm{H}_{2} \underline{S}$}

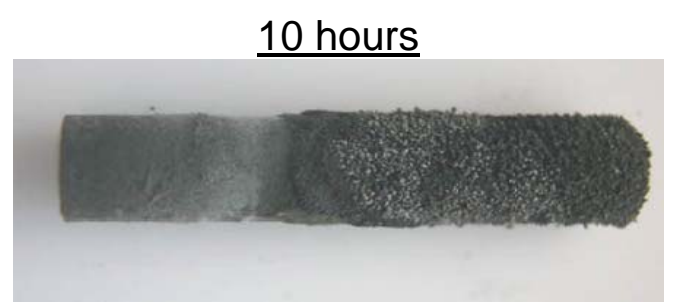

(a) H10D1

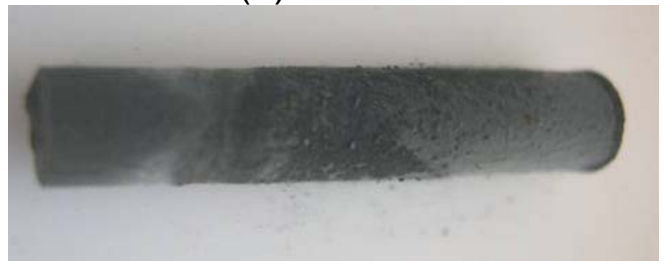

(b) H10D2

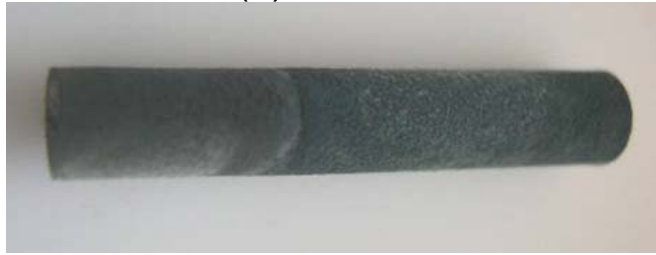

(c) H10D3

$\underline{30 \text { hours }}$

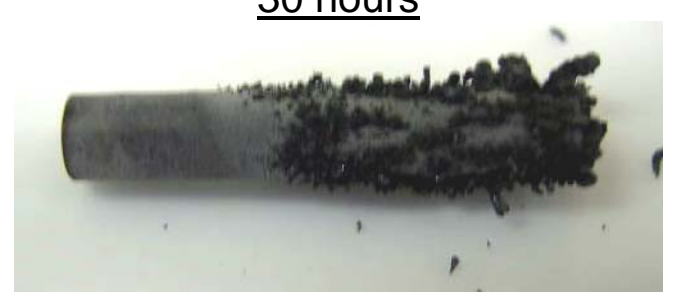

(d) H30D1

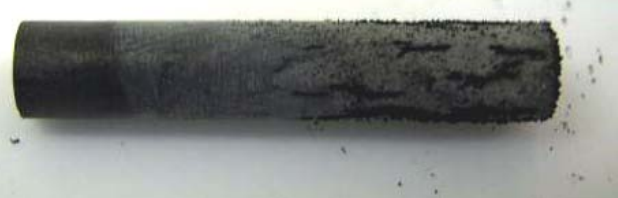

(e) H30D2

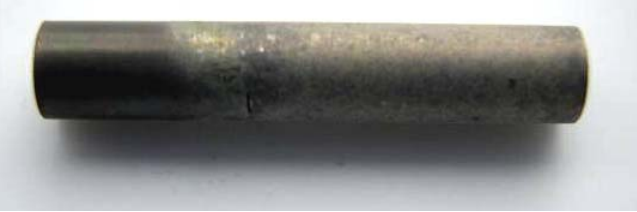

(f) H30D3
100 hours

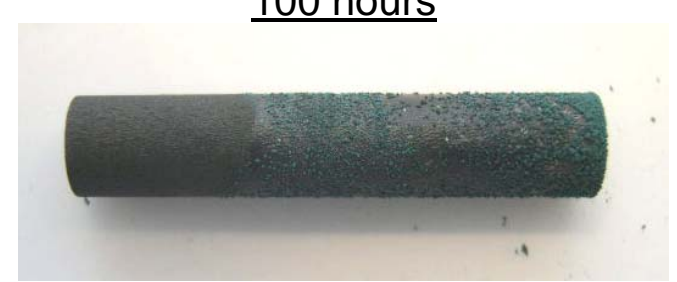

(g) H100D1

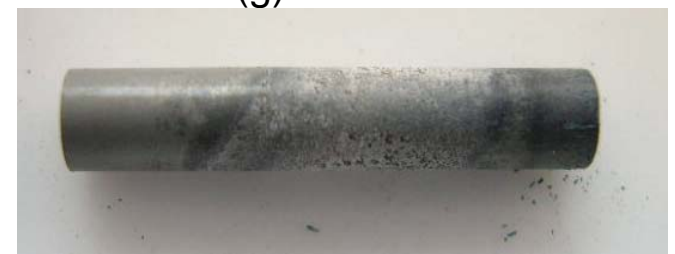

(h) H100D2

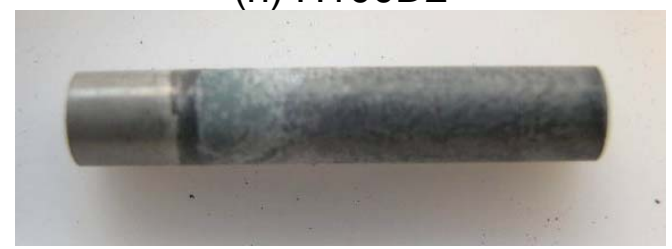

(i) H100D3

300 hours

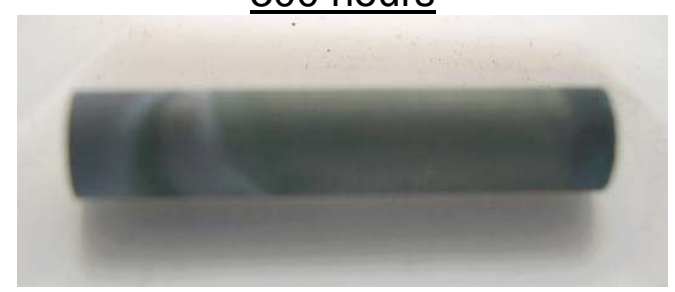

(j) H300D1

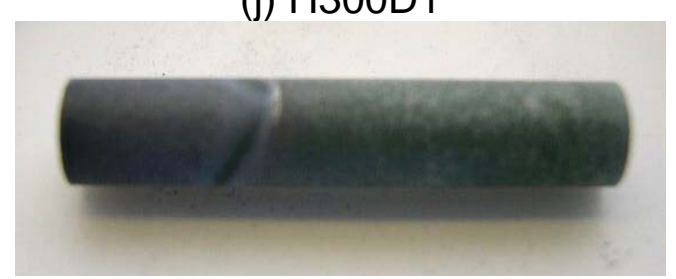

(k) H300D2

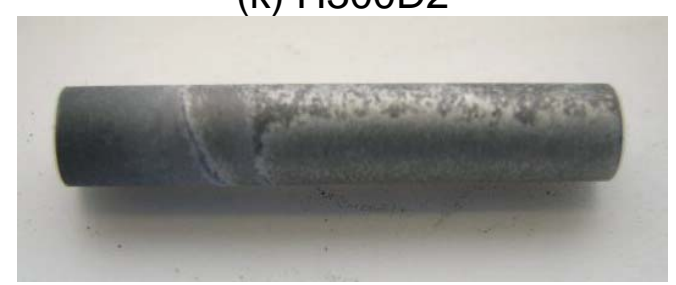

(I) H300D3

Photographs of Triplicate Specimens of Alloy N5 Exposed to $100 \mathrm{ppm}_{2} \mathrm{~S}$ / Balance $\mathrm{N}_{2}$ at $1900^{\circ} \mathrm{F}$ for $10,30,100$ and 300 Hours. Note: Top of Specimens is on the Right in Each Photograph. 


\section{Dry $\mathrm{H}_{2} \underline{S}$}

10 hours

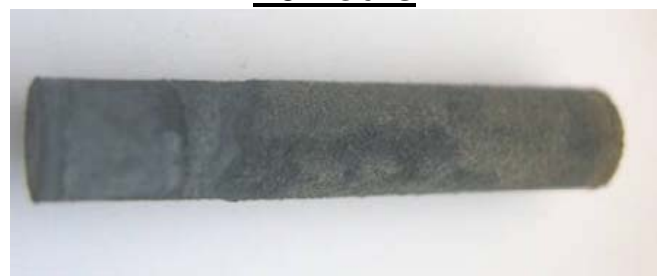

(a) H10E1

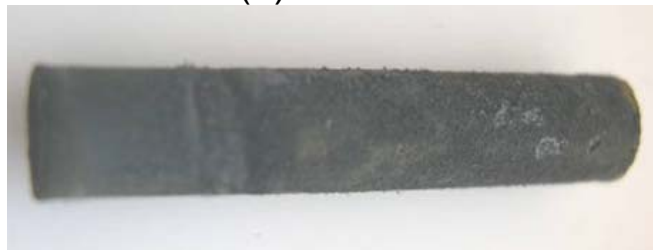

(b) H10E2

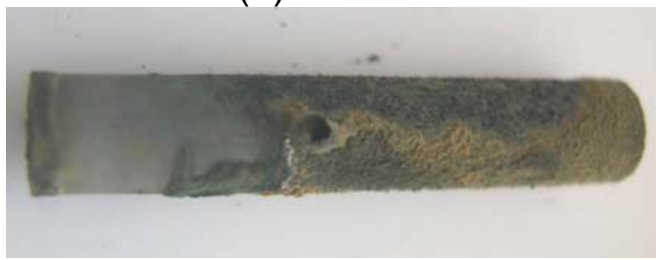

(c) H10E3

$\underline{30 \text { hours }}$

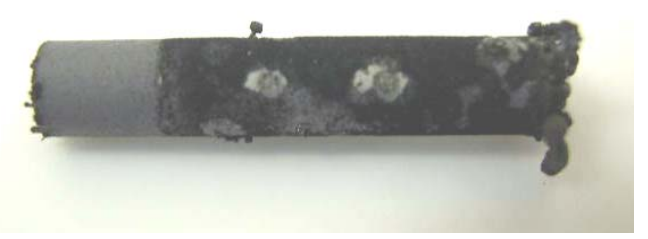

(d) H30E1

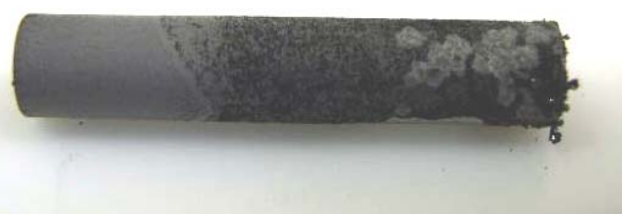

(e) H30E2

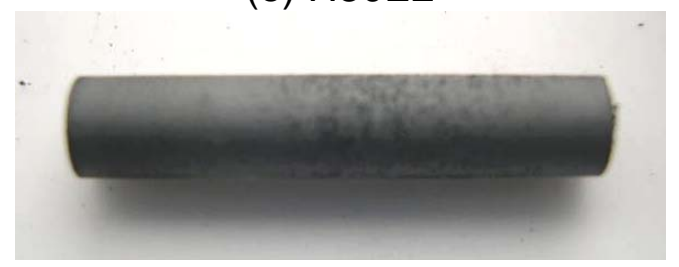

(f) H30E3
100 hours

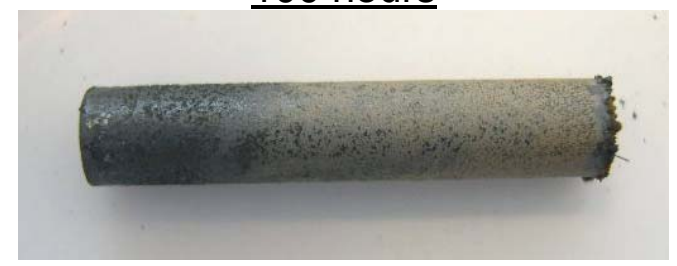

(g) H100E1

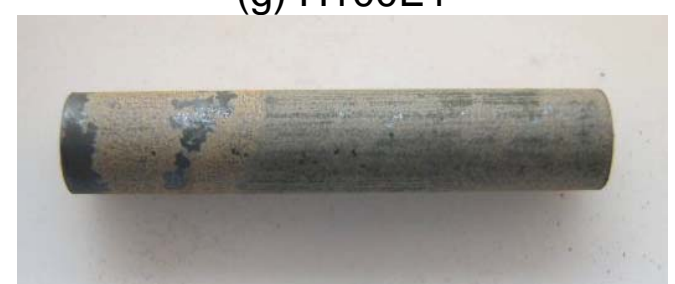

(h) H100E2

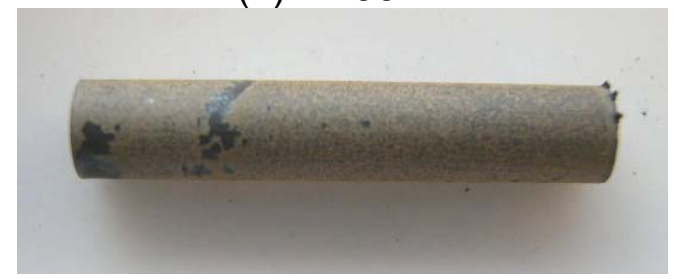

(i) H100E3

$\underline{300 \text { hours }}$

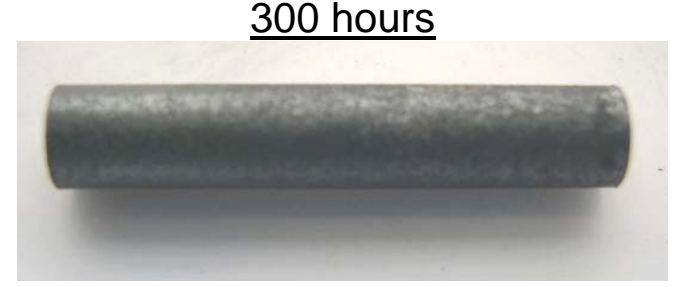

(j) H300E1

(k) H300E2

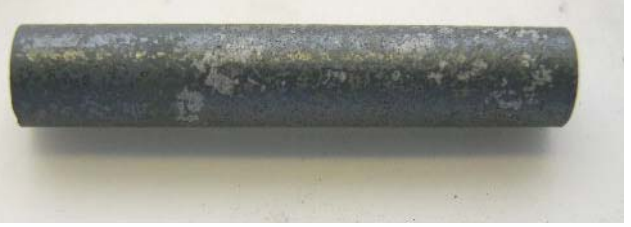

(I) H300E3

Photographs of Triplicate Specimens of Nimonic 263 Exposed to $100 \mathrm{ppm} \mathrm{H}_{2} \mathrm{~S}$ / Balance $\mathrm{N}_{2}$ at $1900^{\circ} \mathrm{F}$ for $10,30,100$ and 300 Hours. Note: Top of Specimens is on the Right in Each Photograph. 


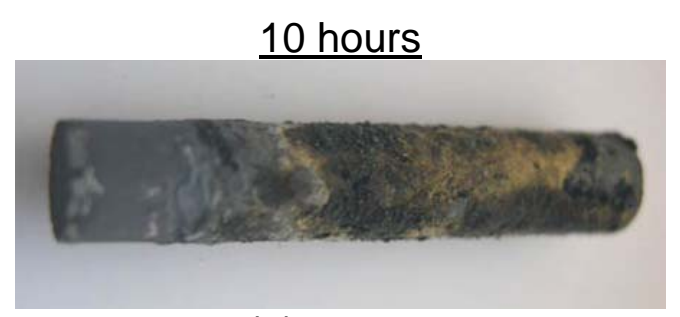

(a) H10F1

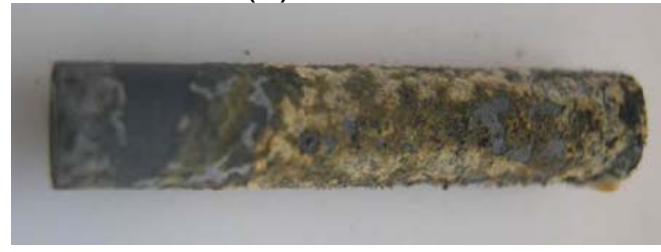

(b) H10F2

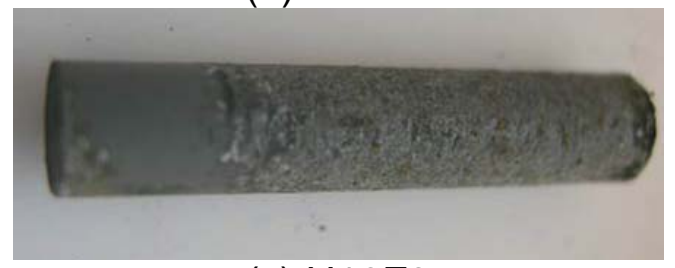

(c) $\mathrm{H} 10 \mathrm{~F} 3$

30 hours

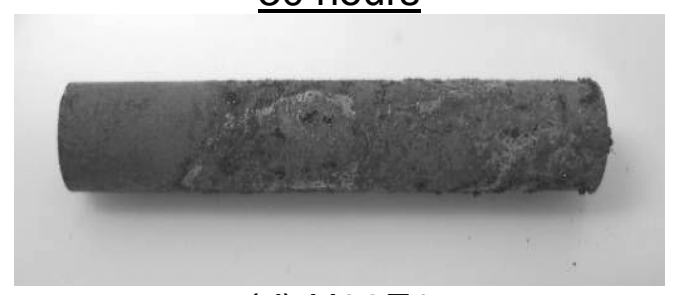

(d) H30F1

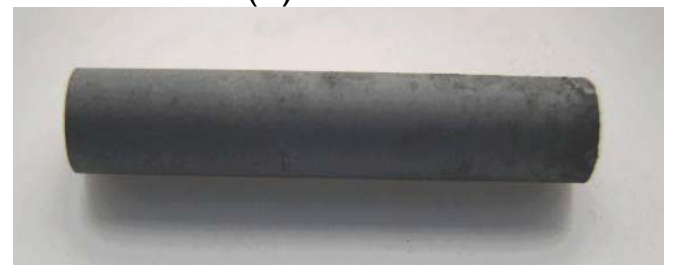

(e) H30F2

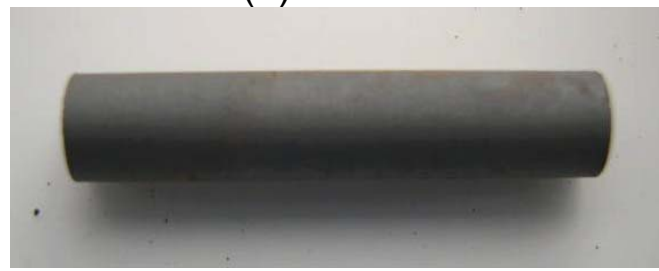

(f) H3OF3

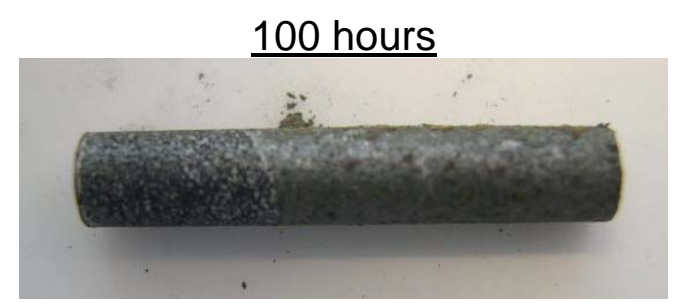

(g) H100F1

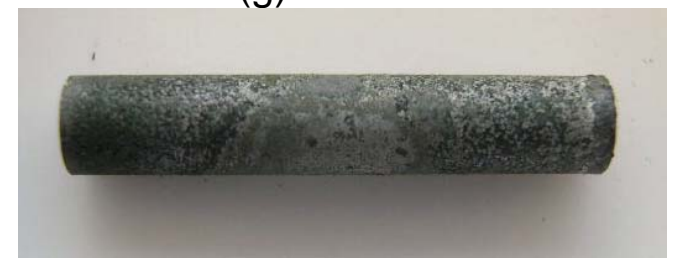

(h) H100F2

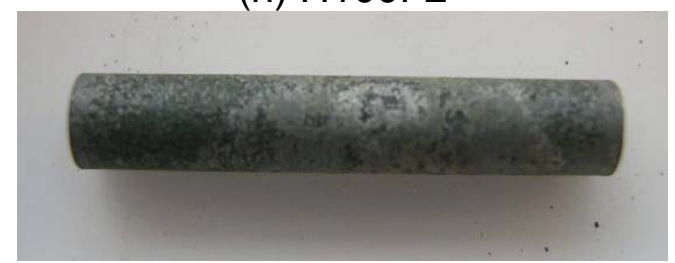

(i) H100F3

300 hours

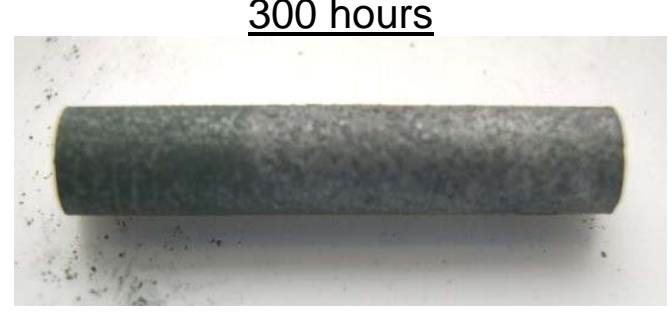

(j) H300F1

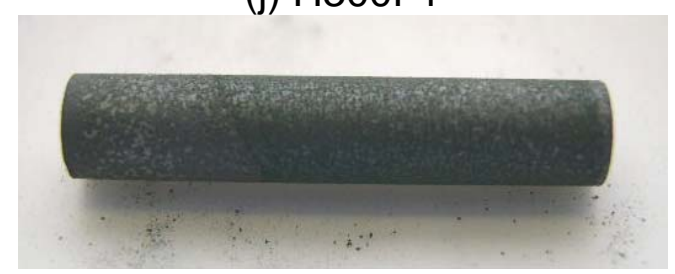

(k) H30OF2

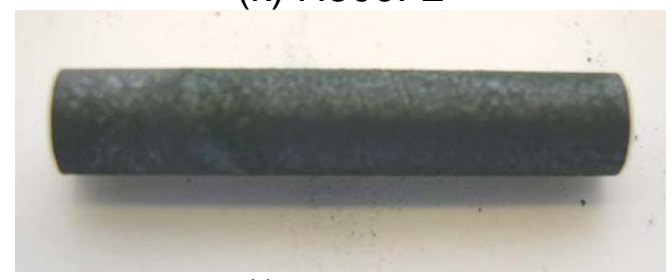

(I) H300F3

Photographs of Triplicate Specimens of GTD111 Exposed to $100 \mathrm{ppm} \mathrm{H}_{2} \mathrm{~S}$ / Balance $\mathrm{N}_{2}$ at $1900^{\circ} \mathrm{F}$ for $10,30,100$ and 300 Hours. Note: Top of Specimens is on the Right in Each Photograph. 


\section{Dry $\mathrm{H}_{2} \underline{S}$}

10 hours

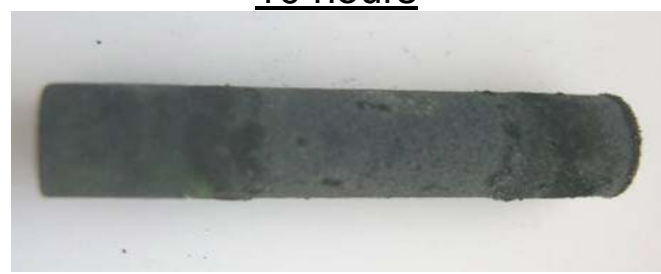

(a) H10G1

(b) H10G2

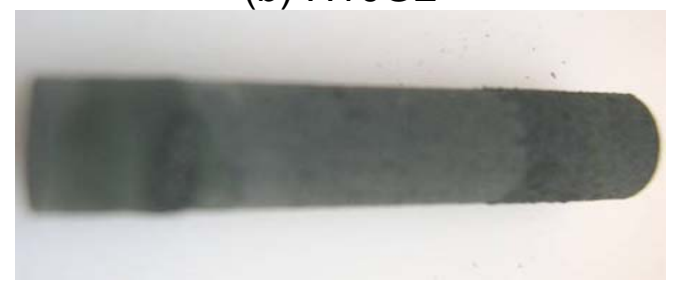

(c) H10G3

30 hours

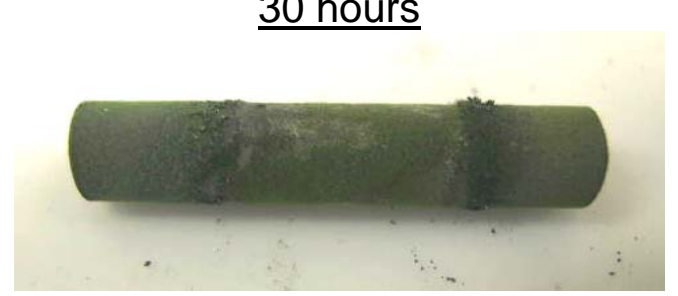

(d) H30G1

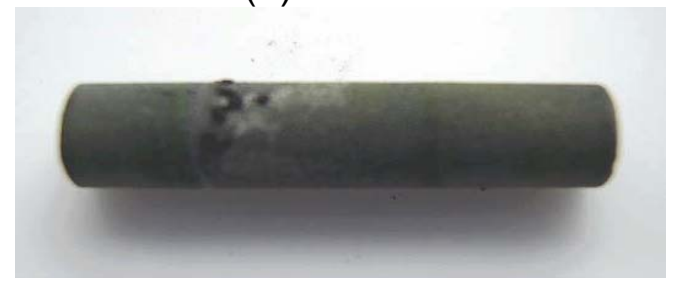

(e) H30G2

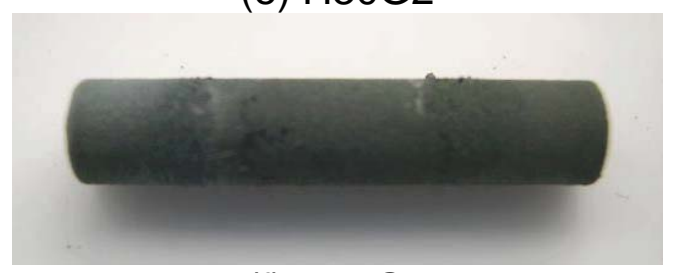

(f) H30G3
100 hours

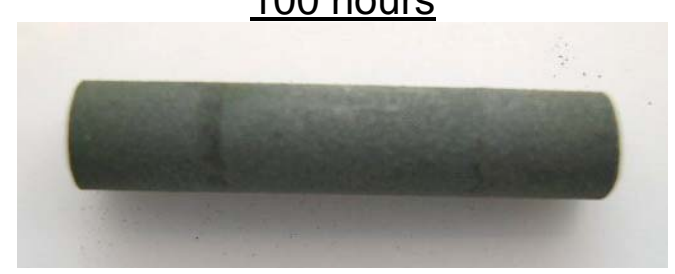

(g) H100G1

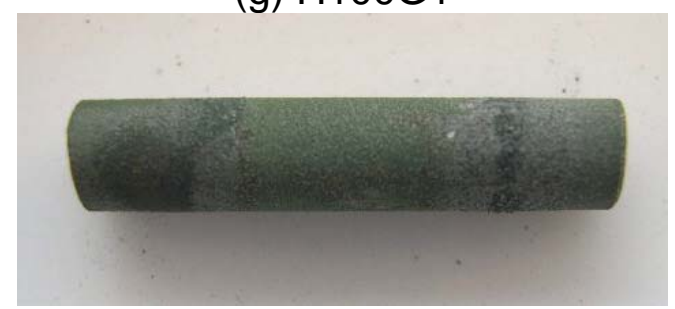

(h) H100G2

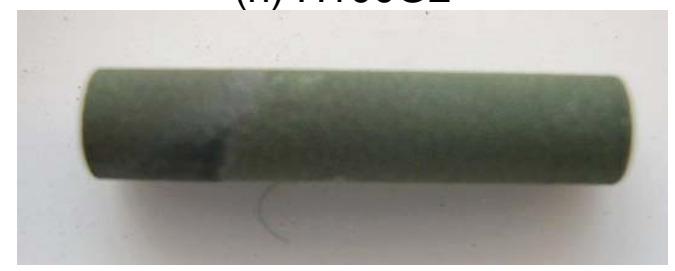

(i) H100G3

300 hours

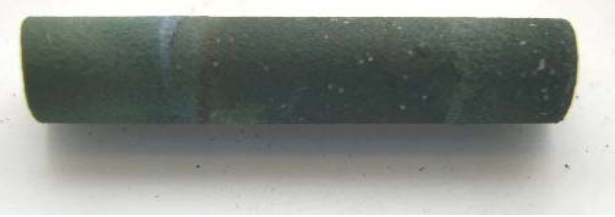

(j) H300G1

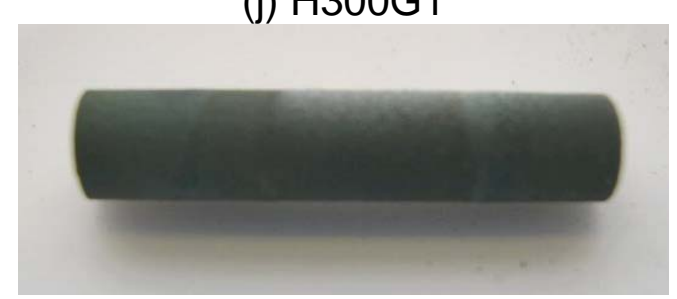

(k) H300G2

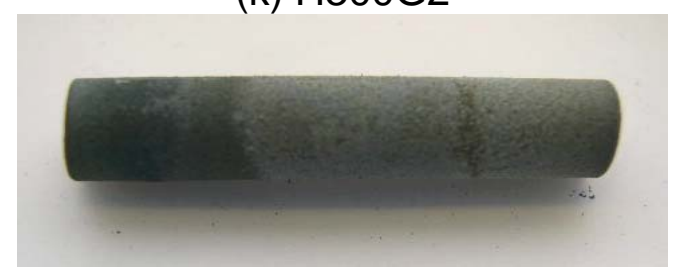

(I) H300G3

Photographs of Triplicate Specimens of Alloy N5 + NiCrAlY Coating Exposed to $100 \mathrm{ppm} \mathrm{H}_{2} \mathrm{~S} /$ Balance $\mathrm{N}_{2}$ at $1900{ }^{\circ} \mathrm{F}$ for 10, 30, 100 and 300 Hours. Note: Top of Specimens is on the Right in Each Photograph. 


\section{Dry $\mathrm{H}_{2} \underline{S}$}

10 hours

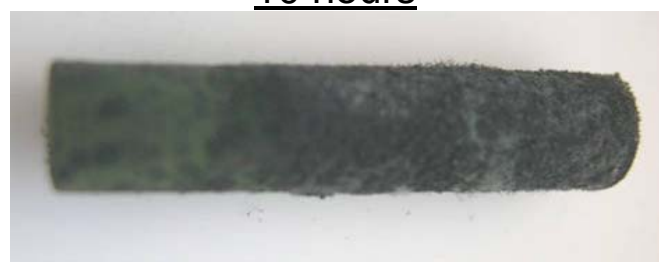

(a) $\mathrm{H} 10 \mathrm{H} 1$

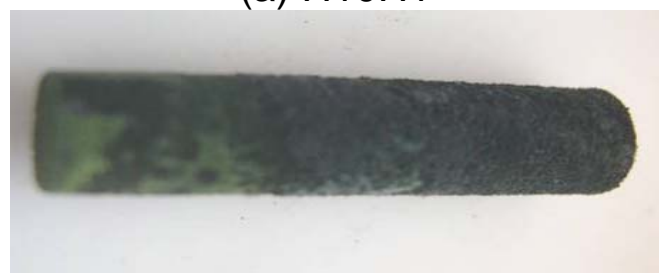

(b) $\mathrm{H} 10 \mathrm{H} 2$

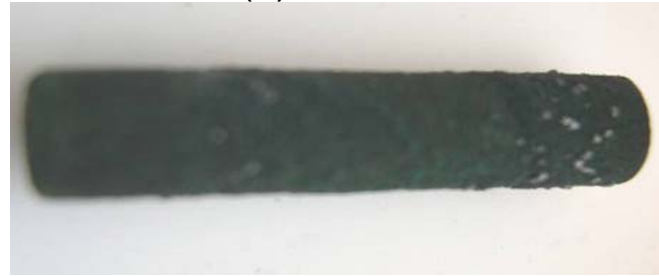

(c) $\mathrm{H} 10 \mathrm{H} 3$

30 hours

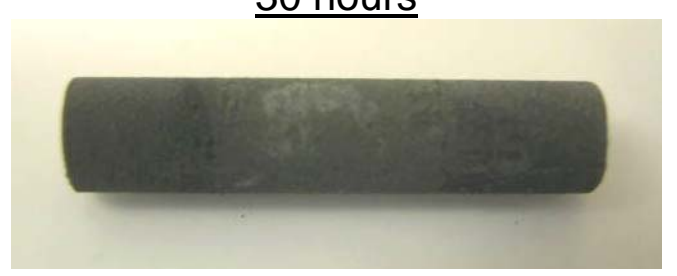

(d) $\mathrm{H} 30 \mathrm{H} 1$

(e) $\mathrm{H} 30 \mathrm{H} 2$

(f) $\mathrm{H} 3 \mathrm{OH} 3$
100 hours

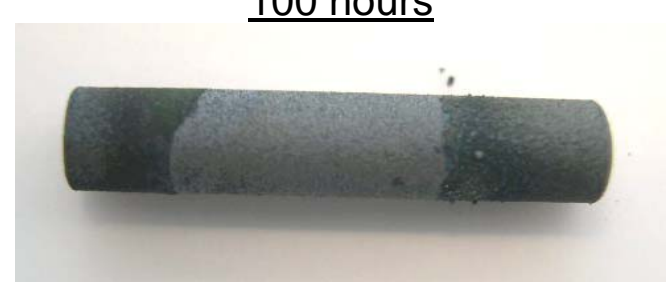

(g) $\mathrm{H} 100 \mathrm{H} 1$

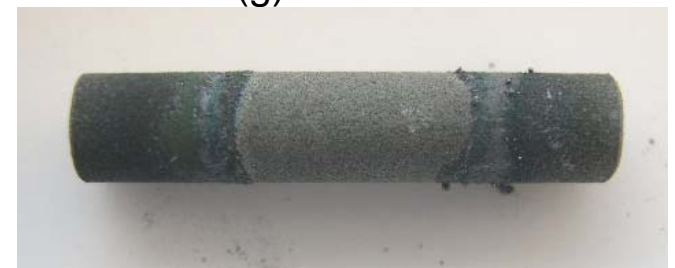

(h) $\mathrm{H} 100 \mathrm{H} 2$

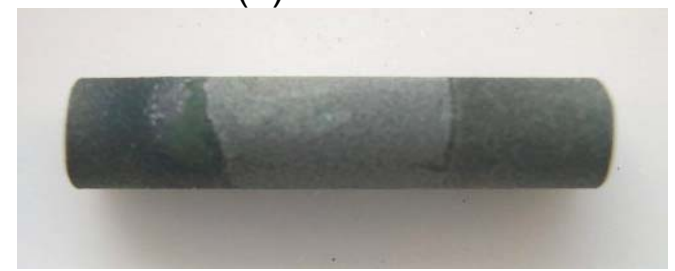

(i) $\mathrm{H} 100 \mathrm{H} 3$

300 hours

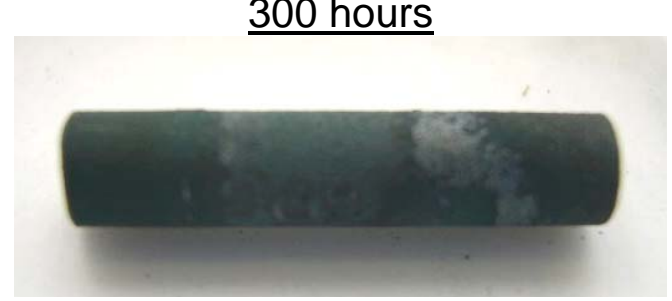

(j) $\mathrm{H} 300 \mathrm{H} 1$

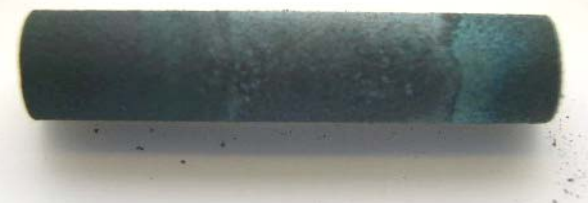

(k) $\mathrm{H} 300 \mathrm{H} 2$

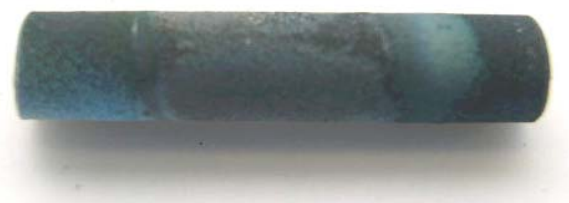

(I) $\mathrm{H} 30 \mathrm{OH} 3$

Photographs of Triplicate Specimens of Nimonic 263 + CoNiCrAlY Coating Exposed to $100 \mathrm{ppm} \mathrm{H}_{2} \mathrm{~S} /$ Balance $\mathrm{N}_{2}$ at $1900{ }^{\circ} \mathrm{F}$ for $10,30,100$ and 300 Hours. Note: Top of Specimens is on the Right in Each Photograph. 


\section{Dry $\mathrm{H}_{2} \underline{S}$}

10 hours

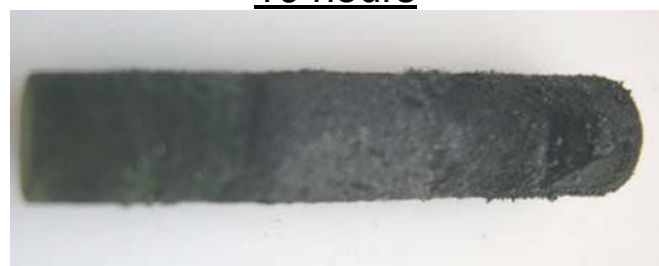

(a) H10K1

(b) $\mathrm{H} 10 \mathrm{~K} 2$

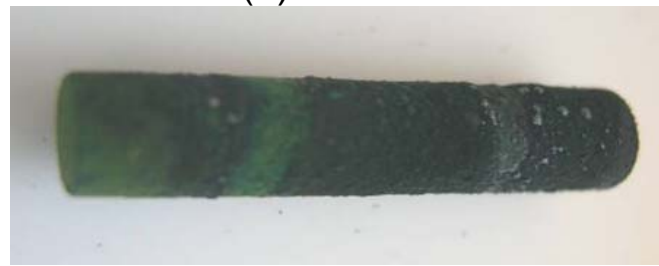

(c) $\mathrm{H} 10 \mathrm{~K} 3$

30 hours

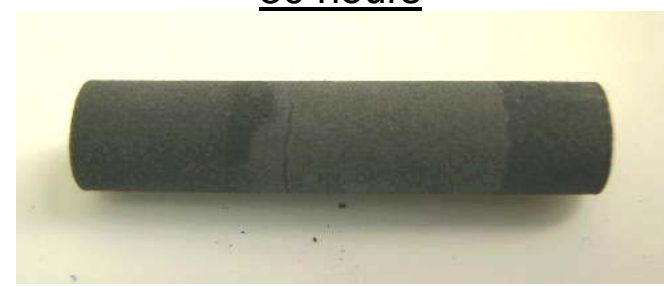

(d) H30K1

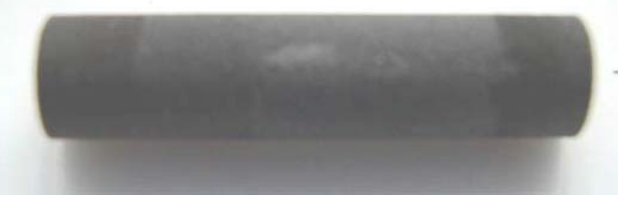

(e) H3OK2

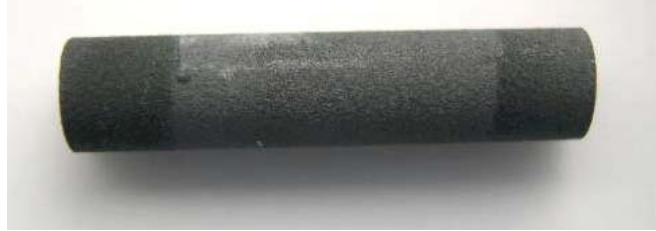

(f) $\mathrm{H} 30 \mathrm{~K} 3$
100 hours

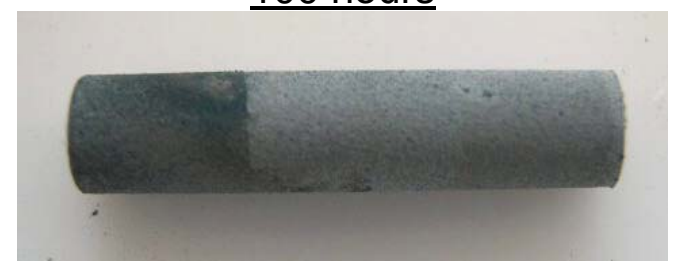

(g) H100K1

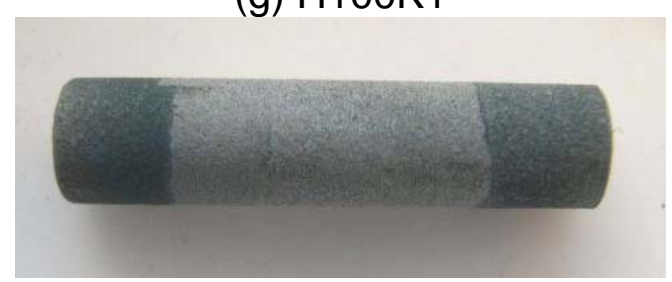

(h) H100K2

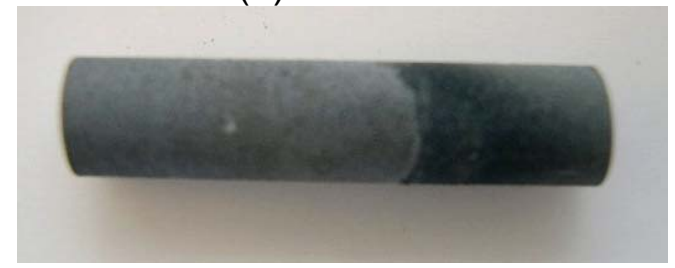

(i) $\mathrm{H} 100 \mathrm{~K} 3$

300 hours

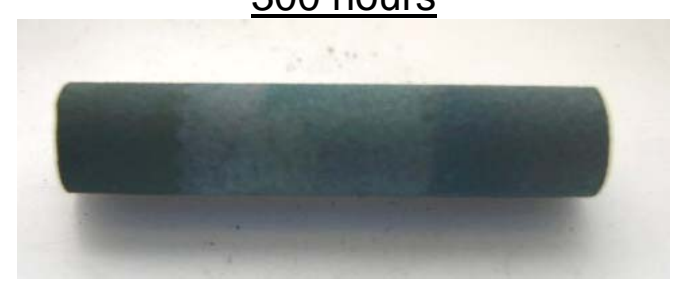

(j) $\mathrm{H} 300 \mathrm{~K} 1$

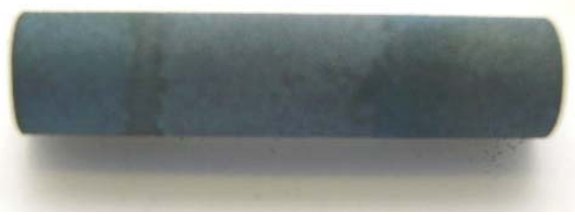

(k) H300K2

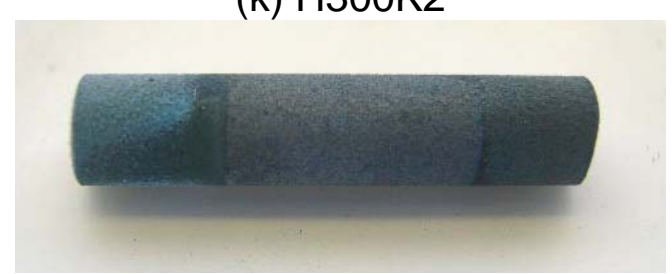

(I) H300K3

Photographs of Triplicate Specimens of GTD111+ CoNiCrAlY Coating Exposed to $100 \mathrm{ppm} \mathrm{H}_{2} \mathrm{~S} /$ Balance $\mathrm{N}_{2}$ at $1900{ }^{\circ} \mathrm{F}$ for 10, 30, 100 and 300 Hours. Note: Top of Specimens is on the Right in Each Photograph. 


\section{Wet $\mathrm{H}_{2} \underline{\mathrm{S}}$}

10 hours

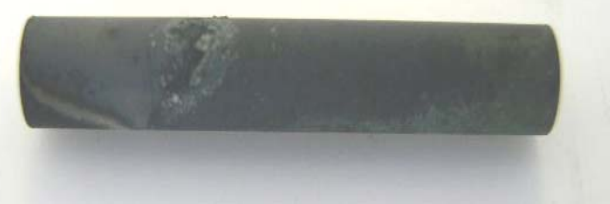

(a) W10A1

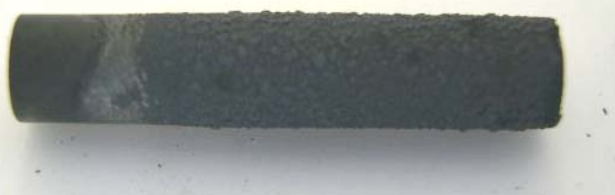

(b) W10A2

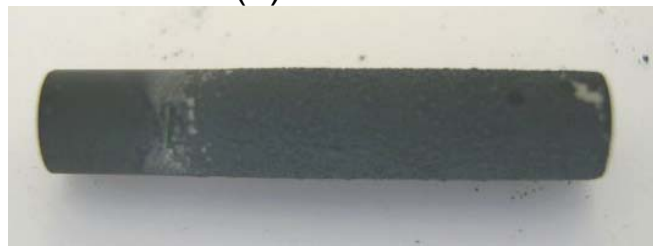

(c) W10A3

$\underline{30 \text { hours }}$

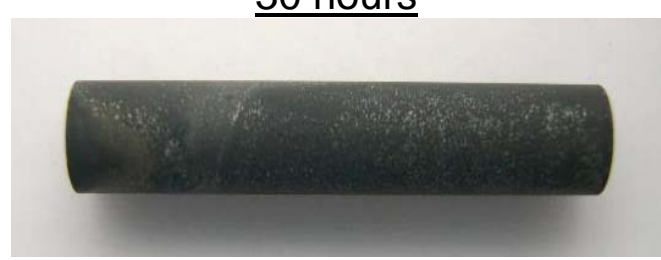

(d) W30A1

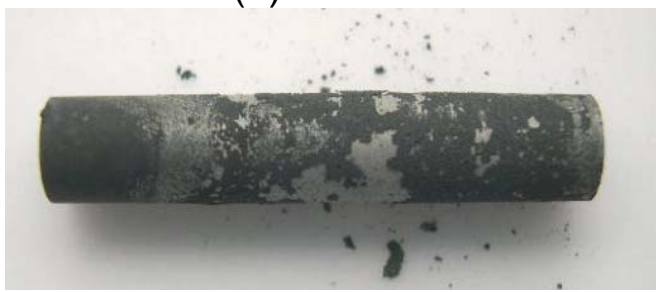

(e) W30A2

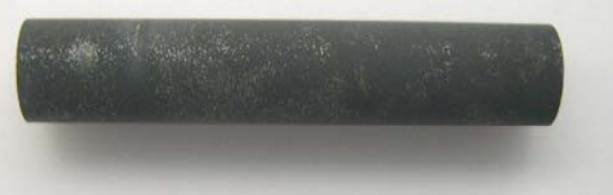

(f) $\mathrm{W} 30 \mathrm{~A} 3$
100 hours

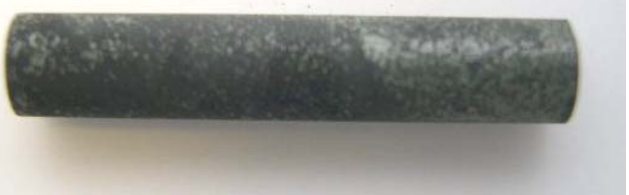

(g) W100A1

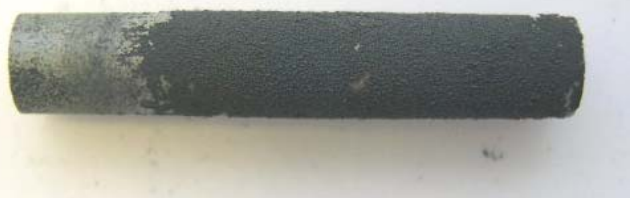

(h) W100A2

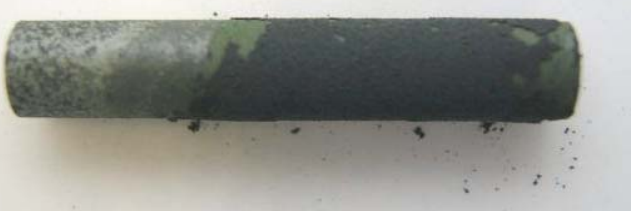

(i) $\mathrm{W} 100 \mathrm{A3}$

300 hours

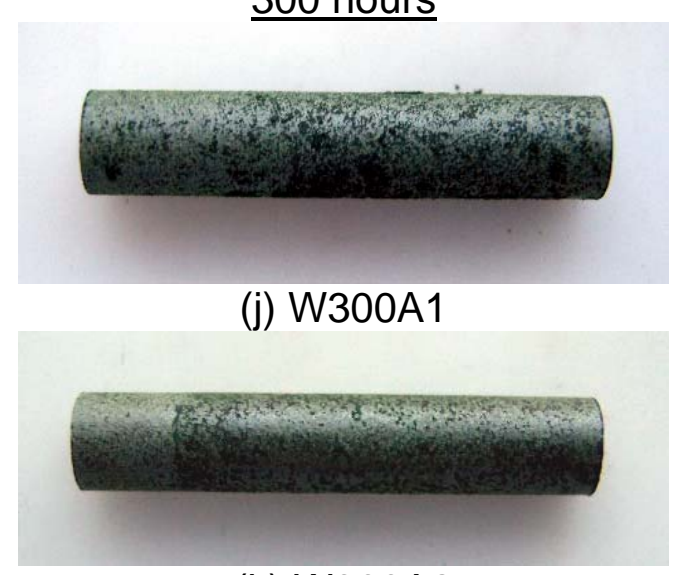

(k) W300A2

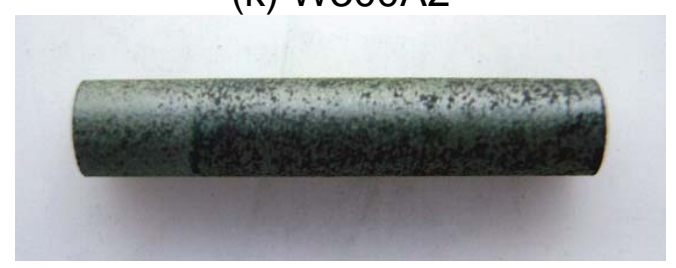

(I) $\mathrm{W} 300 \mathrm{~A} 3$

Photographs of Triplicate Specimens of FSC-414 Exposed to wet $100 \mathrm{ppm} \mathrm{H}_{2} \mathrm{~S}$ / Balance $\mathrm{N}_{2}$ at $1900^{\circ} \mathrm{F}$ for $10,30,100$ and 300 Hours. Note: Top of Specimens is on the Right in Each Photograph. 


\section{Wet $\mathrm{H}_{2} \underline{\mathrm{S}}$}

10 hours

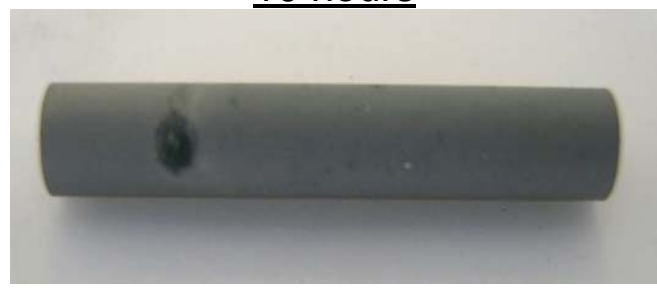

(a) W10B1

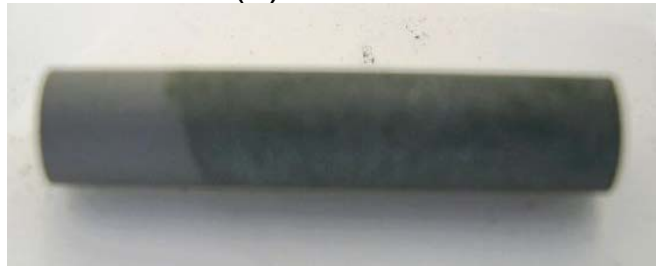

(b) W10B2

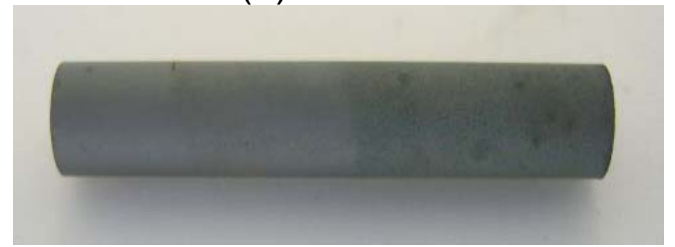

(c) $\mathrm{W} 10 \mathrm{~B} 3$

$\underline{30 \text { hours }}$

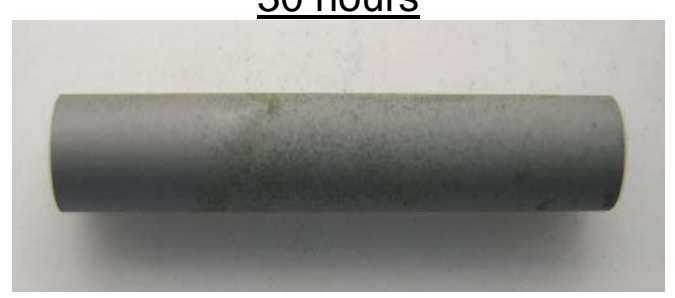

(d) W30B1

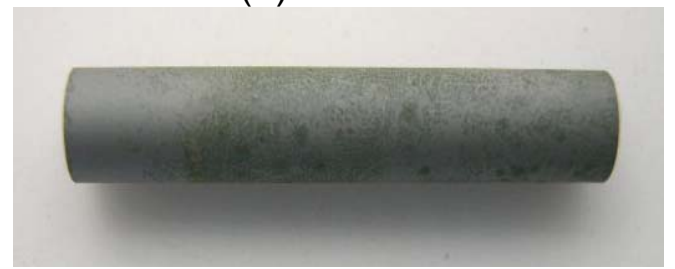

(e) W30B2

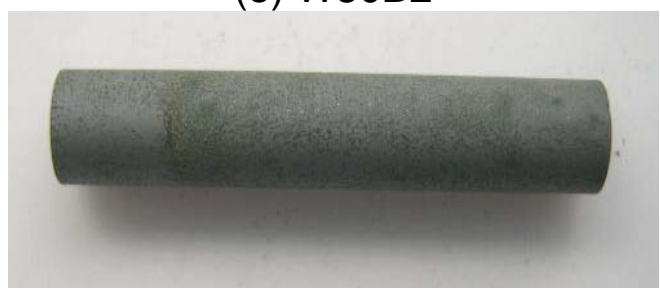

(f) $\mathrm{W} 30 \mathrm{~B} 3$
100 hours

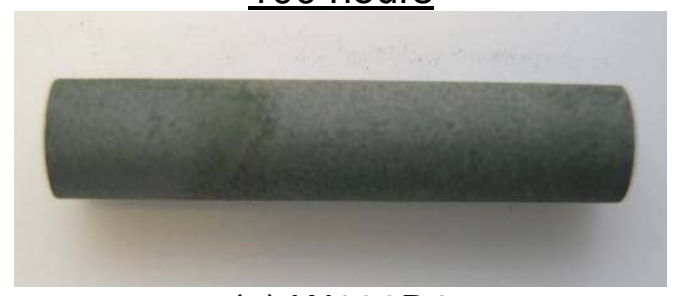

(g) W100B1

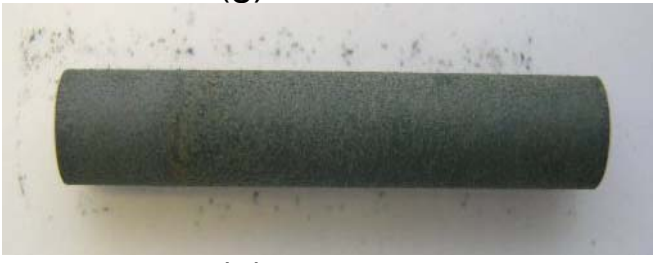

(h) W100B2

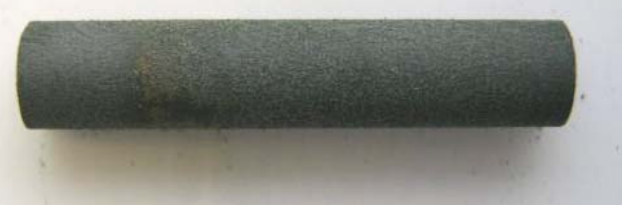

(i) $\mathrm{W} 100 \mathrm{~B} 3$

$\underline{300 \text { hours }}$

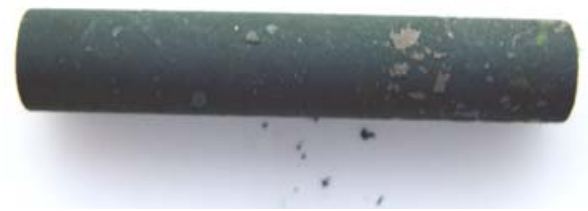

(j) $\mathrm{W} 300 \mathrm{~B} 1$

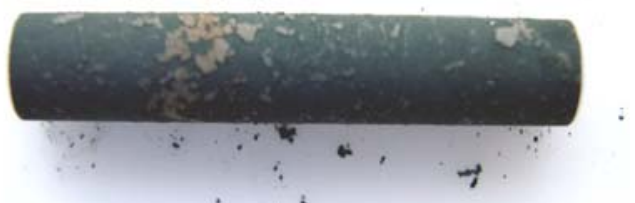

(k) W300B2

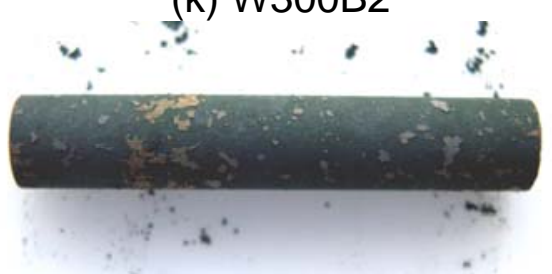

(I) $\mathrm{W} 300 \mathrm{~B} 3$

Photographs of Triplicate Specimens of GTD222 Exposed to wet $100 \mathrm{ppm} \mathrm{H}_{2} \mathrm{~S}$ / Balance $\mathrm{N}_{2}$ at $1900^{\circ} \mathrm{F}$ for $10,30,100$ and 300 Hours. Note: Top of Specimens is on the Right in Each Photograph. 


\section{Wet $\mathrm{H}_{2} \underline{\mathrm{S}}$}

10 hours

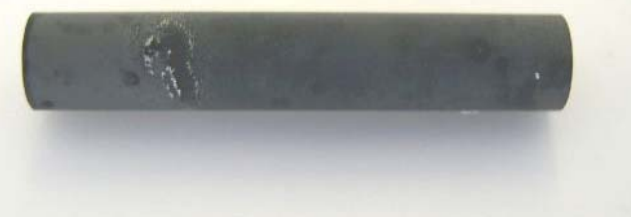

(a) W10C1

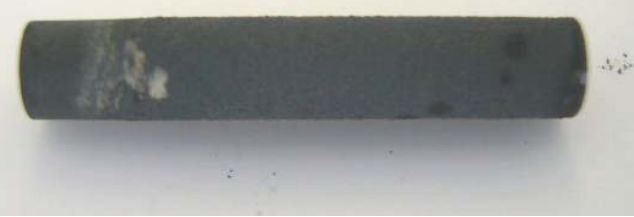

(b) $\mathrm{W} 10 \mathrm{C} 2$

(c) $\mathrm{W} 10 \mathrm{C} 3$

30 hours

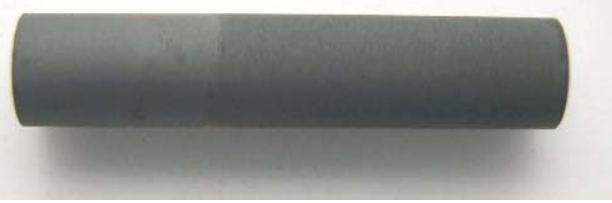

(d) W30C1

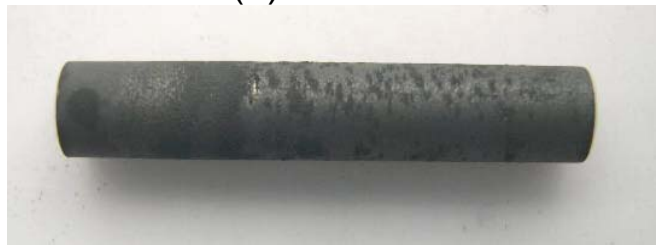

(e) W30C2

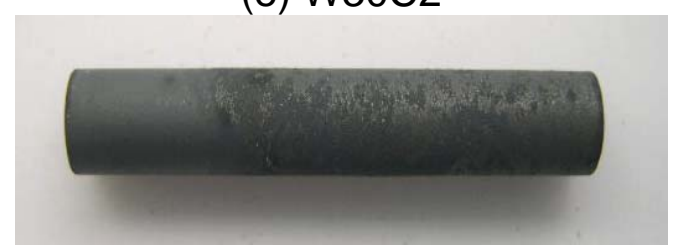

(f) $\mathrm{W} 30 \mathrm{C} 3$
100 hours

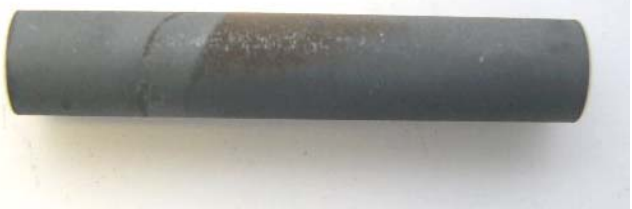

(g) W100C1

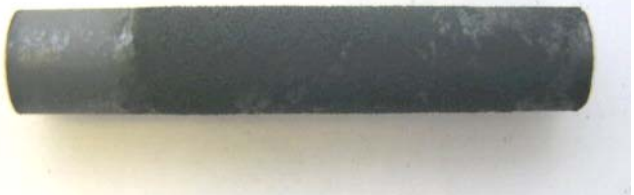

(h) $\mathrm{W} 100 \mathrm{C} 2$

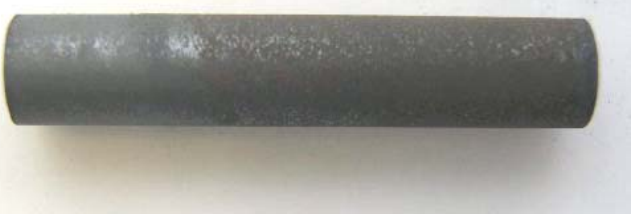

(i) $\mathrm{W} 100 \mathrm{C} 3$

300 hours

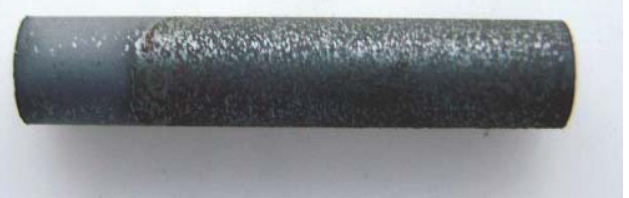

(j) $\mathrm{W} 300 \mathrm{C1}$

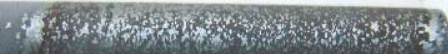

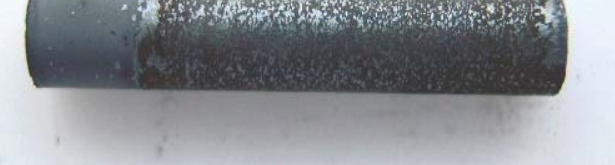

(k) W300C2

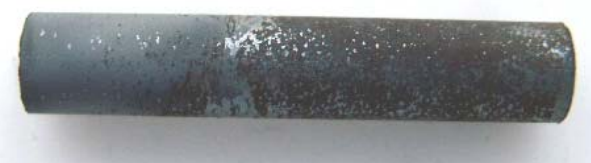

(I) $\mathrm{W} 300 \mathrm{C} 3$

Photographs of Triplicate Specimens of Hastelloy-X Exposed to wet $100 \mathrm{ppm}$ $\mathrm{H}_{2} \mathrm{~S} /$ Balance $\mathrm{N}_{2}$ at $1900{ }^{\circ} \mathrm{F}$ for 10, 30, 100 and 300 Hours. Note: Top of Specimens is on the Right in Each Photograph. 


\section{Wet $\mathrm{H}_{2} \underline{\mathrm{S}}$}

10 hours

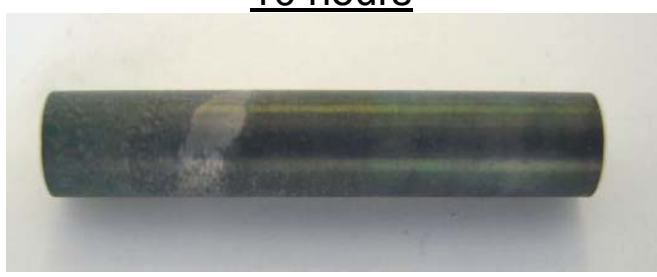

(a) W10D1

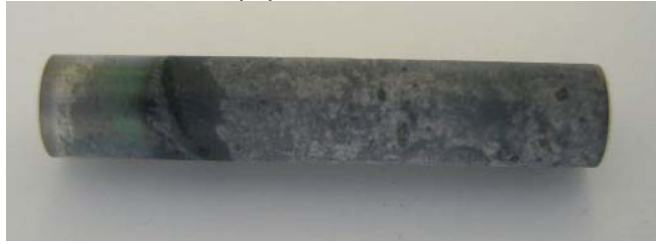

(b) W10D2

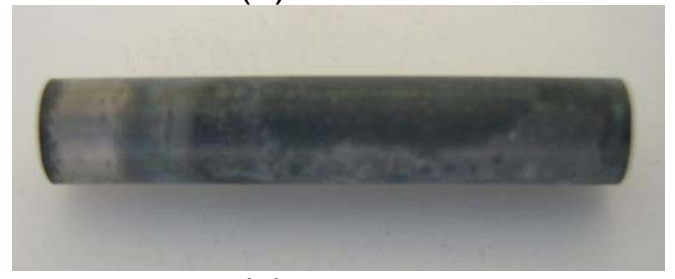

(c) W10D3

$\underline{30 \text { hours }}$

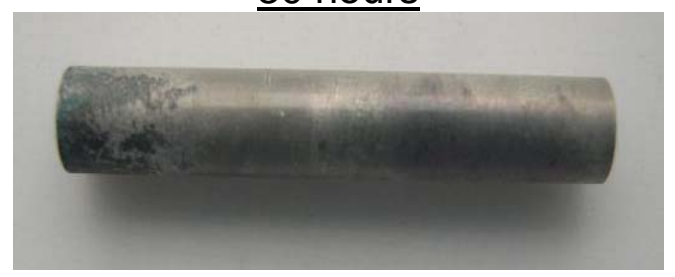

(d) W30D1

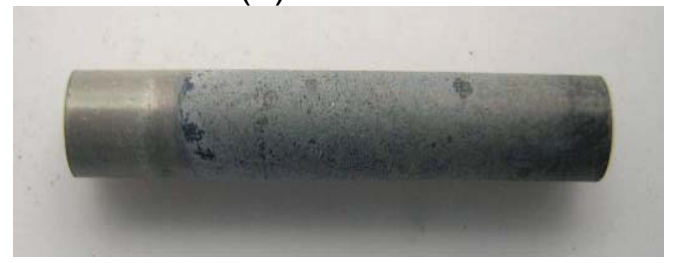

(e) W30D2

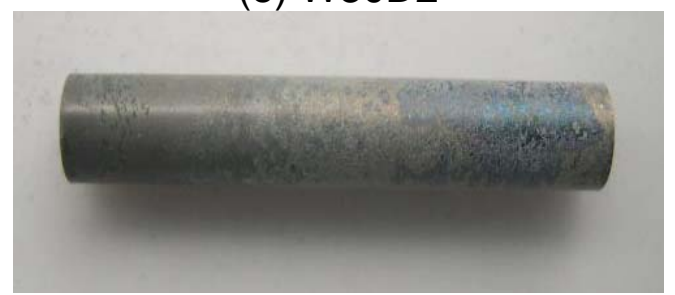

(f) $\mathrm{W} 30 \mathrm{D} 3$
100 hours

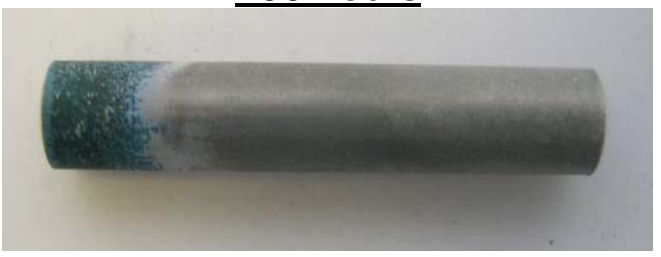

(g) W100D1

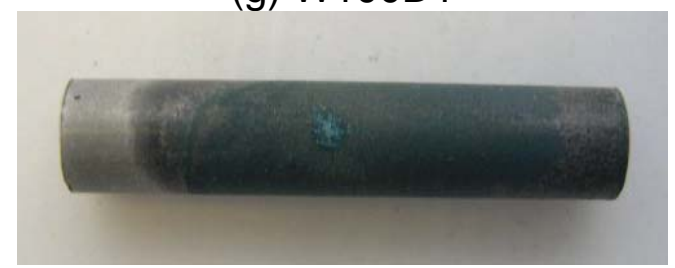

(h) W100D2

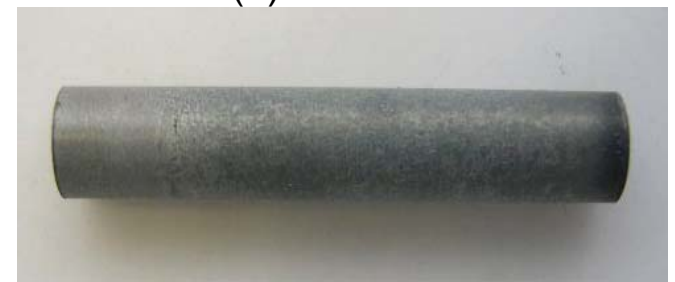

(i) $\mathrm{W} 100 \mathrm{D} 3$

300 hours

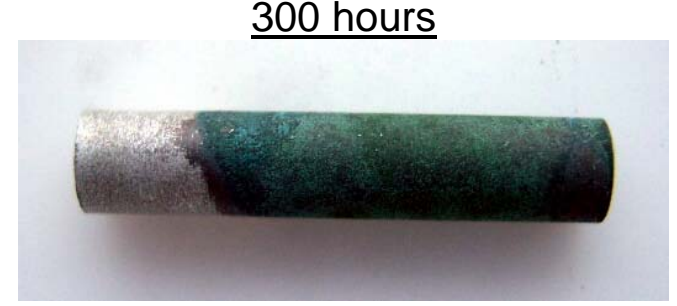

(j) $W 300 \mathrm{D} 1$

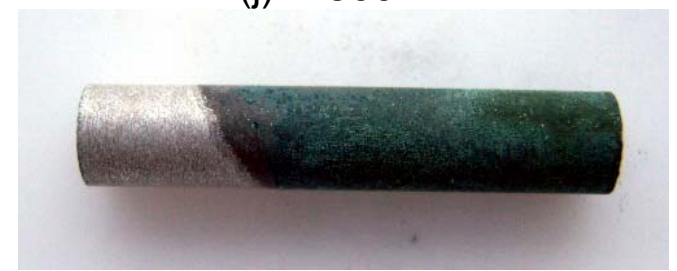

(k) W300D2

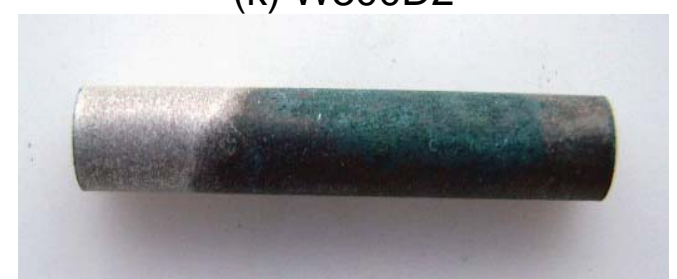

(I) W300D3

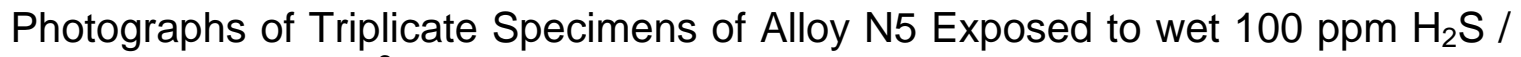
Balance $\mathrm{N}_{2}$ at $1900^{\circ} \mathrm{F}$ for $10,30,100$ and 300 Hours. Note: Top of Specimens is on the Right in Each Photograph. 


\section{Wet $\mathrm{H}_{2} \underline{\mathrm{S}}$}

10 hours

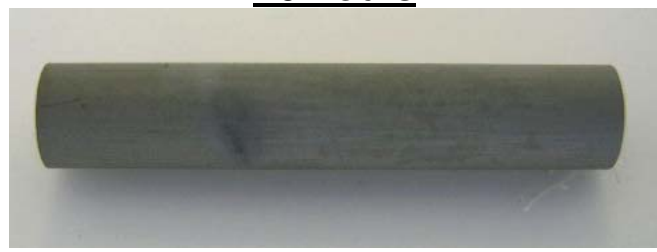

(a) W10E1

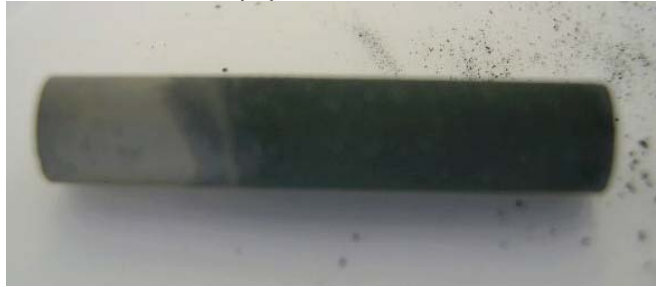

(b) W10E2

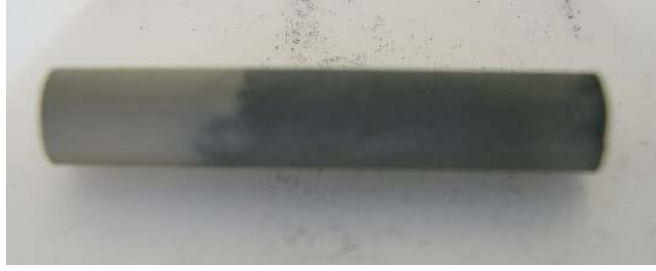

(c) W10E3

30 hours

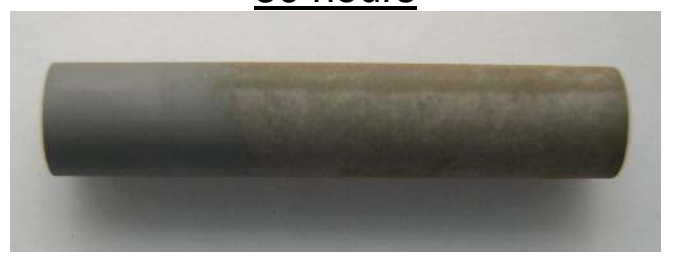

(e) W30E1

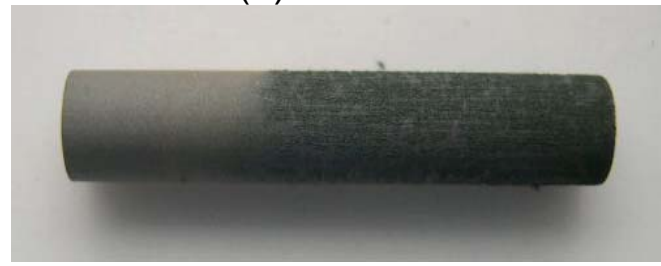

(f) W30E2

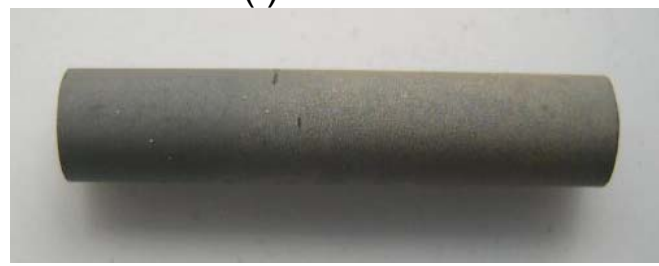

(g) W30E3
100 hours

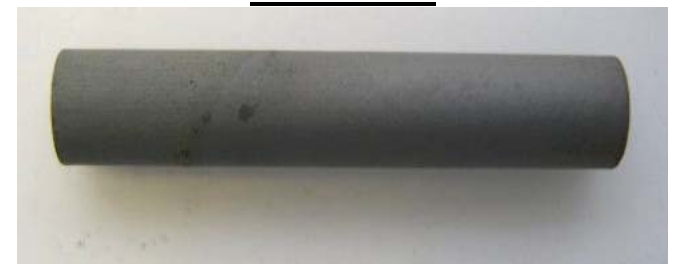

(g) W100E1

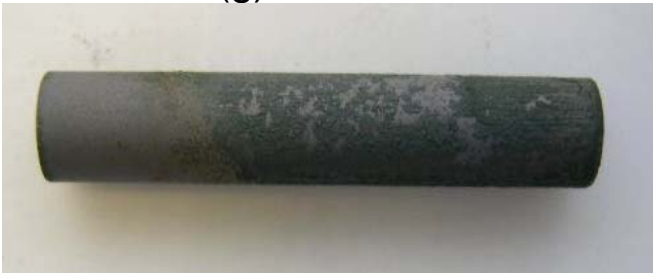

(h) W100E2

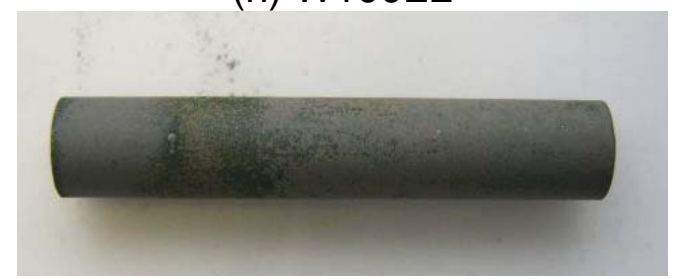

(i) $\mathrm{W} 100 \mathrm{E} 3$

300 hours

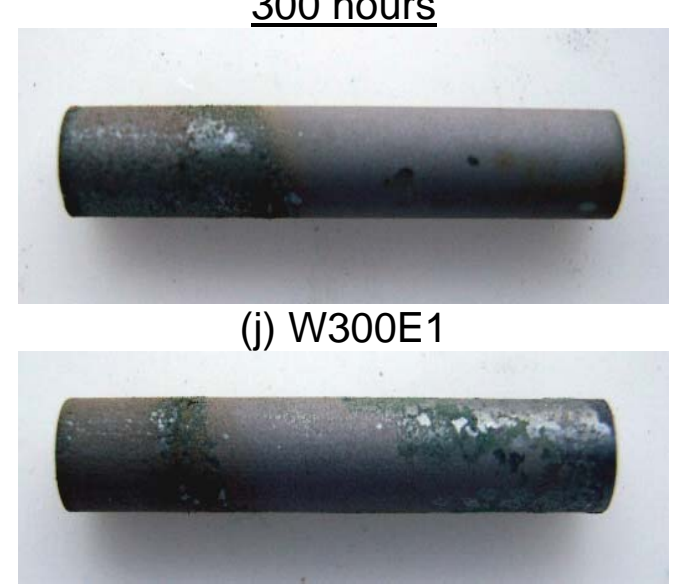

(k) W300E2

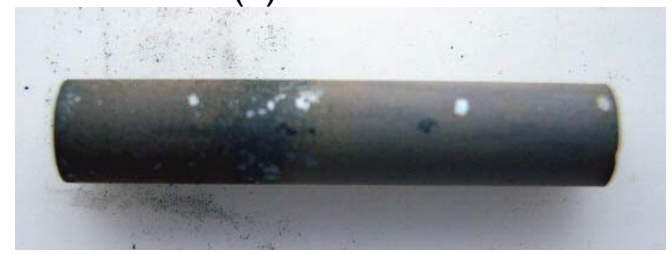

(I) W300E3

Photographs of Triplicate Specimens of Nimonic 263 Exposed to wet $100 \mathrm{ppm}$ $\mathrm{H}_{2} \mathrm{~S} /$ Balance $\mathrm{N}_{2}$ at $1900{ }^{\circ} \mathrm{F}$ for 10, 30, 100 and 300 Hours. Note: Top of Specimens is on the Right in Each Photograph. 


\section{Wet $\mathrm{H}_{2} \underline{\mathrm{S}}$}

10 hours

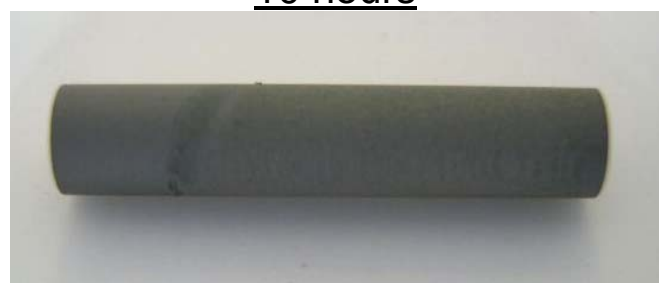

(a) W10F1

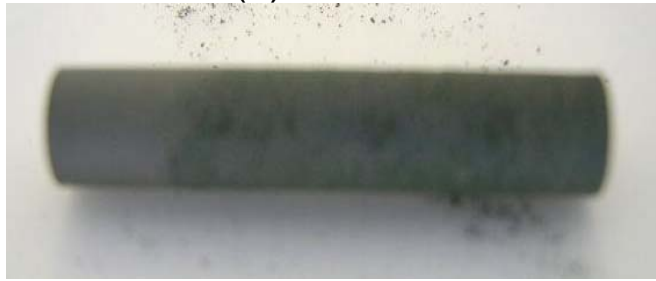

(b) W10F2

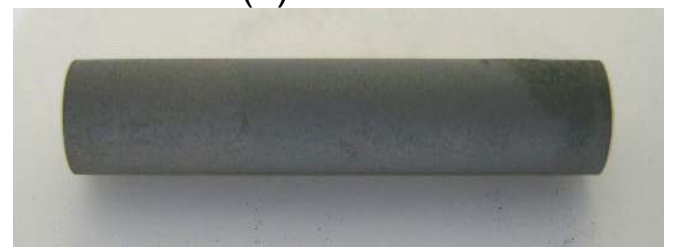

(c) W10F3

$\underline{30 \text { hours }}$

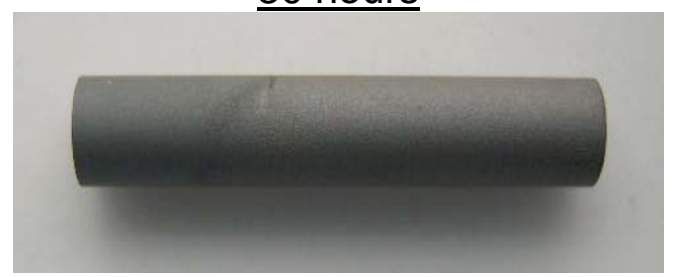

(d) W30F1

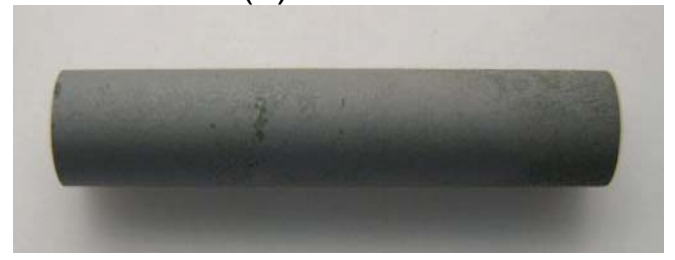

(e) W30F2

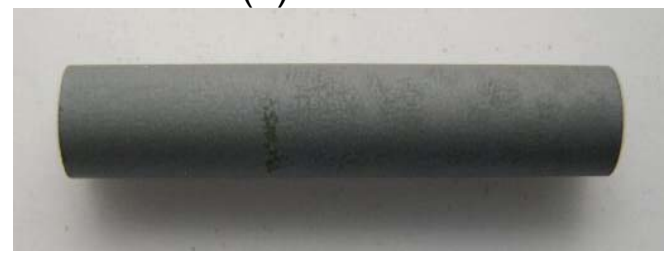

(f) $\mathrm{W} 30 \mathrm{~F} 3$
100 hours

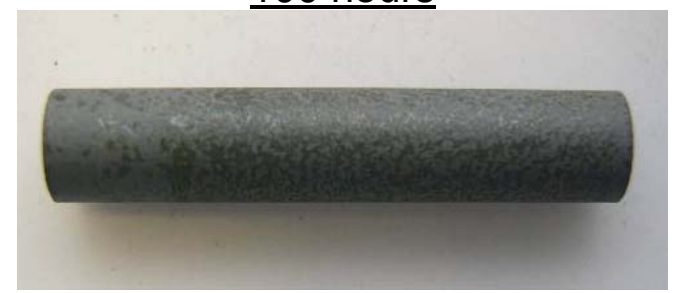

(g) W100F1

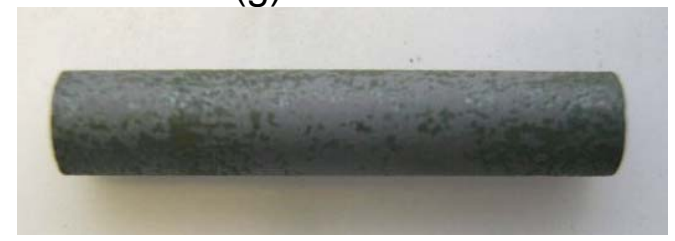

(h) W100F2

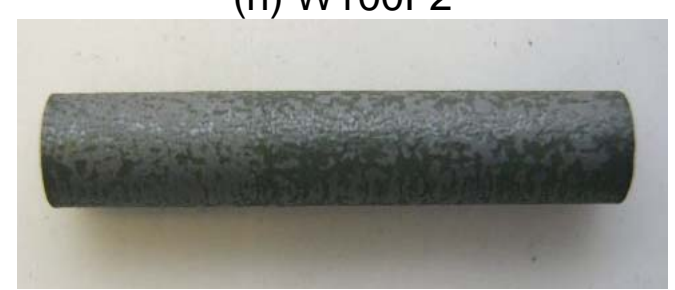

(i) $\mathrm{W} 100 \mathrm{~F} 3$

$\underline{300 \text { hours }}$

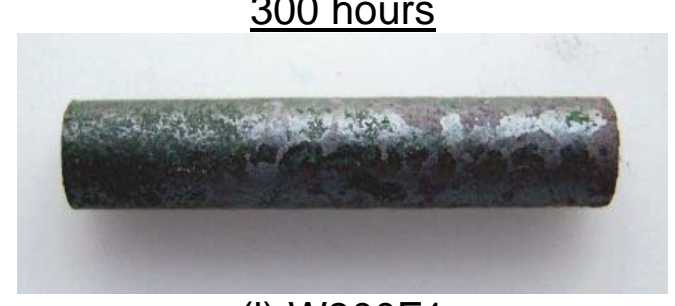

(j) $\mathrm{W} 300 \mathrm{~F} 1$

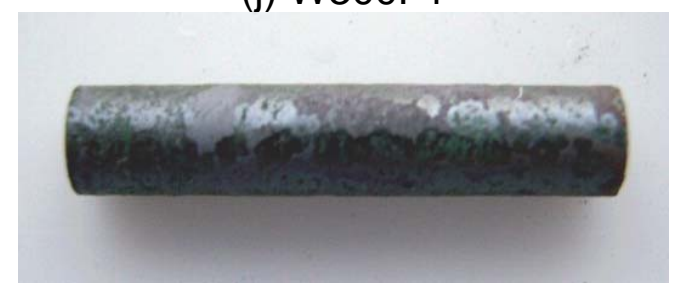

(k) W300F2

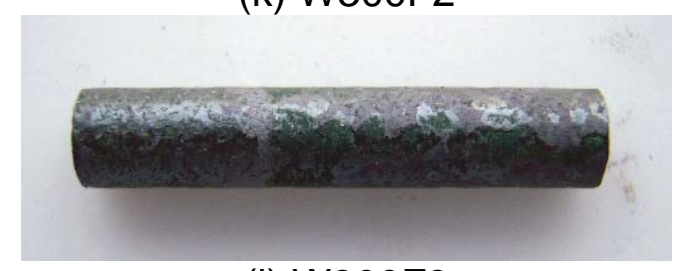

(I) W300F3

Photographs of Triplicate Specimens of GTD111 Exposed to wet $100 \mathrm{ppm} \mathrm{H}_{2} \mathrm{~S}$ / Balance $\mathrm{N}_{2}$ at $1900^{\circ} \mathrm{F}$ for $10,30,100$ and 300 Hours. Note: Top of Specimens is on the Right in Each Photograph. 


\section{Wet $\mathrm{H}_{2} \underline{\mathrm{S}}$}

10 hours

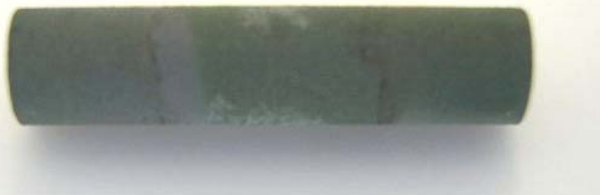

(a) W10G1

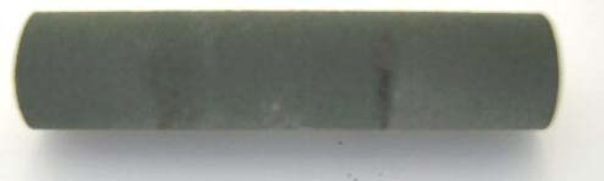

(b) W10G2

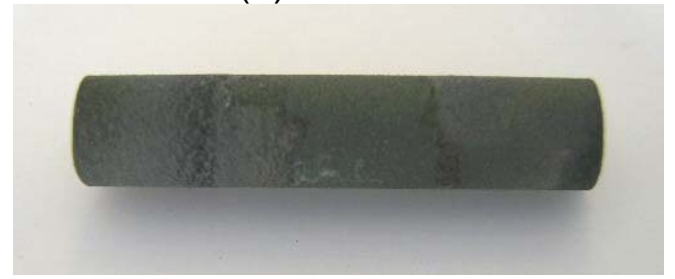

(c) W10G3

30 hours

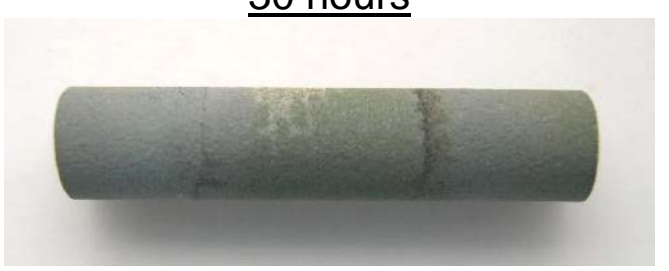

(d) W30G1

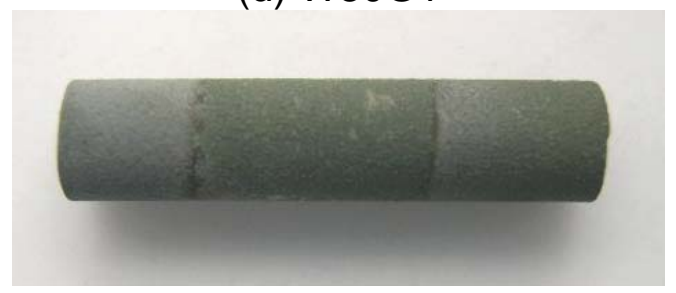

(e) W30G2

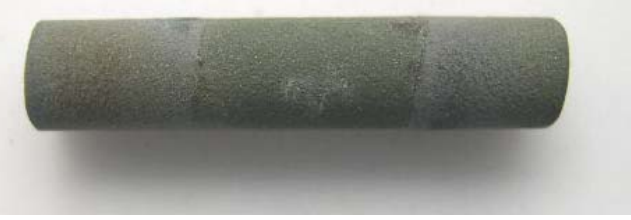

(f) W30G3
100 hours

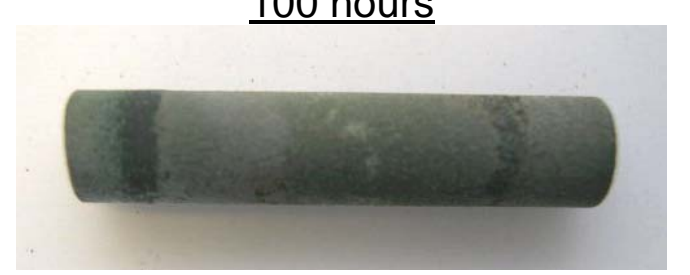

(g) W100G1

(h) W100G2

(i) W100G3

$\underline{300 \text { hours }}$

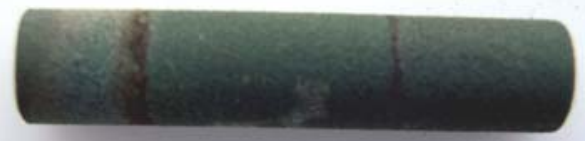

(j) W300G1

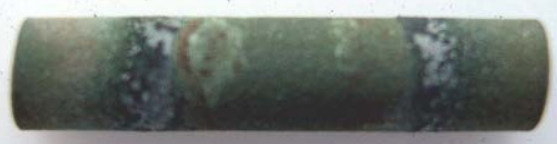

(k) W300G2

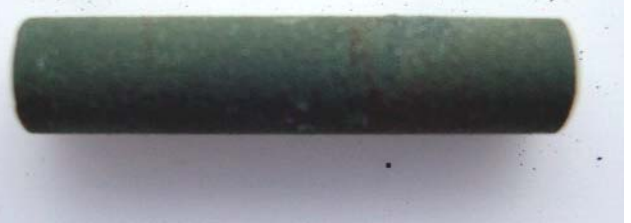

(I) W300G3

Photographs of Triplicate Specimens of Alloy N5 + NiCrAlY Coating Exposed to wet $100 \mathrm{ppm} \mathrm{H} \mathrm{H}_{2} \mathrm{~S} /$ Balance $\mathrm{N}_{2}$ at $1900{ }^{\circ} \mathrm{F}$ for 10, 30, 100 and 300 Hours. Note: Top of Specimens is on the Right in Each Photograph. 


\section{Wet $\mathrm{H}_{2} \underline{\mathrm{S}}$}

10 hours

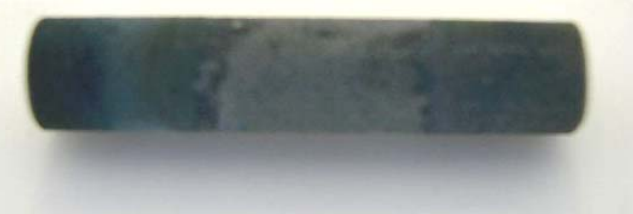

(a) $\mathrm{W} 10 \mathrm{H} 1$

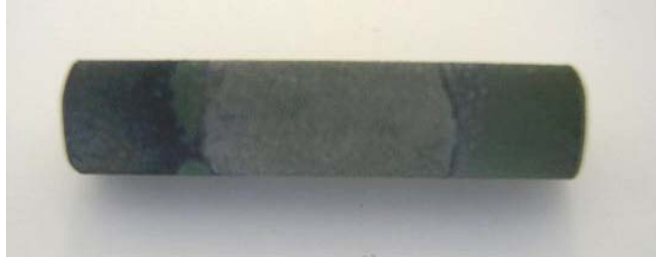

(b) $\mathrm{W} 10 \mathrm{H} 2$

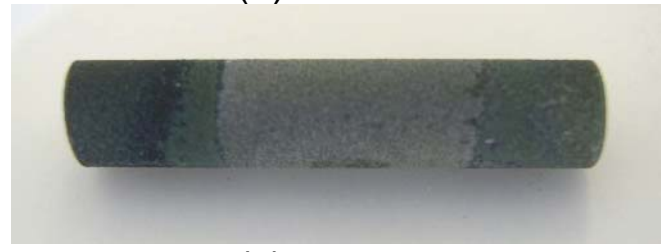

(c) $\mathrm{W} 10 \mathrm{H} 3$

30 hours

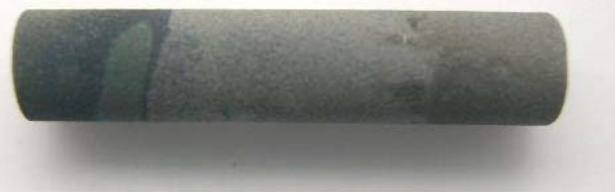

(d) $\mathrm{W} 30 \mathrm{H} 1$

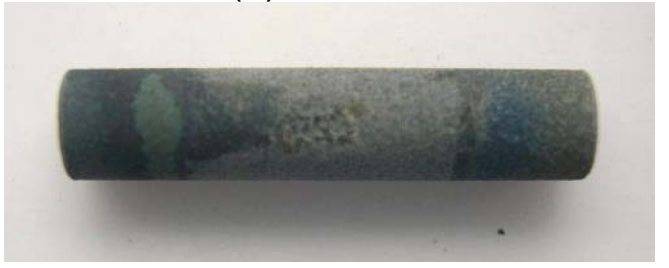

(e) $\mathrm{W} 30 \mathrm{H} 2$

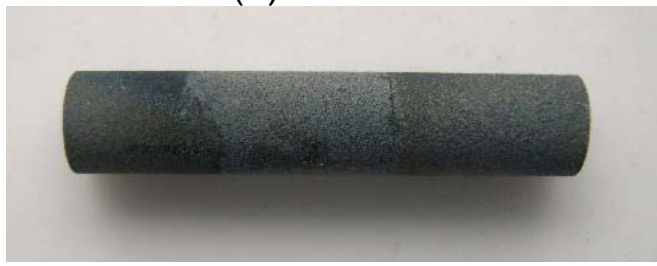

(f) $\mathrm{W} 30 \mathrm{H} 3$
100 hours

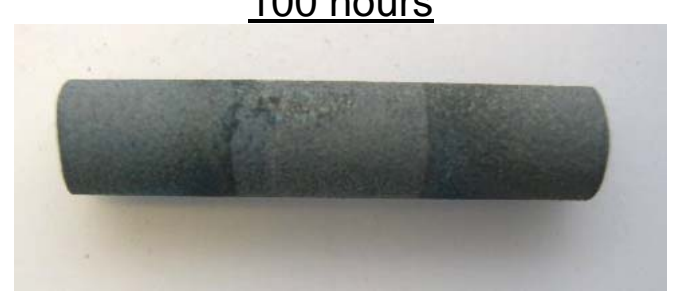

(g) $\mathrm{W} 100 \mathrm{H} 1$

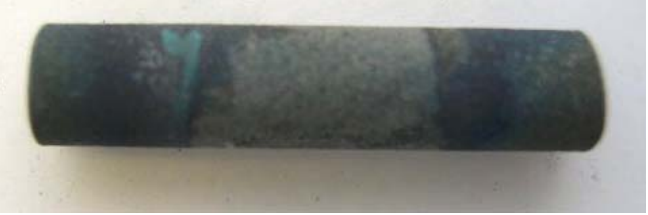

(h) $\mathrm{W} 100 \mathrm{H} 2$

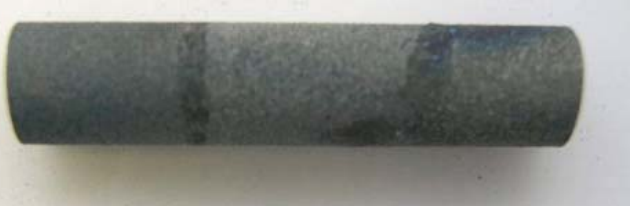

(i) $\mathrm{W} 100 \mathrm{H} 3$

300 hours

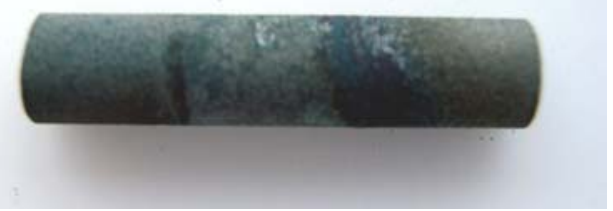

(j) $\mathrm{W} 300 \mathrm{H} 1$

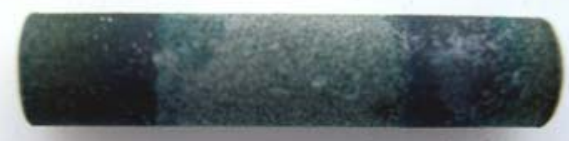

(k) $\mathrm{W} 300 \mathrm{H} 2$

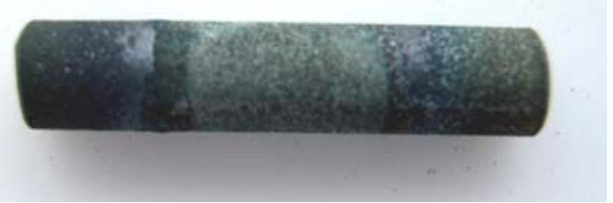

(I) $\mathrm{W} 300 \mathrm{H} 3$

Photographs of Triplicate Specimens of Nimonic 263 + CoNiCrAlY Coating Exposed to wet 100 ppm $\mathrm{H}_{2} \mathrm{~S} /$ Balance $\mathrm{N}_{2}$ at $1900{ }^{\circ} \mathrm{F}$ for $10,30,100$ and 300 Hours. Note: Top of Specimens is on the Right in Each Photograph. 


\section{Wet $\mathrm{H}_{2} \underline{\mathrm{S}}$}

10 hours

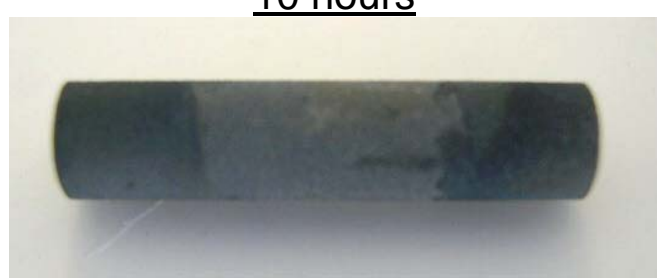

(a) W10K1

(b) W10K2

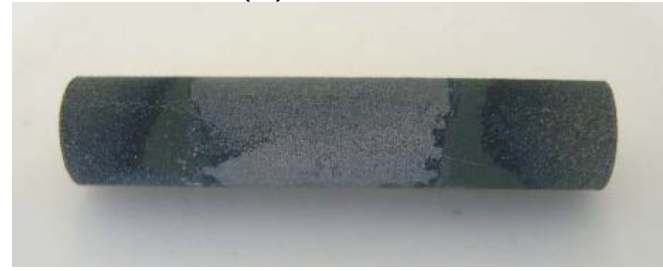

(c) $\mathrm{W} 10 \mathrm{~K} 3$

30 hours

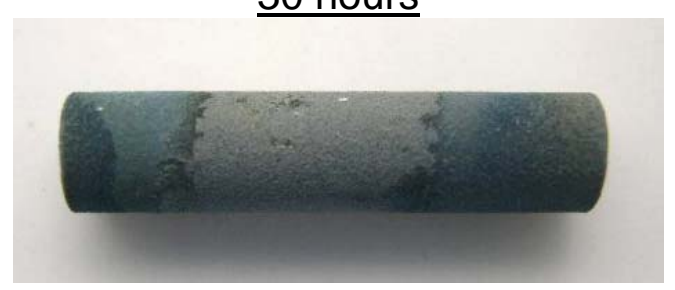

(d) W30K1

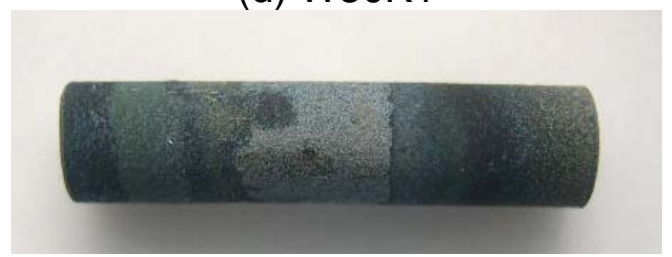

(e) W30K2

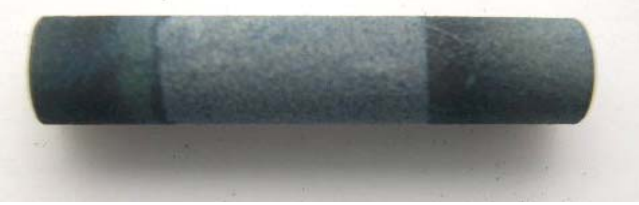

(f) $\mathrm{W} 30 \mathrm{~K} 3$
100 hours

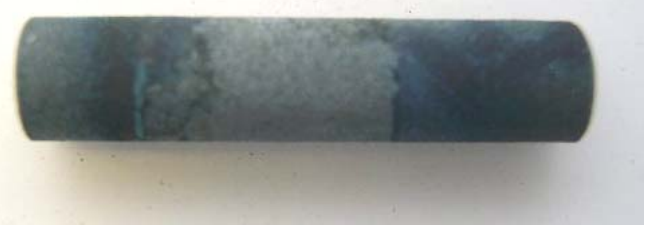

(g) W100K1

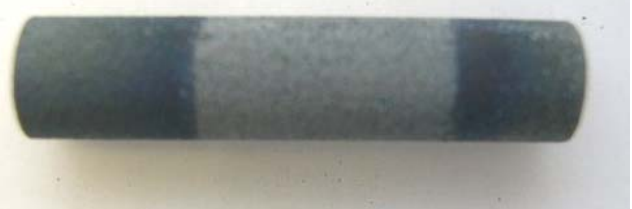

(h) W100K2

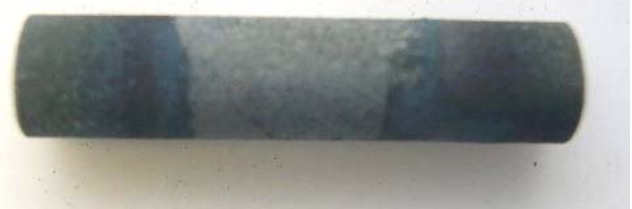

(i) $\mathrm{W} 100 \mathrm{~K} 3$

$\underline{300 \text { hours }}$

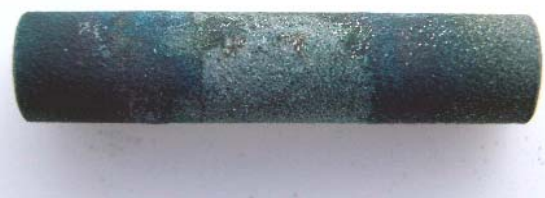

(j) $\mathrm{W} 300 \mathrm{~K} 1$

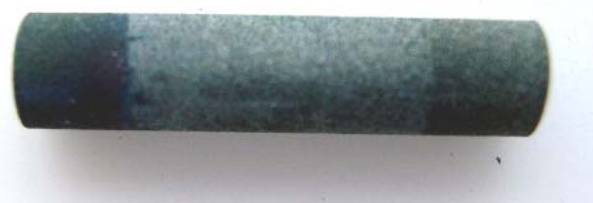

(k) W300K2

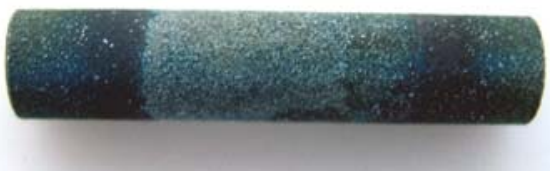

(I) $\mathrm{W} 300 \mathrm{~K} 3$

Photographs of Triplicate Specimens of GTD111 + CoNiCrAlY Coating Exposed to wet $100 \mathrm{ppm} \mathrm{H} \mathrm{H}_{2} \mathrm{~S} /$ Balance $\mathrm{N}_{2}$ at $1900{ }^{\circ} \mathrm{F}$ for 10, 30, 100 and 300 Hours. Note: Top of Specimens is on the Right in Each Photograph. 


\section{APPENDIX C.}

PHOTOMICROGRAPHS OF TRANSVERSE CROSS-SECTIONS OF SPECIMENS SHOWING SCALE THICKNESS AND INTERGRANULAR PENETRATION FOLLOWING EXPOSURE TO DIFFERENT ENVIRONMENTS.

(Photomicrographs are presented in the order of materials, exposure periods, and environments as listed in Table 5) 


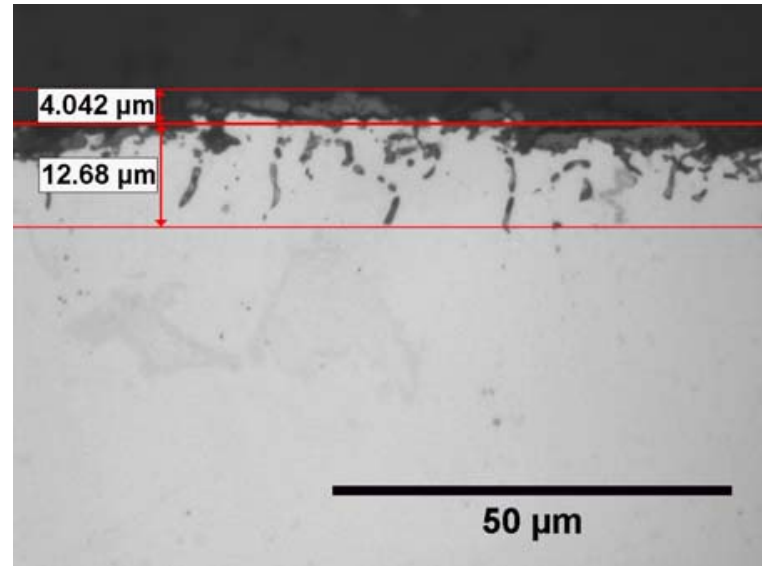

A10A1 - 10 hours

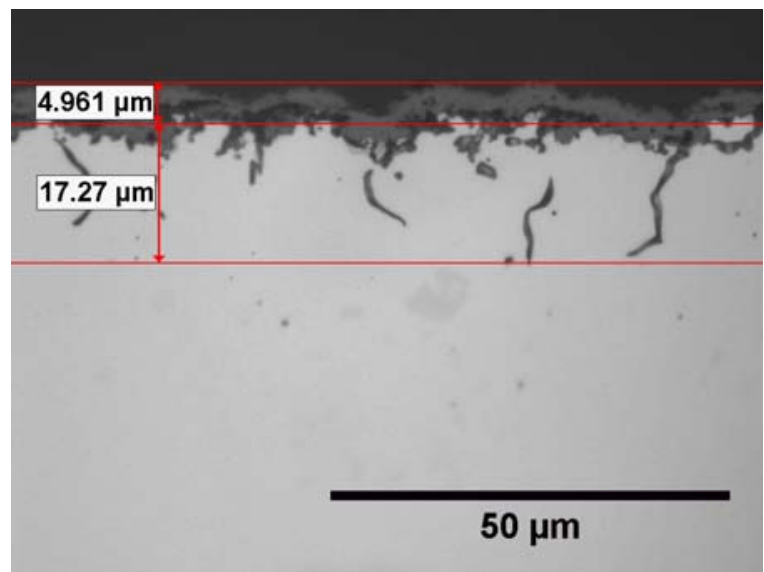

A30A1 - 30 hours

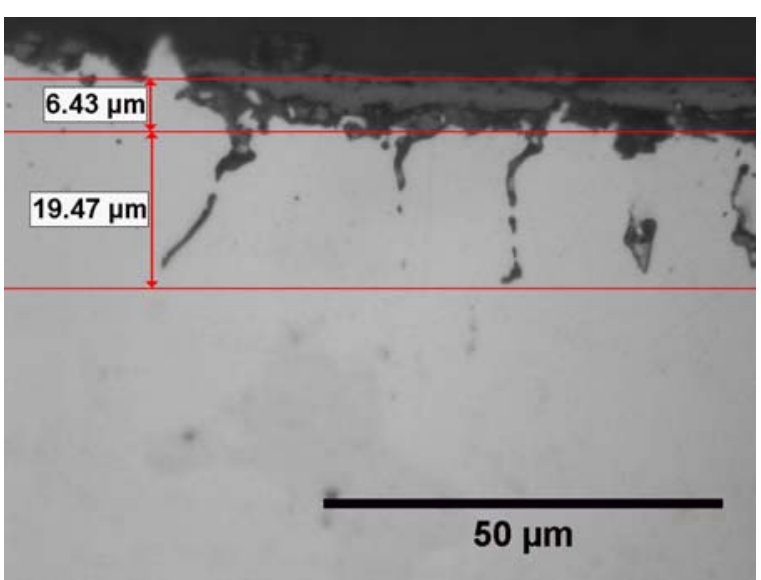

A100A1 - 100 hours

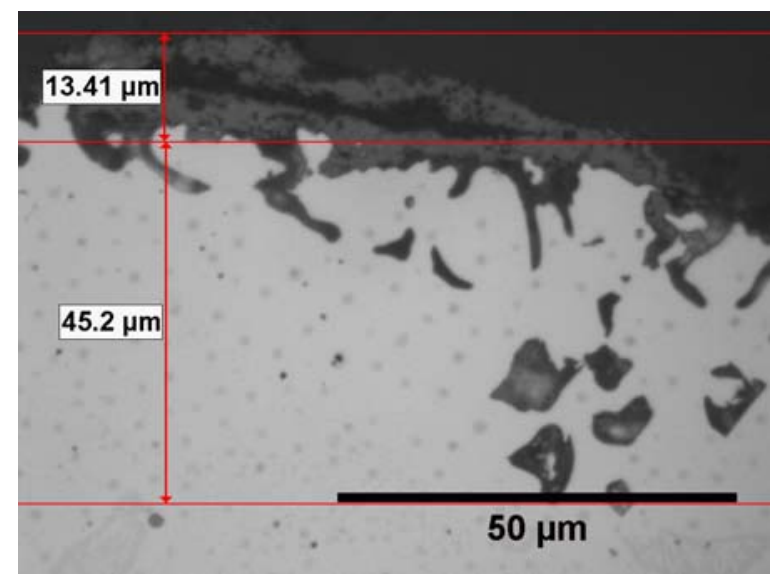

A300A1 - 300 hours

Photomicrographs Showing Surface Scale in Transverse Cross-Sections of FSC-414 Specimens After 10, 30, 100, and 300 Hours in Air. 


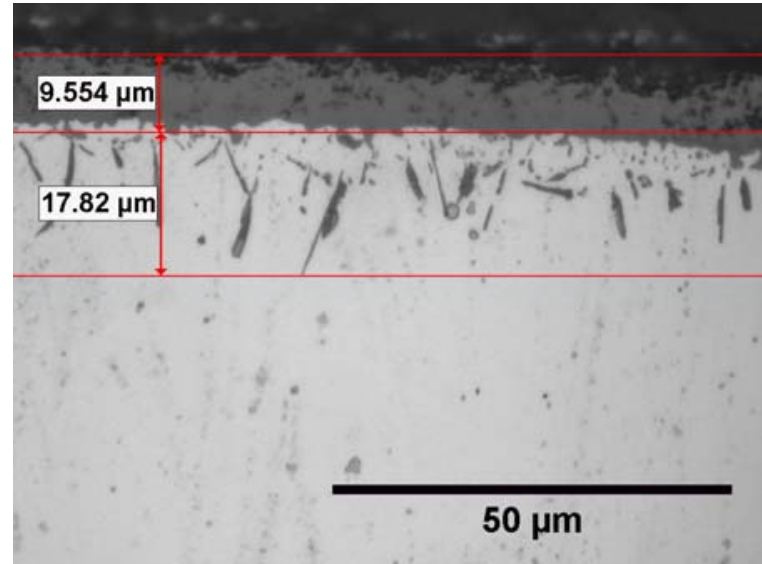

A10B1 - 10 hours

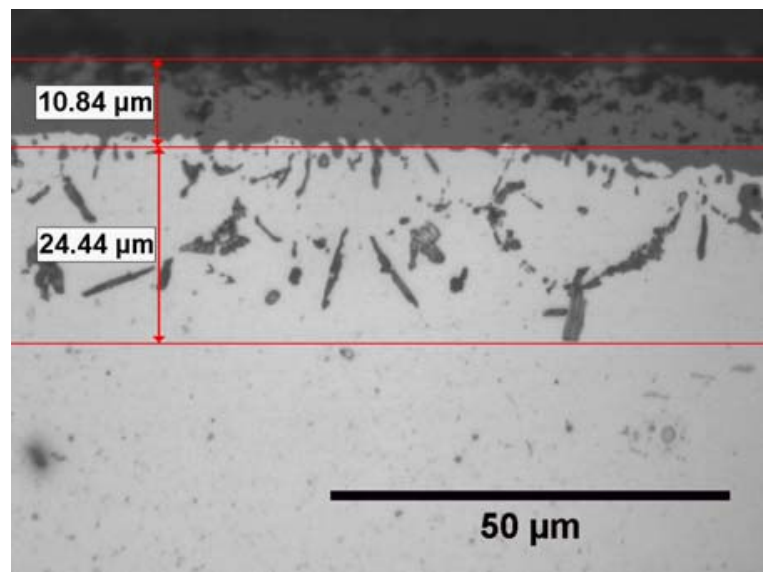

A30B1 -30 hours

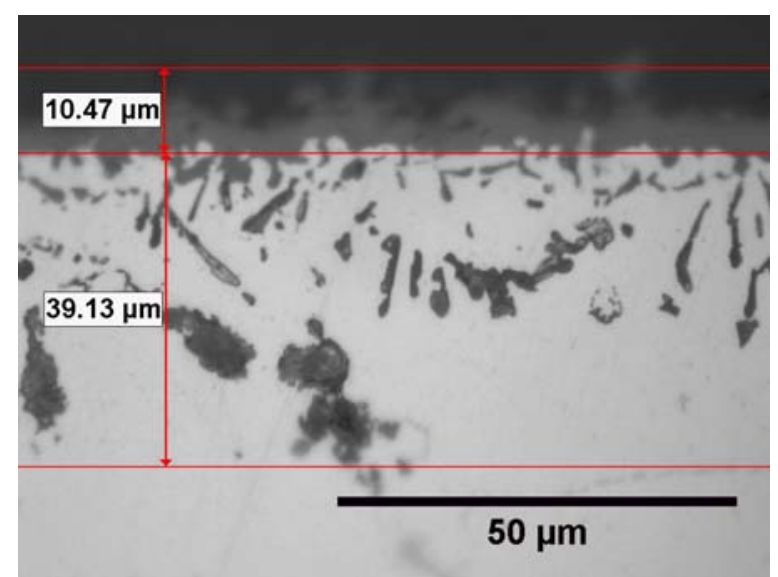

A100B1 -100 hours

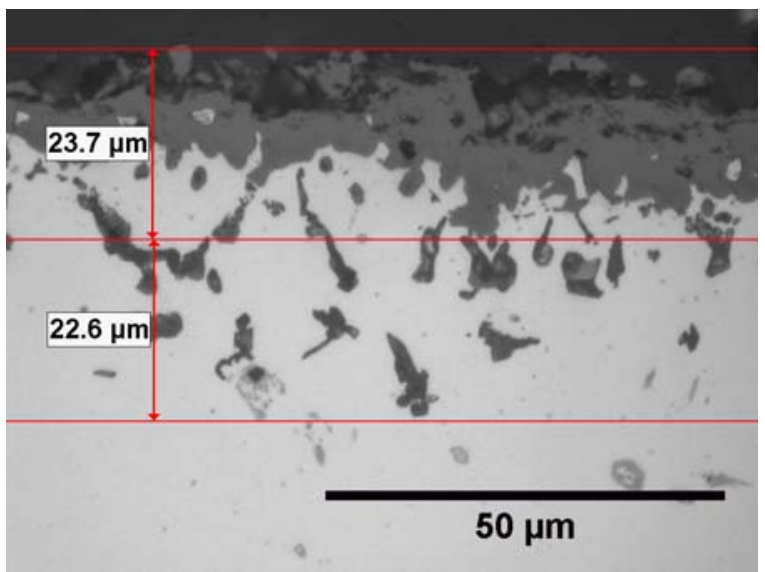

A300B1 - 300 hours

Photomicrographs Showing Surface Scale in Transverse Cross-Sections of GTD-222 Specimens After 10, 30, 100, and 300 Hours in Air. 


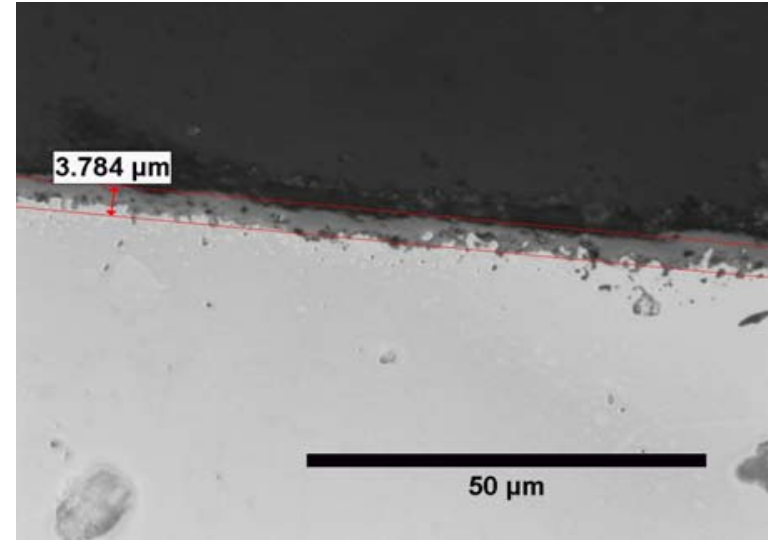

A10C1 - 10 hours

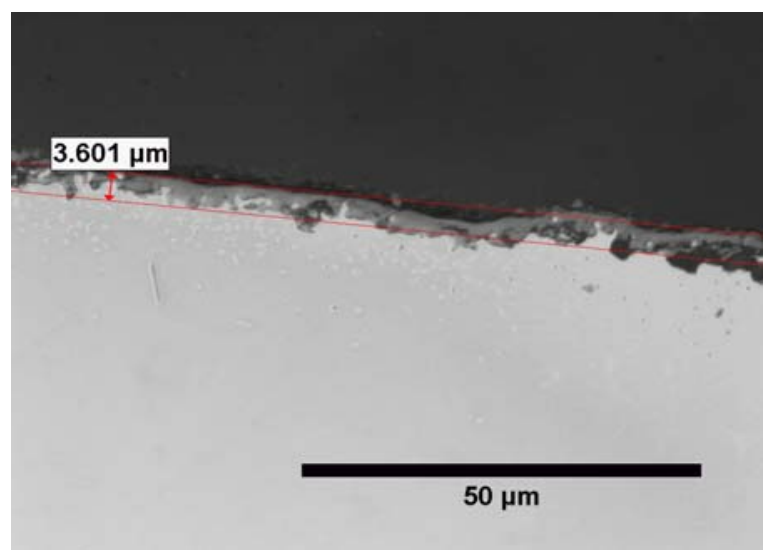

A30C1 - 30 Hours

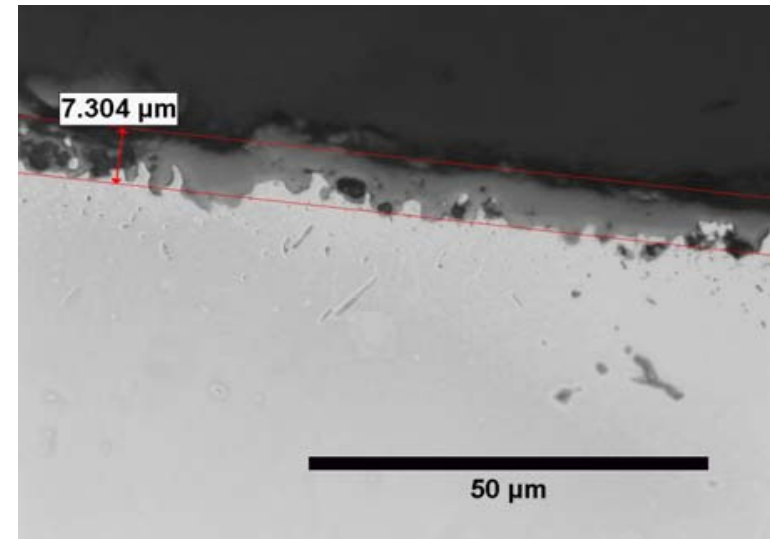

A100C1 - 100 hours

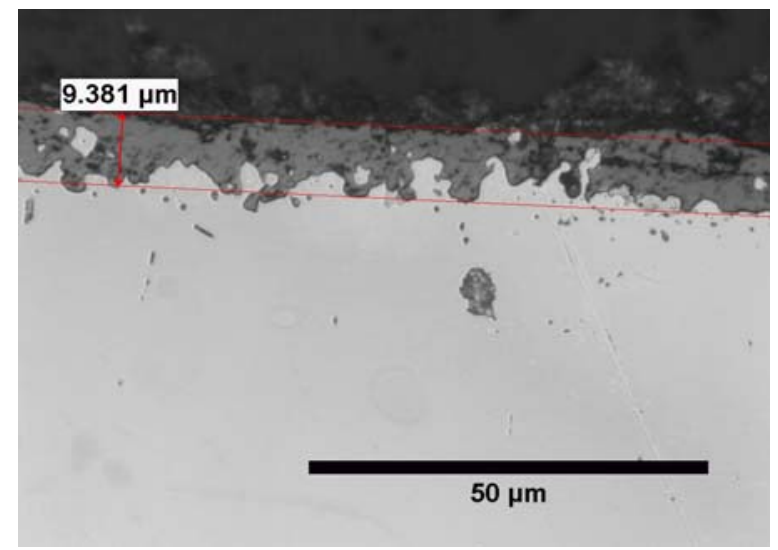

A300C1 - 300 hours

Photomicrographs Showing Surface Scale in Transverse Cross-Sections of Hastelloy-X Specimens After 10, 30, 100, and 300 Hours in Air. 


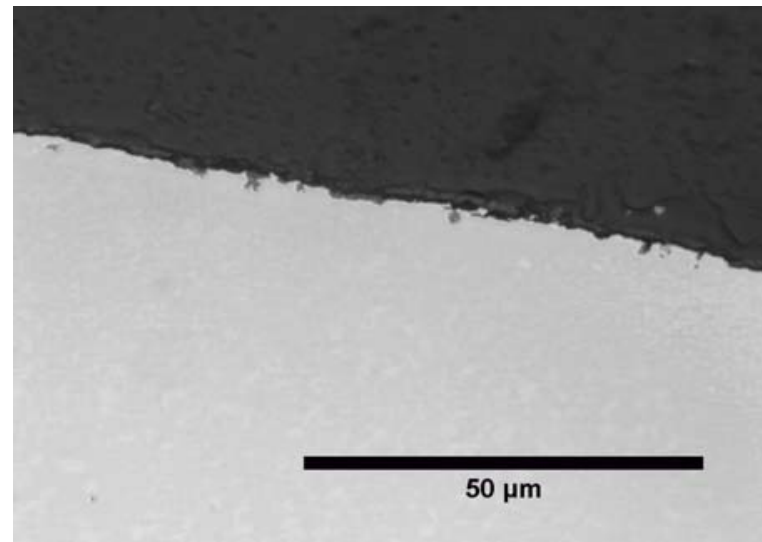

A10D1 - 10 hours

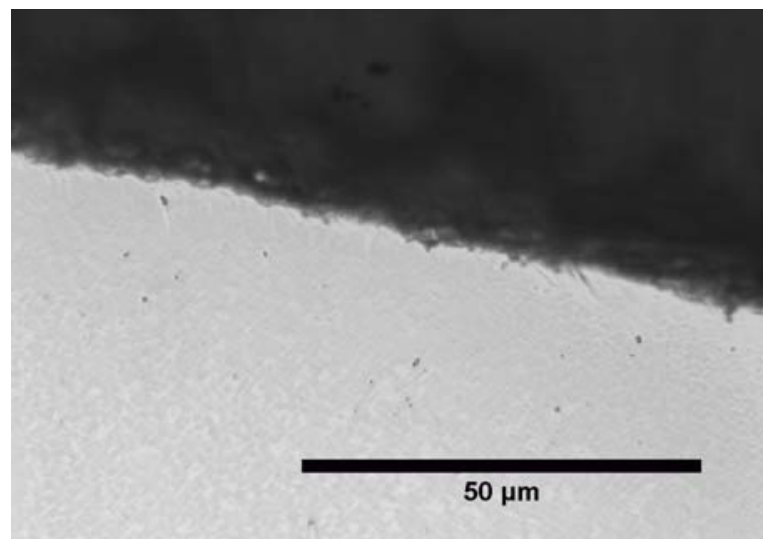

A30D1 - 30 hours

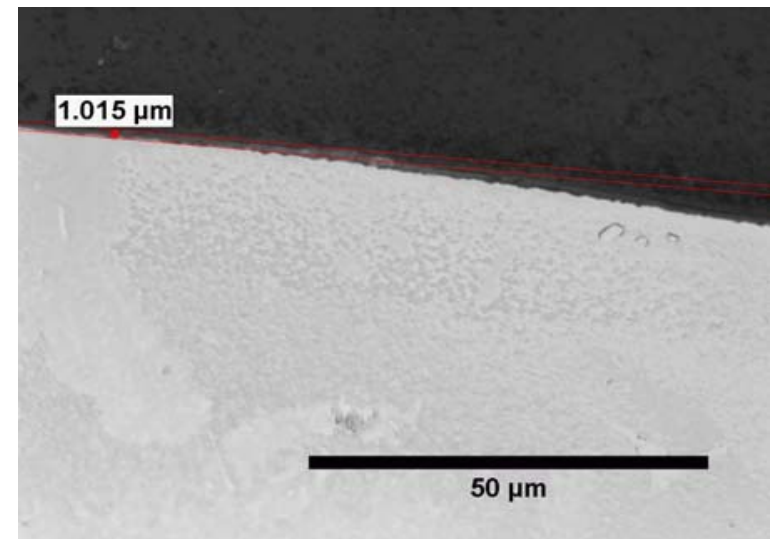

A100D1 - 100 hours

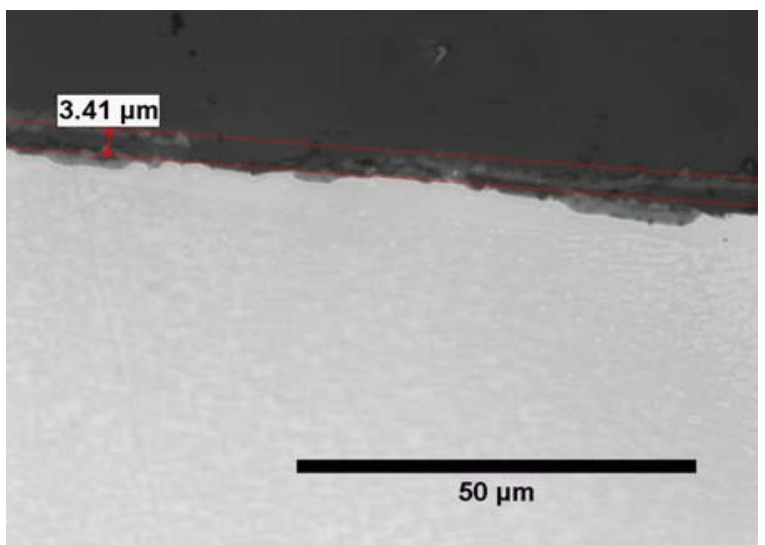

A300D1 - 300 hours

Photomicrographs Showing Surface Scale in Transverse Cross-Sections of Alloy N5 Specimens After 10, 30, 100, and 300 Hours in Air. 


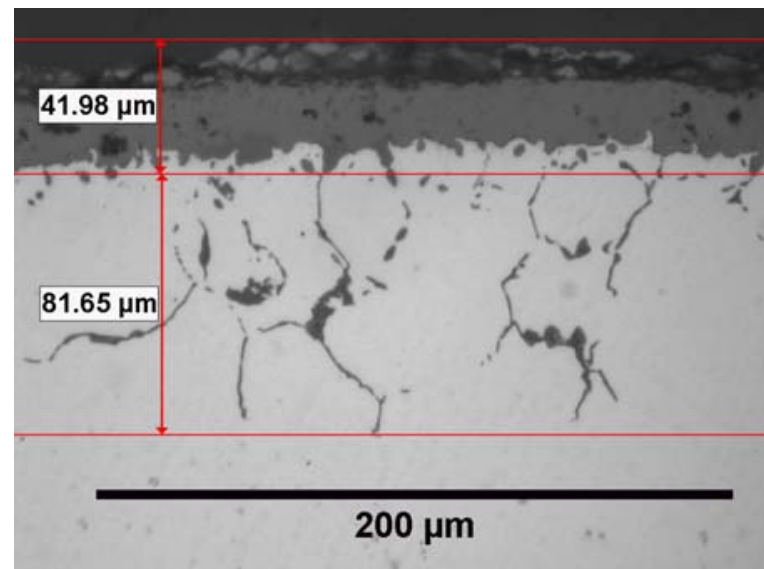

A10E1 - 10 hours

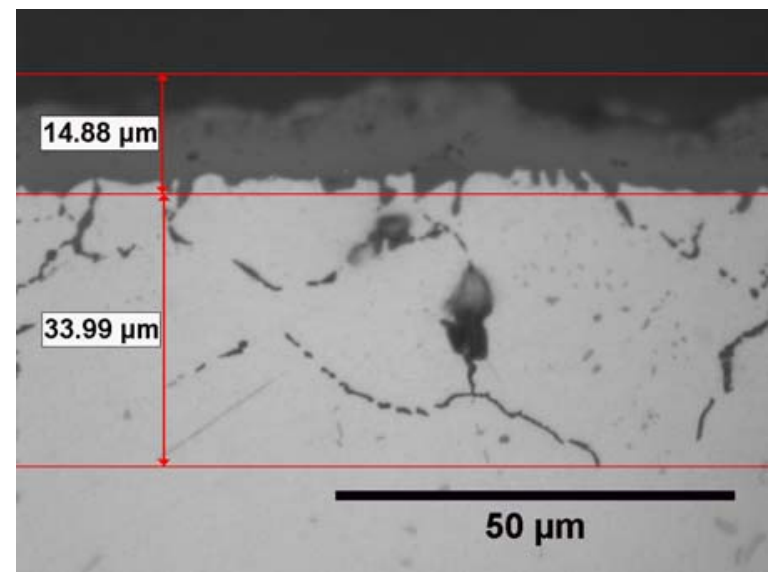

A30E1 - 30 hours

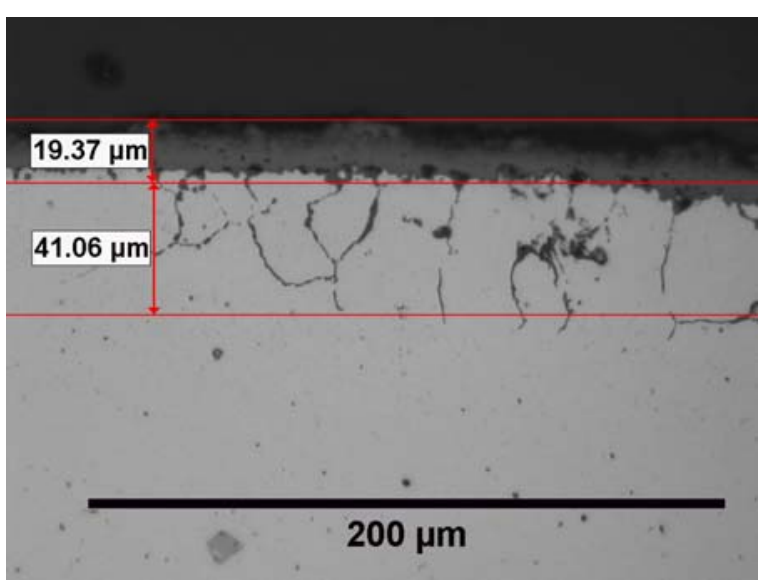

A100E1 - 100 hours

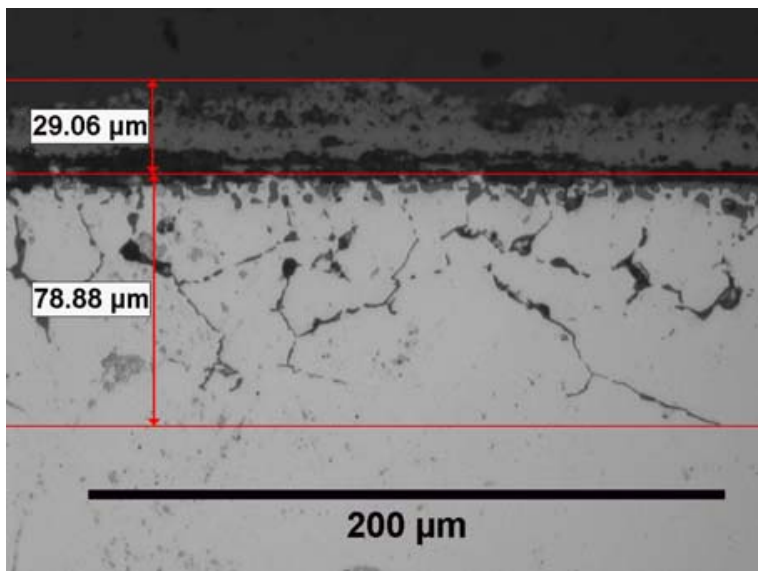

A300E1 - 300 hours

Photomicrographs Showing Surface Scale in Transverse Cross-Sections of Alloy Nimonic 263 Specimens After 10, 30, 100, and 300 Hours in Air. 


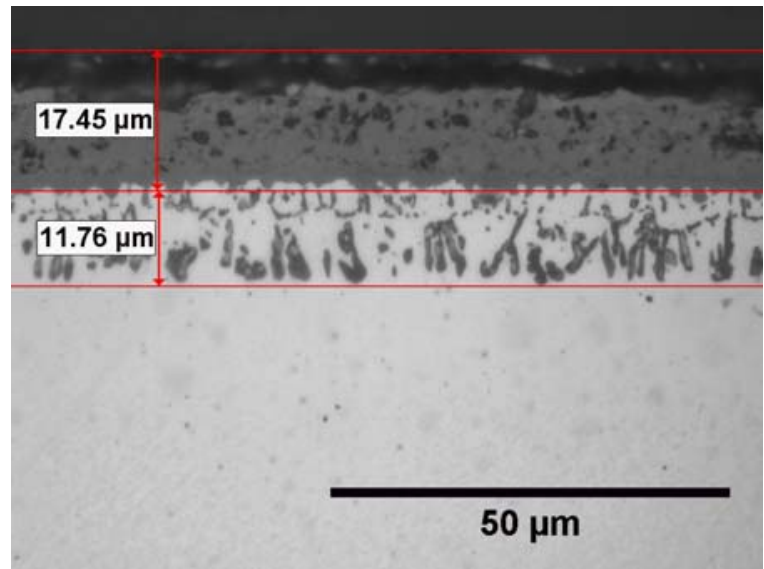

A10F1 - 10 hours

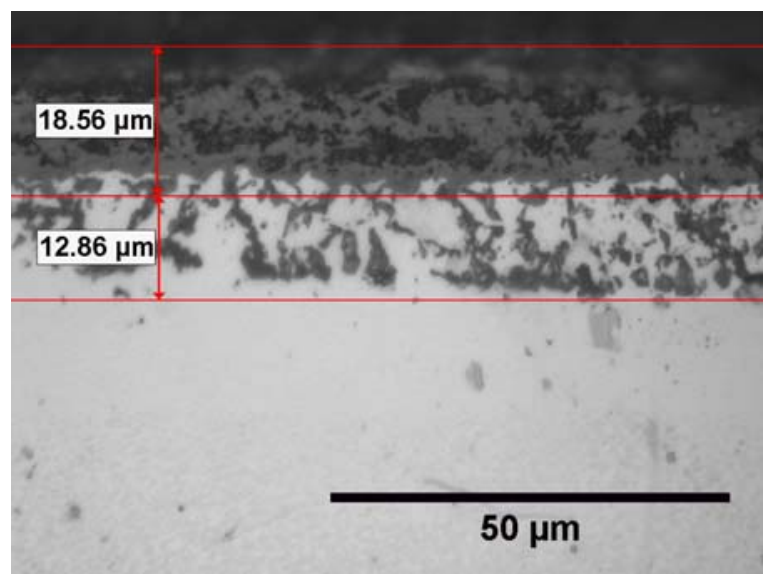

A30F1 - 30 hours

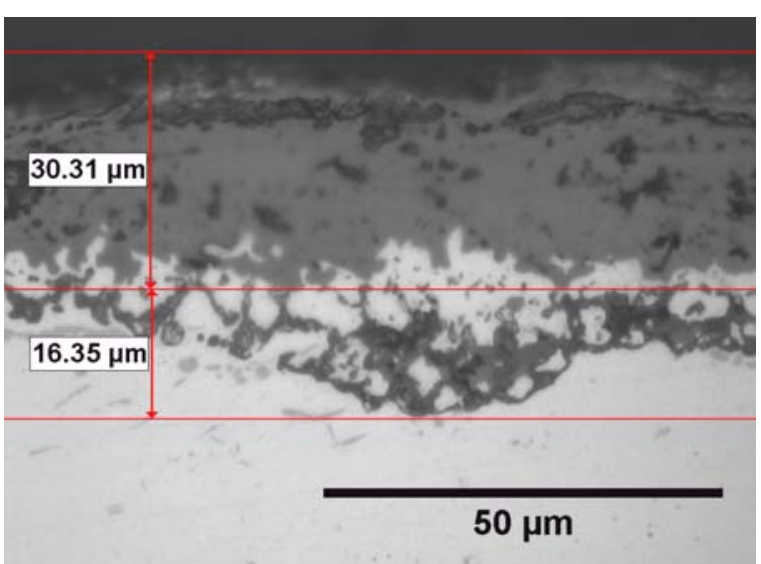

A100F1 - 100 hours

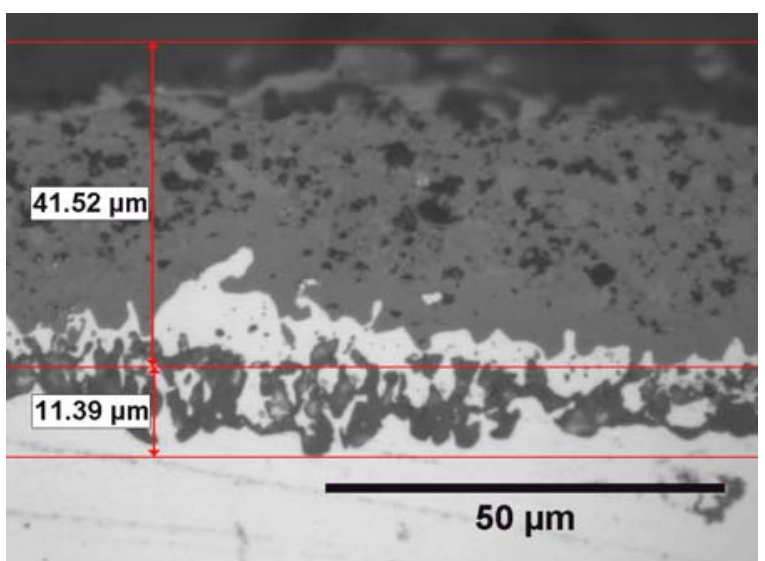

A300F1 - 300 hours

Photomicrographs Showing Surface Scale in Transverse Cross-Sections of Alloy GTD111 Specimens After 10, 30, 100, and 300 Hours in Air. 


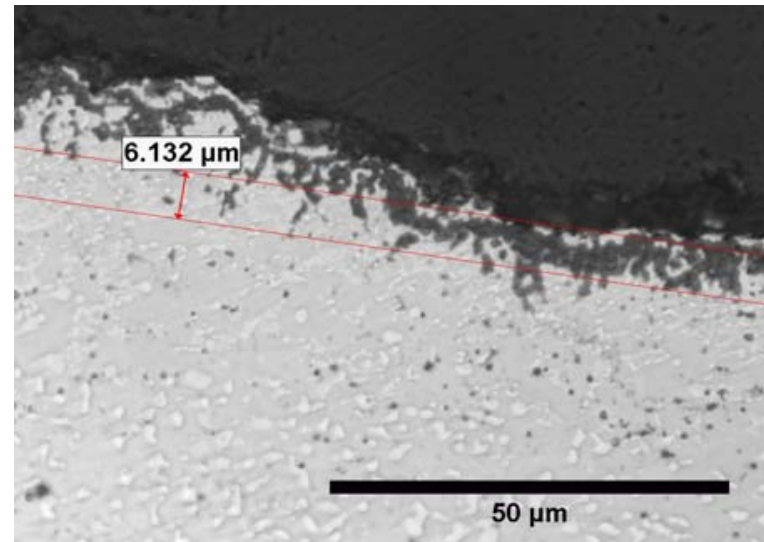

A10G1 - 10 hours

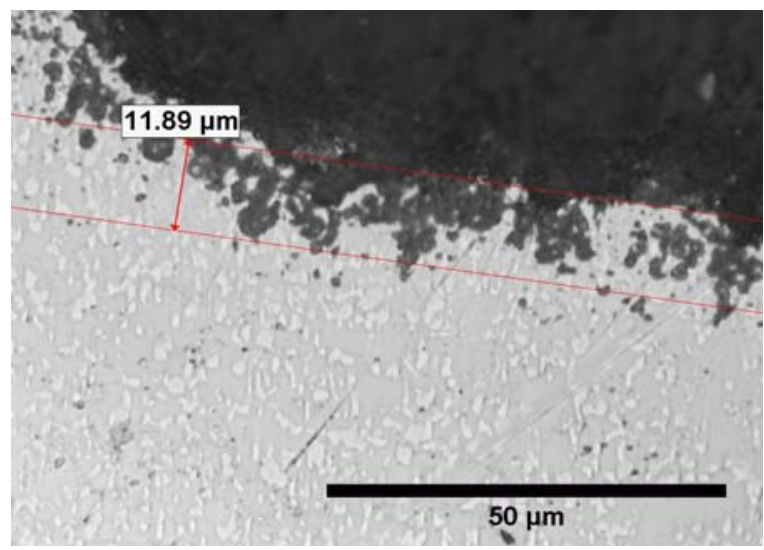

A30G1 - 30 hours

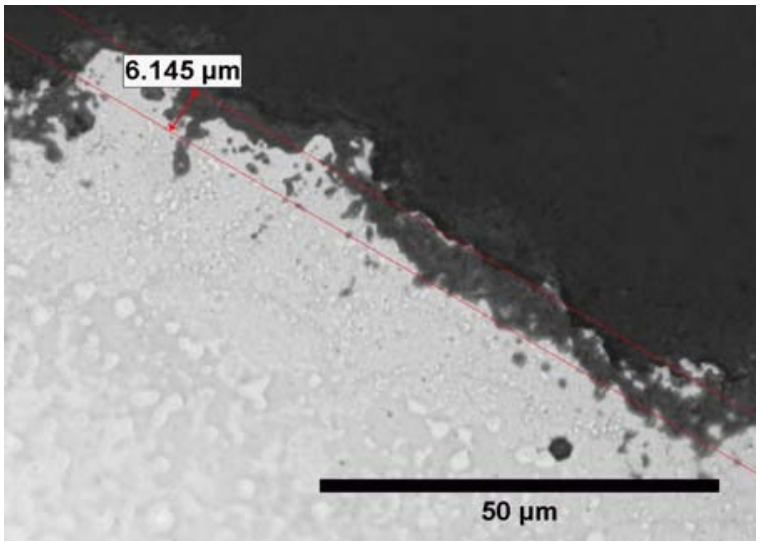

A100G1 - 100 hours

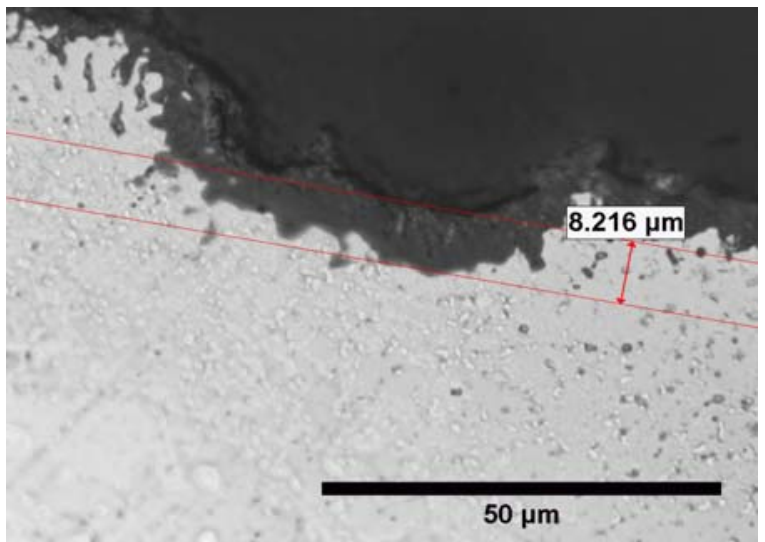

A300G1 - 300 hours

Photomicrographs Showing Surface Scale in Transverse Cross-Sections of Alloy N5 + NiCrAlY Specimens After 10, 30, 100, and 300 Hours in Air. 


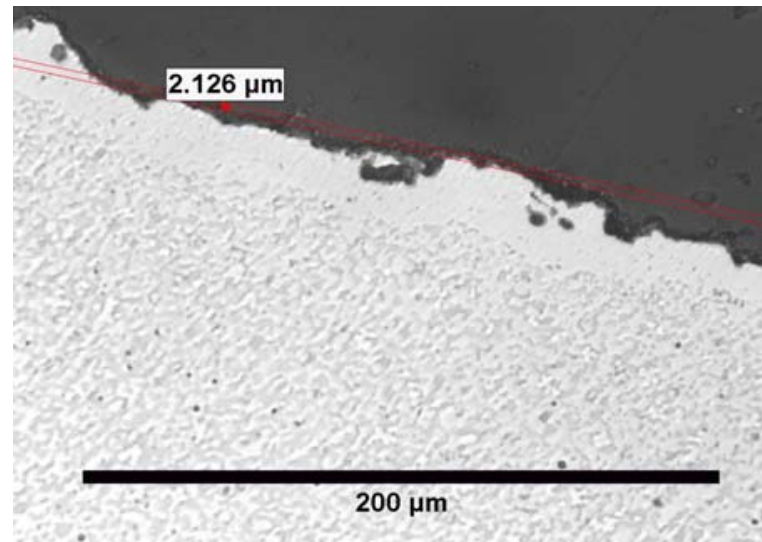

$\mathrm{A} 10 \mathrm{H} 1$ - 10 hours

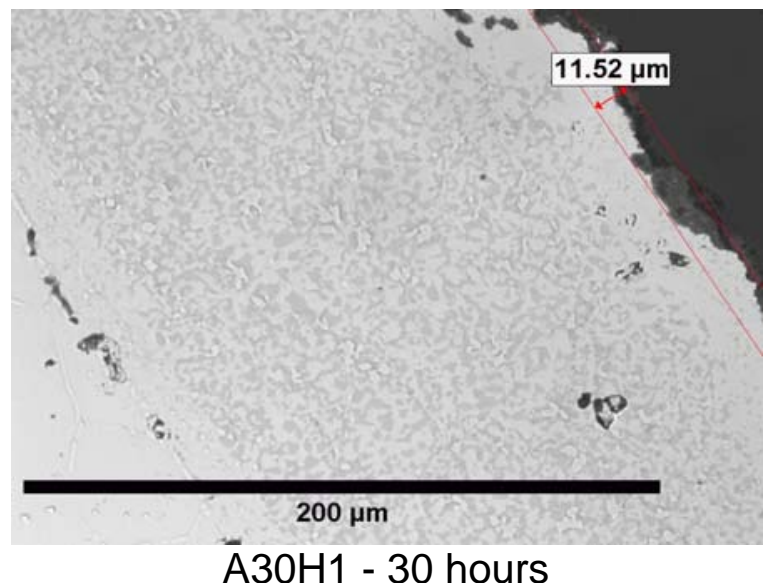

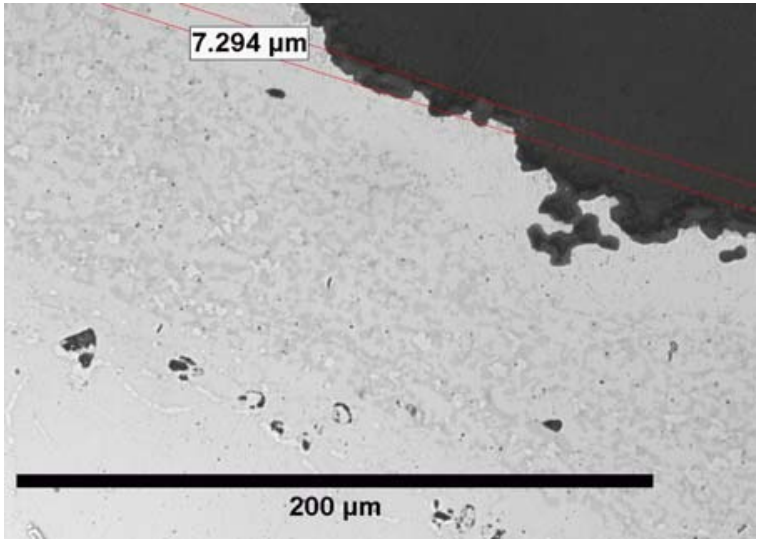

$\mathrm{A} 100 \mathrm{H} 1$ - 100 hours

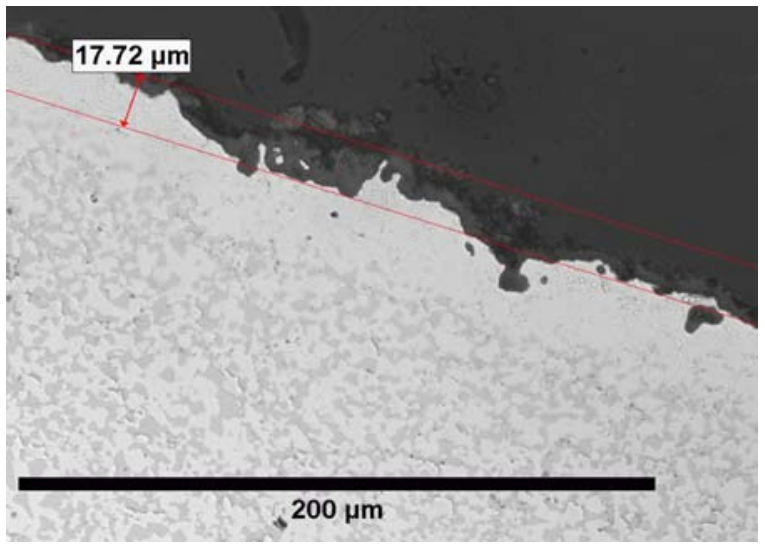

$\mathrm{A} 300 \mathrm{H} 1-300$ hours

Photomicrographs Showing Surface Scale in Transverse Cross-Sections of Alloy Nimonic 263 + CoNiCrAlY Specimens After 10, 30, 100, and 300 Hours in Air. 


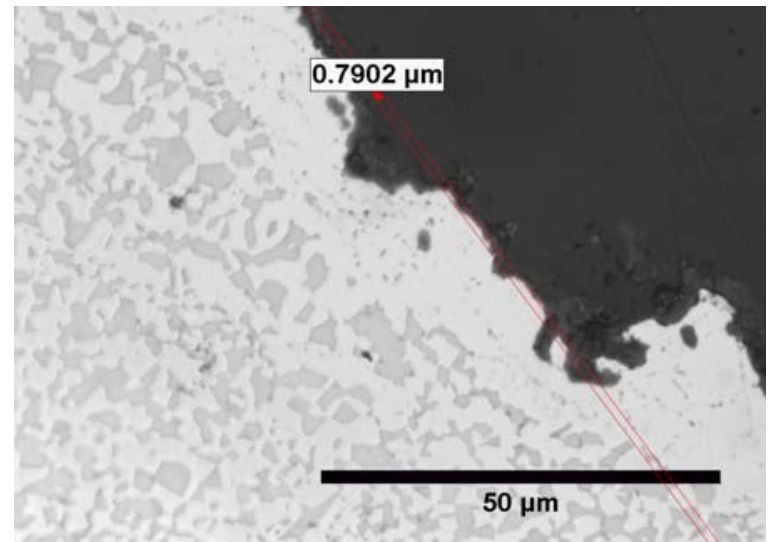

A10K1 - 10 hours

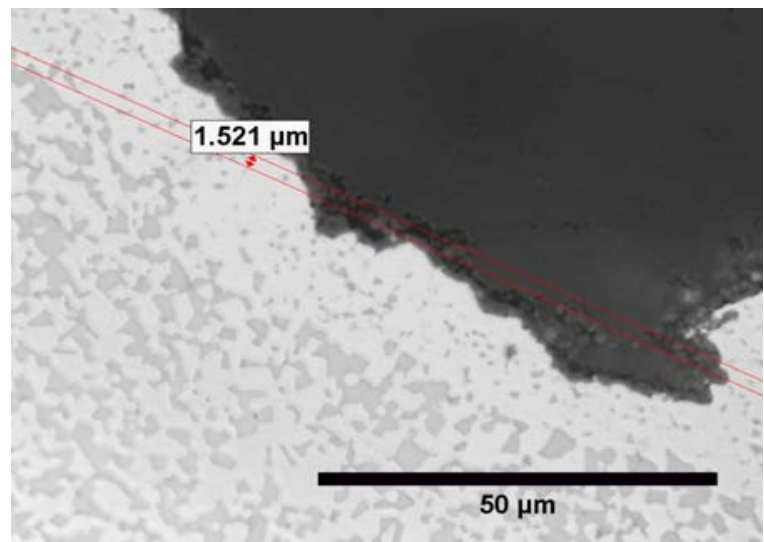

A30K1 - 30 hours

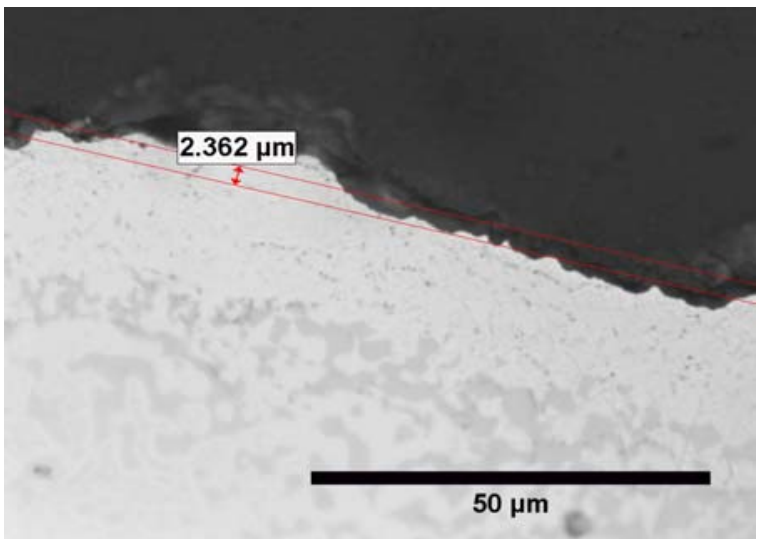

A100K1 - 100 hours

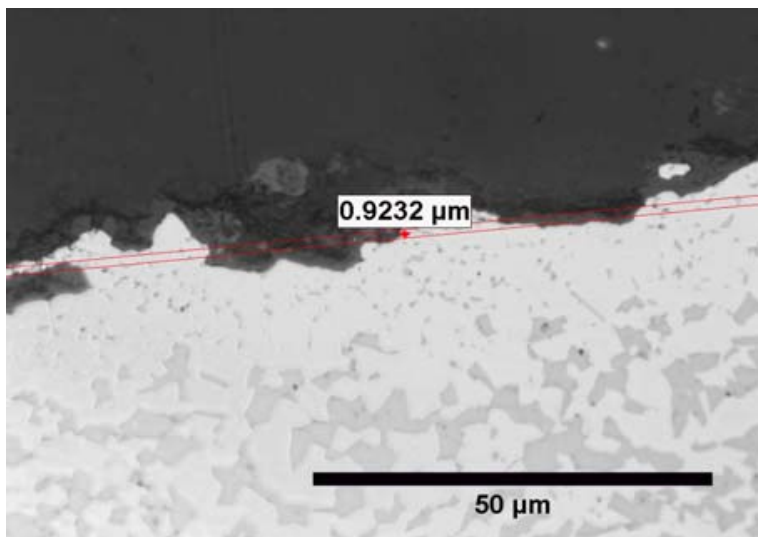

A300K1 - 300 hours

Photomicrographs Showing Surface Scale in Transverse Cross-Sections of Alloy GTD111 + CoNiCrAlY Specimens After 10, 30, 100, and 300 Hours in Air. 


\section{Dry $\mathrm{H}_{2} \underline{S}$}

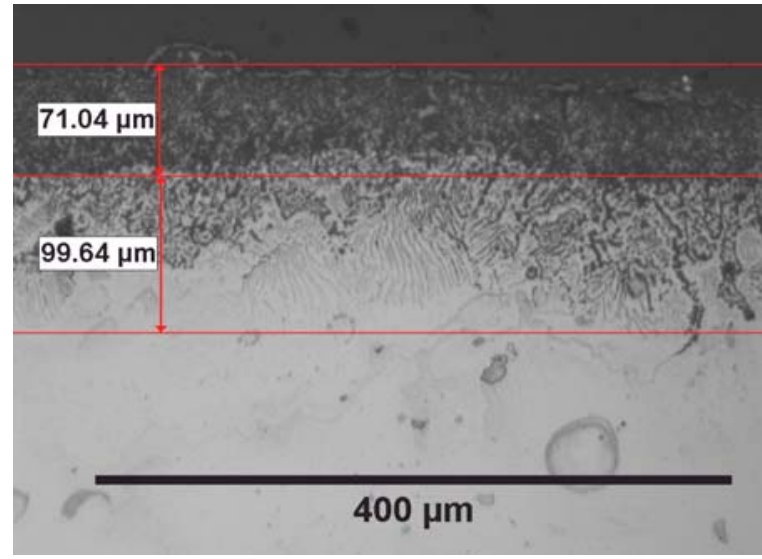

H10A1 - 10 hours

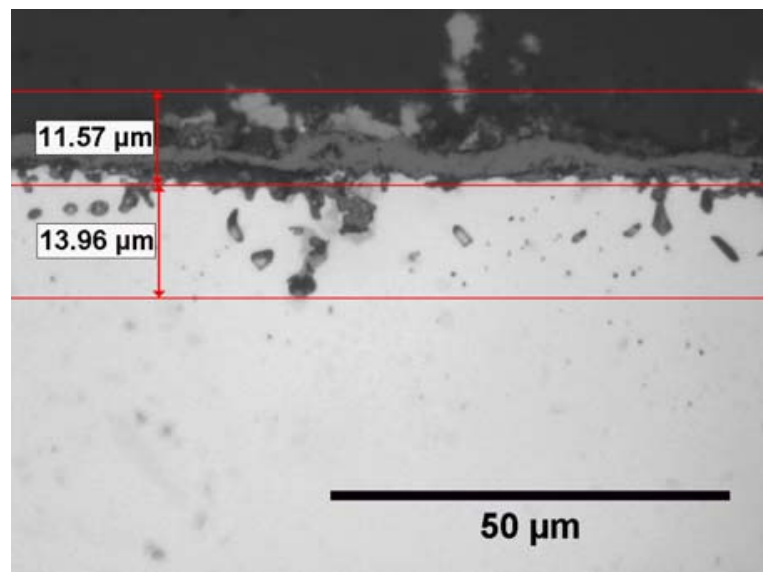

H30A3 - 30 hours

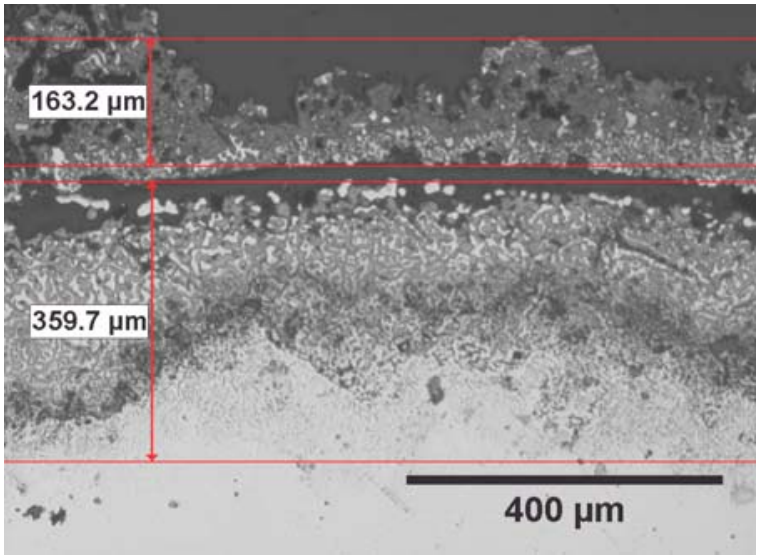

H100A1 - 100 hours

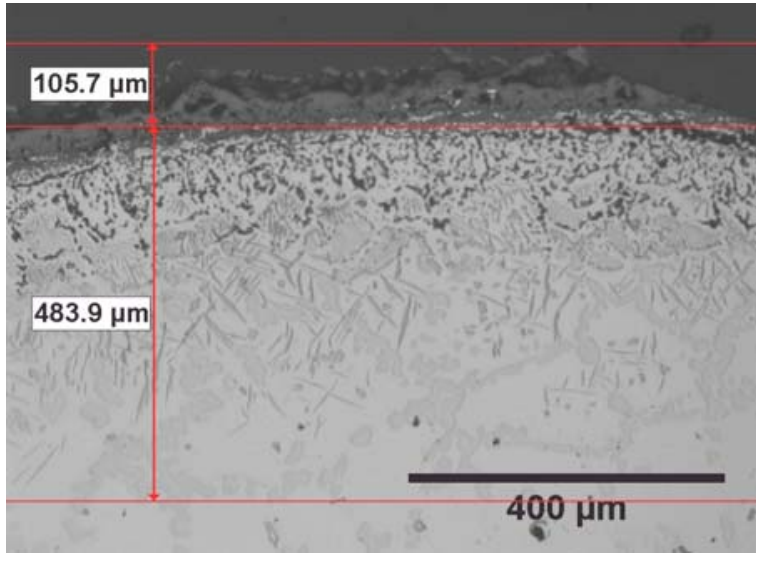

H300A1 - 300 hours

Photomicrographs Showing Surface Scale in Transverse Cross-Sections of FSC-414 Specimens After 10, 30, 100, and 300 Hours in 100 ppm $\mathrm{H}_{2} \mathrm{~S} /$ Balance $\mathrm{N}_{2}$. 


\section{Dry $\mathrm{H}_{2} \underline{\mathbf{S}}$}

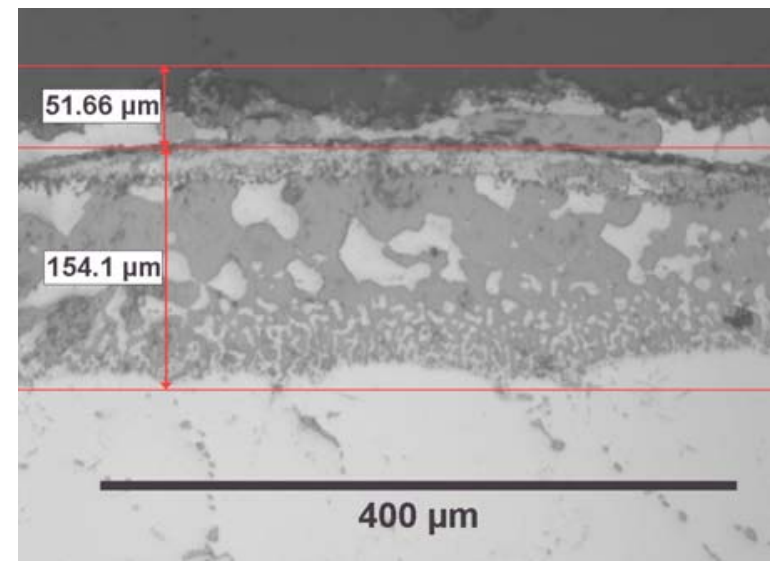

H10B1 - 10 hours

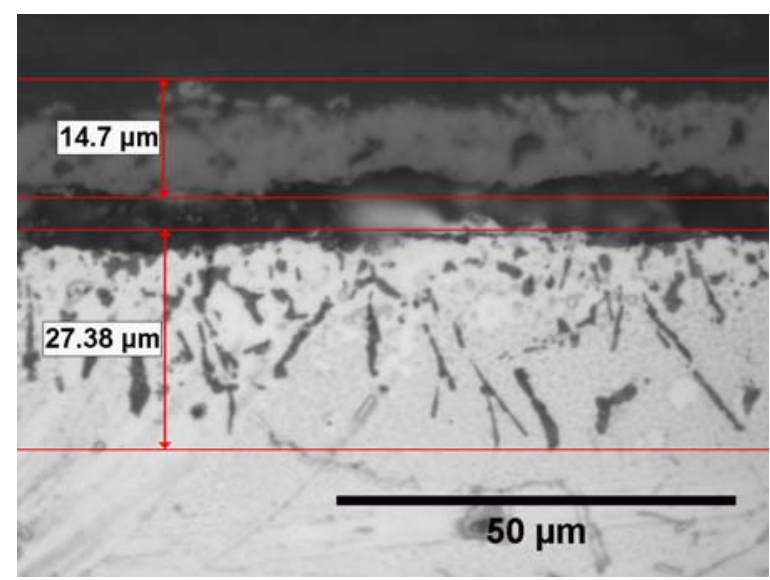

H30B3 - 30 hours

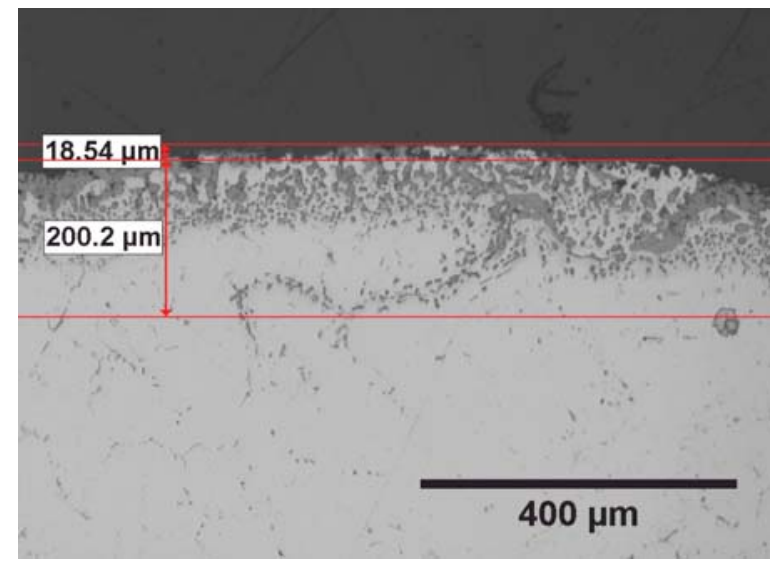

H100B1 - 100 hours

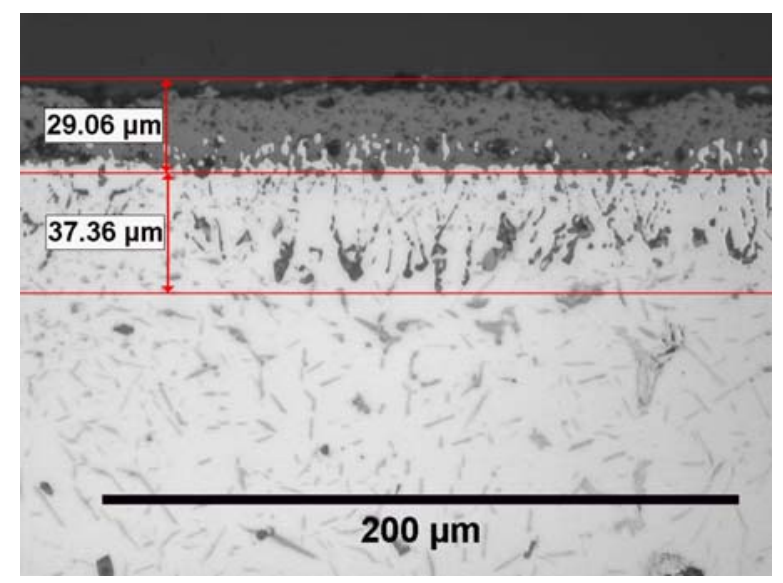

H300B1 - 300 hours

Photomicrographs Showing Surface Scale in Transverse Cross-Sections of GTD-222 Specimens After 10, 30, 100, and 300 Hours in 100 ppm $\mathrm{H}_{2} \mathrm{~S}$ / Balance $\mathrm{N}_{2}$. 


\section{Dry $\mathrm{H}_{2} \underline{S}$}

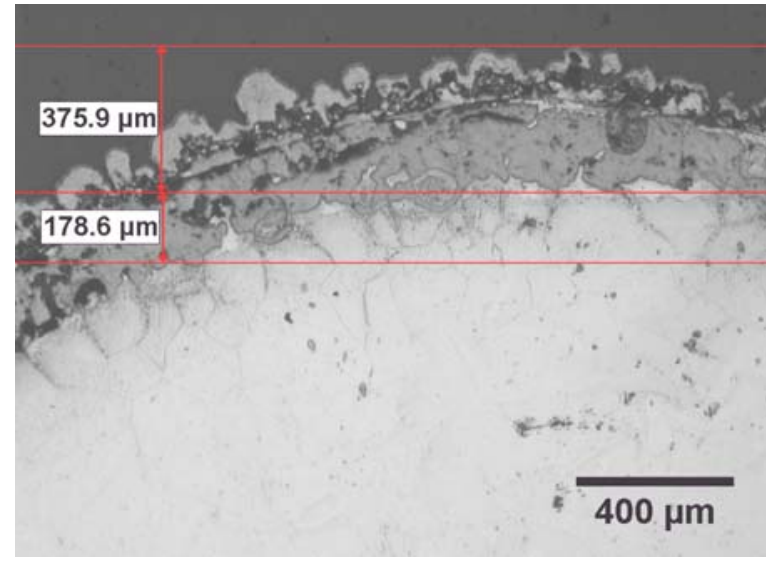

H10C1 - 10 hours

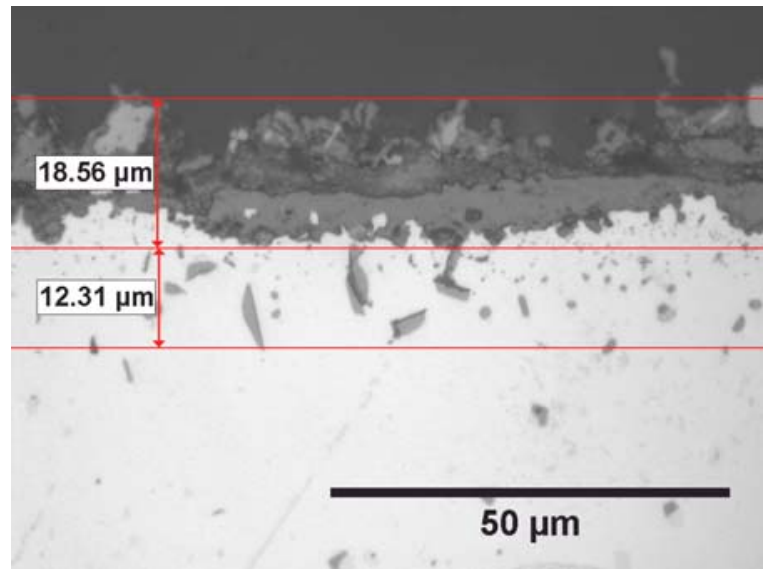

H30C3 - 30 hours

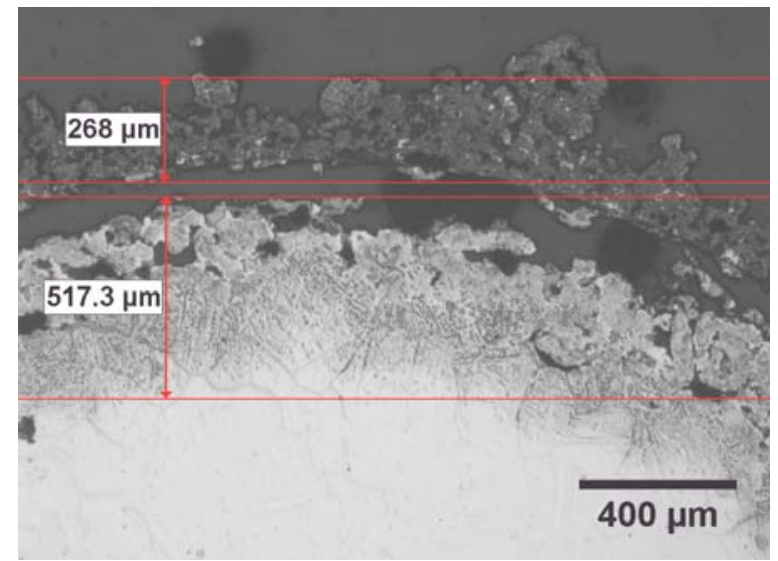

H100C1 - 100 hours

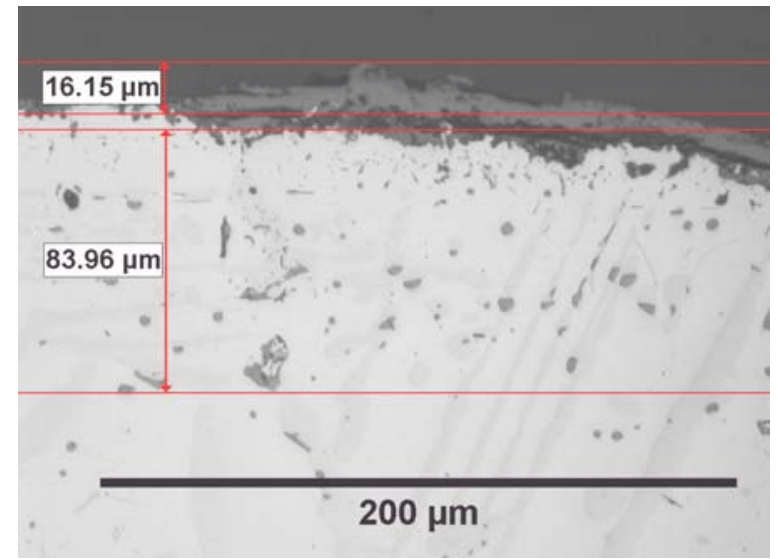

H300C1 - 300 hours

Photomicrographs Showing Surface Scale in Transverse Cross-Sections of Hastelloy- $X$ Specimens After 10, 30, 100, and 300 Hours in 100 ppm $\mathrm{H}_{2} \mathrm{~S}$ / Balance $\mathrm{N}_{2}$. 


\section{Dry $\mathrm{H}_{2} \underline{S}$}

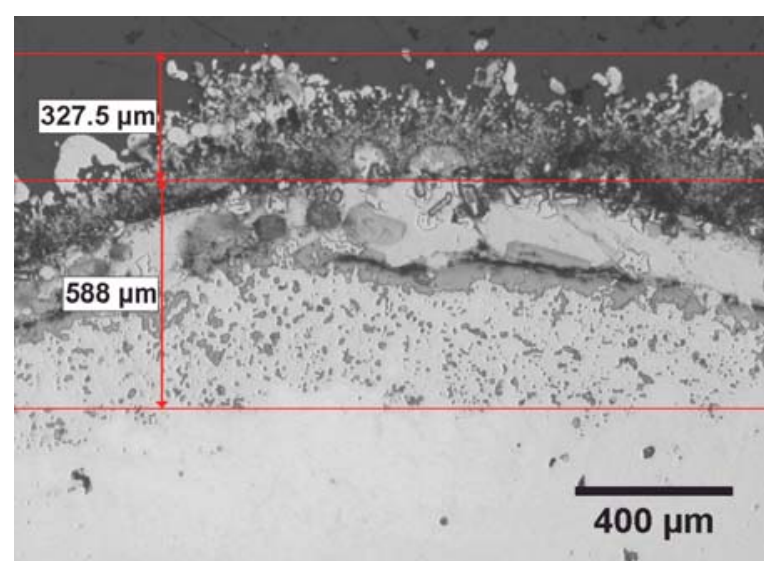

H10D1 - 10 hours

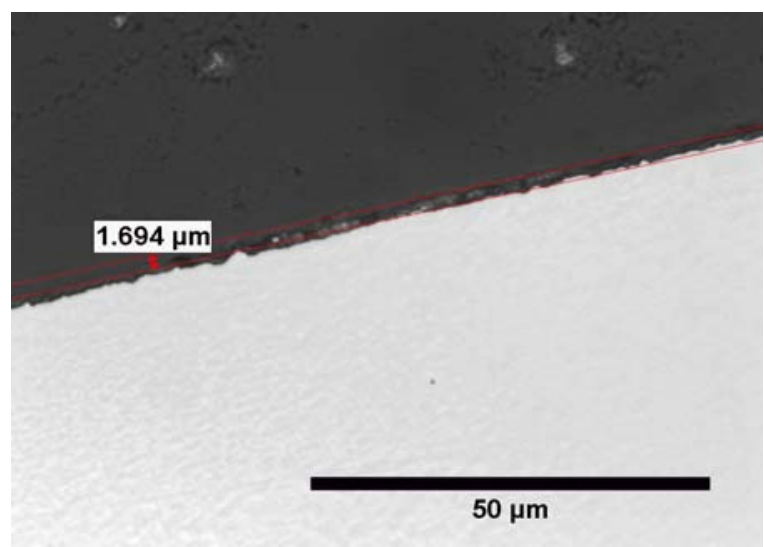

H30D3 - 30 hours

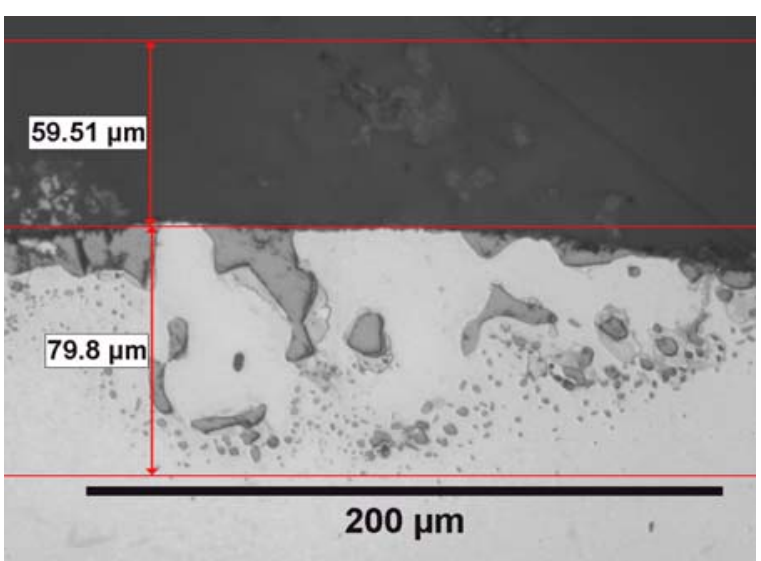

H100D1 - 100 hours

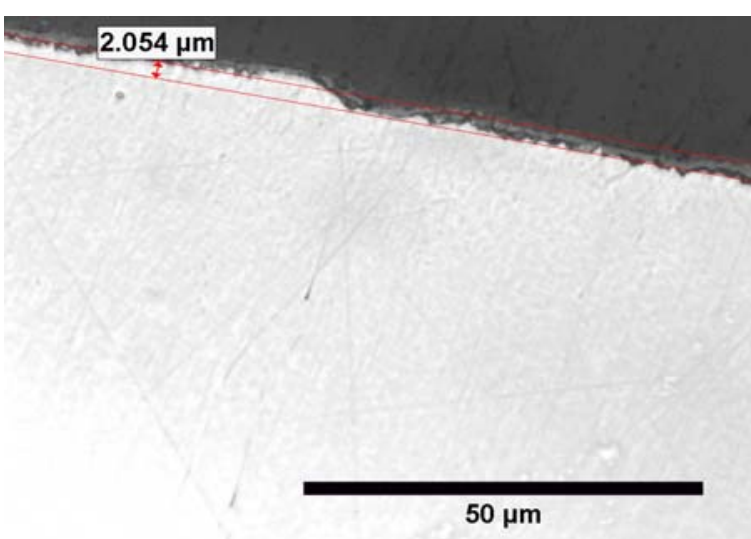

H300D1 - 300 hours

Photomicrographs Showing Surface Scale in Transverse Cross-Sections of Alloy N5 Specimens After 10, 30, 100, and 300 Hours in 100 ppm $\mathrm{H}_{2} \mathrm{~S} /$ Balance $\mathrm{N}_{2}$. 


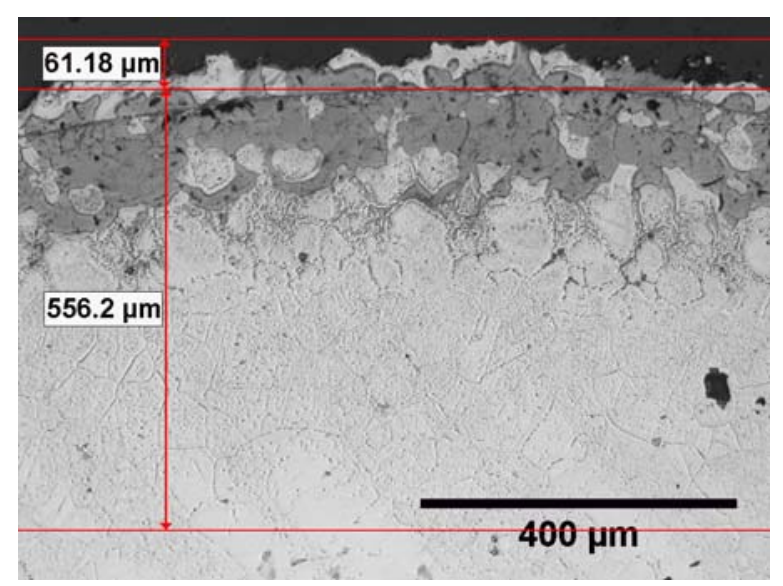

H10E1 - 10 hours

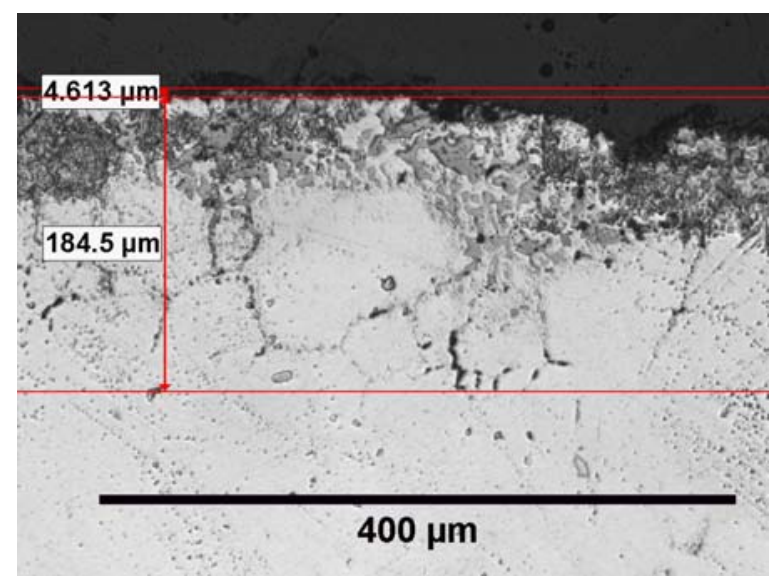

H30E3 - 30 hours

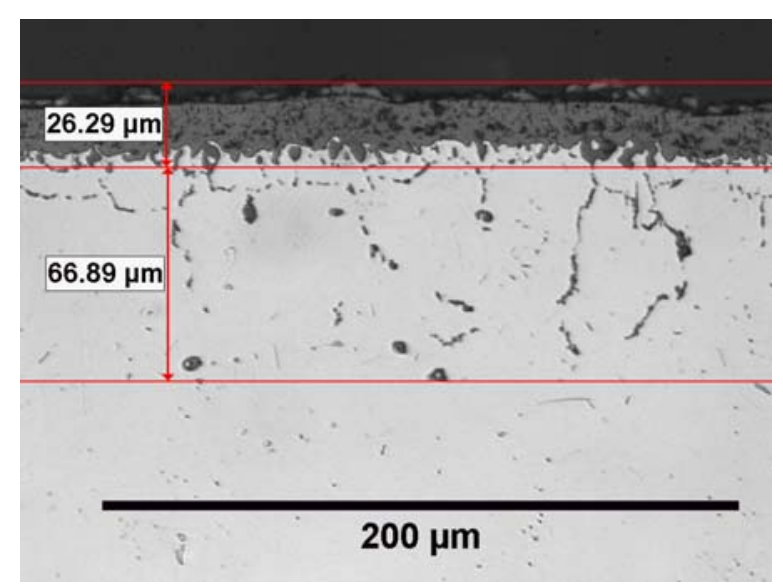

H100E1 - 100 hours

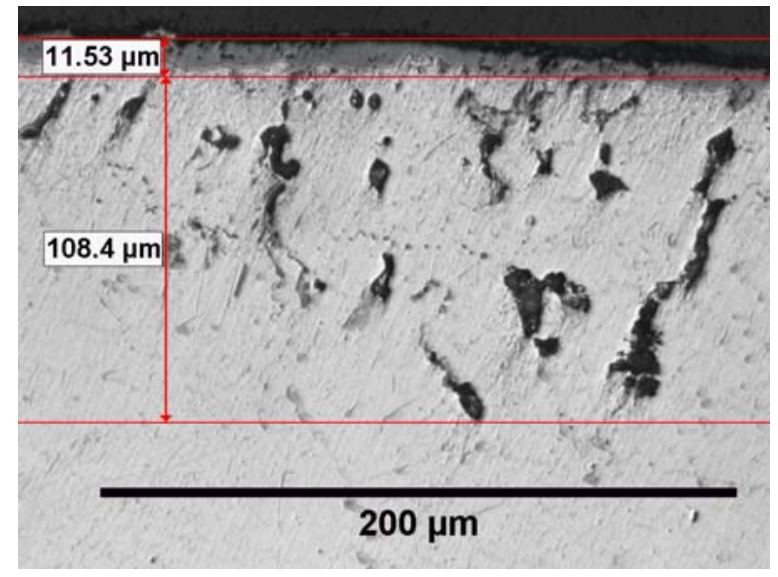

H300E1 - 300 hours

Photomicrographs Showing Surface Scale in Transverse Cross-Sections of Alloy Nimonic 263 Specimens After 10, 30, 100, and 300 Hours in 100 ppm $\mathrm{H}_{2} \mathrm{~S}$ / Balance $\mathrm{N}_{2}$. 


\section{Dry $\mathrm{H}_{2} \underline{\mathrm{S}}$}

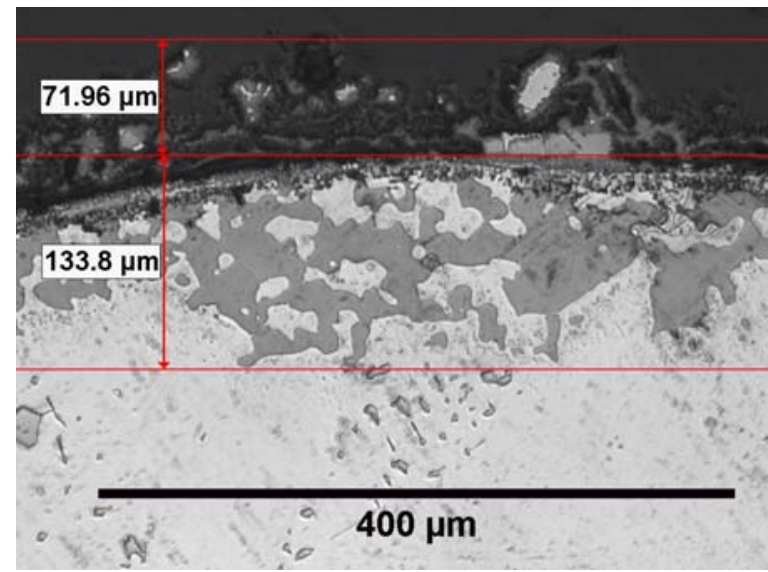

H10F1 - 10 hours

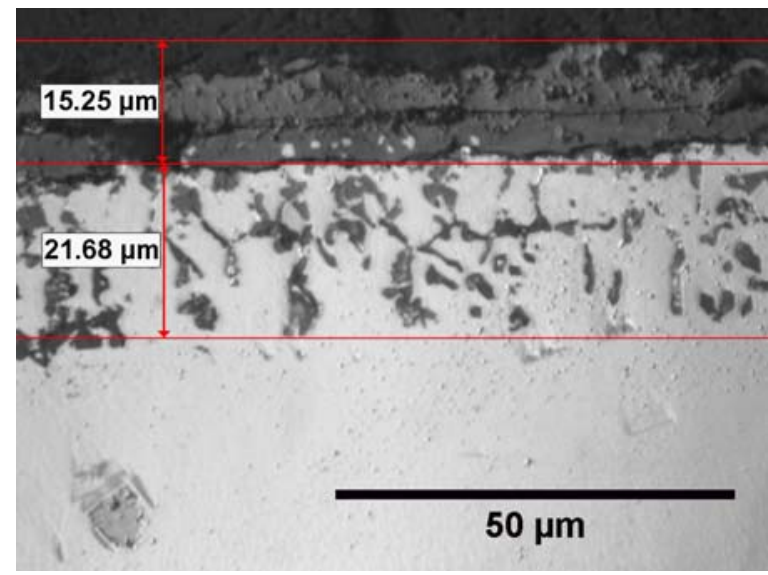

H30F3 - 30 hours

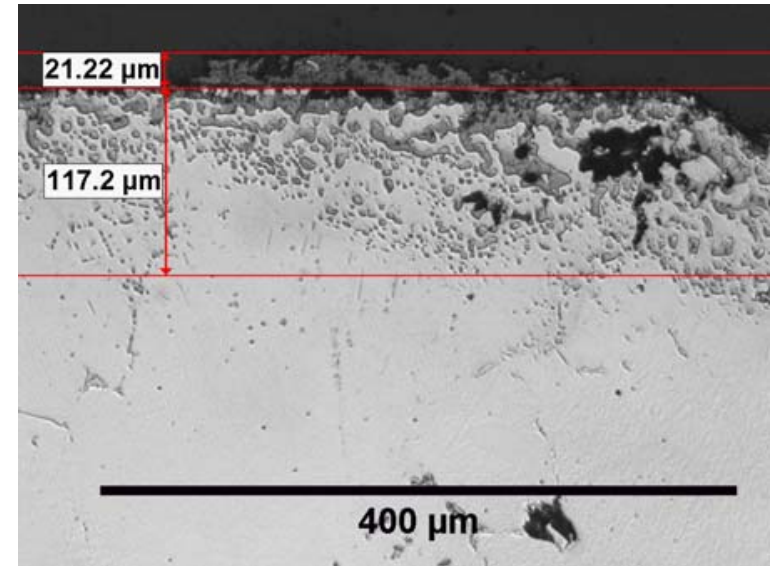

H100F1 - 100 hours

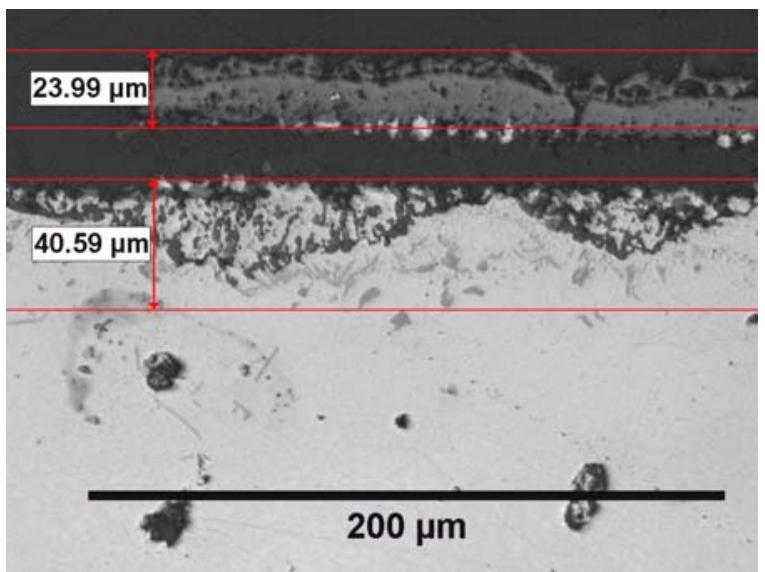

H300F1 - 300 hours

Photomicrographs Showing Surface Scale in Transverse Cross-Sections of Alloy GTD111 Specimens After 10, 30, 100, and 300 Hours in 100 ppm H $\mathrm{H}_{2} \mathrm{~S} /$ Balance $\mathrm{N}_{2}$. 


\section{Dry $\mathrm{H}_{2} \underline{\mathrm{S}}$}

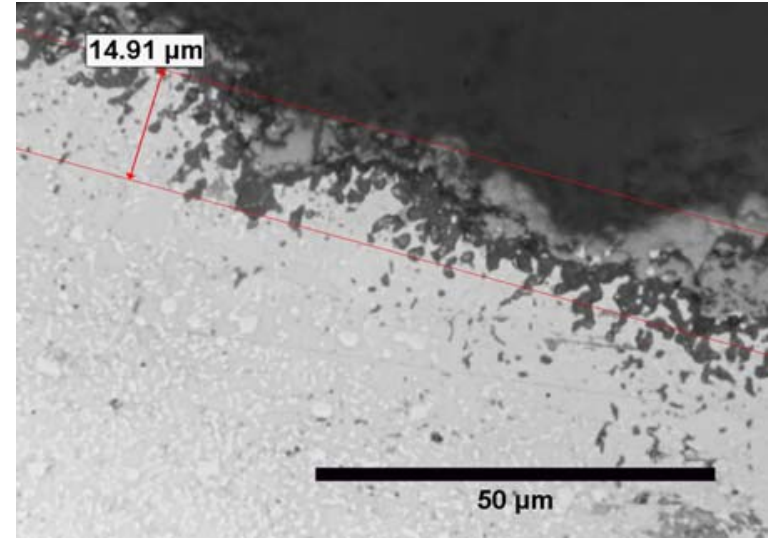

H10G1 - 10 hours

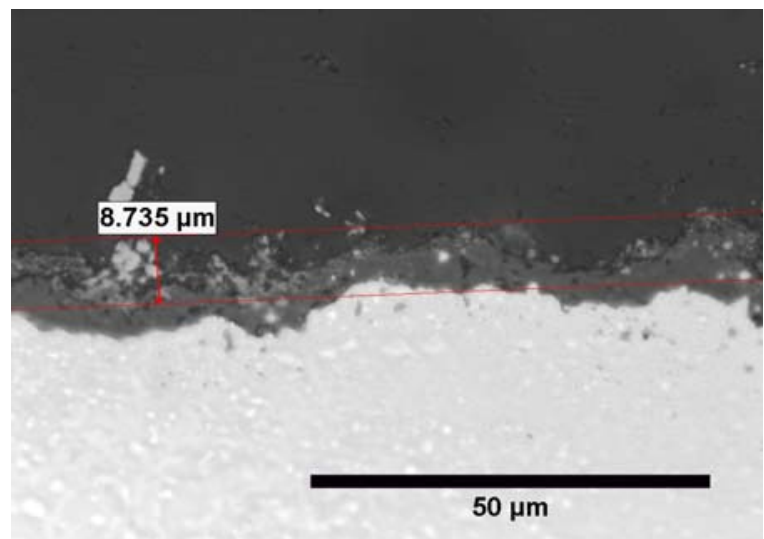

H30G3 - 30 hours

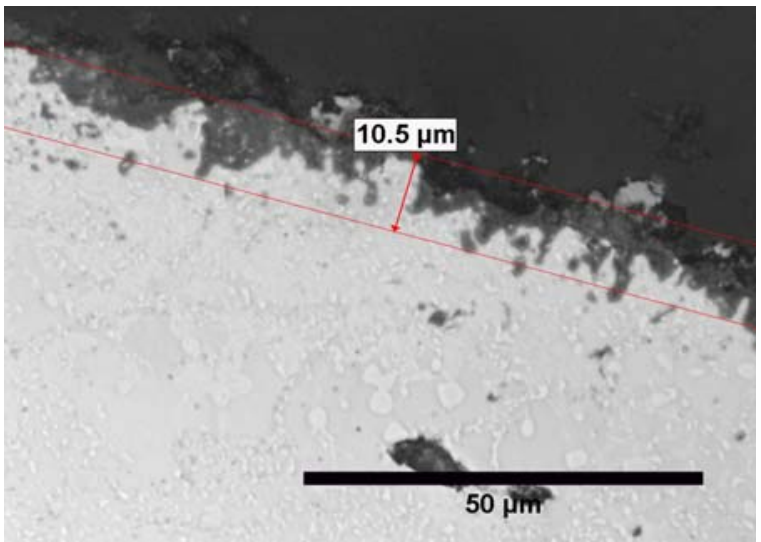

H100G1 - 100 hours

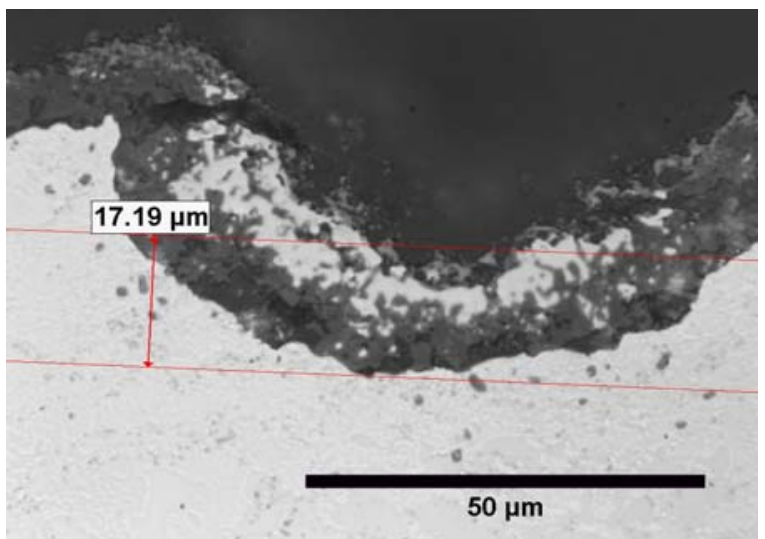

H300G1 - 300 hours

Photomicrographs Showing Surface Scale in Transverse Cross-Sections of Alloy N5 + NiCrAlY Specimens After 10, 30, 100, and 300 Hours in 100 ppm $\mathrm{H}_{2} \mathrm{~S}$ / Balance $\mathrm{N}_{2}$. 


\section{Dry $\mathrm{H}_{2} \underline{\mathrm{S}}$}

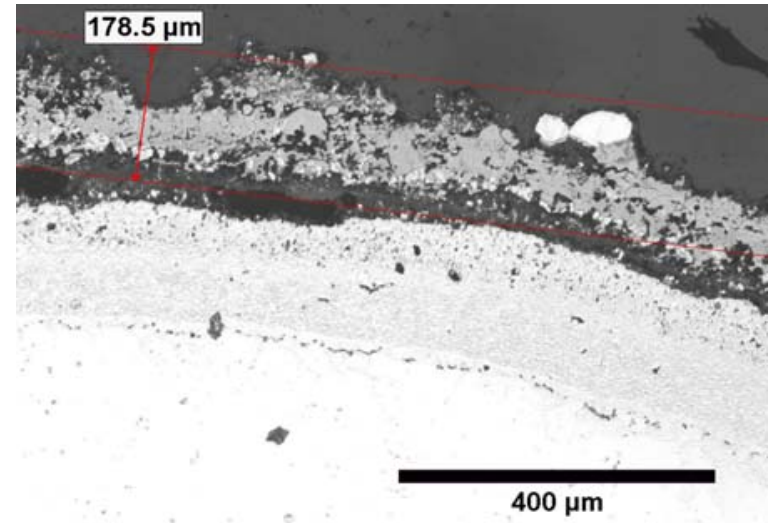

$\mathrm{H} 10 \mathrm{H} 1$ - 10 hours

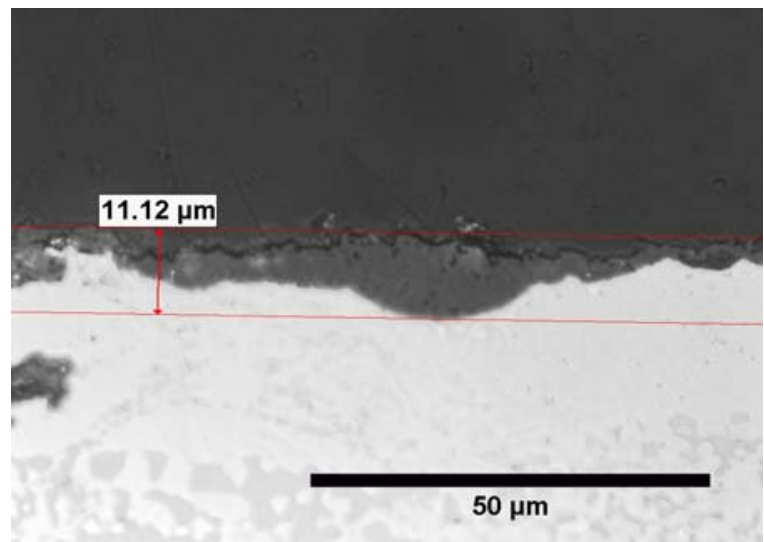

$\mathrm{H} 30 \mathrm{H} 3$ - 30 hours

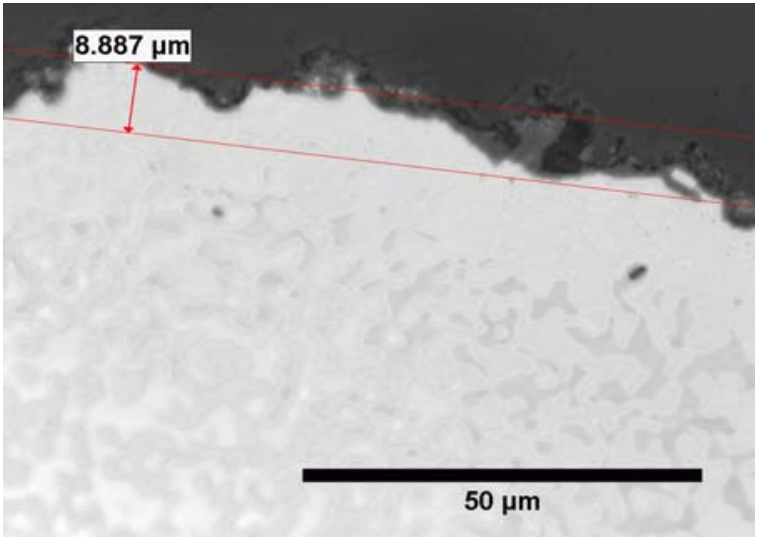

$\mathrm{H} 100 \mathrm{H} 1$ - 100 hours

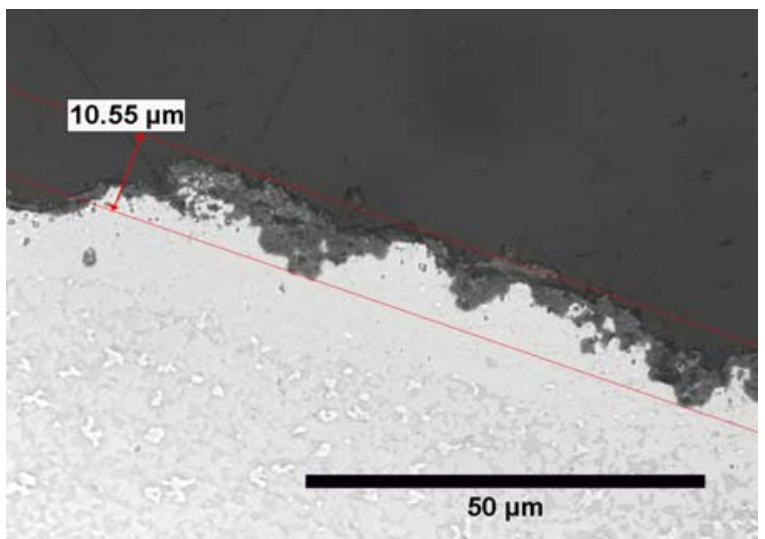

$\mathrm{H} 300 \mathrm{H} 1-300$ hours

Photomicrographs Showing Surface Scale in Transverse Cross-Sections of Alloy Nimonic 263 + CoNiCrAlY Specimens After 10, 30, 100, and 300 Hours in 100 ppm H $\mathrm{H}_{2} \mathrm{~S}$ / Balance $\mathrm{N}_{2}$. 


\section{Dry $\mathrm{H}_{2} \underline{S}$}

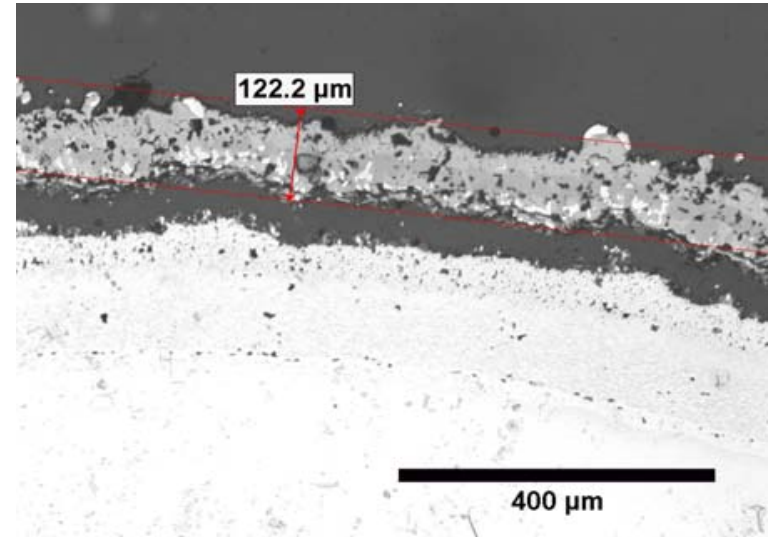

H10K1 - 10 hours

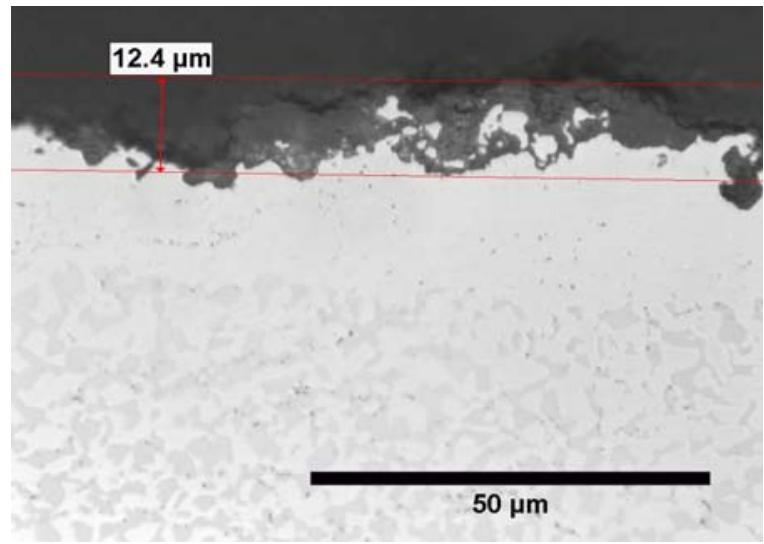

H3OK3 - 30 hours

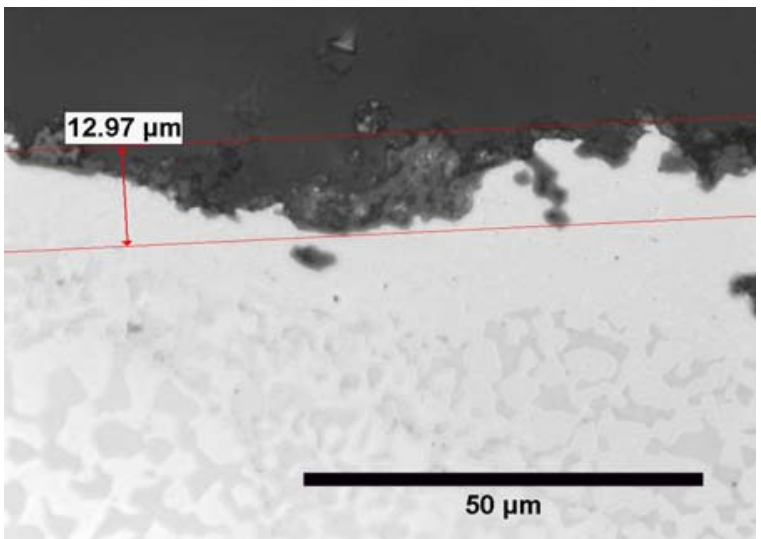

H100K1 - 100 hours

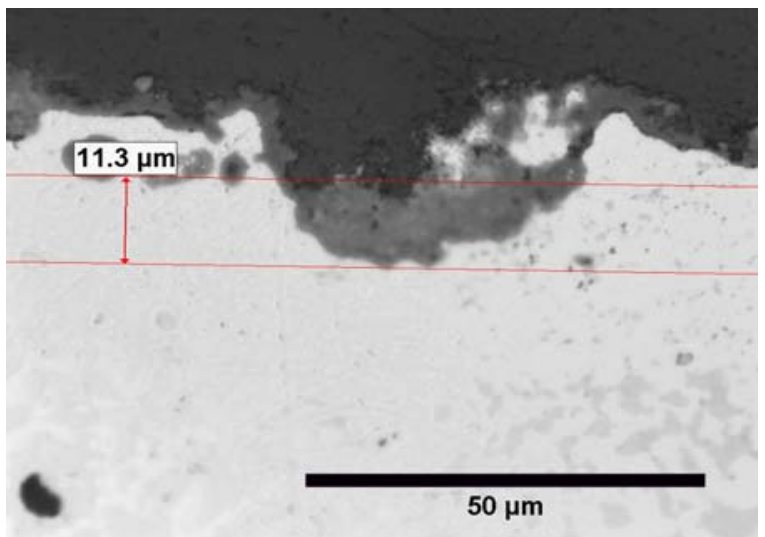

H300K1 - 300 hours

Photomicrographs Showing Surface Scale in Transverse Cross-Sections of Alloy GTD111 + CoNiCrAlY Specimens After 10, 30, 100, and 300 Hours in 100 ppm $\mathrm{H}_{2} \mathrm{~S}$ / Balance $\mathrm{N}_{2}$. 


\section{Wet $\mathrm{H}_{2} \underline{\mathrm{S}}$}

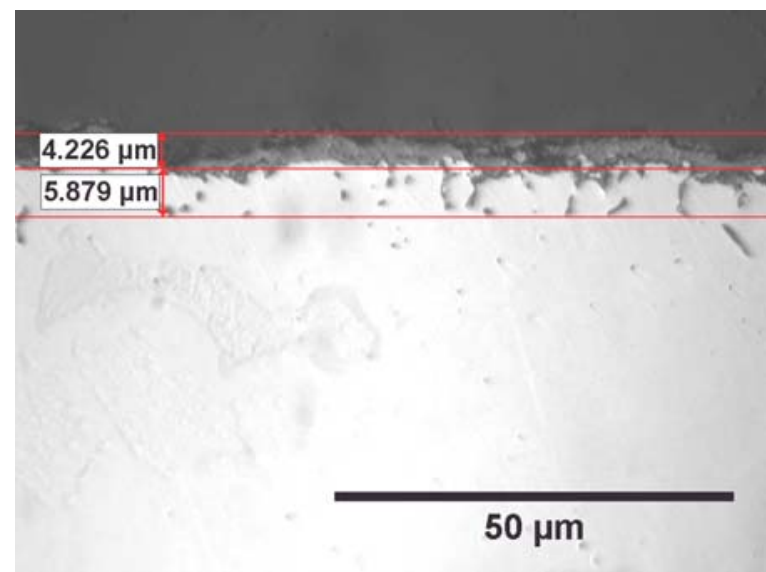

W10A1 - 10 hours

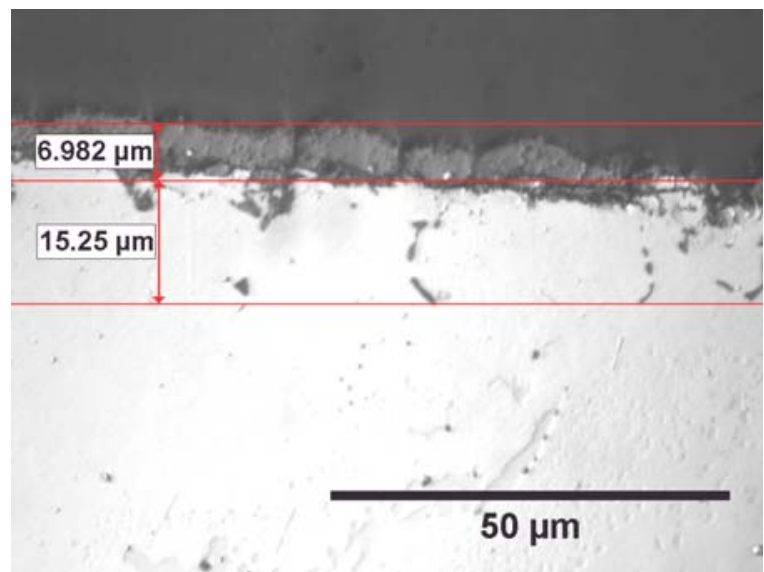

W30A1 - 30 hours

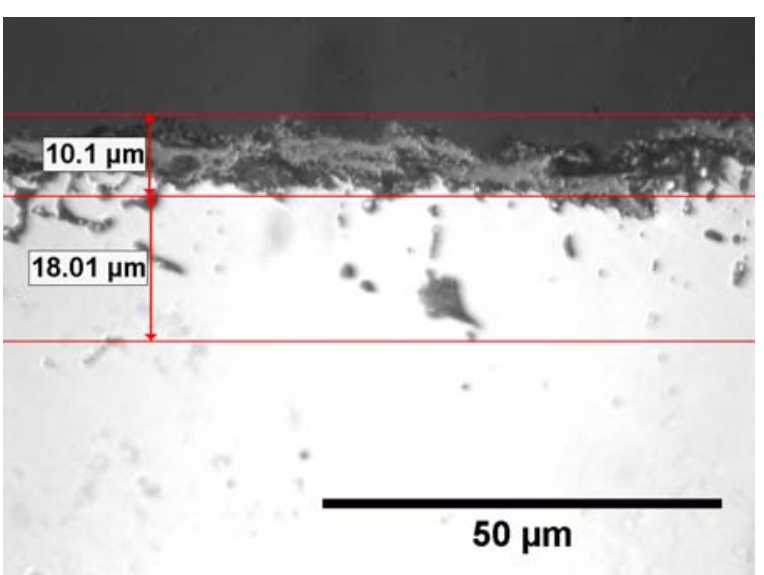

W100A1 - 100 hours

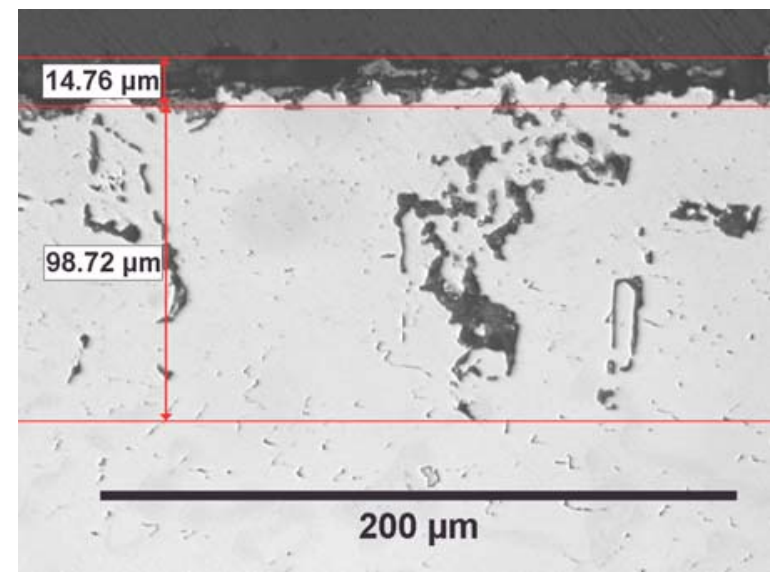

W300A1 - 300 hours

Photomicrographs Showing Surface Scale in Transverse Cross-Sections of FSC-414 Specimens After 10, 30, 100, and 300 Hours in Water-Saturated Mixture of 100 ppm $\mathrm{H}_{2} \mathrm{~S} /$ Balance $\mathrm{N}_{2}$. 


\section{Wet $\mathrm{H}_{2}$ S}

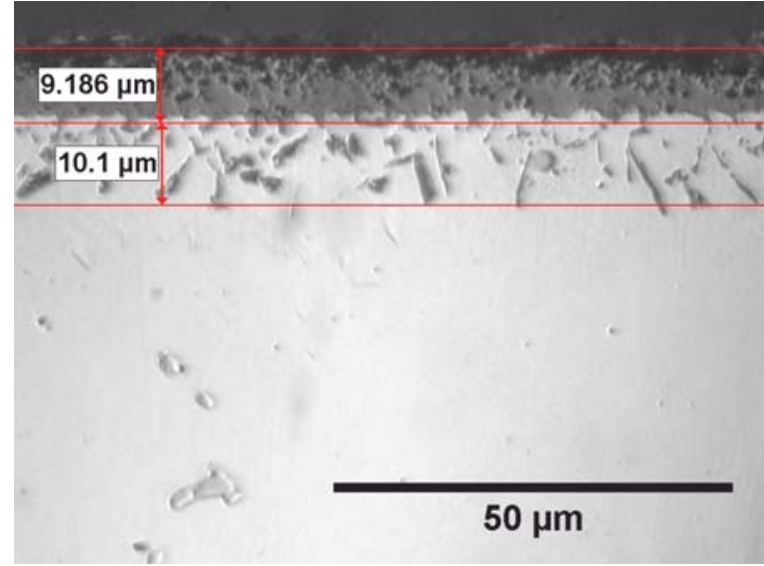

W10B1 - 10 hours

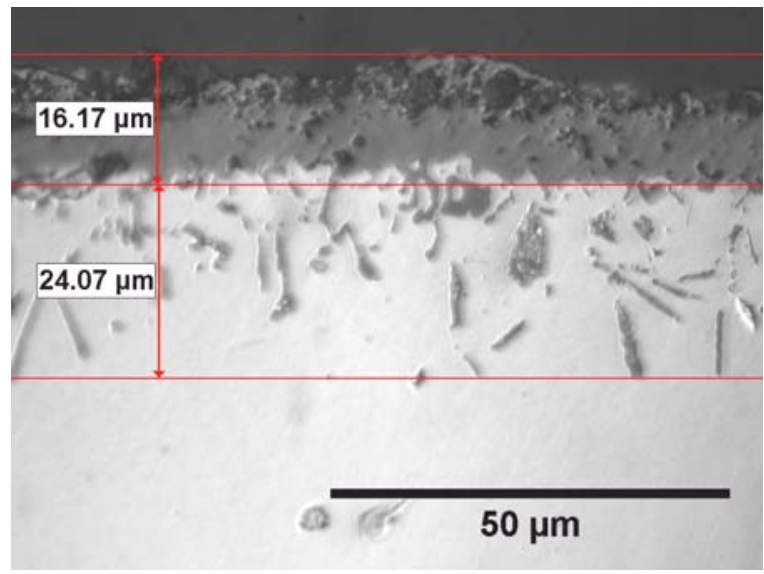

W30B1 - 30 hours

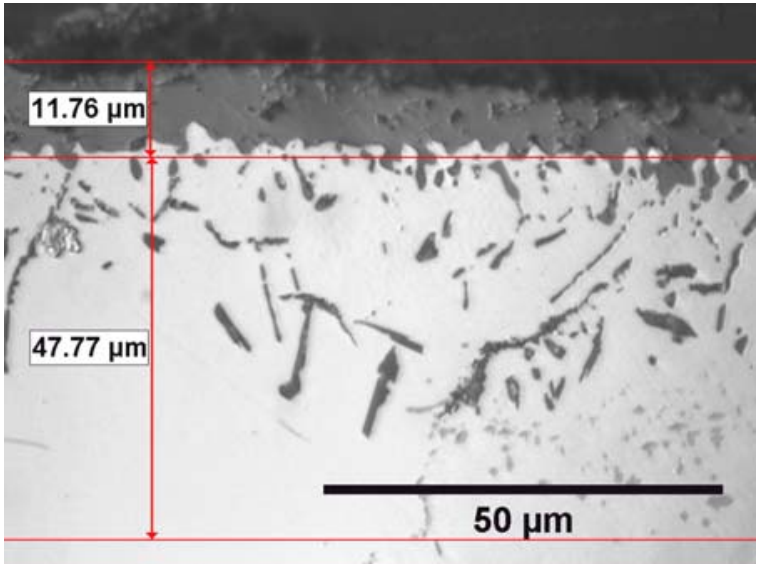

W100B1 - 100 hours

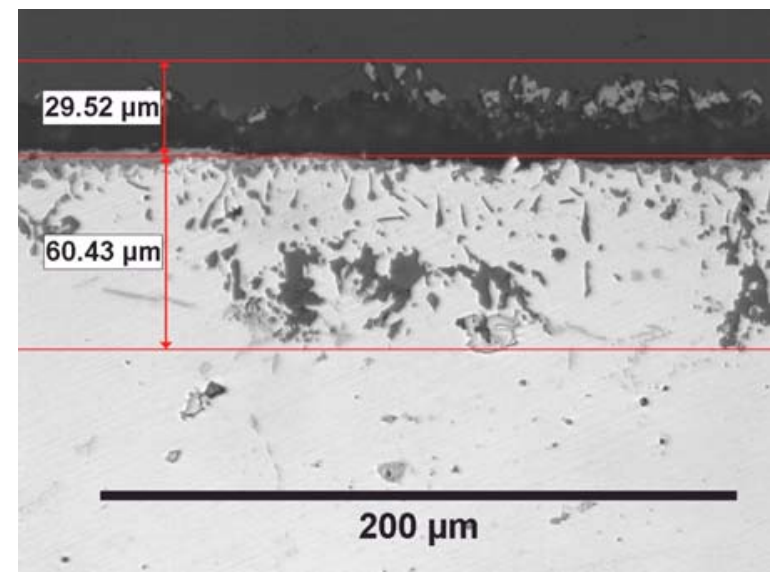

W300B1 - 300 hours

Photomicrographs Showing Surface Scale in Transverse Cross-Sections of GTD-222 Specimens After 10, 30, 100, and 300 Hours in Water-Saturated Mixture of 100 ppm $\mathrm{H}_{2} \mathrm{~S}$ / Balance $\mathrm{N}_{2}$. 


\section{Wet $\mathrm{H}_{2} \underline{\mathrm{S}}$}

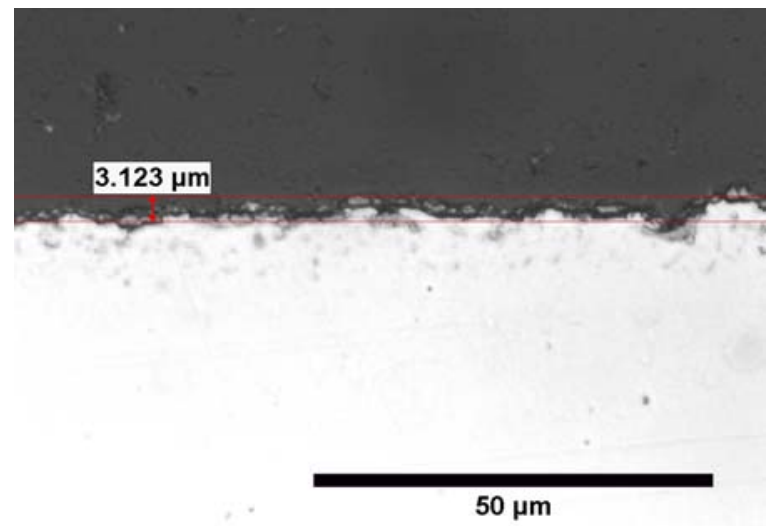

W10C1 - 10 hours

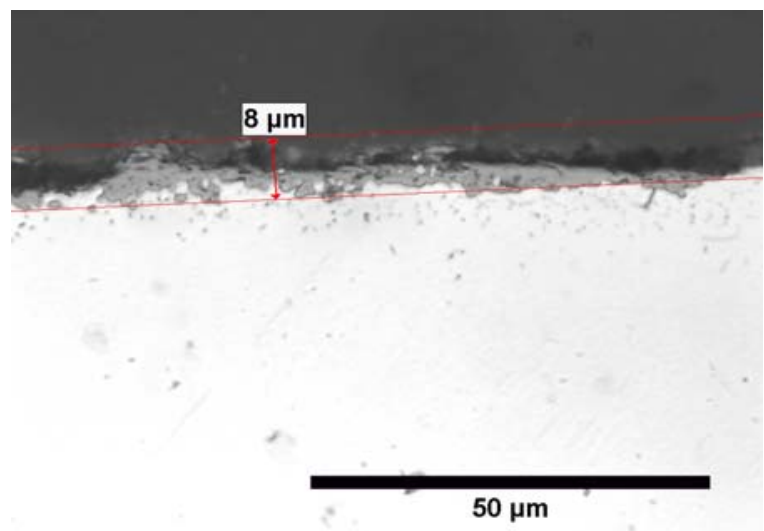

W30C1 - 30 hours

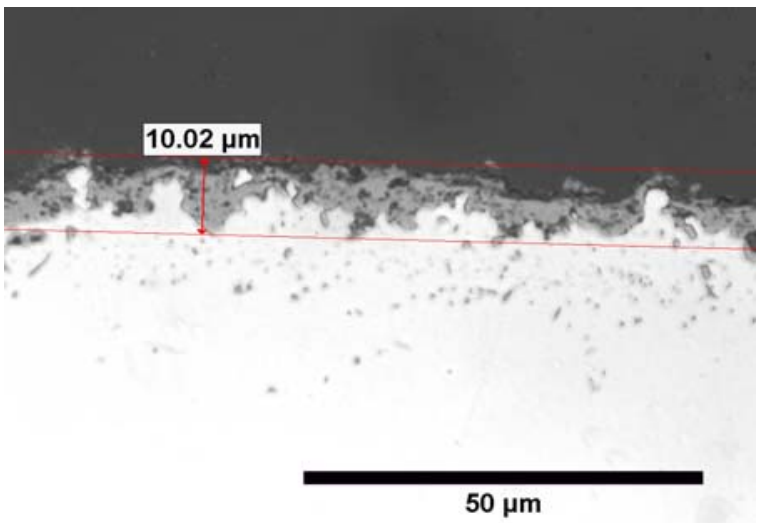

W100C1 - 100 hours

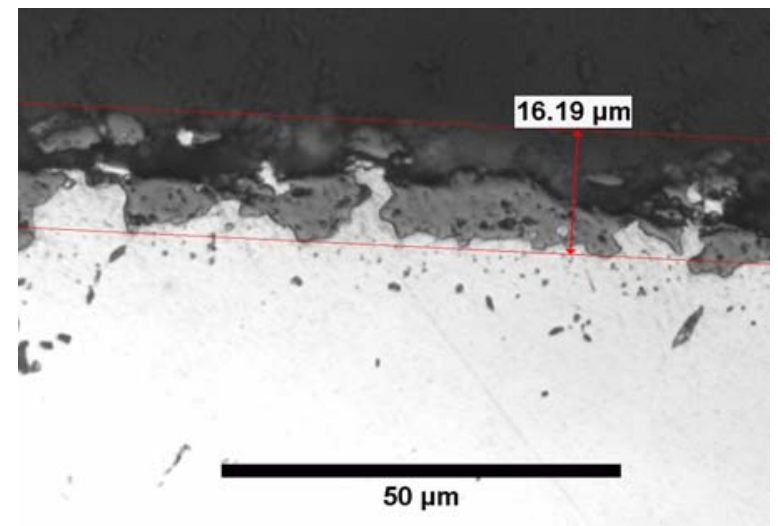

W300C1 - 300 hours

Photomicrographs Showing Surface Scale in Transverse Cross-Sections of Hastelloy- $X$ Specimens After 10, 30, 100, and 300 Hours in Water-Saturated Mixture of 100 ppm $\mathrm{H}_{2} \mathrm{~S} /$ Balance $\mathrm{N}_{2}$. 


\section{Wet $\mathrm{H}_{2} \underline{S}$}

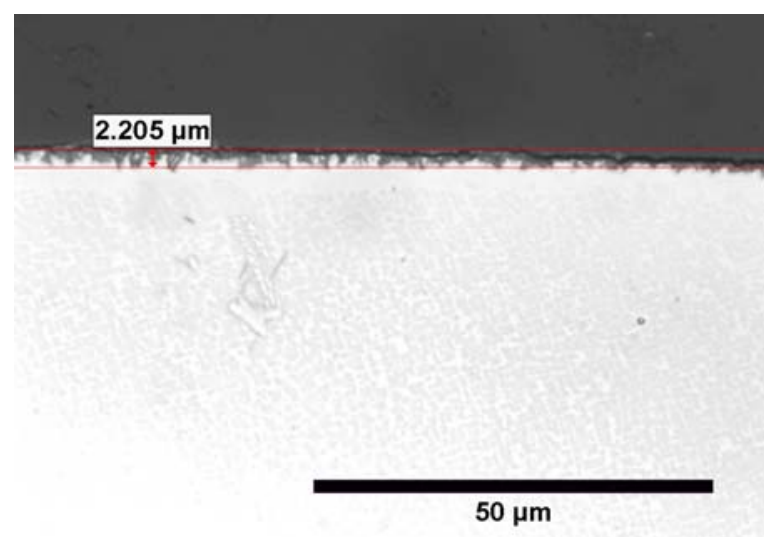

W10D1 - 10 hours

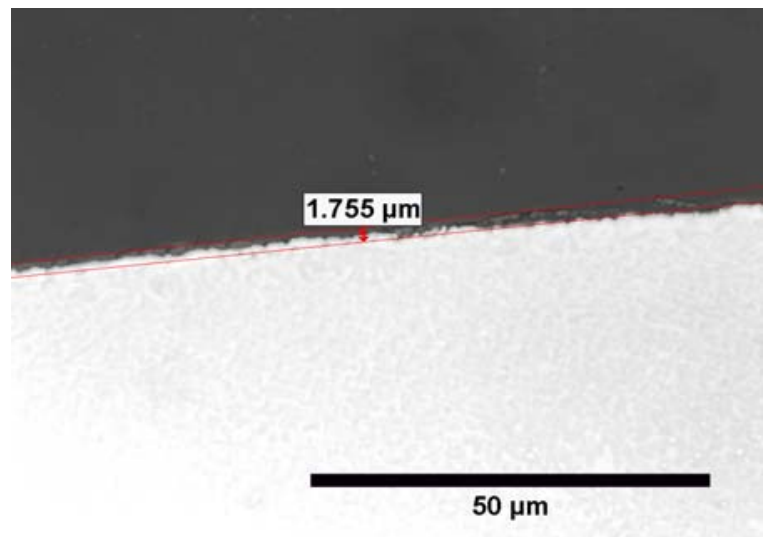

W30D1 - 30 hours

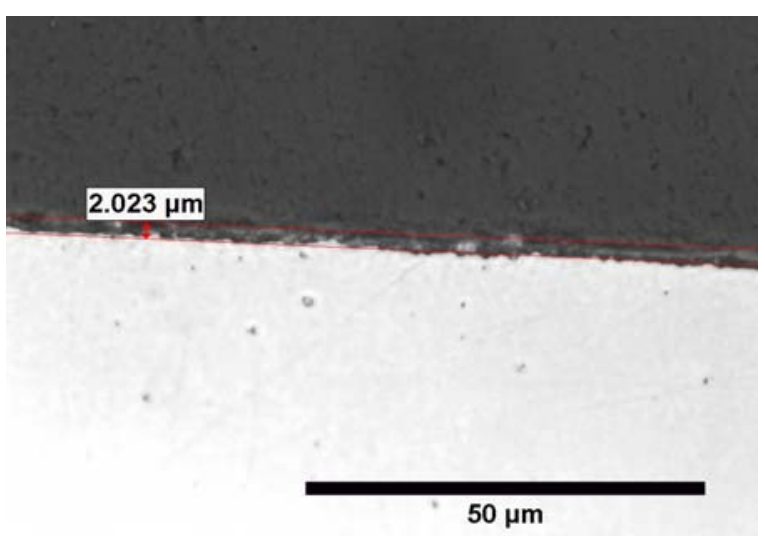

W100D1 - 100 hours

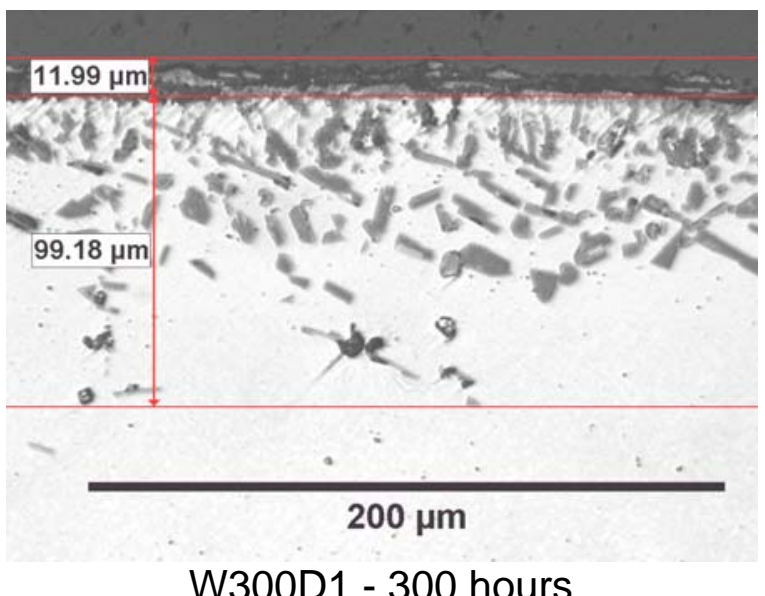

W300D1 - 300 hours

Photomicrographs Showing Surface Scale in Transverse Cross-Sections of Alloy N5 Specimens After 10, 30, 100, and 300 Hours in Water-Saturated Mixture of 100 ppm $\mathrm{H}_{2} \mathrm{~S} /$ Balance $\mathrm{N}_{2}$. 


\section{Wet $\mathrm{H}_{2}$ S}

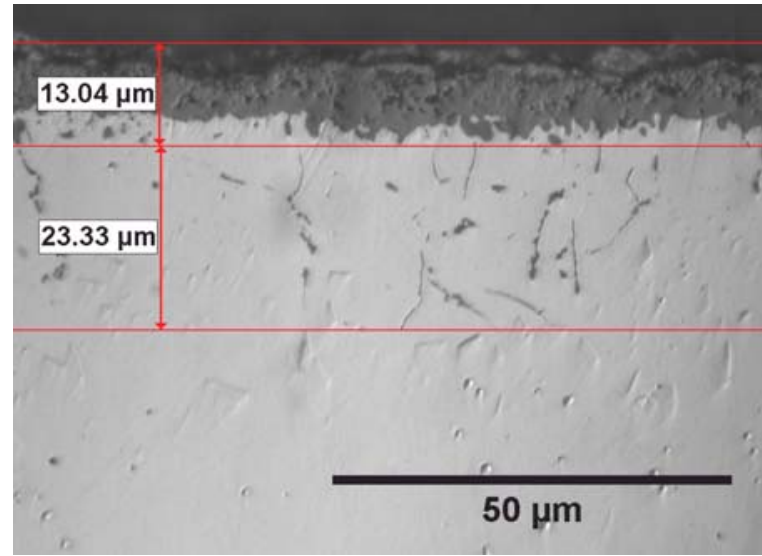

W10E1 - 10 hours

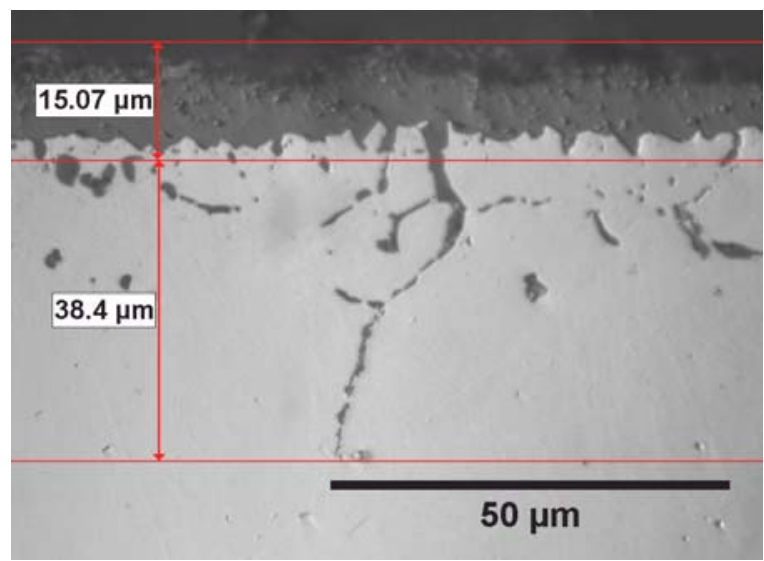

W30E1 - 30 hours

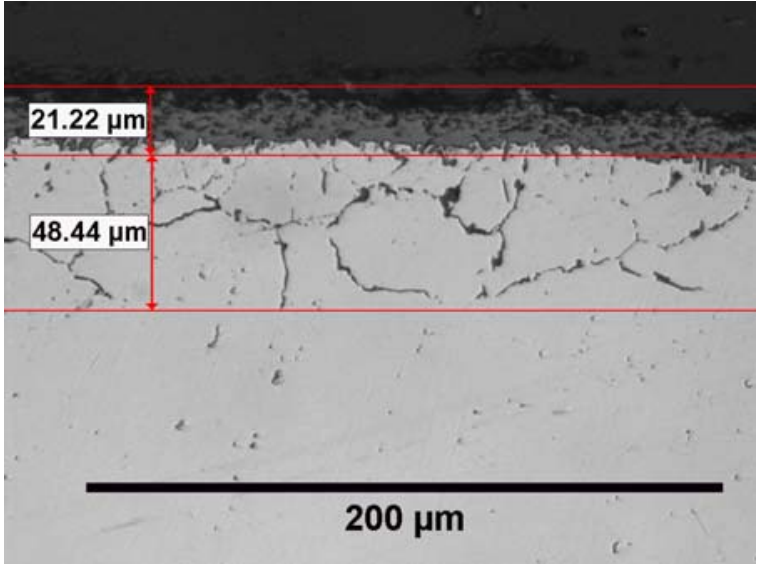

W100E1 - 100 hours

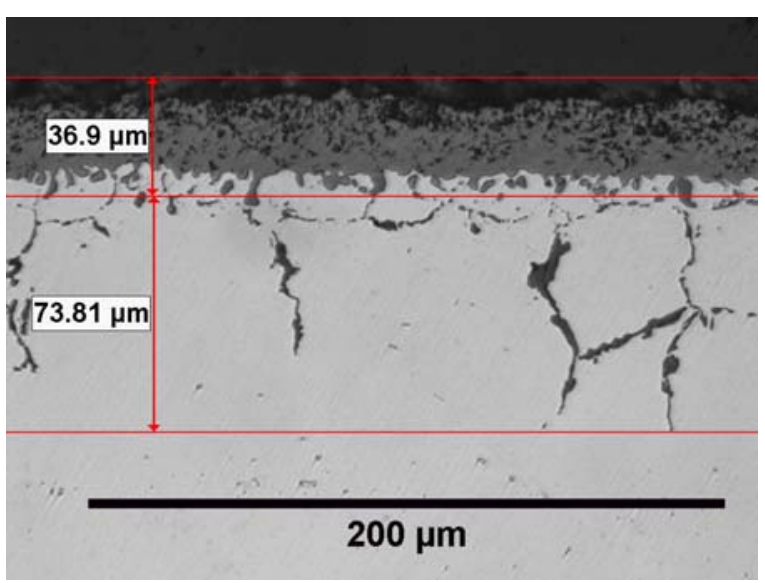

W300E1 - 300 hours

Photomicrographs Showing Surface Scale in Transverse Cross-Sections of Alloy Nimonic 263 Specimens After 10, 30, 100, and 300 Hours in Water-Saturated Mixture of $100 \mathrm{ppm} \mathrm{H}_{2} \mathrm{~S} /$ Balance $\mathrm{N}_{2}$. 


\section{Wet $\mathrm{H}_{2}$ S}

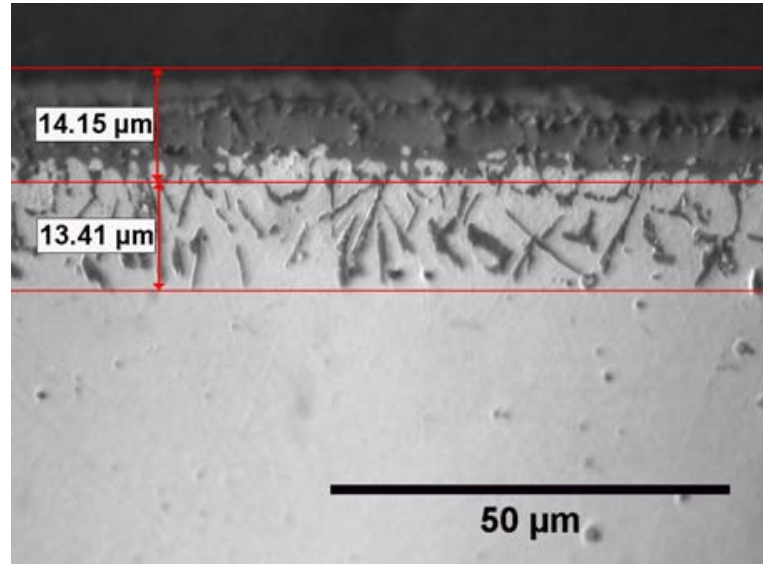

W10F1 - 10 hours

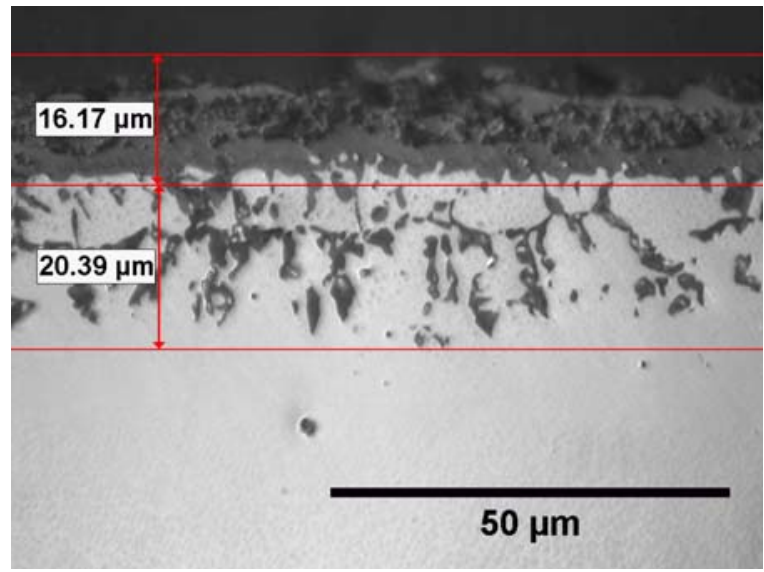

W30F1 - 30 hours

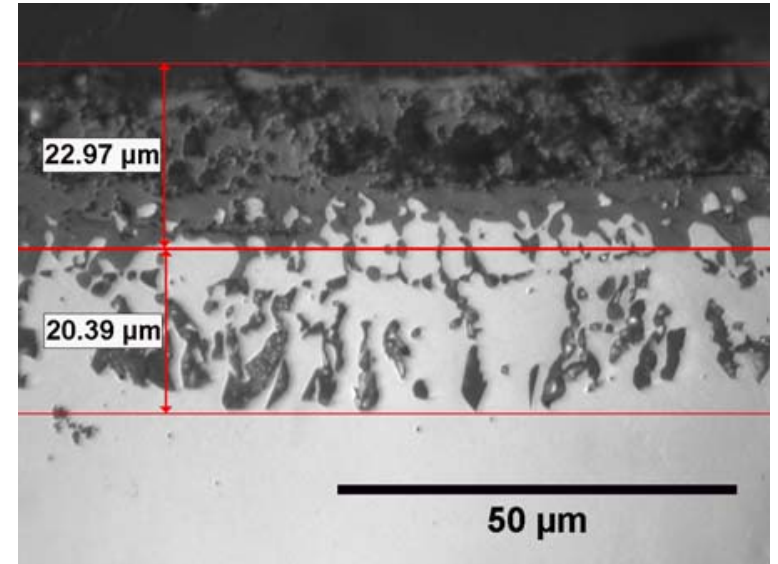

W100F1 - 100 hours

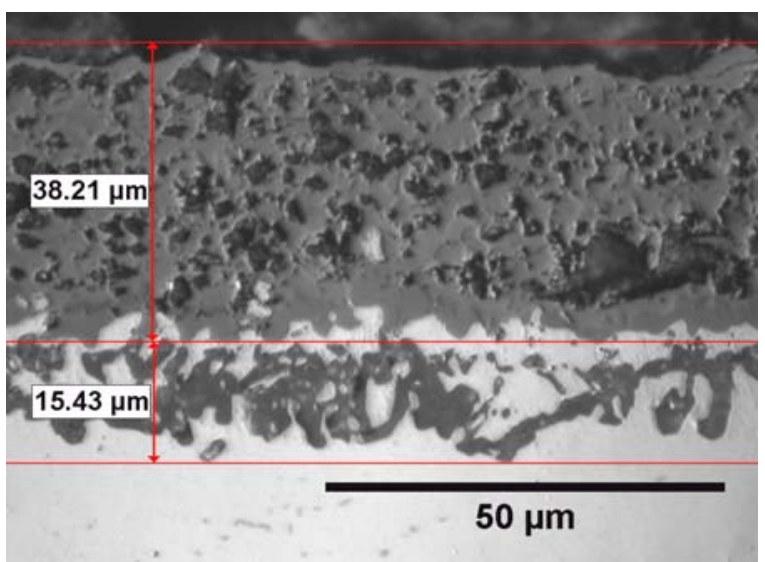

W300F1 - 300 hours

Photomicrographs Showing Surface Scale in Transverse Cross-Sections of Alloy GTD111 Specimens After 10, 30, 100, and 300 Hours in Water-Saturated Mixture of

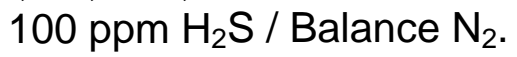




\section{Wet $\mathrm{H}_{2} \underline{\mathrm{S}}$}

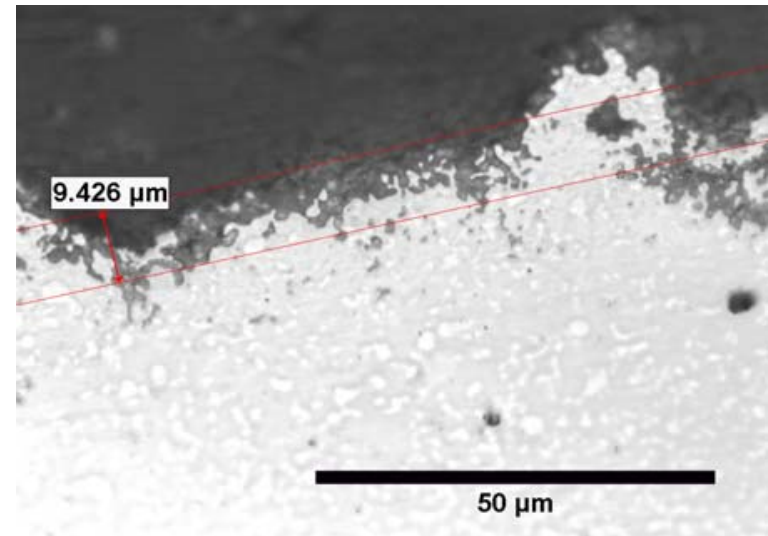

W10G1 - 10 hours

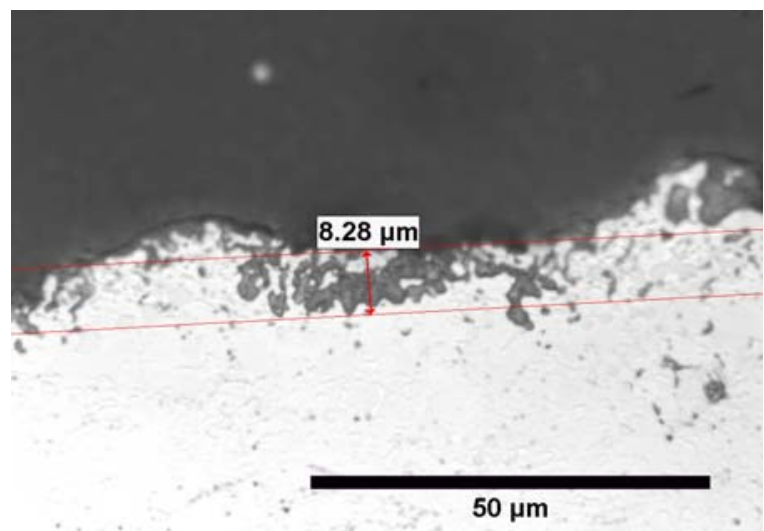

W30G1 - 30 hours

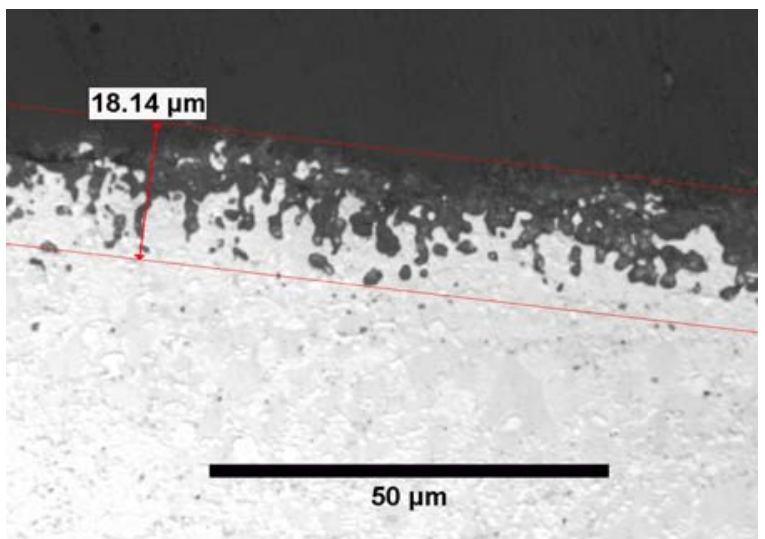

W100G1 - 100 hours

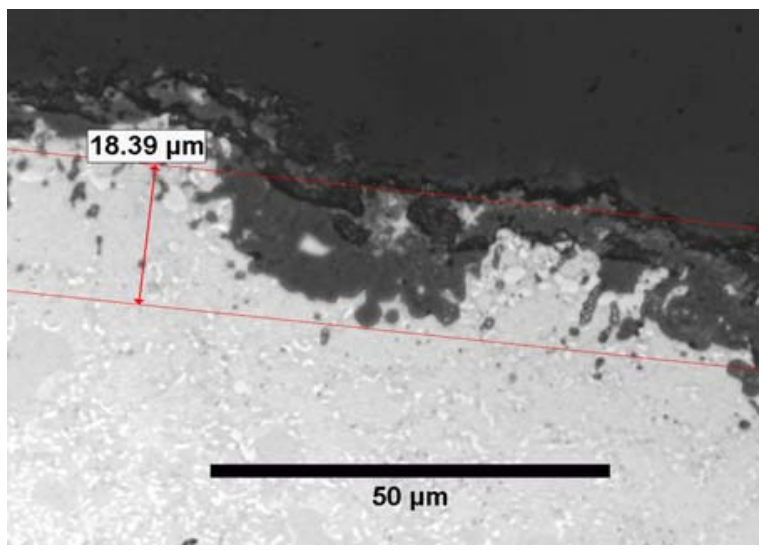

W300G1 - 300 hours

Photomicrographs Showing Surface Scale in Transverse Cross-Sections of Alloy N5 + NiCrAlY Specimens After 10, 30, 100, and 300 Hours in Water-Saturated Mixture of 100 ppm $\mathrm{H}_{2} \mathrm{~S} /$ Balance $\mathrm{N}_{2}$. 


\section{Wet $\mathrm{H}_{2} \underline{\mathrm{S}}$}

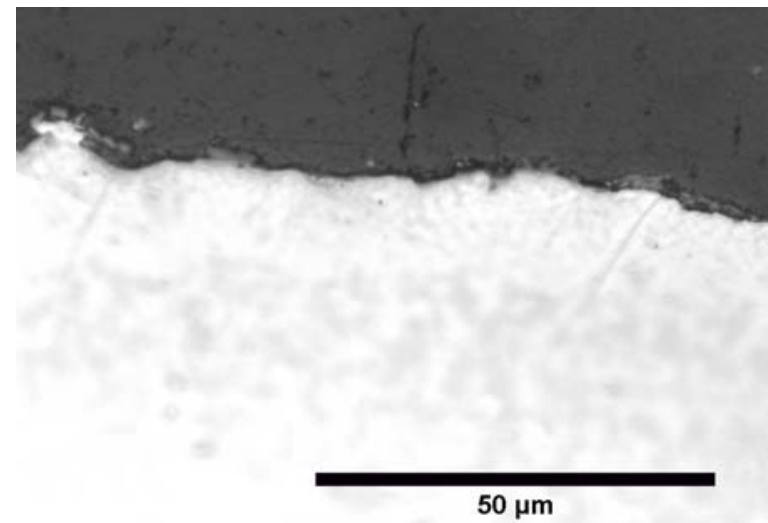

W10H1 - 10 hours

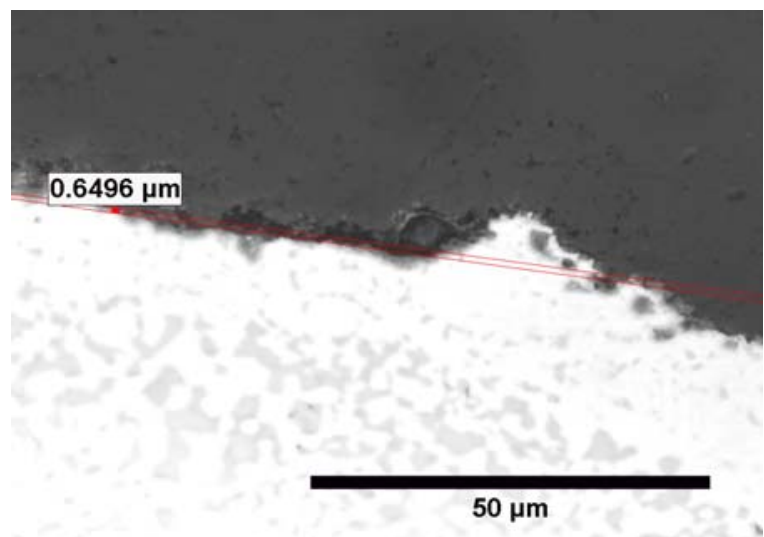

W3OH1 - 30 hours

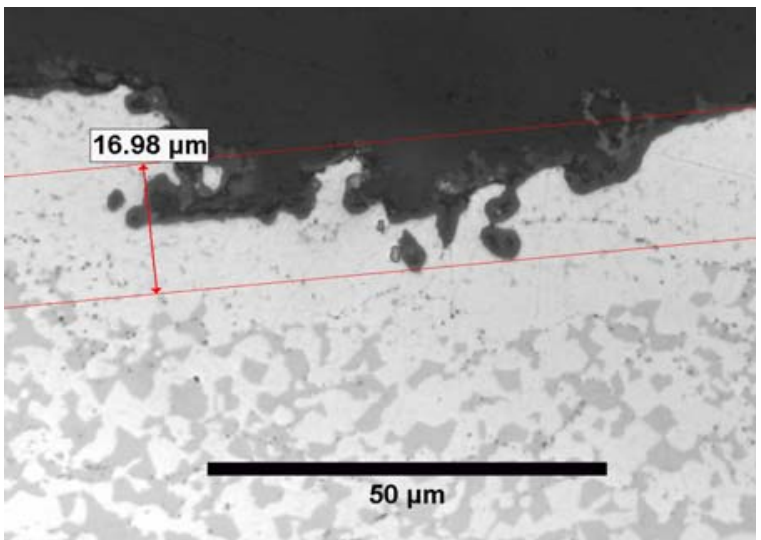

W10OH1 - 100 hours

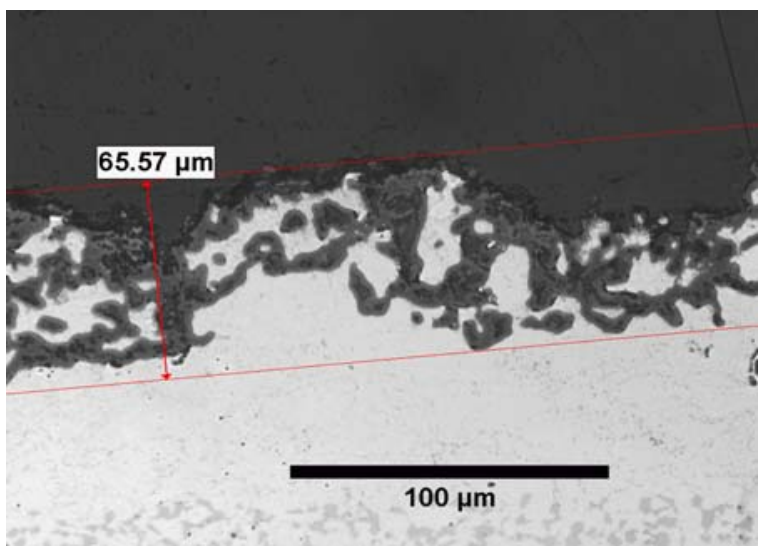

W30OH1 - 300 hours

Photomicrographs Showing Surface Scale in Transverse Cross-Sections of Nimonic $263+$ NiCrAlY Specimens After 10, 30, 100, and 300 Hours in Water-Saturated Mixture of $100 \mathrm{ppm} \mathrm{H}_{2} \mathrm{~S} /$ Balance $\mathrm{N}_{2}$. 


\section{Wet $\mathrm{H}_{2} \underline{\mathrm{S}}$}

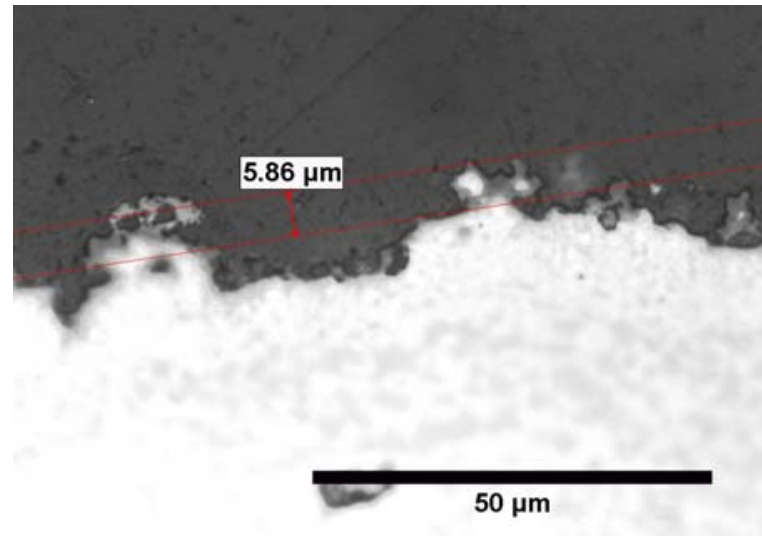

W10K1 - 10 hours

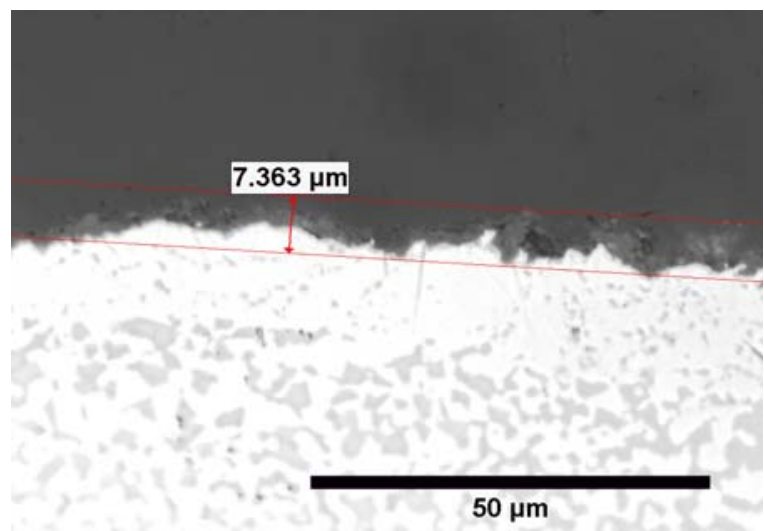

W30K1 - 30 hours

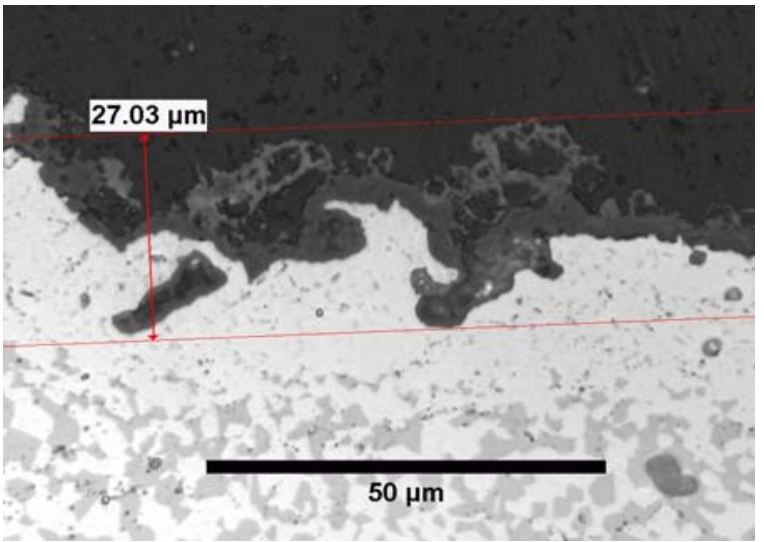

W100K1 - 100 hours

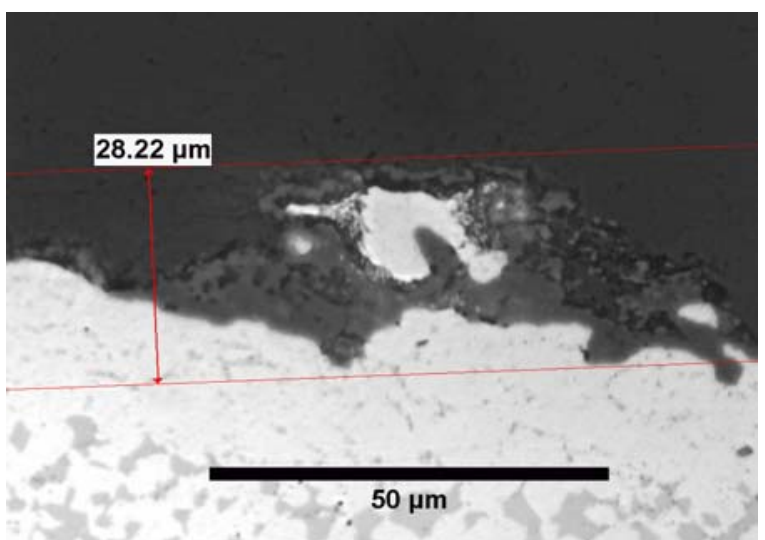

W300K1 - 300 hours

Photomicrographs Showing Surface Scale in Transverse Cross-Sections of Alloy GTD111 + CoNiCrAlY Specimens After 10, 30, 100, and 300 Hours in Water-Saturated Mixture of $100 \mathrm{ppm} \mathrm{H}_{2} \mathrm{~S}$ / Balance $\mathrm{N}_{2}$. 


\title{
Final Technical Report \\ Cooperative Agreement No: DE-FC26-03NT41448
}

\section{Sub-Task 1.1 - High Temperature Environmental Effect Data Collection \\ Sub-Task 1.2. - High Temperature Environmental Effect Mechanism Quantification}

\author{
A. P. Gordon ${ }^{\dagger}$, M. M. Shenoy ${ }^{\dagger}$, M. D. Trexler ${ }^{\ddagger}$, \\ R. W. Neu ${ }^{\dagger \dagger}$, D. L. McDowell ${ }^{\dagger+}$, T. J. Sanders \\ ${ }^{\dagger}$ The George W. Woodruff School of Mechanical Engineering \\ Georgia Institute of Technology, Atlanta, GA 30332-0405, USA \\ ${ }^{\ddagger}$ School of Materials Science and Engineering \\ Georgia Institute of Technology, Atlanta, GA 30332-0245, USA \\ Reporting Period: Sept. 3, 2003 through April 30, 2005
}

\begin{abstract}
Service conditions of turbine blades involve fatigue, creep, and environmental corrosion. The manner in which these mechanisms interact to initiate cracks is complex. These physical mechanisms have been observed through observation and measurement of damage in a directionally-solidified (DS) GTD-111 Ni-base superalloy. Experiments include high temperature low cycle fatigue with and without dwells and thermomechanical fatigue on both longitudinal (L) and transverse ( $\mathrm{T}$ ) specimens. The impact of exposure to different environments on remaining crack initiation is identified. A mechanistic-based crack initiation model correlating the evolution of several possible dominant damage mechanism is proposed. The mechanisms modeled include fatigue, creep-fatigue, and environment-fatigue. The dominant damage mechanism and life results are summarized in damage mechanism and life maps. The model accounts for variations in temperature, orientation, cyclic frequency and deformation rate, stress ratio, sustained-load hold times, environment, and interactions resulting from complex load histories (i.e., high- and lowcycle fatigue, creep-fatigue and thermal-mechanical fatigue).

Georgia Tech PD: R. W. Neu (rick.neu@me.gatech.edu) GEPS Task Technical Lead: David Helmick (david.helmick@ps.ge.com)
\end{abstract}




\section{Sub-task 1.1 - High Temperature Environmental Effect Data Collection}

\section{Background}

Substantive improvements of the thermal efficiency of a gas turbine can be achieved by increasing either the pressure or the temperature directed towards the bucket airfoils. Primary amongst other design considerations, therefore, is the ability of the blades and other hot gas path components to resist high temperature damage mechanisms. Upgrading conventionally cast, polycrystalline (PC) Ni-base superalloy components with higher performance materials has been identified as a means to attain the needed efficiency gains and damage tolerance. Investment casting processes have been modified to enhance the creep strength of Ni-base superalloys beyond what is arguably achievable by means of purely altering the alloying chemistry. Increased resistance to oxidation, hot corrosion, thermal fatigue, and creep in these materials has been achieved by means of directional solidification, a casting technique developed to reduce to the number of grain boundaries (GBs) transverse to the primary stress axis of the bucket.

Even though the high temperature durability of these components have been significantly improved, the combination of cyclic centrifugal forces and temperatures exceeding $600^{\circ} \mathrm{C}$ experienced in buckets can be substantial enough to prompt a myriad of microstructural damage mechanisms. This damage causes the formulation and growth of low cycle fatigue (LCF) cracks which are the primary reason for premature retirement of directionally-solidified (DS) gas turbine blades. Fractographic analyses of failed and retired Ni-base superalloy turbine blades revealed that preferential locations of crack initiation are along the trailing edge near cooling holes, at excessively misaligned GBs intersecting the airfoil tip, and along the suction side of the blade. Potential locations of thermal gradients, e.g. hot-spots, are also susceptible to sustaining LCF cracks. If undetected, these LCF cracks propagate under fatigue until the remaining ligament is overloaded and ruptures. The ultimate consequences are foreign object damage (FOD) to other components, unplanned outages, additional replacement costs, and most precarious, loss of safety.

The majority of the life of a blade is spent in the crack initiation stage; thus, the chief goal of this research will be to characterize the conditions and microstructures associated with crack initiation in directionally-solidified Ni-base superalloys. The goals of this research are as follows:

(1) Distinguish the dominant microstructural mechanisms that develop and enhance damage.

(2) Determine the influence of material orientation on crack initiation.

(3) Define the role of thermal/mechanical pre-exposure on crack initiation life.

(4) Characterize damage mechanisms and relate them to crack initiation behavior.

The content and organization of this section is as follows. Section 2 contains descriptions of the experimental methodologies employed. In Section 3, results in the forms of trends and microstructural characterization obtained from the testing program are described. Also in this section, a mechanistically-based fatigue damage life prediction model is introduced. Conclusions are given in Section 4. Finally, Appendices A, B, C, and D contains graphical test results. 


\section{Experimental Methodology}

A series of experiments have been conducted to fulfill the objectives of this study. Tests were carried out on directionally-solidified nickel based superalloy specimens that are representative of the DS GTD-111 material regularly used for first stage blades and vanes of gas turbines. These tests include isothermal low cycle fatigue (LCF) tests, creep-fatigue tests, thermomechanical fatigue (TMF) tests, and stress-free oxidation tests. While most specimens where tested on samples in the as-cast form, several were preexposed to elevated temperature, stress, and corrosive environments. Following a brief description of the material used in this study, the experimental procedure applied for each type of test is detailed. All experiments were conducted using the facilities within the Mechanical Properties Research Laboratory (MPRL) at Georgia Tech (GT).

Material removed directly from a blade casting would be the most suitable for testing since results could be reported for the exact microstructure and composition as that used in service conditions. Since the shape and size of the blades, however, do not lend themselves to inexpensive machining, test specimens were obtained from several batches of cast rectangular slabs of DS GTD-111. To further ensure that the test specimen microstructures resembled actual blades, the blocks were also processed following casting.

A total of three batches of DS GTD-111 blocks were fabricated to make test specimens. Slabs were specially cast to rectangular shapes comparable in size to first stage turbine blades. The dimensions of these slabs were also designed for machining a large number of specimens and are shown in Table 1. In every case, the solidification axis was along the longest axis of the test blocks. For each of the test batches involved, similar machining and heat treatments were carried out. Longitudinal (L-oriented) specimens are fabricated so that the direction of the columnar grains (i.e., the DS axis) is parallel to the loading axis, whereas transverse (T-oriented) specimens are oriented so that the grains are transverse to the loading direction.

At elevated temperatures, DS GTD-111 components used in gas turbines oxidize. In order to characterize this behavior, oxidation measurements from unstressed, thermally-exposed samples were needed. Small specimen discs measuring $12.7 \mathrm{~mm}$ in diameter and $2.3 \mathrm{~mm}$ in thickness were machined from unused shank sections of fatigue specimens.

All low cycle fatigue (LCF) tests of L and T-oriented DS GTD-111 were performed on a $4.45 \mathrm{kN}$ (10kip) axial servo-hydraulic MTS ${ }^{\circledR}$ testing machine with a dualchannel controller and TestStar software (Testware SX $\left.{ }^{\circledR} 4.0 \mathrm{D}\right)$. The load cell resolution was $\pm 0.22 \mathrm{kN}$. Following the specimen preparation procedure, one set of K-type [Chromel (Yellow/+) and Alumel (Red/-)] thermocouple leads were spot welded to the end of the gage section to monitor the temperature within the gage section. Tests were conducted under mechanical strain control, isothermal conditions, and static laboratory air.

Heat was supplied to the specimen using a radio frequency (RF) induction heater (Ameritherm Single Phase $2 \mathrm{~kW}$ ). Closed-loop feedback control of the specimen temperature was maintained with a PID controller (Watlow Model 945A-2FK5-A000). This controller was designed for K-type thermocouples which have a maximum operating temperature of $1260^{\circ} \mathrm{C}\left(2300^{\circ} \mathrm{F}\right)$. The temperature control resolution was $\pm 1^{\circ} \mathrm{C}$. Forced 
air cooling, which is generally used for tubular specimens, was not applied since heat could be rapidly conducted from the specimen via the water-cooled grips.

Numerous continuously cycled (CC), LCF tests were conducted on L and Toriented specimens sectioned from each batch of DS GTD-111. In isothermal test cases, the mechanical strain range $\left(\Delta \varepsilon_{m}\right)$ is equal to the total strain range $\left(\Delta \varepsilon\right.$ or $\left.\Delta \varepsilon_{t}\right)$. Each test differed by either isothermal temperature $(T)$, strain range, or strain ratio $\left(R_{\varepsilon}=\varepsilon_{\min } / \varepsilon_{\max }\right)$. Configurations and results of these tests are shown in Section 2. Since the majority of the cracks occurring in DS GTD-111 components are transverse to the DS axis, the majority of the experiments were conducted on L-oriented specimens. Initial hysteresis loops and complete stress histories for these experiments are given in Appendix A.

In the current study, crack initiation is assumed to include the process of crack nucleation (i.e., crack incubation) and microcrack growth to a small size (i.e., early propagation). For Ni-base superalloys, an appropriate crack initiation depth $\left(a_{i}\right)$ is between $0.5 \mathrm{~mm}$ and $1 \mathrm{~mm}$. At this length range, the incubation and early propagation of cracks is typically limited to one grain. Allowing longer crack paths could lead out-ofplane crack propagation resulting in the introduction of variability in the estimation of crack initiation life. For this investigation, the cycle during which the tensile load dropped 20\% from initial maximum stabilized tensile load was used to define the crack initiation life $\left(N_{i}^{20 \%}\right.$ or $\left.N_{i}\right)$. In tests designed to be halted prior to specimen fracture, tensile load drops between $5 \%$ and $50 \%$ are typical. At this cycle, the test was halted and the specimen was allowed to cool to room temperature (RT). Afterwards, the specimen was carefully removed from the collets and prepared for microscopy. Whenever possible the crack initiation life at $10 \%$ and $50 \%$ load drop (i.e., $N_{i}^{10 \%}$ and $N_{i}^{50 \%}$, respectively) where also determined.

All isothermal LCF tests were performed according to an ASTM standard (ASTM E606, 2003). Accordingly, the temperature throughout the gage section must be within $T_{n} \pm \Delta T$, where $T_{n}$ is the nominal test temperature in ${ }^{\circ} \mathrm{C}$, and $\Delta T$ is $2^{\circ} \mathrm{C}$ or $1 \% T_{n}$, whichever is greater. Five K-type thermocouples were directly attached to a trial specimen (3 within the gage section) which was then fastened within the collet grips and heated to $T_{n}$. The specimen configuration is shown in Fig. 1. Typical temperature distributions are illustrated in Fig. 2. The temperature distribution is designed so that the maximum temperature occurs in the center of the gage section. This temperature concentration guarantees that crack nucleation is prompted within the extensometer tips. As shown in Fig. 2, for increasing isothermal temperature, the axial temperature gradient within the gage section is higher. During actual tests, however, only one thermocouple was needed to maintain accurate feedback temperature control.

Microstructural damage arising from continuous cycling at elevated temperature is exacerbated by applying either (1) a tensile hold (HT) during the maximum mechanical strain or (2) a compressive hold (HC) during the minimum mechanical strain. For example, subsurface microcracks and creep cavities are predicted to accelerate for cycles with tensile dwell periods. During compressive dwells, however, oxidation embrittlement gives rise to more rapid formation of surface cracks. Under straincontrolled conditions, both the strain range and hold period influence the crack initiation mechanism. A hold period leads to a greater plastic strain range due to the stress relaxation that occurs during the hold. 
Although the equipment used in conducting isothermal low cycle creep-fatigue tests is identical as that used for isothermal LCF testing, there is an exception to the experimental procedure applied. For tests with tensile dwell periods, the specimen is held at the peak tensile strain for a time period, $t_{t}$. Similarly, for tests with compressive holds, the specimen is held at the peak compressive strain for a time period, $t_{c}$. The time to complete one cycle with a tensile hold is given as

$$
t_{t c}=t_{t}+t_{c c}
$$

With a compressive hold it is totaled as

$$
t_{t c}=t_{c}+t_{c c}
$$

where $t_{c c}$ is the loading and unloading time.

Various temperature and strain range combinations were used to conduct tests on L and T-oriented DS GTD-111 specimens. Each test was carried out until a $20 \%$ load drop occurred.

Many engineering components in high-temperature applications undergo thermal stress cycling superimposed on mechanical loading. In laboratory environments, however, isothermal testing may not exercise several of the important microstructural damage mechanisms occurring under varying temperature conditions. By following the procedural guidelines (ASTM E2368, 2004), TMF testing can accurately simulate damage caused by the combined effects of thermal cycling and mechanical fatigue.

In strain-controlled thermomechanical (TMF) tests, a specimen is subjected to simultaneous temperature (i.e., thermal strain) cycling and mechanical strain cycling. Although the frequencies of each cycle type typically have identical frequency, there can exist a difference in phasing between the two. This is defined as the waveform shift ( $\phi$, expressed in degrees) between the maximum temperature response as measured on the specimen and the maximum mechanical strain response. Typically two baseline TMF tests are conducted in the laboratory: linear in-phase (IP), with the maximum mechanical strain at the maximum temperature, and linear out-of-phase (OP), with the maximum mechanical strain reached at the minimum temperature. For reference purpose, $\phi$ is considered positive if the temperature response maximum leads the mechanical strain response maximum by $180^{\circ}$ or less, otherwise the phase angle is considered to be negative. Other TMF cycle types are implemented to simulate other service conditions. In mechanical strain-controlled TMF tests, the mechanical strain component is determined as the difference of total strain measured on the test specimen and the thermal strain, i.e.

$$
\varepsilon_{m}=\varepsilon_{t}-\varepsilon_{t h}
$$

The ideal strain histories for two TMF cycle types are shown in Fig. 3. For each case the mechanical strain range is $1.0 \%$, while the $0.5 \%$ thermal strain peaks and valleys are shifted to cause either IP $\left(\phi=0^{\circ}\right)$ or OP $\left(\phi=180^{\circ}\right)$ cycling. The special case of the thermal strain range $\left(\Delta \varepsilon_{t h}\right)$ is set to zero is an isothermal LCF test. In application, the conventional linear relationship between temperature and thermal strain related by the coefficient of thermal expansion $(\alpha)$ is not sufficient for high temperature ranges, i.e.,

$$
\varepsilon_{t h} \neq \alpha \Delta T
$$

The coefficient of thermal expansion can be a function of temperature for large temperature differences, $\Delta T$. Furthermore, under continuously varying temperature 
cycling, the thermal strains during cooling and heating may differ. For a temperature range of $500^{\circ} \mathrm{C}$ and a cycle time of 3 minutes, $\varepsilon_{t h}$ during cooling and heating are distinct near the minimum and maximum temperatures, as shown in Fig. 4. Axial and radial thermal gradients cause this disparity. The error in thermal strain approximation is interpreted as an over or under-estimation of mechanical strain. This causes additional compressive axial force during heating and additional tensile axial force during cooling. Any uncertainty in either $\varepsilon_{t}$ or $\varepsilon_{t h}$ directly propagates to $\varepsilon_{m}$. Errors in approximating the thermal strain can, therefore, potentially influence deformation and strain-based life estimates.

Strain-controlled TMF tests are more easily executed on axial test frames with dual independent channel servo-loop controllers. The current setup allowed for real-time calculated variable control. Mechanical strain controlled tests were achieved using the independent total strain signal, $\varepsilon$, and calculated thermal strain, $\varepsilon_{t h}$, implemented via Eq. (3). In the identical manner as isothermal LCF tests, axial deformation in the gage section of the specimen is measured with an extensometer contacting the sample at the gage section ends. Only an approximate relationship for thermal strain had to be determined. Free expansion data (no mechanical strain) of the specimen was obtained using a temperature cycle playback identical to that applied during the desired TMF test conditions. The resulting data allowed for the determination of an approximate relationship. As per the TMF standard (ASTM E2368), the explicit temperature-based compensation of thermal strains, shown in Fig. 4, is given as a polynomial, e.g.

$$
\varepsilon_{t h}=\sum_{i=0}^{n} a_{i}\left(T_{T / C}\right)^{i}
$$

The approximated thermal strain compensation nearly bisects the free expansion thermal strain data. The specimen temperature reading from the thermocouple $\left(T_{T / C}\right)$ was retransmitted to the TestStar controller and used to calculate the components of thermal and thus mechanical strain. Equation (5) is implemented prior to test initiation.

Using the independent total strain reading, $\varepsilon_{t}$, and calculated variable, $\varepsilon_{t h}$, the current experimental setup allows for real-time calculated $\varepsilon_{m}$-control. The $\varepsilon_{t h}$ can be compensated for as a function of temperature or time. Free expansion data (no mechanical strain) of the specimen must be obtained using temperature cycle playback identical to that applied during the desired TMF test conditions. Only an approximate relationship for thermal strain remains to be determined. A $3^{\text {rd }}$ order polynomial relationship based on temperature is used to fit 5 cycles of free expansion thermal strain data. It was later verified that the thermal strain hysteresis during the zero-load cycling was below $5 \% \Delta \varepsilon_{t h}$.

With this experimental setup, two or more devices in the arrangement were connected to a common ground through different paths, causing a ground loop to occur. To avoid this problem, the signals from these devices were isolated. An isolation amplifier was fabricated at Georgia Tech for this purpose. The circuit diagram for this appliance is given in Fig. 5. Without correcting these grounding issues inaccurate signals could have been transmitted to the heating device which can create a lethal hazard. A flow chart illustrating the equipment and the transmitted signals used for this experimental setup is shown in Fig. 6. Both the thermal and mechanical closed-loop feedback loops were digitally controlled. 
In TMF experiments, the test variables were configured to draw out, or in some cases deemphasize, particular microstructural deformation mechanisms contributing to failure. With exception to the phasing, $\phi$, and cycling time, $t_{c c}$, all TMF test variables are either exclusively related to the mechanical cycling or thermal cycling. In this study, the mechanical strain cycles were either in-phase $\left(0^{\circ}\right)$ or out-of-phase $\left(180^{\circ}\right)$ with the thermal strain cycles. For every experiment a cycle time of 180 s was applied. This quantity was selected based on the maximum capable heating rate of the induction heating system and the maximum cooling rate using unforced laboratory air. Since no hold times were applied, from either Eq. (1) or Eq. (2), $t_{t c}=t_{c c}$ under TMF.

The identical test parameters from isothermal LCF experiments (i.e., strain range, $\Delta \varepsilon$, strain ratio, $R_{\varepsilon}$, and strain rate, \&) correspond to those of the mechanical cycling for TMF experiments (i.e., mechanical strain range, $\Delta \varepsilon_{m}$, mechanical strain ratio, $R_{\varepsilon_{m}}$, and strain rate, $\underset{m}{\&}$ ). Since each of these tests is non-isothermal, the mechanical strain range $\left(\Delta \varepsilon_{m}\right)$ is not equal to the total strain range $\left(\Delta \varepsilon_{t}\right)$. Completely reversed (i.e., $R_{\varepsilon_{m}}=-1$ ), mechanical strain ranges of $0.5 \%$ or $1.0 \%$ were used, and since the total cycle time was fixed at $180 \mathrm{~s}, \&_{m}^{\&}$ during loading or unloading was either $0.0056 \% / \mathrm{s}$ or $0.0111 \% / \mathrm{s}$.

Cyclic thermal strain parameters (i.e., thermal strain range, $\Delta \varepsilon_{t h}$, and thermal strain rate, $\underset{t h}{\&}$ ) are dependent on cyclic temperature variables (i.e., minimum temperature, $T_{\min }$, and maximum temperature, $T_{\max }$ ). Both $T_{\min }$ and $T_{\max }$ were selected to accurately simulate the temperature range in nominal service conditions of $1^{\text {st }}$ stage $\mathrm{Ni}$ base turbine buckets. For a typical TMF experiment, temperature was continuously cycled at $538^{\circ} \mathrm{C}\left(1000^{\circ} \mathrm{F}\right) \leftrightarrows 927^{\circ} \mathrm{C}\left(1700^{\circ} \mathrm{F}\right)$ or $538^{\circ} \mathrm{C} \leftrightarrows 1038^{\circ} \mathrm{C}\left(1900^{\circ} \mathrm{F}\right)$. These temperature ranges correspond to thermal strain ranges of $0.55 \%$ and $0.89 \%$, respectively. Since a fixed 3 min cycle period was used, the rates during either heating or cooling had a magnitude of either $4.32^{\circ} \mathrm{C} / \mathrm{s}\left(7.78^{\circ} \mathrm{F} / \mathrm{s}\right)$ or $5.55^{\circ} \mathrm{C} / \mathrm{s}\left(10^{\circ} \mathrm{F} / \mathrm{s}\right)$. Stress histories and stress-total/mechanical/thermal strain hysteresis curves for each of the tests are given in Appendix B. All TMF tests were performed according to an ASTM standard (ASTM E2368, 2004). The same techniques used to minimize static axial temperature gradients in isothermal LCF testing were used for minimizing dynamic axial temperature gradients in TMF testing.

Turbines are typically initialized, operated, and checked prior to final delivery and installation to ensure operability. To simulate this early usage and its effect on fatigue crack initiation life, several specimens were exposed to pre-exposure. This pre-exposure consisted of a specified temperature $\left(982^{\circ} \mathrm{C}\right.$ for all cases), mechanical load (0, $\pm 100 \mathrm{MPa}$ ), and environment (air or syngas) for $100 \mathrm{hr}$. In the stressed cases, creep deformation occurred and was recorded. The specimens were subsequently tested under LCF conditions. Fatigue results for each of the experiments are given in Appendix C.

Corrosion of superalloys is complicated in the case of pre-service and in-service exposure of engines parts to the syngas environment. Syngas, which is the product of coal gasification, is quite caustic as it is comprised of large amounts of $\mathrm{H}_{2} \mathrm{~S}$ and coal particulates. To simulate these conditions in laboratory experiments $\mathrm{N}_{2}$ with $100 \mathrm{ppm}$ $\mathrm{H}_{2} \mathrm{~S}$ can be applied. In reality the coal particles add a large wear factor to corrosion rate. Sulfur attacking superalloys is far more aggressive that oxygen ingression as evidenced by the parabolic rate constants listed in Table 2 . Generally, the rate of corrosion in sulfur 
is often several orders of magnitude greater than that in corrosion in air. For reasons noted by Khanna (Khanna, 2002): higher diffusion rates of sulfur, low melting eutectic formation, low solubility, similar energies of formation, high dissolution pressure.

Determination of oxidation and sulfidation growth kinetics of DS GTD-111 was identified as a means to benchmark surface-accumulated layers observed on gage sections of fatigued samples (Fig. 7a). For this purpose, small samples were machined from unstressed material. Disc-shaped specimens were obtained from shank sections of fatigue-tested specimens (Fig. 7b). of unused material. After the small specimens were polished and cleaned, the samples were individually heated in a 3-zone tubular electric resistance furnace (Lindberg $240 \mathrm{~V}$ Model: 59744-A). The test variations included various combinations of temperature [from $871^{\circ} \mathrm{C}\left(1600^{\circ} \mathrm{F}\right)$ to $1093^{\circ} \mathrm{C}\left(2000^{\circ} \mathrm{F}\right)$ ] and time (from $1.0 \mathrm{hr}$ to $1000.0 \mathrm{hr}$ ) in static air. A K-type thermocouple and open loop control was used to maintain the desired specimen temperature. Since a significant length of the thermocouple was subjected to high temperature, thermocouple wiring insulted with temperature resistance insulation (Omega Model XC-K-24) was necessary.

Comparing microstructures from tested samples will directly characterize the interaction of creep-fatigue-environment damage mechanisms. Analysis of the tested specimens was performed using Light Optical Microscopy (LOM) and Scanning Electron Microscopy (SEM). The specimen was prepared in a number of steps: sectioning, mounting, grinding, and polishing. A schematic of the sections are shown in Fig. 7. Although the plane with the maximum crack length is not known a priori, a general rule of thumb is to axially section the specimen in the midsection of the dominant surface crack, which at times could be visually observed. Typically, only two or three cuts were necessary to remove the desired samples from the gage section of the fatigue-tested specimen. A similar cutting procedure was applied for stress-free tested samples. After sectioning, samples were mounted for ease of further processing. In order to optically study the microstructural features, mounted samples were ground and polished using a multi-step procedure. Scanning electron microscopy (SEM) was used to visually observe microstructural features (e.g. voids and individual precipitate particles) that were too small to be clearly seen in an optical microscope.

\section{Results and Discussion}

Numerous experiments were conducted under both $\mathrm{L}$ and T-oriented specimens of DS GTD-111 in order to characterize its fatigue behavior. This section details the results for each of the types of experiments. Data curves for each of the experiments conducted in this study are given in the Appendix. Following the discussion of the experimental results is the description of pertinent microstructural observation(s).

The largest number of experiments in this study was conducted under isothermal LCF conditions. Of these experiments, the most were conducted at $871^{\circ} \mathrm{C}\left(1600^{\circ} \mathrm{F}\right)$. This temperature is of particular importance since the behavior of the material in both orientations undergoes a transition in its yield strength and oxidation behavior. Above $871^{\circ} \mathrm{C}$, inelastic deformation in DS GTD-111 is predominantly accommodated by quasi cubic slip within the matrix channels. Whereas below this temperature, deformation is accommodated by both mechanisms (i.e., octahedral and cube slip). Also at this temperature there is a noticeable decrease is the material's ability to develop surface oxides. For these reasons, $871^{\circ} \mathrm{C}\left(1600^{\circ} \mathrm{F}\right)$ is considered the benchmark temperature. 


\section{Orientation Effects}

A total of twelve strain-controlled isothermal fatigue tests were conducted at $871{ }^{\circ} \mathrm{C}$ under continuous cycling (CC) conditions. A strain rate of $0.5 \% / s$ and strain ratio $\left(R_{\varepsilon}=\varepsilon_{\min } / \varepsilon_{\max }\right)$ of -1 were maintained for all tests. Six were conducted for samples in each grain orientation. The stress histories for the $\mathrm{L}$ and $\mathrm{T}$-oriented material subjected to a total (or mechanical) strain range $(\Delta \varepsilon)$ of $1.0 \%$ are shown in Fig. 8. In this case, the fatigue life until fracture of the L-oriented specimen outlasted that of the T-oriented specimen by nearly a factor of three. The discrepancy in the cyclic softening behavior of the two materials leading up to final failure is significant. While the L-oriented sample fractures suddenly, the T-oriented sample exhibits a gradual loss in load carrying capability. This gradual loss indicates that there may have been more than one crack initiated and failure was due to the propagation of the dominant one(s). This is plausible since T-oriented specimens contain numerous grain boundaries transverse to the stress axis. These grain boundaries represent potential locations of crack initiation that are not present in L-oriented DS GTD-111.

The results of isothermal LCF tests are summarized in Table $3 \mathrm{a}$ for L-oriented material and Table $3 \mathrm{~b}$ for T-oriented material. The appendix figure number of the each data curve (initial hysteresis loops and stress history) is given in the last column of each table. Based on the initial stabilized maximum tensile load, $P_{i, \max }$, the load drop can be calculated (i.e., $90 \% P_{i, \max }, 80 \% P_{i, \max }, 50 \% P_{i, \max }, 0 \% P_{i, \max }$ ). Using the stress history, the life can be determined for a particular load drop (e.g. $N_{i}$ at $90 \% P_{i, \max }$ ). The compared results of Tables 2 and 3 indicate as the strain range is increased for each material orientation the cycles to a particular load drop decreased. The total strain lives of Loriented specimens exceeded that of T-oriented samples by a factor of generally between 2 and 4 except for the higher strain ranges.

Since the objective of the current investigation is to quantify the mechanisms of crack initiation, the fatigue cycle in which the material exhibited a $20 \%$ drop in the maximum initial stabilized tensile load, denoted as $80 \% P_{i, \max }$, was used as the criterion for measuring crack initiation in the gage section of the specimen. When compared at the $20 \%$ load drop condition, crack initiation lives of L-oriented material exceeds that of the T-oriented material at $871^{\circ}$ by at least a factor of 3. Figure 9 compares $\Delta \varepsilon$ versus $N_{i}$ for the benchmark conditions of $871^{\circ} \mathrm{C}, R_{\varepsilon}=-1, \&=0.5 \% / \mathrm{s}$, and $t_{h c}=t_{h t}=0$.

The plastic component of the strain range is determined as the difference between the mechanical strain range and the elastic component of the strain range, i.e.,

$$
\Delta \varepsilon_{p l}=\Delta \varepsilon_{m}-\Delta \varepsilon_{e l}=\Delta \varepsilon_{m}-\frac{\Delta \sigma}{E_{i}}
$$

where $\Delta \sigma$ is the stress range $\left(\sigma_{\max }-\sigma_{\min }\right)$, determined from stabilized stress history data. Table 4 contains the plastic and elastic strain ranges for each of the continuous cycling tests conducted at $871^{\circ} \mathrm{C}$. The orientation of the material is given in the last column. At high mechanical strain ranges $\left(\Delta \varepsilon_{e l}+\Delta \varepsilon_{p l} \geq 1.0 \%\right)$, the plastic portion of strain range is significantly higher than that obtained for low $\Delta \varepsilon_{m}$, and vice versa for low mechanical strain ranges. The crack initiation life, $N_{i}$, of each material orientation decreased with increasing $\Delta \varepsilon_{p l}$ or $\Delta \varepsilon_{e l}$. The plastic strain range versus crack initiation life curves of DS GTD-111 are compared with two polycrystalline Ni-base superalloys in Fig. 10. The 
close proximity of the data points between individual curves indicates that the difference in fatigue crack initiation lives of $\mathrm{L}$ and $\mathrm{T}$ orientations is mostly due to the elastic response of the material and not the inelastic properties.

The transition crack initiation life, $N_{i}^{(t)}$ is defined to occur when the amount of the amount of inelastic deformation work is approximately equal to that of the elastic work (or when $\Delta \varepsilon_{e l}=\Delta \varepsilon_{p l}$ ). These points were be determined as: $N_{i}^{(t)}=65, \Delta \varepsilon_{m}=1.0 \%$ (L-oriented), and $N_{i}^{(t)}=38, \Delta \varepsilon_{m}=0.7 \%$, (T-oriented). These low transition life values are typical of a higher strength alloy.

\section{Temperature Effects}

Continuous cycling (CC), mechanical strain-controlled tests were also conducted at temperatures above and below the benchmark temperature with $\Delta \varepsilon_{m}$ ranging from $0.5 \%$ to $2.0 \%$. The isothermal temperatures ranged from $427^{\circ} \mathrm{C}\left(800^{\circ} \mathrm{F}\right)$ to $1038^{\circ} \mathrm{C}\left(1900^{\circ} \mathrm{F}\right)$ for the L-oriented material and $649^{\circ} \mathrm{C}\left(1200^{\circ} \mathrm{F}\right)$ to $1038^{\circ} \mathrm{C}$ for the T-oriented material. The crack initiation lives and decomposed plastic and elastic strain ranges from these experiments are listed in Table 5. This table contains a summary of these CC tests results (excluding the experiments that were conducted at $871^{\circ} \mathrm{C}$ ).

Figures 11 and 12 illustrate the temperature-dependent strain-life curves for L and T-oriented DS GTD-111, respectively. Also included in each of these graphs are data obtained from monotonic experiments (Ibanez, 2003). For any series of tests on Loriented DS GTD-111 at a constant mechanical strain range, the number of cycles needed to nucleate a crack decreased with increasing temperature. For example, cycling the mechanical strain range at $0.8 \%$, and increasing the temperature from $760^{\circ} \mathrm{C}$ to $982^{\circ} \mathrm{C}$ caused the crack initiation life to decrease by a factor of 21 . When cycling at $1.0 \%$, increasing the temperature from $649^{\circ}$ to $1038^{\circ} \mathrm{C}$, caused $N_{i}$ to decrease by a factor of 27 .

Similar observation can be made for data from T-oriented DS GTD-111 specimens contained in the lower portion of Table 6 . Increasing the temperature from $871^{\circ} \mathrm{C}$ to $982^{\circ} \mathrm{C}$ at $\Delta \varepsilon_{m}=1.0 \%$, resulted in the decrement of $N_{i}$ by 2.7 . Varying the temperature from $761^{\circ} \mathrm{C}$ to $1038^{\circ} \mathrm{C}$ at $1.0 \%$ caused the crack initiation life of the material to decrease by a factor of 7.4. Under CC loading with a constant strain rate $(0.5 \% / s)$, the plastic strain range characterizes the fatigue-dominated crack initiation of DS GTD-111 under isothermal LCF testing. At temperatures of $871^{\circ} \mathrm{C}, 982^{\circ} \mathrm{C}$, and $1038^{\circ} \mathrm{C}$, the decrease in yield strength caused the increase of the cyclic plastic part of total strain range for both $\mathrm{L}$ and $\mathrm{T}$ specimen samples, as shown in Fig. 4. At the stated temperatures, $N_{i}$ can be correlated with $\Delta \varepsilon_{p l}$ at $R^{2}>0.9$ for each orientation. Since the correlations hold for both long and short-lived tests, the influences of the other damage forms (i.e., environmental and creep) are, therefore, somewhat limited.

Figure 13 shows that for different test temperatures, but otherwise similar testing conditions, increasing the temperature only slightly altered the crack initiation mechanism. At $760^{\circ} \mathrm{C}\left(1400^{\circ} \mathrm{F}\right)$ only a small amount of oxidation occurred at the exposed surface and along the nucleated crack. This crack followed a slightly more tortuous path that was more influenced by subsurface carbides compared to the surfaceinitiated crack which occurred at $871^{\circ} \mathrm{C}\left(1600^{\circ} \mathrm{F}\right)$. At temperatures above $871{ }^{\circ} \mathrm{C}$, 
oxidation has an increasing role in crack incubation and early propagation. Oxidized crack tips tended to propagate in the direction perpendicular to the remotely applied load.

\section{Mean Strain Effects}

The effect of mean stress was determined for L-oriented DS GTD-111 using a series of completely reversed $\left(R_{\varepsilon}=-1\right)$ and zero-to-tension $\left(R_{\varepsilon}=0\right)$ strain-controlled low cycle fatigue tests at temperatures ranging from $760^{\circ} \mathrm{C}$ to $871^{\circ} \mathrm{C}$. The test results of each of these experiments are given in Table 6. The mean stress effect was negligible for the strain ranges considered. At $871^{\circ} \mathrm{C}$, the crack initiation life of L-oriented specimens decreased slightly with a nominally tensile mean load as shown schematically in Fig. 14. In cases where the plastic strain range was significant, mean stress relaxation occurred and the mean stress converged toward zero or a tensile stress level allowing cyclically stability. This typically occurred within the first 100 reversals. Based on these results the mean stress effects can be neglected for cases where the strain range exceeds $0.01 \%$.

\section{Frequency Effects}

The effect of strain rate, \& (strain range per half unit of total cycle period) was investigated for L and T-oriented DS GTD-111 using a series of isothermal tests conducted at $982^{\circ} \mathrm{C}$. For the strain range considered $(1.0 \%)$, the two strain rates of $\&=0.50 \% / s$ and $\&=0.01 \% / s$, corresponded to either short $(4 s)$ or long (180s) cycle times. The test results of each of these experiments are given in Table 7.

By increasing the cycle time, DS GTD-111 sustains additional viscoplastic deformation, resulting in a small increase of the plastic strain range. As shown in Fig. 15, the stress range decreased for both orientations due to time dependent stress relaxation. Accordingly, the calculated proportion of elastic strain range decreased even though the elastic modulus, as expected, remained unchanged for the two orientations. The $\Delta \varepsilon-N_{i}$ curves for fast and slow strain rates are compared in Figure 16. It can be observed that for either for orientation there is at most only a $20 \%$ increase in the plastic strain range that corresponding to the sizable $70 \%$ reduction on crack initiation life. This indicates that there is a crack initiation mechanism aside from the "fatigue" mechanism lowering the service life of the material. Figure 17 shows the microstructures of fatigue-tested $\mathrm{L}$ and T-oriented DS GTD-111 under the two strain rates considered. At the more rapid strain rate of $0.5 \% / \mathrm{s}$ (Fig. 17a,b), carbides, even those in the vicinity of the surface (Fig. $17 \mathrm{~b})$ are less likely to participate in the crack incubation and early propagation process. Comparatively, under slow strain rates subsurface eutectics (Fig. 17c) and carbides (Fig. 17d) are favorable locations for crack initiation.

\section{Dwell Effects}

Cyclic experiments for DS GTD-111 under creep-fatigue loading conditions to investigate the effects additional damage mechanisms, such as surface oxidation and/or subsurface cracking at inclusions. The results of these experiments are listed in Table 8 and graphed in Fig. 18. By incorporating hold times in tension (HT) or compression (HC), $N_{i}$ was shortened even though the plastic strain ranges were comparable to those from CC conditions. For example, in HT cases samples exhibited lives shortened by a factor between one-fourth and one-half of those of CC specimens. Also, HC loading 
resulted in lives shorter by one-third of those from the CC samples under the identical conditions. At $871^{\circ} \mathrm{C}, \mathrm{HC}$ leads to the shortest life regardless of material orientation; however, as temperature was increased to $982^{\circ} \mathrm{C}$, HT cycling lead to the shortest lives. With increasing temperature $N_{i}$ under $\mathrm{HC}$ converged to $N_{i}$ under CC. This trend continued for DS GTD- 111 at $1038^{\circ} \mathrm{C}$ when cycled at a strain range of $1.0 \%$ (Table 8 ).

Visual inspection of profiles of fatigue-tested samples of L-oriented DS GTD111, in Fig. 19, indicates that there is a changeover in the mechanism of crack initiation when cycling under $\mathrm{CC}, \mathrm{HC}$, and $\mathrm{HT}$. At $871^{\circ} \mathrm{C}$ with $\Delta \varepsilon=0.8 \%$, continuously cycled samples display devoid of surface cracks and has a slightly non-planar fracture surface; therefore under $\mathrm{CC}$ conditions at high temperatures oxidation plays a negligible role. Under HC conditions, surface cracks are plentiful and the final fracture surface is approximately perpendicular to the stress axis. In this case, crack initiation was dominated by surface cracks initiated at oxides. In the HT case, the final fracture surface is the jagged. There is a limited amount of surface cracking. Similar to slow strain rate cases, HT cases exhibit inclusion dominated crack initiation behavior.

The surface-initiated damage mechanism was drawn out by modifying the test configurations to higher temperatures and low stress (e.g. low strain range) conditions with compressive dwells. For tests under these conditions, at least $97 \%$ of the life of the specimen is spent in compression; furthermore, the low stress condition limits the involvement of fatigue or inclusion dominated. During cyclic reversals the typically brittle accumulated oxide layer fractures in tension and exposes subjacent virgin material. Once this newly-oxidized material fractures and the process repeats. The result is the formation of a series of oxide spikes propagating transverse to the direction of the applied load, as shown in Fig. 20. The accumulated oxidation layer at the exposed surface or the fracture surface consists of several distinct layers. The outer layer is a homogeneous mixture of Ti- and Cr-rich oxides, while the inner layer is Al-rich and is intermingled with matrix material. Separating the oxide layers from the unaffected metal is a matrix layer denuded of primary precipitate particles.

Figure 21a shows the result of increasing the mechanical strain range from $0.5 \%$ to $1.0 \%$. In this case the plastic strain range was higher than the previous figure and oxide spikes tended to be shorter. There was some evidence of subsurface void nucleation at carbide inclusions. The microstructure in Fig. $21 \mathrm{~b}$ was subjected to the identical conditions, with the exception that HC was replaced with HT. Subsurface carbides were the main cause of crack initiation. Cracks propagated by coalescing with adjacent microcracks. In nearly all cases the carbides themselves did not fracture.

\section{Non-Isothermal Effects}

Both L and T-oriented DS GTD-111 specimens have been subjected to TMF cycling. Each of the results is listed in Table 9. For a typical TMF experiment, temperature was continuously cycled at $538^{\circ} \mathrm{C} \leftrightarrows 927^{\circ} \mathrm{C}$ (at $538^{\circ} \mathrm{C} \leftrightarrows 1038^{\circ} \mathrm{C}$ in one case), and the cycle period was $180 \mathrm{~s}$. Completely reversed mechanical strain ranges of $0.5 \%$ or $1.0 \%$ were either IP $\left(0^{\circ}\right)$ or OP $\left(180^{\circ}\right)$ with the thermal strain cycles. The coefficient of thermal expansion (CTE) of oxidized material is $9 \times 10^{-6} /{ }^{\circ} \mathrm{C}$, which is nearly $75 \%$ of that for the base material. Oxide scales grown on the metal during maximum tensile conditions of fatigue reversals experience the largest compressive and shear stresses at the minimum strain level. Superimposing a compressive stress with a 
temperature drop, such as the case in IP TMF cycling, results in uneven thermal strain distribution across the oxide/metal interface, which accelerates the accumulation of oxide spallation. Adhesive oxide scales, like those grown on DS GTD-111 are prone to developing shear cracks that cause wedging. A typical spalled surface is shown in Fig. 22a.

Whereas the oxide spalling mechanism is a consequence of shear cracking and wedging, oxidation spiking (shown in Fig. 22b) results from oxide formation under compressive conditions at high temperatures. Such is the case with OP TMF cycling. The oxidized material fractures upon cyclic reversals much like isothermal continuously cycled cases.

The stress response of DS GTD-111 under TMF is plotted with respect to the mechanical component of strain for each test case in Fig. 23a,b. Since both the modulus and the yield strength of the material are temperature dependent, the stresses required to achieve the maximum and minimum strains are not equal. The consequence is positive mean stresses for OP cycling, and negative mean stresses for IP cycling. Increasing the mechanical strain range caused an increase in the fraction of plastic to total mechanical strain amplitude for both $\mathrm{L}$ and $\mathrm{T}$ cases. Mechanical strain-life curves for DS GTD-111 under TMF are shorter than those under LCF, primarily due to the additional microstructural mechanisms brought about in the presence of non-isothermal cycling (e.g. oxide spiking for OP cases and spallation for IP cases), as well as increased cycle time that caused subsurface damage in IP cases.

\section{Pre-Exposure Effects}

Several specimens of L and T-oriented DS GTD-111 were subjected pre-exposure prior to fatigue cycling. Each of the test configurations is listed in Table 10. While most specimens where pre-exposed in static air, two specimens where exposed to wet H2S that simulated the gasified operating environment of DS GTD-111 turbine blades. A common pre-exposure time of $100 \mathrm{hr}$ and temperature of $982^{\circ} \mathrm{C}$ was applied to all cases. Afterwards, each specimen was tested continuously cycled with $\Delta \varepsilon=0.8 \%$ at $871^{\circ} \mathrm{C}$ and with $R_{\varepsilon}=-1$, \& $\&=0.5 \% / \mathrm{s}$ until crack initiation occurred. The stress/strain histories for each of the tests are given in Appendix C.

Two L-oriented specimens were mechanically loaded during the pre-exposure. One was loaded in compression and the other in tension. Because of the high temperature and the sustained stress, creep deformation occurred. Figure 24 shows the effect of the pre-exposure on the initial hysteresis loops. When compared with unexposed L-oriented material subjected to the same fatigue conditions, the material pre-exposed in compression (Fig. 24a) shows virtually no alteration in the initial mechanical properties. Conversely the material pre-exposed in tension (Fig. 24b) has a slightly lower elastic modulus and lower yield strength. Whereas the pre-exposure in compression only reduced the crack initiation life by a factor of 2 , the pre-exposure in tension reduced the life by factor of 10 .

Two specimens (one L and one T) were subjected to an environment with 100ppm $\mathrm{H}_{2} \mathrm{~S}$ and $0 M P a$ (e.g. stress-free). For comparison purposes an additional L-oriented sample was subjected to static air and $0 M P a$. Each sample was subsequently fatigue tested under identical conditions. The initial hysteresis loops corresponding to each of these samples is illustrated in Fig. 24c. The $\mathrm{H}_{2} \mathrm{~S}$ environment lowered both the elastic 
modulus and the yield strength of the L-oriented material. As a result, the tensile stress carrying capability of the material was reduced by nearly $30 \%$. Introducing $\mathrm{H}_{2} \mathrm{~S}$ into the environment, caused the plastic strain range to increase by nearly a factor of 10 . The $\mathrm{T}-$ oriented material also had a substantial plastic strain range when cycled. Mechanical strain lives of the material are illustrated in Fig. 25. Of all of the L-oriented cases considered, pre-loading at $100 \mathrm{MPa}$ in tension had the most detrimental effect on the crack initiation life of the material. Pre-exposing the material to either $0 \mathrm{MPa}$ or $-100 \mathrm{MPa}$ had approximately the identical effect, i.e., a factor of 2 reduction in life.

Microscopy was conducted to characterize the underlying mechanisms responsible for the reduction in mechanical strain life with pre-exposure at $982^{\circ} \mathrm{C}$ for $100 \mathrm{hr}$. Figure 26 compares the microstructures of the two pre-crept and fatigued samples to an L-oriented sample that was pre-crept in tension, but not subjected to any fatigue cycling whatsoever. Common to the surface of each material is the accumulated oxidation layer. A thinner layer was obtained for the case pre-crept in compression (c). The surfaces of the cracks of the cycled cases (b and c) have similar accumulated oxidation layers. The thicknesses of the layers on the external vertical surface exceeds those on the fracture surfaces since the material was fatigued at a lower temperature than its pre-exposure temperature. For the two cases pre-crept in tension ( $a$ and $b$ ), numerous microcracks were found in both the cycled (a) and un-cycled (b) case. Upon fatigue cycling cracks emanating from the surface coalesced with those initiated at the subsurface, thus creating a non-planar fracture path. In each of the cases rafting occurred either transversely ( $a$ and $b$ ) or along (c) the pre-stress axis. These raft-shaped particles measure five times the original length of the cuboidal precipitate particles.

Microscopy of unstressed, air or $\mathrm{H}_{2} \mathrm{~S}$ pre-exposed DS GTD-111 indicates that surface damage was amplified in the case of hydrogen sulfide due to hydrogen embrittlement which weakens the resistance of DS GTD-111 to corrosion thus causes stress corrosion cracking. In both cases shown in Fig. 27, surface initiated cracking was the dominant cause of damage. Multiple hairline cracks emanated from surface-formed oxide pits and extended into the bulk of the material. Very little oxidation formed on the fracture surface in either case since the fatigue temperature was low. Since the surface degradation was more aggressive in the $\mathrm{H}_{2} \mathrm{~S}$-exposed case, the accumulated layer was thicker. Internal corrosion was rarely observed and is not expected in DS GTD-111.

\section{Stress-Free Oxidation and Sulfidation}

Isothermal stress-free heating tests were conducted using a 3-zone tubular electric resistance furnace. Specimen discs were heated to temperatures between $871^{\circ} \mathrm{C}$ and $1093^{\circ} \mathrm{C}$ in static laboratory air. The exposure time ranged from $1 \mathrm{hr}$ to $1000 \mathrm{hr}$. DS GTD111 was also exposed to $\mathrm{H}_{2} \mathrm{~S}$ for $100 \mathrm{hrs}$ at $982^{\circ} \mathrm{C}$. Reported values of oxidation surface penetration depth, $L_{O X}$, and $\gamma^{\prime}$ depletion (GPD) depth, $L_{G P D}$, are the averages of several direct measurements from multiple locations of micrographs of samples and are shown in Table 11.

The surface layers accumulated as a result of exposure in the simulated syngas environment (i.e., $\mathrm{H}_{2} \mathrm{~S}$ ) are slightly thinker than that occurring with exposure in air, shown in Fig. 28a,b. The existence of multiple layers occurring at the surfaces of each specimen are identical, however. The collection of oxidized samples indicates time and temperature-dependent oxidation accumulation. Specimens subjected to longer exposure 
times and/or higher temperatures displayed an increased depth in oxide formation, as shown in Fig. 28c-e. Generally, the development of the inner and outer oxide layers were comparable, and the thickness of the Al-rich layer typically exceeded that of the Cr/Tirich layer. Initially, the accumulated thickness of the $\mathrm{Al} / \mathrm{Ti} / \mathrm{Cr}$ scale was very thin; diffusion though the scale was rapid, establishing steady-state oxidation at the scaleenvironment interface. As the reaction proceeded at a constant rate, the increase in scale layer thickness resulted in a reduction in metal diffusion across the scale and, consequently, to a reduction in the reaction rate. In other words, after an initial oxidation scale develops, one or more of the reactants must be transported across the accumulated scale for the oxidation process to continue. At some locations, a limited amount of $\mathrm{Cr}_{2} \mathrm{O}_{3}$ flaked off the surface.

Using the listed measurements, the transient and steady state oxidation accumulation stages were correlated using a quartic law of the form

$$
L_{O X}=\alpha_{O X} f\left(\sigma_{m}, \Delta \varepsilon_{i n}\right)\left[\Theta_{O X}(T) t\right]^{\frac{1}{4}} .
$$

We define $f$ as a factor that accounts for oxidation enhancement resulting from mechanical loading. Under unstressed conditions, $f=1$. An evolution model was also developed for $\gamma^{\prime}$ depletion by replicating the procedure for determination of the oxidation model. As indicated by the data in Table 11, GPD depths were generally larger than oxidation depths. The presence of two diffusion stages (transient and steady state), however, was common to both reactions. The quartic formulation given in Eq. (7) correlates matrix layer evolution well, i.e.,

$$
L_{G P D}=\alpha_{G P D} f\left(\sigma_{m}, \Delta \varepsilon_{i n}\right)\left[\Theta_{G P D}(T) t\right]^{\frac{1}{4}} .
$$

In Eqs. (7) and (8), the temperature dependence of the diffusivity is expressed via the Arrhenius term

$$
\Theta=\Theta_{0} \exp \left(-\frac{Q}{R T}\right)
$$

where $\Theta_{0}$ is a material property, $Q$ is the activation energy (minimum energy needed), $T$ is the material temperature measured in degrees Kelvin, and $R$ is the universal gas constant (8.314 $\mathrm{J} / \mathrm{mol}-\mathrm{K}$ ). Using regression analyses, the constants for these relations were determined as listed in Table 12. Because of the slight difference in activation energy, less heat is required to bring about matrix layer formation than is necessary to cause oxide formation. The matrix layer beneath the cumulative scale consists of less $\mathrm{Cr}$ and $\mathrm{Ti}$ and more $\mathrm{Al}$ than the virgin material.

Isothermal tests were carried out using a vertical tube furnace in which samples were hung using a Pt wire. The samples were held at two test temperatures for times ranging from 1-14 hours in dry gas with a composition of $100 \mathrm{ppm} \mathrm{H}_{2} \mathrm{~S}$ in $\mathrm{N}_{2}$. Figure 30a shows the results of these experiments. The rate of the mass gain at the higher temperature is greater as is expected during oxidation testing. Figure $30 \mathrm{~b}$ shows the mass gain squared versus time in which the data points fall along a straight line. This indicates that the oxidation behavior is parabolic and follows the equation:

$$
x^{2}=k t
$$

where $x$ is the mass gain, $k$ is the parabolic rate constant, and $t$ is time. Using a log plot of the parabolic rate constant versus $1 / T$, the activation energy of the reaction can be estimated via 


$$
k=k_{0} \exp \left(\frac{-Q}{R T}\right)
$$

where $k_{o}$ is a constant, $R$ is the gas constant, $T$ is temperature measured in Kelvin, and $Q$ is the activation energy. The value for the activation energy was found to be equal to $4.02 J$.

The amount of weight gain is very small and the corresponding sulfide scale is barely detectable. Similar observations have been made based on the wet $\mathrm{H}_{2} \mathrm{~S}$ preexposed samples from GE, these samples still show very little corrosion damage even after $100 \mathrm{hr}$ at $982^{\circ} \mathrm{C}\left(1900^{\circ} \mathrm{F}\right)$. This may suggest that GTD-111 DS is resistive to sulfur attack. However, literature suggests that the high temperature oxidation of many superalloy and steels in $\mathrm{H}_{2} \mathrm{~S}$ is noticeable after at least 500 hours. It should also be noted that the $\mathrm{H}_{2} \mathrm{~S}$ concentration of $0.01 \%$ or $100 \mathrm{ppm}$ is extremely low and will hinder the corrosion reactions [4].

\section{Conclusions}

In order to develop a crack initiation model that accurately accounts for variations in temperature, orientation, cyclic frequency, stress ratio, firing gas composition, sustained-load hold times and interactions resulting from complex load histories (i.e. high and low-cycle fatigue, creep-fatigue, thermal-mechanical fatigue, and environmental degradation. Several series of elevated temperature low cycle fatigue experiments were conducted. Based on the mechanical responses and corroborating microscopic observation obtained. Several conclusions were achieved:

1. Orientation Effects: When placed under identical conditions, crack initiation life of L-oriented DS GTD-111 always exceeded that of the T-oriented material generally by a factor of 2 . Under continuous cycling (CC) conditions the mechanical strain range versus crack initiation responses were decomposed into elastic and plastic strain range components. A two-parameter, power law regression analyses indicated that the individual $\Delta \varepsilon_{p l}-N_{i}$ responses of the two materials at $871^{\circ} \mathrm{C}$ could be unified with one curve fit resulting in $R^{2}=0.88$.

2. Temperature and Mean Stress Effects: Additional isothermal CC LCF tests were conducted with either (1) temperatures ranging from $649^{\circ} \mathrm{C}$ to $1038^{\circ} \mathrm{C}$ or (2) mean tensile stresses $\left(R_{\varepsilon}=0\right)$ at $982^{\circ} \mathrm{C}$. For both cases the $\Delta \varepsilon_{p l}-N_{i}$ responses for L and T-oriented DS GTD-111 were curve-fitted to the previous power law resulting in $R^{2}=0.86$.

3. Frequency Effects: Lowering the strain rate to $\&=0.01 \% / s$ allowed the introduction of creep-induced stress relaxation to occur during fatigue reversals. The additional viscoplastic straining accommodated decreased stress ranges and subsurface microcracking that lowered $N_{i}$ from those obtained from tests with $\&=0.50 \% / s$ but otherwise identical conditions.

4. Dwell Effects: Introducing hold times in either tension or compression introduced inclusion-dominated and surface-dominated damage, respectively. In both cases, crack initiation lives were shortened from those obtained from $\mathrm{CC}$ tests but otherwise identical conditions.

5. Non-isothermal Effects: Observations from TMF experiments that isolated the microstructural mechanisms of creep, fatigue, and environment were conducted 
on both L and T-oriented DS GTD-111. Under non-isothermal conditions with large plastic strain ranges, fatigue damage dominates. Tests with small $\Delta \varepsilon_{p}$, are subject to environmental-fatigue damage since the surface-related mechanisms are active at long exposure times. For identical conditions, the lives of T-oriented specimens outlasted those of L-oriented samples.

6. Pre-Exposure Effects: Crack initiation behavior of pre-exposed DS GTD-111 was investigated. The damage mechanism responsible for crack initiation in the material was moderated by the pre-exposure condition. For example, the most malevolent pre-exposure configuration was that in which a sample was subjected to a sustained tensile load at high temperature. In this case cracks were nucleated by coalescence of microcracks that had formed near inclusion particles during the pre-exposure period. It was also found that the sulfidizing environment was more detrimental to the crack initiation life than the oxidizing environment.

7. Oxidation and Sulfidation Kinetics: Results show kinetics of oxidation in DS GTD-111 parabolic oxidation are parabolic and have an estimated activation energy of $4.02 \mathrm{~J}$ in dry $100 \mathrm{ppm} \mathrm{H}_{2} \mathrm{~S}-\mathrm{N}_{2}$ gas. Thin sulfide scales suggest that corrosion is minimal. This is in accordance with sulfidation behavior of samples pre-exposed in wet $\mathrm{H} 2 \mathrm{~S}$ for $100 \mathrm{hr}$ at $982^{\circ} \mathrm{C}$. Based on these observations it can be concluded that 100 hours is not a sufficient time to investigate the kinetics base on the low $\mathrm{H}_{2} \mathrm{~S}$ concentration. Additionally, a concentration of $0.01 \%$ is too low for adequate characterization of sulfidation testing. Typically concentration between $1-40 \%$ is used.

To further investigate the behavior of DS GTD-111 DS in the $\mathrm{H}_{2} \mathrm{~S}$ atmosphere, thermogravimetric analysis (TGA) will be performed using a McBain-Bakr (McBain and Bakr, 1926) quartz spring balance, diagramed in Fig. 30. These tests include 100ppm $\mathrm{H}_{2} \mathrm{~S}$ in $\mathrm{N}_{2}$ balance at test temperatures ranging from $1200^{\circ} \mathrm{F}-1800^{\circ} \mathrm{F}$ where $1800^{\circ} \mathrm{F}$ is the upper limit on our furnace. All samples, ranging in mass between $5-8 g$, have been prepared by mechanically polishing the surfaces using 1200 grit paper. Additional tests in dry and wet air will be conducted in summer 2005 and reported in the dissertation of M. Trexler.

\section{References}

1. A.S. Khanna. Introduction to High Temperature Oxidation and Corrosion. ASM International, 2002.

2. McBain, J. W., and Bakr, A. M., J. Amer. Chem. Soc.,48, 698. (1926). 


\section{Tables}

Table 1: Slab dimensions in units of $m m$ (in)

\begin{tabular}{cccc}
\hline Batch & Length & Width & Depth \\
\hline 1 & 178 & 76 & 16 \\
& $(7.00)$ & $(3.00)$ & $(0.63)$ \\
2 & 254 & 197 & 32 \\
& $(10.00)$ & $(7.75)$ & $(1.25)$ \\
3 & 229 & 127 & 16 \\
& $(9.00)$ & $(5.00)$ & $(0.63)$ \\
\hline
\end{tabular}

Table 2: Parabaolic rate contsants for diffusion of various metals

\begin{tabular}{cccccc}
\hline \multirow{2}{*}{ Type of Diffusion } & \multicolumn{5}{c}{ Metal } \\
& & $\mathrm{Ni}$ & $\mathrm{Co}$ & $\mathrm{Fe}$ & $\mathrm{Cr}$ \\
\hline Oxidation & $\left(\mathrm{gm}^{2} \mathrm{~cm}^{-4} \mathrm{~s}^{-1}\right)$ & $9.10 \mathrm{E}-11$ & $1.60 \mathrm{E}-09$ & $5.50 \mathrm{E}-08$ & $4.50 \mathrm{E}-12$ \\
& $\left({ }^{\circ} \mathrm{C}\right)$ & 1000 & 950 & 800 & 1000 \\
Sulfidation & $\left(\mathrm{gm}^{2} \mathrm{~cm}^{-4} \mathrm{~s}^{-1}\right)$ & $8.50 \mathrm{E}-04$ & $6.70 \mathrm{E}-06$ & $8.10 \mathrm{E}-06$ & $8.10 \mathrm{E}-07$ \\
& $\left({ }^{\circ} \mathrm{C}\right)$ & 650 & 720 & 800 & 1000 \\
\hline
\end{tabular}

Table 3a: Fatigue life of L-oriented DS GTD-111 at $871^{\circ} \mathrm{C}$ under continuous cycling

\begin{tabular}{|c|c|c|c|c|c|c|}
\hline \multirow{2}{*}{$\begin{array}{c}\text { Total Strain } \\
\text { Range, } \Delta \varepsilon(\%)\end{array}$} & \multicolumn{4}{|c|}{ Life, $N$} & \multirow{2}{*}{ Specimen ID } & \multirow{2}{*}{ Figure } \\
\hline & $90 \%$ & $80 \%$ & $50 \%$ & Fracture & & \\
\hline 0.5 & 29159 & 29159 & 29159 & 29159 & L8-3 & A. 14 \\
\hline 0.8 & 3699 & 3699 & 3699 & 3699 & GTD-CC04 & A. 3 \\
\hline 0.9 & 867 & 987 & 1010 & 1054 & A27-L-F6 & A. 38 \\
\hline 1.0 & 315 & 1357 & 1409 & 1409 & GTD-CC05 & A. 2 \\
\hline 2.0 & - & $\sim 81$ & - & 250 & GTD-CC10 & A. 1 \\
\hline 4.0 & 10 & 11 & 23 & 23 & L8-2A & A. 10 \\
\hline
\end{tabular}

Table 3b: Fatigue life of T-oriented DS GTD-111 at $871^{\circ} \mathrm{C}$ under continuous cycling

\begin{tabular}{|c|c|c|c|c|c|c|}
\hline \multirow{2}{*}{$\begin{array}{c}\text { Total Strain } \\
\text { Range, } \Delta \varepsilon(\%)\end{array}$} & \multicolumn{4}{|c|}{ Life, $N$} & \multirow{2}{*}{ Specimen ID } & \multirow{2}{*}{ Figure } \\
\hline & $90 \%$ & $80 \%$ & $50 \%$ & Fracture & & \\
\hline 0.5 & 16069 & 16069 & 16069 & 16069 & T8-2A & A.13 \\
\hline 0.8 & 564 & 598 & 721 & 1091 & T8-5 & A. 25 \\
\hline 0.9 & 188 & 271 & 302 & 429 & B17-T-F2 & A. 37 \\
\hline 1.0 & 266 & 300 & 333 & 333 & T8-2 & A. 17 \\
\hline 2.0 & 28 & 28 & 28 & 28 & T8-1A & A. 12 \\
\hline 4.0 & 3 & 3 & 3 & 3 & T8-1 & A. 11 \\
\hline
\end{tabular}


Table 4: Crack Initiation life of $L$ and T-oriented DS GTD-111 at $871^{\circ} \mathrm{C}$

\begin{tabular}{cccc}
\hline $\begin{array}{c}\text { Plastic Strain } \\
\text { Range, } \Delta \varepsilon_{p l}(\%)\end{array}$ & $\begin{array}{c}\text { Elastic Strain } \\
\text { Range, } \Delta \varepsilon_{\text {el }}(\%)\end{array}$ & $\begin{array}{c}\text { Crack Initation } \\
\text { Life, } N_{i}\end{array}$ & $\begin{array}{c}\text { Specimen ID } \\
\text { (Orientation) }\end{array}$ \\
\hline$<0.01$ & 0.50 & 29159 & L8-3 (L) \\
0.01 & 0.79 & 3699 & GTD-CC04 (L) \\
0.08 & 0.82 & 987 & A27-L-F6 (L) \\
0.03 & 0.97 & 1357 & GTD-CC05 (L) \\
0.80 & 1.20 & - & GTD-CC10 (L) \\
2.810 & 1.19 & 11 & L8-2A (L) \\
$<0.01$ & 0.50 & 16069 & T8-2A (T) \\
0.07 & 0.73 & 598 & T8-5 (T) \\
0.22 & 0.68 & 271 & B17-T-F2 (T) \\
0.22 & 0.78 & 300 & T8-2 (T) \\
1.46 & 0.54 & 28 & T8-1A (T) \\
2.87 & 1.13 & 3 & T8-1 (T) \\
\hline
\end{tabular}


Table 5: Crack Initiation life of $L$ and T-oriented DS GTD-111 above and below $871^{\circ} \mathrm{C}$

\begin{tabular}{|c|c|c|c|c|c|c|c|}
\hline $\begin{array}{l}\text { Tempe } \\
\left({ }^{\circ} \mathrm{C}\right)\end{array}$ & $\begin{array}{l}\text { rature } \\
\left({ }^{\circ} \mathrm{F}\right)\end{array}$ & $\begin{array}{c}\text { Total Strain } \\
\text { Range, } \\
\Delta \varepsilon_{m}(\%)\end{array}$ & $\begin{array}{c}\text { Plastic } \\
\text { Strain } \\
\text { Range, } \\
\Delta \varepsilon_{p l}(\%)\end{array}$ & $\begin{array}{c}\text { Elastic } \\
\text { Strain } \\
\text { Range, } \\
\Delta \varepsilon_{\text {el }}(\%)\end{array}$ & $\begin{array}{c}\text { Crack } \\
\text { Initation } \\
\text { Life, } N_{i}\end{array}$ & $\begin{array}{l}\text { Specimen ID } \\
\text { (Orientation) }\end{array}$ & Figure \\
\hline 427 & 800 & 2.0 & 0.55 & 1.45 & 30 & L8-4 (L) & A.16 \\
\hline 649 & 1200 & 1.0 & 0.09 & 0.91 & 14915 & A27-L-F10 (L) & A.52 \\
\hline 649 & 1200 & 1.5 & 0.05 & 1.45 & 394 & A27-L-F5 (L) & A.51 \\
\hline 760 & 1400 & 0.8 & 0.04 & 0.76 & 19403 & B21-L-F3 (L) & A.47 \\
\hline 760 & 1400 & 2.0 & 0.32 & 1.68 & 105 & L8-3A (L) & A. 15 \\
\hline 871 & 1600 & 0.5 & $<0.01$ & 0.50 & 29159 & L8-3 (L) & A.14 \\
\hline 871 & 1600 & 0.8 & 0.01 & 0.79 & 3699 & GTD-CC04 (L) & A. 3 \\
\hline 871 & 1600 & 0.9 & 0.08 & 0.82 & 987 & A27-L-F6 (L) & A.38 \\
\hline 871 & 1600 & 1.0 & 0.03 & 0.97 & 1357 & GTD-CC05 (L) & A. 2 \\
\hline 871 & 1600 & 2.0 & 0.80 & 1.20 & $\sim 81$ & GTD-CC10 (L) & A. 1 \\
\hline 871 & 1600 & 4.0 & 2.81 & 1.19 & 11 & L8-2A (L) & A. 10 \\
\hline 982 & 1800 & 0.8 & 0.27 & 0.53 & 912 & L8-9A (L) & A. 36 \\
\hline 982 & 1800 & 1.0 & 0.35 & 0.65 & 509 & L8-8A (L) & A.34 \\
\hline 982 & 1800 & 1.0 & 0.43 & 0.57 & 562 & L8-4A (L) & A. 19 \\
\hline 982 & 1800 & 2.0 & 1.19 & 0.81 & 112 & L8-8 (L) & A.35 \\
\hline 1038 & 1900 & 1.0 & 0.45 & 0.55 & 539 & L8-7A (L) & A. 29 \\
\hline 649 & 1200 & 1.2 & 0.35 & 0.85 & 194 & B18-T-F3 (T) & A.53 \\
\hline 760 & 1400 & 1.0 & 0.15 & 0.85 & 1033 & A27-T-F3 (T) & A.45 \\
\hline 871 & 1600 & 0.5 & $<0.01$ & 0.50 & 16069 & T8-2A (T) & A.13 \\
\hline 871 & 1600 & 0.8 & 0.07 & 0.73 & 598 & T8-5 (T) & A. 25 \\
\hline 871 & 1600 & 0.9 & 0.22 & 0.68 & 271 & B17-T-F2 (T) & A. 37 \\
\hline 871 & 1600 & 1.0 & 0.22 & 0.78 & 300 & T8-2 (T) & A. 17 \\
\hline 871 & 1600 & 2.0 & 1.46 & 0.54 & 28 & T8-1A (T) & A.12 \\
\hline 871 & 1600 & 4.0 & 2.87 & 1.13 & 3 & T8-1 (T) & A. 11 \\
\hline 982 & 1800 & 0.8 & 0.27 & 0.53 & 219 & T8-4 (T) & A. 24 \\
\hline 982 & 1800 & 1.0 & 0.42 & 0.58 & 207 & $\mathrm{~T} 8-3 \mathrm{~A}(\mathrm{~T})$ & A.18 \\
\hline 1038 & 1900 & 1.0 & 0.73 & 0.27 & 140 & T8-7 (T) & A.28 \\
\hline
\end{tabular}


Table 6: Comparison of mean strain effects of DS GTD-111 (0.5\%/s for each case)

\begin{tabular}{ccccccccc}
\hline Temperature & $\begin{array}{c}\text { Strain } \\
\text { Ratio, } \mathrm{R}_{\varepsilon}\end{array}$ & $\begin{array}{c}\text { Total } \\
\text { Strain } \\
\text { Range, } \\
\Delta \varepsilon_{m}(\%)\end{array}$ & $\begin{array}{c}\text { Plastic } \\
\text { Strain } \\
\text { Range, } \\
\Delta \varepsilon_{p l}(\%)\end{array}$ & $\begin{array}{c}\text { Elastic } \\
\text { Strain } \\
\text { Range, } \\
\Delta \varepsilon_{\text {el }}(\%)\end{array}$ & $\begin{array}{c}\text { Crack } \\
\text { Initation } \\
\text { Life, } N_{i}\end{array}$ & $\begin{array}{c}\text { Specimen ID } \\
\text { (Orientation) }\end{array}$ & Figure \\
\hline 760 & 1400 & -1 & 0.8 & 0.04 & 0.76 & 19403 & B21-L-F3 (L) & A.47 \\
760 & 1400 & 0 & 1.0 & 0.07 & 0.93 & 4114 & B21-L-F2 (L) & A.46 \\
871 & 1600 & -1 & 0.5 & $\sim 0.01$ & 0.5 & 29159 & L8-3 (L) & A.14 \\
871 & 1600 & -1 & 0.8 & 0.01 & 0.79 & 3699 & GTD-CC04 (L) & A.3 \\
871 & 1600 & -1 & 0.9 & 0.08 & 0.82 & 987 & A27-L-F6 (L) & A.38 \\
871 & 1600 & -1 & 1.0 & 0.03 & 0.97 & 1357 & GTD-CC05 (L) & A.2 \\
871 & 1600 & 0 & 0.5 & $\sim 0.01$ & 0.5 & 24000 & A27-L-F6 (L) & A.49 \\
871 & 1600 & 0 & 0.8 & 0.05 & 0.75 & 2990 & A27-L-F12 (L) & A.40 \\
871 & 1600 & 0 & 1.2 & 0.32 & 0.88 & 454 & B21-L-F1 (L) & A.44 \\
927 & 1700 & - & 0.8 & - & - & 785 & A27-L-F5 (L) & A.39 \\
\hline
\end{tabular}

Table 7: Comparison of strain rate dependence of DS GTD-111 (for each case $R_{\varepsilon}=-1$ )

\begin{tabular}{ccccccccc}
\hline Temperature & $\begin{array}{c}\text { Strain } \\
\text { Rate } \\
(\% / \mathrm{s})\end{array}$ & $\begin{array}{c}\text { Total } \\
\text { Strain } \\
\text { Range, } \\
\Delta \varepsilon_{m}(\%)\end{array}$ & $\begin{array}{c}\text { Plastic } \\
\text { Strain } \\
\text { Range, } \\
\Delta \varepsilon_{p l}(\%)\end{array}$ & $\begin{array}{c}\text { Elastic } \\
\text { Strain } \\
\text { Range, } \\
\Delta \varepsilon_{\text {el }}(\%)\end{array}$ & $\begin{array}{c}\text { Crack } \\
\text { Initation } \\
\text { Life, } N_{i}\end{array}$ & $\begin{array}{c}\text { Specimen ID } \\
\text { (Orientation) }\end{array}$ & Figure \\
\hline 982 & 1800 & 0.01 & 1.0 & 0.48 & 0.52 & 169 & A27-L-F13 (L) & A.42 \\
982 & 1800 & 0.5 & 0.8 & 0.27 & 0.53 & 912 & L8-9A (L) & A.36 \\
982 & 1800 & 0.5 & 1.0 & 0.43 & 0.57 & 562 & L8-4A (L) & A.19 \\
982 & 1800 & 0.5 & 1.0 & 0.35 & 0.65 & 509 & L8-8A (L) & A.34 \\
982 & 1800 & 0.5 & 2.0 & 1.19 & 0.81 & 112 & L8-8 (L) & A.35 \\
\hline 982 & 1800 & 0.01 & 1.0 & 0.55 & 0.45 & 52 & A27-T-F2 (T) & A.43 \\
982 & 1800 & 0.5 & 0.8 & 0.27 & 0.53 & 219 & T8-4 (T) & A.24 \\
982 & 1800 & 0.5 & 1.0 & 0.42 & 0.58 & 207 & T8-3A (T) & A.18 \\
\hline
\end{tabular}


Table 8: Crack initiation life of DS GTD-111 under isothermal creep-fatigue conditions

\begin{tabular}{|c|c|c|c|c|c|c|c|c|}
\hline$\left({ }^{\circ} \mathrm{C}\right)$ & $\left({ }^{\circ} \mathrm{F}\right)$ & $\begin{array}{l}\text { Hold Type } \\
\text { (Hold } \\
\text { Time) }\end{array}$ & $\begin{array}{c}\text { Total } \\
\text { Strain } \\
\text { Range, } \\
\Delta \varepsilon_{m}(\%)\end{array}$ & $\begin{array}{l}\text { Plastic } \\
\text { Strain } \\
\text { Range, } \\
\Delta \varepsilon_{p l}(\%)\end{array}$ & $\begin{array}{l}\text { Elastic } \\
\text { Strain } \\
\text { Range, } \\
\Delta \varepsilon_{\text {el }}(\%)\end{array}$ & $\begin{array}{c}\text { Crack } \\
\text { Initation } \\
\text { Life, } N_{i}\end{array}$ & $\begin{array}{l}\text { Specimen ID } \\
\text { (Orientation) }\end{array}$ & Figure \\
\hline 649 & 1200 & $\mathrm{HC}(2 \mathrm{~min})$ & 1.0 & 0.01 & 0.99 & 6768 & L8-6 (L) & A.23 \\
\hline 871 & 1600 & $\mathrm{HT}$ (2min) & 0.8 & 0.08 & 0.72 & 678 & GTD-HT04 (L) & A. 9 \\
\hline 871 & 1600 & $\mathrm{HT}$ (2min) & 1.0 & 0.11 & 0.89 & 590 & GTD-HT05 (L) & A. 6 \\
\hline 871 & 1600 & $\mathrm{HT}$ (2min) & 2.0 & 0.69 & 1.31 & 84 & GTD-HT10 (L) & A. 4 \\
\hline 871 & 1600 & $\mathrm{HC}(2 \mathrm{~min})$ & 0.8 & 0.12 & 0.68 & - & GTD-HC04 (L) & A. 8 \\
\hline 871 & 1600 & $\mathrm{HC}(2 \mathrm{~min})$ & 1.0 & 0.16 & 0.84 & $>250$ & GTD-HC05 (L) & A. 7 \\
\hline 871 & 1600 & $\mathrm{HC}(2 \mathrm{~min})$ & 2.0 & 0.68 & 1.32 & 49 & GTD-HC10 (L) & A. 5 \\
\hline 982 & 1800 & $\mathrm{HT}$ (2min) & 1.0 & 0.31 & 0.69 & 331 & L8-5 (L) & A. 21 \\
\hline 982 & 1900 & $\mathrm{HC}(2 \mathrm{~min})$ & 0.5 & 0.05 & 0.45 & 1537 & L8-6A (L) & A. 24 \\
\hline 982 & 1900 & $\mathrm{HC}(2 \mathrm{~min})$ & 1.0 & 0.37 & 0.63 & 406 & L8-5A (L) & A. 23 \\
\hline 1038 & 1900 & $\mathrm{HC}(2 \mathrm{~min})$ & 1.0 & 0.46 & 0.54 & 464 & L8-7 (L) & A.30 \\
\hline 871 & 1600 & $\mathrm{HT}$ (2min) & 1.0 & 0.32 & 0.68 & 150 & T8-5A (T) & A. 26 \\
\hline 871 & 1600 & $\mathrm{HC}(2 \mathrm{~min})$ & 1.0 & 0.35 & 0.65 & 87 & T8-6A (T) & A. 27 \\
\hline 982 & 1800 & $\mathrm{HT}$ (2min) & 1.0 & 0.38 & 0.62 & 47 & $\mathrm{~T} 8-4 \mathrm{~A}(\mathrm{~T})$ & A. 20 \\
\hline 982 & 1800 & $\mathrm{HC}(2 \mathrm{~min})$ & 1.0 & 0.42 & 0.58 & 115 & T8-3 (T) & A. 22 \\
\hline 1038 & 1900 & $\mathrm{HC}(2 \mathrm{~min})$ & 1.0 & 0.27 & 0.73 & 152 & T8-7A (T) & A.31 \\
\hline
\end{tabular}


Table 9: Crack initiation life of DS GTD-111 under TMF conditions (Re=-1)

\begin{tabular}{|c|c|c|c|c|c|c|c|c|c|c|c|}
\hline \multicolumn{2}{|c|}{ Minimum Temp. } & \multicolumn{2}{|c|}{ Maximum Temp. } & \multirow{2}{*}{$\begin{array}{l}\text { Phase } \\
\text { Shift }\left(^{\circ}\right)\end{array}$} & \multirow{2}{*}{$\begin{array}{c}\text { Total } \\
\text { Strain } \\
\text { Range, } \\
\Delta \varepsilon_{m}(\%)\end{array}$} & \multirow{2}{*}{$\begin{array}{l}\text { Plastic } \\
\text { Strain } \\
\text { Range, } \\
\Delta \varepsilon_{p l}(\%)\end{array}$} & \multirow{2}{*}{$\begin{array}{l}\text { Elastic } \\
\text { Strain } \\
\text { Range, } \\
\Delta \varepsilon_{\text {el }}(\%)\end{array}$} & \multirow{2}{*}{$\begin{array}{l}\text { Thermal } \\
\text { Strain } \\
\text { Range, } \\
\Delta \varepsilon_{\text {el }}(\%)\end{array}$} & \multirow{2}{*}{$\begin{array}{c}\text { Crack } \\
\text { Initation } \\
\text { Life, } N_{i}\end{array}$} & \multirow{2}{*}{$\begin{array}{l}\text { Specimen ID } \\
\text { (Orientation) }\end{array}$} & \multirow{2}{*}{ Figure } \\
\hline$\left({ }^{\circ} \mathrm{C}\right)$ & $\left({ }^{\circ} \mathrm{F}\right)$ & $\left({ }^{\circ} \mathrm{C}\right)$ & $\left({ }^{\circ} \mathrm{F}\right)$ & & & & & & & & \\
\hline 538 & 1000 & 927 & 1700 & 180 & 1.0 & 0.19 & 0.81 & 0.53 & 148 & L8-11A (L) & B. 3 \\
\hline 538 & 1000 & 927 & 1700 & 180 & 0.5 & 0.02 & 0.48 & 0.61 & 1270 & L8-10A (L) & B. 2 \\
\hline 538 & 1000 & 1038 & 1900 & 0 & 1.0 & 0.13 & 0.87 & 0.53 & 58 & L8-9 (L) & B. 1 \\
\hline 538 & 1000 & 927 & 1700 & 180 & 1.0 & 0.44 & 0.56 & 0.53 & 17 & $\mathrm{~T} 8-10(\mathrm{~T})$ & B.4 \\
\hline 538 & 1000 & 927 & 1700 & 180 & 0.5 & 0.20 & 0.30 & 0.53 & 173 & $\mathrm{~T} 8-10 \mathrm{~A}(\mathrm{~T})$ & B.5 \\
\hline
\end{tabular}

Table 10: Crack initiation life of pre-exposed DS GTD-111 (For each case $t_{\text {pre }}=100 \mathrm{hr}$ )

\begin{tabular}{|c|c|c|c|c|c|c|c|c|c|c|c|c|}
\hline \multicolumn{4}{|c|}{ Pre-Exposure } & \multicolumn{9}{|c|}{ Fatigue Test } \\
\hline \multicolumn{2}{|c|}{ Temperature } & \multirow{2}{*}{$\begin{array}{l}\text { Environ- } \\
\text { ment }\end{array}$} & \multirow{2}{*}{$\begin{array}{l}\text { Load } \\
\text { (MPa) }\end{array}$} & \multicolumn{2}{|c|}{ Temperature } & \multirow{2}{*}{$\begin{array}{l}\text { Environ- } \\
\text { ment }\end{array}$} & \multirow{2}{*}{$\begin{array}{c}\text { Total } \\
\text { Strain } \\
\text { Range, } \\
\Delta \varepsilon_{m}(\%)\end{array}$} & \multirow{2}{*}{$\begin{array}{l}\text { Plastic } \\
\text { Strain } \\
\text { Range, } \\
\Delta \varepsilon_{p l}(\%)\end{array}$} & \multirow{2}{*}{$\begin{array}{c}\text { Elastic } \\
\text { Strain } \\
\text { Range, } \\
\Delta \varepsilon_{\text {el }}(\%)\end{array}$} & \multirow{2}{*}{$\begin{array}{l}\text { Crack } \\
\text { Initation } \\
\text { Life, } N_{i}\end{array}$} & \multirow{2}{*}{$\begin{array}{l}\text { Specimen ID } \\
\text { (Orientation) }\end{array}$} & \multirow{2}{*}{ Figure } \\
\hline$\left({ }^{\circ} \mathrm{C}\right)$ & $\left({ }^{\circ} \mathrm{F}\right)$ & & & $\left({ }^{\circ} \mathrm{C}\right)$ & $\left({ }^{\circ} \mathrm{F}\right)$ & & & & & & & \\
\hline 982 & 1800 & Air & 100 & 871 & 1600 & Air & 0.8 & 0.22 & 0.58 & 167 & A27-L-F1 (L) & C. 2 \\
\hline 982 & 1800 & Air & -100 & 871 & 1600 & Air & 0.8 & $<0.01$ & 0.8 & 1857 & A27-L-F8 (L) & C. 3 \\
\hline 982 & 1800 & Wet $\mathrm{H}_{2} \mathrm{~S}$ & 0 & 871 & 1600 & Air & 0.8 & 0.23 & 0.57 & 979 & A27-L-F15 (L) & C.5 \\
\hline 982 & 1800 & Wet $\mathrm{H}_{2} \mathrm{~S}$ & 0 & 871 & 1600 & Air & 0.8 & 0.11 & 0.69 & 84 & A27-T-F4 (T) & C. 6 \\
\hline 982 & 1800 & Air & 0 & 871 & 1600 & Air & 0.8 & $<0.01$ & 0.8 & 1958 & B17-L-F3 (L) & C.4 \\
\hline \multicolumn{4}{|c|}{ No Pre-Exposure } & 871 & 1600 & Air & 0.8 & 0.01 & 0.79 & 3699 & GTD-CC04 (L) & A. 3 \\
\hline \multicolumn{4}{|c|}{ No Pre-Exposure } & 871 & 1600 & Air & 0.8 & 0.07 & 0.73 & 598 & T8-5 (T) & A. 25 \\
\hline
\end{tabular}


Table 11: Test configurations for isothermal, unstressed samples

\begin{tabular}{ccccc}
\hline Specimen ID & $\begin{array}{c}\text { Exposure Time } \\
(\mathrm{hr})\end{array}$ & $\begin{array}{c}\text { Temperature } \\
\left({ }^{\circ} \mathrm{C}\right)\end{array}$ & $L_{\text {ox }}(\mu \mathrm{m})$ & $\begin{array}{c}L_{G P D} \\
(\mu \mathrm{m})\end{array}$ \\
\hline LT-16-1 & 1 & 871 & 3 & 5 \\
LT-16-6 & 314 & 871 & 5 & 10 \\
LT-18-1 & 1 & 982 & 3 & $<1$ \\
LT-18-2 & 3 & 982 & 5 & 10 \\
LT-18-3 & 10 & 982 & 7 & 13 \\
LT-18-4 & 32 & 982 & 13 & 15 \\
LT-18-6 & 34 & 982 & 22 & 22 \\
LT-18-7 & 1006 & 982 & 25 & 23 \\
LT-19-2 & 3 & 1038 & 6 & 10 \\
LT-19-3 & 10 & 1038 & 10 & 12 \\
LT-19-4 & 32 & 1038 & 16 & 18 \\
LT-19-5 & 100 & 1038 & 19 & 23 \\
LT-19-6 & 316 & 1038 & 23 & 32 \\
LT-19-7 & 1182 & 1038 & 32 & 64 \\
TL-18-2 & 3 & 982 & 6 & 8 \\
TL-18-3 & 10 & 982 & 7 & 10 \\
TL-18-5 & 100 & 982 & 18 & 18 \\
TL-18-6 & 316 & 982 & 29 & 27 \\
TL-18-7 & 1182 & 982 & 30 & 35 \\
\hline
\end{tabular}

Table 12: Unstressed oxidation and $\gamma$ ' depletion depth model constants

\begin{tabular}{ccccc}
\hline Model & $\alpha(\mu \mathrm{m})$ & $\theta(1 / \mathrm{hr})$ & $Q(\mathrm{~J} / \mathrm{mol})$ & $m$ \\
\hline Oxidation & 1000 & 115 & 275376 & 0.25 \\
GPD & 1000 & 115 & 264462 & 0.25 \\
\hline
\end{tabular}




\section{Figures}

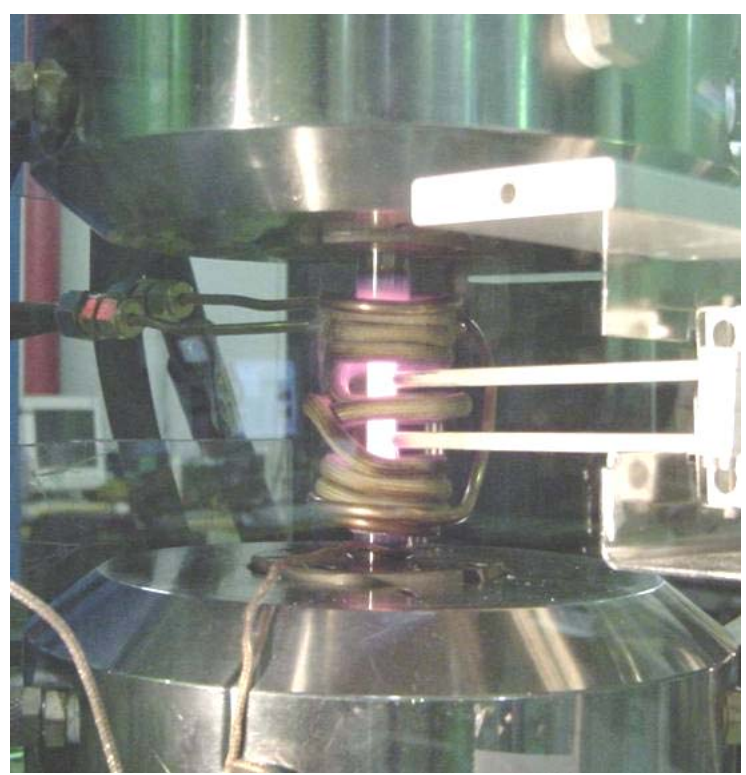

Figure 1: Arrangement of specimen within the collet grips and induction coils.

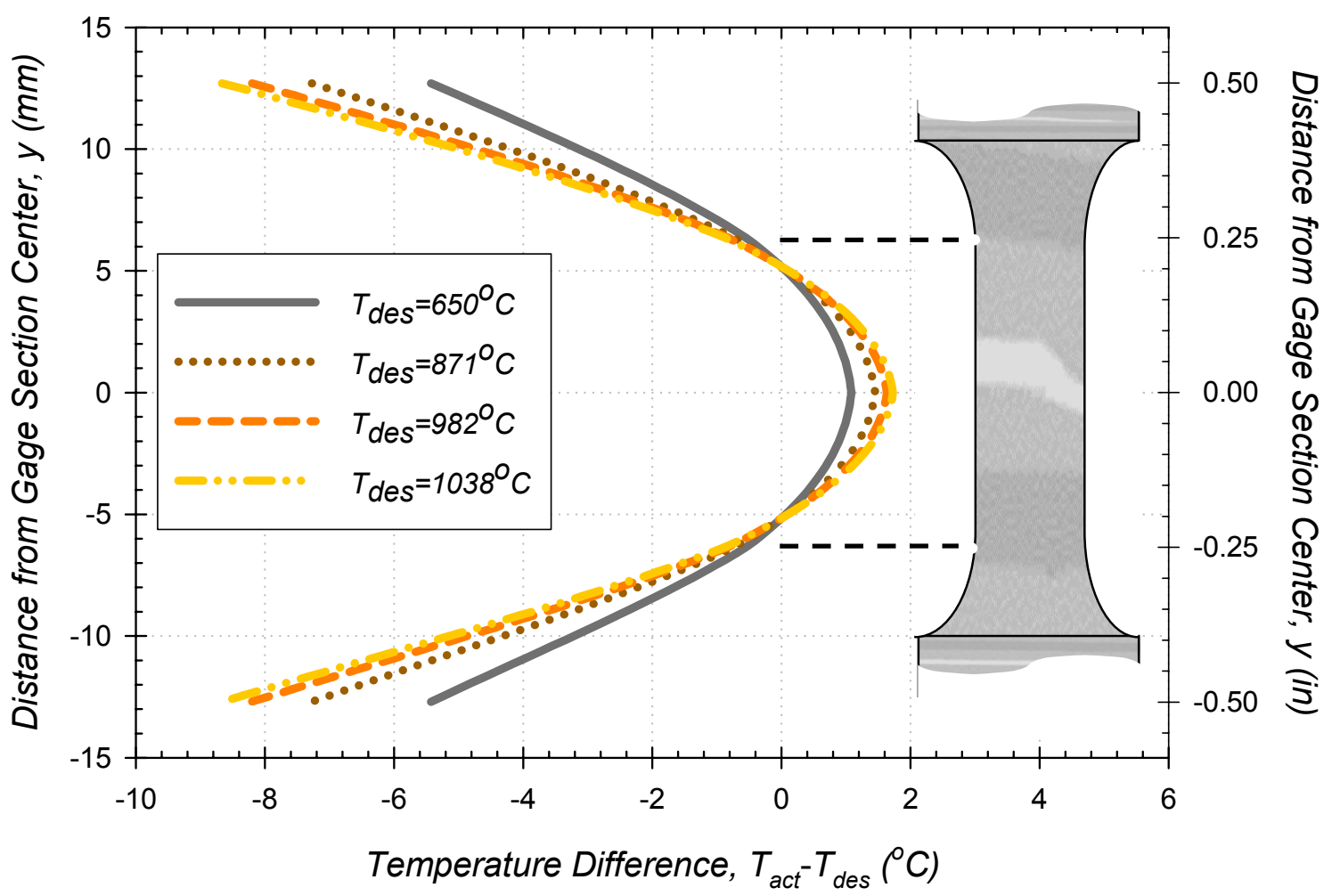

Figure 2: Typical axial temperature gradients within specimen gage section for various desired temperature set points (specimen not drawn to scale). 

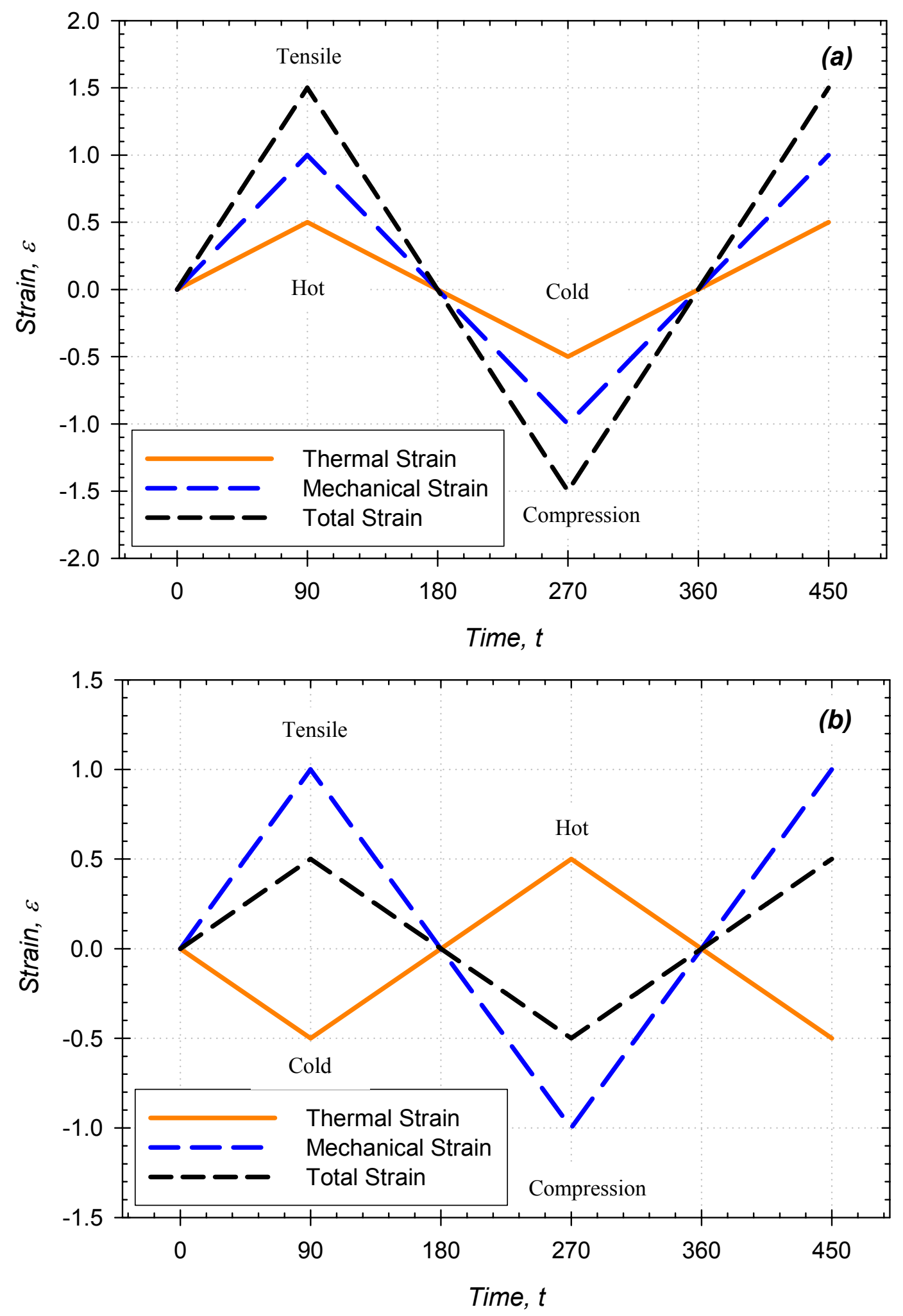

Figure 3: Strain history for (a) in-phase and (b) out-of-phase straincontrolled thermomechanical fatigue cycling. 


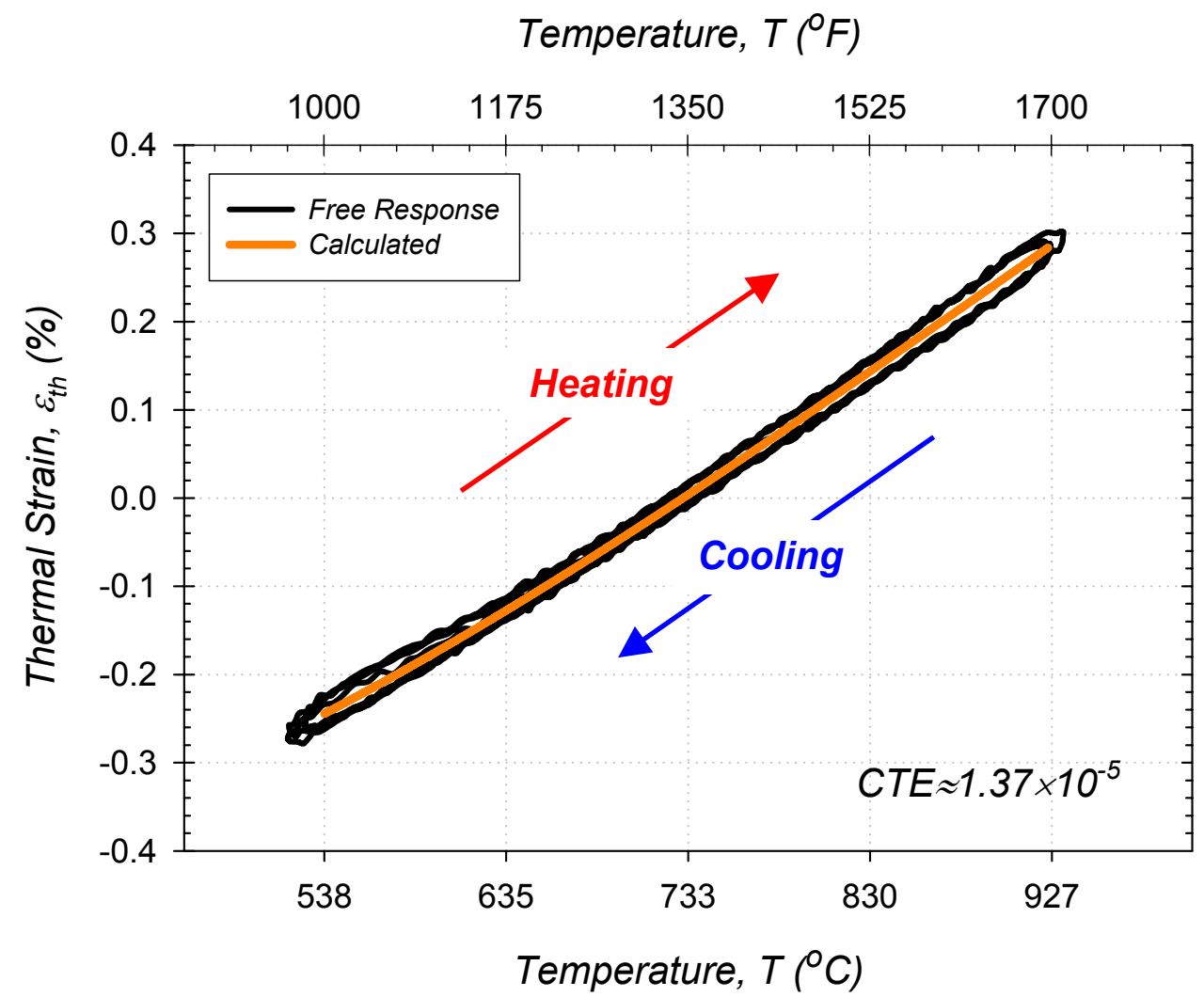

Figure 4: Thermal strain generated during free expansion under temperature cycling at zero load.

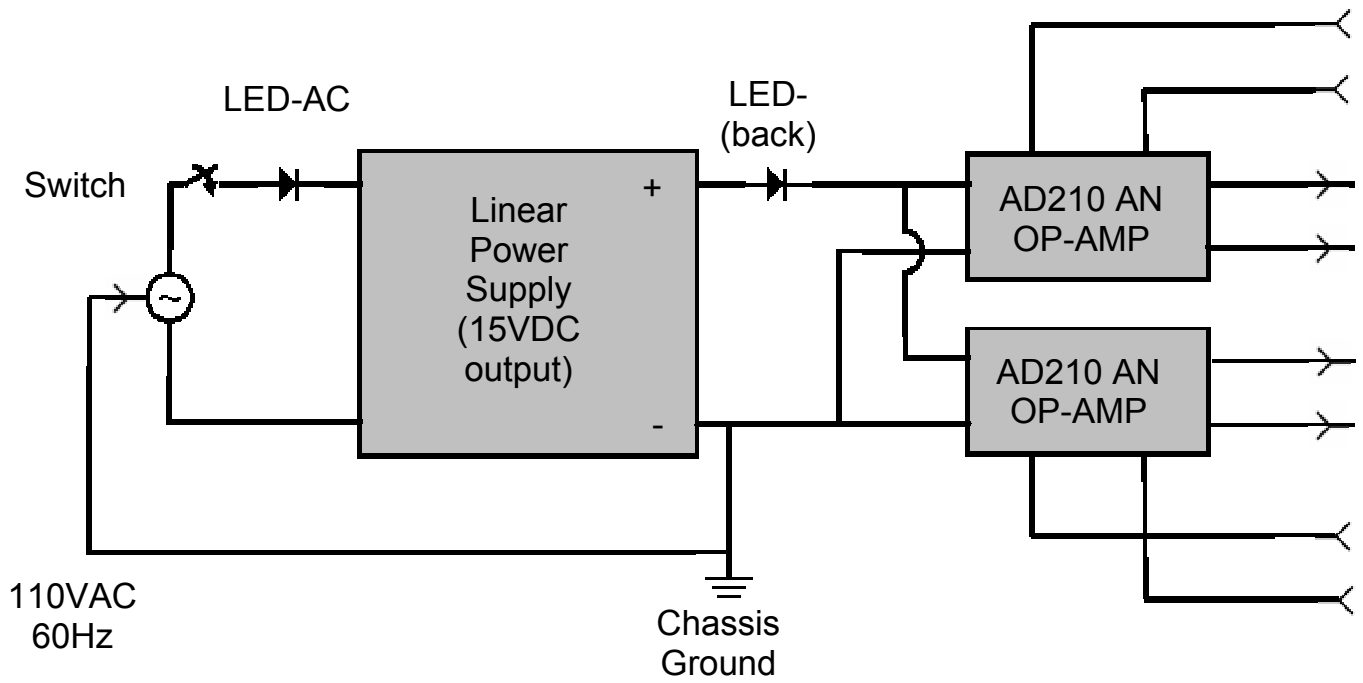

Figure 5: Circuit diagram for isolation device. 


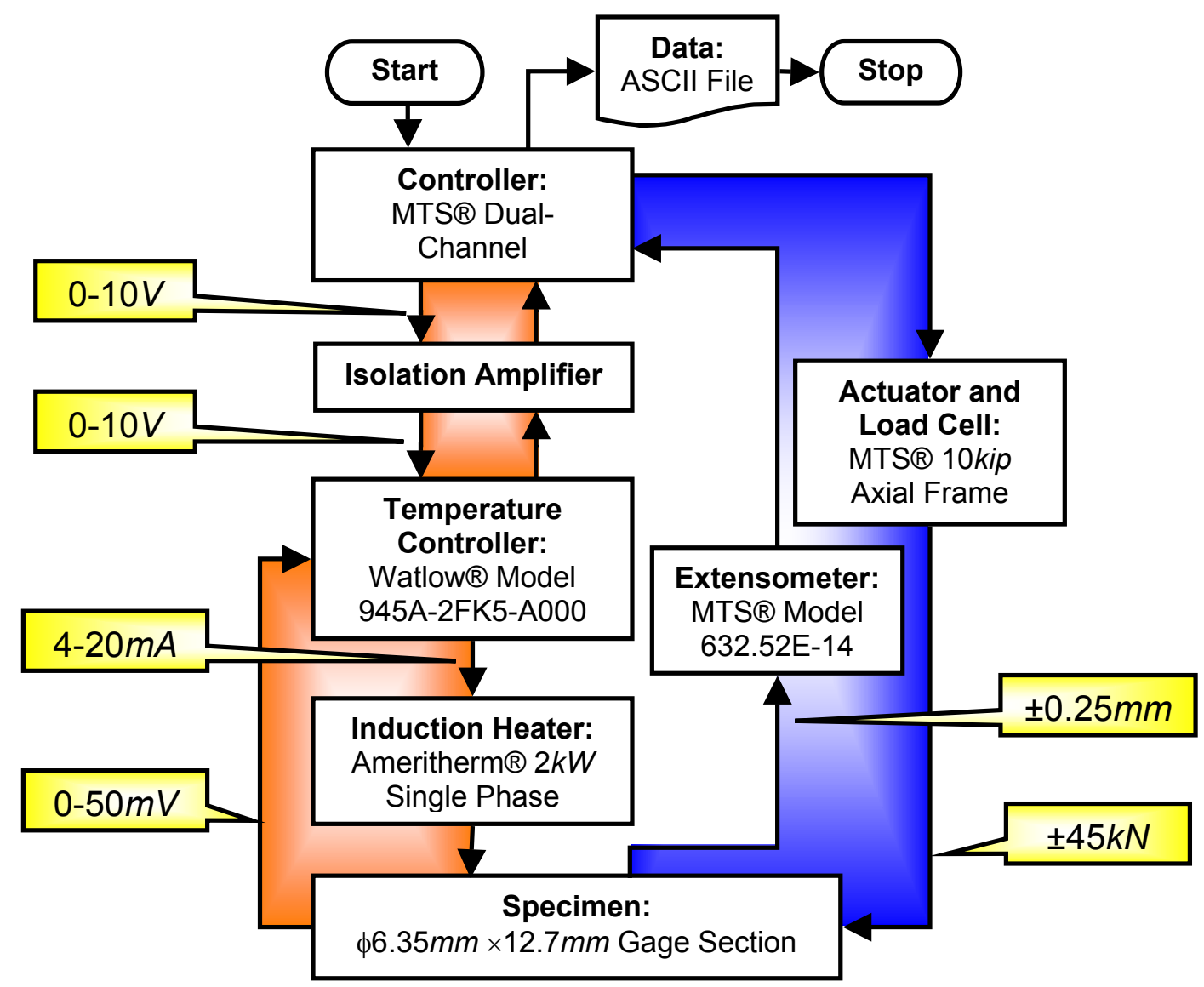

Figure 6: Components and respective signals transmitted in dual closed loop feedback control, thermomechanical fatigue testing system.
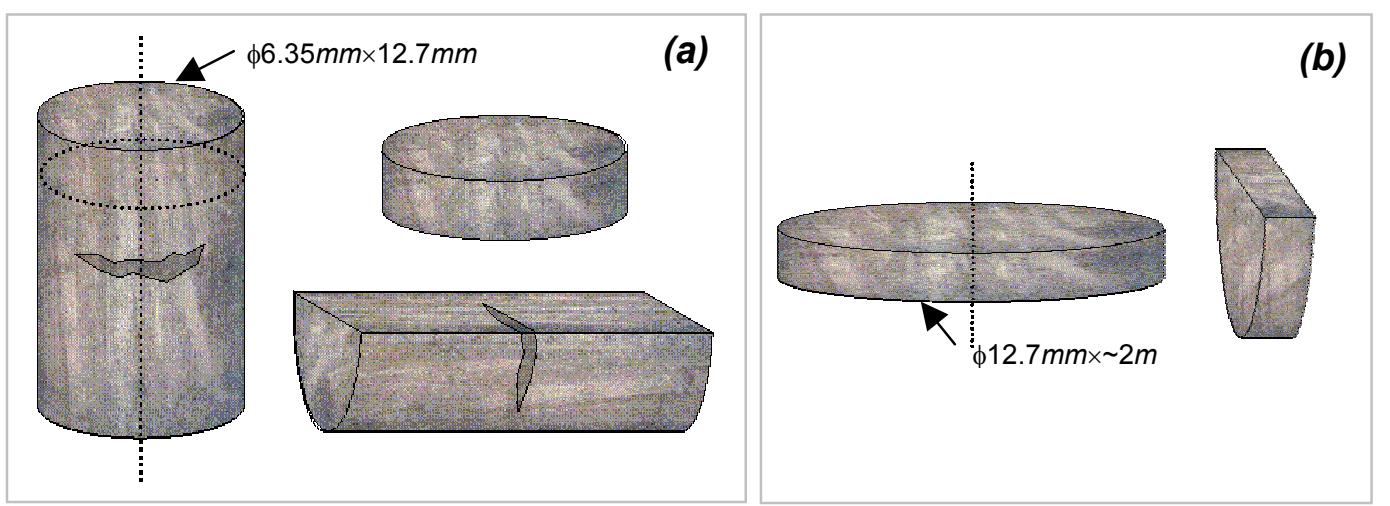

Figure 7: Sectioning convention for (a) fatigue and (b) oxidation specimens. 


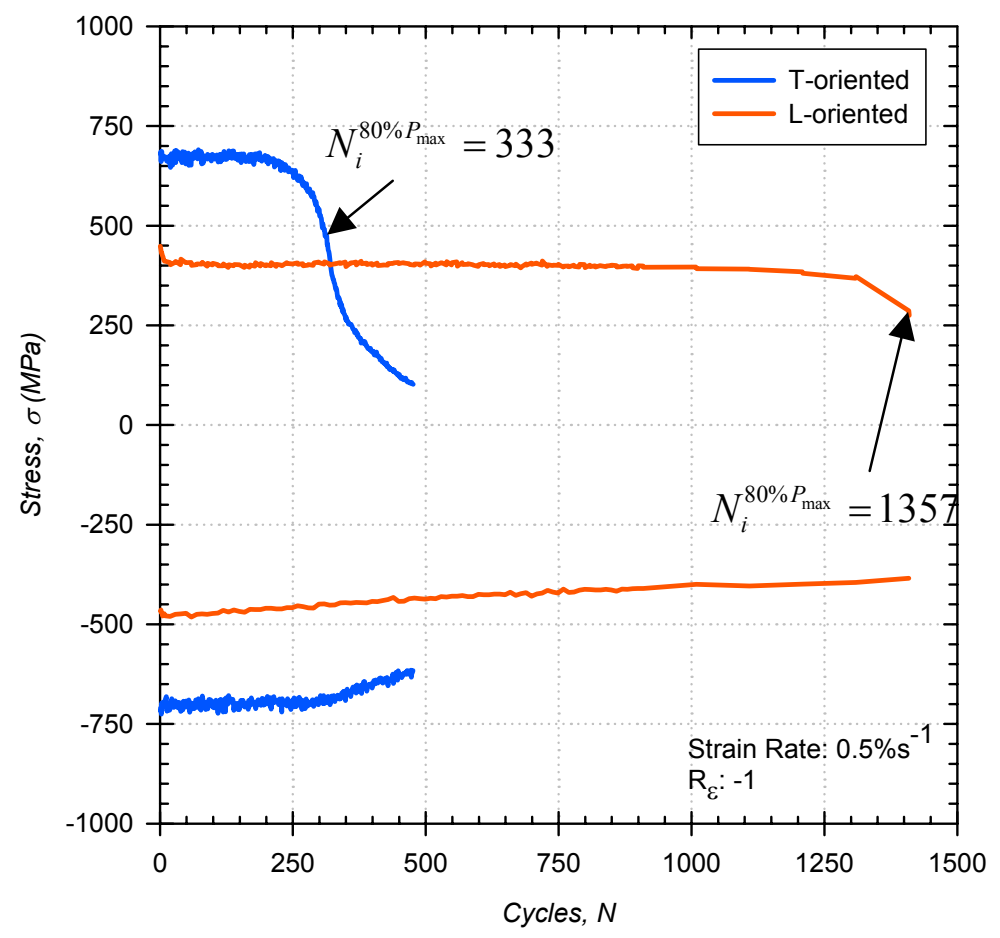

Figure 8: Stress histories for $L$ and T-oriented DS GTD-111 at $\Delta \varepsilon=1.0 \%$ and $T=871^{\circ} \mathrm{C}\left(1600^{\circ} \mathrm{F}\right)$.

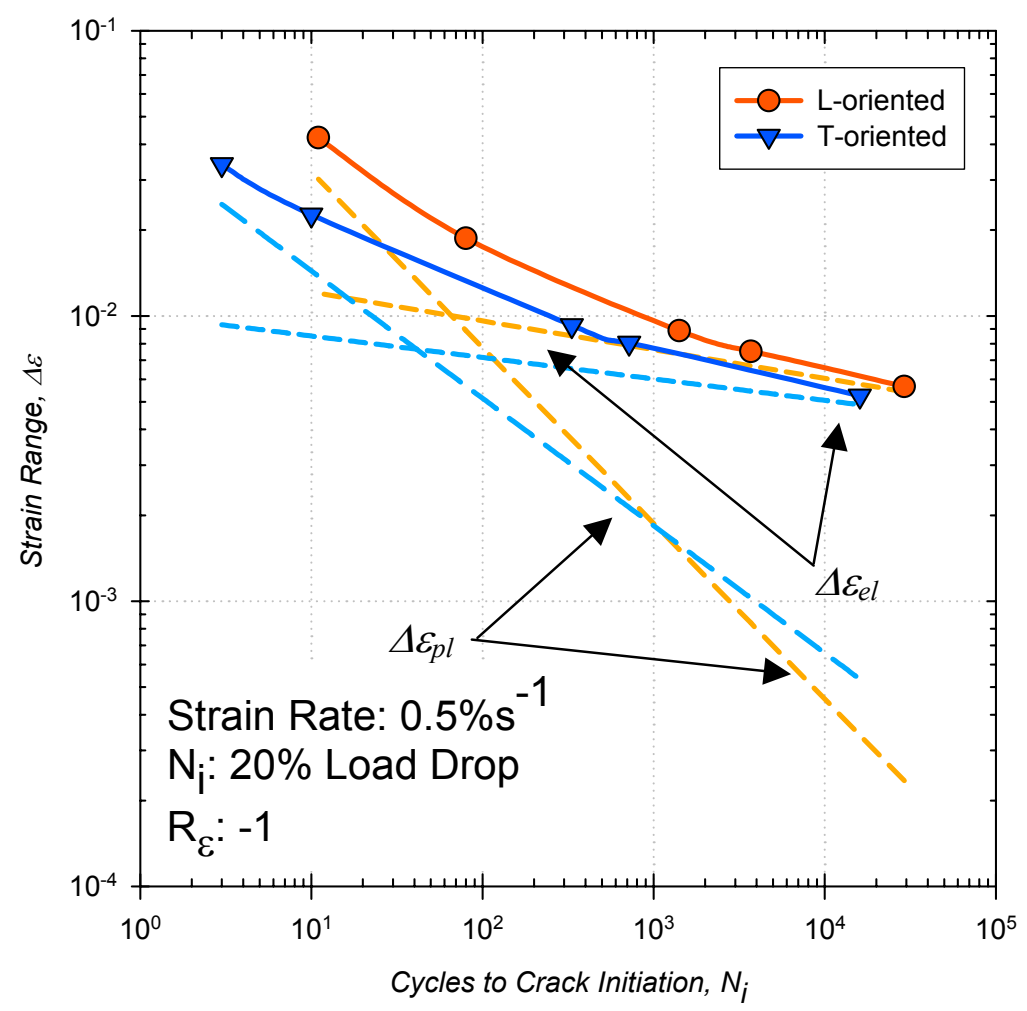

Figure 9: Strain life curves of crack initiation lives of $L$ and T-oriented DS GTD-111 at $T=871^{\circ} \mathrm{C}\left(1600^{\circ} \mathrm{F}\right)$. 


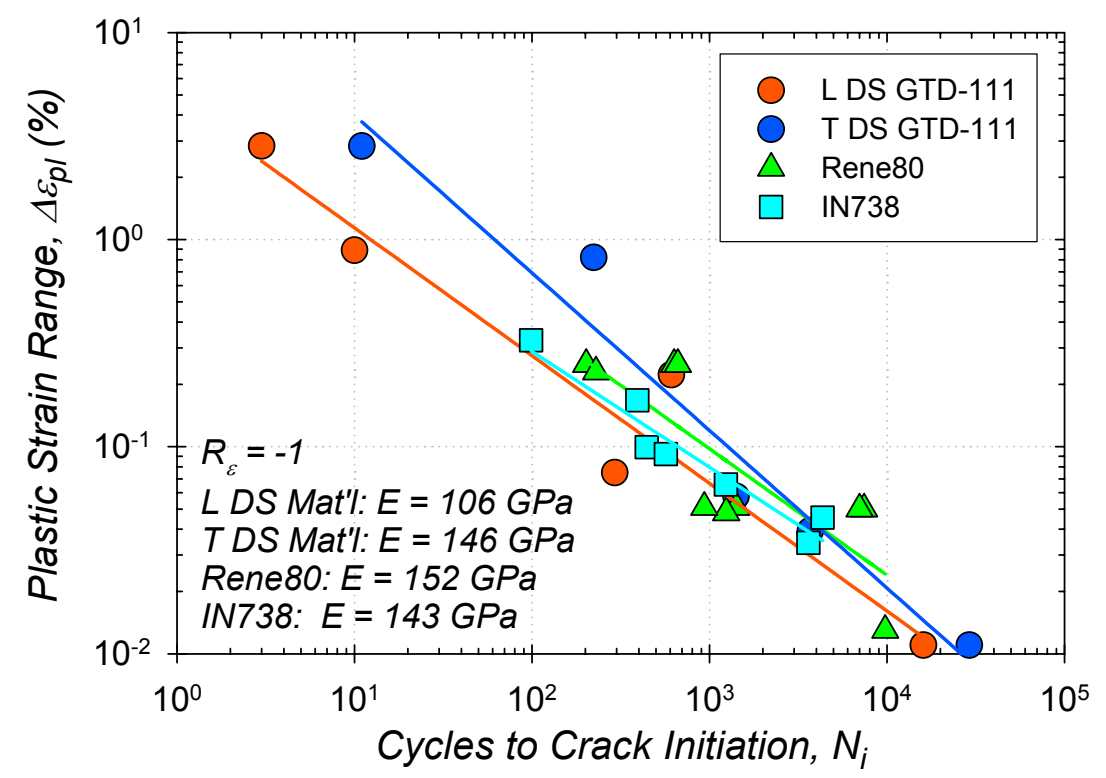

Figure 10: Comparison of plastic strain crack initiation lives of DS GTD111 with PC Ni-base superalloys at 871C. 

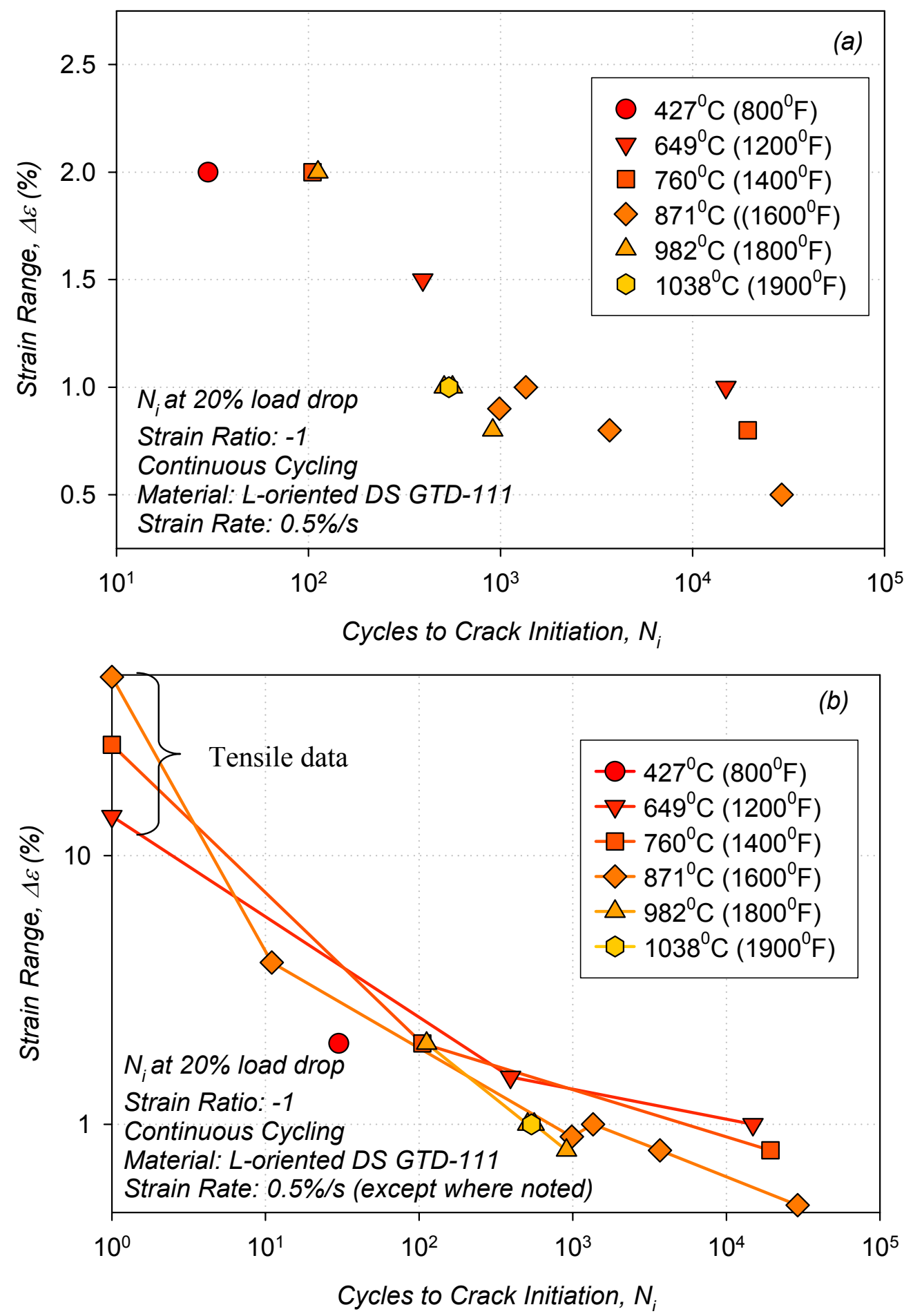

Figure 11: Temperature dependence of crack initiation life of Loriented DS GTD-111 under continuous cycling conditions, strain rate of $0.5 \% / \mathrm{s}$, and strain ratio of $R_{\varepsilon}=-1$. (a) Semi-log plot (b) log-log plot compared with tensile data at $0.0167 \%$ (Ibanez, 2003). 

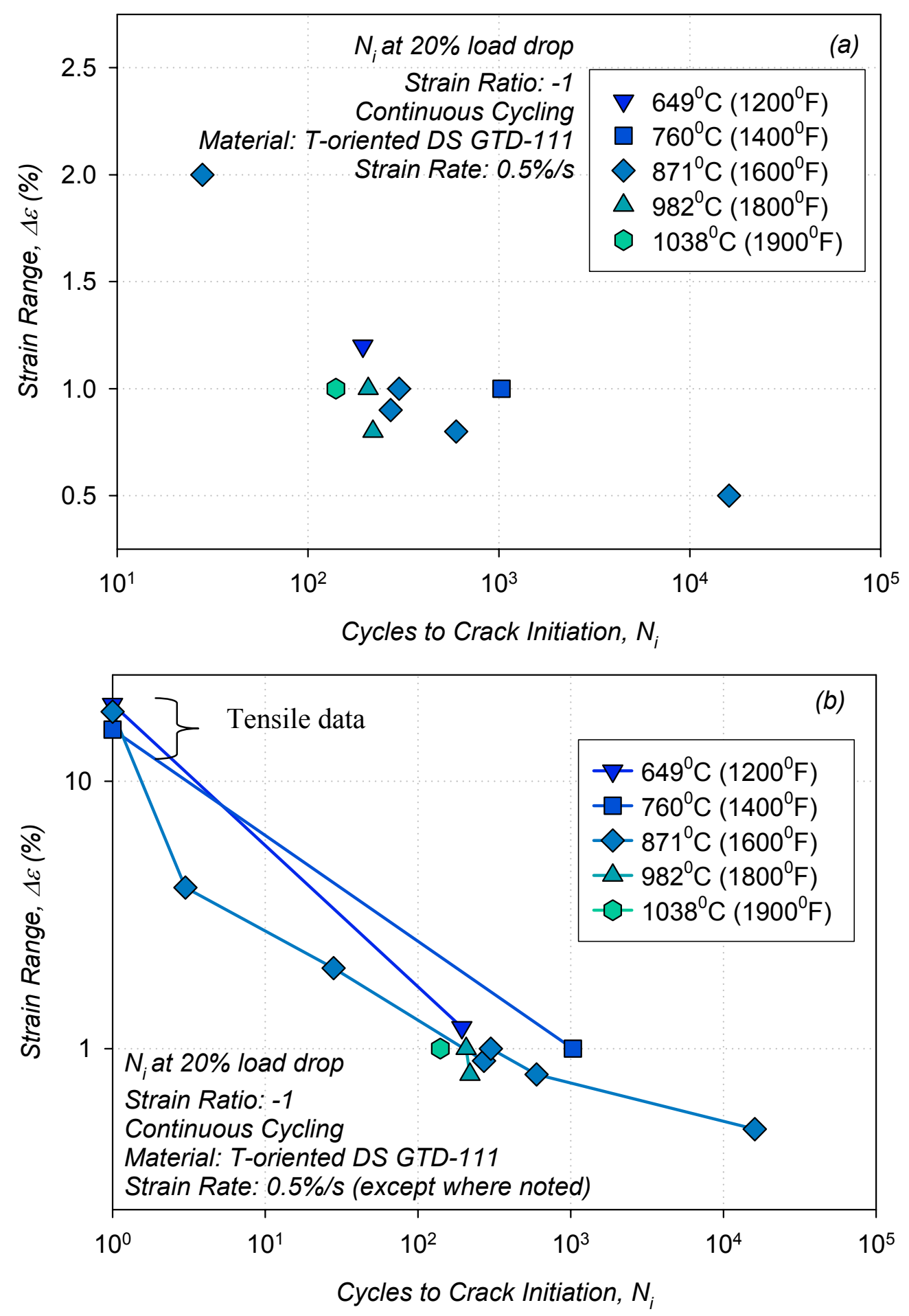

Figure 12: Temperature dependence of crack initiation life of Loriented DS GTD-111 under continuous cycling conditions, strain rate of $0.5 \% / \mathrm{s}$, and strain ratio of $R_{\varepsilon}=-1$. (a) Semi-log plot (b) log-log plot compared with tensile data at $0.0167 \%$ (Ibanez, 2003). 

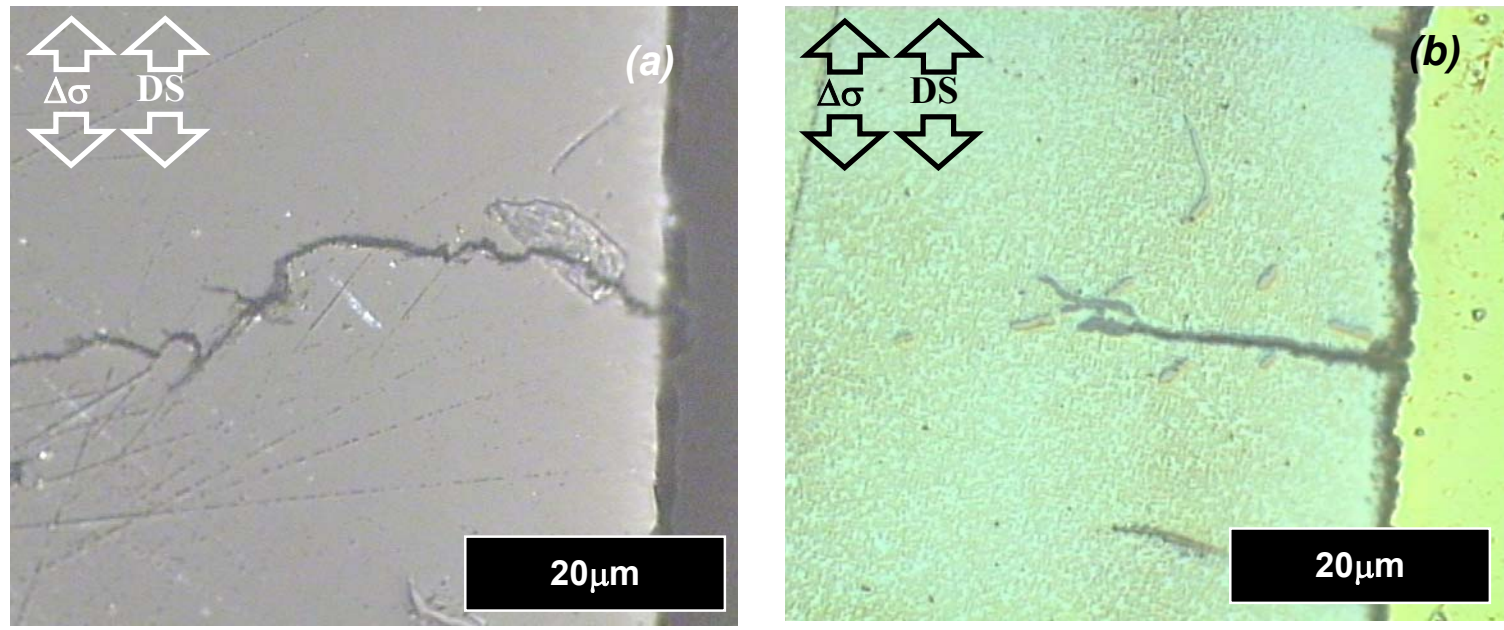

Figure 13: Comparison of cracks in L-oriented DS GTD-111 subjected to: (a) $\Delta \varepsilon=0.8 \%$ and $760^{\circ} \mathrm{C}$ and (b) $\Delta \varepsilon=0.9 \%$ and $871{ }^{\circ} \mathrm{C}$. For each case $R_{\varepsilon}=-1$ and $\&=0.5 \% / s$.

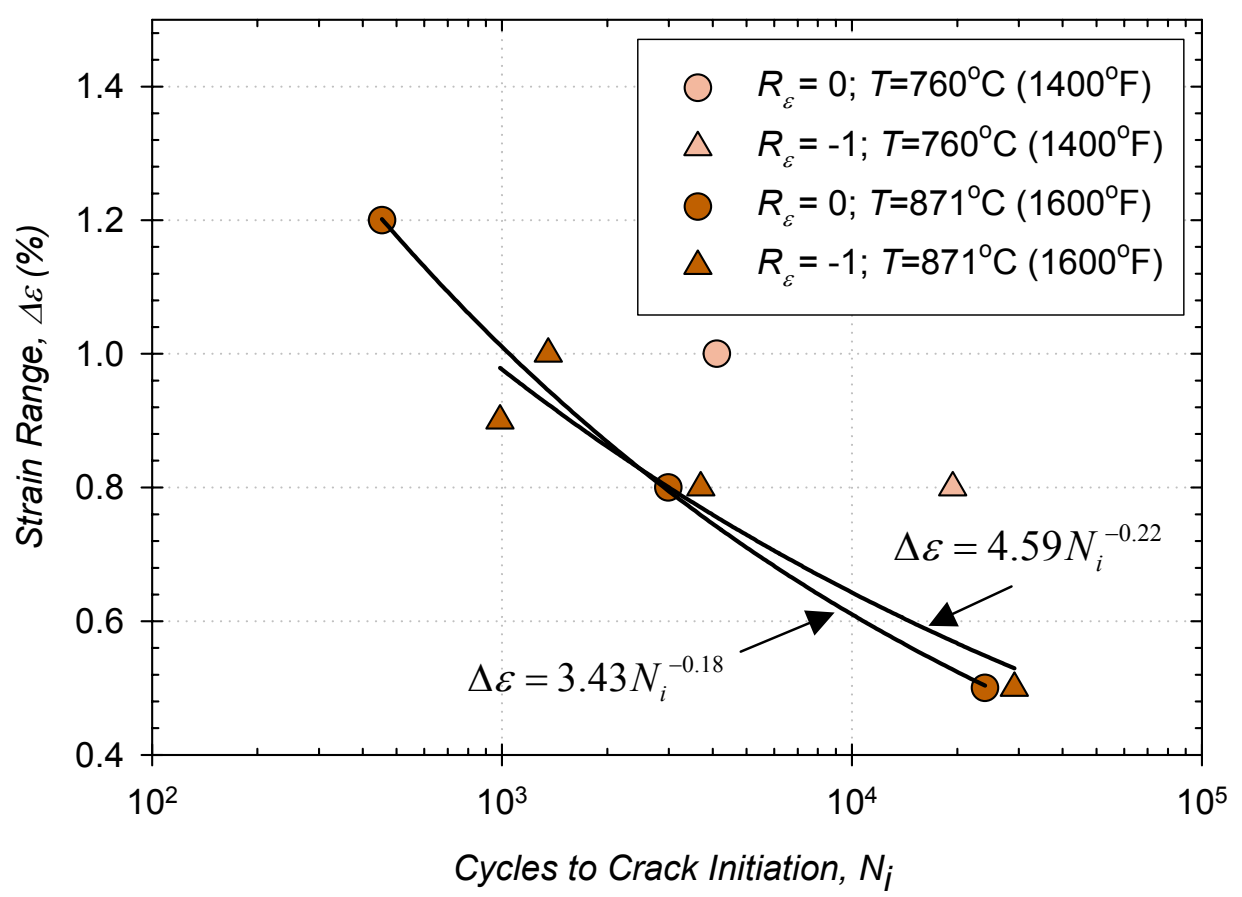

Figure 14: Mean stress effects on crack initiation life of L-oriented DS GTD-111 with \& $=0.5 \% / s$. 


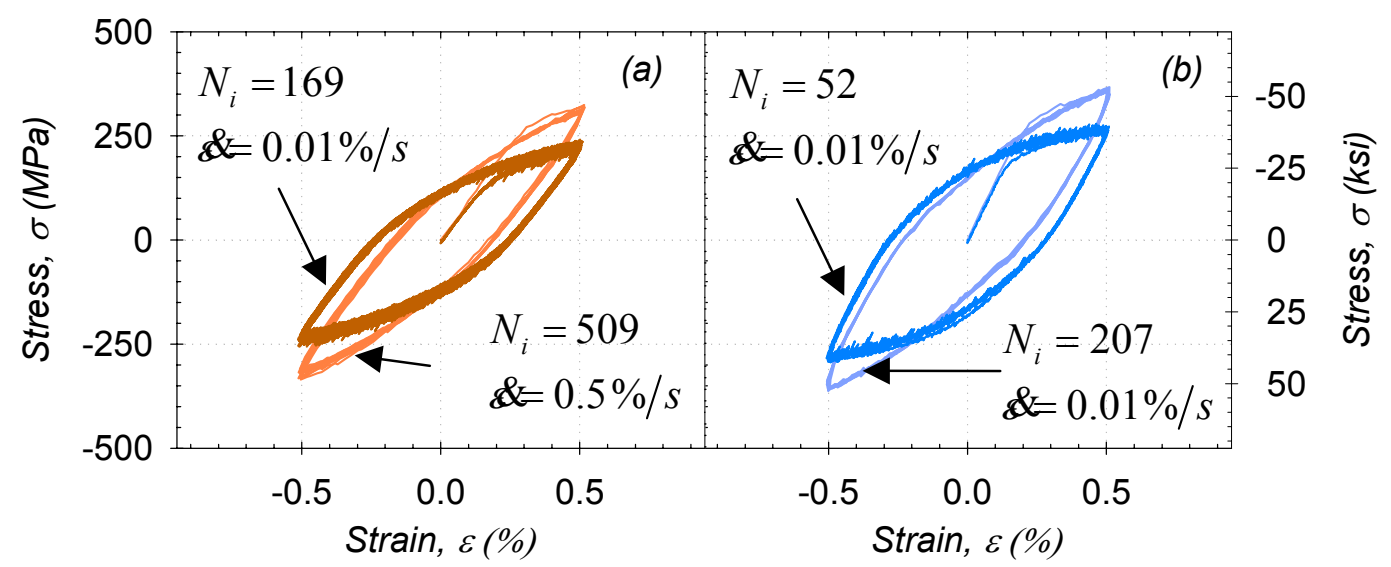

Figure 15: Initial hysteresis loops of (a) L-oriented and (b) T-oriented DS GTD-111 subjected to dissimilar strain rates with $R_{\varepsilon}=-1$ and $T=982^{\circ} \mathrm{C}$.

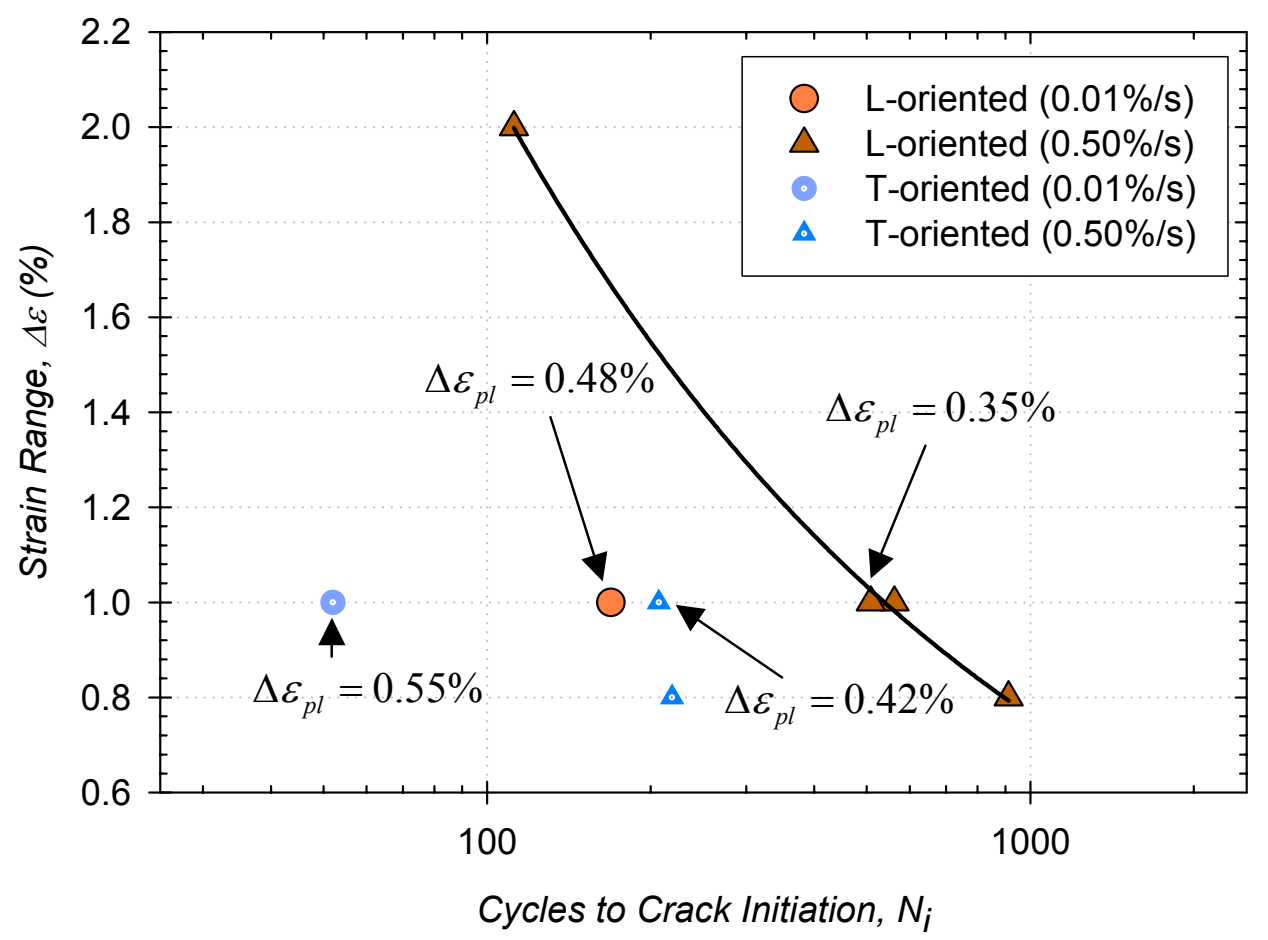

Figure 16: Strain rate effects on the crack initiation life of $L$ and $T$ oriented DS GTD-111 with $R_{\varepsilon}=-1$. 

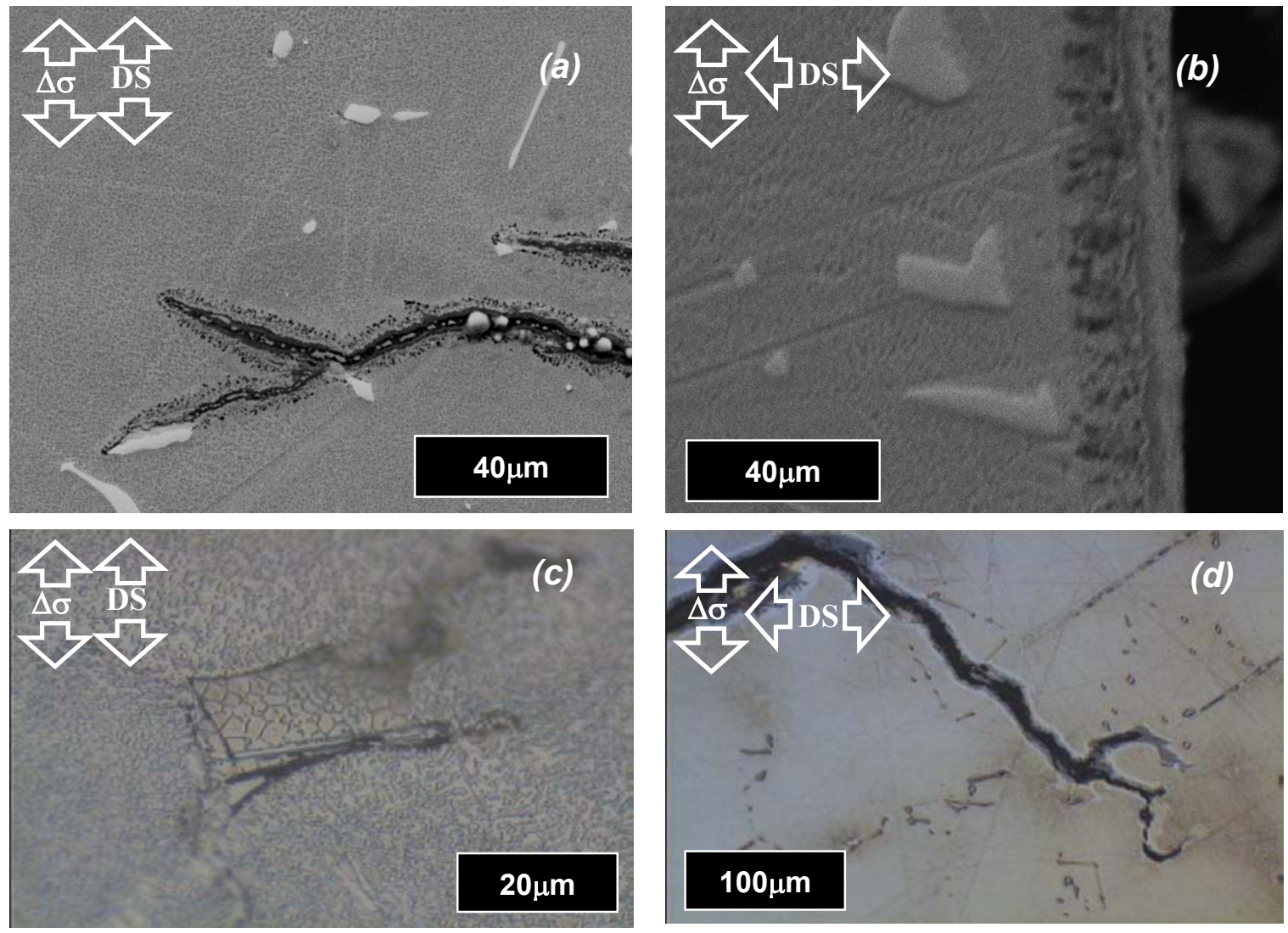

Figure 17: Effect of strain rate on crack initiation life of continuously cycled $(a, c) L$ and $(b, d)$ T-oriented DS GTD-111 under $(a, b)$ $\&=0.5 \% / s$ and $(c, d) \&=0.01 \% / s$. For each case $R_{\varepsilon}=-1$ and $T=982^{\circ} \mathrm{C}$. 

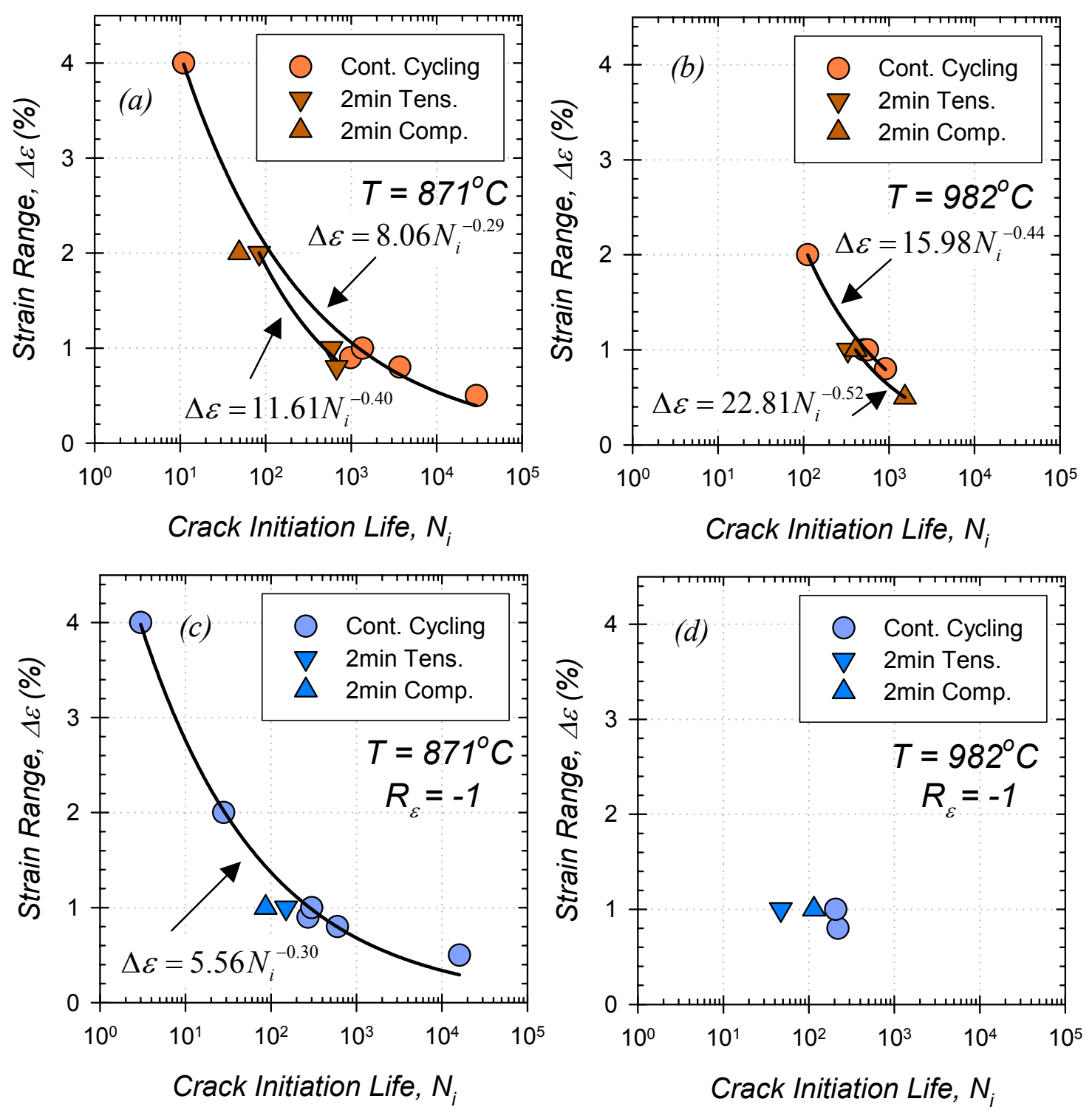

Figure 18: Effects of dwells on crack initiation life of $(a, b) L$ and $(c, d)$ T-oriented DS GTD-111 at $(a, c) 871^{\circ} \mathrm{C}\left(1600^{\circ} \mathrm{F}\right)$ and $(b, d) 982^{\circ} \mathrm{C}$ $\left(1800^{\circ} \mathrm{F}\right)$. 


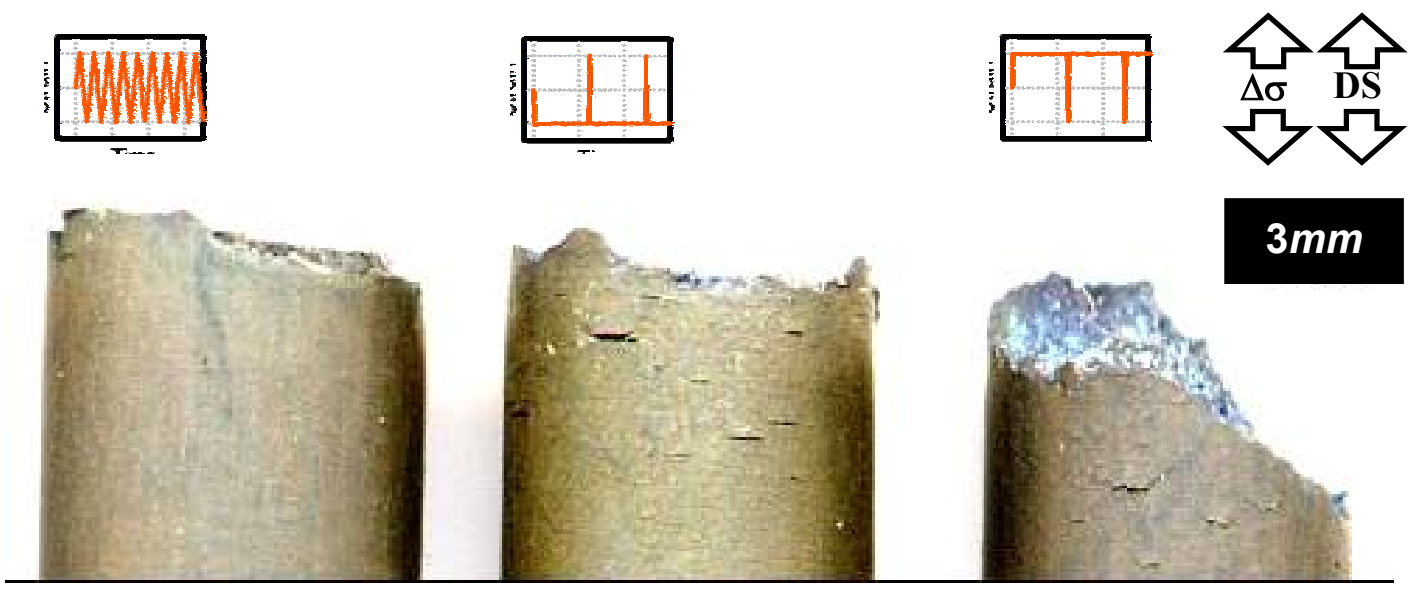

Figure 19: Effect of cycle type on fracture profile of L-oriented DS GTD111 with $\Delta \varepsilon=0.8 \%, R_{\varepsilon}=-1, \quad \&=0.5 \% / s$, and $871^{\circ} \mathrm{C}\left(1600^{\circ} \mathrm{F}\right)$.
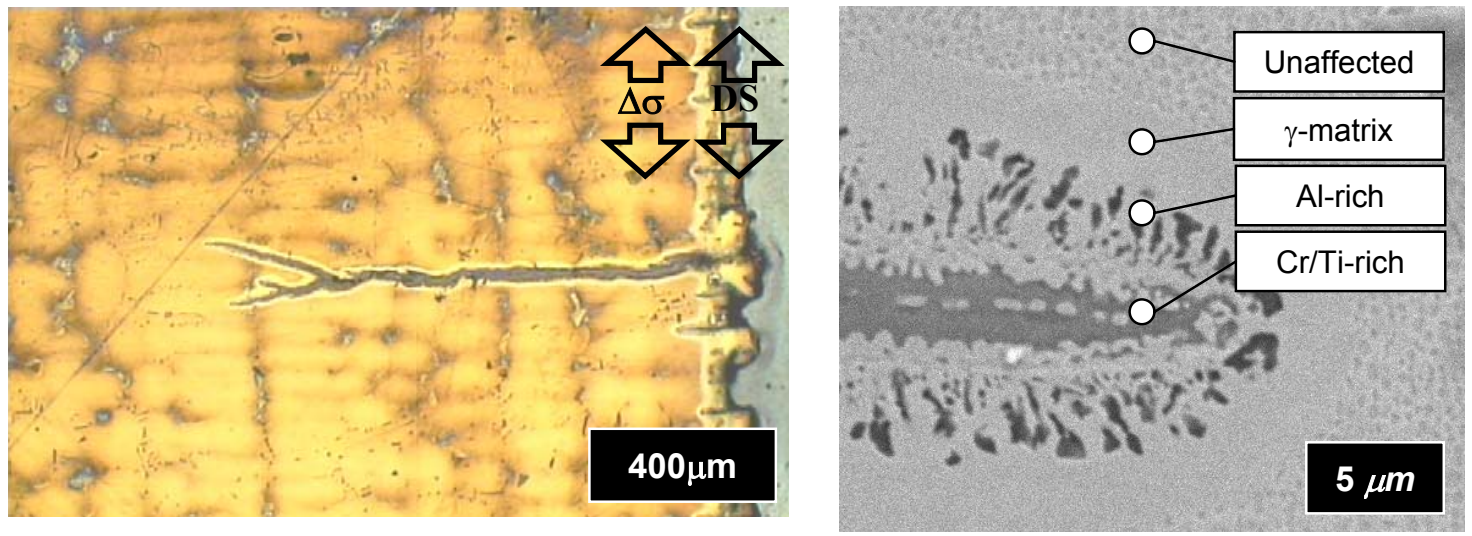

Figure 20: Oxide spiking observed in L-oriented DS GTD-111 with $982^{\circ} \mathrm{C}, \Delta \varepsilon=0.5 \%, R_{\varepsilon}=-1$, and $2 \mathrm{~min}$ compressive holds. 

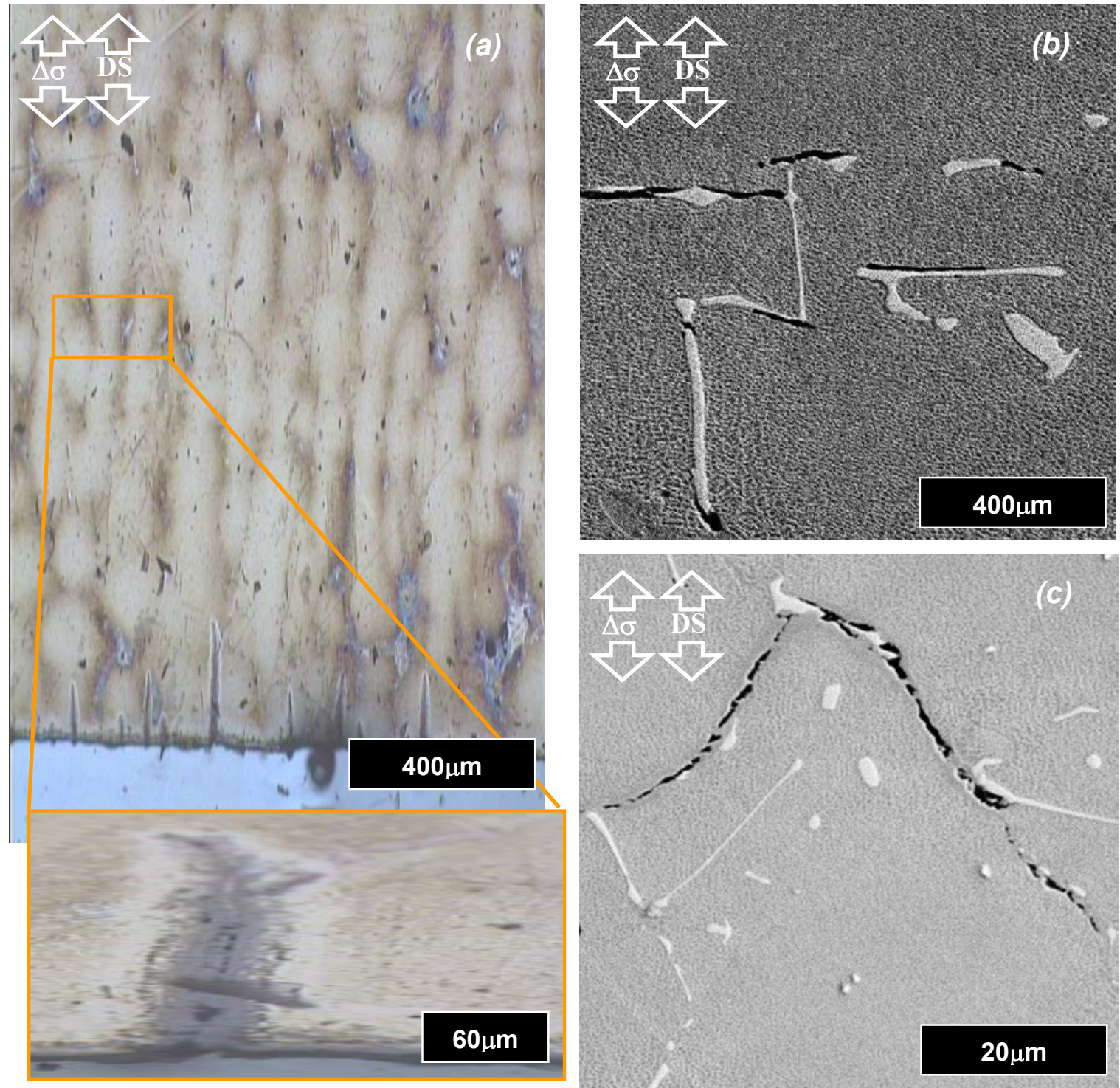

Figure 21: Dwell effects on crack initiation of L-oriented DS GTD-111 under (a) 2 min dwell in compression and (b, c) 2 min dwell in tension. For each case $\Delta \varepsilon=1.0 \%, R_{\varepsilon}=-1, T=982^{\circ} \mathrm{C}$, and $\&=0.5 \% / \mathrm{s}$. 

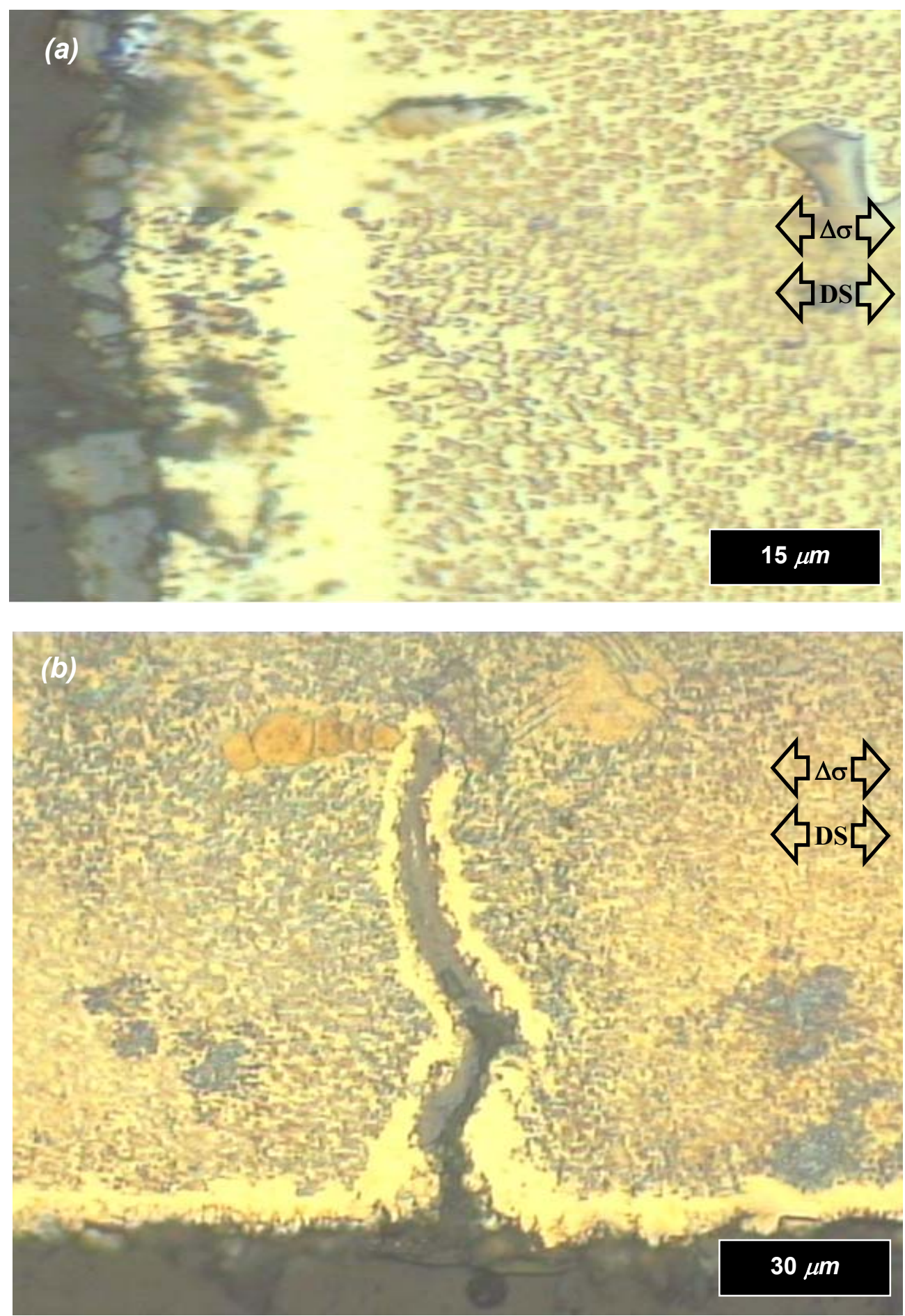

Figure 22: Oxide (a) spallation and (b) spiking observed in L-oriented DS GTD-111 resulting from (a) in-phase TMF cycling with $\Delta \varepsilon=1.0 \%$, $T_{\max }=1038^{\circ} \mathrm{C}$, and (b) out-of-phase $T M F$ cycling with $\Delta \varepsilon=0.5 \%$, $T_{\max }=927^{\circ} \mathrm{C}$. For each case, $R_{\varepsilon}=-1, T_{\min }=538^{\circ} \mathrm{C}$, and $t_{t c}=180 \mathrm{~s}$. 

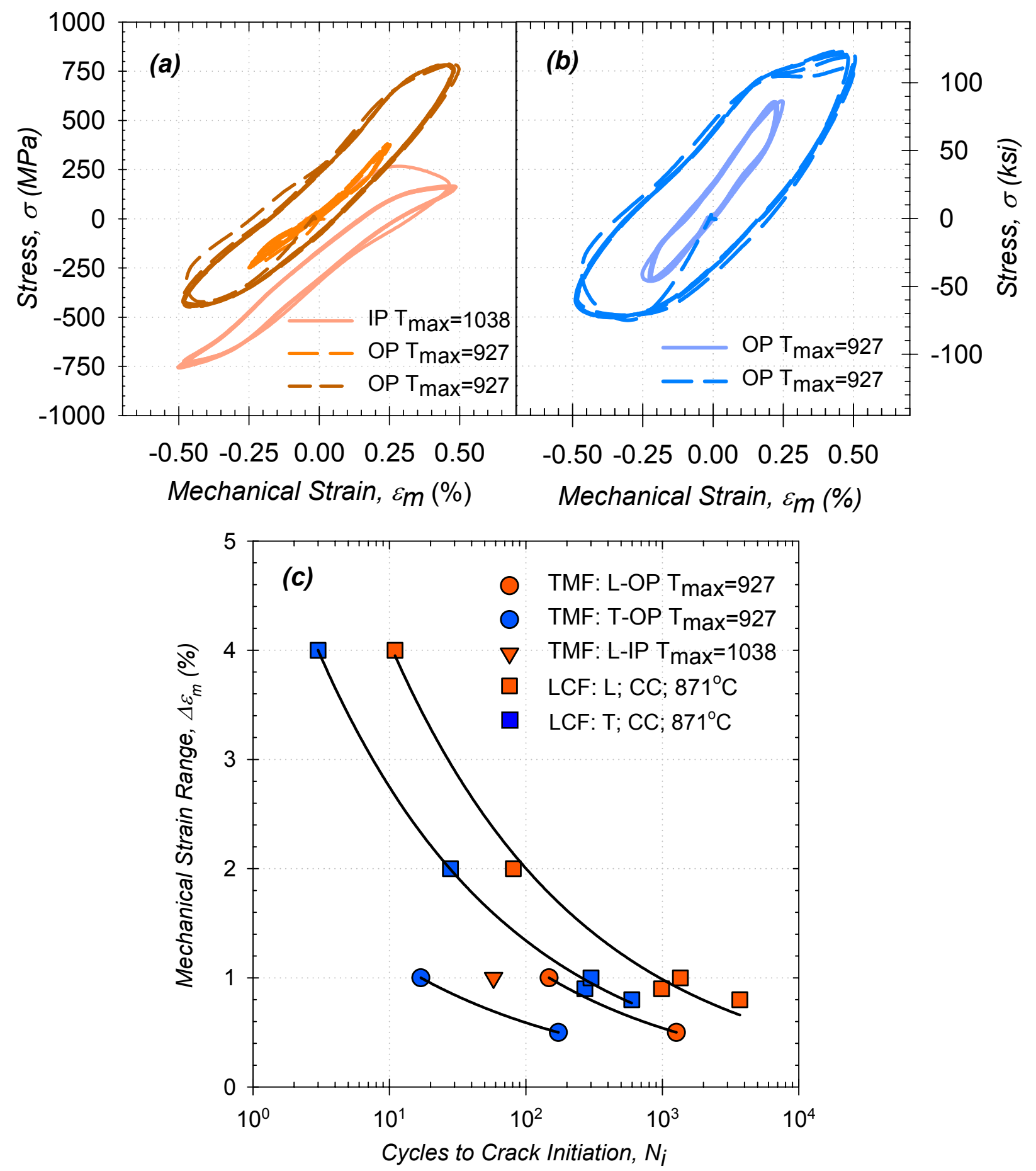

Figure 23: (a, b) Comparison of initial stress-mechanical strain responses of TMF cycled (a) $L$ and (b) T DS GTD-111 between $T_{\min }=538^{\circ} \mathrm{C} \leftrightarrows T_{\max }=927^{\circ} \mathrm{C}$. In one case $T_{\max }=1038^{\circ} \mathrm{C}$ and (c) comparison of mechanical strain crack initiation lives. For each case $R_{\varepsilon}=-1$. 

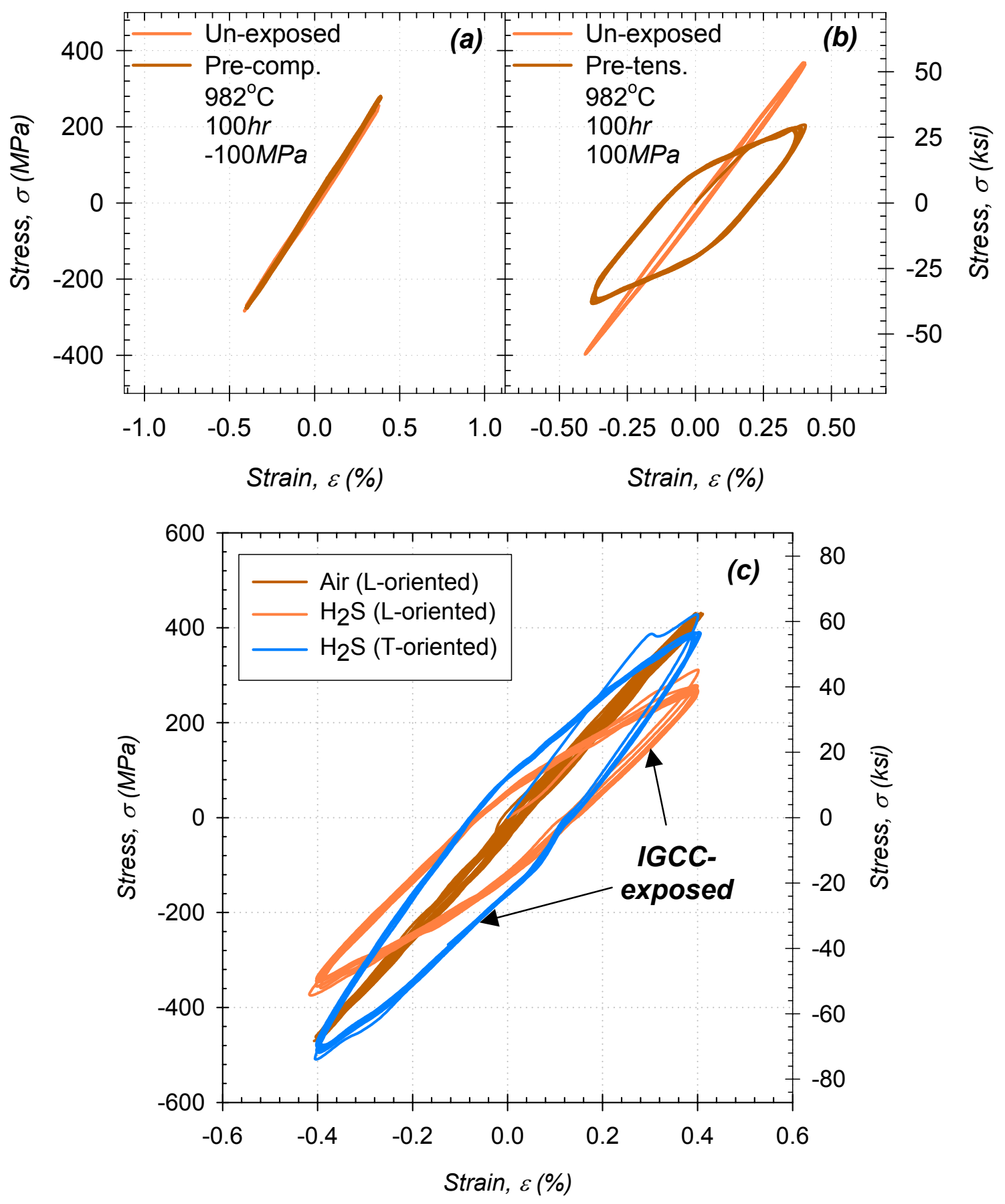

Figure 24: Pre-exposure effects on initial hysteresis loops of $L$ and $T$ oriented DS GTD-111 under continuous cycling conditions with $R_{\varepsilon}=-$ $1, \&=0.5 \% / \mathrm{s}$, and $871^{\circ} \mathrm{C}\left(1600^{\circ} \mathrm{F}\right)$ : (a) $\Delta \varepsilon=0.5 \%$, (b) $\Delta \varepsilon=0.8 \%$, and (c) $\Delta \varepsilon=0.8 \%$ (pre-exposed in air and $\mathrm{H}_{2} \mathrm{~S}$ with no load). 


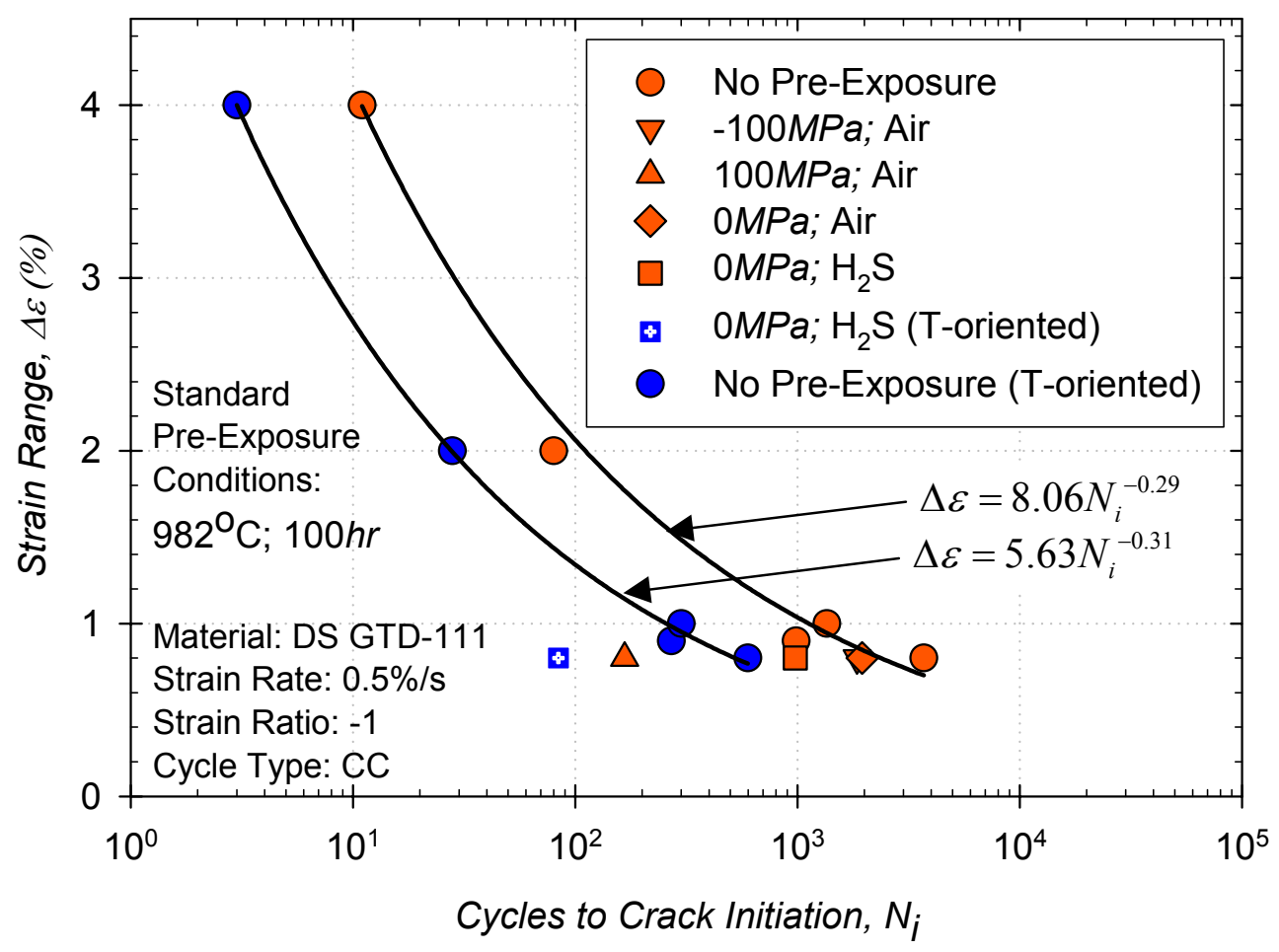

Figure 25: Effects of pre-exposure on crack initiation life of L-oriented DS GTD-111 under continuous cycling conditions with $R_{\varepsilon}=-$ $1, \&=0.5 \% / s$, and $871^{\circ} \mathrm{C}\left(1600^{\circ} \mathrm{F}\right)$. 

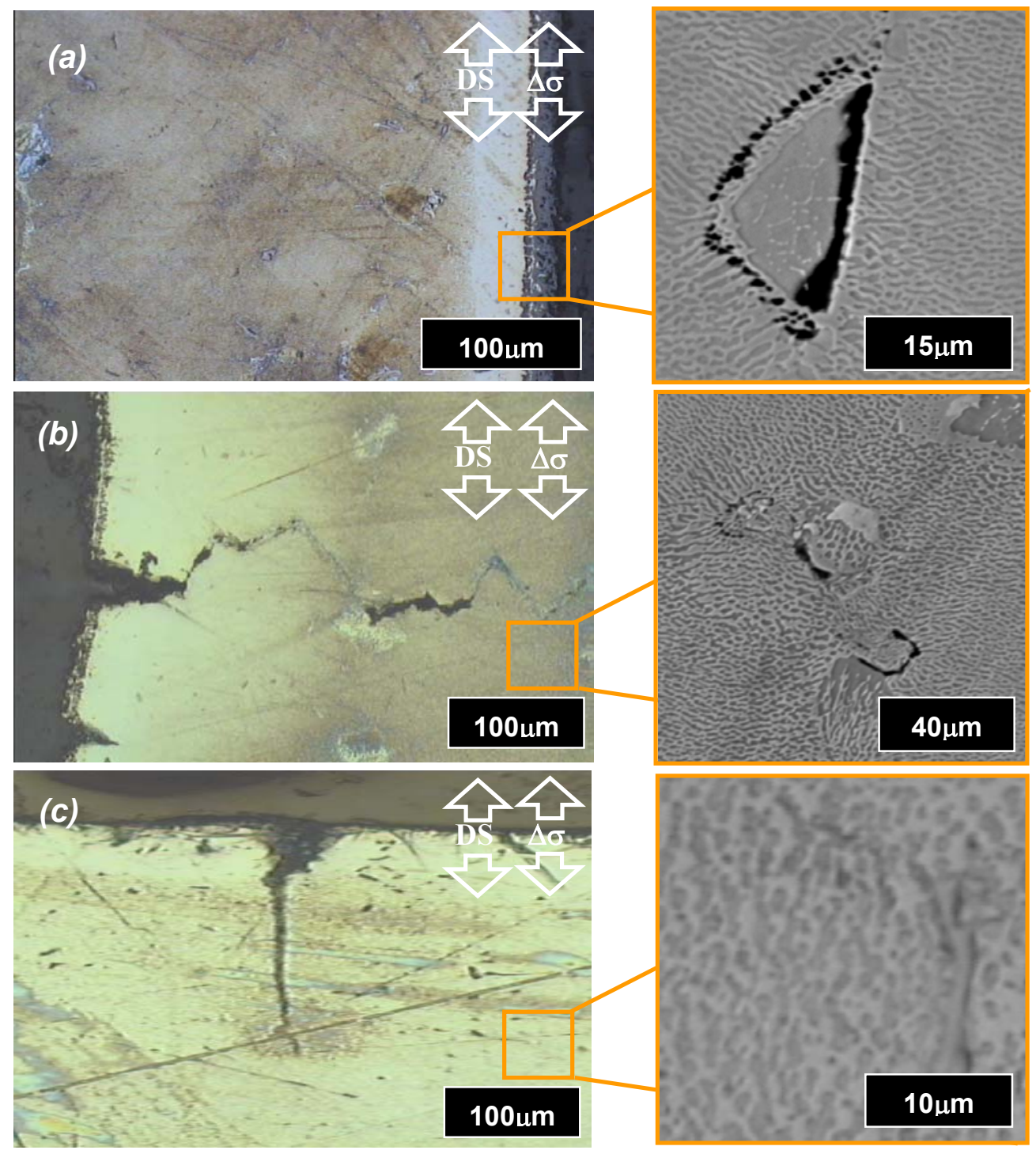

Figure 26: Pre-exposure effects on crack initiation behavior of Loriented DS GTD-111 in static air under (a) pre-exposure only (100hr, $\left.982^{\circ} \mathrm{C}, 100 \mathrm{MPa}\right)$, (b) pre-exposure $\left(100 \mathrm{hr}, 982^{\circ} \mathrm{C}, 100 \mathrm{MPa}\right)$ and fatigue $\left(\Delta \varepsilon=0.5 \%\right.$ and $\left.871^{\circ} \mathrm{C}\right)$, and (c) pre-exposure (100hr, $\left.982^{\circ} \mathrm{C},-100 \mathrm{MPa}\right)$ and fatigue $\left(\Delta \varepsilon=0.8 \%\right.$ and $\left.871^{\circ} \mathrm{C}\right)$. 


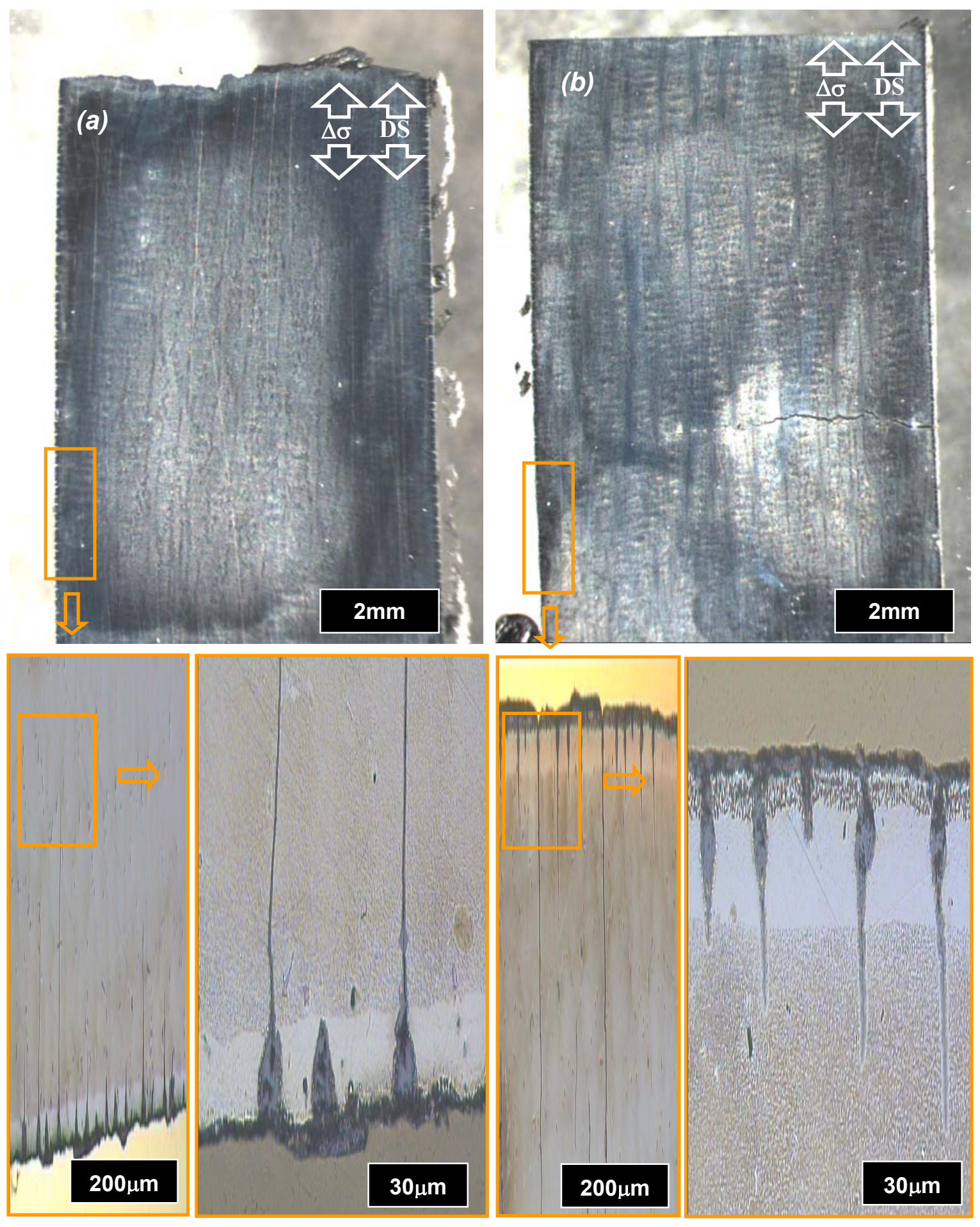

Figure 27: Comparison of microstructures of (a) air and (b) $\mathrm{H}_{2} \mathrm{~S}$ exposed L-oriented DS GTD-111. Pre-exposure conditions: $982^{\circ} \mathrm{C}$, 100hr, OMPa. Fatigue cycling conditions: $\Delta \varepsilon=0.8 \% 871^{\circ} \mathrm{C}$, and $\&=0.5 \% / \mathrm{s}$. 

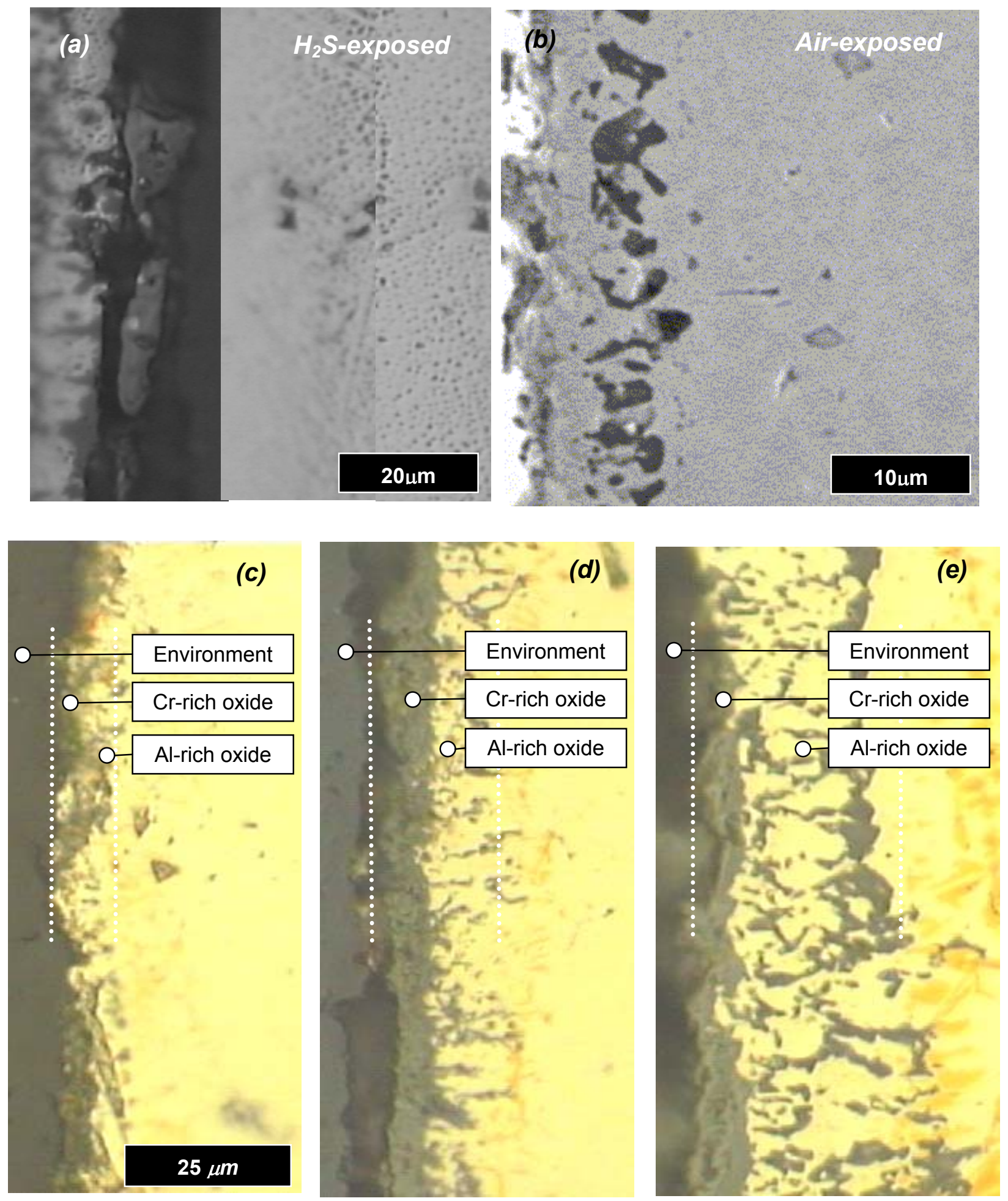

Figure 28: Surface oxidation observed in an unstressed DS Ni-base superalloy. Specimens were subjected to (a) $\mathrm{H}_{2} \mathrm{~S}$ at $982^{\circ} \mathrm{C}$ for $100 \mathrm{hr}$, (b) air at $982^{\circ} \mathrm{C}$ for $100 \mathrm{hr}$, (c) air at $1038^{\circ} \mathrm{C}$ for $3.2 \mathrm{hr}$, (d) air at $1038^{\circ} \mathrm{C}$ for $31.6 \mathrm{hr}$, and (e) air at $1038^{\circ} \mathrm{C}$ for $316 \mathrm{hr}$. 


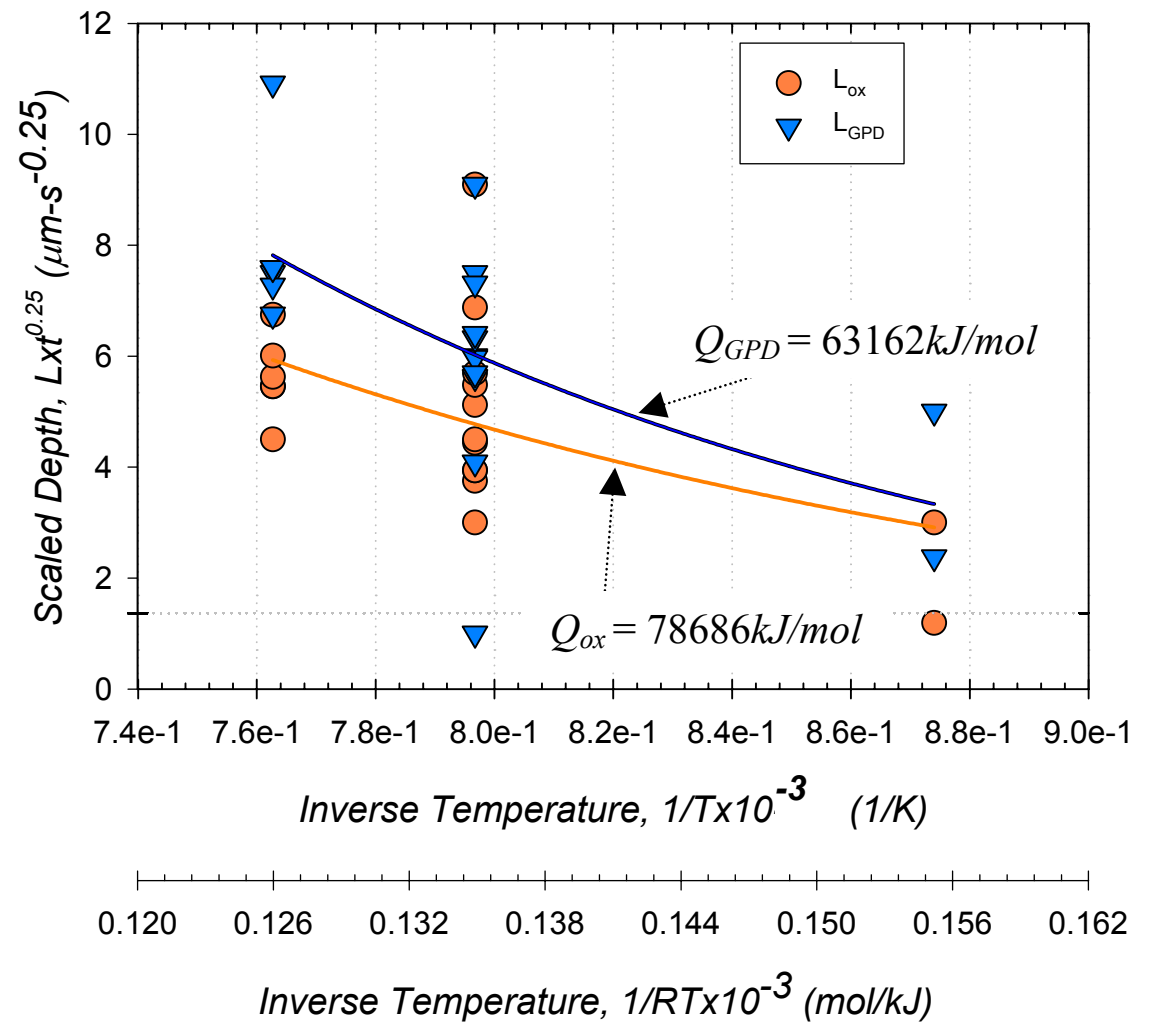

Figure 29: Regression modeling of oxidation growth kinetics of $D S$ GTD-111 above $649^{\circ} \mathrm{C}$. 

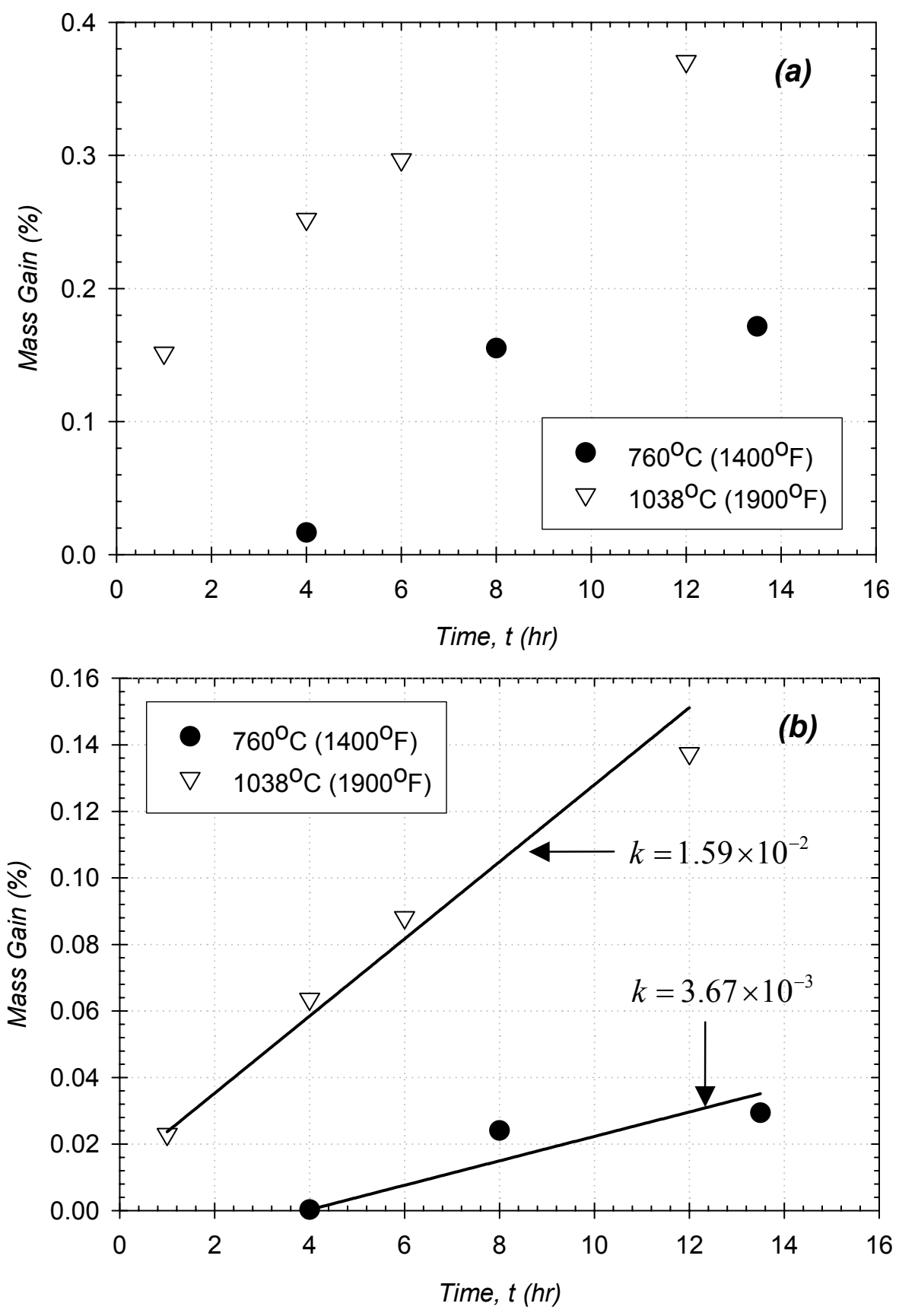

Figure 30: (a) Mass gain and (b) mass gain squared histories of parabolic weight gain of DS GTD-111 in isothermal 100ppm $\mathrm{H}_{2} \mathrm{~S}$ gas. 


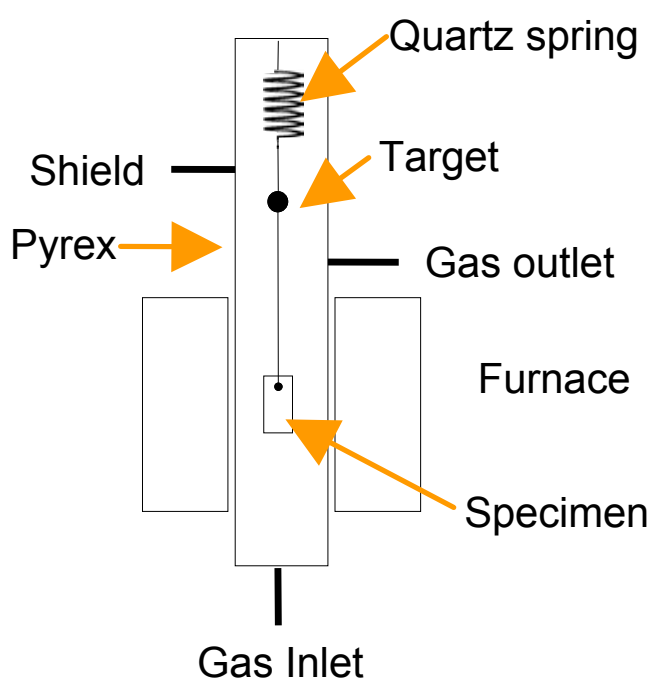

Figure 31: Experimental setup of a thermogravimetric analyzer (TGA) using a quartz spring measuring system. 


\section{Sub-Task 1.2. - High Temperature Environmental Effect Mechanism Quantification}

\section{Background}

This section outlines the formulation of two constitutive models which can capture the stress-strain behavior in directionally solidified (DS) GTD111. A crystal plasticity approach is used in the first mode with the capability to capture the grain orientation dependent stress strain response in creep and fatigue. Even though it is easier to incorporate the deformation physics at the slip system level which should lead to better prediction, the slip system level computations are very time intensive. Therefore, a macroscopic transversely isotropic model has also been formulated, which leads to a reduction in the associated computational costs when compared to the crystal plasticity model. These models have been implemented in ABAQUS and can be used to predict the stress-strain response for different temperature and mechanical loading histories.

A physically-based crack initiation model has been developed to facilitate estimation of crack initiation behavior under both isothermal and non-isothermal LCF. Data has been coupled with the formulation to make useful crack initiation life predictions. This crack initiation model has been developed based on mechanical results from a variety of fatigue experiments and metallurgical microstructural observations from tested samples. In application, the model can be applied to represent the physics of damage accumulation and failure seen in service exposed materials including geometry effects specific to a given component design. It can also be used to correlate microstructural conditions to exposure conditions and residual useful life

The content and organization of this report is as follows. Section 2 describes the constitutive model formulation and implementation of the constitutive model. The model calibration is also included in this section. The mechanistically-based crack initiation model is developed in Section 3. Predictions for uniaxial fatigue behavior of DS GTD111 compared with experiments is also included in this section. Conditions that were included are those with or without hold times, isothermal and non-isothermal conditions Conclusions are given in Section 4.

\section{Methodology}

DS GTD111 is used extensively in applications that require a material with high strength, good creep, fatigue, and corrosion resistance. The operating temperatures of these components can range from room temperature (RT) to very high temperatures $\left(>1000{ }^{\circ} \mathrm{C}\right.$ ) and they are exposed to complex mechanical loading superimposed with thermal transients. In recent years, emphasis has been placed on enhancing the material properties in turbine blades along preferential directions using manufacturing techniques such as directional solidification. Directional solidification leads to elimination of grain boundaries along the axis of the blades (the loading direction), which effectively increases the creep resistance of the material at high temperatures. Nickel base superalloys consist of FCC unit cells, thus, each grain consists of crystals with cubic symmetry along a specified orientation. In polycrystalline superalloys, the grains are randomly oriented resulting in an averaged isotropic macroscopic response, which can be modeled using an isotropic elastoviscoplastic model. However, in DS (and single crystal) superalloys, the macroscopic material stress-strain response is anisotropic and it changes 
as a function of the loading orientation. It is essential to capture this anisotropy in any constitutive model, which introduces new challenges in constitutive modeling of the deformation behavior and life prediction.

The observed deformation response in DS GTD-111 is consistent with that of other Nickel base superalloys and can be summarized as follows [(Westbrook 1996)]:

1) The yield strength increases with increasing temperature up to a peak temperature of $750^{\circ} \mathrm{C}$ beyond which it decreases.

2) Slip is generally dictated by the resolved shear stresses along the favorable crystallographic slip planes. However, Nickel base superalloys do not obey Schmid's law in all orientations and slip on any given plane can also depend on the resolved stresses on other planes (e.g. dislocation cross slip planes) due to dislocation core spreading effects in $\gamma^{\prime}$.

3) There is tension (T)-compression (C) asymmetry of the flow stress. The nature of this asymmetry is orientation and temperature dependent [(Lall 1979; Paidar 1984; Vitek 1996)].

4) The initial yield behavior is nearly temperature path history independent, i.e., if a sample is first deformed at a high temperature at which the yield strength is high and then deformed at a low temperature, the material response is similar to a virgin material deformed at the lower temperature [(Vitek 1996)].

The deformation mechanisms change with varying loads, orientations, strain rates, and temperatures. A physically-based constitutive relation that can simulate the structural response in order to facilitate component life prediction should incorporate the active deformation mechanisms as a function of stress level and temperature.

The crystal plasticity approach provides good predictive capabilities, especially when modeling components where the size, morphology and crystallographic orientation of the grains control the anisotropic deformation behavior since it is easier to incorporate the deformation mechanisms at the slip system level. A physically-based crystal plasticity constitutive model is outlined to capture the homogenized deformation response in DS GTD-111. The crystal plasticity models are probably the most accurate method to capture the material behavior but they lead to an increase in the computational cost in addition to the requirement of knowing the grain orientations in advance. A conservative design would be based on the weakest grain orientation but it might not always be possible to determine this orientation beforehand for complex loading and temperature histories. In Figure 1, the Young's modulus is plotted as a function of the loading orientation for DS GTD-111 at $871{ }^{\circ} \mathrm{C}$ (a) using the experimental data, (b) using the crystal plasticity model and (c) assuming transverse isotropy about the solidification direction. The Young's modulus for the crystal plasticity model is obtained from the uniaxial stress-strain response in the elastic regime using a 3-D model with 20 grains in the transverse section (perpendicular to the solidification direction). These grains are given a random transverse orientation which is a good assumption since the rotational axis about the [001] axis cannot be controlled during directional solidification of the DS plates. The Young's modulus is also determined by considering the material as transversely isotropic by rotating the transversely isotropic stiffness matrix along the specified orientation. In Figure 1, there is a very good agreement for the Young's modulus with the experimental data for both the crystal plasticity and the macroscopic transversely isotropic models. A study of the elastic compliance matrix (and the Young's modulus) in a DS Ni-base 
superalloy, MAR M247 by Hasebe et al. (Hasebe 1992; Shirafuji 1998) showed that the elastic compliance matrix reduced to a transversely isotropic matrix when the number of grains were high enough. The error in assuming a transversely isotropic compliance matrix was less than $10 \%$ when five columnar grains were used with random rotation angles about the [001] axis. It is assumed that the transversely isotropic model should be a suitable representation for DS GTD-111 since there are 6-10 grains in the test specimen gage section in the transverse orientation which should be sufficient to give the averaged transversely isotropic response. It should be noted that the number of grains would be much larger in the actual industry component (turbine blade).

Both the crystal plasticity based constitutive model and the transversely isotropic model have been have been implemented as ABAQUS User MATerials (UMAT) using a semi-implicit integration scheme. The predictive capabilities of these models are tested using experimental in-phase (IP) and out-of-phase (OP) thermomechanical fatigue data.

\section{Thermodynamics}

When a material is subjected to an inelastic deformation it undergoes irreversible changes in its microstructure. The changing internal structure of the body can be characterized by its state. Virtually any aspect of microstructure that can undergo irreversible rearrangement is a candidate for description by an internal state variable, e.g. dislocation density, lattice orientation etc. (McDowell 1991; McDowell 1992). ISV constitutive theory is based on the notion that a general non-equilibrium, irreversible process can be treated as a sequence of constrained equilibrium states. The path history dependence of the irreversible process is embedded in the evolution of the internal state variables, thus, extending the equilibrium state space to non-equilibrium processes.

For any irreversible quasi-static process, the non-negative intrinsic energy production rate per unit volume is given by

$$
\rho \gamma_{\text {loc }}=\rho r \&-\frac{1}{T}(\rho \gamma-\underset{\% \%}{\nabla} \cdot q) \geq 0
$$

where $\rho$ is the density, $\eta$ is the entropy per unit mass, $\gamma$ is the internal rate of heat supply extrinsic to dissipation associated with microstructural rearrangement and $\underset{0 \text {, }}{q}$ is the heat flux vector. The inequality $\rho \gamma_{l o c} \geq 0$ is one way of expressing the Clausius-Duhem inequality, where the existence of both entropy and temperature is assumed to continue into the domain of irreversible processes. The local form of the first law of thermodynamics is written as

$$
\rho \gamma-\underset{0 / q 0 /}{\nabla} q=\rho \&-\sigma: \&
$$

where $u$ is the internal energy per unit mass. Consider the thermodynamic potential (Helmholtz free energy) $\psi\left(\underset{\%}{\varepsilon}, T, \xi_{i}\right)$ with the path history dependence embedded in the evolution of the internal state variables, $\xi_{i}$, which may be a scalar, vector or any higher order tensor. From the definition of Helmholtz free energy

$$
\psi=u-T \eta
$$

Expanding the state function as a total differential,

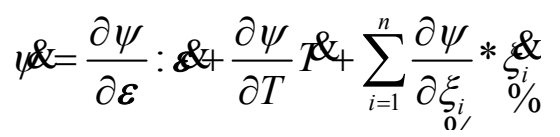


Differentiating Equation (3) and substituting Equation (2) and (4) gives

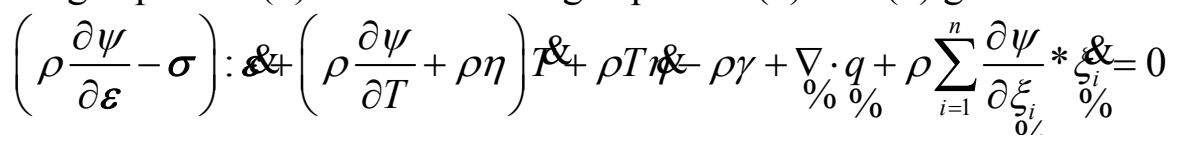

$\varepsilon_{0}$, and $T$ are independent variables therefore each term in the parenthesis should vanish independently, giving the relations

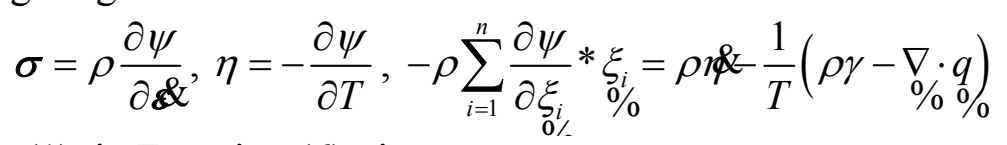

Using Equation (1) in Equation (6) gives

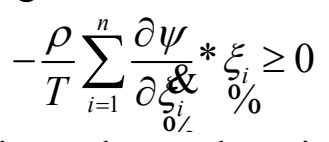

Using small strain assumptions, the total strain is decomposed into an elastic and plastic strain component, i.e.

$$
\varepsilon=\varepsilon^{e}+\varepsilon^{p}
$$

Therefore,

$$
\rho \frac{\partial \psi}{\partial \&}=\rho \frac{\partial \psi}{\partial \&}=-\rho \frac{\partial \psi}{\partial \&}=\sigma
$$

Using the inelastic strain as a state variable in Equation (7) gives the relation

$$
\sigma: \mathbb{Z}-\rho \sum_{i=1}^{n-1} \frac{\partial \psi}{\partial \xi_{i}^{\mathbf{k}},} * \xi_{i} \geq 0
$$

The intrinsic dissipation inequality in Equation (9) should be satisfied in any irreversible process. The evolution equations for the state variables are expressed as

and

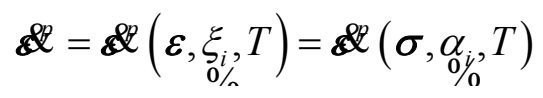

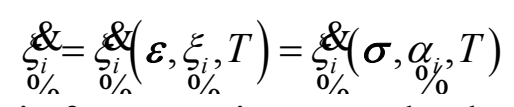

where $\underset{0 \%}{\alpha}$ is the thermodynamic forces conjugate to the thermodynamic displacement $\xi_{i}$. Care should be taken to ensure that the constitutive equations do not violate the restrictions laid out by the intrinsic dissipation inequality.

\section{Crystal Plasticity Model}

\section{Formulation}

A homogeneous constitutive model is formulated for which no explicit distinction is made between the matrix and precipitate phases. A crystal plasticity framework is employed to capture the orientation dependent material response. As the temperature increases, strain rate effects are increasingly important and a rate dependent method is employed. Crystal plasticity approaches have been used extensively in modeling single crystal superalloys [(Meric 1991; Vitek 1996; Osterle 2000)] and the general methodology can be briefly described as follows. A multiplicative rule is used for the deformation gradient, F, given as [(Bilby 1955; Lee 1969; Sheh 1988)]

$$
\mathbf{F}=\mathbf{F}^{\mathrm{e}} \cdot \mathbf{F}^{\mathrm{p}}
$$


As shown in Figure 2, the deformation gradient is separated into two parts: (i) the plastic deformation gradient $\left(\mathbf{F}^{\mathbf{p}}\right)$ formed due to dislocation motion, associated with a change in the shape of the grain but not its crystal lattice and (ii) the elastic deformation gradient $\left(\mathbf{F}^{\mathbf{e}}\right)$, which essentially models reversible elastic stretch and rigid body rotation of the lattice. For any given lattice, the close packed planes act as the slip planes with unit normal vector $\mathrm{m}_{0 \rho}^{\alpha}$ in the reference configuration for each of the $\alpha$ slip systems, along which the dislocations move in the slip direction in the reference configuration with unit vector $\mathrm{s}_{09}^{\alpha}$. The shearing rates along each of the active slip systems ( $\alpha$ ) depend on the resolved shear stress on the slip systems. The macroscopic plastic velocity gradient is obtained by summing over all slip systems in the intermediate relaxed configuration according to

$$
\mathbf{L}^{\mathbf{p}}=\mathbf{D}^{\mathbf{p}}+\mathbf{W}^{\mathbf{p}}=\sum_{\alpha=1}^{\mathrm{N}_{\text {slip }}} \&\left(\left(\mathrm{~s}_{0 \%}^{\alpha} \otimes \underset{\%}{\mathrm{~m}_{0}^{\alpha}}\right)\right.
$$

where the plastic rate of deformation $\left(\mathbf{D}^{\mathrm{p}}\right)$ and plastic spin $\left(\mathbf{W}^{\mathrm{p}}\right)$ are given by the symmetric and anti-symmetric parts of the plastic velocity gradient, i.e.,

$$
D_{i j}^{p}=\frac{1}{2}\left(L_{i j}^{p}+L_{i j}^{p}\right), \quad W_{i j}^{p}=\frac{1}{2}\left(L_{i j}^{p}-L_{i j}^{p}\right)
$$

It should be noted that the intermediate, relaxed configuration in Figure 2 is not unique since any superimposed rigid body rotation on the deformation gradient will also satisfy Equation (11). Uniqueness of the intermediate configuration is obtained by assuming that the substructure spin is co-rotational with the lattice.

\section{Constitutive Model}

A rate-dependent crystal plasticity formulation is used. The overall velocity gradient is computed by summing the shearing rates along the active slip systems as given in Equation (12). All slip systems are assumed active above the threshold stress and the shearing rate of the $\alpha^{\text {th }}$ system is related to its associated viscous overstress by

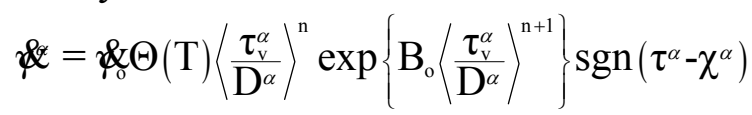

where the slip system viscous overstress $\tau_{v}^{\alpha}$ is given in terms of the resolved shear stress $\tau^{\alpha}$ by

$$
\tau_{\mathrm{v}}^{\alpha}=\left|\tau^{\alpha}-\chi^{\alpha}\right|-\kappa^{\alpha} \frac{\mu}{\mu_{\mathrm{o}}}
$$

Here, $\chi^{\alpha}$ is the backstress and $\kappa^{\alpha}$ is the threshold stress on each slip system. Increase in the threshold stress leads to an increase in size of the viscoplastic flow potential, while the back stress results in a shift of the potential surface. The threshold stress may be viewed as the resistance to plastic flow arising from statistical strengthening mechanisms associated with increase of dislocation density, solid solution strengthening, etc. The back stress displays a directional dependence and is associated with several features of the heterogeneous material at the microscale, including internal stresses that develop with deformation due to dislocation pile-up at obstacles such as precipitate particles, grain or phase boundaries, differential yielding with strain in hard (dislocation walls) and soft regions of the microstructure, and statistical interactions of dislocations bypassing barriers. Both back stress, $\chi^{\alpha}$, and the threshold stress, $\kappa^{\alpha}$, depend on the history of temperature and viscoplastic deformation. 
Equations (14) and (15) contain several other additional parameters defined as follows: \& is a reference shearing rate, $n$ governs the power law creep regime, $\mathrm{B}_{\mathrm{o}}$ and $(n+1)$ collectively govern the power law breakdown behavior at higher strain rates where the response becomes nearly rate independent, $\mathrm{D}^{\alpha}$ is the drag stress which is weakly dependent on the history of temperature and viscoplastic deformation when the behavior of the $\gamma$ and $\gamma^{\prime}$ phases are homogenized, $\mu$ is the shear modulus, with $\mu_{\mathrm{o}}$ being the value of the shear modulus at absolute zero, and $\Theta(T)$ is the diffusivity parameter given by

$$
\begin{aligned}
& \Theta(T)=\exp \left(-\frac{Q_{o}}{R T}\right) \quad \text { for } T \geq \frac{T_{m}}{2} \\
& \text { and } \\
& \Theta(T)=\exp \left\{-\frac{2 Q_{o}}{R T_{m}}\left[\ln \left(\frac{T_{m}}{2 T}\right)+1\right]\right\} \quad \text { for } T \leq \frac{T_{m}}{2}
\end{aligned}
$$

where $Q_{0}$ is the activation energy for thermally-activated dislocation bypass of obstacles, $\mathrm{R}$ is the universal gas constant, and $\mathrm{T}_{\mathrm{m}}$ is the absolute melting point temperature.

The effective strain rate sensitivity exponent on the stress (inverse strain rate sensitivity) is given by

$$
\mathrm{m}=\frac{\partial \ln \psi}{\partial \ln \tau^{\alpha}}=\frac{\partial \ln \&}{\partial \ln \tau_{\mathrm{v}}^{\alpha}}=\mathrm{n}+\frac{\mathrm{B}_{\mathrm{o}}(\mathrm{n}+1)}{\hat{\tau}_{\mathrm{o}} \mu / \mu_{\mathrm{o}}}\left\langle\frac{\tau_{\mathrm{v}}^{\alpha}}{\hat{\tau}_{\mathrm{o}} \mu / \mu_{\mathrm{o}}}\right\rangle^{\mathrm{n}}
$$

The anomalous yield behavior of $\gamma-\gamma^{\prime}$ Nickel base superalloys, i.e., the yield stress increases with temperature in the intermediate temperature regime, is incorporated in the threshold stress. The anomalous behavior has been attributed to mechanisms involving $\{111\}\langle 110\rangle$ octahedral slip within the $\gamma^{\prime}$ precipitates. Several models attempt to explain this mechanism, including the Takeuchi and Karamoto model [(Takeuchi 1971; Takeuchi 1971)], the Paidar-Pope-Vitek (PPV) model [(Paidar 1984)] and the model proposed by Hirsch [(Hirsch 1992)]. Though recent experimental evidence [(Sun 1996)] seems to support the Hirsch theory, the PPV model has perhaps been the most widely used model, at least in the anomalous temperature regime.

According to the PPV model [(Paidar 1984)], in the anomalous behavior temperature regime $\langle 10 \overline{1}\rangle$ screw dislocations on (111) planes split into two superShockley partials separated by an APB. There are two configurations of the $a / 2\langle 10 \overline{1}\rangle$ screw dislocation: a glissile configuration with its core spread in the (111) plane and a sessile configuration with a non-planar core spread in the (1i1) plane. The core transformation is explained in three steps: (i) constriction of the glissile core on the (111) plane, (ii) movement of the constricted dislocation along the (010) plane, and (iii) spreading of the dislocation on the $(1 \overline{1} 1)$ plane. The glissile core has to constrict first for this to happen; hence, any shear stress that aids in the constriction of the partial dislocations will assist cross-slip, leading to formation of sessile locks which act as obstacles to the glissile dislocations. Slip on the octahedral planes will be influenced by the stresses on the primary (step (i)) and secondary cross slip planes (step (iii)), along the direction perpendicular to the partial dislocations, as well as the stresses acting on the cube plane (step (ii)). The additional dependence of the activation enthalpy for the glissile 
to sessile transformation on the shear stress components $\left(\tau_{p e}, \tau_{s e}\right.$ and $\left.\tau_{c b}\right)$ leads to a nonSchmid effect. Since the enthalpy is also dependent on the sign of the stress components for steps (i) and (ii), it will differ in tension and compression. This leads to a tensioncompression asymmetry that is orientation dependent. It should be noted that step (iii) is independent of the sense of loading.

To include the non-Schmid effects, the yield criterion for octahedral slip systems is modified based on the PPV theory as proposed by Qin and Bassani [(Qin 1992)]. Each octahedral slip system is active only when the Schmid stress and a linear combination of the additional stress terms exceed a critical value, i.e.,

$$
\tau^{\alpha}=\mathrm{h}_{\mathrm{pe}} \tau_{\mathrm{pe}}^{\alpha}+\mathrm{h}_{\mathrm{se}} \tau_{\mathrm{se}}^{\alpha}+\mathrm{h}_{\mathrm{cb}}\left|\tau_{\mathrm{cb}}^{\alpha}\right|=\tau_{\mathrm{cr}}
$$

where $\tau_{\mathrm{pe}}, \tau_{\mathrm{se}}$ and $\tau_{\mathrm{cb}}$ are the shear stresses on the primary, secondary and cube slip systems, respectively, and $\tau_{\text {cr }}$ is the critical resolved shear stress. This formulation is embedded in the current set of constitutive relations through a critical threshold stress $\left(\kappa^{\alpha}\right)$, i.e.,

where

$$
\kappa^{\alpha}=\kappa_{\mathrm{c}}^{\alpha}+\kappa_{\mathrm{e}}^{\alpha}
$$

and

$$
\kappa_{\mathrm{c}}^{\alpha}=\kappa_{\mathrm{o}}^{\alpha}(\mathrm{T})+\mathrm{h}_{\mathrm{pe}} \tau_{\mathrm{pe}}^{\alpha}+\mathrm{h}_{\mathrm{se}} \tau_{\mathrm{se}}^{\alpha}+\mathrm{h}_{\mathrm{cb}}\left|\tau_{\mathrm{cb}}^{\alpha}\right|
$$

$$
\mathbb{R}_{\mathrm{e}}^{\mathcal{Q}}=\mathrm{h}_{\mathrm{o}} \sum_{\beta=1}^{\mathrm{N}_{\text {slip }}} \mathrm{q}^{\alpha \beta}\left|\not k^{\beta}\right|-\mathrm{h}_{\kappa \mathrm{s}} \kappa^{\alpha} \sum_{\beta=1}^{\mathrm{N}_{\text {slip }}}\left|\&^{\beta}\right|-\mathrm{h}_{\mathrm{s}} \Theta(\mathrm{T})\left\langle\kappa^{\alpha}-\kappa_{\mathrm{th}}\right\rangle^{\mathrm{r}_{\mathrm{s}}}
$$

The back stress evolution for homogenized $\gamma-\gamma^{\prime}$ models can be written in the hardening-dynamic recovery plus static thermal recovery format as

$$
\not \&=\mathrm{h}_{\chi}|\&| \operatorname{sgn}\left(\tau^{\alpha}-\chi^{\alpha}\right)-\mathrm{h}_{\chi \mathrm{d}} \chi^{\alpha} \mid \psi\left(\frac{1}{\mathrm{R}_{\chi}} \frac{\partial \mathrm{R}_{\chi}}{\partial \mathrm{T}}+\frac{1}{\mathrm{~h}_{\chi \mathrm{d}}} \frac{\partial \mathrm{h}_{\chi \mathrm{d}}}{\partial \mathrm{T}}\right) \chi^{\alpha} \&-\Omega_{\chi}^{\alpha}
$$

where $\Omega_{\chi}^{\alpha}=\mathrm{h}_{\chi \mathrm{s}}(\mathrm{T})\left|\chi^{\alpha}\right|^{\mathrm{r}_{\chi \mathrm{s}}}$ is the static thermal recovery term, $\left(\mathrm{h}_{\chi}, \mathrm{h}_{\chi \mathrm{d}}, \mathrm{h}_{\chi \mathrm{s}}, \mathrm{r}_{\chi \mathrm{s}}\right)$ are material parameters, and $\mathrm{R}_{\chi}=\mathrm{h}_{\chi} / \mathrm{h}_{\chi \mathrm{d}}$. The temperature rate dependent term is necessary to properly model hysteresis behavior under thermomechanical fatigue (TMF) [(McDowell 1992)], since the model parameters that govern the back stress evolution rates are themselves functions of temperature. Note that for single phase models, the back stress evolution equation can likely be neglected, but it is essential to describe the overall average behavior of the crystallographic grains in homogenized $\gamma-\gamma^{\prime}$ models. It is a very significant fraction of the flow stress (typically $>30 \%$ ) for Ni-base superalloys [(Castelli 1993)]. It should be noted that the Eqs. 14-20 are thermodynamically admissible and satisfy the dissipation inequality laid out in Eq. 9. The constitutive equations are summarized in Table 1.

\section{Implementation}

The constitutive model is implemented as an User Material subroutine (UMAT) in ABAQUS, based on the implicit integration scheme of McGinty [(McGinty 2001)].. This subroutine is called at each integration point and at each time step increment. The subroutine must perform two functions; (a) it should update the stresses and the internal 
state variables to their values at the end of the increment and (b) it should update the material Jacobian matrix.

A hyperelastic formulation is used which is valid for arbitrary finite strains. A time step sub-incrementation scheme and a linear search algorithm were employed in the constitutive subroutine to ensure convergence. The flow rule in Eq. (14) should be satisfied at the end of the time step. A Newton Raphson scheme is used to solve the plastic shear strain increment along each slip system, $\alpha$

$$
\left(\Delta \gamma^{\beta}\right)^{i+1}=\left(\Delta \gamma^{\beta}\right)^{i}-\left(\frac{g^{\alpha}}{g^{\alpha \beta^{\prime}}}\right)^{i}
$$

where

$$
\left(g^{\alpha}\right)^{i}=0=\operatorname{Sgn}\left(\tau^{\alpha}-\chi^{\alpha}\right) \frac{\mathcal{E}^{\&}}{\chi_{o} \Theta(T)}-\left\langle\frac{\tau_{\mathrm{v}}^{\alpha}}{\mathrm{D}^{\alpha}}\right\rangle^{\mathrm{n}} \exp \left\{\mathrm{B}_{\mathrm{o}}\left\langle\frac{\tau_{\mathrm{v}}^{\alpha}}{\mathrm{D}^{\alpha}}\right\rangle^{\mathrm{n}+1}\right\}
$$

and

$$
\left(g^{\alpha \beta}\right)^{i}=\left(\frac{\partial g^{\alpha}}{\partial \Delta \gamma^{\beta}}\right)^{i}
$$

The Jacobian matrix is obtained as

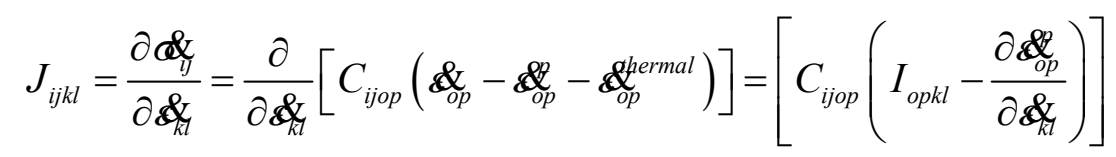

where $I_{o p k l}$ is the $4^{\text {th }}$ order symmetric identity tensor i.e.

$$
\begin{gathered}
I_{o p k l}=\frac{\delta_{o p} \delta_{k l}+\delta_{o k} \delta_{p l}}{2} \\
\frac{\partial \&_{o p}}{\partial \&_{k l}^{\&}}=\frac{\partial \&_{o p}^{p}}{\partial \sigma_{m n}} \frac{\partial \sigma_{m n}}{\partial \&_{s t}} \frac{\partial \&_{s t}^{\&}}{\partial \&_{k l}}
\end{gathered}
$$

But

$$
\sigma_{n+1}=\sigma_{n}+\alpha t
$$

and

$$
\frac{\partial \sigma_{m n}}{\partial \alpha_{s t}}=I_{m n s t} \Delta t
$$

Using this in Eq. (28) and substituting it in Eq. (27) the Jacobian is obtained as

$$
J_{i j k l}=\left(C_{i j k l}^{-1}+\frac{\partial \underset{i j}{\&}}{\partial \sigma_{k l}} \Delta t\right)^{-1}
$$

where

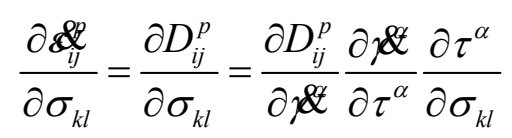

\section{Transverse Isotropy Model}




\section{Formulation}

The kinematics of finite elastoplastic deformation is prescribed by the multiplicative decomposition of the deformation gradient $\boldsymbol{F}$ (Lee 1969)

$$
\boldsymbol{F}=\boldsymbol{F}^{e} \cdot \boldsymbol{F}^{p}
$$

The material point is first mapped from the undeformed configuration, $\beta_{o}$, to the intermediate unstressed configuration, $\beta_{i}$, by the plastic part $\boldsymbol{F}^{p}$ and then carried to the current configuration by the elastic part, $\boldsymbol{F}^{e}$ as shown in Figure 3 . The intermediate configuration determined by $\boldsymbol{F}^{p}$ is defined in such a way that the axis of symmetry, ${ }_{0} \dot{m}$, at $\beta_{i}$ with respect to the global system is the same as the corresponding orientation in the reference configuration $\beta_{o}$. The intermediate configuration is the isoclinic configuration' introduced by Mandel (Mandel 1971) and is uniquely defined to within a rigid rotation about the local axis of symmetry. The equations are implemented in the intermediate configuration. However, it should be noted that the constitutive equations can be written in any one of the three configurations and can be appropriately transformed to the other two. A hyperelastic formulation is used in which the second Piola-Kirchhoff stress, $\sigma^{P K 2}$, is determined in the intermediate configuration using Equation (32).

$$
\sigma_{i j}^{P K 2}=C_{o i j k l} \varepsilon_{k l}^{e}
$$

where $\boldsymbol{C}_{\boldsymbol{o}}$ is the forth rank elasticity tensor in the undeformed configuration. The plastic velocity gradient in the intermediate configuration, $L_{0}^{p}$, is given by

$$
\boldsymbol{L}_{i}^{p}=\frac{1}{2}\left(\boldsymbol{L}_{i}^{p}+\boldsymbol{L}_{i}^{p T}\right)+\frac{1}{2}\left(\boldsymbol{L}_{i}^{p}-\boldsymbol{L}_{i}^{p T}\right)=\boldsymbol{D}_{i}^{p}+\boldsymbol{W}_{i}^{p}
$$

where $\boldsymbol{D}_{i}^{p}$ is the rate of plastic deformation and $\boldsymbol{W}_{i}^{p}$ is the plastic spin in the intermediate configuration. Since the intermediate configuration is assumed to be isoclinic, the direction of isotropy in the intermediate configuration, ${ }_{0} \dot{i}$, , is given by Aravas (Aravas 1992) as

$$
\underset{0 \%}{\underset{\alpha}{\&}}=\left(\boldsymbol{W}_{i}^{p}+\boldsymbol{D}_{i}^{p} \cdot(\underset{0 \%}{d} \otimes \underset{0 \%}{d})-(\underset{0 \%}{d} \otimes \underset{0 \%}{d}) \cdot \boldsymbol{D}_{i}^{p}\right)=0
$$

where ${ }_{0}$ \% is the direction of anisotropy in the reference configuration. From Equation (34), the plastic spin can be determined in the intermediate configuration as

$$
\boldsymbol{W}_{i}^{p}=\left(\underset{0 \%}{d_{0}} \otimes \underset{0 \%}{d}\right) \cdot \boldsymbol{D}_{i}^{p}-\boldsymbol{D}_{i}^{p} \cdot\left(\underset{\mathbf{0} \%}{d_{2}} \otimes \underset{\mathbf{0} \%}{d_{2}}\right)
$$

It should be noted that all the equations are formulated in the intermediate configuration using the second Piola-Kirchhoff stress as the stress measure (Dafalius 1998).

\section{Constitutive Equations}

In this section a form will be developed for the evolution equations in Equation (10) which satisfy the restrictions on the constitutive equations that were laid out in Equation (9). Each element in the material is assumed to carry no stress in the initial stress free state. The material being considered comprises grains which are randomly oriented in the transverse orientation, thus, giving an average stress-strain response in the transverse orientation. Therefore, the material is considered to be an elastoplastic solid, 
which is characterized by persistent transversely isotropic symmetries. At each material point we specify the axis of transverse isotropy, ${ }_{0}^{d}$ and the mechanical behavior at each point depends not only on the stress and deformation history but also on this local preferential direction.

The inelastic behavior is generally idealized as rate independent elastoplastic at lower homologous temperatures and by a power law creep model at higher homologous temperatures (Nan 1991). In these approaches, the transition from one representation to another provides difficulties since creep laws are not mathematical limits of rateindependent plasticity and vice-versa. Unified material approaches do not separately postulate constitutive laws for creep and plasticity and consider all deformation as rate dependent with varying degrees of rate dependence. The transition from low to high temperature is characterized by a change in strength and rate dependence which can be embedded in the parameters in the constitutive equations by making them temperature dependent. A rate dependent unified formulation is used without a yield criterion and the plastic deformation rate (symmetric part of the plastic velocity gradient) is related to the effective stress by

$$
\boldsymbol{D}_{i}^{p}=\boldsymbol{N}=A \Theta\left\langle\frac{\bar{\sigma}-K}{D_{o}}\right\rangle^{n} \boldsymbol{N}
$$

where $\bar{\sigma}$ is the effective stress, $\mathrm{K}$ is the threshold stress and $D_{o}$ is the drag stress. $\Theta$ is the diffusivity parameter and $N$ gives the direction of plastic flow. The effective stress depends on the viscous overstress, $\quad \Sigma\left(\Sigma=\sigma^{\prime P K 2}-\alpha\right)$ where $\sigma^{\prime P K 2}=\sigma^{P K 2}-1 / 3 \operatorname{tr}\left(\sigma^{P K 2}\right) \delta$ is the deviatoric second Piola-Kirchoff stress, $\delta$ is the $2^{\text {nd }}$ rank identity tensor, and $\alpha$ is the backstress tensor) and the direction of grain growth ${ }_{0}^{d}$. The sense of ${ }_{0 \%}$ is immaterial, therefore the dependence is taken in terms of the dyadic product $\boldsymbol{M}={\underset{0}{0} \text {. }}_{0 \%} \otimes d_{0}$. In order to maintain form invariance under arbitrary rigid body rotations, the effective stress $\bar{\sigma}(\Sigma, \boldsymbol{M})$ must be expressible in terms of the principal invariants of its tensorial arguments and invariants involving the products based on the theory of tensorial invariants (Spencer 1962; Spencer 1971). Therefore, the admissible invariants are given as

$$
\operatorname{tr}(\boldsymbol{M}), \operatorname{tr}\left(\boldsymbol{M}^{2}\right), \operatorname{tr}\left(\boldsymbol{M}^{3}\right)
$$

where

$$
\begin{gathered}
\operatorname{tr}(\boldsymbol{M})=\operatorname{tr}\left(\boldsymbol{M}^{2}\right)=\operatorname{tr}\left(\boldsymbol{M}^{3}\right)=1 \\
\operatorname{tr}(\Sigma), \operatorname{tr}\left(\Sigma^{2}\right), \operatorname{tr}\left(\Sigma^{3}\right)
\end{gathered}
$$

where

$$
\operatorname{tr}(\Sigma)=0
$$

and

$$
\operatorname{tr}(\boldsymbol{M} \cdot \Sigma), \operatorname{tr}\left(\boldsymbol{M}^{2} \cdot \Sigma\right), \operatorname{tr}\left(\boldsymbol{M} \cdot \Sigma^{2}\right)
$$

where

$$
\operatorname{tr}(\boldsymbol{M} \cdot \Sigma)=\operatorname{tr}\left(\boldsymbol{M}^{2} \cdot \Sigma\right)
$$


Restricting the invariants to only the quadratic arguments and neglecting the trivial terms, the effective stress can be represented as given in (Robinson 1983; Robinson 2001)

$$
\bar{\sigma}=\sqrt{3\left(J_{2}-\xi\left(J-J_{o}^{2}\right)-\frac{3}{4} \varsigma J_{o}^{2}\right)}
$$

where

$$
J_{2}=\frac{1}{2} \Sigma_{i j} \Sigma_{i j}, J_{o}=M_{i j} \Sigma_{i j}
$$

and

$$
J=M_{i j} \Sigma_{j k} \Sigma_{k i} \text {. }
$$

Here $\xi$ and $\varsigma$ are temperature dependent material parameters which are determined from experimental data. In Equation 36, $\boldsymbol{N}$ is a unit vector in the inelastic strain rate direction and is given as

$$
N=\frac{\nabla \bar{\sigma}}{\|\nabla \bar{\sigma}\|}=\frac{\Gamma}{\|\Gamma\|}
$$

where

$$
\Gamma_{i j}=\Sigma_{i j}-\xi\left(M_{k i} \Sigma_{j k}+M_{j k} \Sigma_{k i}-2 J_{o} M_{i j}\right)-\frac{3}{2} \varsigma J_{o}\left(M_{i j}-\frac{1}{3} \delta_{i j}\right)
$$

and

$$
\|\Gamma\|=\left(\Gamma_{i j} \Gamma_{i j}\right)^{\frac{1}{2}}
$$

Hydrostatic stress independence is observed in most metals; this implies inelastic strain incompressibility which is ensured since $\operatorname{tr}(\Gamma)=\Gamma_{i i}=0$. The diffusivity parameter, $\Theta$, is given as

$$
\Theta=\exp \left(-\frac{Q}{R T}\right)
$$

where $Q$ is the energy for thermal activation and $R$ is the universal gas constant. Two macroscopic internal state variables are used; they are the threshold stress $(\mathrm{K})$ and the backstress $(\alpha)$. The threshold stress $(\mathrm{K})$ is a scalar variable which is used to model isotropic hardening i.e. an increase in the yield surface radius with cycling. DS GTD111 shows a cyclically stable stress-strain response and there is no isotropic hardening therefore a constant value of the threshold stress is used to capture the initial yield behavior, which is depends only on the temperature. The backstress is used to capture the directional effects of pre-strain on the kinetics of plastic flow (also called the Bauschinger effects). The backstress introduces the effects of storage of elastic energy due to heterogeneity of the microstructure. Physically, grain boundaries are the main sources of backstresses in polycrystalline materials, however, there are several sources of backstress in single crystal and DS Ni-base superalloys even though the grain boundaries are absent or very less in number. Milligan and Antolovich (Milligan 1989) showed in their study of deformation behavior in single crystal Ni-base superalloy PWA1480, that when dislocations emerge from precipitates, constrictions of the dislocations occur because of high anti-phase energy (APBE). This shows that portions of the dislocation which exit the precipitate are split because of elastic repulsion and suggests that a form of the backstress should be a component in the local force equilibrium equation. Backstress 
is also generated due to pile-ups of dislocations at hard boundaries such as $\gamma^{\prime}$ precipitates. The evolution equation for the backstress is

$$
\alpha_{i j}^{\&}=H\left(L \underset{i j}{\mathbb{2}}-\pi_{i j} \& \&\right)-R_{\alpha} \bar{\sigma}^{m} \pi_{i j}
$$

where

$$
\pi_{i j}=\alpha_{i j}-\xi\left(M_{k i} \alpha_{j k}+M_{j k} \alpha_{k i}-2 \hat{J}_{o} M_{i j}\right)-\frac{3}{2} \varsigma \hat{J}_{o}\left(M_{i j}-\frac{1}{3} \delta_{i j}\right)
$$

and

$$
\hat{J}_{o}=M_{i j} \alpha_{i j}
$$

where $H, L$ and $R_{\alpha}$ are constants. The backstress evolution in Equation (41) is of the Bailey-Orowan type which presumes that high temperature deformation occurs under the action of two simultaneously competing mechanisms; a hardening process proceeding with accumulated deformation characterized by the first term and a recovery term characterized by the second term. A static thermal recovery term is also used, which is important at moderate to low strain rates, especially at high temperatures. It should be noted that even though $\xi$ and $\varsigma$ are determined experimentally, there are certain restrictions placed on their values so that Equation (9) is satisfied. The constitutive equation relating the backstress $(\alpha)$ to its thermodynamic conjugate $(\xi)$, which is a strain like quantity is given as

where

$$
\alpha=H L \xi
$$

$$
\xi_{i j}=\underset{i j}{\&}-\frac{\pi_{i j}}{L} \&-\frac{R_{\alpha}}{H L} \bar{\sigma}^{m} \pi_{i j}
$$

Substituting the backstress in the intrinsic dissipation inequality gives

$$
\sigma_{i j}^{\prime P K 2} \underset{i j}{\&}-\alpha_{i j} \&
$$

Substituting Equations (36) and (42) into Equation (43) and doing further simplification places the following restrictions on the parameters

$$
\xi \leq 1 \text { and } \xi \geq \frac{3}{8} \varsigma
$$

The equations are summarized in Table 2.

\section{Implementation}

For each increment, the constitutive equations are integrated from an initial state at $t=t_{n}$ to a final state at $t=t_{n}+\Delta t=t_{n+1}$. The inputs of the integration scheme are the prescribed total strain increments for that time step and the stress $\left(\sigma_{n}\right)$ and the internal state variables $\left(k_{n}\right.$ and $\left.\alpha_{n}\right)$ from the previous time step. It is assumed that the thermoelastic properties and the model parameters remain constant through the time step. The flow rule in Eq. (36) should be satisfied at the end of the time step. A Newton Raphson scheme is used to solve the plastic strain increment

$$
\Delta p^{i+1}=\Delta p^{i}-\frac{f^{i}}{f^{\prime i}}
$$

where 


$$
f^{i}=\$ \Delta t-A \Theta^{i}\left\langle\frac{\bar{\sigma}^{i}-K^{i}}{D_{o}}\right\rangle^{n} \text { and } f^{\prime i}=\frac{\partial f^{i}}{\partial \Delta p^{i}}
$$

A subincrementation scheme is used along with the Newton Raphson scheme to help in convergence based on McGinty (McGinty 2001). The direction of plastic flow ( $N$ ) is kept constant for the Newton Raphson iterations in each subincrement. The Jacobian matrix is obtained as given in Equation (29). Using Equations (36), this simplifies to

$$
J_{i j k l}=\left(C_{i j k l}^{-1}+\frac{A n \Theta}{2 D \bar{\sigma}}\left\langle\frac{\bar{\sigma}-K}{D}\right\rangle^{n-1} \Gamma_{i j} N_{k l} \Delta t\right)^{-1}
$$

\section{Crystal Plasticity Model}

The twelve octahedral (Bettge) $<110>$ slip systems are appropriate for FCC single crystals and are active for the entire temperature range. The six cube slip systems $\{100\}<110>$ type (three planes and two directions = six systems) may be active at high homologous temperatures and high resolved shear stress (in $\gamma^{\prime}$ phase), but their role is less well understood and characterized. A precise mechanism is still largely conjectural, since the identification of the role of cube slip is challenging. For homogenized $\gamma-\gamma^{\prime}$ single crystals, the role of cube slip is usually assessed by considering the breakdown of the capability of octahedral slip to model the stress-strain-time behavior as a function of temperature, which of course depends on other elements of the constitutive framework. The work of Bettge and Osterle [(Bettge 1999)] on SC16 have revealed that macroscopic cube slip is a manifestation of octahedral slip and takes place by multiple cross slip events on octahedral planes, producing zig-zag dislocation lines. This enables the dislocations to travel large distances along matrix channels, thus effectively shearing material along the cube planes. In addition, actual cube slip has been observed [(Sun 1996)] in the $\gamma^{\prime}$ - precipitates at higher temperatures and along orientations closer to [111]. Experimentally, softening occurs as the orientation is rotated from the [100] orientation [(Mielek 1997)]. When only octahedral slip is active, hardening is predicted as the loading axis is rotated from the [100] direction, which is inconsistent with experimental observation. Both sources of cube slip, zig-zag cross slip in the $\gamma$ phase and motion of dislocation segments on cube planes in the $\gamma^{\prime}$ phase, are lumped together in the present model, treated via the activation of the cube slip systems. Both the octahedral and cube slip systems are assumed to be active in this model at all times.

The material parameters for the octahedral slip systems are determined from data obtained from uniaxial cyclic loading of longitudinal specimens along the [100] orientation, for which the loading axis is oriented along the grain growth direction. Cube slip is not activated in this orientation for small deformations. The material parameters for the cube slip systems are obtained using data from transverse specimens (loading perpendicular to the grain growth direction) due to lack of data in other orientations. It should be noted that the response of directionally solidified material in the transverse orientation is an average response comprising several grains. A total of thirty grains with random transverse orientations are used to obtain this average behavior along the loading axis. The Taylor constraint for intergranular interactions is assumed in this averaging process, such that each grain (with different orientations along the transverse direction) is 
subjected to the same deformation gradient; this approach is convenient in that a single element can be used to obtain the initial estimate of the material parameters, and is sufficiently accurate for slip of polycrystals with high symmetry cubic structures. A more rigorous finite element analysis is subsequently run with 30 hexagonal grains, each containing 96 elements, to optimize the fit of material parameters.

Under cyclic loading, lattice rotation may be insignificant, but in the presence of creep or cyclic ratcheting, lattice rotation may build up at sites of stress concentration or near interfaces such as grain boundaries. However, the creep and ratcheting strains are small in the cyclic loading experiments conducted to determine the material parameters. So these lattice rotations are neglected for the purpose of estimating the material parameters. Several other simplifications can be made regarding the deformation behavior of this material at this temperature. In the temperature range under consideration, the precipitate structures (size, shape, morphology) are stable so higherscale microstructural changes such as coarsening of precipitates can be neglected in the evolution equations.

From the cyclic deformation data, it is observed that the initial yield strength does not change significantly with cyclic loading. This indicates that the threshold and drag stress are nearly constant and that the backstress is the primary internal state variable that evolves with deformation. The threshold stress is mainly limited to the critical value $\kappa_{\mathrm{c}}^{\alpha}$ that incorporates non-Schmid terms as previously explained, and the drag stress $\left(\mathrm{D}^{\alpha}\right)$ is kept constant. The remaining material parameters must be physically admissible. This calls for a systematic procedure for determining the constants. First, certain physical parameters such as elastic properties and activation energies of thermally activated diffusional processes are obtained from literature. The activation energy is set to $\mathrm{Q}=309$ $\mathrm{kJ} / \mathrm{mol}$, based on the data from Daleo and Wilson on GTD-111 [(Daleo 1998)]. Estimating the remaining material parameters by using graphical methods in the analysis of deformation data is often not possible with viscoplasticity models because there is a strong coupling between creep and cyclic plasticity. Approximate values of the material parameters can often be obtained, but a trial-and-error approach almost always must be employed to refine the parameters to accurately capture the experimental deformation response. A more robust and efficient approach is to integrate the constitutive model, simulating the actual loading histories, using an optimization scheme to iteratively evaluate the model parameters [(Tanner 1999)].

The general optimization scheme involves running several simulations involving different thermomechanical histories including cyclic deformation with and without hold periods, as well as creep deformation, using a set of parameters suggested by the optimization code. Then the model response is compared to the actual experimental response generating a value of an objective function defined on the basis of the norm of the error between the experimental and predicted deformation responses. The Epogy code by Synaps [(Epogy 2004)] is used to minimize the objective function by iteratively modifying the material parameters based on minimization of the objective function. Epogy employs four widely used search methods (linear simplex, downhill simplex, gradient, and genetic algorithms) to assure stability and enhance the probability that an optimum set of material parameters will be obtained among various local minima. The rate of convergence and effectiveness of the optimization is highly dependent on the 
objective function, which in turn depends on the type of loading history. For uniaxial cyclic loading, the error function is defined as

$$
\text { Error }=\sum_{\mathrm{i}}\left(\sigma_{\exp }^{\mathrm{i}}-\sigma_{\text {pre }}^{\mathrm{i}}\right)^{2} \mathrm{~W}_{\mathrm{i}}
$$

where $\sigma_{\text {exp }}^{\mathrm{i}}$ is the experimentally measured stress along the loading direction, $\sigma_{\text {pre }}^{\mathrm{i}}$ is the stress predicted by the model at the same strain, and $\mathrm{W}_{\mathrm{i}}$ is a weighting parameter for the $\mathrm{i}^{\text {th }}$ strain value. The weighting parameter is varied from 0.8 to 1.2 , depending on which region of the stress-strain response is being simulated. Less weight is placed on the elastic portions of the deformation $\left(\mathrm{W}_{\mathrm{i}}=0.8\right)$ while $\mathrm{W}_{\mathrm{i}}=1.2$ is used during inelastic flow. During strain hold periods in which inelastic deformation and stress relaxation occurs, $\mathrm{W}_{\mathrm{i}}=1.0$.

Since the static thermal recovery terms are influential only for long hold periods and into the steady state creep regime, these terms can be neglected in the preliminary parameter estimation exercise that uses only continuous cycling data without hold times. The results of this preliminary exercise provides tighter bounds on those parameters that are less sensitive to rate for purposes of later optimization exercises that include cyclic data with strain holds and creep data intended to refine the estimates of material parameters. The error norm used for estimating parameters associated with creep behavior is

$$
\text { Error }_{\text {creep }}=\sum_{\mathrm{i}}\left(\varepsilon_{\text {exp }}^{\mathrm{i}}-\varepsilon_{\text {pre }}^{\mathrm{i}}\right)^{2} \mathrm{E}_{\mathrm{av}}^{2}
$$

where $\varepsilon_{\exp }^{\mathrm{i}}-\varepsilon_{\text {pre }}^{\mathrm{i}}$ is the difference between experimentally measured strain and predicted strain at a given time instant. This error norm employs the average value of the Young's modulus, $\mathrm{E}_{\mathrm{av}}$, in the specified orientation to scale the errors in terms of pseudo-stresses. For this final optimization step, the total error is the sum of the errors for the cyclic and creep deformation data,

$$
\text { Error }=\text { Error }_{\text {cyclic }}+\text { Error }_{\text {creep }}
$$

The material parameters obtained are listed in Table 3. Some of the material parameters are not listed due to unavailability of experimental data for fitting parameters. Static thermal recovery effects in evolution of $\kappa$ are omitted, for example. Accordingly, dashed entries in Table 4 signify zero values of the parameters.

The most sensitive material parameters for this data set as determined by the optimization scheme are $\mathrm{h}_{x \mathrm{~d}}$ and $\mathrm{h}_{x \mathrm{~s}}$, which control the backstress evolution. It should be noted that backstress plays an important role in Ni-base superalloys. Based on the tests by Ferney et al. [(Ferney 1991)] and Latif et al. [(Latif 1994)], it could be to the order of 50$60 \%$ of the flow stress. Experimental data used to fit parameters and correlated simulations based on the constitutive model are compared in Figures 4-12.

\section{Transversely Isotropic Model}

For the transversely isotropic stiffness matrix, there are five independent material parameters: the elastic modulus along the solidification axis $E_{L}$, the elastic modulus 
transverse to the solidification direction $E_{T}$, the Poisson's ratios, $v_{L}$ and $v_{T}$, and the shear modulus, $G_{L}$, which are determined experimentally through the entire temperature range. The material parameters in the inelastic strain evolution equation are determined from the experimental isothermal fatigue and creep tests. The Epogy code by Synaps (Epogy 2004) is used to obtain a best fit curve by iteratively modifying the material parameters based on minimization of the objective function. Epogy employs four widely used search methods (linear simplex, downhill simplex, gradient, and genetic algorithms) to assure stability and enhance the probability that an optimum set of material parameters will be obtained among various local minima. The material parameters are listed in Table 4; some of the material parameters are left blank due to unavailability of experimental data for fitting parameters. It should be noted that parameter, $\xi$, does not influence the stress strain response in the longitudinal and transverse orientation for uniaxial tension-

compression loading. It is assumed to be equal to $\frac{3}{8} \varsigma$ in order to satisfy Eq. (9) due to lack of any torsion data. It is emphasized that this choice is based on convenience and should be experimentally determined. Experimental data used to fit parameters and correlated simulations based on the constitutive model are compared in Figures 13-26.

\section{TMF Predictions}

For TMF, a deformation gradient $\mathbf{F}^{\theta}$ based on the thermal expansion is included in the multiplicative decomposition of the deformation gradient. An intermediate, thermally expanded, hot unstressed configuration and a hot plastically deformed relaxed configuration are introduced [(Shrikanth 1999)]. Thus the total deformation gradient is given by $\mathbf{F}=\mathbf{F}^{\mathrm{e}} \cdot \mathbf{F}^{\mathrm{p}} \cdot \mathbf{F}^{\theta}$, where the linearized elastic deformation is described by $\mathbf{F}^{\mathrm{e}}$, the plastic deformation by $\mathbf{F}^{\mathrm{p}}$, and the thermal expansion/contraction by $\mathbf{F}^{\theta}$. Assuming isotropic thermal expansion, the velocity gradient associated with thermal expansion effects is given as

$$
\mathbf{L}_{\mathrm{o}}^{\theta}=\left(\mathbf{F}^{\theta}\right)^{-1}=\alpha \otimes \mathbf{I}
$$

where $\alpha$ is the thermal expansion coefficient and $\mathbf{I}$ is the $2^{\text {nd }}$ rank identity tensor. The coefficient of thermal expansion, $\alpha_{T}$, varies with temperature but is independent of the history of the deformation parameters and is assumed to be an isotropic scalar quantity, i.e. it does not vary significantly with direction of heat flow. Solving the differential equation over a time step gives

$$
\mathbf{F}_{\mathrm{t}+\Delta \mathrm{t}}^{\theta}=\exp \left(\mathbf{L}_{\mathrm{o}}^{\theta} \Delta \mathrm{t}\right) \mathbf{F}_{\mathrm{t}}^{\theta}
$$

Material parameters are assigned temperature dependence using third order interpolative polynomial fits of values determined for isothermal cyclic and creep loading cases. The temperature is updated and held constant for each Newton Raphson step and the material parameters relevant to that temperature are used.

The model is used to predict the stress-strain response for both in-phase (IP) and outof-phase (OP) thermomechanical fatigue (TMF) tests for both the crystal plasticity and the transversely isotropic formulation. These TMF simulations can be considered as true predictions and validate the model calibration. The model predictions correlate reasonably well with the experimental results for loading in both the longitudinal and 
transverse orientations, as shown in Figures 27-29 for the crystal plasticity based model and Figures 30-31 for the transversely isotropic model.

\section{Mechanistic-based Crack Initiation Model}

Predictions of crack initiation life of L and T-oriented DS GTD-111 have been made using both experimentally-determined lives of DS GTD-111 specimens and data generated from elastic viscoplastic deformation modeling. The mechanistically-based crack initiation model is based on that developed by Neu and Sehitoglu (Neu and Sehitoglu, 1989). Assuming a unique relationship between the damage fraction and cycle fraction with respect to cycles to crack initiation for each damage mode, the total crack initiation life can be represented in terms of the individual damage components (fatigue, creep-fatigue and oxidation-fatigue respectively), and can be written as

$$
\frac{1}{N_{i}^{\text {tot }}}=\frac{1}{N_{i}^{\text {fat }}}+\frac{1}{N_{i}^{c r}}+\frac{1}{N_{i}^{o x}}
$$

Relationships for each of these components were determined by conducting LCF and TMF experiments to isolate the individual damage mechanisms. Mathematic formulations were developed to relate each mechanism (fat, $\mathrm{cr}$, and $e n v$ ) to temperature, orientation, cyclic frequency, hold time, etc. Fatigue damage is related as,

$$
N_{i}^{\text {fat }}=C_{1} f_{1}\left(\Delta \varepsilon_{p l}\right)^{C_{2}}
$$

where $C_{1}, C_{2}$, and $f_{1}$ are constants. To characterize this relation, data was needed from experiments conducted at low temperatures (e.g. $871^{\circ} \mathrm{C}$ and below), high strain rates $(0.5 \% / \mathrm{s})$, and continuous cycling conditions. At these conditions, both oxidation-assisted cracking at the surface is limited. Tests conducted at and below $871^{\circ} \mathrm{C}$ were used to correlate Eq. (53). Identical sets of tests conducted on L and T-oriented DS GTD-111 specimens generally led to comparable plastic strain ranges in each material. The elastic viscoplasticity UMAT modeled deformation accurately for each orientation. Thus, in addition to capturing the effects of varying temperature, strain range, cycle time, etc., crystallographic orientation is incorporated in the formulation via the inelastic strain range. The slight difference observed in the plastic strain versus life curves of L and Toriented material is captured by the factor, $f_{l}$.

The creep-fatigue interaction mechanism was found to correlate according to

$$
N_{i}^{c r}=C_{3} \Theta_{c r}(T, t)\left(\frac{t_{c}+t_{h}}{t_{c}+2 t_{h}}\right)^{f_{2}}\left(\Delta \varepsilon_{i n}\right)^{C_{4}}
$$

where $C_{3}, C_{4}$, and $f_{2}$ are constants. The inelastic strain range, $\Delta \varepsilon_{\text {in }}$, is used here since it captures orientation dependence. The constant $f_{2}$ also accounts for material orientation. Both the diffusion coefficient, $\Theta_{c r}$, and the parenthetical factor involving cycle time, $t_{c}$, and dwell time, $t_{h}$, help to account for the temperature-dependent viscoplasticity not captured in Eq. (53). The diffusion term, similar to the one introduced by Neu and Sehitoglu, is applicable with or without temperature cycling, i.e.,

$$
\Theta_{c r}(T, t)=\frac{1}{t_{c}} \int_{0}^{t_{c}} \exp \left(-\frac{Q_{c r}}{R T(t)}\right) d t
$$

Here $Q_{c r}$ is the activation energy for creep, $T(t)$ is the temperature history, and $R$ is the gas constant. Specimens subjected to dwell times experience the most significant stress relaxation caused by viscoplastic deformation. The correlation of Eq. (55) involved HT 
and $\mathrm{HC}$ tests above $871^{\circ} \mathrm{C}$. The inelastic deformation in these cases, namely the plastic deformation associated with dislocation activity and the viscous deformation associated with creep microcracking, cannot be completely isolated; however, each is individually accounted for in Eqs. (53) and (54).

The term relating environmental-fatigue interaction to crack initiation was based on direct observations of micrographs of polished samples where surface cracking was predominant,

$$
N_{i}^{o x}=C_{5}\left[\Phi_{o x} \Theta_{o x}\left(t_{c}+t_{h}\right)\right]^{C_{6}} \Delta \varepsilon_{m}{ }^{C_{7}}
$$

Here $C_{5}, C_{6}$, and $C_{7}$ are constants. The cycle time and diffusion term capture the oxidation growth kinetics. While both the $\Delta \varepsilon_{m}$ and the phasing factor, $\Phi_{\text {ox }}$, capture the rate at surface damage, i.e.,

$$
\Phi_{o x}=\exp \left[-C_{8}(\underset{\mathrm{in}}{\&} / \underset{m}{\&}+1)^{2}\right]
$$

This term is designed to be maximum when the ration of thermal strain rate, \& to mechanical strain rate, $\underset{m}{\mathbb{2}}$, is equal to -1 . This is the case of out-of-phase TMF cycling. where the effects of surface cracking at surface-grown oxides are the most detrimental to crack initiation life.

\section{Results and Discussion}

Simulated hysteresis data (i.e., $\Delta \varepsilon_{p l}$ and $\sigma$ ) of a stabilized cycle was used to compute the accumulated effects of fatigue, creep-fatigue, and environmental-fatigue damage on crack initiation life based on the formulation given in Eqs. (53)-(57). Based on the results of experiments and simulations, predictions for crack initiation lives of $\mathrm{L}$ and T-oriented DS GTD-111 have been made for continuously cycled specimens. With increasing temperature, lower crack initiation lives were predicted for both materials, as shown in Fig. 33. In most cases, the fatigue damage term dominates; however, the strain range versus life predictions illustrate the changeover from predominantly fatigue to coupled environmental-fatigue by decreasing the applied strain range. As the temperature is decreased below $871^{\circ} \mathrm{C}$, there is insufficient thermodynamic driving force for oxidation and the role of environment is limited as a result. These predicted curves compare well with test results obtained for a variety of temperature and mechanical strain range combinations.

The crack initiation life predictions for both orientations are also illustrated in contour plots of mechanical strain versus temperature, Fig. 34. Based on the balance of the contributions of each of the damage mechanisms, the fatigue-related damage mechanism dominates over the creep-fatigue and oxidation-fatigue mechanism in most $\mathrm{CC}$ cases. For low mechanical strain ranges, the oxidation-fatigue mechanism dominates only when the temperature is high enough to lead to surface-accumulated oxides that participate in the fracture process

In addition to isothermal cases, predictions for crack initiation lives of L-oriented DS GTD-111 were developed for cycling under non-isothermal conditions. Predicted trends for the mechanical strain life of the material are illustrated in Fig. 35a. Under TMF conditions at lower mechanical strain ranges, the OP cycle is predicted to be more damaging than the IP cycle. This prediction is consistent with experiments since the oxide spiking mechanism, which is more detrimental to life than damage initiated at 
subsurface inclusions, is experienced during OP cycling. During IP cycling with $\Delta \varepsilon_{m}<1.0 \%$, life is dominated by the fatigue mechanism. With the increase of $\Delta T$ from $389^{\circ} \mathrm{C}$ to $500^{\circ} \mathrm{C}$, the constitutive model predicts $\Delta \varepsilon_{\text {in }}$ to increase. Tests conducted at same temperature and total strain amplitudes on longitudinal and transverse oriented DS GTD111 specimens lead to larger inelastic strain ranges for transverse specimens since transverse specimens have a larger elastic stiffness. Consequently, the lives of longitudinal specimens typically outlast those of transverse specimens when tested at the same total strain range. The crystallographic orientation dependence in the constitutive model captures this phenomenon, predicting the trends especially at the higher mechanical strain ranges. Crack initiation life predictions are also illustrated on a contour plot Fig. 35 b. For mechanical strain ranges above $1.0 \%$, the fatigue damage mechanism dominates and crack initiation life is independent of the phase shift between thermal and mechanical cycling. At low strain ranges, however, the type of damage that is the most detrimental to crack initiation life is predicated by the phasing. In-phase cycling lead to creep-fatigue dominated damage, while out-of-phase cycling lead to oxide-fatigue damage. These life predictions agree with crack initiation life results from experiments as well as the corresponding observations of damage using microscopy.

\section{Conclusions}

A crystal plasticity based model and a transversely isotropic constitutive model are formulated to capture the stress strain behavior in DS GTD111. Isothermal fatigue and creep data are used to calibrate the models. The models are implemented as an UMAT in ABAQUS. In-phase and out-of-phase thermomechanical fatigue responses are adequately predicted by both the models. The transversely isotropic model is shown to cut down the analysis time when compared to the crystal plasticity model. However, some of the limitations of the models and future suggestions are as follows:

\section{Crystal Plasticity Model:}

1. Most of the experiments are conducted for isothermal fatigue in the longitudinal and transverse orientations for uniaxial fatigue. The validity of the model should be tested at other orientations and for multi-axial fatigue. The parameters for cube slip have been obtained using the macroscopic averaged response from randomly oriented grains in the transverse orientation. It should be noted that this might introduce errors in the parameters; ideally, they should be determined from experiments conducted on single crystals in the [111] orientation, where cube slip is the dominant active slip system.

2. Crystal plasticity based approaches can account for the effect of grain orientation on the stress-strain response. It would be very useful to obtain the grain orientation map using electron back scatter diffraction (EBSD) and study the effect of the orientation distribution (in the transverse orientation) on the constitutive stress-strain response.

\section{Transversely Isotropic Model:}

1. The validity of the model should be tested for other orientations and also for multiaxial fatigue. Multiaxial fatigue testing is a non-trivial task since there are no set standards for conducting these tests in DS materials. ASME standards for 
multi-axial testing sets a limit on the minimum number of grains along the thickness of the thin tube, which is difficult to maintain in DS GTD-111. Extensive testing would be required to develop these standards and get useful data; this was not feasible in this project but it would be of considerable interest for future work.

2. Even though an effort has been made to encapsulate the physics of the deformation behavior into the formulation, the macroscopic transversely isotropic formulation smears the influence of individual grains and gives an averaged response.

3. While the model captures the first three points in Section 3; it does not have the capability to capture the tension-compression (TC) asymmetry, which could be significant in the anomalous temperatures regime. However, it should be noted that DS GTD-111 is mainly used in second stage turbine blades and the temperature range of interest is mainly above the peak anomalous temperature $\left(750^{\circ} \mathrm{C}\right)$, where $\mathrm{TC}$ asymmetry is non-existent or negligible.

In general, the analysis time for the transversely isotropic Umat is comparable to the crystal plasticity Umat. The analyses are conducted for a square plate with a circular hole subjected to uniaxial tension-compression as shown in Figure 32. Generalized plane strain elements are used and the analysis are conducted for one fatigue cycle using 600 elements in the longitudinal orientation and the transverse orientation ((110) orientation in the case of the crystal plasticity simulations). The average time for the analysis is observed to be a factor of three lower for the transversely isotropic model simulation as compared to the crystal plasticity model, thus showing a significant saving in computational time.

The deformation and failure mechanisms occurring in a directionally solidified Ni-base superalloy, DS GTD-111, have been characterized as a function of temperature and loading history. Observations from experiments that isolated the microstructural damage mechanisms were applied to mathematically relate crack initiation behavior of both L and T-oriented DS GTD-111. Three primary damage mechanisms are identified: fatigue, creep-fatigue and oxidation-fatigue induced damage. Using numerical simulations of the thermomechanical behavior of DS GTD-111, damage and crack initiation life predictions have been made. Isothermal and thermomechanical uniaxial fatigue conditions with hold times at temperatures ranging from room temperature (RT) to $1038^{\circ} \mathrm{C}$ were considered. Under high frequency and isothermal conditions with large plastic strain ranges and low temperatures, fatigue damage dominates. The contribution of the coupled creep-fatigue mechanism appears by increasing either the temperature or cycle time. Tests with small $\Delta \varepsilon_{p}$, including those with superimposed thermal cycling, are subject to environmental-fatigue damage since the surface-related mechanisms are active at long exposure times. For identical conditions, the lives of $\mathrm{L}$ specimens outlast those of T. Each of these phenomena is accounted for in the above formulation that facilitates accurate crack initiation prediction for various conditions. Crack initiation life predictions, based on the life models for each damage mechanism, follow the experimental trends.

\section{References}


Aravas, N. (1992). "Finite elastoplastic transformations of transversely isotropic metals." International Journal of Solids and Structures 29(17): 2137-2157.

Bettge, D. O., W (1999). "Cube slip in near-[111] oriented specimens of a single-crystal nickel-base superalloy." Scripta Materialia (USA) 40(4): 389-395.

Bilby, B. B., R; Smith, E (1955). "Continuous Distributions of Dislocations: A New Application of the Methods of Non-Riemannian Geometry." Proc. Royal Society A231: 263-273.

Castelli, M. E., JR (1993). "Improved techniques for thermomechanical testing in support of deformation modeling." Symposium on Thermomechanical Fatigue Behavior of Materials (STP 1186); H. Sehitoglu. Ed., San Diego, CA, ASTM: 195-211.

Dafalius, Y. (1998). "Plastic Spin: Necessity or Redundancy." International Journal of Plasticity 14(9): 909-931.

Daleo, J. W., JR (1998). "GTD-111 Alloy material study." J. engineering for gas turbines and power 120: 375-382.

Epogy (2004). "Synaps Inc., Atlanta, GA, USA, v2004A."

Ferney, V. H., M; Clavel, M (1991). "Multiaxial cyclic behavior in two precipitates strengthened alloys: Influence of the loading path and microstructure." Memoires et Etudes Scientifiques de la Revue de Metallurgie 88: 441-451.

Hasebe, T. S., M; Ohnami, M (1992). "Elastic anisotropy of directionally solidified superalloy." JEMT, Transactions of the ASME 114(2): 141-146.

Hirsch, P. (1992). " A new theory of the anomalous yield stress in LI2 alloys." Philosophical Magazine A 62(3): 569-612.

Lall, C. C., S; Pope, D (1979). "Orientation and temperature dependence of the yield stress of Ni3 (al, nb) single crystals." Metallurgical Transactions A 10A: 1323.

Latif, A. C., M; Ferney, V; Saanouni, A (1994). "On the modeling of non-proportional cyclic plasticity of Waspaloy." ASME J. Eng. Mat. Techn. 116: 35-44.

Lee, H. (1969). "Elastic plastic deformations at finite strains." Journal of applied mechanics, ASME 36: 1-6.

Mandel, J. (1971). "Plasticite classique et viscoplasticite." Courses and Lectures, ICMS, Udine, Springer, Wien, New York(97). 
McDowell, D. (1991). "Micromechanical implications for state variable viscoplasticity." American Society of Mechanical Engineers, Materials Division (Publication) MD 26: 103-114.

McDowell, D. (1992). "A nonlinear kinematic hardening theory for cyclic thermoplasticity and thermoviscoplasticity." Int. J. Plasticity 8: 695-728.

McGinty, R. (2001). "Multiscale Representation of Polycrystalline Inelasticity." Ph.D. thesis(Georgia Institute of Technology).

Meric, L. P., P; Cailletaud, G (1991). "Single Crystal Modeling for Structural Calculations Part 1 - Model Presentation." Journal of Engineering Materials and Technology (Transactions of the ASME) 162(113): 162-170.

Mielek, J. N., V; Zarubova, N; Gemberle, A (1997). "Orientation dependence of plastic deformation in NiAl single crystals." Mater. Sci. Eng. A234-236: 410-413.

Milligan, W. A., SD (1989). "Deformation modeling and constitutive modeling for anisotropic superalloys." NASA Report 4215.

Nan, M. K., E (1991). "Thermoviscoplasticity based on overstress applied to the analysis of fibrous metal matrix composites." Journal of composite materials 26(7): 969-990.

Neu, R. W., and Sehitoglu, H. (1989). "Thermomechanical Fatigue, Oxidation, and Creep: Part II. Life Prediction." Metallurgical Transactions A 20.

Osterle, W. B., D; Fedelich, B; Klingelhoffer, K (2000). "Modelling the orientation and direction dependence of the critical resolved shear stress of Nickel-base superalloy single crystals." Acta Materialia 48: 689-700.

Paidar, V. P., DP; Vitek, V (1984). "A theory of the anomalous yield behavior in LI2 ordered alloys." Acta Metallurgica 32(3): 435-448.

Qin, Q. B., JL (1992). "Non-Schmid yield behavior in single crystals." J. Mech. Phys. Solids 40(4): 813-833.

Robinson, D. (1983). "Constitutive relationships for anisotropic high temperature alloys." NSA Technical Memorandum 83437.

Robinson, D. B., WK (2001). "Model of viscoplasticity for transversely isotropic inelastically compressible solids." Journal of Engineering Mechanics 127(6): 567-573.

Sheh, M. S., DC (1988). "Anisotropic constitutive model for Nickel-base Single Crystal Superalloys." NASA Report No. CR-182157. 
Shirafuji, N. K., S; Masao, S; Ohnami, M (1998). "Tension torsion multiaxial low cycle fatigue of MAR-M247 LC directionally solidified superalloy at elevated temperatures." Journal of Engineering Materials and Technology, ASME 120.

Shrikanth, A. Z., N (1999). "A computational model for the finite element analysis of thermoplasticty coupled with ductile damage at finite strains." International Journal for Numerical Methods in Engineering 45: 1569-1605.

Spencer, A. (1971). "Theory of invariants." Continuum Physics 1: 240-253.

Spencer, A. R., RS (1962). "Isotropy integrity bases for vectors and second order tensors." Arch. Ration. Mech. Anal. 9: 45-63.

Sun, Y. H., PM (1996). "Geometry of dislocation glide in L12 - phase: TEM observations." Dislocations in Solids 10: 27-68.

Takeuchi, S. K., E (1971). "Anomalous temperature dependence of the yield stress in $\mathrm{Ni}_{3}$ Ga." J. Phys. Soc. Japan 31: 1282.

Takeuchi, S. K., E (1971). "Temperature and Orientation Dependence of the Yield Stress in Ni3Ga Single Crystals." Acta Matellurgica 21: 415 -425.

Tanner, A. M., RD; McDowell, D. L. (1999). "Modeling Temperature and Strain Rate Sequence Effects on OFHC Copper." Int. J. Plasticity 15: 575-603.

Vitek, V. P., DP; Bassani, JL (1996). "Superalloys (Ni base) and dislocations - An Introduction." Dislocations in Solids 10: 135-186.

Westbrook, J. (1996). "Superalloys (Ni base) and dislocations - An Introduction." Dislocations in Solids 10: 1-25. 


\section{Tables}

Table 1. Summary of crystal plasticity based constitutive model.

Flow Rule:

$\&=\psi_{0} \Theta(T)\left\langle\frac{\tau_{v}^{\alpha}}{D^{\alpha}}\right\rangle^{n} \exp \left\{\mathrm{B}_{\mathrm{o}}\left\langle\frac{\tau_{\mathrm{v}}^{\alpha}}{\mathrm{D}^{\alpha}}\right\rangle^{\mathrm{n}+1}\right\} \operatorname{sgn}\left(\tau^{\alpha}-\chi^{\alpha}\right)$

where

$\tau_{\mathrm{v}}^{\alpha}=\left|\tau^{\alpha}-\chi^{\alpha}\right|-\kappa^{\alpha} \frac{\mu}{\mu_{\mathrm{o}}} \quad$ and $\quad \mathrm{D}^{\alpha}=\mathrm{D}_{\mathrm{o}} \frac{\mu}{\mu_{\mathrm{o}}}$

$\Theta(T)=\exp \left(-\frac{Q_{o}}{R T}\right) \quad T \geq \frac{T_{m}}{2}$

$\Theta(T)=\exp \left\{-\frac{2 \mathrm{Q}_{\mathrm{o}}}{\mathrm{RT}_{\mathrm{m}}}\left[\ln \left(\frac{\mathrm{T}_{\mathrm{m}}}{2 \mathrm{~T}}\right)+1\right]\right\} \quad \mathrm{T} \leq \frac{\mathrm{T}_{\mathrm{m}}}{2}$

Evolution Equations for Hardening Variables:

Back stress

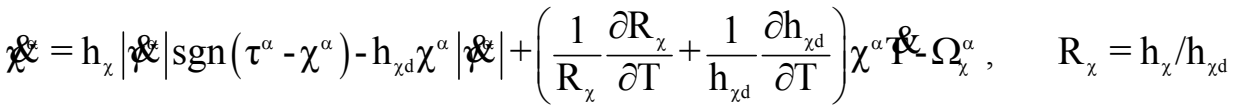

where $\Omega_{\chi}^{\alpha}=\mathrm{h}_{\chi s} \Theta_{\chi s}(\mathrm{~T})\left|\chi^{\alpha}\right|^{\mathbf{r}_{1 s}}, \quad \Theta_{\chi s}(\mathrm{~T})=\Theta(\mathrm{T})$

Threshold Stress

$\kappa^{\alpha}=\kappa_{\mathrm{c}}^{\alpha}+\kappa_{\mathrm{e}}^{\alpha}, \quad \kappa_{\mathrm{c}}^{\alpha}=\kappa_{\mathrm{o}}^{\alpha}(\mathrm{T})+\mathrm{h}_{\mathrm{pe}} \tau_{\mathrm{pe}}^{\alpha}+\mathrm{h}_{\mathrm{se}} \tau_{\mathrm{se}}^{\alpha}+\mathrm{h}_{\mathrm{cb}}\left|\tau_{\mathrm{cb}}^{\alpha}\right|$

$\mathrm{h}_{\mathrm{o}} \sum_{\beta=1}^{\mathrm{N}_{\text {slip }}} \mathrm{q}^{\alpha \beta}\left|\&^{\beta}\right|-\mathrm{h}_{\kappa \mathrm{s}} \mathrm{\kappa}^{\alpha} \sum_{\beta=1}^{\mathrm{N}_{\text {slip }}}\left|\beta^{\beta}\right|-\mathrm{h}_{\mathrm{s}} \Theta(\mathrm{T})\left\langle\kappa^{\alpha}-\mathrm{\kappa}_{\mathrm{th}}\right\rangle^{\mathrm{r}_{\mathrm{s}}}$

Initial conditions: $\chi^{\alpha}(0)=0, \kappa_{\mathrm{e}}^{\alpha}(0)=0$ 
Table 2. Summary of transversely isotropic constitutive model.

Flow Rule:

$\boldsymbol{D}_{i}^{p}=\boldsymbol{N}=A \Theta\left\langle\frac{\bar{\sigma}-K}{D_{o}}\right\rangle^{n} \boldsymbol{N}$

where

$\bar{\sigma}=\sqrt{3\left(J_{2}-\xi\left(J-J_{o}^{2}\right)-\frac{3}{4} \varsigma J_{o}^{2}\right)} \quad$ and $\quad N=\frac{\nabla \bar{\sigma}}{\|\nabla \bar{\sigma}\|}=\frac{\Gamma}{\|\Gamma\|}$

$\Sigma=\sigma^{\prime P K 2}-\alpha \quad$ and $\quad \sigma^{\prime P K 2}=\sigma^{P K 2}-1 / 3 \operatorname{tr}\left(\sigma^{P K 2}\right) \delta$

$\Gamma_{i j}=\Sigma_{i j}-\xi\left(M_{k i} \Sigma_{j k}+M_{j k} \Sigma_{k i}-2 J_{o} M_{i j}\right)-\frac{3}{2} \varsigma J_{o}\left(M_{i j}-\frac{1}{3} \delta_{i j}\right)$ and $\|\Gamma\|=\left(\Gamma_{i j} \Gamma_{i j}\right)^{\frac{1}{2}}$

$J_{2}=\frac{1}{2} \Sigma_{i j} \Sigma_{i j}, J_{o}=M_{i j} \Sigma_{i j}$ and $J=M_{i j} \Sigma_{j k} \Sigma_{k i}$ and $\Theta(\mathrm{T})=\exp \left(-\frac{\mathrm{Q}_{\mathrm{o}}}{\mathrm{RT}}\right)$

Evolution Equations for Hardening Variables:

Back stress

$a \underset{l y}{\&}=H\left(L \underset{i j}{\& 2}-\pi_{i j} \&\right)-R_{\alpha} \bar{\sigma}^{m} \pi_{i j}$

where

$\pi_{i j}=\alpha_{i j}-\xi\left(M_{k i} \alpha_{j k}+M_{j k} \alpha_{k i}-2 \hat{J}_{o} M_{i j}\right)-\frac{3}{2} \varsigma \hat{J}_{o}\left(M_{i j}-\frac{1}{3} \delta_{i j}\right)$ and $\hat{J}_{o}=M_{i j} \alpha_{i j}$ 
Table 3. Material Parameters

(a) Octahedral Slip System Constants $\quad \chi^{\alpha}(0)=0, \kappa_{\mathrm{e}}^{\alpha}(0)=0$

\begin{tabular}{ccccccccccc}
\hline $\begin{array}{c}\text { Temperature } \\
\left({ }^{\circ} \mathrm{C}\right)\end{array}$ & $\begin{array}{c}\mathrm{h}_{\chi} \\
(\mathrm{MPa})\end{array}$ & $\begin{array}{c}\mathrm{R}_{\chi} \\
(\mathrm{MPa})\end{array}$ & $\mathrm{h}_{\chi \mathrm{s}}$ & $\mathrm{r}_{\chi \mathrm{s}}$ & $\begin{array}{c}\kappa_{\mathrm{o}}^{\alpha} \\
(\mathrm{MPa})\end{array}$ & $\begin{array}{c}\mathrm{h}_{\mathrm{o}} \\
(\mathrm{MPa})\end{array}$ & $\mathrm{h}_{\text {кs }}$ & $\mathrm{h}_{\mathrm{s}}$ & $\mathrm{r}_{\mathrm{s}}$ & $\mathrm{q}^{\alpha \beta}$ \\
\hline 427.0 & 55160 & 110.0 & - & - & 93.0 & 6895 & 135 & - & - & 1 \\
760.0 & 186165 & 148.0 & $2.37 \mathrm{E}-7$ & 1 & 148.0 & 0 & 0 & - & - & - \\
871.1 & 137900 & 92.0 & $7.77 \mathrm{E}-8$ & 1 & 70.0 & 0 & 0 & - & - & - \\
982.2 & 41370 & 69.0 & - & - & 14.0 & 0 & 0 & - & - & - \\
1037.8 & 17238 & 64.3 & - & - & 14.0 & 0 & 0 & - & - & - \\
\hline
\end{tabular}

(b) Cube slip system constants $\chi^{\alpha}(0)=0, \kappa_{\mathrm{e}}^{\alpha}(0)=0$

\begin{tabular}{ccccccccccc}
\hline $\begin{array}{c}\text { Temperature } \\
\left({ }^{\circ} \mathrm{C}\right)\end{array}$ & $\begin{array}{c}\mathrm{h}_{\chi} \\
(\mathrm{MPa})\end{array}$ & $\begin{array}{c}\mathrm{R}_{\chi} \\
(\mathrm{MPa})\end{array}$ & $\mathrm{h}_{\chi \mathrm{s}}$ & $\mathrm{r}_{\chi \mathrm{s}}$ & $\begin{array}{c}\kappa_{\mathrm{o}}^{\alpha} \\
(\mathrm{MPa})\end{array}$ & $\begin{array}{c}\mathrm{h}_{\mathrm{o}} \\
(\mathrm{MPa})\end{array}$ & $\mathrm{h}_{\mathrm{ks}}$ & $\mathrm{h}_{\mathrm{s}}$ & $\mathrm{r}_{\mathrm{s}}$ & $\mathrm{q}^{\alpha \beta}$ \\
\hline 427.0 & - & - & - & - & - & - & - & - & - & - \\
760.0 & - & - & - & - & - & - & - & - & - & - \\
871.1 & 172400 & 81.2 & - & 1 & 64.2 & 0 & 0 & - & - & - \\
982.2 & 55160 & 54.5 & - & - & 11.0 & 0 & 0 & - & - & - \\
1037.8 & 17238 & 44.8 & - & - & 11.0 & 0 & 0 & - & - & - \\
\hline
\end{tabular}

(c) Common constants

\begin{tabular}{|c|c|c|c|c|c|c|c|c|c|}
\hline $\begin{array}{c}\text { Temperature } \\
\left({ }^{\circ} \mathrm{C}\right)\end{array}$ & $\begin{array}{c}\mathrm{C}_{11} \\
(\mathrm{MPa})\end{array}$ & $\begin{array}{c}\mathrm{C}_{12} \\
(\mathrm{MPa})\end{array}$ & $\begin{array}{c}\mathrm{C}_{44} \\
(\mathrm{MPa})\end{array}$ & $\mathrm{n}$ & $\mathrm{B}_{\mathrm{o}}$ & \multicolumn{2}{|c|}{$\begin{array}{c}\mu \\
(\mathrm{MPa})\end{array}$} & $\mathrm{h}_{\mathrm{pe}}$ & $\mathrm{h}_{\mathrm{cb}}$ \\
\hline 427.0 & 169617 & 70675 & 132000 & 5 & 0.05 & \multicolumn{2}{|c|}{132000} & 0.15 & 0.1 \\
\hline 760.0 & 151690 & 70329 & 125750 & 5 & 0.05 & \multicolumn{2}{|c|}{125750} & 0.15 & 0.1 \\
\hline 871.1 & 138830 & 69980 & 120360 & 4 & 0.05 & \multicolumn{2}{|c|}{120360} & 0.0 & 0.0 \\
\hline 982.2 & 134450 & 69980 & 113880 & 4 & 0.05 & \multicolumn{2}{|c|}{113880} & 0.0 & 0.0 \\
\hline \multirow[t]{3}{*}{1037.8} & 131000 & 69980 & 108180 & 4 & 0.05 & \multicolumn{2}{|c|}{108180} & 0.0 & 0.0 \\
\hline & $\begin{array}{c}\mathrm{Q}_{\mathrm{o}} \\
(\mathrm{KJ} / \mathrm{mol})\end{array}$ & $\begin{array}{c}\mathrm{R} \\
(\mathrm{J} / \mathrm{mol} \mathrm{K})\end{array}$ & $\begin{array}{c}\mu_{\mathrm{o}} \\
\text { (MPa) }\end{array}$ & & $\begin{array}{c}\text { \& } \\
\text { \& } \\
\left(\mathrm{s}^{-1}\right)\end{array}$ & & $\begin{aligned} & \kappa_{\text {th }} \\
& \text { (MP }\end{aligned}$ & & $\begin{array}{c}\mathrm{D}_{\mathrm{o}} \\
(\mathrm{MPa})\end{array}$ \\
\hline & 309 & 8.314 & 166000 & & $1.15 \mathrm{x}$ & & 0.0 & & 102.0 \\
\hline
\end{tabular}


Table 4. Material Parameters

At time $=0, \sigma=\mathbf{0}, \alpha=\mathbf{0}$

\begin{tabular}{cccccc}
\hline $\begin{array}{c}\text { Temperature } \\
\left({ }^{\circ} \mathrm{C}\right)\end{array}$ & $\begin{array}{c}\mathrm{E}_{\mathrm{L}} \\
(\mathrm{MPa})\end{array}$ & $\begin{array}{c}\mathrm{E}_{\mathrm{T}} \\
(\mathrm{MPa})\end{array}$ & $v_{\mathrm{L}}$ & $v_{\mathrm{T}}$ & $\begin{array}{c}\mathrm{G}_{\mathrm{L}} \\
(\mathrm{MPa})\end{array}$ \\
\hline 427 & 126340 & 159048 & 0.195 & 0.4 & 104000 \\
650 & 120930 & 156190 & 0.195 & 0.4 & 97900 \\
760 & 103425 & 146667 & 0.195 & 0.4 & 95200 \\
871 & 95200 & 126000 & 0.195 & 0.4 & 89650 \\
982 & 89650 & 121505 & 0.195 & 0.4 & 82740 \\
1038 & 82740 & 121505 & 0.195 & 0.4 & 75850 \\
\hline
\end{tabular}

\begin{tabular}{ccrcccccc}
\hline $\begin{array}{c}\text { Temperature } \\
\left({ }^{\circ} \mathrm{C}\right)\end{array}$ & $\begin{array}{c}\mathrm{K} \\
(\mathrm{MPa})\end{array}$ & $\mathrm{n}$ & $\xi$ & $\varsigma$ & $\mathrm{H}$ & $\begin{array}{c}\mathrm{L} \\
(\mathrm{MPa})\end{array}$ & $\begin{array}{c}\mathrm{R}_{\alpha} \\
\left(\mathrm{MPa}^{-3}\right)\end{array}$ & $\mathrm{M}$ \\
\hline 427 & 100 & 30 & 0.165 & 0.44 & 130 & 180 & - & - \\
650 & 175 & 22 & 0.165 & 0.44 & 150 & 310 & $6.00 \mathrm{E}-15$ & 3 \\
760 & 150 & 8 & 0.083 & 0.22 & 500 & 275 & $1.00 \mathrm{E}-13$ & 3 \\
871 & 100 & 4 & 0.028 & 0.075 & 200 & 250 & $5.75 \mathrm{E}-13$ & 3 \\
982 & 20 & 4 & 0.028 & 0.075 & 225 & 190 & $4.00 \mathrm{E}-12$ & 3 \\
1038 & 20 & 4 & 0.028 & 0.075 & 125 & 190 & - & - \\
\hline
\end{tabular}

\begin{tabular}{cccc}
\hline $\begin{array}{c}\mathrm{Q} \\
(\mathrm{KJ} / \mathrm{mol})\end{array}$ & $\begin{array}{c}\mathrm{R} \\
(\mathrm{J} / \mathrm{mol} \mathrm{K})\end{array}$ & $\mathrm{A}$ & $\begin{array}{c}\mathrm{D}_{\mathrm{o}} \\
(\mathrm{MPa})\end{array}$ \\
\hline 319 & 8.314 & $3.3174 \mathrm{E}+7$ & 270 \\
\hline
\end{tabular}

Table 5. Constants for the crack initiation model.

\begin{tabular}{|c|c|c|c|c|c|}
\hline \multicolumn{2}{|c|}{ Fatigue Term } & \multicolumn{2}{|c|}{ Creep-Fatigue Term } & \multicolumn{2}{|c|}{ Environment-Fatigue Term } \\
\hline Symbol & Value & Symbol & Value & Symbol & Value \\
\hline $\mathrm{C}_{1}$ & 0.15 & $\mathrm{C}_{3}$ & 60 & $\mathrm{C}_{5}$ & 0.10 \\
\hline $\mathrm{C}_{2}$ & -0.65 & $\mathrm{C}_{4}$ & -1.10 & $\mathrm{C}_{6}$ & -0.57 \\
\hline \multirow[t]{3}{*}{$\mathrm{f}_{1}$} & 0.78 & $\mathrm{f}_{2}$ & 1.00 & $\mathrm{C}_{7}$ & -0.45 \\
\hline & & $\mathrm{Q}_{\mathrm{cr}}(\mathrm{KJ} / \mathrm{mol})$ & 6.75 & $\mathrm{C}_{8}$ & 0.50 \\
\hline & & & & $\mathrm{Q}_{\mathrm{ox}}(\mathrm{KJ} / \mathrm{mol})$ & 164 \\
\hline
\end{tabular}




\section{Figures}

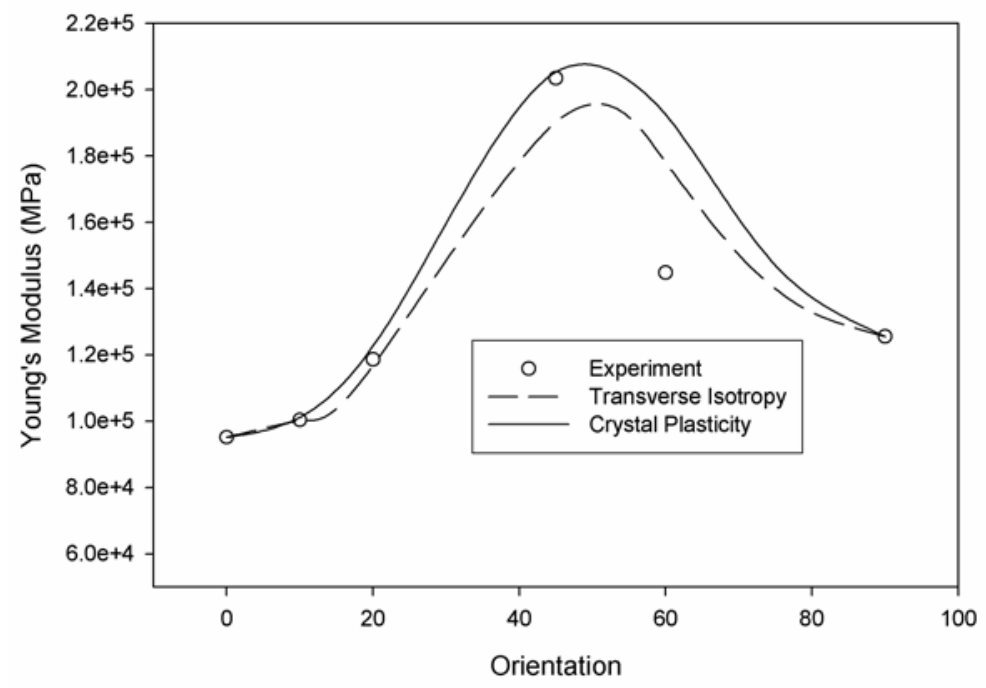

Figure 1 Plot of Young's modulus as a function of loading orientation with respect to the solidification direction. 


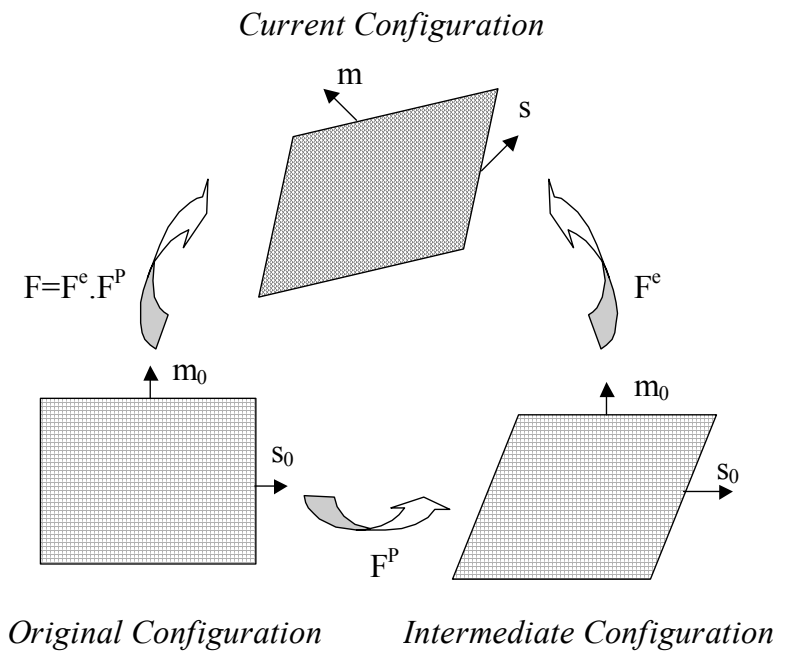

Figure 2 Elastoplastic decomposition of the deformation gradient. 

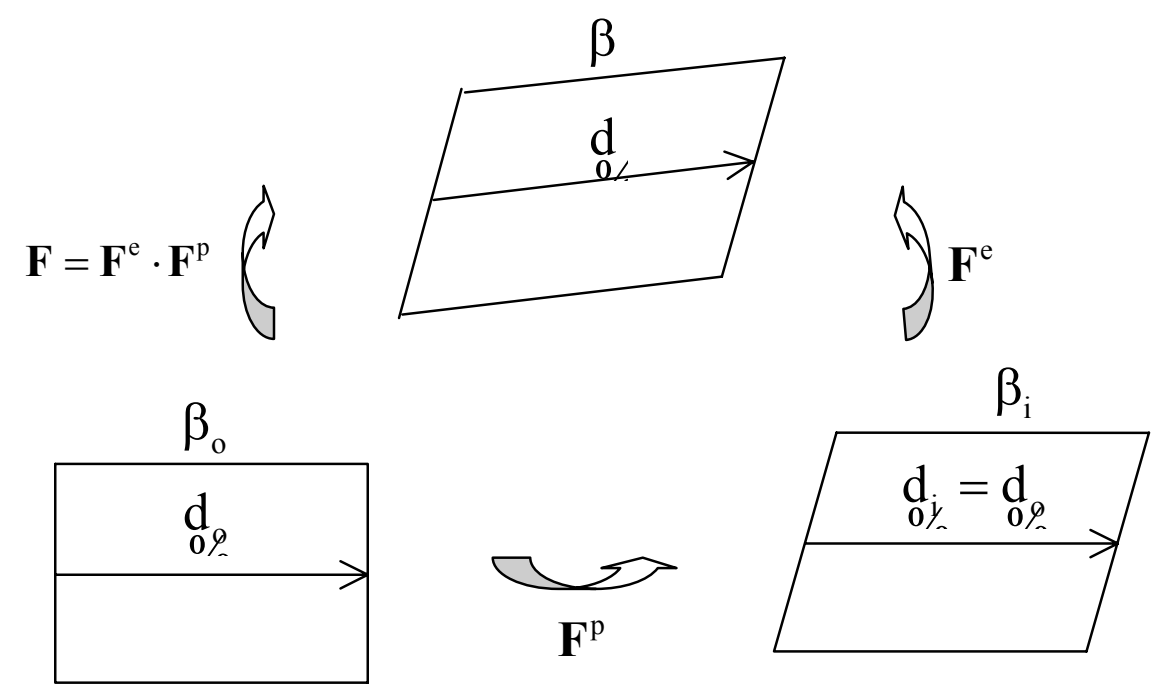

Figure 3 Multiplicative decomposition of the displacement gradient. 


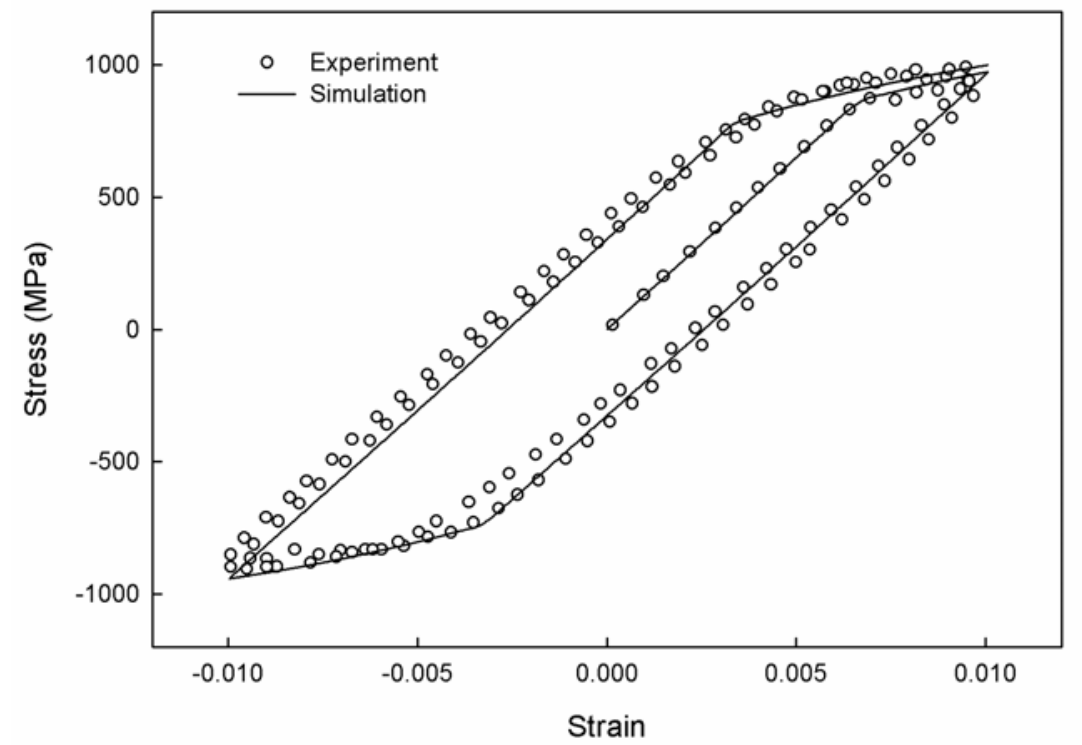

Figure 4 Stress-strain response: experimental data and correlated simulations at $427^{\circ} \mathrm{C}$ (longitudinal, $\mathrm{CC}, 1^{\text {st }}$ cycle). 


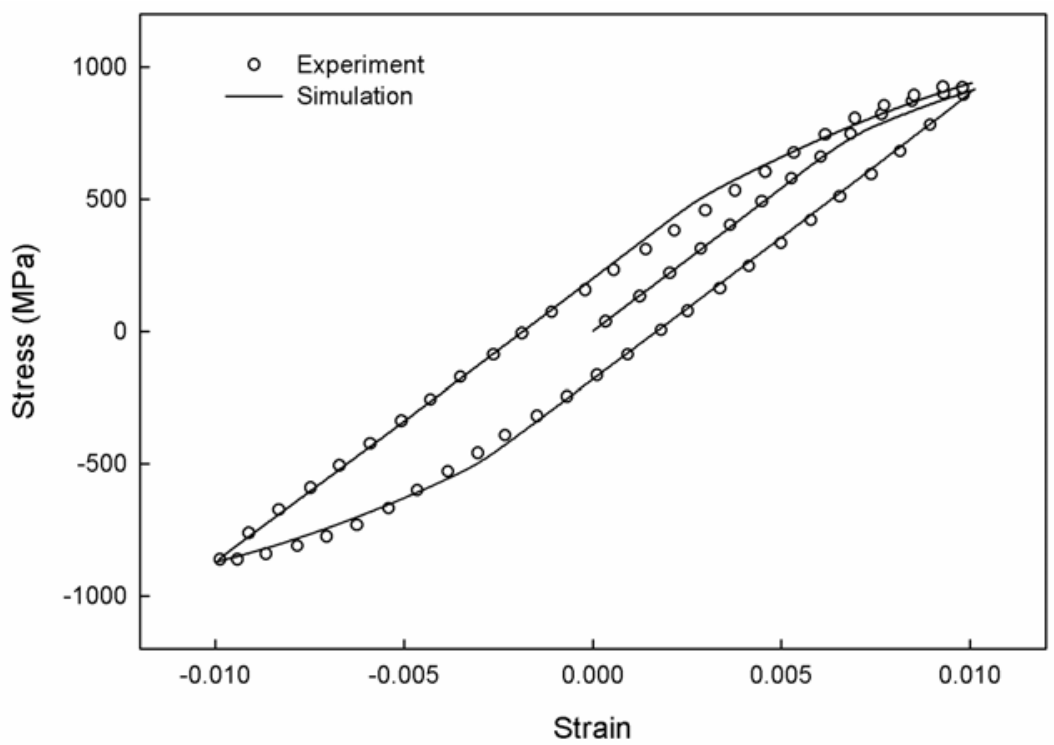

Figure 5 Stress-strain response: experimental data and correlated simulations at $760^{\circ} \mathrm{C}$ (longitudinal, $\mathrm{CC}, 1^{\text {st }}$ cycle). 


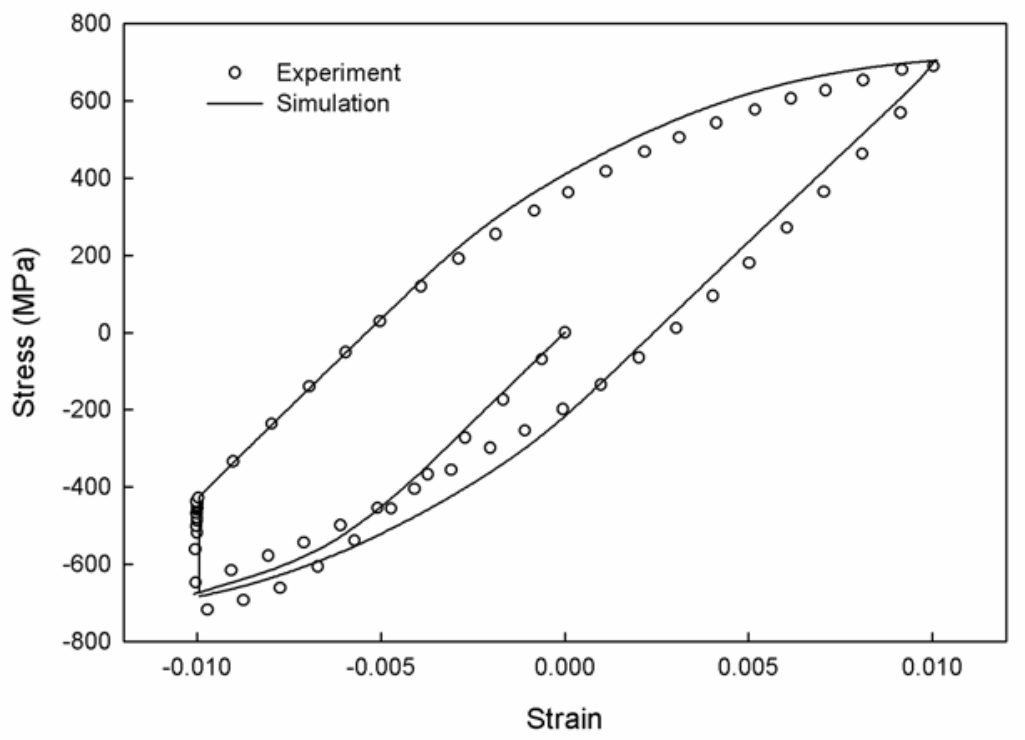

Figure 6 Stress-strain response: experimental data and correlated simulations at $871{ }^{\circ} \mathrm{C}$ (longitudinal, $\mathrm{HC}, 1^{\text {st }}$ cycle). 


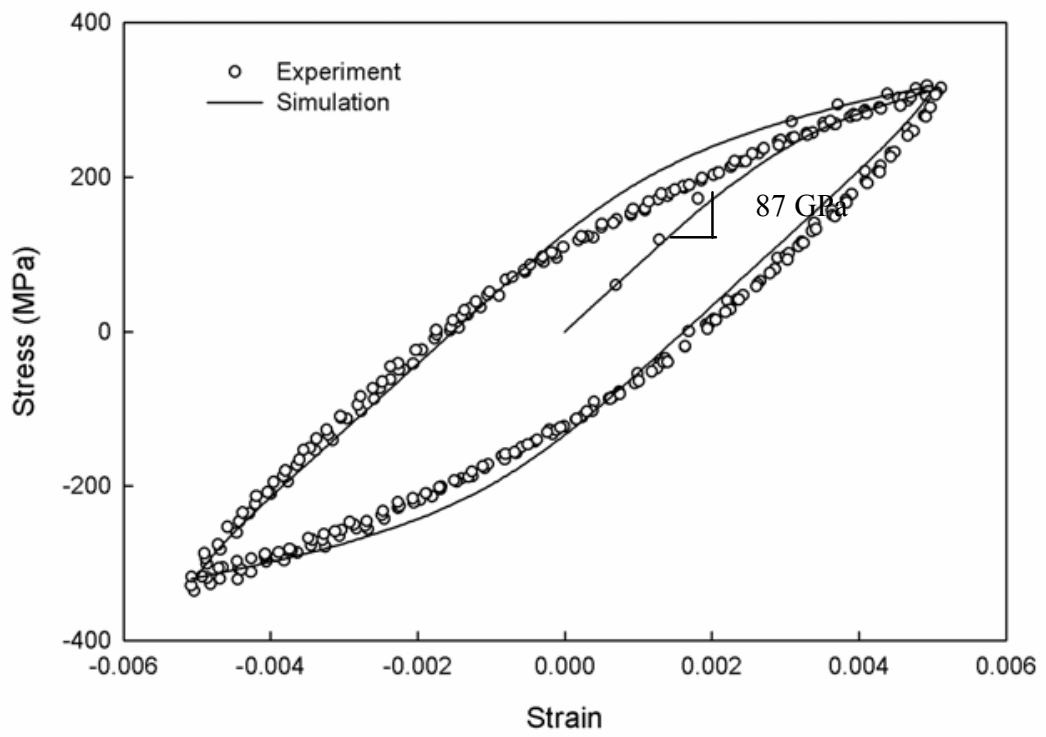

Figure 7 Stress-strain response: experimental data and correlated simulations at $982^{\circ} \mathrm{C}$ (longitudinal, $\mathrm{CC}, 1^{\text {st }}$ cycle). 


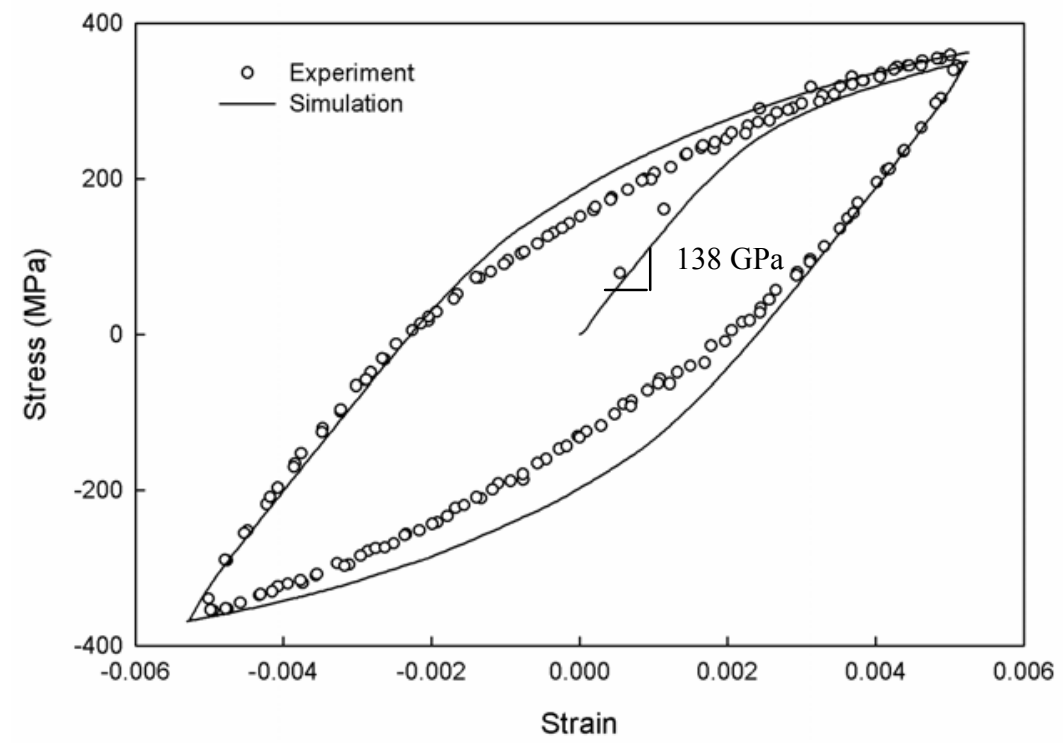

Figure 8 Stress-strain response: experimental data and correlated simulations at $982^{\circ} \mathrm{C}$ (Transverse, CC, $1^{\text {st }}$ cycle). 


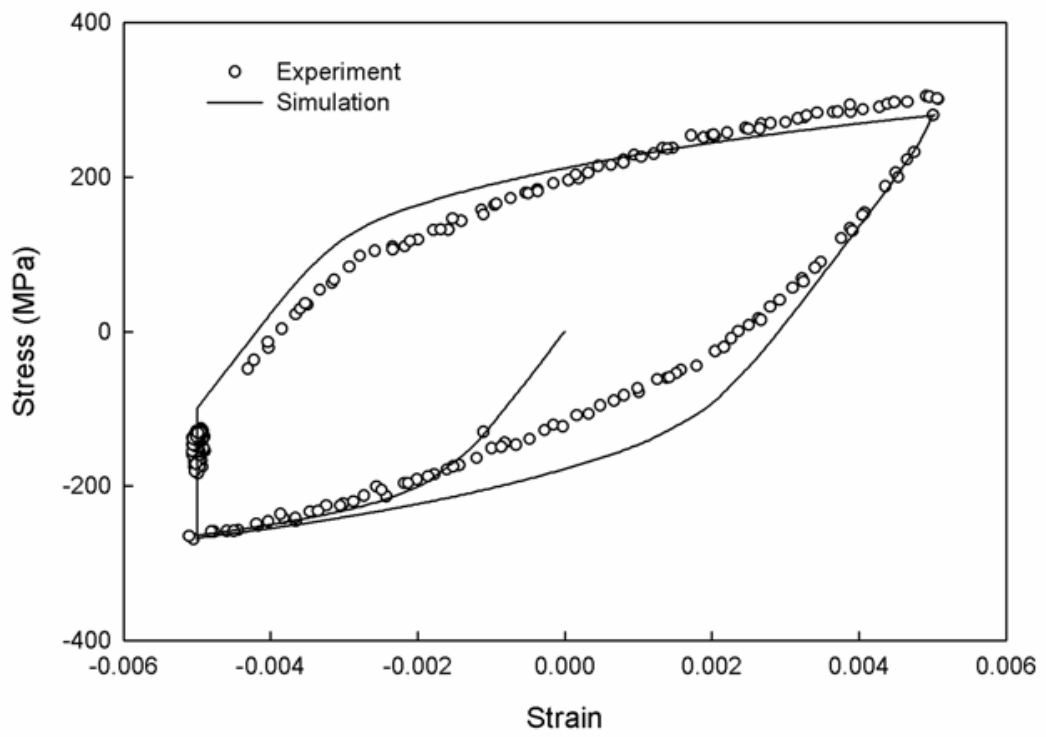

Figure 9 Stress-strain response: experimental data and correlated simulations at $1038^{\circ} \mathrm{C}$ (transverse, $\mathrm{CC}, 1^{\text {st }}$ cycle). 


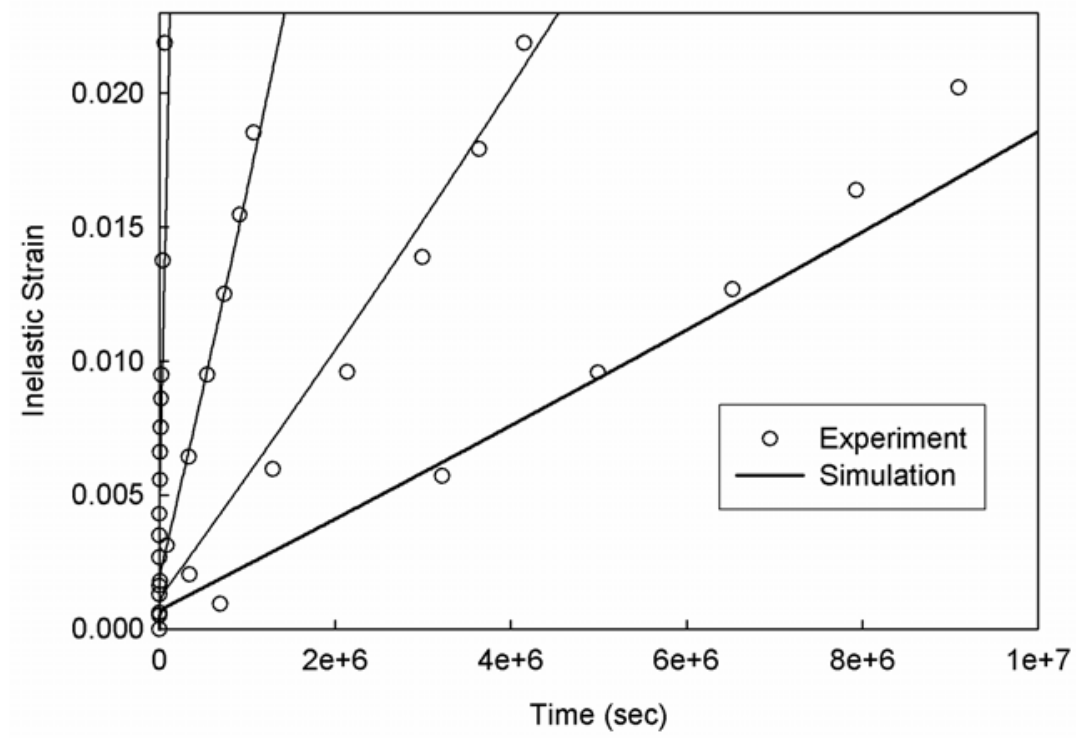

Figure 10 Primary and secondary creep responses: experimental data and correlated simulations at $871{ }^{\circ} \mathrm{C}$ (longitudinal). 


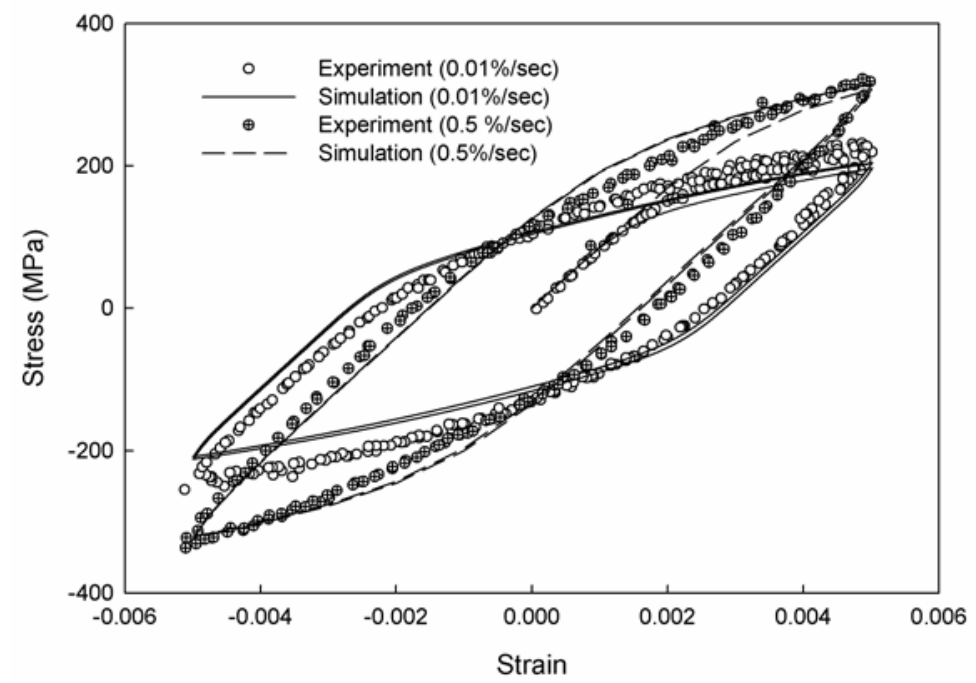

Figure 11 Stress-strain data: experimental data and predicted simulations at $982^{\circ} \mathrm{C}$ for strain rates at $0.01 \% / \mathrm{sec}$ and $0.5 \% / \mathrm{sec}$ (longitudinal, $\mathrm{CC}, 1^{\text {st }}$ cycle) 


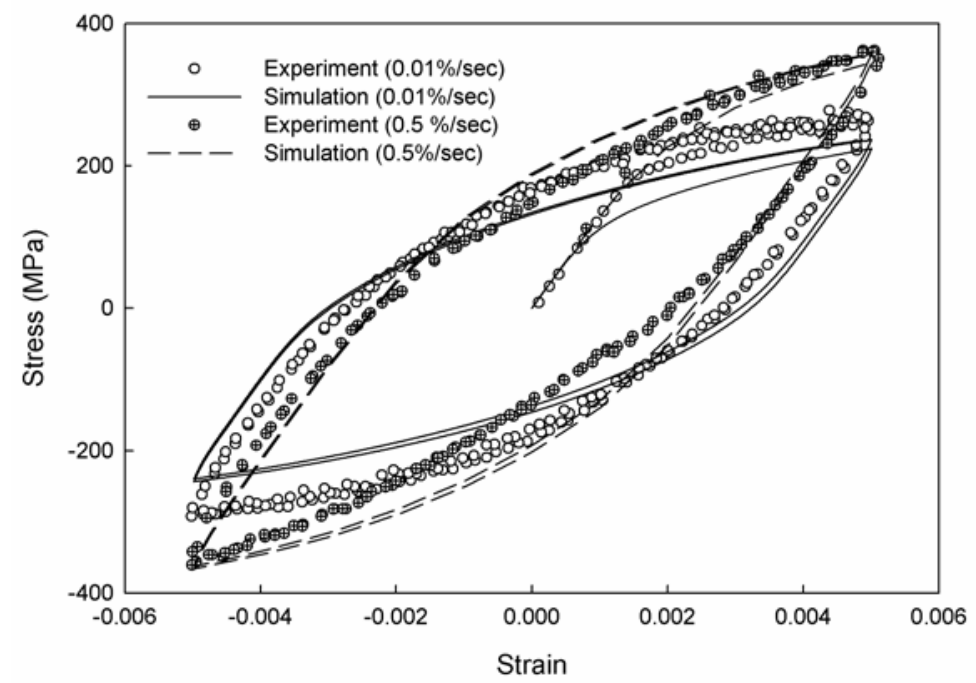

Figure 12 Stress-strain data: experimental data and predicted simulations at $982^{\circ} \mathrm{C}$ for strain rates at $0.01 \% / \mathrm{sec}$ and $0.5 \% / \mathrm{sec}$ (transverse, $\mathrm{CC}, 1^{\text {st }}$ cycle) 


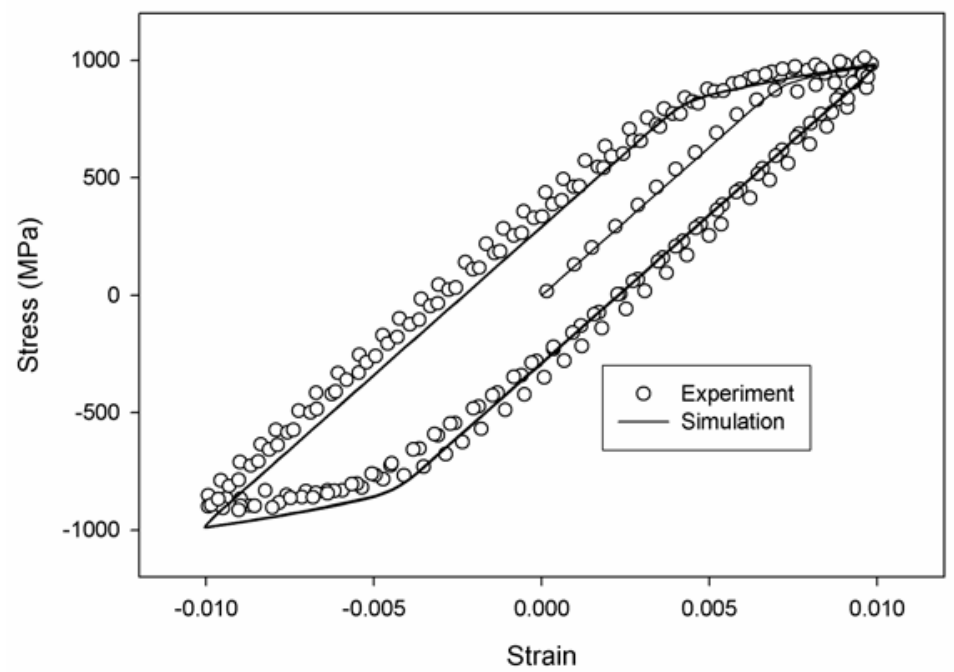

Figure 13 Stress strain response at $426^{\circ} \mathrm{C}\left(1^{\text {st }}\right.$ cycle $)$ in the longitudinal orientation. 


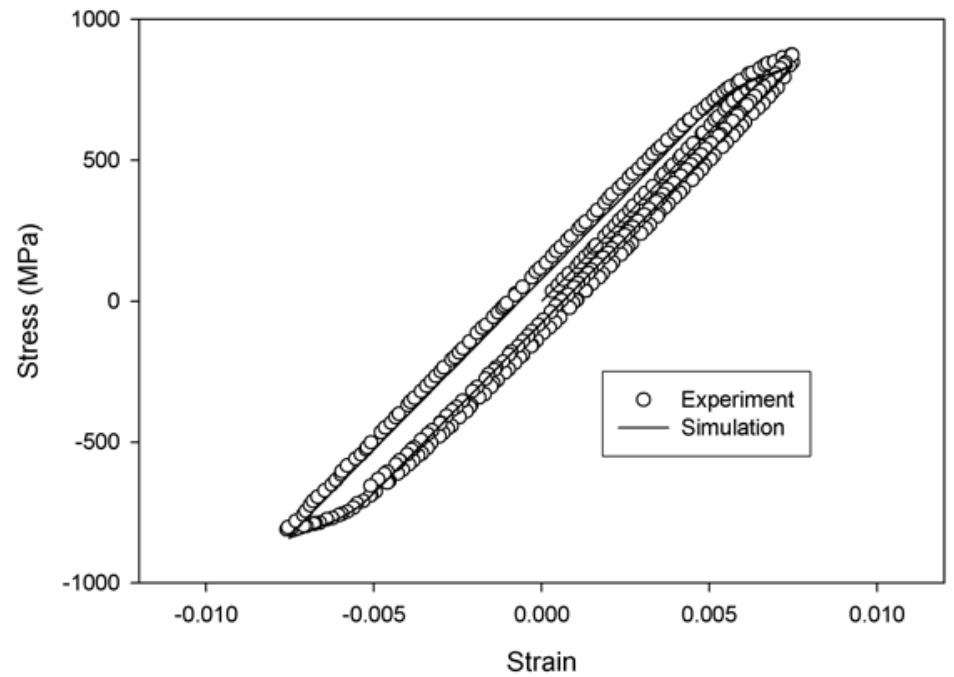

Figure 14 Stress strain response at $650{ }^{\circ} \mathrm{C}\left(1^{\text {st }}\right.$ cycle $)$ in the longitudinal orientation. 


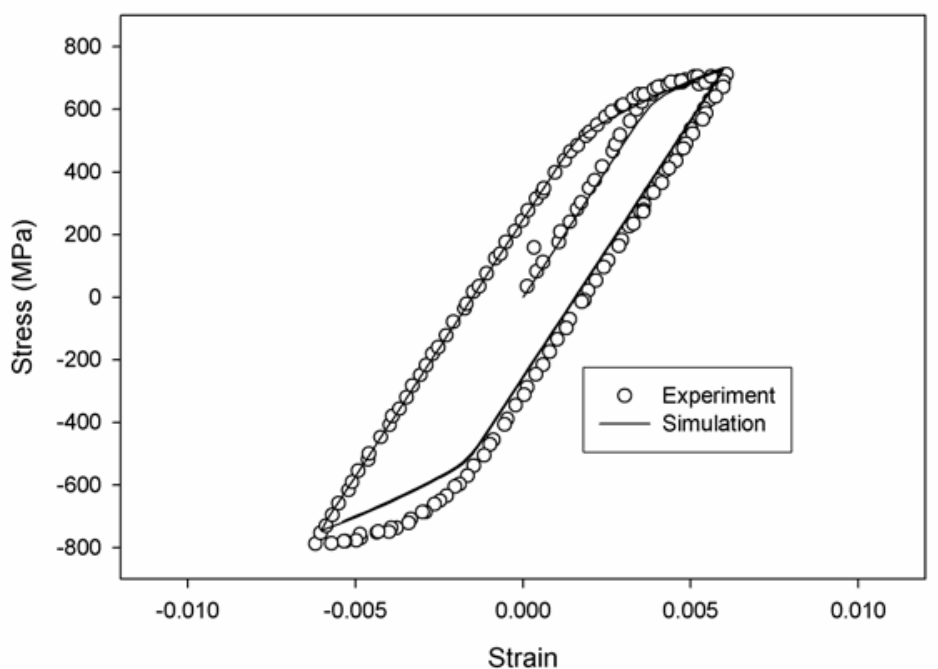

Figure 15 Stress strain response at $650{ }^{\circ} \mathrm{C}\left(1^{\text {st }}\right.$ cycle $)$ in the transverse orientation. 


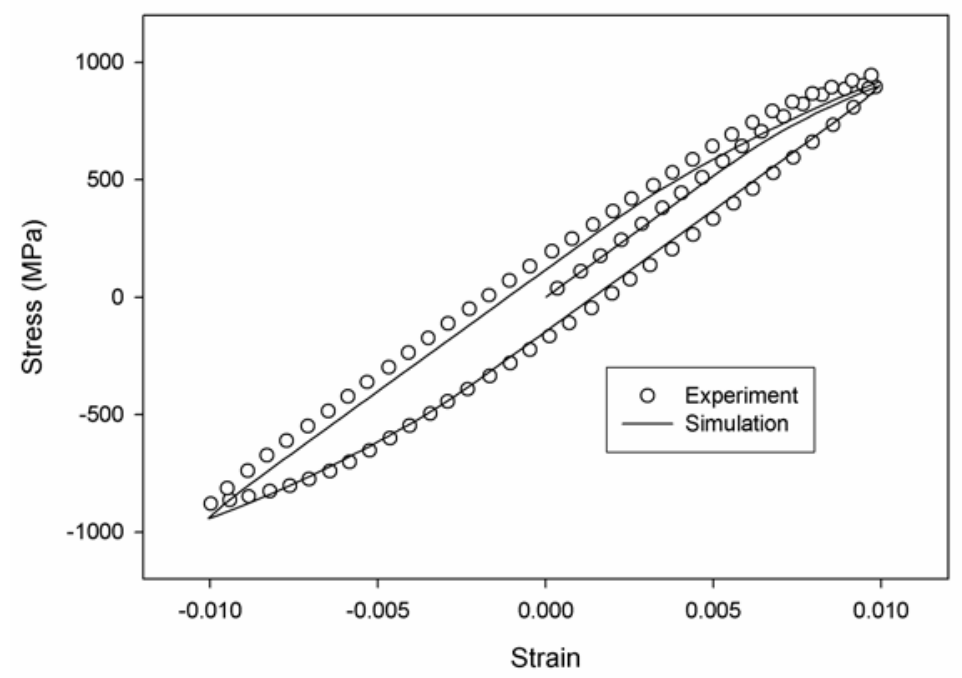

Figure 16 Stress strain response at $760{ }^{\circ} \mathrm{C}\left(1^{\text {st }}\right.$ cycle $)$ in the longitudinal orientation. 


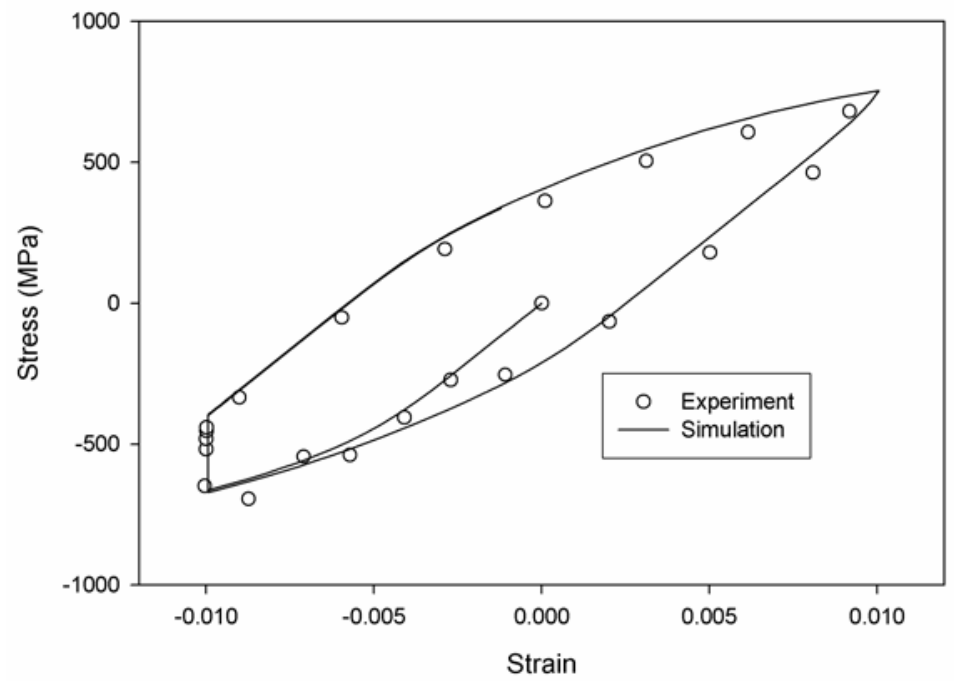

Figure 17 Stress strain response at $871^{\circ} \mathrm{C}\left(1^{\text {st }}\right.$ cycle $)$ in the longitudinal orientation with a $120 \mathrm{sec}$ hold time at the peak strain in compression. 


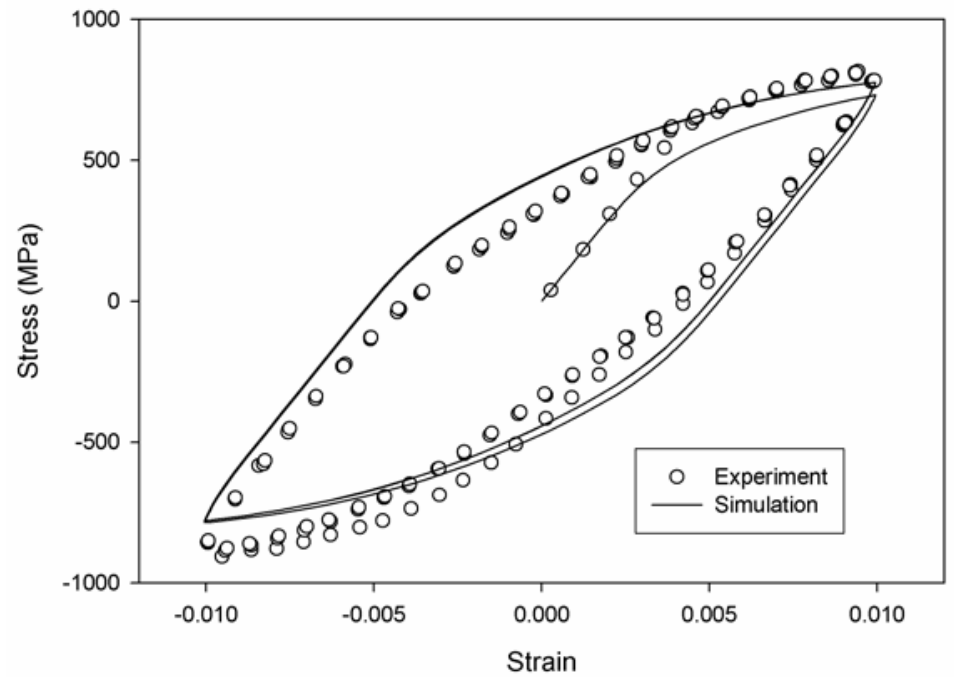

Figure 18 Stress strain response at $871{ }^{\circ} \mathrm{C}\left(1^{\text {st }}\right.$ cycle $)$ in the transverse orientation. 


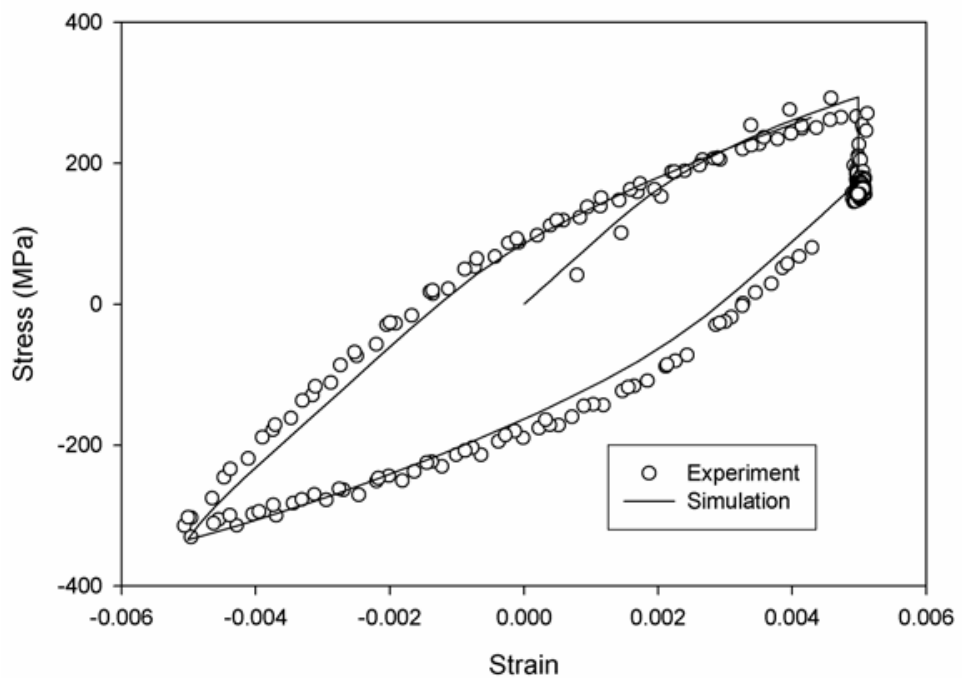

Figure 19 Stress strain response at $982{ }^{\circ} \mathrm{C}\left(1^{\text {st }}\right.$ cycle $)$ in the longitudinal orientation with a $120 \mathrm{sec}$ hold time at the peak strain in tension. 


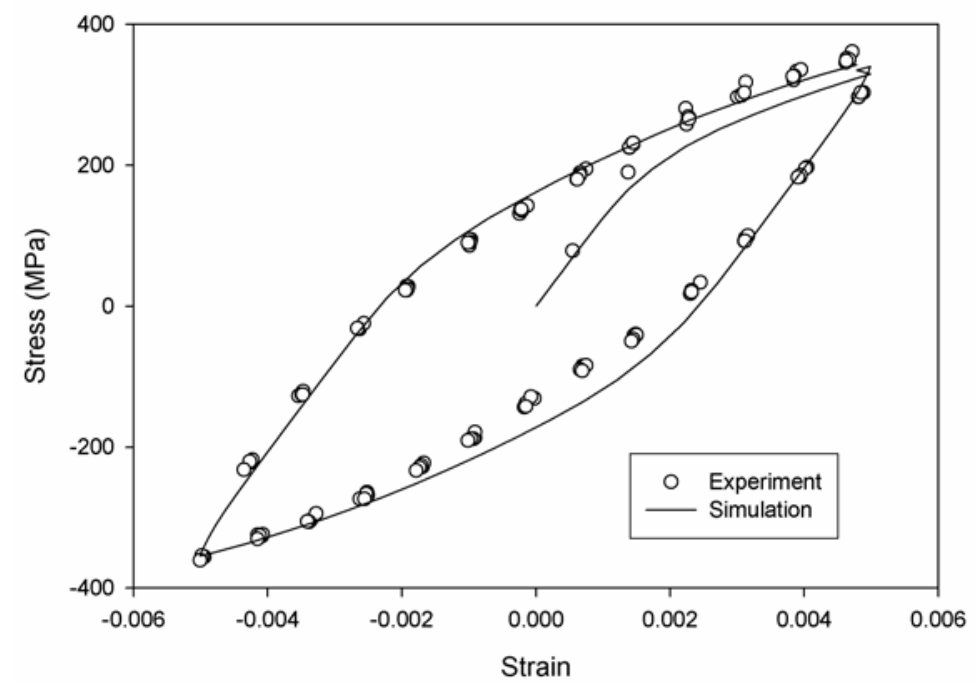

Figure 20 Stress strain response at $982{ }^{\circ} \mathrm{C}\left(1^{\text {st }}\right.$ cycle $)$ in the transverse orientation. 


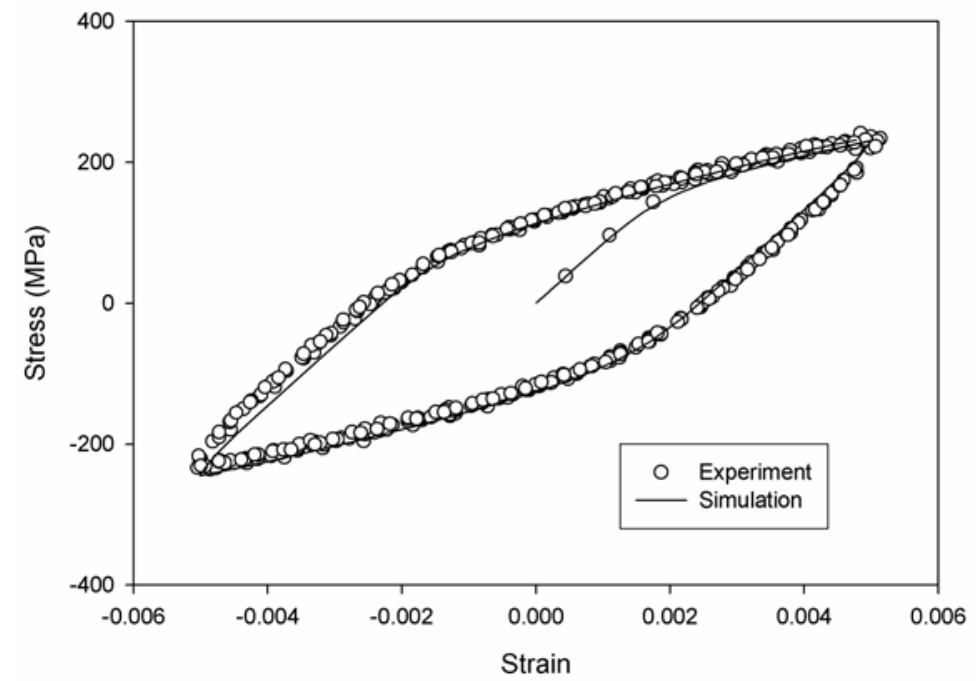

Figure 21 Stress strain response at $1038^{\circ} \mathrm{C}\left(1^{\text {st }}\right.$ cycle $)$ in the longitudinal orientation. 


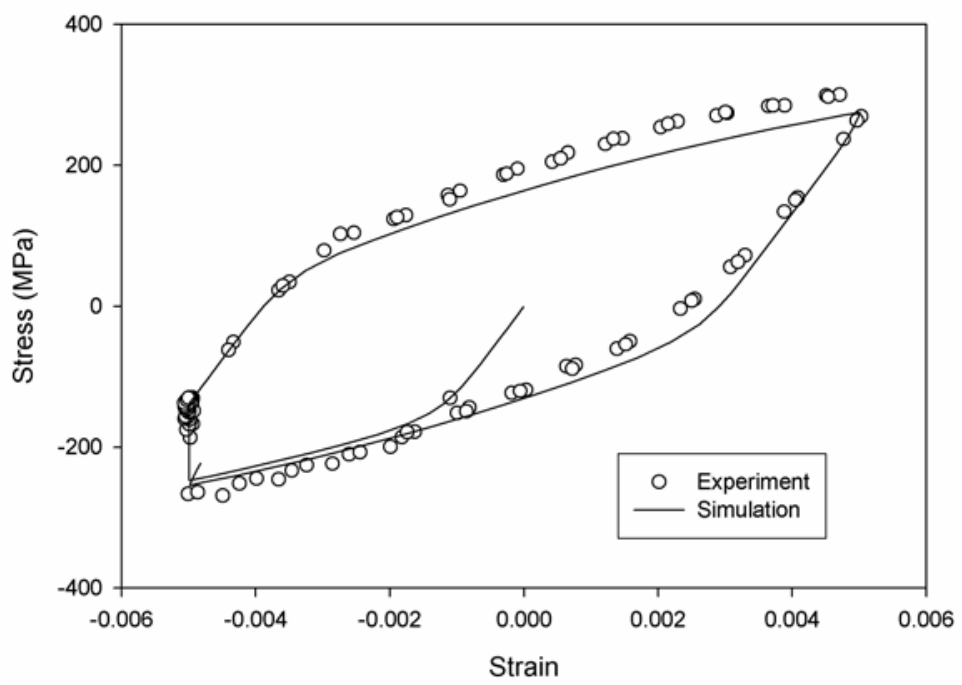

Figure 22 Stress strain response at $1038^{\circ} \mathrm{C}\left(1^{\text {st }}\right.$ cycle $)$ in the transverse orientation with a $120 \mathrm{sec}$ hold time at the peak strain in compression. 


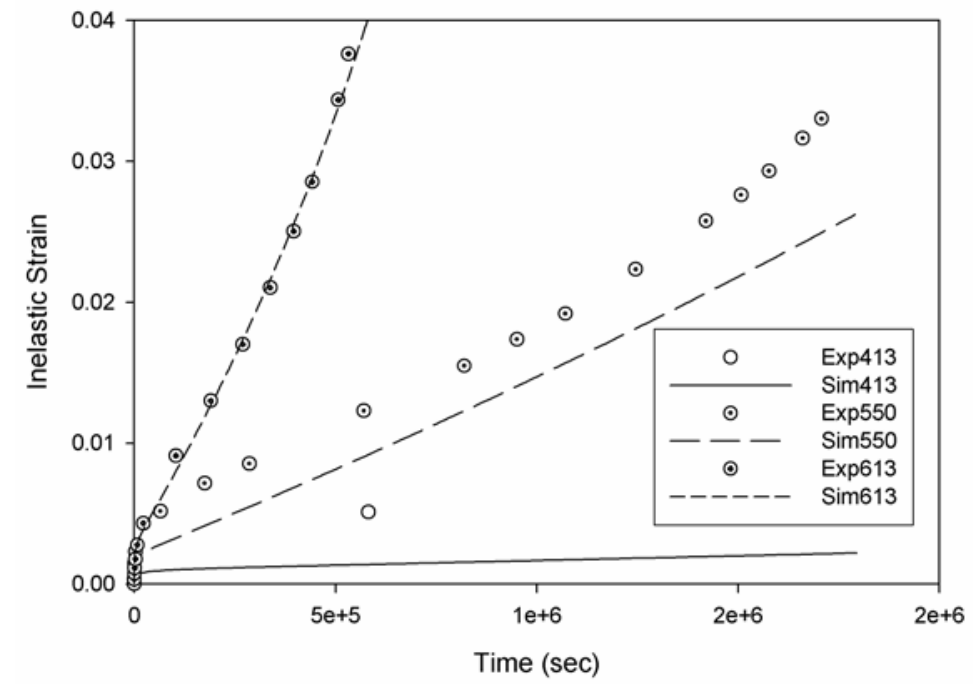

Figure 23 Creep response at $760{ }^{\circ} \mathrm{C}$ in the longitudinal orientation with stress held constant at 413, 550 and $613 \mathrm{MPa}$. 


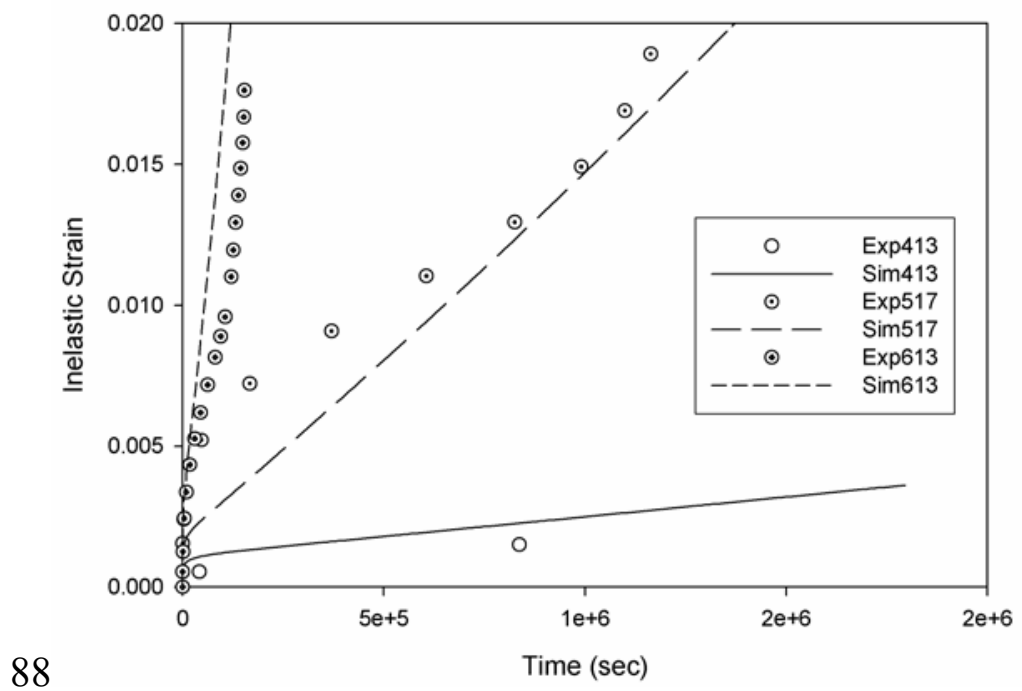

Figure 24 Creep response at $760{ }^{\circ} \mathrm{C}$ in the transverse orientation with stress held constant at 413, 517 and $613 \mathrm{MPa}$. 


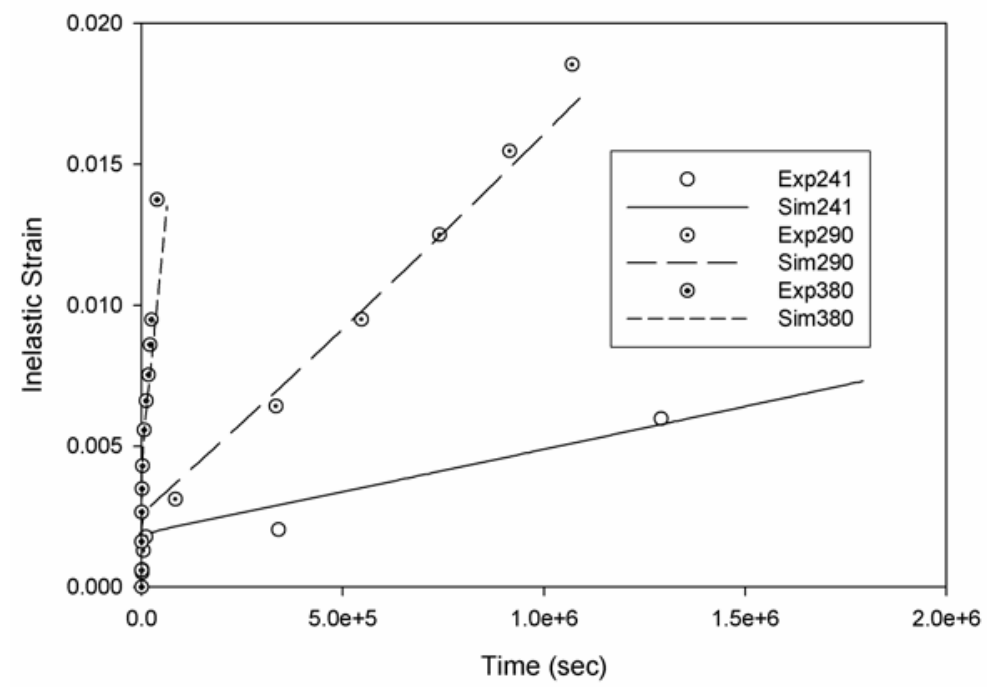

Figure 25 Creep response at $871{ }^{\circ} \mathrm{C}$ in the longitudinal orientation with stress held constant at 241, 290 and $380 \mathrm{MPa}$. 


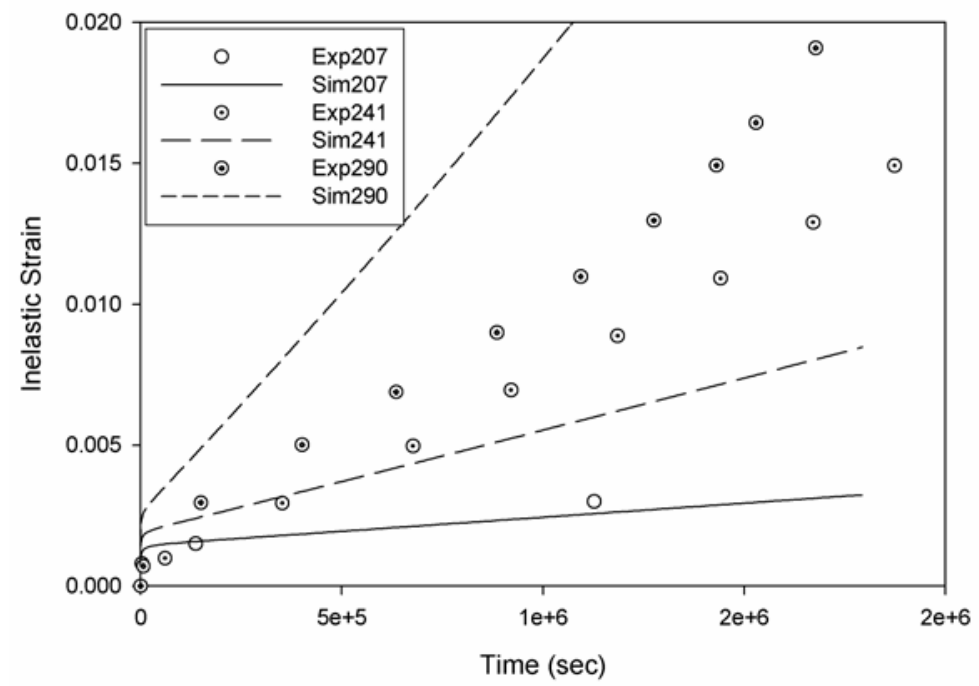

Figure 26 Creep response at $871{ }^{\circ} \mathrm{C}$ in the transverse orientation with stress held constant at 207, 241 and $290 \mathrm{MPa}$. 


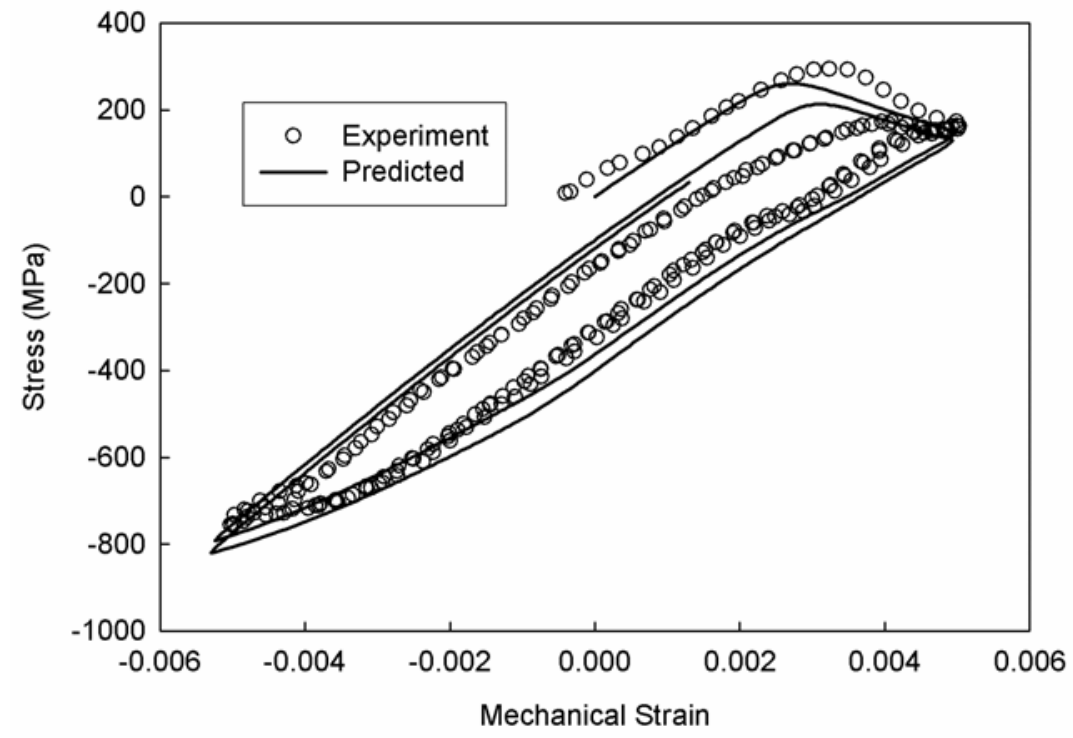

Figure 27 Stress-strain response: comparison of experimental data with model predictions for in-phase (IP) $\mathrm{TMF} 538{ }^{\circ} \mathrm{C}-1038{ }^{\circ} \mathrm{C}$ in the longitudinal orientation $\left(1^{\text {st }}\right.$ cycle). 


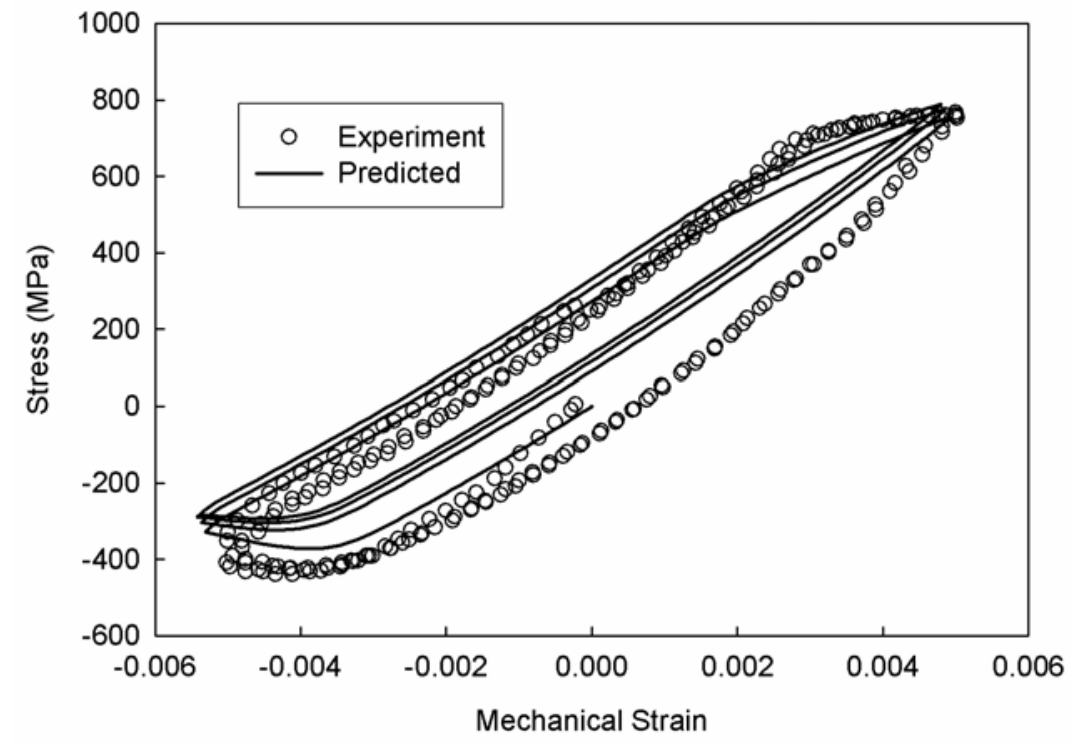

Figure 28 Stress-strain response: comparison of experimental data with model predictions for out-of-phase (OP) TMF $538^{\circ} \mathrm{C}-927^{\circ} \mathrm{C}$ in the longitudinal orientation. 


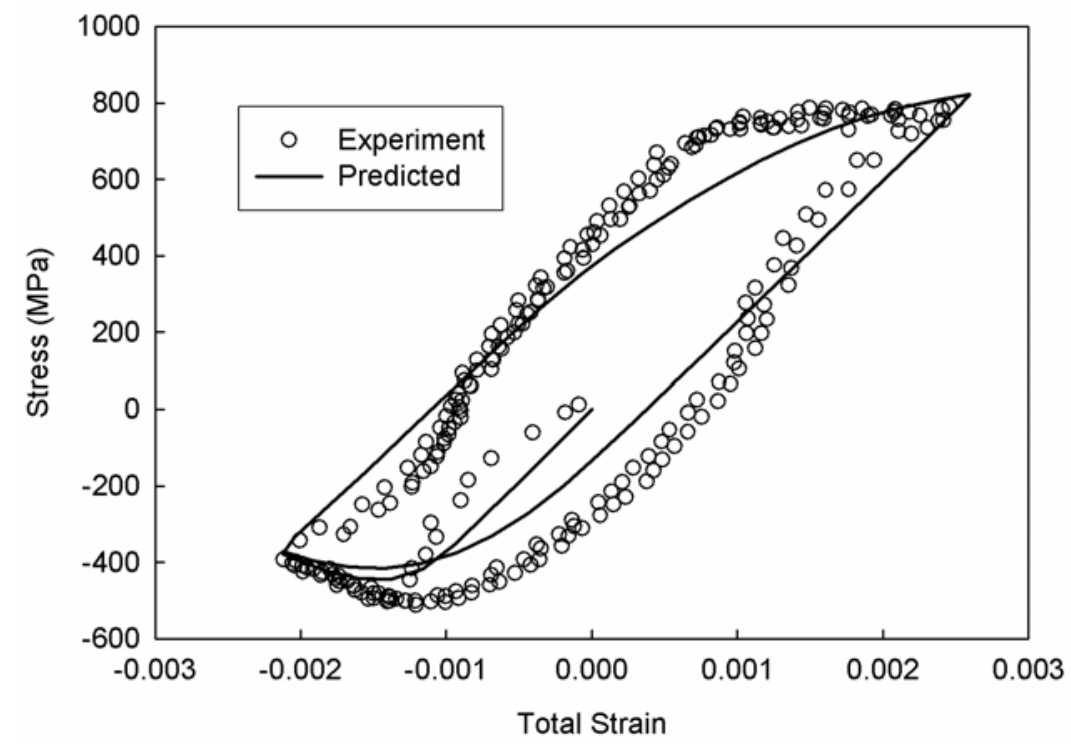

Figure 29 Stress-strain response: comparison of experimental data with model predictions for out-of-phase (OP) TMF $538^{\circ} \mathrm{C}-927{ }^{\circ} \mathrm{C}$ in the transverse orientation. 


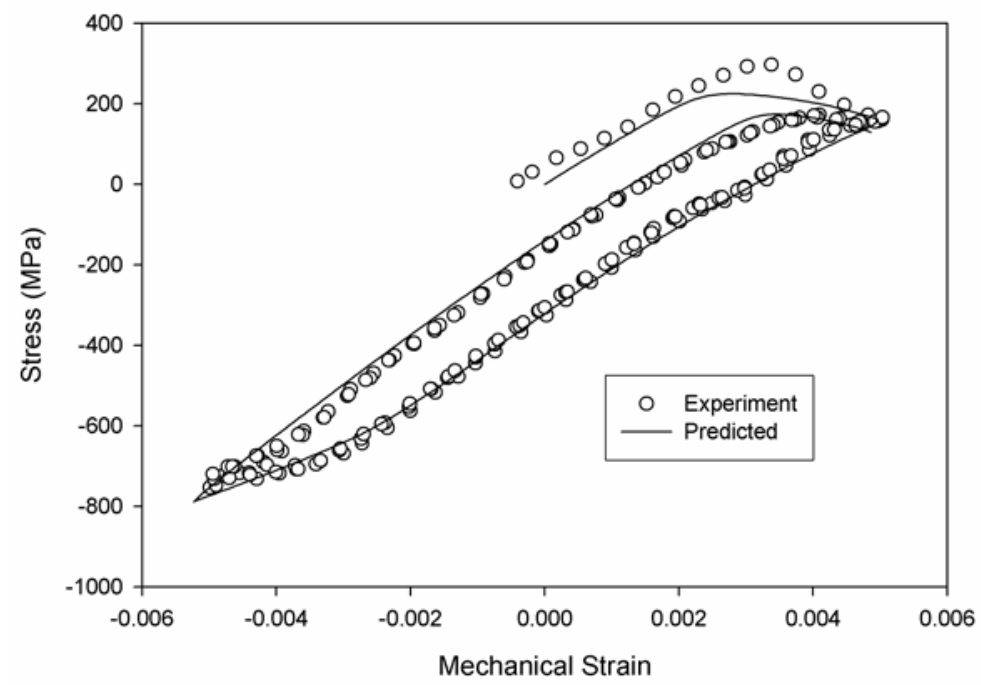

Figure 30 Stress-strain response for in-phase (IP) TMF $538{ }^{\circ} \mathrm{C}-1038{ }^{\circ} \mathrm{C}$ in the longitudinal orientation $\left(1^{\text {st }}\right.$ cycle $)$. 


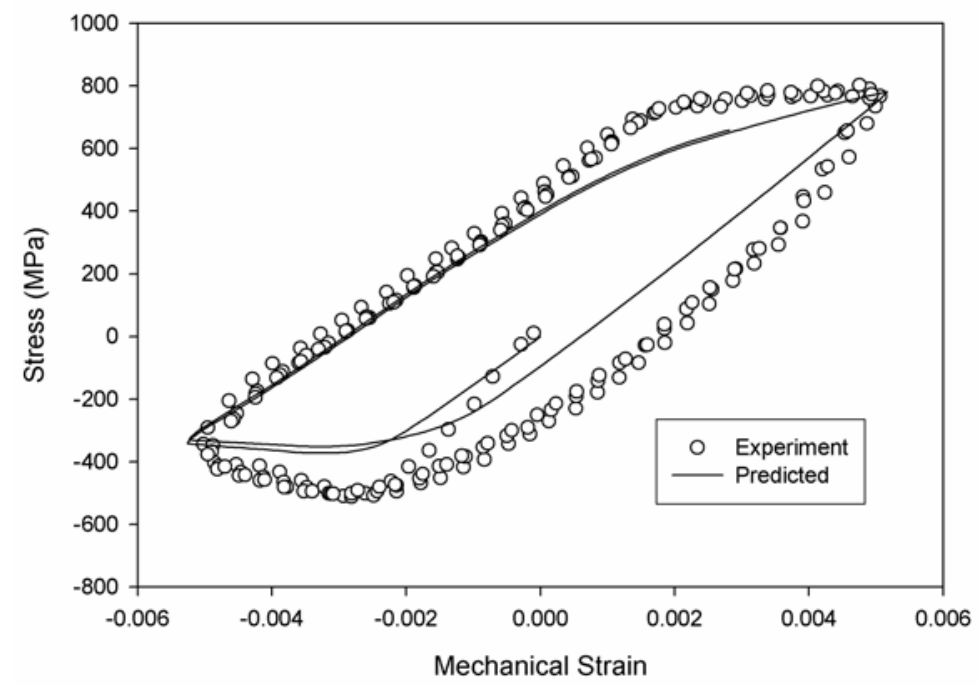

Figure 31 Stress-strain response for out-of-phase (OP) TMF $538{ }^{\circ} \mathrm{C}-927{ }^{\circ} \mathrm{C}$ in the transverse orientation $\left(1^{\text {st }}\right.$ cycle $)$. 


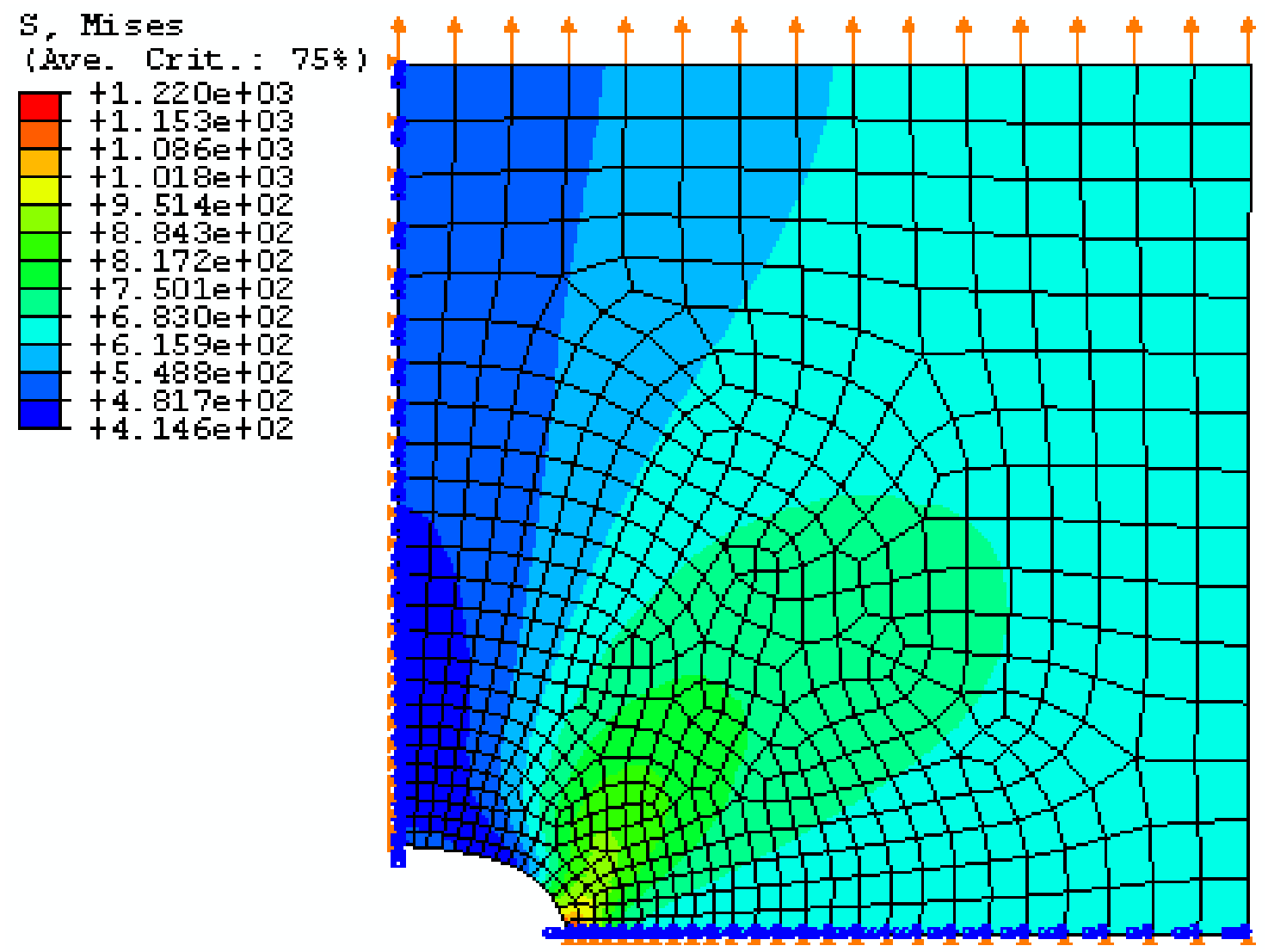

Figure 32 Mesh to study the analysis time for the crystal plasticity and transversely isotropic model. 

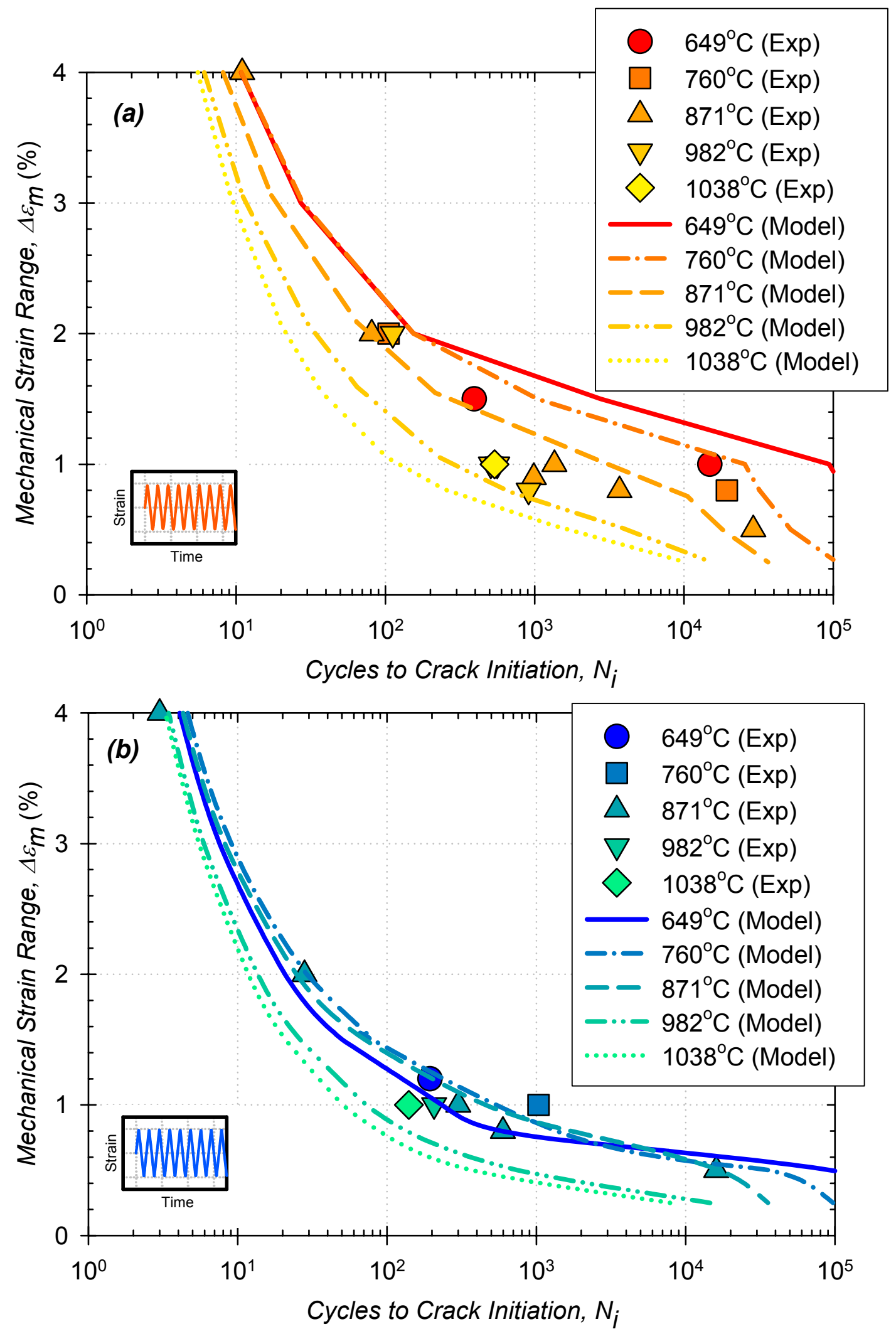

Figure 33: Predictions of total life based of (a) $L$ and (b) T-oriented $D S$ GTD-111 under $L C F$ conditions. For each case $R_{\varepsilon}=-1$, and \& $\&=0.5 \% / \mathrm{s}$. 

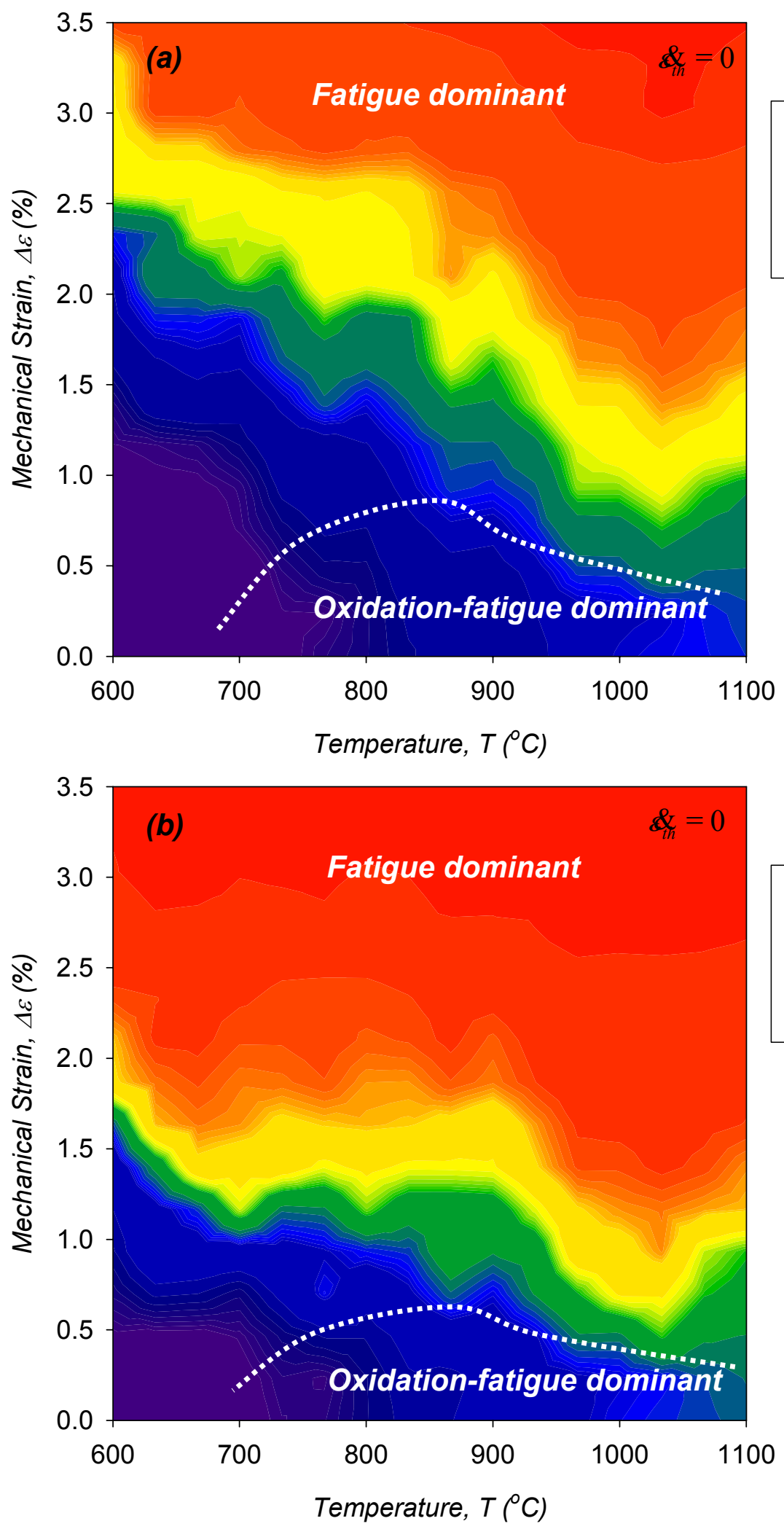

Figure 34: Predictions of total life based of (a) $L$ and (b) T-oriented DS GTD-111 under continuously cycling conditions. For each case $R_{\varepsilon}=-1$, \& $=0$, and \& $=0.5 \% / \mathrm{s}$. 

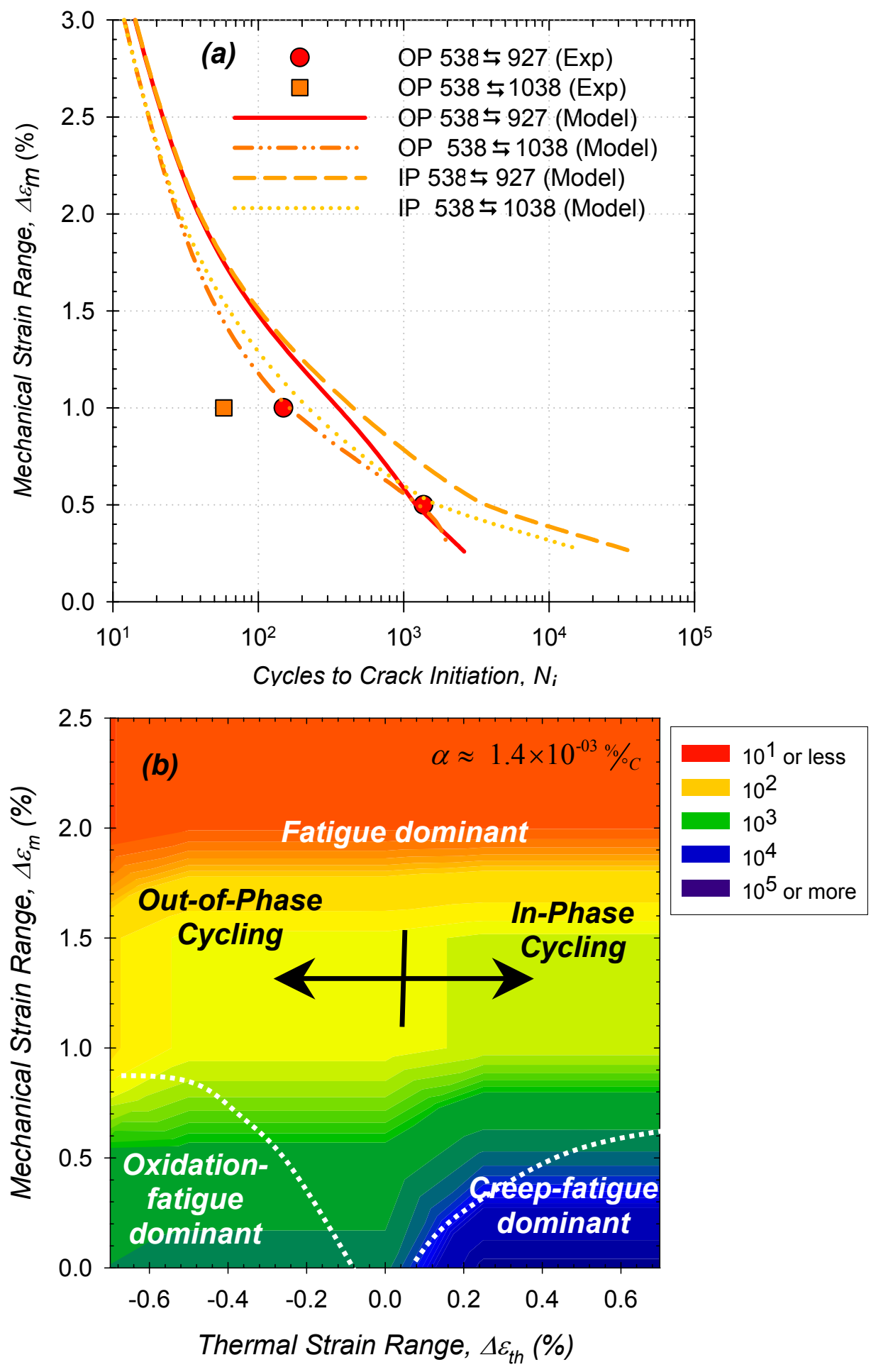

Figure 35: Predictions of total life based of L-oriented DS GTD-111 (a) under LCF conditions $(R \varepsilon=-1, \quad \&=0.5 \% / s)$ and (b) under TMF conditions $\left(R_{\varepsilon}=-1, T_{\min }=538^{\circ} \mathrm{C}, t_{t c}=180 \mathrm{~s}\right)$. 